DOE/ET/51013--T244

DOE/ET/51013--T244

DE92 002787

PFC/RR-91-11

Study of ICRF Wave Propagation and Plasma

Coupling Efficiency in a Linear Magnetic

Mirror Device

S. Peng

July 1991

Plasma Fusion Center

Massachusetts Institute of Technology

Cambridge, MA 02139 USA

This work was supported by NASA under Contract\# NAS-9-18372. Reproduction, translation, publication, use, and disposal, in whole or in part, by or for the US Government is permitted. 
The Goverment reserves for itself and others acting on its behalf a royalty free, nonexclusive, irrevocable, world-wide license for governmental purposes to publish, distribute, translate, duplicate, exhibit, and perform any such data copyrighted by the contractor.

\section{DISCLAIMER}

This report was prepared as an account of work sponsored by an agency of the United States Government. Neither the United States Government nor any agency thereof, nor any of their employees, makes any warranty, express or implied, or assumes any legal liability or responsibility for the accuracy, completeness, or usefulness of any information, apparatus, product, or process disclosed, or represents that its use would not infringe privately owned rights. Reference herein to any specific commercial product, process, or service by trade name, trademark, manufacturer, or otherwise does not necessarily constitute or imply its endorsement, recommendation, or favoring by the United States Government or any agency thereof. The views and opinions of authors expressed herein do not necessarily state or reflect those of the United States Government or any agency thereof. 


\title{
Study of
}

\section{ICRF Wave Propagation and Plasma Coupling}

\section{Efficiency}

\section{In a Linear Magnetic Mirror Device}

\author{
by
}

Scott Y. Peng

B.S.N.E., Texas A\&M University (1983)

Submitted to the Department of Nuclear Engineering in partial fulfillment of the requirements for the degree of

Doctor of Philosophy

at the

\section{MASSACHUSETTS INSTITUTE OF TECHNOLOGY}

July 1991

(C) Massachusetts Institute of Technology 1991

Signature of Author

Department of Nuclear Engineering July 26, 1991

Certified by

Jeffrey Freidberg

Professor of Nuclear Engineering

Thesis Supervisor

Certified by

Ted F. Yang Research Scientist, MIT Plasma Fusion Center Thesis Supervisor

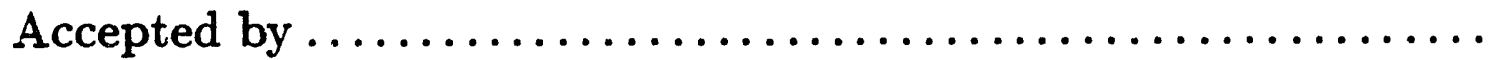

Allan F. Henry Chairman, Departmental Committee on Graduate Students 


\title{
Study of
}

\section{ICRF Wave Propagation and Plasma Coupling Efficiency}

\section{In a Linear Magnetic Mirror Device}

\author{
by \\ Scott Y. Peng \\ Submitted to the Department of Nuclear Engineering \\ on July 26, 1991, in partial fulfillment of the \\ requirements for the degree of \\ Doctor of Philosophy
}

\begin{abstract}
Ion Cyclotron Range of Frequency (ICRF) wave propagation in an inhomogeneous axial magnetic field in a cylindrical plasma-vacuum system has historically been inadequately modelled. Previous works either sacrifice the cylindrical geometry in favor of a simpler slab geometry [1], concentrate on the resonance region (mode conversion)[2], use a single mode to represent the entire field structure[3], or examine only radial propagation[4]. This thesis performs both analytical and computational studies to model the ICRF wave-plasma coupling and propagation problem. Experimental analysis is also conducted to compare experimental results with theoretical predictions.

Both theoretical as well as experimental analysis are undertaken as part of the thesis. The theoretical studies simulate the propagation of ICRF waves in an axially inhomogeneous magnetic field and in cylindrical geometry. Two theoretical analysis are undertaken - an analytical study and a computational study. The analytical study treats the inhomogeneous magnetic field by transforming the $(r, z)$ coordinate into another coordinate system $(\rho, \xi)$ that allows the solution of the fields with much simpler boundaries (Plasma-vacuum boundary at $\rho=1$, conducting wall at $\rho=\kappa)$. The plasma fields are then Fourier transformed into two coupled convolution-integral equations which are then differenced and solved for both the perpendicular mode number $\alpha$ as well as the complete EM fields.

The computational study involves a multiple eigenmode computational analysis of the fields that exist within the plasma-vacuum system. The inhomogeneous axial field is treated by dividing the geometry into a series of transverse axial slices and using a constant dielectric tensor in each individual slice. The slices are then connected by longitudinal boundary conditions.
\end{abstract}

The experimental accomplishment of this thesis include the design, construc- 
tion, and operation of a linear magnetic mirror device, the PPEX machine. A full set of heating systems has been installed on the PPEX device - 400kW of ICRF power, $2 \mathrm{~kW}$ of ECH startup power. Diagnostics to monitor different plasma properties have been either designed and constructed from scratch or modified from existing designs. Experiments to examine ICRF wave propagation were conducted.

Thesis Supervisor: Jeffrey Freidberg

Title: Professor of Nuclear Engineering

Thesis Supervisor: Ted F. Yang

Title: Research Scientist, MIT Plasma Fusion Center 


\section{Acknowledgements}

First, I would like to thank Dr. Ted Yang, the head of the Propulsion Group at the MIT Plasma Fusion Center for taking me on as a student and for giving me the freedom to develop and learn at my own pace. I am deeply indebted to Professor Jeff Freidberg for all his help and for his guidance in working with me as my co-supervisor to develop insight into a uniquely new ICRF wave propagation theory. Haryey Lander must be thanked for what his assistance in changing me from a neophyte experimentalist into someone who sort of knows what he's doing. I would also like to thank the co-head of our group, Astronaut Dr. Franklin Chang-Diaz for his assistance and support over the course of this thesis. Dr. Rich Myer is instrumental in giving me a coherent picture of ICRF heating and propagation. The BEACH code was Rich's original idea and I merely filled in the colors. Professor Dieter Sigmar and Professor Kim Molvig must also be thanked for their comments and pointers and also for being members of my thesis committee.

Many people contributed to the construction and running of the PPEX miror machine. Frank Silva and his crew (Caes, Bill, and Ralph) must be thanked for their skillful assistance and guidance and all sorts of help. Bob Childs for educating me on the importance of having a CLEAN vacuum system. David Griffin for teaching me the basics of ICRF amplifier technology and for his politeness and wisdom. Dr. Steven Golovato for his assistance in customizing and tuning our ICRF matchbox. Lorenzo Pires for showing me the ropes of the archaic Continental RF amplifier system and for helping us debug the system when it was down. John Urbahn for his contributions to the creation of the PPEX machine by his design and construction of the PPEX coil set and many other vital subsystems. Tom Hsu for bringing me up to speed on plasma diagnostics experimental techniques and methods. Dr. Tom Moran for assisting me in setting up our spectroscopic system, Dr. Dan Goodman for discussions of mirror ICRF heating, and Professor Ian Hutchinson for making insightful suggestions and comments that allowed me to gain further insight into the experimental aspect of the thesis.

Many other people rnust also be thanked for contributing to the theoretical portion of this thesis. To James Wei for helping me greatly in my understanding of Green's function, Elliptic Integral, LU decompositions and many other problems. Dr. John Machuzak for his helpful suggestions, his IDL FFT code and keen insight into mirror physics.

To the other wonderful friends I made at the MIT Plasma Fusion Center Dave, Yoon-Hee, Bobby and Sandy Rhee who overwhelmed me with their hospitality. To Professor Bob Witt and our trips to the GB. To Dr. Mark Foord, who showed me by example to take it easy. To Dr. Ken Pendergast for our many CQ quests and movie trips. To Dr. Manos Chaniotakis, who was my comrade in suffering and who helped me greatly in my times of pain. To Sam Chu for being a good sport. To Mathias Koch for always being encouraging. To Pei-Wen Wang for his infinite patience and good advice. And to Dr. Kevin Wenzel, who 
has influenced me immeasurably, and who, as my first officemate at MIT, helped me to open up and see myself and others better.

I would also like to thank my friends at the Graduate Student Council who made my tenure there a most enjoyable one. To Ying-Ying, you were the best (and only) administrative assistant I ever worked with. To the members of my executive committee - Drs. John Scott-Thomas, Marik Hansen, Mike Warwick, Mike Grossberg, and Jong-On Hahm. You guys were the greatest bunch of folks I have ever worked with. And finally thanks to Bert Hootsmans, my wonderful treasurer and the sharpest dresser I know.

To my other friends at MIT and elsewhere whose shoulders I have often leaned upon to help me make it through difficult times - Vera Talanker, Dr. Yu-Hao Huang, Bonnie Kao, Daniel Lee, Dr. Chi-Chen Cheng, Cynthia Chuang, HyeSung Ha, Kim Russel, Mohammed Moghimi, Joseph Arias, Ricardo Yupari and many many others. Thank you.

To my very very good friend Dr. John Massidda who listened to me when I was down, who helped me see things clearer, and whose free spirit is always an inspiration to me. Thanks, Fred!

Many thanks to the wonderful people at First Boston who stayed in touch with me in times of trouble and especially to Marie, Paul, Nestor and Walid for keeping the faith with me for so long.

To my girlfriend Julia Hsu. In good times and bad, through trials and tribulations, she has always stood by me and helped me through it all. Mere words cannot thank her enough for her warm loving support.

Finally, I want to thank my family - my parents Gary and Shirley Peng, and brother Stony. For their constant support and patience in putting up with me in moods good and bad, for cheering me up when I was miserable, and for always being there when I needed them. I would never be where I am without their loving support and nurture. I would like to especially thank my mother Shirley for teaching me the meaning of life by living it herself with courage and conviction. Her loving wisdom has often led me out of the darkness of despair and is always a source of comfort and inspiration to me.

This thesis is dedicated to the spirit of graduate students everywhere - the dogged dedication, the constant drive and struggle to overcome those seemingly insurmountable obstacles that are part of graduate school, the will to excel, and the fountainhead of hope and youth that drives all new endeavers. To those grad students who come before and after me, this is to you.

"This is my morning, my day begins: rise up now, rise up, great noontide!"

- Friedrich Nietzsche (Thus Spake Zirathustra) 


\section{Contents}

1 INTRODUCTION 17

1.1 History of Fusion . . . . . . . . . . . . . . . . 17

1.2 Mirror Machine Principles . . . . . . . . . . . . . . 18

1.3 Thesis Motivation . . . . . . . . . . . . . . . 18

1.4 Organization of Thesis .................. 20

2 The PPEX Tandem Mirror Machine 21

2.1 Major Machine Systems . . . . . . . . . . . . . 25

2.1.1 Vacuum System .................. 25

2.1 .2 Magnets ........................... 25

2.1 .3 Gas Feed...................... 29

2.1 .4 Plasma Heating .................. 30

2.2 Plasma Heating Systems . . . . . . . . . . . . . . 31

$2.2 .1 \mathrm{ECRH} \ldots \ldots \ldots . \ldots \ldots \ldots \ldots$

$2.2 .2 \mathrm{ICRF} \ldots \ldots \ldots \ldots \ldots$

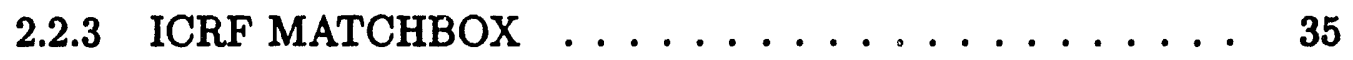

2.2 .4 ICRF Antenna . . . . . . . . . . . . 37

2.3 Diagnostics ........................ 42

2.3.1 Langmuir Probe . . . . . . . . . . . . . . . . 42

2.3.2 Interferometer . . . . . . . . . . . . . . 43

2.3.3 Spectrometer ..................... 45

2.3.4 $H_{\alpha}$ Detector .................... 48 
2.3.5 Diamagnetic Loop $\ldots \ldots \ldots \ldots \ldots$. . . . . . 48

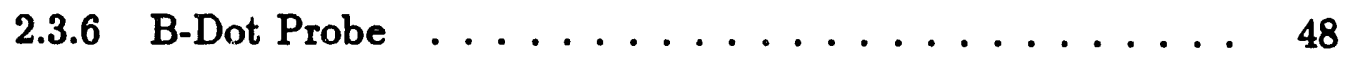

2.4 Data Acquisition . . . . . . . . . . . . . 52

2.5 PPEX OPERATIONS $\ldots \ldots \ldots \ldots \ldots \ldots \ldots \ldots$

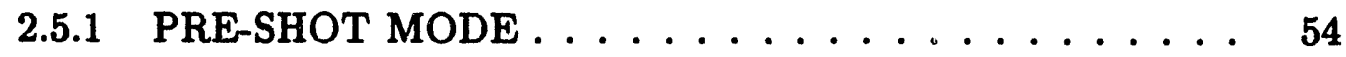

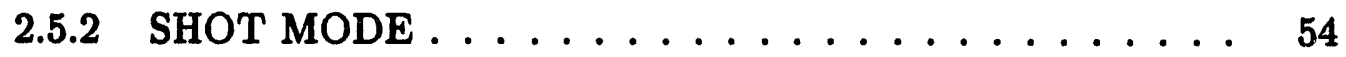

2.6 Systems Designed and Fabricated . . . . . . . . . . 56

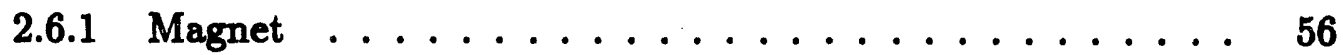

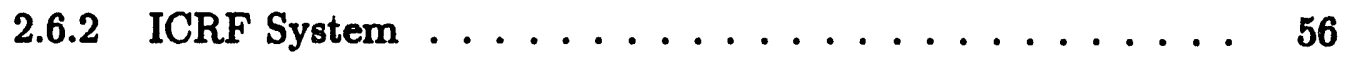

2.6.3 Diagnostics . . . . . . . . . . . . . 56

2.6 .4 Instrumentation $\ldots \ldots \ldots \ldots \ldots \ldots \ldots \ldots$

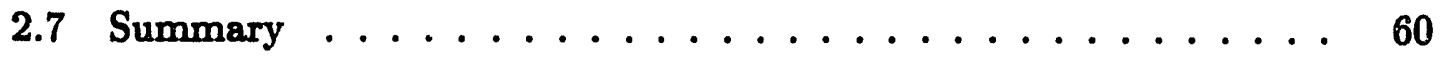

3 ICRF THEORY 61

3.1 Motivation for Study . . . . . . . . . . . . 63

3.2 ICRF Background Formulation $\ldots \ldots \ldots \ldots \ldots \ldots$

3.2.1 Standard Formulation . . . . . . . . . . . 65

3.2.2 Method of Solution . . . . . . . . . . . . 67

3.3 Assumptions and Geometry used in Axially Varying Cylindrical Analysis . . . . . . . . . . . . . . . 70

3.4 External Source Fields . . . . . . . . . . . . . . . . 72

3.5 Vacuum Fields $\ldots \ldots \ldots \ldots \ldots \ldots \ldots \ldots$

3.5.1 Change of Variable . . . . . . . . . . . . 79

3.6 Boundary Condition $\ldots \ldots \ldots \ldots \ldots \ldots$

3.6.1 Boundary I: Vacuum chamber wall $\rho=\kappa \ldots \ldots \ldots . \ldots 83$

3.6.2 Boundary II: Plasma Vacuum Interface $\rho=1 \ldots \ldots 3$

3.6.3 Curve fitting to $\Psi_{\text {wire }}(\rho=1, \xi)$ and $\delta \Psi_{\text {wire }} / \partial \rho$ for semianalytical Fourier Transform . . . . . . . . . . . 84

3.7 Plasma Fields . . . . . . . . . . . . . . . . . 91 


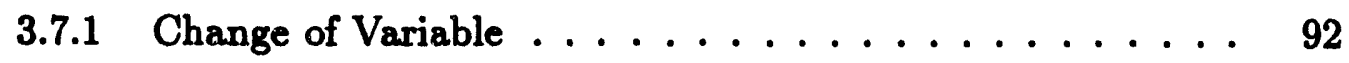

3.7.2 Fourier Transform of $\hat{K}_{1 R 2}$ and $\hat{K}_{2 R 2}$ terms . . . . . 96

3.7.3 Solution of Coupled Integral Equations . . . . . . . 110

3.7.4 Alternative Method of Solution of $\alpha \ldots \ldots \ldots \ldots$

3.7.5 Figure of Merit Calculation . . . . . . . . 116

3.8 Code Benchmarking and Comparison to Literature . . . . . . 121

3.8.1 Benchmark: Straight Cylinder Case . . . . . . . . 121

3.8.2 Comparison of CYLWAVE results with literature . . . . 124

3.9 Discussion of Results . . . . . . . . . . . . . 126

3.9.1 Resonance in chamber vs. Resonance out of chamber . . 126

3.9.2 Mirror Ratio Scan . . . . . . . . . . . . 133

3.9.3 Resonance Location Scan . . . . . . . . . . . . 136

3.9.4 Density Scan . . . . . . . . . . . . . . 138

3.10 Conclusion . . . . . . . . . . . . . . . 140

4 Computational ICRF Analysis 142

4.1 BEACH Code Theory . . . . . . . . . . . . 144

4.1.1 Formulation of Field Solution . . . . . . . . . . . 144

4.1.2 Boundary Conditions . . . . . . . . . . . . . 148

4.1.3 Absorbed Power Calculation . . . . . . . . . . . . 149

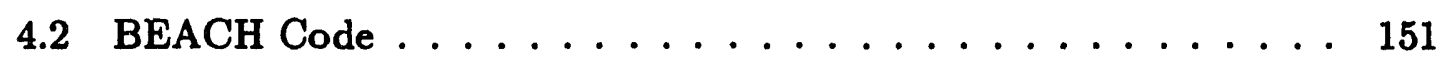

4.2.1 Background . . . . . . . . . . . . . . 152

4.2.2 Theory \& Algorithm . . . . . . . . . . . . . . 152

4.2.3 BEACH Subcodes . . . . . . . . . . . . . . . . . 154

4.3 Physics Issues . . . . . . . . . . . . . . . . . . . 154

4.4 Computational Results . . . . . . . . . . . . . . . 161

4.4.1 Baseline Model . . . . . . . . . . . . . . . . . . 162

4.4.2 Collisionless vs. Collisional . . . . . . . . . . . 167

4.4.3 Full Turn Loop vs. Dual Half Turn Loop . . . . . . . 175 
4.4.4 Effects of Density on Propagation . . . . . . . . . 186

4.4.5 Effects of Temperature on Propagation . . . . . . . . 191

4.4.6 Effects of $\omega / \omega_{c i}$ on Propagation . . . . . . . . . 195

4.4.7 Comparison of BEACH Results to Other Experimental Results - B-66 . . . . . . . . . . . . . . 197

4.5 Differences between BEACH and CYLWAVE . . . . . . . . 209

4.6 Summary of Computational Study . . . . . . . . . . 212

5 Experimental Results 214

5.1 Baseline ECRH Shots . . . . . . . . . . . . . 215

5.2 ICRF Shots $\ldots \ldots \ldots \ldots \ldots \ldots \ldots \ldots \ldots \ldots \ldots$

5.2.1 Classification of ICRF Discharge $\ldots \ldots \ldots \ldots \ldots 218$

5.2.2 Verification of the BEACH code . . . . . . . . . 220

5.3 TYPE I Discharge $-\omega>\omega_{\text {ci }} \ldots \ldots \ldots \ldots \ldots \ldots 223$

5.3.1 H- $\alpha$ and Interferometry Results . . . . . . . . 223

5.3.2 Langmuir Probe Results . . . . . . . . . . . . 225

5.3.3 B-Dot Probe Results for $\omega>\omega_{c i} \ldots \ldots \ldots \ldots \ldots$

5.3.4 $B_{z}$ Measurements in Three Regions of ICRF discharge . . 230

5.4 TYPE II Discharge $-\omega<\omega_{c i} \ldots \ldots \ldots \ldots \ldots \ldots$

5.4.1 H- $\alpha$ and Interferometry Results . . . . . . . . . 235

5.4.2 Langmuir Probe Results for TYPE II Discharge . . . . . 236

5.4.3 B-Dot Probe Results - The Radial Scan . . . . . . . 239

5.5 Summary of Experimental Results . . . . . . . . . . . . 242

6 Conclusions and Recommendations 244

6.1 Conclusions . . . . . . . . . . . . . . . . 244

6.1.1 Analytical Study . . . . . . . . . . . . . . . 244

6.1.2 Computational ICRF Study . . . . . . . . . . 245

6.1.3 Experimental Results . . . . . . . . . . . . . . . 247

6.1.4 General Conclusion . . . . . . . . . . . . . . 248 
6.2 Recommendations for Further Studies . . . . . . . . . . . . . 249

6.2.1 Theoretical - Analytical (CYLWAVE) . . . . . . . 249

6.2.2 Theoretical - Computational (BEACH) . . . . . . . . 249

6.2.3 Experimental . . . . . . . . . . . . . 250

A Useful Plasma Definitions

A.1 Plasma Dispersion Function $Z(\xi) \ldots \ldots . \ldots \ldots 2$

A.2 The Plasma Dielectric Tensor . . . . . . . . . . . . . 253

A.2.1 Expansion of the Plasma Dielectric Tensor for a cold collisional plasma ...................... 254

A.2.2 Real and Imaginary parts . . . . . . . . . . . 262

A.2.3 Perpendicular wave number $K_{\mathrm{x}}$ for TE Mode . . . . . . 263

B Current Driven Boundary Conditions 267

B.1 Source Term, Full Turn Loop . . . . . . . . . . . . . 267

B.2 Source Term, Half Turn Loop . . . . . . . . . . . . . . . 268

B.3 The Radial Current Feeder Problem . . . . . . . . . . . . . . 270

B.3.1 Modified Solution of the Vacuum-Plasma Eigenmodes . . . 270

C Transverse Plasma Fields and Excitation Coefficient Definitions 274

D ICRF Wave Propagation in Slab Geometry 277

D.1 Full Slab Model: $k_{z}=0 \ldots 278$

D.1.1 Field Formulation . . . . . . . . . . . . 278

D.1.2 Plasma Dispersion Relation for TE and TM Modes . . . . 282

D.1.3 Boundary Conditions . . . . . . . . . . . . . . 282

D.1.4 Dissipation and Figure of Merit for TE Mode . . . . . . 285

D.1.5 Discussion of Results . . . . . . . . . . . . . . 292

D.2 Full Slab Theory of $k_{z} \neq 0 \mathrm{TE} / \mathrm{TM}$ Waves $\ldots \ldots \ldots . . \ldots . . . .293$

D.2.1 Formulation ................... 293

D.2.2 Plasma Dispersion for $k_{z} \neq 0 \ldots \ldots 295$ 
D.2.3 Vectorization . . . . . . . . . . . . . 257

D.2.4 Boundary Condicion . . . . . . . . . . . . 299

D.2.5 Figure of Merit Calculation . . . . . . . . . 303

D.2.6 Variational Solution of $\eta \ldots \ldots \ldots \ldots$. . . . 306

E Derivations used in the Analytical ICRF Analysis 310

E.1 Boundary Condition for the zalytical Model . . . . . . . 310

E.1.1 Boundary I: Vecuum chamber wall $\rho=\kappa \ldots \ldots \ldots 310$

E.1.2 Boundary II: Plasma Vacuum Interface $p=1 \ldots 311$

E.2 Curve Fitting of Vacuum Field Source Terms . . . . . . . . 315

F Diagnostic Theory \& Calibration $\quad 319$

F.1 Diamagnetic Loop . . . . . . . . . . . . . . . 319

F.1.1 Basic Theory . . . . . . . . . . . . . . . 319

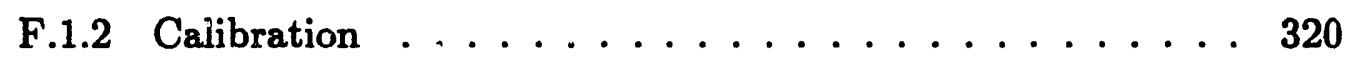

F.2 Spectrometer $\ldots \ldots \ldots \ldots \ldots \ldots \ldots \ldots \ldots \ldots$

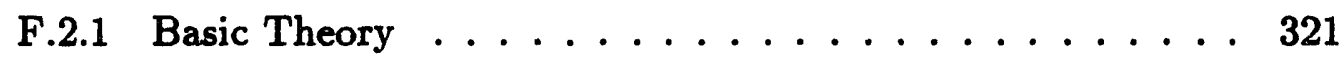

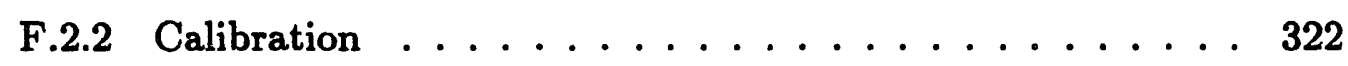

F.3 Interferometer . . . . . . . . . . . . . 322

F.3.1 Basic Theory . . . . . . . . . . . . . 322

F.3.2 Calibration . . . . . . . . . . . . . 324

F.3.3 Problems . . . . . . . . . . . . . . . 324

F.4 Langmuir Probe . . . . . . . . . . . . . . . . 324

F.4.1 Basic Theory . . . . . . . . . . . . . . 324

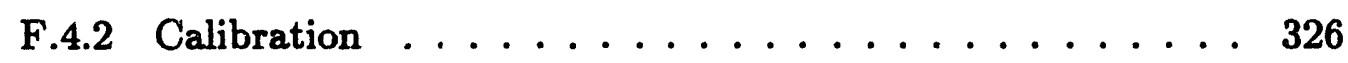

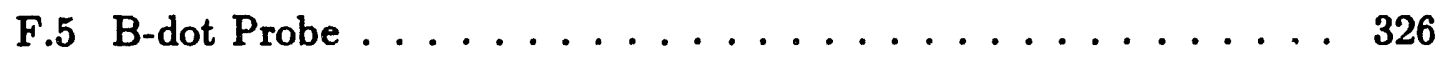

F.5.1 Basic Theory . . . . . . . . . . . . 326

F.5.2 Calibration $\ldots \ldots \ldots \ldots \ldots \ldots \ldots \ldots$ 
G Problems Encountered during PPEX Experiment $\quad 327$

G.1 Heating Systems . . . . . . . . . . . . . . . 327

G.1.1 Incorrect ECRH Microwave frequency . . . . . . . . 327

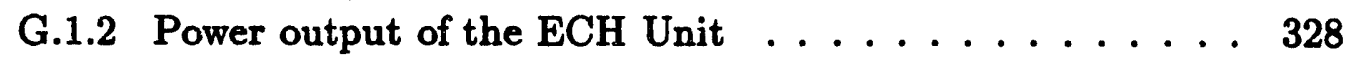

G.1.3 ICRF System . . . . . . . . . . . . . . . . 328

G.2 Diagnostics . . . . . . . . . . . . . . . . . . . . 329

G.3 Ground Loop . . . . . . . . . . . . . . . . . 329 


\section{List of Figures}

1-1 Mirror Machine Coil Schematic . . . . . . . . . . . . 19

2-1 PPEX Tandem Mirror Experiment Layout . . . . . . . . . . 22

2-2 PPEX Design and Construction Timetable . . . . . . . . . . 24

2-3 PPEX Vacuum System Schematic . . . . . . . . . . . . 26

2-4 EFFI Computed $B_{z 0}(z)$ profile for PPEX configuration . . . . . 27

2-5 PPEX Machine Layout, Axial Cross-Sectional View . . . . . . . 28

2-6 ICRF Heating System Schematic . . . . . . . . . . . 33

2-7 ICRF Matchbox Schematic . . . . . . . . . . . . 36

2-8 Schematic of the Center Cell ICRF Antenna in Vacuum Chamber 39

2-9 Schematic of the Center Cell ICRF Antenna on PPEX . . . . . 40

2-10 Langmuir Probe Schematic . . . . . . . . . . . . . . 44

2-11 PPEX $35 \mathrm{GHz}$ Interferometer Geometry . . . . . . . . . 46

2-12 Spectromeier System Schematic . . . . . . . . . . . 4 47

2-13 H $\alpha$ Detector Schematic . . . . . . . . . . . . . . . 49

2-14 B-Dot Probe Schematic . . . . . . . . . . . . 51

2-15 Instrumentation Schematic . . . . . . . . . . . . . 53

3-1 Dispersion of ICRF Waves . . . . . . . . . . . . 68

3-2 Geometry of Cylindrical Plasma-Vacuum System . . . . . . 71

3-3 $\psi_{\text {wire }}(\rho, \xi)$ in vacuum for a dipole current source . . . . . . . 77

3-4 $\Psi$ and $\Psi_{f i t}$ for a dipole current source . . . . . . . . . 86

3-5 Fourier Transform of $\Psi_{\text {fit }}$ for a Dipole Current Source. . . . . . 90 
3-6 $R(\xi)$ and $z(\xi)$ for geometry modeled in analysis . . . . . . . 101

3-7 Complex $\xi$-plane integral . . . . . . . . . . . . . 105

3-8 Complex $\xi$-plane integral for $\xi_{\text {res }}=0 \ldots 108$

3-9 ICRF $B_{z}$ along the surface of a straight plasma cylinder . . . . 123

3-10 Comparison of field profile calculated from CYLWAVE and literature125

3-11 Real, Im $\alpha$ versus $k_{\xi} \ldots \ldots 127$

3-12 Cross-sectional View of ICRF $B_{z}$ (Resonance is OUTSIDE machine)128

3-13 View of ICRF $B_{z}$ propagation. (Resonance is OUTSIDE machine) 129

3-14 Real, Im $\alpha$ versus $k_{\xi}$ for Resonance-in-chamber case . . . . . . . 130

3-15 Cross Sectional view of ICRF $B_{z}$ (Resonance is IN Chamber) . . . 131

3-16 ICRF $B_{z}$ Propagation (Resonance is IN chamber) . . . . . . . 132

3-17 $R_{p l}$ and Resonance Volume versus Mirror Ratios . . . . . . . 135

3-18 $R_{\text {pl }}$ versus resonance location $\xi_{\text {res }} \ldots \ldots \ldots$. . . . . . . . 137

3-19 Plasma Impedence Calculation for different Densities . . . . . 139

4-1 Cylindrical ICRF Geometry . . . . . . . . . . . . . . . 145

4-2 BEACH Code Algorithm . . . . . . . . . . . . 155

4-3 Sample output from BEACH Code . . . . . . . . . . . 156

4-4 PPEX Axial Magnetic Field Profile $B_{z 0}(z) \ldots \ldots$

4-5 $J_{\theta}\left(k_{z}\right)$ Spectrum for the Baseline Full Turn Loop Antenna . . . 164

4-6 ICRF $\mathrm{B}_{\mathrm{z}}$ Propagation in a Cold Collisionless Plasma, Full Turn Loop Antenna,w/wci0 $=0.788 \ldots \ldots 165$

4-7 ICRF $\mathbf{E}_{\mathrm{z}}$ Propagation in a Cold Collisionless Plasma, Full Turn Loop Antenna, $\omega / \omega c i 0=0.788 \ldots \ldots 166$

4-8 ICRF $\mathrm{B}_{\mathrm{z}}$ Propagation in a Cold Collisional Plasma, Full Turn Loop Antenna, $\omega / \omega_{c i 0}=0.788 \ldots \ldots . \ldots . \ldots 170$

4-9 ICRF $\mathrm{E}_{\mathrm{z}}$ Propagation in a Cold Collisional Plasma, Full Turn Loop Antenna, $\omega / \omega_{c i 0}=0.788 \ldots \ldots 171$ 
4-10 BEACH computed ICRF $\int \vec{B}_{z} \cdot d \vec{A}$, for Collisionless vs. Collisional Plasmas ........................ 174

4-11 Full Turn Loop and Dual Half Turn Loop Schematic . . . . . . 176

4-12 ICRF B. Propagation in a Cold Collisionless Plasma, Full Turn Loop Antenna,w/wci0 $=0.788 \ldots \ldots 177$

4-13 ICRF E. Propagation in a Cold Collisionless Plasma, Full Turn Loop Antenna, $\omega / \omega c i 0=0.788 \ldots \ldots 178$

4-14 ICRF B. Propagation in a Cold Collisionless Plasma, Dual Half Turn Loop Antenna, $\omega / \omega c i 0=0.788 \ldots \ldots$

4-15 ICRF E $\mathbf{E}_{\mathbf{2}}$ Propagation in a Cold Collisionless Plasma,Dual Half Turn Loop Antenna, $\omega / \omega c i 0=0.788 \ldots \ldots 181$

4-16 Radial Field profiles for $E_{+}, E_{-}, E_{z}, B_{z}$, Cold Collisionless Plasma, Full Turn Loop Antenna, $\omega / \omega c i 0=0.788 \ldots . . . . . . . . .183$

4-17 Radial Field profiles for $E_{+}, E_{-}, E_{z}, B_{z}$, Cold Collisionless Plasma,

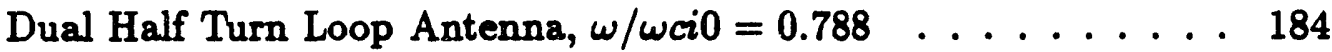
4-18 ICRF $\int \vec{B}_{z} \cdot d \vec{A}$ for a $1 \times 10^{12} \mathrm{~cm}^{-3}$ vs. $5 \times 10^{12} \mathrm{~cm}^{-3}$ plasma . . 189 $4-19 k_{z}$ roots for a $1 \times 10^{12} \mathrm{~cm}^{-3}$ vs. $5 \times 10^{12} \mathrm{~cm}^{-3}$ plasma . . . . . . 190 4-20 ICRF $\int \overrightarrow{B_{z}} \cdot d \vec{A}$ for a $10 \mathrm{eV}$ plasma and a $50 \mathrm{eV}$ plasma . . . . . . 193 $4-21 k_{z}$ roots for a $10 \mathrm{eV}$ vs. $50 \mathrm{eV}$ plasma . . . . . . . . . . . . 194 4-22 BEACH computed ICRF $\int B_{z} r d r$ for different $\omega / \omega_{\text {cio }} \ldots \ldots$ 4-23 B-66 Schematic . . . . . . . . . . . . . . . . 200 4-24 B-66 $B_{20}(z)$ Field Profile . . . . . . . . . . . . . . 201 4-25 ICRF Wave $\int \vec{B}_{z} \cdot d \vec{A}$ for Magnetic Field Geometry of the B-66 Machine, Collisionless . . . . . . . . . . . . . . . 203 4-26 BEACH $\int B_{z} r d r$ Simulation. $T_{\|}=7 \mathrm{eV}, n_{\mathrm{e}}=1.75 \times 10^{13} \ldots 204$ 4-27 B-66 Data and Finite Temperature Modeling . . . . . . . . . 205 4-28 B-66 vs. BEACH Simulation. $T_{\|}=5 \mathrm{eV}, n_{e}=2 \times 10^{13} \ldots \ldots$ 4-29 B-66 vs. BEACH Simulation. $T_{\|}=10 \mathrm{eV}, n_{e}=2 \times 10^{13} \ldots \ldots 207$ 4-30 B-66 vs. BEACH Simulation. $T_{\|}=10 \mathrm{eV}, n_{e}=0.9 \times 10^{13} \ldots 208$ 
4-31 ICRF $\int B_{z} r d r$ Propagation in a Cold Collisional Plasma . . . . . 211

5-1 Typical PPEX ECH Shot Diagnostic Data . . . . . . . . . . 217

$5-2$ TYPE I \& TYPE II Discharges . . . . . . . . . . . . . . . 219

5-3 H- $\alpha$ Comparison between TYPE I \& TYPE II Discharges . . . . . 221

5-4 TYPE I ICRF Diagnostic Data . . . . . . . . . . . . . 224

5-5 Langmuir Probe Results for a TYPE I Discharge . . . . . . . 226

5-6 Fourier Spectral Broadening of a Plasma 3MHz CC-ICRF Shot . . 229

5-7 ICRF Fourier Spectrum for Ramp-Up, Oscillatory, and Quiescence Regions ....................... 232

5-8 CC-ICH Diagnostic Data during a TYPE II Shot . . . . . . . 237

5-9 Langmuir Probe Data during TYPE II ICRF shot . . . . . . . . 238

5-10 PPEX Results vs. BEACH, $I_{\infty}=680 \mathrm{~A}, I_{\text {mirr }}=1600 \mathrm{~A} \ldots \ldots 241$

B-1 Geometry of Current Source . . . . . . . . . . . . . 269

D-1 Geometry of TE and TM wave incident on vacuum-plasma interface 286

D-2 $\operatorname{Re}\left(\psi_{T E}\right)$ and $|T|^{2}$ versus $\omega_{c i} \ldots \ldots \ldots \ldots$

D-3 Figure of Merit calculation for $k_{z}=0$ versus $\omega_{c i} \ldots \ldots 289$

D-4 $\operatorname{Re}\left(\psi_{T E}\right)$ and $|T|^{2}$ versus density ............. 290

D-5 Figure of Merit for $k_{z}=0$ at different densities . . . . . . . . 291

D-6 Figure of Merit calculation for $k_{z} \neq 0$ at different ion cyclotron

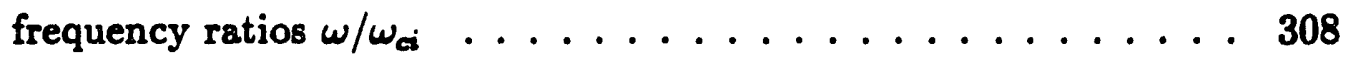

D-7 Figure of Merit calculation for $k_{z} \neq 0$ versus $n_{e} \ldots \ldots 309$ 


\section{Chapter 1}

\section{INTRODUCTION}

\subsection{History of Fusion}

Unlimited energy has been the quest of man ever since the discovery of fire. As he learned to make use of his energy resources over the centuries, his demand for energy has increased exponentially. Over the course of the last century alone, the world population has roughly quadrupled, while the world's energy usage has increased more than 100 times. While the world's current energy reserves are calculated to be able to provide man with about 500 years of use, it is not inconceivable that at some rapidly approaching time in the future, man will be unable to drive a car, heat his home, or cook his dinner with electricity based upon fossil fuel. Many sources of future energies have been explored, but thus far, fusion remains as one of the most promising, but most technically challenging sources of energy.

Fusion energy was harnessed in the explosion of the first hydrogen bomb in 1949. However, by 1955, a more peaceful way of harnessing this energy was already being attempted by scientists at laboratories world wide. Initially, the plasma would be heated by increasing the confining magnetic field rapidly, thus increasing the density of the plasma and subsequently the temperature. This was called adiabatic compression, which was widely adopted in the pinch experiments 
at Los Alamos National Lab. However, as the experiments became more successful and confinement of the plasma attained better temperatures and densities, the need to heat the plasmas to higher temperatures arose.

\subsection{Mirror Machine Principles}

A magnetic mirror is a device used in magnetic confinement of plasmas. The basic principle of the device is as follows: an ion traverses the length of the device along a axial magnetic field that is changing along $z$. As the ion approaches the magnetic field maximum, the conservation of the magnetic moment $\mu$ dictates that the particle lose parallel energy and gain perpendicular energy. At a certain turning point, the particle has lost all of its parallel energy and is then forced to turn back into the device. A measure of the confinement ability of the mirror machine is the mirror ration $R_{m}$, which is defined as the ratio of the maximum to minimum magnetic fields. Particles lying inside this mirror loss cone will be lost out of the machine, while those that lie outside the loss cone will be confined.

Figure 1-1 shows the basic layout of the magnetic coils that are required for the generation of this type of magnetic field.

\subsection{Thesis Motivation}

The motivation for this thesis is the study of ICRF wave propagation along an inhomogeneous axial magnetic field. Although many studies on ICRF have been performed over the past 30 years, an adequate treatment of both the field inhomogeneity and the cylindrical geometry of the plasma-vacuum system has not been done. This goal of this thesis is to study this problem. Two theoretical studies and one experimental study is undertaken. The theoretical studies consists of an analytical study and a subsequent multimode eigenmode computational study. The experiment involves the design, construction, and operation of a small mirror 


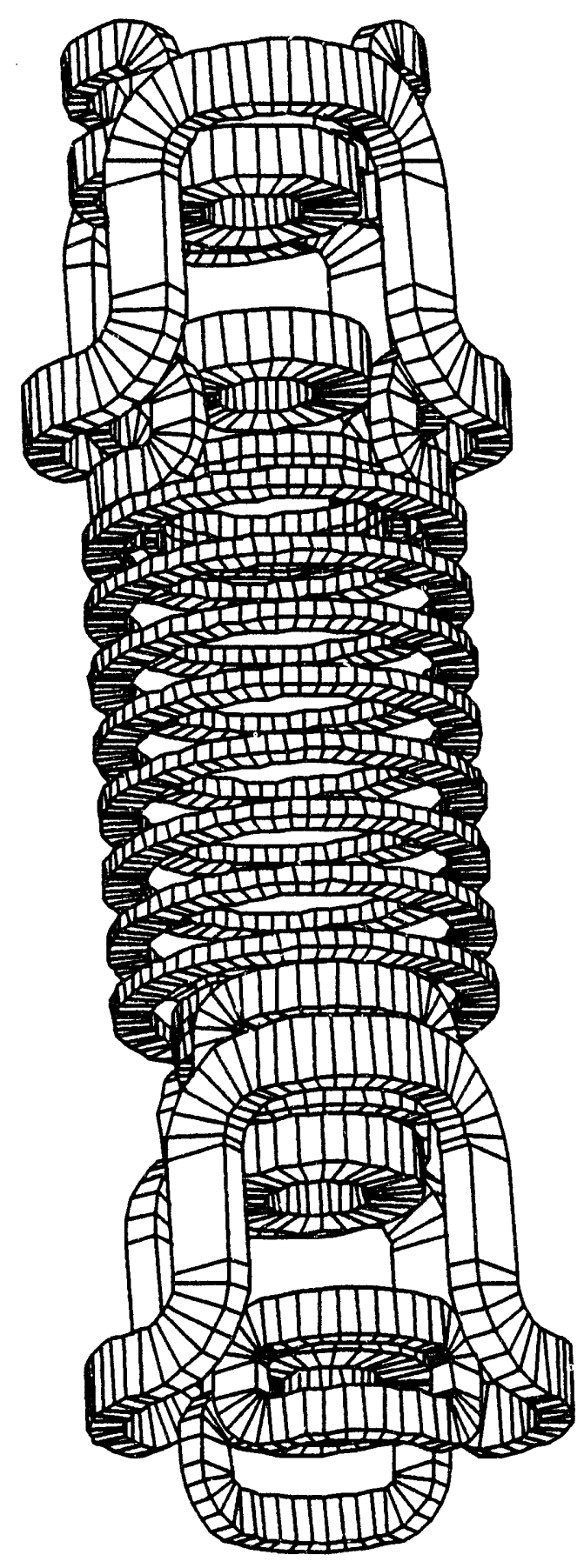

Figure 1-1: Magnetic Coil layout of a mirror machine. 
machine.

\subsection{Organization of Thesis}

The thesis consists of six chapters. Chapter 2 will introduce the experiment, the PPEX machine, which was designed and constructed as part of the thesis. Chapter 3 describes the analytical study and the new techniques that were employed to obtain the solution of the wave propagation problem. Chapter 4 describes the multimodal computational study to simulate ICRF wave propagation. Chapter 5 presents the experimental results that were obtained from the PPEX machine. Chapter 6 contains the conclusions and recommendations drawn from this thesis. 


\section{Chapter 2}

\section{The PPEX Tandem Mirror Machine}

The basic physics issues and engineering design criteria of a tandem mirror machine have been studied since $1981[5,6,7]$. This thesis studies ICRF wave propagation in a linear mirror geometry both theoretically and experimentally.

The experiments conducted in the course of this thesis were performed on the Plasma Propulsion Experiment (PPEX) at MIT. The PPEX machine is a tandem mirror device that is currently under operations at MIT. Its purpose is to study plasma heating and plasma-gas interactions. The machine was designed, constructed and made operational as part of this thesis. Figure 2-1 shows schematically some of the major systems that are employed on the PPEX machine for purposes of data acquisition or heating. These systems will be discussed in detail in the next few sections.

The PPEX machine was designed, constructed, and made operational due to the combined effort of 2 full time staff and 2 grad students. The timetable for the PPEX's design, construction, and experimentation phases can be seen on Fig. 2-2

The PPEX design incorporates features such as a tandem magnetic field and ICRF heating at the B-field minimu. These are also found on other larger tandem mirror experiments such as MIT's TARA $[8,9,10]$, LLNL's TMX-U $[11,12]$, 


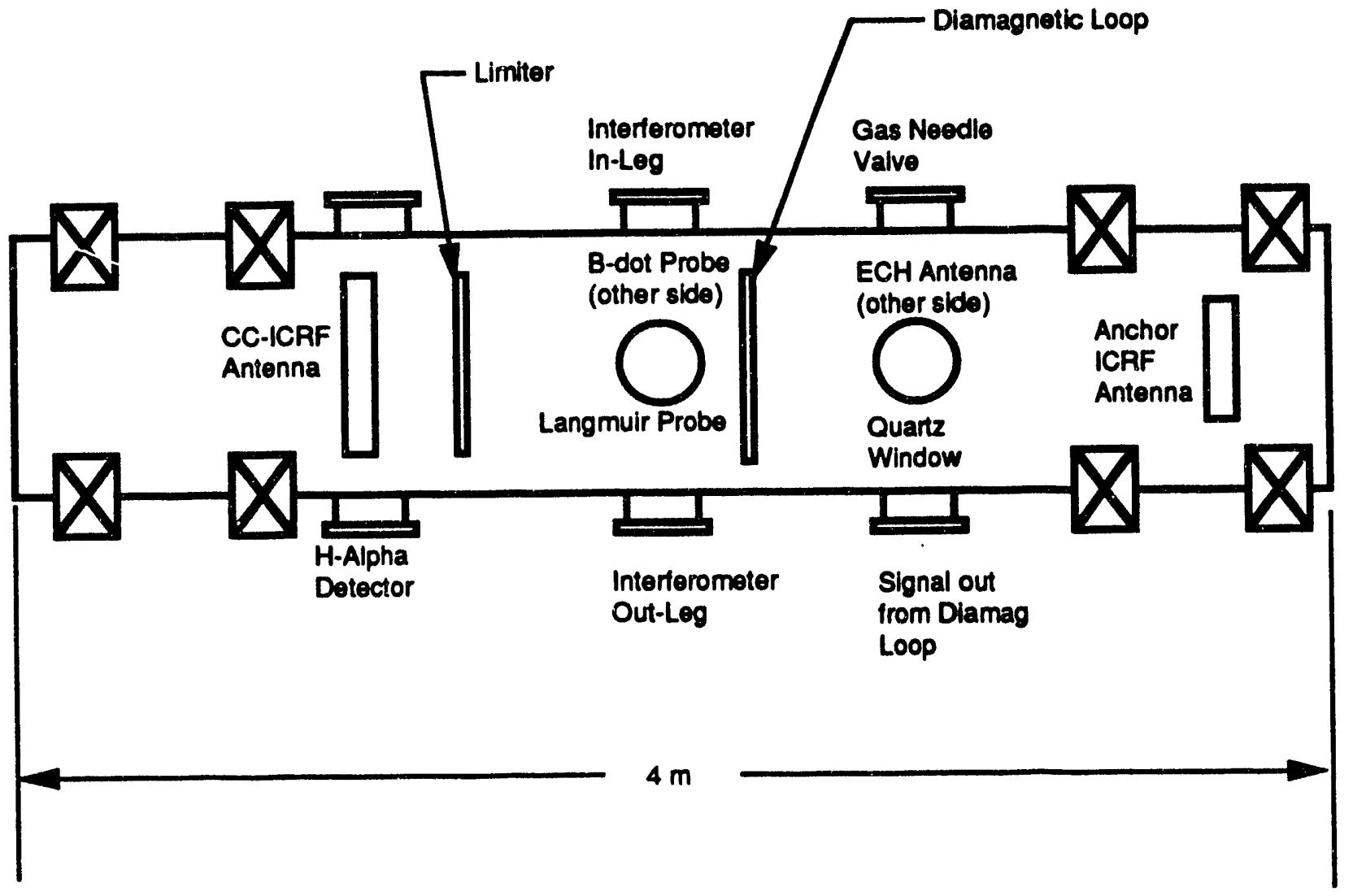

Figure 2-1: PPEX Tandem Mirror Experiment Layout. The center cell solenoid coils are not shown for clarity. 
Japan's Gamma 10 [13] and RFC-XX[14], and University of Wisconsin's Phaedrus [15]. PPEX utilizes electron and ion cyclotron heating and makes use of the plasma end losses particular to mirror devices for propulsion studies. The machine was designed to operate at a center cell field of about 1-2kG and a maximum mirror field of approximately $12 \mathrm{kG}$ utilizing 8 convection cooled Center Cell solenoid coils, 2 convection cooled Transition Boost solenoid coils and 4 liquid nitrogen cooled (vacuum dewered) mirror solenoid coils. Two kilowatts of electron cyclotron resonant heating (ECRH) power is used to initiate gas break down and pre-ionization. (Helium, hydrogen, and argon gases have been used) Up to two hundred kilowatts of Ion Cyclotron Resonant Frequency (ICRF) power can be injected via two ICRF antennas during the plasma discharge cycle to provide the main heating of the plasma.

The working gas for the PPEX machine is usually hydrogen. Other gases have been used (Argon and Helium), but for the main heating experiments, hydrogen was the preferential working gas.

This chapter discusses the PPEX machine. First, the four major sub-systems making up the PPEX are described in Section 2.1. These four major sub-systems are Vacur n, Magnets, Gas Feed, and Plasma Heating. Because this thesis studies ICRF heating, the Heating system is especially important and is discussed in greater detail in Section 2.2. The diagnostics package is examined in Section 2.3. The data acquisition equipment assembled on the PPEX is discussed in Section 2.4. Next, the overall operations of the PPEX will be presented. Section 2.6 will describe briefly the design and fabrication work that was directly performed as part of this thesis. Finally, a brief summary of the work performed in the design, construction and operation of the machine is provided. 


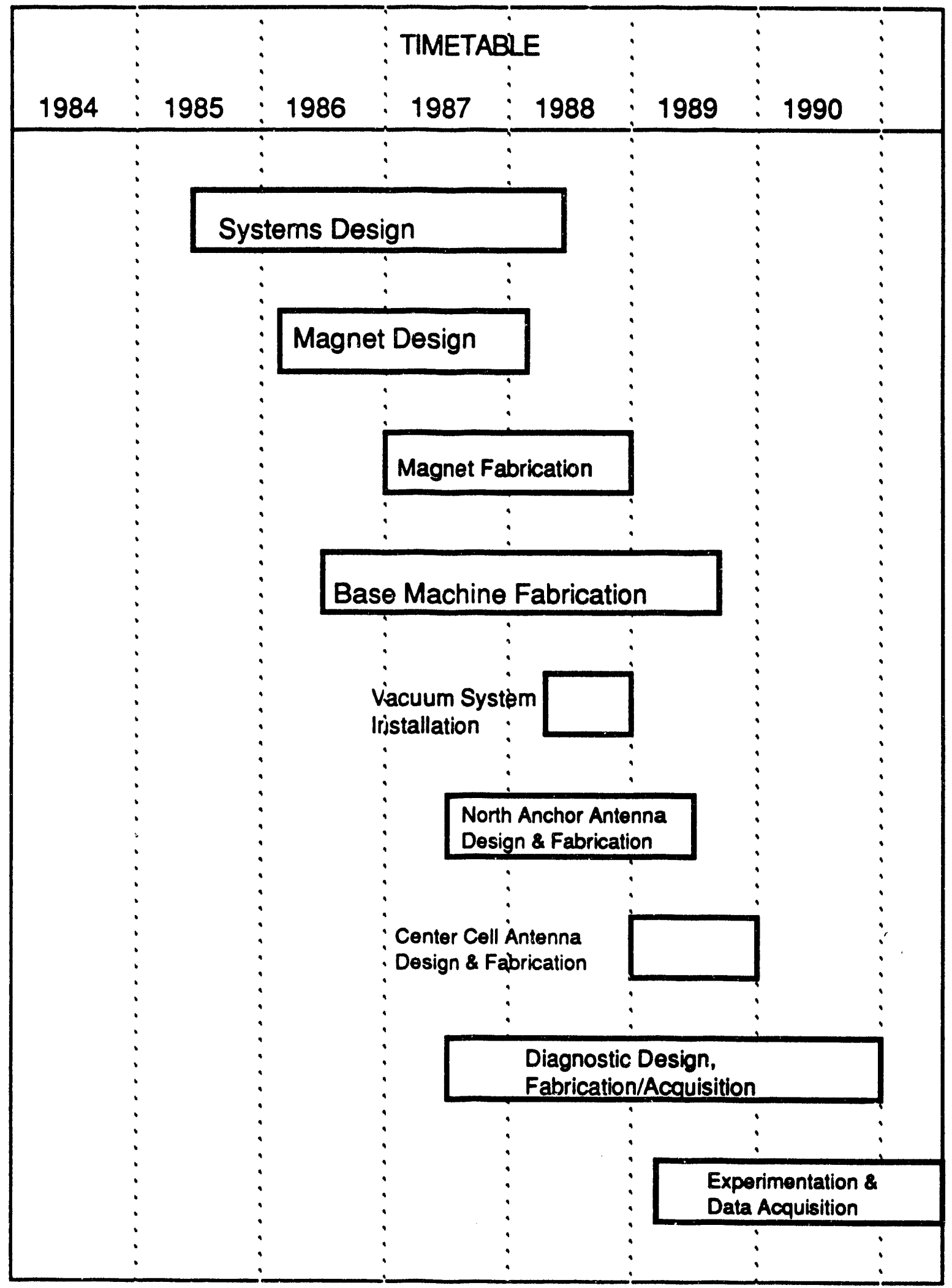

Figure 2-2: PPEX Design and Construction Timetable. The time from initial design to computerized data acquisition is approximately 5 years. 


\subsection{Major Machine Systems}

\subsubsection{Vacuum System}

As seen in Fig. 2-3, the PPEX Vacuum System consists of two mechanical forepumps and two turbo-molecular pumps. The mechanical pumps are sufficient to pump the chamber assembly down to about $10^{-3}$ Torr, at wh . int the turbo pumps are used to bring the vacuum down to the $10^{-8}$ range. There is also a natural cryopump that is created when the mirror coils are nitrogen cooled. $10^{-8}$ vacuum is readily obtainable when this cryopump is activated.

\subsubsection{Magnets}

The PPEX m. gnet layout was designed as part of this thesis using the code EFFI [16]. EFFI allowed the computation of field profiles and distributions for a given coil configuration. A set of typical coil current settings is provided on Table 2.1. Using these typical design settings, the EFFI code computes the axial $B_{z 0}(z)$ field for a typical PPEX configuration and the result is plotted on Figure 2-4. The PPEX machine axiai cisss sectional view is given on Figure 2-5.

The magnets currently installed on the PPEX machine are simple solenoiods. Original design plans call for the addition of ioffe and saddle coils, but currently the PPEX uses only 3 sets of solenoids - center cell solenoids, transitional boost solenoids and mirror solencids. The center cell and transition solenoids are cooler? by natural convection, while the mirror solenoids are cooled by either forced air convection or liquid nitrogen. Since the resistance of copper decreases as its temperature decreases, using liquid nitrogen provides an added advantage of reducing the magnet power required in addition to its main function of cooling.

Although the ioffe and saddle coils were included in the original design, the advantages of having such coils have not been conclusively proven. Results from other mirror experiments (Phaedrus $[17,18,19]$, TARA [20]) indicate that the 


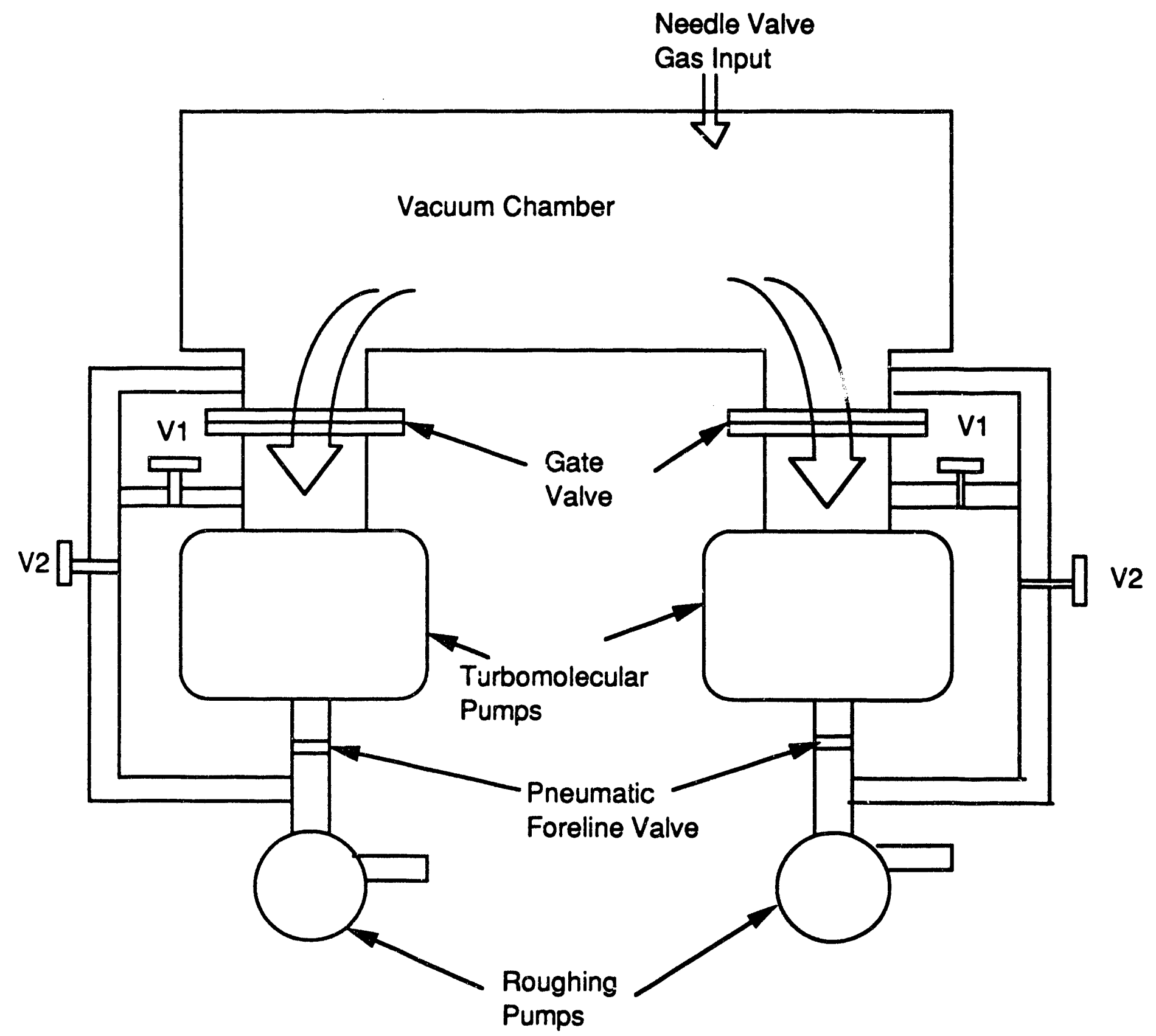

Figure 2-3: PPEX Vacuum System Schematic. Valves V1 and V2 are manual valves that can be opened or closed to pump down the chamber even with the gate valve closed. 
NIT PHAEDRUS/EXPLORER

19:08:31E 05/06/90

FIELD MAGNITUDE ALONG FLUX LINES 16 THROUGH 18

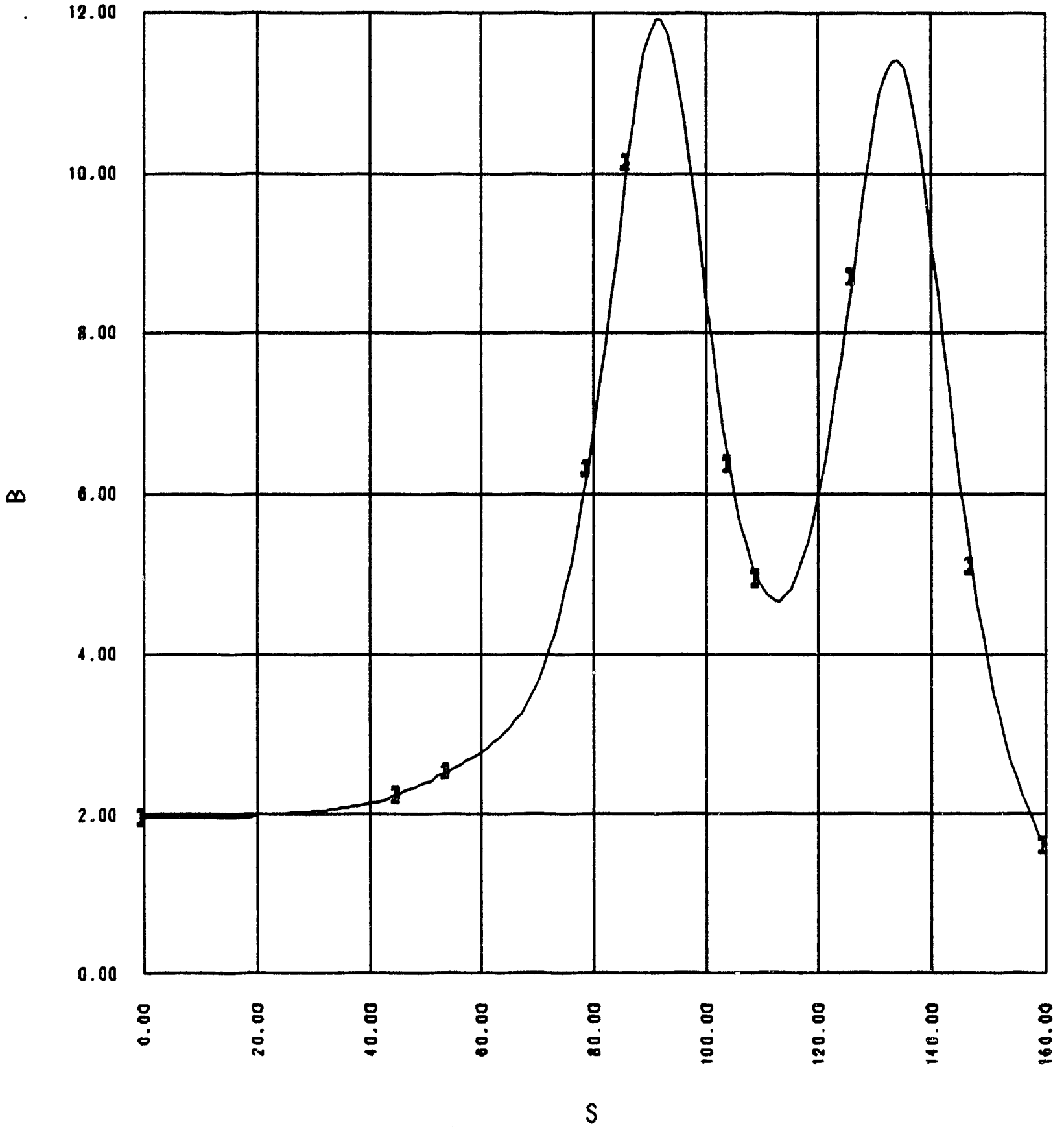

Figure 2-4: EFFI [16] computed $B_{z 0}(z)$ profile for typical PPEX configuration $I_{c c}=640 \mathrm{~A}, I_{\text {mirr }}=1600 \mathrm{~A}$ 


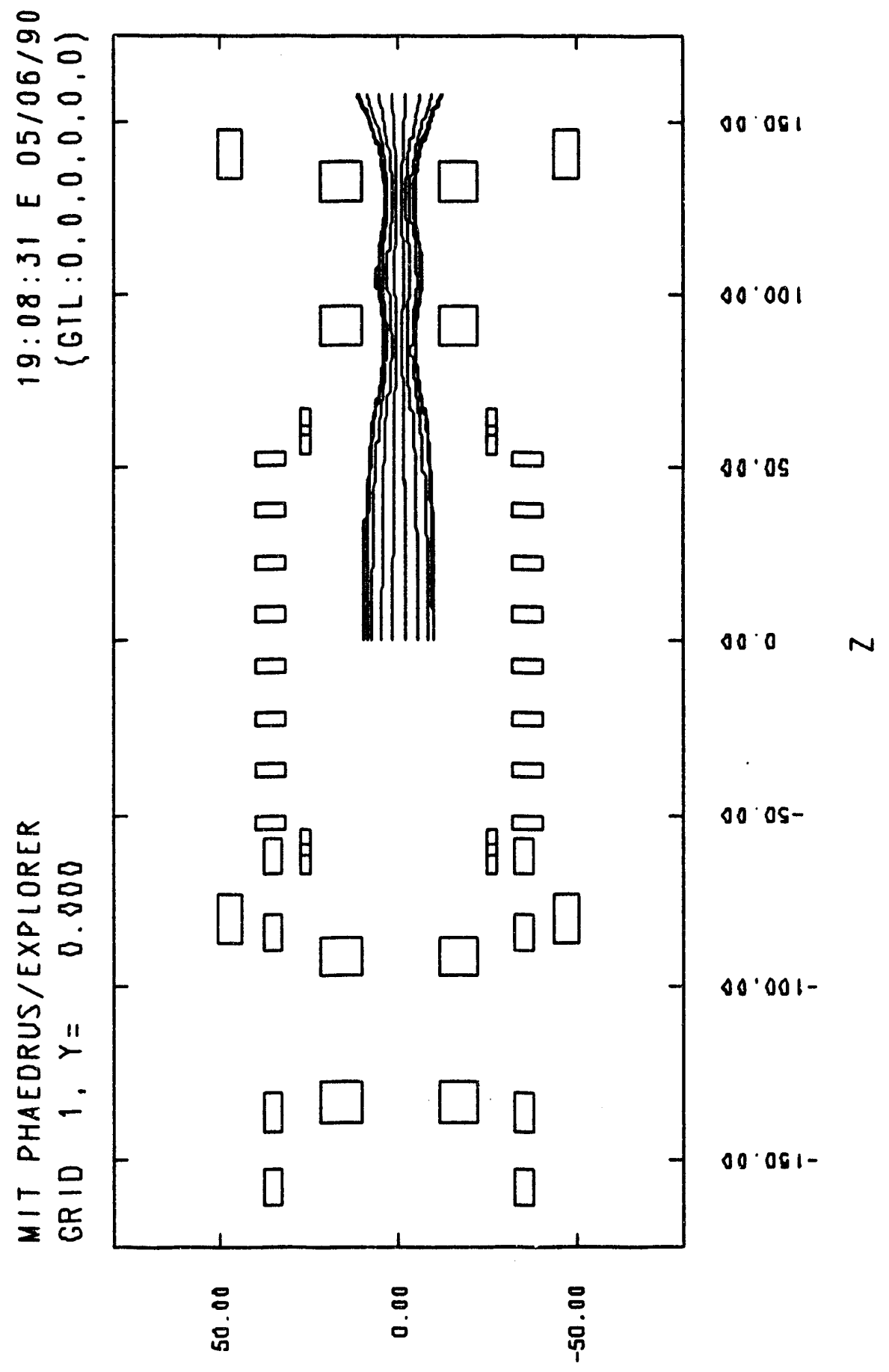

Figure 2-5: PPEX Machine Layout, ${ }^{X}$ Axial Cross-Sectional View. Some field lines are traced out to show the magnetic geometry of the system. 
presence of ICRF can provide a ponderomotive force that stabilizes the plasma against interchange modes caused by bad curvature, thus making the necessity of the minimum-B coils less critical than initially believed.

\subsubsection{Gas Feed}

While other machines generally use a gas-box for flow regulation and distribution [21], the PPEX machine is unable to utilize a gas box because of its compact size. Rather a gas needle valve is employed as the means of gas distribution. The gas feeding system location on the PPEX is shown schematically in Figure 2-3. The gas flow needle valve is located on one of the northern $\mathrm{CC}$ access ports. The gas can either be puffed into the closed chamber or kept flowing during the discharge by opening the two bypass valves to the turbomolecular pump. For gas puff fueling, the pressure is usually in the $10^{-4}$ to $10^{-3}$ Torr range. For a flow discharge, the pressure for optimum heating is found to be in the $10^{-5}$ to $10^{-4}$ Torr range.

\section{PUFF FUELING}

Puff fueling refers to the act of filling a chamber with low pressure gas and then closing all the ports to ensure a constant pressure discharge medium. However, other gases and vapors are also entering the system either due to leakage or outgassing. Thus a puff fueled plasma is generally kept at a higher pressure in order to ensure that the majority of the gases in the chamber is the desired fuel gas and not water vapor and other gases that outgas or leak into the chamber. This method of fueling is not often used. The great majority of the shots employ flow fueling as the preferred fueling scheme. 


\section{FLOW FUELING}

Flow fueling refers to the constant pumping out of the gases in the system. In this fueling scheme, a needle valve is kept open to provide the gas source, and some bypass valves are opened to continuously pump out some of the gas. This creates a flow of gas in the vacuum chamber, hence the term "flow fueling". Two schemes are used in PPEX flow fueling : North Pumping, or South Pumping. The North Pumping scheme simply refers to the case where the refueling gas is pumped out the same axial end of the machine as the needle feed valve. South pumping refers to the fact that the refueling gas is drawn across the centerline of the central cell towards the vacuum pump on the other end of the machine.

The North Pumping Scheme is less desirable because the flowing gas is pumped out away from the point of maximum ion heating (at $\mathrm{Z}=0$ ), while the South Pumping Scheme pulls the fueling gas across the resonance point, thereby fueling the plasma.

The flow fueling process is as follows: first, the gate valve (See Figure 2-3) to the turbomolecular pumps are shut. Then Bypass Valve V1 is opened to allow slow pumping via turbomolecular pump. Finally, the needle valve is engaged to let in the working gas at a constant flow rate. Eventually the chamber equilibriates to a working pressure that is kept at approximately 5 to $10 \times 10^{-5}$ Torr.

\subsubsection{Plasma Heating}

Three heating systems are currently deployed on the PPEX - one ECRH unit (Center Cell) and two ICRF units (One in North Anchor, one in Center Cell). Altogether these systems provide up to $200 \mathrm{~kW}$ of heating power to the plasma. The heating systems are discussed in further details in Section 2.2. 


\subsection{Plasma Heating Systems}

\subsubsection{ECRH}

The Electron Cyclotron Resonant Heating (ECRH) system consists of a Raytheon $2 \mathrm{KW}$ system running at a fixed frequency of $2.1 \mathrm{GHz}$ and is used for breakdown and plasma preionization. The $2.1 \mathrm{GHz}$ frequency corresponds to a resonant field of $750 \mathrm{G}$ for a pure hydrogen plasma.

The ECH microwave power that is emitted by the Raytheon ECRH unit is modulated by a half rectified sinusoidal waveform of approximately $5 \mathrm{~ms}$ in duration and repeating once every $8 \mathrm{~ms}$. Although it would be better for our purpose to have a fully rectified power output, it was practically impossible to modify the unit because it would have required extensive redesign.

\section{OPERATIONS}

During normal operations, the ECRH transmitter is triggered $\mathrm{ON}$ at a preprogrammed time after the magnets begin to be energized (Usually about $100 \mathrm{~ms}$ ). This is to ensure that the magnet coil currents have flattopped, providing the ECH with a resonance surface upon which to absorb the microwave power. This is desirable because if there is no electron resonance surface, much of the ECH power would be reflected back towards the transmitter, possibly causing damage.

\subsubsection{ICRF}

Since the main experimental purpose of this thesis is to study the ion cyclotron heating of the mirror plasma, the ICRF system is one of the most important systems of the PPEX machine. 


\section{ICRF SYSTEM COMPONENTS}

A complete set of the equipment that the PPEX ICRF System consists of is listed in Table 2.2.

The primary Ion Cyclotron Resonant Frequency (ICRF) amplifiers consists of two $200 \mathrm{~kW}$ Continental Electric Transmitters, one used ir heating the center cell plasma at $3 \mathrm{MHz}$, the other used in heating the anchor at $7.5 \mathrm{MHz}$. The Continental Electric Transmitter Basic Schematic is shown in Fig. 2-6.

\section{ICRF TRANSMITTER}

The ICRF Transmitter/Amplifier is a Continental Electric amplifier that is capable of outputting $500 \mathrm{~kW}$ of ICRF power. The amplification is performed by a Class $\mathrm{C}$ Tetrode RF Tube. A tube operating in Class $\mathrm{C}$ has a conduction angle of less than 180 degrees [22]. This means that out of a full cycle, the tube puts out power less than $1 / 2$ of the time. Because high power RF operation necessitates a Class $\mathrm{C}$ operation, the frequency of the output will not be pure. However, since all class C RF amplifier tubes are coupled to an output tuning circuit, the output waveform can be tuned to minimize the distortion of the base harmonic. Nevertheless, it must be noted that because of the imperfections of the tuning circuit, multiple harmonics and other frequencies will be present in the output of the ICRF transmitter, albeit excited at a much lower amplitude than the base ICRF frequency component.

\section{ICRF TRANSMITTER TUNING}

The ICRF transmitters have to be tuned for optimum performance at each frequency. Each transmitter has two sets of tuning capacitors, one set used to tune the intermediate stage, and the other to match the transmitter to the $50 \Omega$ transmission line. A sample tuning sequence would consist of the following steps: 
Signal Level Genarator

Kenwood TS-440

(100 W Output)

Amplifier

Continental Electric

RF Amplifier

(Output $=0$ to $200 \mathrm{~kW}$ )

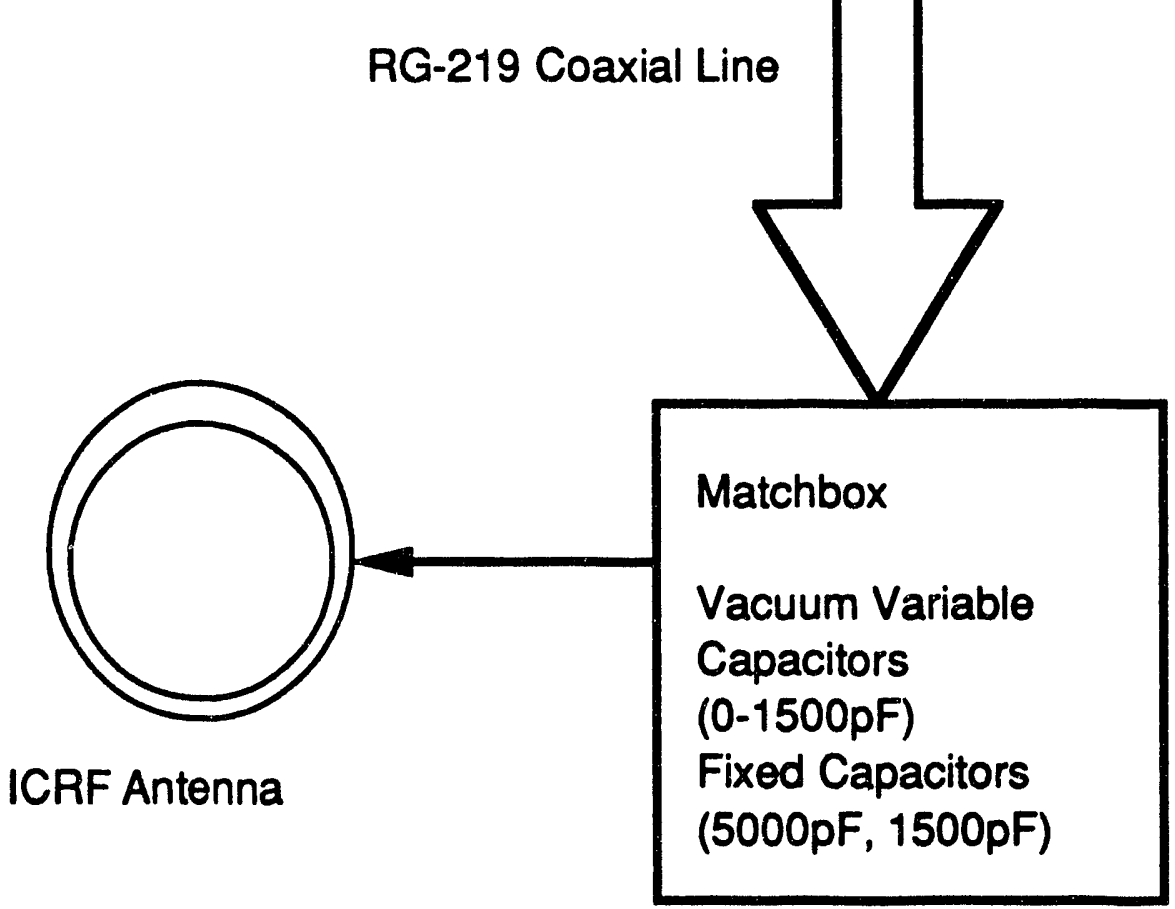

Figure 2-6: ICRF Heating System Schematic. A pulse from an HP function generator is sent to the signal level generator which gates a pulse of a given length to the Continental amplifier. 
1. Set frequency output on the signal level RF generator (Kenwood TS-440) and lock in the frequency.

2. Warm up transmitter and RF tube.

3. Turn on the RF cathode power supply.

4. Gate in short RF pulses (Generated by an HP pulse generator) for tuning. Typical pulses occur twice a second for approximately $0.5 \mathrm{~ms}$.

5. Vary the intermediate stage capacitors to maximize the Driver Grid Current to tune the intermediate stage and achieve resonance at this frequency.

6. After intermediate stage has been tuned, switch on HV (high voltage) to about $1 \mathrm{kV}$. Monitor the Forward-Reflected Power levels from the $-70 \mathrm{~dB}$ directional couplers on the dummy load. Gate in tuning pulses of about $0.5 \mathrm{~ms}$ long twice a second. Tune by maximizing the forward power and minimizing the reflected power. Obtaining the best sinusoidal waveform is also a criterion for resonance tuning.

7. If the forward-reflected voltage ratio seen on the scope is $>10$, then the transmitter is considered tuned.

\section{ICRF OPERATIONS}

During a shot, when ICRF heating is not needed, the ICRF transmitters are kept in STANDBY mode. In this STANDBY Mode, the transmitter and the RF tube are both warmed up and ready to be fired. The RF tube cathode is biased at a high voltage of $1-10 \mathrm{kV}$, but the tube screen is biased negatively enough to prevent electron cascade and amplification.

When a request for ICRF power is issued through the MDS data acquisition system and software [23], a gated pulse lowers the negative screen bias, thus permitting electron amplification. The on-time of the system is pre-programmed into 
the MDS software and controlled through a Jorway J221 Controller. A maximum on-time of $100 \mathrm{~ms}$ is selected to ensure a $0.1 \%$ duty cycle for the ICRF system. (100ms ON, 100s OFF)

\subsubsection{ICRF MATCHBOX}

The ICRF system requires an impedence matching network to match the plasma load to the $50 \Omega$ load of the RG-219 transmission line that brings the ICRF power from the Continental Transmitters to the antenna. The set of capacitors that control the impedence and resonant matching makes up the matchbox. The matchbox schematic is shown in Figure 2-7.

The matchbox consists of essentially two capacitors - a tuning capacitor $C_{T}$, and a load matching capacitor $C_{L}$. The tuning capacitor is used to tune a resonant LC circuit to the frequency

$$
\omega^{2}=\frac{1}{L C_{T}}
$$

where $L$ includes the antenna as well as the plasma inductance. The load capacitor $C_{L}$ is used to match the entire load to the $50 \Omega$ RF Coax line.

Because of the low frequencies that are required for ion resonance in the central cell, the tank capacitance $C_{T}$ required for resonance is high (since $C_{T}=1 /\left(\omega^{2} L\right)$ ). This requirement makes the addition of fixed capacitance a necessity. Three sets of fixed capacitors are therefore installed in parallel with the variable capacitor $C_{T}$. These fixed capacitors are banked into and out of the matching circuit by a vacuum interruptor. These vacuum interruptors are switched $\mathrm{ON}$ by applying pressurized air. For the type of vacuum interruptor that was used in the central cell matchbox (Jennings RP233B), approximately 25 PSI of air pressure was required to close the contact.

The matchbox operates as follows: the tuning capacitor $C_{T}$ is first tuned so that resonance at the desired ICRF frequency is reached. After the resonance has been achieved, the tuning capacitor is then detuned slightly off resonance. 
From

Transmitter
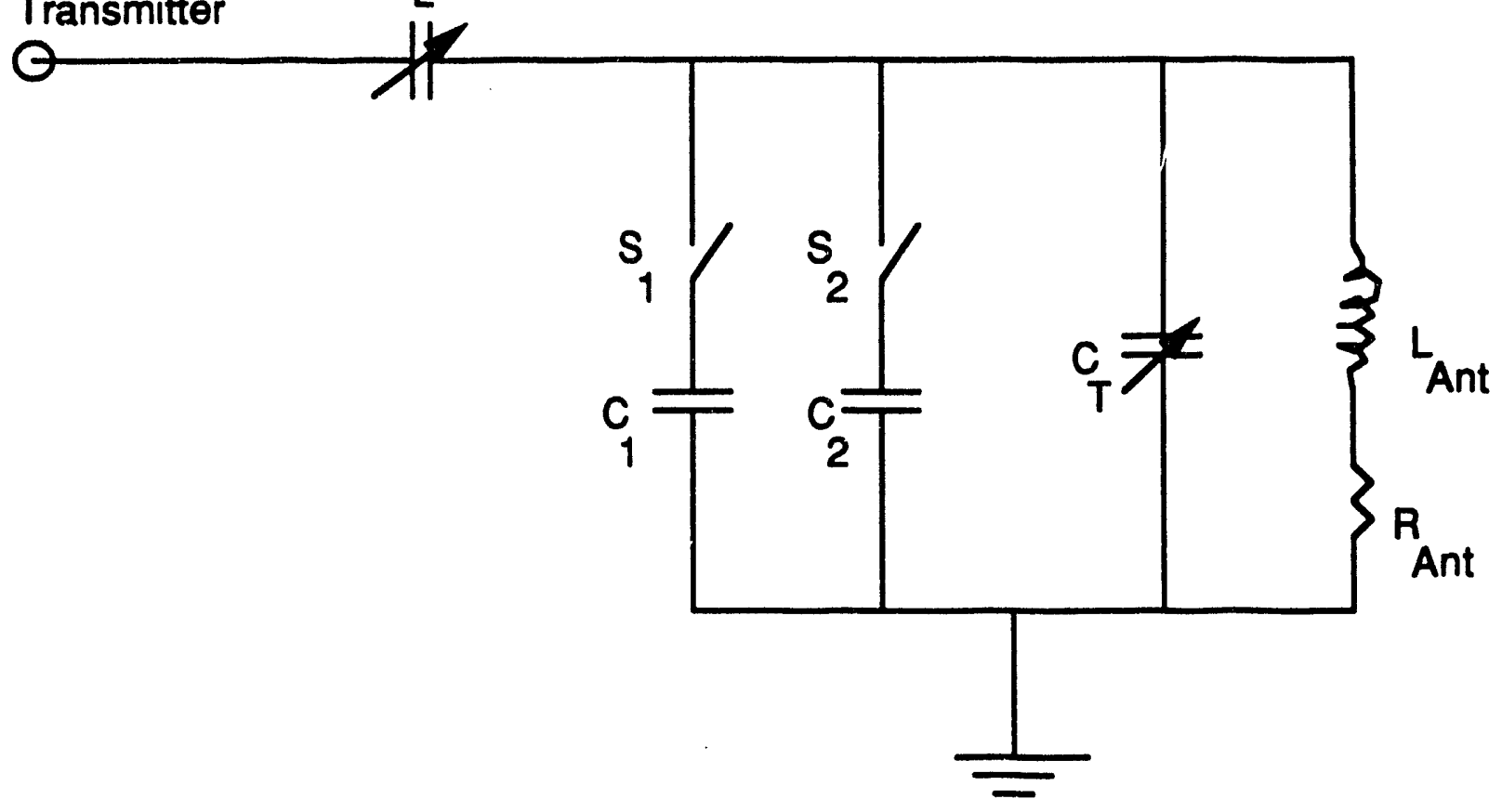

C = Load matching capacitor

$\mathrm{L}$

C = Fixed capacitors

1,2

$S=$ Vacuum Switches

1,2

C = Tuning capacitor

$T$

$L$ - Antenna inductance

Ant

$R=$ Antenna Resistance

Ant

Figure 2-7: ICRF Matchbox Schematic. Switches S1 and S2 can be closed to gate in additional capacitance if lower resonant frequency is required. The variable tank and loading capacitors $C_{T}$ and $C_{L}$ are both vacuum variable capacitors. 
The net impedence of $Z_{1}$ that results is then seen to be largely resistive and slightly inductive. The load capacitor $C_{L}$ is then used to cancel out the inductive impedence and bring the total impedence of the entire load $Z_{2}$ to be approximately $50 \Omega$.

\section{NORTH ANCHOR MATCHBOX}

The north anchor matchbox consists of two vacuum variable capacitors, one responsible for tank resonance, $C_{T}$, the other for matching the plasma to the $50 \Omega$ transmission line, $C_{L}$. The tank capacitor consists of a Jennings vacuum variable capacitor, CV2C-2000, with a capacitance range of $100-2000 \mathrm{pF}$ at a working voltage of $35 \mathrm{kV}$. The load capacitor is another Jennings CVDD vacuum variable.

\section{CENTRAL CELL MATCHBOX.}

The variable tank capacitor $C_{T}$ consists of a Comet CV2C-2000 Vacuum Variable Capacitor. The variable load capacitor $C_{L}$ consists of a smaller Jenning CVDD capacitor with a capacitance range of $50-750 \mathrm{pF}$ at $15 \mathrm{kV}$. The fixed capacitors used to increase the overall tank resonating capacity consist of three types of capacitors: two Murata 1500pF capacitors and one Murata water-cooled 4500pF capacitor. The vacuum interruptors are the Jennings RP233B type.

\subsubsection{ICRF Antenna}

The ICRF Antenna used in the PPEX machine is the dual half-turn loop (DHTL) antenna. The design and construction of the antenna are made with the following considerations in mind.

1. Vacuum compatibility - The antenna must be soldered with vacuum compatible solder and solder must be allowed to flow smoothly so that no air pockets remain after solidification. Vacuum solder is used to minimize outgassing. 
2. Arc prevention - All edges and corners must be rounded and deburred. This is to ensure that no arcing takes place that may damage the antenna or other items within the chamber to which the antenna may arc.

3. Ease of assembly inside chamber - Becuse of its size, the antenna must be assembled inside the vacuum chamber. Thus ease of assembly and installation is of primary concern.

A schematic of the Center Cell ICRF antenna in the vacuum chamber is shown on Figure 2-8. The view shows how the ICRF antenna must be designed to couple with the other components of the ICRF system. Only two bolts are necessary to fasten the antenna into place. A schematic of design of the center cell ICRF antenna is given on Figure 2-9. 


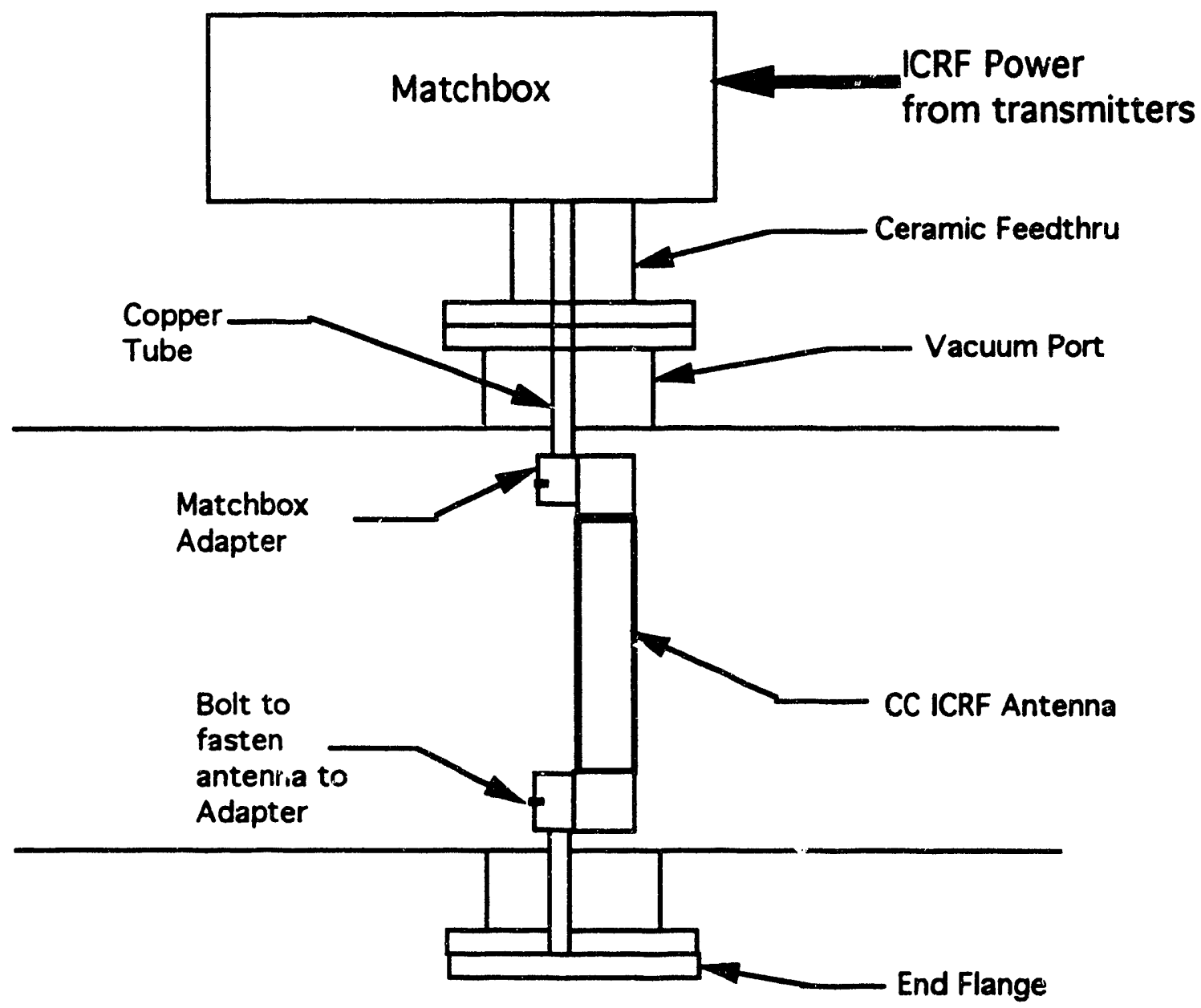

CC ICRF Antenna in Vacuum Chsimber Schematic

Note: NOT TO SCALE

Figure 2-8: Schematic of the Center Cell ICRF Antenna in the Vacuum Chamber of PPEX. This view shows how the ICRF antenna must couple together with other components of the ICRF system. Only two bolts, one through each of the matchbox adapter are required to hold the antenna in place. 


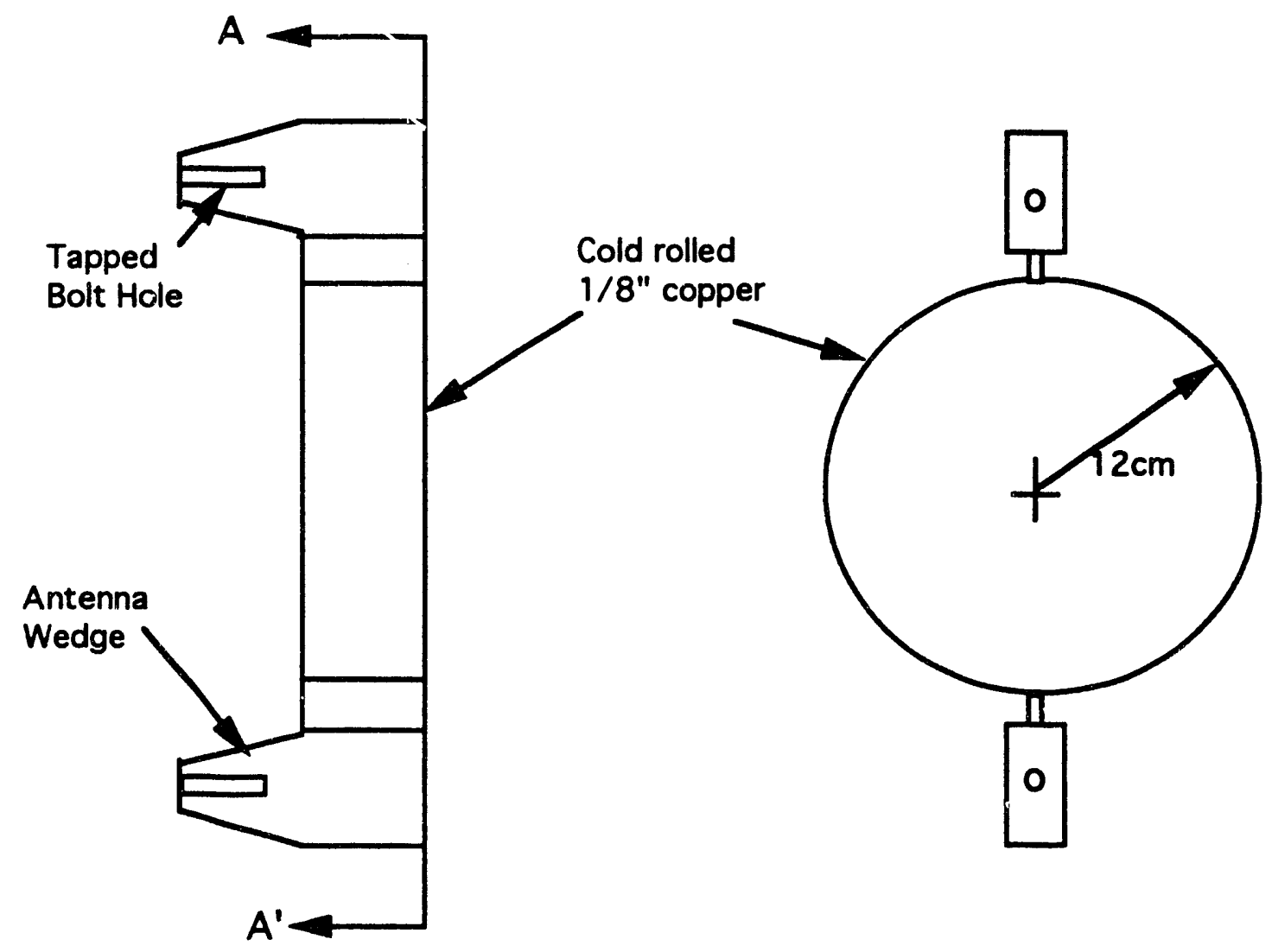

CC ICRF Antenna: Side View

Front View A-A'

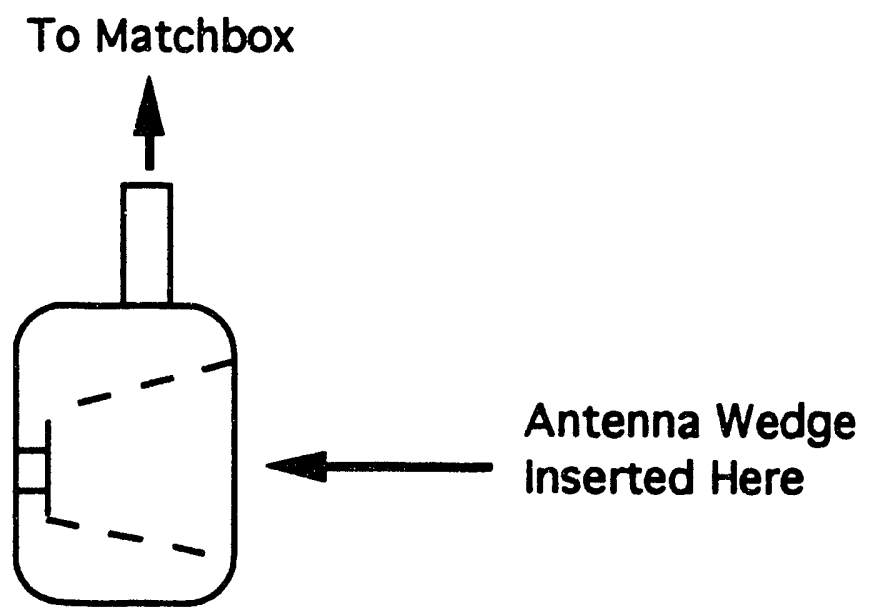

\section{Side View of Matchbox Adapter}

NOTE: Drawings NOT TO SCALE

Figure 2-9: Schematic of the Center Cell ICRF Antenna on PPEX. The wedge shape of the connector is designed to slot into the connector from the matchbox. Only two screws are required for the assembly of this antenna. 
Table 2.1: PPEX Coil Set

\begin{tabular}{|l|l|l|l|}
\hline COIL & COIL TYPE & $\begin{array}{l}\text { Design Current Density } \\
\left(\mathrm{A} / \mathrm{cm}^{2}\right)\end{array}$ & $\begin{array}{l}\text { Typical Operating } \\
\text { Current (A) }\end{array}$ \\
\hline CENTER CELL & SOLENOID & 800 & 600 \\
BOOST & SOLFNOID & 800 & 600 \\
MIRROR & SOLENOID & 2700 & 1600 \\
\hline
\end{tabular}

Table 2.2: ICRF System Components

\begin{tabular}{|c|c|c|}
\hline ICRF System Component & Typical Equipment & Notes \\
\hline Signal Generator & $\begin{array}{l}\text { Ham Radio- } \\
\text { Kenwood TS440 }\end{array}$ & $\begin{array}{l}\text { Generates the RF Signal to } \\
\text { be amplified. Frequericy } \\
\text { range is from } 2 \mathrm{MHz} \text { to } 32 \mathrm{MHz} \text {. }\end{array}$ \\
\hline Amplifier & $\begin{array}{l}\text { Continental Electric } \\
\text { Transmitter }\end{array}$ & $\begin{array}{l}\text { Provides the RFamplification } \\
\text { from signal level (100W) } \\
\text { to } 200 \mathrm{KW} \text {. Duty cycle } \\
\text { is } 0.1 \%\end{array}$ \\
\hline Transmission Line & RG219 Coaxial Line & $\begin{array}{l}\text { Transmits power from } \\
\text { Continental transmitter to the } \\
\text { RF load. }\end{array}$ \\
\hline Matchbox & $\begin{array}{l}2 \text { Vacuum Variable } \\
\text { Capacitors, } C_{L} \text { and } C_{T} .\end{array}$ & $\begin{array}{l}\text { Matches impedence of the load to } \\
\text { transmission line impedence }(50 \Omega) \text {. } \\
\text { Matchbox also contains directional } \\
\text { couplers for observing forward and } \\
\text { reflected power. }\end{array}$ \\
\hline Antenna & $\begin{array}{l}\text { Full Turn or } \\
\text { Dual Half Turn Loops. } \\
\text { Copper }\end{array}$ & Transmits power to plasma \\
\hline
\end{tabular}




\subsection{Diagnostics}

In order to determine the plasma characteristics of the discharge, an adequate set of diagnostics must be assembled to measure some basic plasma parameters. These parameters include the ion temperature $T_{i}$, electron temperature $T_{e}$, and electron and ion densities $n_{i}, n_{e}$. A system of diagnostics was designed, fabricated or reconstructed, calibrated and used to determine plasma properties as part of this thesis. This section discusses the diagnostic systems that were fabricated and/or reconstructed for use on the PPEX.

The diagnostics currently operating on PPEX are listed in Table 2.3 along with the plasma properties that they measure.

Table 2.3: PPEX Diagnostic Set

\begin{tabular}{|l|l|l|}
\hline DIAGNOSTIC & LOCATION & PLASMA PROPERTY MEASURED \\
\hline LANGMUIR PROBE & CC, $\mathrm{z}=0 \mathrm{~cm}$ & $T_{e}(a), n_{e}(a)$ \\
INTERFEROMETER & CC, $\mathrm{z}=0 \mathrm{~cm}$ & $n_{e}$ \\
$H_{\alpha}$ Detector & $\mathrm{CC}, \mathrm{z}=-50 \mathrm{~cm}$ & $\mathrm{n}(\mathrm{r})$ \\
SPECTROMETER & $\mathrm{CC}, \mathrm{z}=+50 \mathrm{~cm}$ & $T_{i}, n_{i}$, Plasma Rotation \\
DIAMAGNETIC LOOP & $\mathrm{CC}, \mathrm{z}=3 \mathrm{~cm}$ & $\beta, n_{e}, T_{i}+T_{e}$ \\
B-DOT PROBE & $\mathrm{CC}, \mathrm{z}=0 \mathrm{~cm}$ & ICRF $B_{z}$ radial profile \\
\hline
\end{tabular}

Because the theory for these diagnostics has been investigated throughly over the past 30 years, a brief theory of each of the diagnostics used on the PPEX machine is given in Appendix $\mathrm{F}$ for completeness.

\subsubsection{Langmuir Probe}

\section{LANGMUIR PROBE SETUP}


The Langmuir Probes used in the experiment is the standard single probe seen in the top part of Figure 2-10. The driving circuitry is the standard high voltage driver circuitry [24] seen in the lower half of the same figure. The variable capacitor $C$ shown is used to eliminate the capacitive pickup of the transmission line, which can be considerable.

The probe is powered by a KEPCO amplifier power supply that is capable of supplying up to \pm 500 volts. The ramp signal is a triangular wave generated by a function generator. The scanning frequency was found to be optimum at approximately $1 \mathrm{kHz}$.

The power supply itself must be electrically isolated from the probe to minimize the plasma feedback to the supply. Two 1:1 isolation transformers are used for this purpose, and the drive circuitry is shown in the bottom half of Figure 2-10

\subsubsection{Interferometer}

\section{INTERFEROMETER SETUP}

The interferometer used on the PPEX is a heterodyne transceiver system. The interferometer system is comprised of a Gunn Oscillator, a double-balanced mixer, a ramp generator providing a fixed sweeping frequency $(80 \mathrm{KHz})$ to the Gunn Oscillator, a constant voltage supply that provides both $\pm 5 \mathrm{~V}$ and $\pm 15 \mathrm{~V}$, and a Jorway 1808 Phase Detector. The interferometer used has a $35 \mathrm{GHZ}$ base frequency oscillator, although a $60 \mathrm{GHz}$ unit is also available. The waveguides used in the system are either the WR-28 type (35GHZ oscillator) or the WR-15 (60GHZ). The reference leg of the interferometer is simulated by taking a -10dB signal from the plasma leg through a phase shifter inside the interferometer box.

The microwave transmitter-receiver geometry for the PPEX machine is shown in Figure 2-11. As is seen, the receiver horn $(+20 \mathrm{~dB})$ is place inside the vacuum and samples the microwaves that pass through the plasma. A phase-shifter is inserted into the return leg of the plasma-leg receiver to compensate for the different 

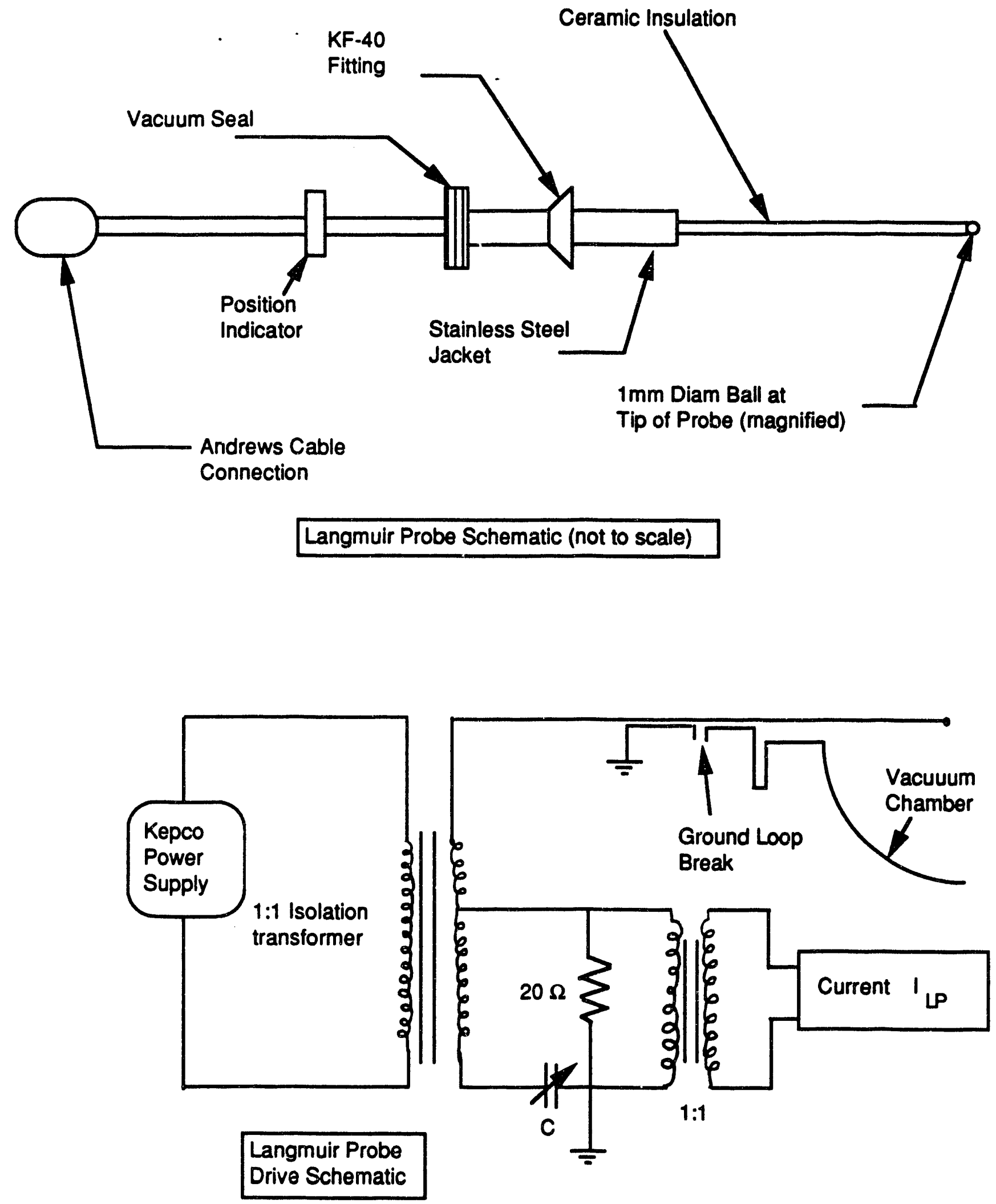

Figure 2-10: Standard Lamguir Probe Schematic as described by Labombard [24] 
lengths of travel between the plasma leg and the vacuum leg.

\subsubsection{Spectrometer}

\section{SPECTROMETER SETUP}

The spectrometer system consists of a $0.5 \mathrm{~m}$ focal length Ebert scanning spectrometer (Jarrel Ashe Model 82000 Spectrometer), a photomultiplier assembly, a high voltage power supply, a rotating mirror assembly, and an Arora A12 digitization module. The system schematic is shown in Fig. 2-12.

The spectrometer has an inlet slit width of $25 \mu$ and an adjustable outlet slit coupled to a photomultiplier detector. Optimum results were obtained for an outlet slit width of $25 \mu$.

The base unit spectrometer is coupled to an RCA 1P28 photomultiplier tube assembly powered by a high voltage (negative) power supply operating between 700-1000V. Calibration was performed using both a hydrogen lamp $\left(H_{\beta}\right.$ line at $4863 \AA$ ) and a mercury lamp ( $\mathrm{Hg}$ line at $4358.3 \AA$ ).

The scanning rotating mirror assembly consists of a glass slide mounted atop a variable speed motor that is driven at $9 \mathrm{~V}$. One rotation of the mirror corresponds to two scans of $14 \AA$ each. The minimum scan period is about $3 \mathrm{~ms} / \mathrm{scan}$. This corresponds to a motor rotation rate of about $160 \mathrm{~Hz}$. This $3 \mathrm{~ms}$ scan period would then allow a maximum of about 5-10 scans during the ICRF heating period of $30 \mathrm{~ms}$.

The light from the discharge is led to the spectrometer via either fiber-optic line or a focusing lens. Care must be taken to remove the spectrometer sufficiently far away from the machine lest the fringing magnetic field distort the data obtained.

The light to be examined with this scanning spectrometer system was selected to be one that reflects most accurately the state of the plasma temperature. The first line that is observed using the spectrometer system was the H- $\beta$ line, but since it was not most reflective of the bulk plasma temperature, other lines were also examined. A good line is the C III line at $4647.4 \AA$. 


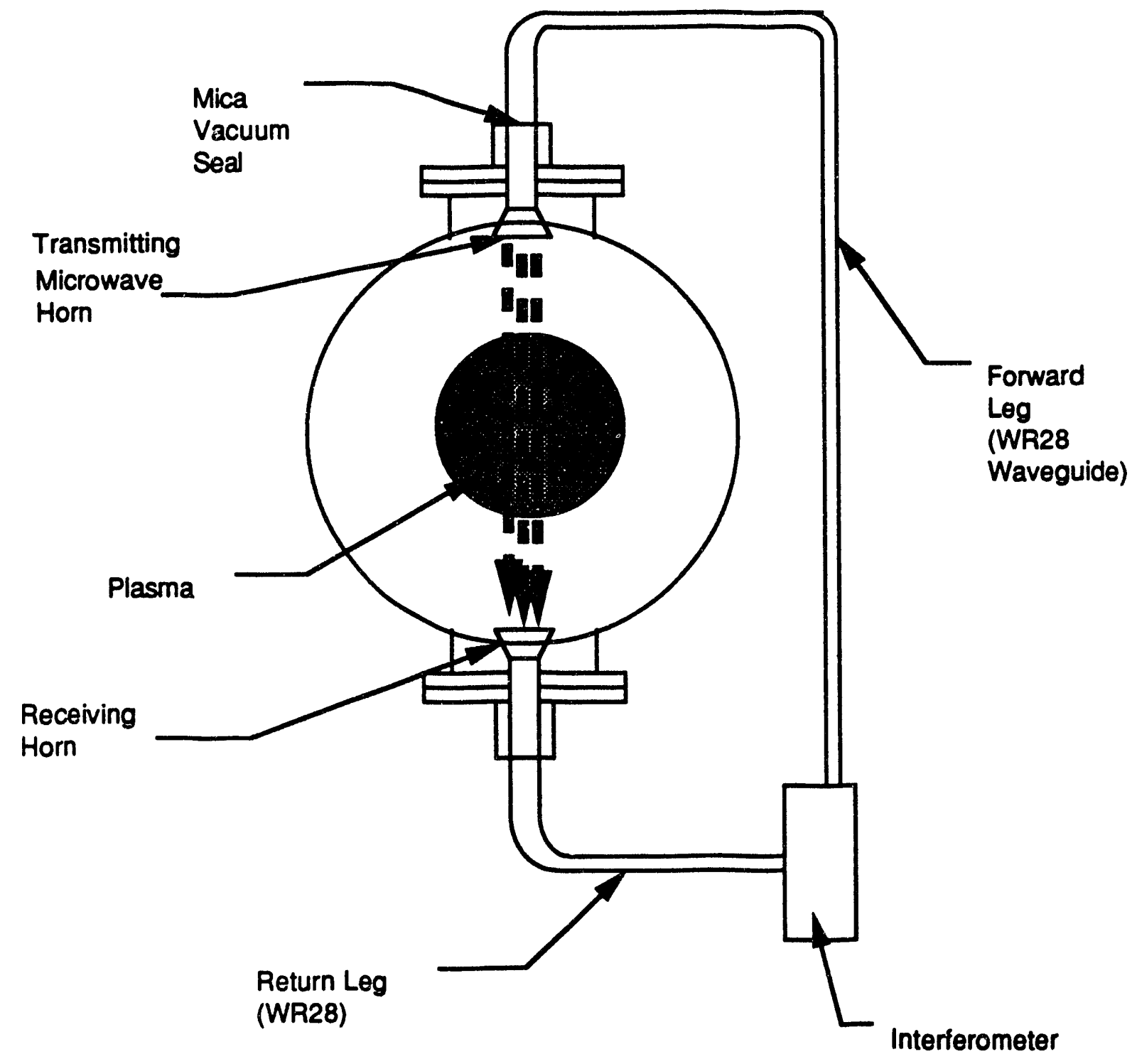

Figure 2-11: PPEX $35 \mathrm{GHz}$ Interferometer Geometry 


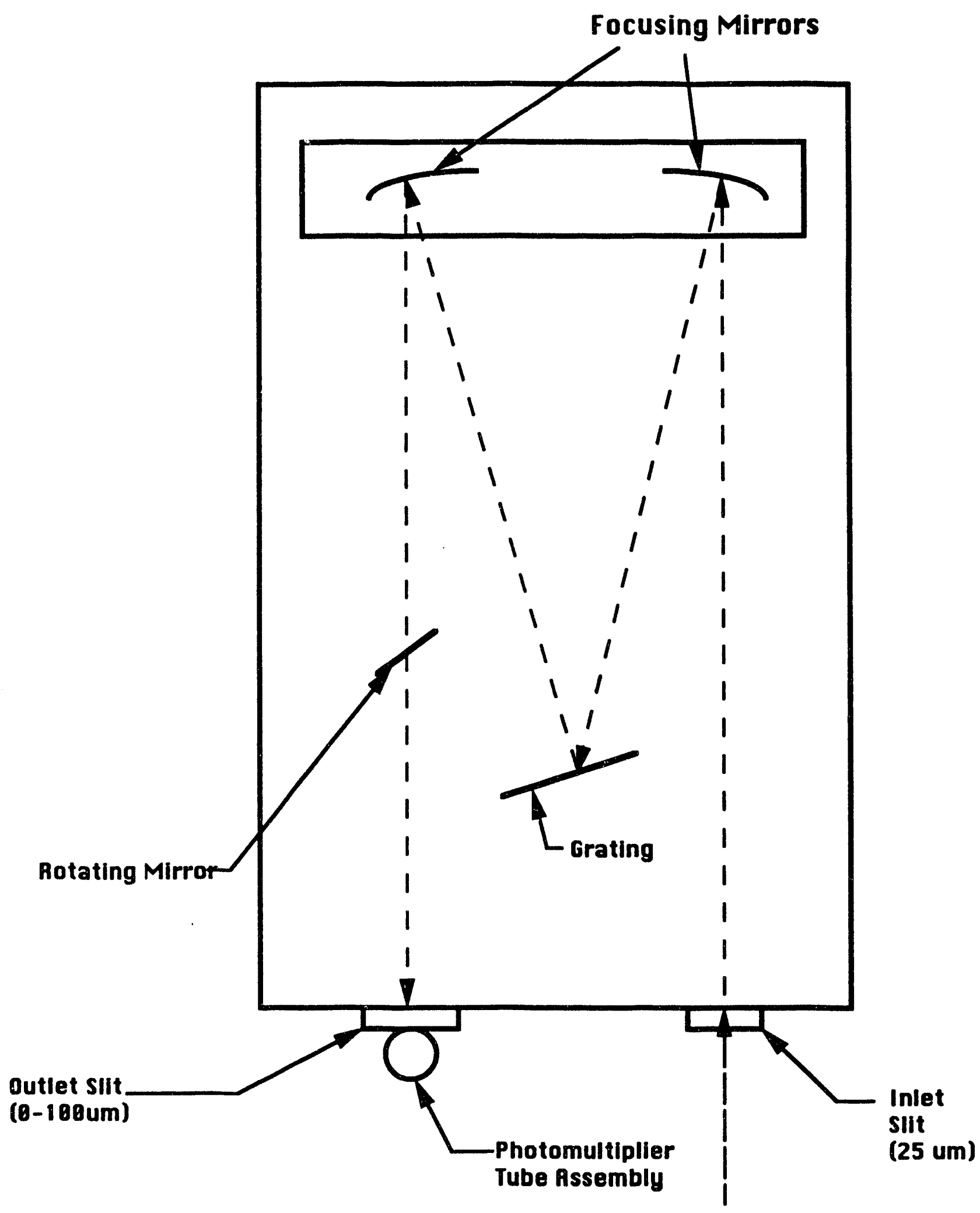

Figure 2-12: Spectrometer System Schematic. The rotating mirror is used to shift the beam by \pm 7 Angstroms so that a scan in wavelength is possible. 


\subsection{4 $H_{\alpha}$ Detector}

\section{H- $\alpha$ Setup}

The basic $H_{\alpha}$ detector used on the PPEX is a single photodetector system that was used on the TARA mirror machine. The H- $\alpha$ geometry is seen in Figure 2-13.

The $\mathrm{H}-\alpha$ detector is basically a light detector that is connected to the vacuum chamber via a KF-40 connection attached externally to a quartz window flange on one of the central cell ports. The fitting between the quartz flange and the $\mathrm{H}-\alpha$ detector is optically blackened to minimize reflection and multiple imaging.

\subsubsection{Diamagnetic Loop}

\section{Experimental Setup}

A typical diagmagnetic loop is installed in the central cell of the PPEX machine. 20 turns of vacuum compatible teflon coated wire is wound inside a hollow stainless steel cylinder that is rolled so that it surrounds the plasma. The entire loop is mounted on a set of two rails that sit at the bottom of the vacuum chamber. The signal is transmitted via a twisted pair cable to an $\mathrm{RC}$ integrator, with the result digitized by an Aurora-12 digitizer at $100 \mathrm{kHz}$.

\subsubsection{B-Dot Probe}

\section{B-Dot Probe SETUP}

The B-Dot Probes used in this thesis were constructed for the TARA Tandem Mirror Experiment and are described in detail by Chen [25]. Briefly, each probe consists of 3 orthogonal loops of double-turn insulated copper wire held together by epoxy. The 3 loops, located in the tip of the B-Dot probe, are enclosed by

a glass envelope that shields the probe from contact with the plasma. The 3 loops are also electrostatically shielded by a set of copper covering that is gapped to allow penetration of the desired electromagnetic fields but which exclude the 


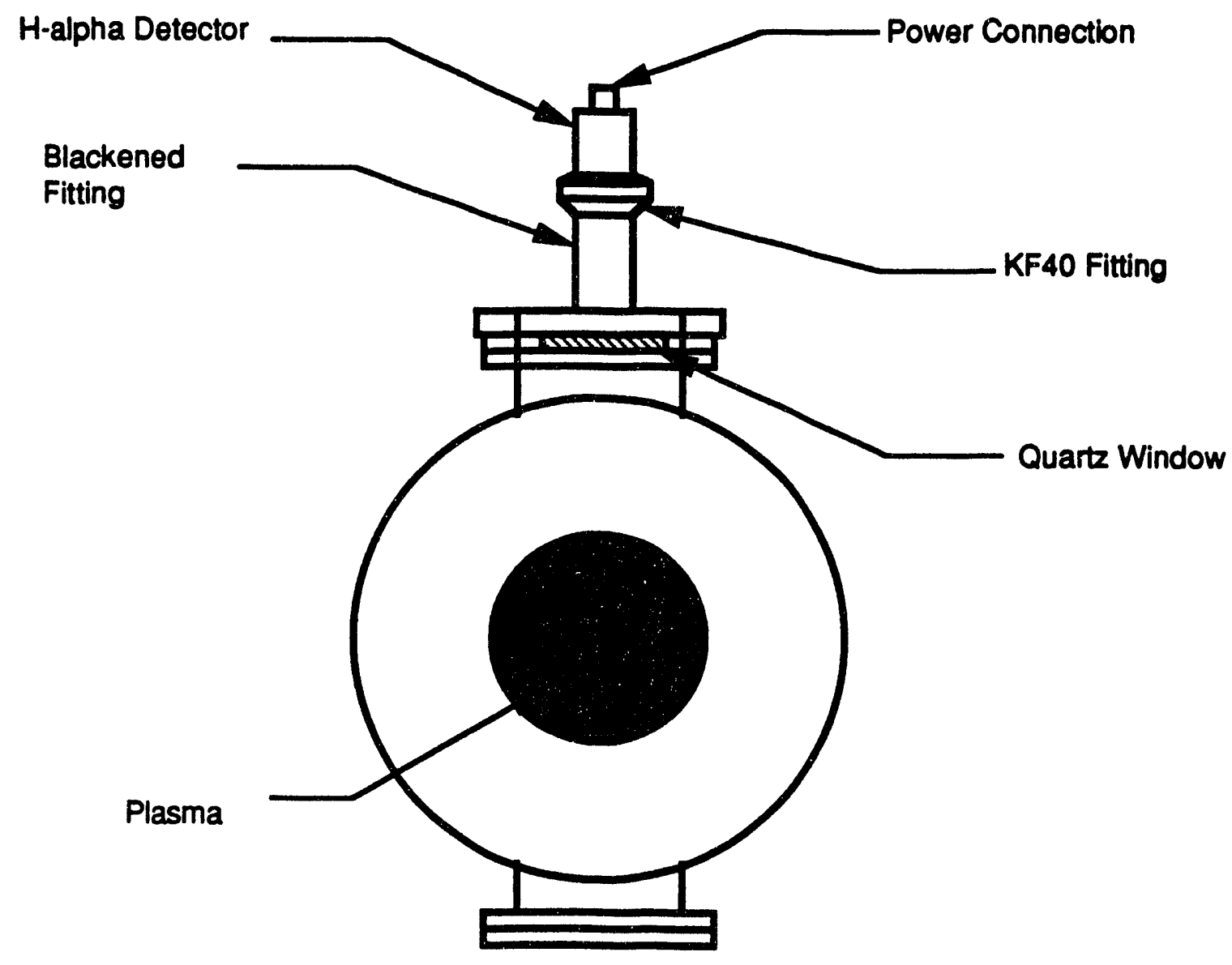

Figure 2-13: Ha Detector Schematic 
electrostatic fields. The basic schematic of the B-Dot Probe (BDP) can be seen on Figure 2-14. 


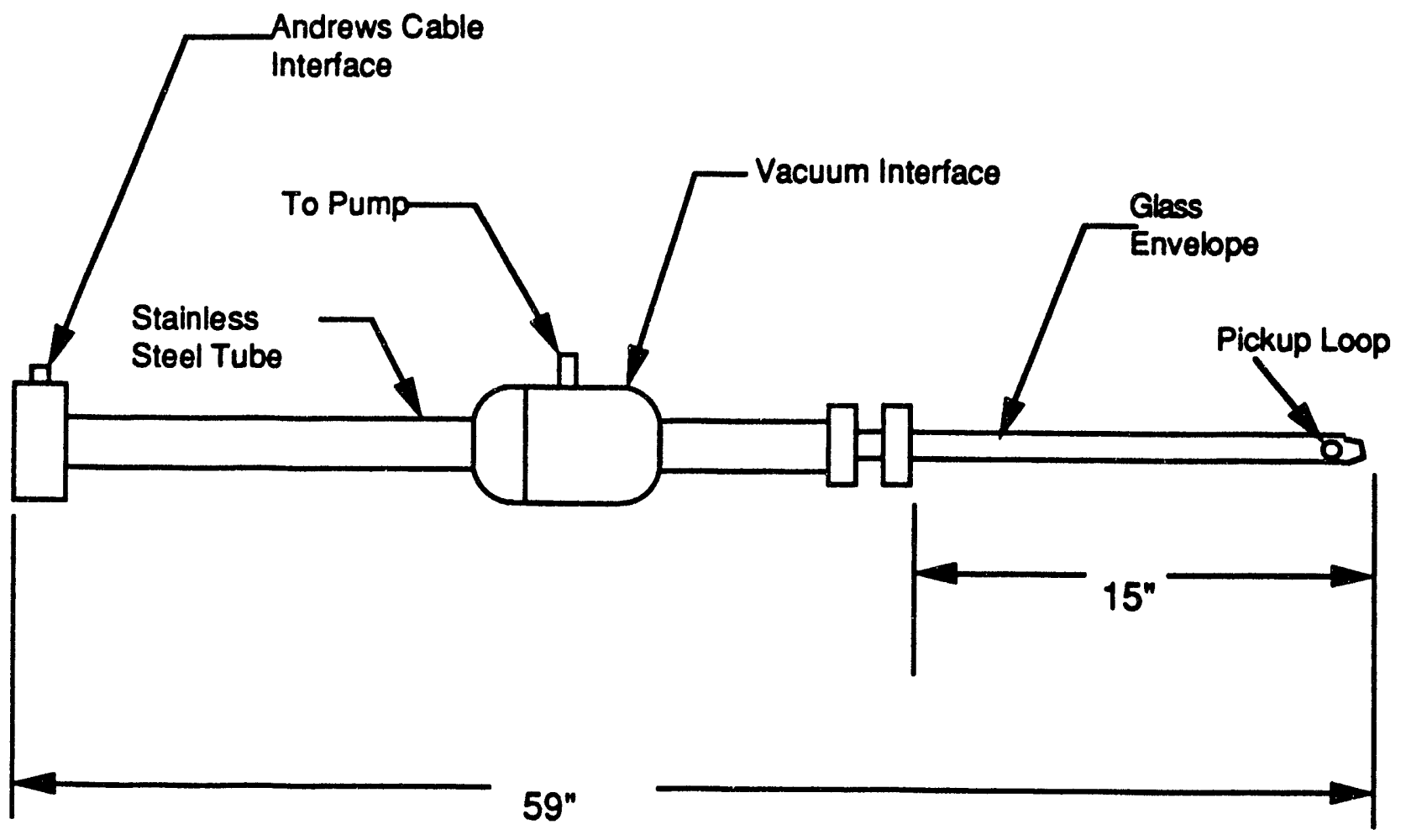

Note: Figure not to scale

Figure 2-14: B-Dot Probe Schematic. The probe can be moved radially in or out of the plasma without breaking vacuum. The pickup loop at the tip of the probe has a diameter of $1 / 4$ inch. 


\subsection{Data Acquisition}

To coordinate the flow of information from the various diagnostics, a CAMAC Data Acquisition System was set up. The CAMAC modules currently used on the PPEX are shown in Table 2.4.

Table 2.4: CAMAC Modules Used on the PPEX

\begin{tabular}{|c|c|c|}
\hline CRATE & Characteristic & Purpose \\
\hline Jorway 221 & 12 Channel Trigger/Gate output & $\begin{array}{l}\text { Sequencing Module controls } \\
\text { Magnet On-Time, ICH on-time, } \\
\text { DAQ Module triggering. }\end{array}$ \\
\hline Aurora 12 & $\begin{array}{l}8 \text { Channel } 0-100 \mathrm{KHz} \text { A-D } \\
\text { Convertor }\end{array}$ & $\begin{array}{l}\text { Digitize Diamagnetic Loop } \\
\text { voltage, Langmuir Probe Voltage, } \\
\text { other lower frequency diagnostics. }\end{array}$ \\
\hline Jorway 1808 & $\begin{array}{l}\text { Single Channel } 0-100 \mathrm{KHz} \\
\text { Phase detector }\end{array}$ & $\begin{array}{l}\text { Measures phase difference } \\
\text { between a given clock and a signal. } \\
\text { Measures interferometer phase shift. }\end{array}$ \\
\hline LeCroy 8501 & Multifrequency Clock & $\begin{array}{l}\text { Generates clock for } \\
\text { Aurora } 12 \text { for slower speed DAQ. }\end{array}$ \\
\hline LeCroy 2264 & $\begin{array}{l}8 \mathrm{Channels}, 40 \mathrm{KHz}(8 \mathrm{Ch})- \\
2 \mathrm{MHz}(1 \mathrm{Channel})\end{array}$ & Interferometer/LP \\
\hline LeCroy 8100 & Programmable Amplifier & $\begin{array}{l}\text { Amplifies low level signals. } \\
\text { Used with B-dot probe. }\end{array}$ \\
\hline LeCroy 8818 & $\begin{array}{l}100 \mathrm{MHz}, 32 \mathrm{~KB} \text { Memory } \\
\text { Single Channel A-D Converter }\end{array}$ & $\begin{array}{l}\text { Measures signal from } \\
\text { B-Dot probe. Max 512mV p-p. }\end{array}$ \\
\hline
\end{tabular}

The entire instrumentation package for PPEX is shown in Fig 2-15

The software used to tie the CAMAC instruments to the VAX is the MDS system developed at the MIT Plasma Fusion Center. The data processing is performed using the IDL [26] software by Research Systems, Inc. 


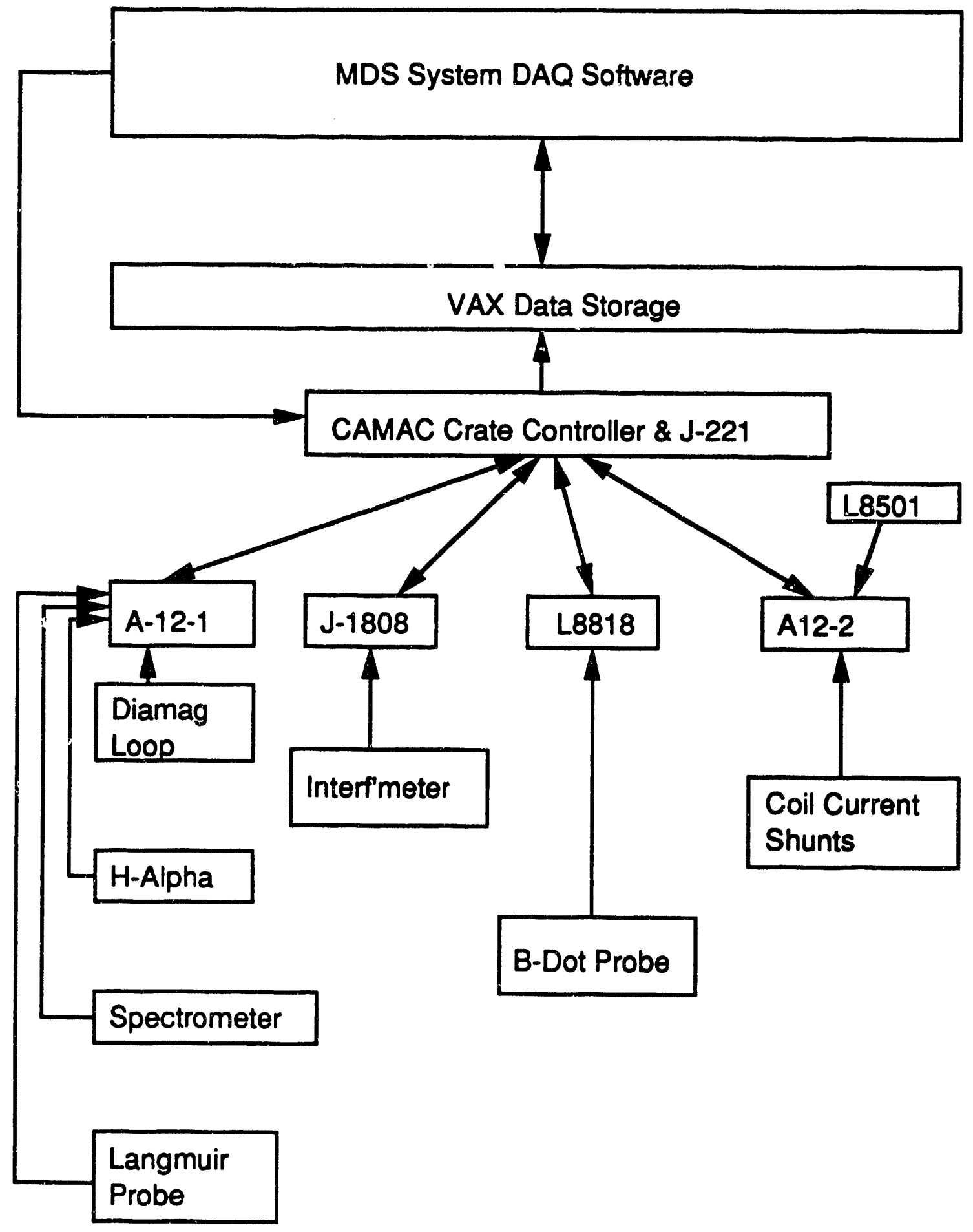

Figure 2-15: Instrumentation Schematic. The digitizers used are the A-12 $(100 \mathrm{kHz}$ Max), J-1808 (phase digitizer) and the L8818 (100MHz Max). 


\subsection{PPEX OPERATIONS}

The PPEX operates in a pulsed mode. An entire run is about 5 seconds from beginning to end. The mean time between shots (MTBS) is approximately 3-15 minutes, depending upon the on-time of the systems. The primary issue of concern of the runs is the duty cycle of the equipment. Specifically, if the magnets were being forced-air cooled, the MTBS is limited to greater than 10 minutes due to the resistive heating of the magnets. If the magnets were being cooled by vapor/liquid nitrogen, the MTBS could be much less ( 5 minutes).

\subsubsection{PRE-SHOT MODE}

When no shots are being run on the PPEX machine, the vacuum chamber is pumped down to a pressure of $10^{-7}$ Torr to minimize the stray gas and water vapor in the chamber. The pre-shot sequence of events and checklist typically proceeds as follows:

1. Energize $13.8 \mathrm{kV}$ magnetic power supply.

2. Warm up ECRH source (warmup time 1 minute) and ICRF supply (warmup time 20 minutes).

\section{Energize diagnostic power supplies}

4. Close turbomolecular pump gate valves and use needle valve to puff in controlled amounts of gas - either hydrogen, helium or argon.

5. Open up bypass valve to the turbopumps to do flow pumping, maintaining the pressure at about $7-10 \times 10^{-5}$ Torr.

\subsubsection{SHOT MODE}

The magnet coils are first pulsed on at time $t=0 \mathrm{~ms}$. These magnets have a typical magnet rise time of about $200 \mathrm{~ms}$. The $\mathrm{ECH}$ unit is turned on at $100 \mathrm{~ms}$ to generate 
a source of pre-ionized plasma. Because of the frequency mismatch of the ECH mentioned before, one ionization gauge is always kept energized to provide a source of free electrons for ionization. At $600 \mathrm{~ms}$, the ICRF unit is gated on to provide up to $100 \mathrm{~kW}$ of ICRF heating power for about $10-30 \mathrm{~ms}$. Data Acquisition normally occurs between $500-800 \mathrm{~ms}$. The system is then powered down at $1000 \mathrm{~ms}$. 


\subsection{Systems Designed and Fabricated}

This section describes the systems that were designed and fabricated as part of this thesis. The experiment itself was designed and constructed through the collaboration of 2 staff and 2 graduate students (including the author).

\subsubsection{Magnet}

The work performed in this thesis includes designing the magnets of PPEX using the EFFI code. Field and stress calculations were performed to ensure that the magnets could withstand the hoop stress. The magnets were also tested using a current source as well as applying a high voltage source (Hi-poting) to make sure that there is no unintentional grounds and that the insulation is adequate. Finally the magnets were assembled onto the machine.

\subsubsection{ICRF System}

Various parts of the ICRF system were designed. These are given in Table 2.5.

\subsubsection{Diagnostics}

Many diagnostics were acquired from the TARA program. However, much customization, testing, and adaptation of the various diagnostics were required.

\subsubsection{Instrumentation}

When the PPEX machine first started acquiring data, only two oscilloscopes and a film camera were available to take data. Part of the work performed in this thesis was to acquire, assemble, and integrate CAMAC modules into a coherent data acquisition system. Using the MDS software written at MIT, a software package was written to automatically trigger modules, acquire data from modules, and store the data in accessible form. Programs to process these data were also 
Table 2.5: ICRF Subsystems Designed and Constructed

\begin{tabular}{|c|c|}
\hline COMPONENT of ICRF SYSTEM & WORK PERFORMED \\
\hline Antenna & $\begin{array}{l}\text { Design and fabricated both ICRF antennas } \\
\text { used in the center cell and end cell. } \\
\text { Design emphasized ease of assembly within small } \\
\text { space of vacuum chamber. Design also must be } \\
\text { compatible with existing vacuum feedthru. } \\
\text { Fabrication using copper sheet metal was } \\
\text { performed at the MIT Machine Shop }\end{array}$ \\
\hline Vacuum Feedthru & $\begin{array}{l}\text { The existing vacuum feedthru from the TARA } \\
\text { machine were tested and altered to match } \\
\text { the vacuum ports and antenna geometry }\end{array}$ \\
\hline Matchbox & $\begin{array}{l}\text { Existing matchbox were fitted with special } \\
\text { extensions and connectors to connect antenna } \\
\text { to matchbox. Designed and constructed suppor } \\
\text { structures to hold the matchbox ( 100lbs) } \\
\text { in place against the side of the PPEX machine. } \\
\text { Matchboxes were also customized with different } \\
\text { vacuum capacitors to the desired ICRF frequency }\end{array}$ \\
\hline
\end{tabular}


Table 2.6: Work Performed on Diagnostics Set

\begin{tabular}{|c|c|}
\hline Diagnostic & Work Performed \\
\hline Interferometer & $\begin{array}{l}\text { Assembled set of waveguide path and phase } \\
\text { shifter. Constructed feedthrus and made design } \\
\text { modifications to the KF- } 40 \text { feedthru to enable insertion } \\
\text { of microwave horns into vacuum }\end{array}$ \\
\hline H- $\alpha$ Detector & $\begin{array}{l}\text { Designed and assembled system to detect } \\
\text { H- } \alpha \text { light emissions from plasma. A focusing system } \\
\text { using a lens and a blackened feedthru tube was constructed. }\end{array}$ \\
\hline Langmuir Probe & Designed circuit to drive langmuir probe. \\
\hline Spectrometer & $\begin{array}{l}\text { Constructed scanning system to scan } \pm 7 \mathrm{~A} \\
\text { using an existing interferometer. Calibrated and alligned } \\
\text { spectrometer using mercury lamp and laser. }\end{array}$ \\
\hline B-Dot Probe & Reconditioned and calibrated existing B-Dot probes \\
\hline
\end{tabular}


written. These programs include FFT routines to process the Fourier spectrum of B-dot probe data, routines to process the interferometer phase data, and routines to analyze the Langmuir probe data. 


\subsection{Summary}

In summary, this thesis contributed to the design, construction and operation of a small tandem mirror machine at MIT. The purpose of this machine (as pertaining to this thesis) is to study ICRF wave propagation phenomena. The major systems used in the study of ICRF propagation (antenna, b-dot probe, digitizers, software) were all either designed and constructed from scratch or customized as part of this thesis.

In constructing the PPEX machine, many existing systems and parts were utilized to save time and reduce the cost of construction. The result is an experiment that is constructed inexpensively, versatile enough to perform many physics studies yet small enough that a 2 man crew could effectively run the entire machine. 


\section{Chapter 3}

\section{ICRF THEORY}

This chapter discusses the theoretical analysis performed in the thesis. The first section provides a brief survey of some historical ICRF literature and points out the motivation for performing the theoretical analysis. The next section introduces the basic equations to be solved describes in general terms the steps required to obtain the solution. Next, the basic assumptions underlying the cylindrically bounded ICRF wave propagation problem are addressed in Section 3.3. The geometry of the axially varying cylindrical system is also introduced. Section 3.4 solves for the source fields that are generated by the dipole loop launching antenna. Section 3.5 then solves for the total self consistent vacuum fields. The boundary conditions that link the vacuum, source, and plasma fields are discussed in Section 3.6. Next, the functional dependence of the plasma fields are derived in Section 3.7. The code that is developed as part of this work is benchmarked and compared to literature in Section 3.8. Finally, the ICRF field profiles and other results obtained from the axially varying cylindrical analysis performed are discussed in Section 3.9.

The analysis described in this chapter contains significant features and approximations which were made in order to simplify the algebra. A slow $z$ approximation reduces the coupled differential equations to two integral equations which are relatively straight forward to solve. Several coordinate transformations are 
also required to reduce the complex boundary of a varying radius plasma cylinder to a simple boundary. The almost-TM mode is neglected in order to treat the almost-TE mode in a coherent manner. The combination of both the slow-z approximation and the coordinate transformation also allows a large portion of the theory to be treated analytically. Finally, because of the complications generated by the axial inhomogeneity of the system, this theory assumes a cold plasma with collisions and a sharp boundary radial profile model in order to simplify the rest of the calculations.

The power of the analysis is evident even in spite of these limitations and simplifications. The theory treats the resonance poles in a coherent fashion, allowing the propagation of waves past the resonance locations. The code also allows the computation of the ICRF EM fields for arbitrary mirror ratios, arbitrary $\beta$ (subject to the cold plasma approximation). Finally, it treats the axial inhomogeneity and the cylindrical geometry of the system in a self consistent manner that has not been accomplished before.

Note that the problem of the full slab model with $k_{z}=0$ is examined in Appendix D.1. This simple analysis is shown for the purpose of providing a flavor of the method of solution that will be followed in the general cylindrical case. A $k_{z} \neq 0$ analysis for a full slab model is also developed and presented in Appendix D.2 for the sake of completeness. It is seen that $k_{z} \neq 0$ introduces coupling between otherwise decoupled TE and TM modes. 


\subsection{Motivation for Study}

The ICRF wave-plasma coupling problem is one that has been often examined during the past 30 years. Among these the pioneering theoretical work of Stix[2, 27, 28, 29], Swanson[30], and Bernstein [31] along with the experiments performed on B-66 $[3,32]$ and the C-Stellerator $[33,34]$ in the early and late 60's paved the way for ICRF as it is generally studied today. However, there are yet some problems in ICRF that remain largely unsolved. One major issue that has not been adequately addressed is the propagation characteristics of ICRF waves in an axially inhomogeneous magnetic field in a cylindrical geometry. More recent tokamak [35, 36] or mirror [1] ICRF studies, though sophisticated, often sacrifice either field inhomogeneity or cylindrical geometry in order to render the problem analytically solveable. Seldom are both subjects treated together coherently. Since both the presence of a magnetic beach and the cylindrical geometry of the system affect ICRF propagation characteristics in a linear magnetic mirror, overlooking either the nonuniform $B_{z}$ or the cylindrical nature of the system can result in a distorted view of ICRF wave propagation and heating. The thrust of this chapter, is thus to examine ICRF wave propagation and plasma heating in an axially varying cylindrical geometry including a resonance beach. By studying ICRF wave propagation characteristics in realistic physical and magnetic geometries, one can better understand the process of ICRF beach heating.

Much work on mode conversion and resonance tunneling $[37,38,39]$ has also been performed over the last thirty years and is summarized in a review article by Swanson [40]. The theoretical work performed in the thesis does not consider those effects because of the regime to which the analysis is applied. Specifically, the work performed in this chapter assumes a cold plasma with collisions - conditions which are valid for the plasmas encountered in the MIT PPEX linear mirror machine. The implication is that the plasma regime of interest is too cold for finite temperature effects or tunneling to be important. However, the theoretical 
work performed here could conceivably be modified and extended to include these additional effects. This is further discussed in Chapter 6.

It should be stressed that the unique aspect of the work undertaken in this chapter is the inclusion of the axially varying magnetic field geometry in an analytical treatment of the ICRF wave propagation problem. It is a difficult problem that has historically been unsatisfatorily treated, and the work performed here offers a good starting point to achieve the inclusion of both the magnetic field inhomogeneity and the cylindrical geometry in ICRF wave-plasma coupling calculations. 


\subsection{ICRF Background Formulation}

Recent conventional tokamak ICRF studies such as ones by McVey [41] and Bers [42] use ray-tracing techniques because of the assumption of relatively small wavelength of the ICRF wave as compared to the plasma radius. However, for the magnetic mirror geometry under study, the magnetic field is such that $\lambda \geq a$. This rules out the use of ray-tracing techniques in the analysis, particularly for the cylindrical geometry case. For the cylindrical geometry studied here, the general approach of Lam [43] is adopted in which a combination of radial standing wave and axial propagating wave is selected as the form of the field solutions.

\subsubsection{Standard Formulation}

The basic formulation of the ICRF plasma coupling problem begins with the standard approach using Maxwell's Equations The technique that is commonly used in analyses of homogeneous systems is the Fourier transform in time:

$$
\frac{\partial}{\partial t} \rightarrow-i \omega
$$

Maxwell's equations can then be written as

$$
\nabla \times \vec{B}=\frac{-i \omega}{c^{2}} \underline{\underline{\epsilon}} \cdot \vec{E}
$$

where $\underline{\underline{\epsilon}}$ is known as the dielectric tensor and is given in vacuum by [44]

$$
\underline{\underline{\epsilon}} \equiv\left[\begin{array}{lll}
1 & 0 & 0 \\
0 & 1 & 0 \\
0 & 0 & 1
\end{array}\right]
$$

In a cold plasma, $\underline{\underline{\epsilon}}$ is given by [45]

$$
\underline{\underline{\epsilon}} \equiv\left[\begin{array}{ccc}
S & -i D & 0 \\
i D & S & 0 \\
0 & 0 & P
\end{array}\right]
$$


For a warm or hot plasma, Stix [2] showed that the zero terms in the tensor given above become non-zero. The theoretical analysis taken in this chapter assumes a cold plasma with collisions.

The elements of the dielectric tensor $\underline{\underline{\epsilon}}$ for finite temperature plasmas are [2]

$$
\begin{gathered}
S=\frac{1}{2}(R+L) \\
D=\frac{1}{2}(R-L) \\
R=1+\sum_{\alpha} \frac{\omega_{p \alpha}^{2}}{\omega k_{z} v_{\alpha}} Z\left(\xi_{1}\right) \\
L=1+\sum_{\alpha} \frac{\omega_{p \alpha}^{2}}{\omega k_{z} v_{\alpha}} Z\left(\xi_{-1}\right) \\
P=1-\sum_{\alpha} \frac{\omega_{p \alpha}^{2}}{\left(k_{z} v_{\alpha}\right)^{2}} \frac{Z^{\prime}\left(\xi_{0}\right)}{1+\frac{i q_{\alpha}}{h_{z} v_{\alpha}} Z\left(\xi_{0}\right)}
\end{gathered}
$$

where

$$
\xi_{n}=\frac{\omega+i \nu_{\alpha}+n \omega_{c \alpha}}{k_{z} v_{\alpha}}
$$

Where $\nu_{\alpha}$ is the collision frequency. $Z\left(\xi_{n}\right)$ is the plasma dispersion function as documented by Fried and Conte [46]. Its definition and expansion in the limit of a cold plasma is shown in Appendix I.

The power density absorbed by the plasma is determined by the induced plasma current flowing in phase with the electric field $[44,47]$

$$
\begin{gathered}
p_{a b s}\left(W / m^{3}\right)=\frac{1}{2} \operatorname{Re}\left(\vec{E} \cdot \vec{J}^{*}\right) \\
P_{a b s}(W)=\frac{1}{2} \operatorname{Re}\left(\int \vec{E} \cdot \vec{J}^{*} d V\right)
\end{gathered}
$$

The goal of the theoretical analysis is to study the propagation characteristics of ICRF waves in a bounded plasma-vacuum system and to determine optimal coupling of the wave to the plasma. To determine optimal coupling, a figure of merit is required for comparison. One that is mostly commonly used as a figure of merit in coupling calculations is the plasma impedence $R_{p l}$, where

$$
R_{p l} \equiv \frac{2 P_{a b}}{I^{2}}
$$


Thus one of the goals of this study is to determine how to optimize $R_{p l}$. The steps to be taken in the solution of the fields and subsequently the determination of $R_{p l}$ are outlined in the next section.

\section{Infinite Plasma Dispersion Relation}

The infinite plasma dispersion relation is given by $[2,45]$

$$
S k_{\perp}^{4}+k_{\perp}^{2}\left[k_{z}^{2}(S+P)-k_{0}^{2}(S P+R L)\right]+P\left(k_{z}^{2}-k_{0}^{2} R\right)\left(k_{z}^{2}-k_{0}^{2} L\right)=0
$$

For each value of $k_{z}$, there exists two $k_{\perp}$ roots, referred to as the "Almost TM" Mode (large $k_{\perp}$ ), and the "Almost TE" Mode (smaller $k_{\perp}$ ). These are shown in Fig. 3-1. The analysis performed here considers only the "almost TE" mode and neglects the "almost TM" mode in order to minimize the algebra involved. The "almost TM" mode couples almost all of its energy to the electrons since its $E_{z}$ is mostly given up to the electrons in the plasma. Therefore, the result of neglecting this "almost TM" mode is to neglect the fact that a significant portion of the ICRF energy is given up to the electrons, thus overestimating the ion coupling.

\subsubsection{Method of Solution}

The basic steps taken in this chapter to solve for the EM fields in the plasmavacuum system are as follows:

- Solve for EM fields in vacuum - source and induced oscillations

- Obtain and solve differential equation for the functional dependence of the plasma fields using slow-z approximation and coordinate transformation

- Obtain transverse plasma and vacuum fields from Maxwell's equations.

- Take Fourier transform to permit use of plasma-vacuum boundary condition

- Use boundary conditions to solve field coefficients. 


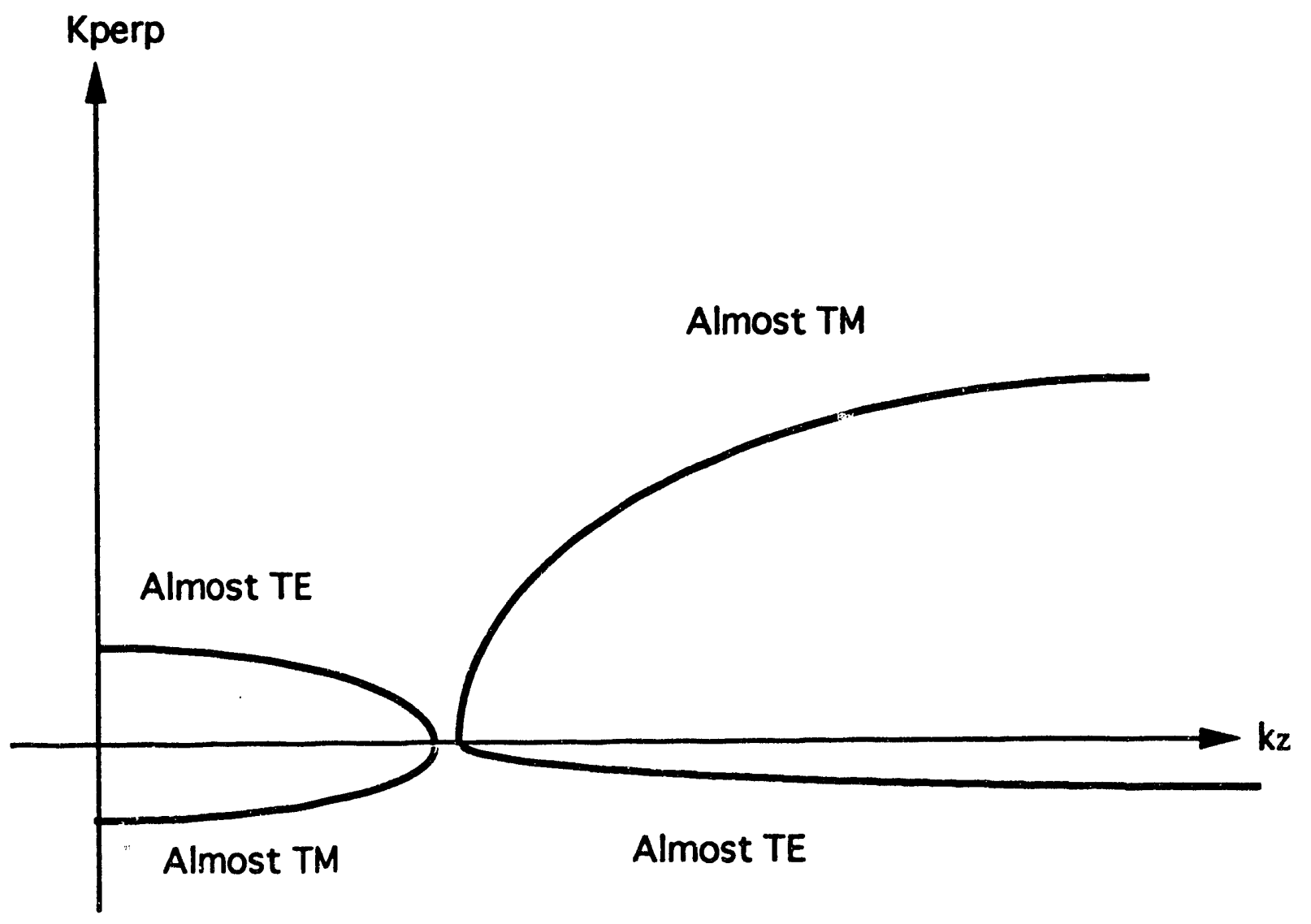

Infinite Plasma Dispersion Relation

Figure 3-1: Dispersion of ICRF Waves 
- Compute power absorbed by plasma and plasma loading impedence for different plasma and geometric parameters. 


\subsection{Assumptions and Geometry used in Axially Varying Cylindrical Analysis}

As mentioned before, the unique aspect of the work performed in this thesis is the inclusion of both the nonuniform axial magnetic field and the cylindrical geometry. In order to make the problem tenable and to place emphasis on the new approach taken to solve the problem, some assumptions are made:

1. Axial magnetic field has a finite axial variation. This means not only that $B_{z 0}=B_{z 0}(r, z)$, but also that there exists a radial field $B_{r}$ because of the zero divergence Maxwell's equation $(\nabla \cdot \vec{B}=0)$.

2. $m=0$. No azimuthal variation in the EM fields. Corresponds to a simple loop antenna. This assumption simplifies the problem and still allows the examination of the axisymmetric EM waves. The method can be generalized to $m \neq 0$.

3. $K_{3} \equiv P \rightarrow \infty, K_{3} E_{z}$ is finite, but $E_{z} \simeq 0$. This assumption implies that the $E_{z}$ field is much smaller than either the $E_{r}$ or $E_{\theta}$ fields.

4. Surface current MHD model. This model assumes a macroscopic (i.e. nonRF) surface current on the plasma-vacuum boundary. The presence of a surface current results in an MHD pressure balance that is able to support a radially constant plasma pressure profile $[48,49]$. This produces a constant radial density profile which is useful in simplifying the radial structure of the solutions.

5. Plasma medium is a cold plasma with collisions. This assumes that for the plasmas of interest, $k_{\perp} v_{t e}<\omega$. This limits the upper bound of applicable temperatures to approximately $10-100 \mathrm{eV}$.

The geometry of the cylindrical system is shown in Figure 3-2. The launching antenna structure is a simple dipole loop. 


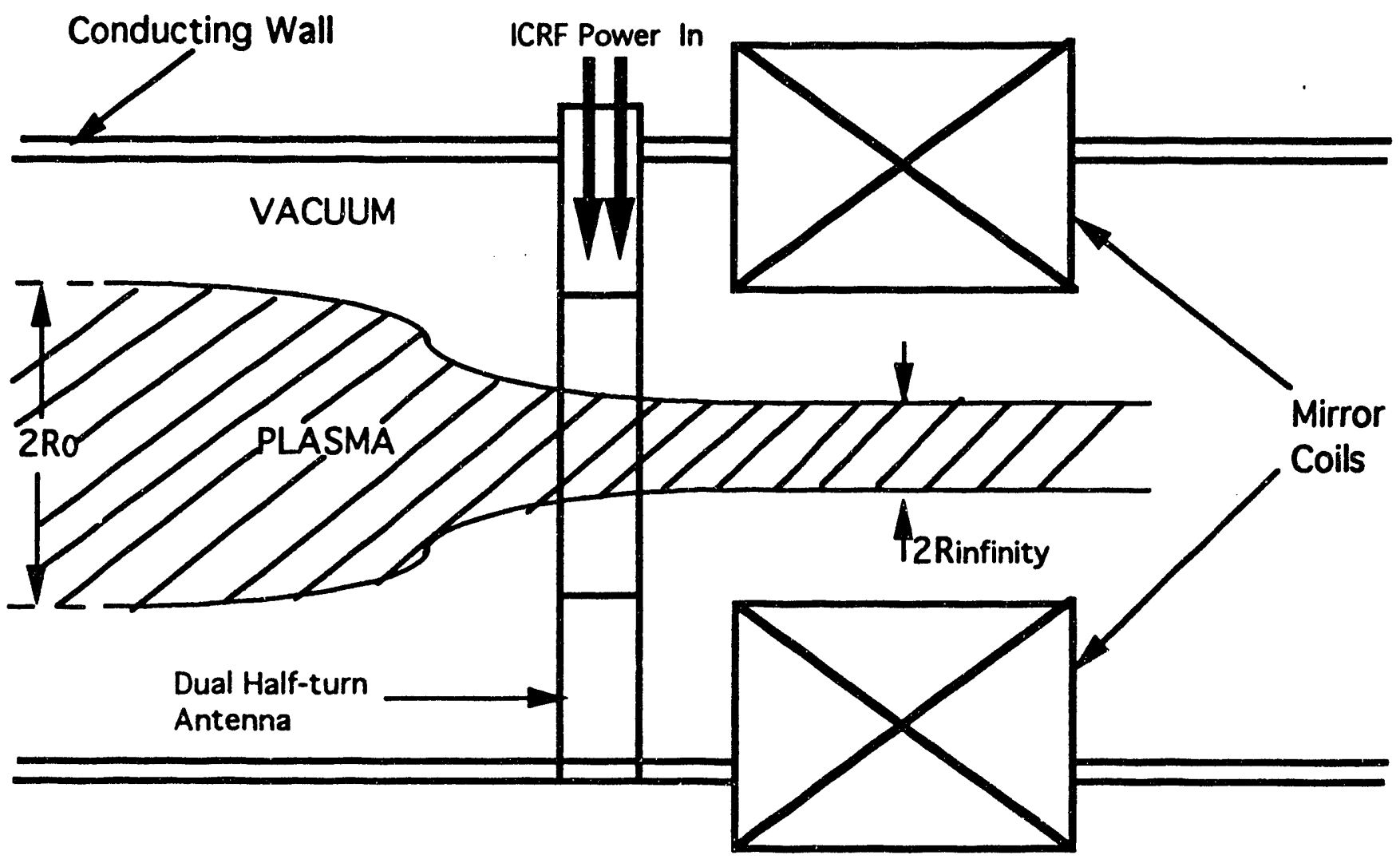

Actual Experimental Geometry of Mirror Machine

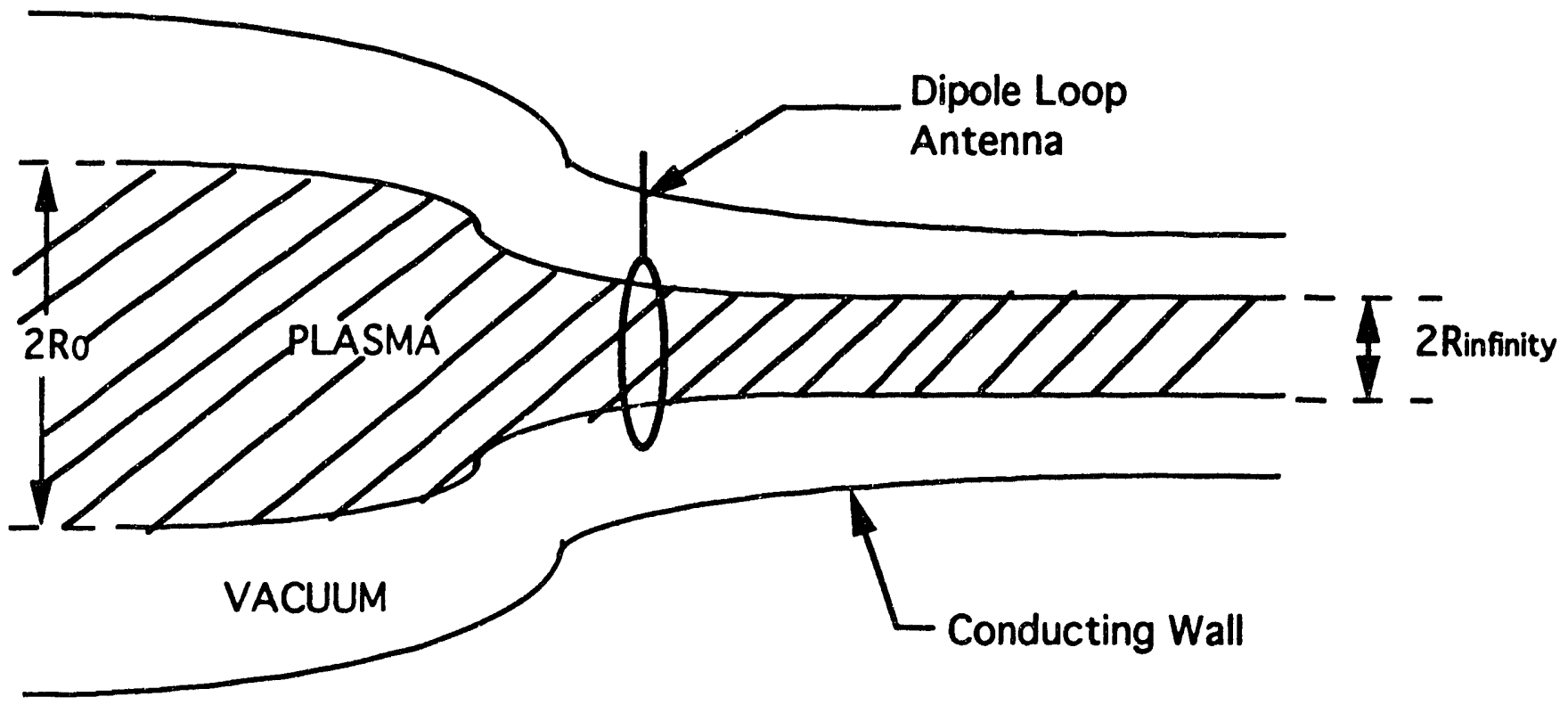

\section{Model of Actual Experimental Geometry}

Figure 3-2: Geometry of Cylindrical Plasma-Vacuum System. The actual experimental geometry is shown at top, the modelled geometry used in the analysis of this chapter is shown at bottom. The source antenna is the dual half-turn loop for the actual experiment and a dipole loop for the model. 


\subsection{External Source Fields}

The fields present in the vacuum part of the system as shown in Figure 3-2 consist of both the natural harmonics that exist within the geometry and the fields generated by the antenna source current. Consider first the source fields.

Assumption: Dipole Source

For a magnetic dipole of current I, the fields can be found by

$$
\vec{B}=\nabla \times \vec{A}
$$

where

$$
\vec{A}=\frac{\mu_{0} I}{4 \pi} \oint \frac{d \vec{l}}{r^{\prime}}
$$

For $r \gg a$

In the limit of $r \gg a$, it has been shown by Jackson [44], Kraus [50], and Lorrain [47] that the fields in spherical geometry are:

$$
\begin{aligned}
& B_{r}=\frac{\mu_{0}}{4 \pi} \frac{2 m}{r^{3}} \cos \theta \\
& B_{\theta}=\frac{\mu_{0}}{4 \pi} \frac{m}{r^{3}} \sin \theta \\
& B_{\phi}=0
\end{aligned}
$$

where $\phi$ is the direction of the current flow, $\theta$ is the angle from the perpendicular axis of the face of the current loop to the radial vector $\hat{r}$. However, since the geometry of the problem is cylindrical, the source fields in cylindrical geometry must be calculated.

The vector potential is given by

$$
\vec{A}=\frac{\mu_{0} I}{4 \pi} \int \frac{\overrightarrow{d l}}{r^{\prime}}
$$

It can be shown that this is equivalent to

$$
\vec{A}=\frac{\mu_{0}}{4 \pi} \frac{\vec{m} \times \hat{r}}{\left(r^{\prime}\right)^{2}}
$$


where $\hat{r}$ is the unit vector from the origin to the observer, $r^{\prime}$ is the distance from the origin to the observer, and $\vec{m}$ is the dipole moment of the loop. $\vec{r}$ and $\vec{m}$ are given by

$$
\begin{gathered}
\hat{r}=\frac{r}{\sqrt{r^{2}+z^{2}}} \hat{r}+\frac{z}{\sqrt{r^{2}+z^{2}}} \hat{z} \\
\vec{m} \equiv \pi a^{2} I \hat{z}
\end{gathered}
$$

The vector potential $\vec{A}$ is then calculated to be:

$$
\begin{gathered}
\vec{A}=\frac{\mu_{0} a^{2} I}{4\left(r^{2}+z^{2}\right)^{3 / 2}}(r \hat{\theta}) \\
\left(r^{\prime}\right)^{2}=\left(r^{2}+z^{2}\right)
\end{gathered}
$$

Recall that the magnetic field generated by this source loop is defined as

$$
\vec{B}=\nabla \times \vec{A}
$$

After some algebra, it can be shown that the source fields of the simple dipole loop for $r \gg a$ are given by

$$
\begin{aligned}
& B_{r, \text { ocource }}=\frac{3 \mu_{0} a^{2} I r z}{4\left(r^{2}+z^{2}\right)^{5 / 2}} \\
& B_{\theta, \text { oource }}=0 \\
& B_{z, \text { ocource }}=\frac{\mu_{0} a^{2} I}{4\left(r^{2}+z^{2}\right)^{3 / 2}}\left[2-\frac{3 r^{2}}{\left(r^{2}+z^{2}\right)}\right] \\
& E_{r, \text { ource }}=0 \\
& E_{\theta, \text { oource }}=\frac{i \omega \mu_{0} a^{2} I}{4} \frac{r}{\left(r^{2}+z^{2}\right)^{3 / 2}} \\
& E_{z, \text { oource }}=0
\end{aligned}
$$


For distances at which $r \sim a$, where $a$ is the radius of the dipole loop, the above derivation is invalid. The treatment of Jackson[44] is used. Jackson showed that the vector potential for a current dipole loop is given (in spherical coordinates) by elliptic integrals $\mathrm{K}$ and $\mathrm{E}$ as follows:

$$
\begin{gathered}
A_{\phi}=\frac{4 \mu_{0} I a}{\sqrt{a^{2}+\mathcal{P}^{2}+2 a \mathcal{P} \sin \theta}}\left[\frac{\left(2-k_{A}^{2}\right) K\left(k_{A}\right)-2 E\left(k_{A}\right)}{k_{A}^{2}}\right] \\
k_{A}^{2}=\frac{4 a \mathcal{P} \sin \theta}{a^{2}+\mathcal{P}^{2}+2 a \mathcal{P} \sin \theta}
\end{gathered}
$$

where $E$ and $K$ are the Complete Elliptic Integrals tabulated by Abramowitz and Stegun [51], and $\mathcal{P}$ is the radial distance from the origin to the observer point in spherical geometry.

Expressing this in cylindrical geometry:

$$
\begin{gathered}
r \equiv \mathcal{P} \sin \theta \quad ; \quad \mathcal{P}^{2}=r^{2}+z^{2} \\
\theta \equiv \phi \\
A_{\theta}=\frac{4 \mu_{0} I a}{\sqrt{a^{2}+r^{2}+z^{2}+2 a r}}\left[\frac{\left(2-k_{A}^{2}\right) K\left(k_{A}\right)-2 E\left(k_{A}\right)}{k_{A}^{2}}\right]
\end{gathered}
$$

where

$$
k_{A}^{2} \equiv \frac{4 a r}{a^{2}+r^{2}+z^{2}+2 a r}
$$

Given the vector potential $\vec{A}$, the induced magnetic field can be found by

$$
\begin{aligned}
\vec{B} & =\nabla \times \vec{A} \\
B_{r} & =\frac{\partial A_{\theta}}{\theta_{\theta}} \\
B_{\theta} & =0 \\
B_{z} & =\frac{1}{\partial \frac{\partial}{\partial r}\left(+A_{\theta}\right)}
\end{aligned}
$$


From the source magnetic fields, the source electric fields can also be found by Maxwell's equation

$$
\nabla \times E=-\frac{\partial}{\partial t} \vec{B}
$$

The r-component becomes

$$
\begin{aligned}
i \omega B_{r} & =-\frac{\partial}{\partial z} E_{\theta} \\
-i \omega \frac{\partial}{\partial z} A_{\theta} & =-\frac{\partial}{\partial z} E_{\theta} \\
\Rightarrow E_{\theta} & =i \omega A_{\theta}+c_{r}(r)
\end{aligned}
$$

What is $c_{r}(r)$ ? Using the z-component yields

$$
\begin{aligned}
i \omega B_{z} & =\frac{1}{r} \frac{\partial}{\partial r}\left(r E_{\theta}\right) \\
i \omega \frac{1}{r} \frac{\partial}{\partial r}\left(r A_{\theta}\right) & =\frac{1}{r} \frac{\partial}{\partial r}\left(r E_{\theta}\right) \\
\Rightarrow E_{\theta} & =A_{\theta}
\end{aligned}
$$

Therefore $c_{r}(r)=0$.

Define:

$$
\Psi \equiv r A_{\theta}
$$

\section{Change of Variable}

A change of variable is next performed here in order to simplify the boundary condition. The rationale for selecting this particular set of variables will be given in the next section.

The selected transform is:

$$
\rho \equiv \frac{r}{R(z)} \quad ; \quad d \xi \equiv \frac{d z}{R(z)}
$$

where $R(z)$ is the plasma-vacuum boundary. $k_{A}$ from Equation 3.16 then becomes

$$
\begin{aligned}
k_{A}^{2} & \equiv \frac{4 a r}{a^{2}+r^{2}+z^{2}+2 a r} \\
& =\frac{4 a \rho R(\xi)}{a^{2}+\rho^{2} R^{2}(\xi)+z(\xi)^{2}+2 a \rho R(\xi)}
\end{aligned}
$$


Therefore

$$
\Psi_{\text {wire }}(\rho, \xi)=\frac{4 \mu_{0} \operatorname{Ia\rho R}(\xi)}{\sqrt{a^{2}+\rho^{2} R^{2}(\xi)+z^{2}(\xi)+2 a \rho R(\xi)}}\left[\frac{\left(2-k_{A}^{2}\right) K\left(k_{A}\right)-2 E\left(k_{A}\right)}{k_{A}^{2}}\right]
$$

It must be noted that the $z$ here refers to the distance from the antenna location. Therefore $z \rightarrow\left(z-z_{\text {ant }}\right)$, where $z_{\text {ant }}$ is the z-location of the dipole loop antenna. $z=0$ is the center of the machine where the slope of the axial magnetic field is zero. The equation for $\Psi_{\text {wire }}$ then becomes

$$
\Psi_{\text {wire }}(\rho, \xi)=\frac{4 \mu_{0} \operatorname{Ia\rho } R(\xi)}{\sqrt{a^{2}+\rho^{2} R^{2}(\xi)+\left[z(\xi)-z_{\text {ant }}\right]^{2}+2 a \rho R(\xi)}}\left[\frac{\left(2-k_{A}^{2}\right) K\left(k_{A}\right)-2 E\left(k_{A}\right)}{k_{A}^{2}}\right]
$$

and

$$
k_{A}^{2}=\frac{4 a \rho R(\xi)}{a^{2}+\rho^{2} R^{2}(\xi)+\left[z(\xi)-z_{a n t}\right]^{2}+2 a \rho R(\xi)}
$$

Figure 3-3 shows a 3-d plot of the $\Psi$ computed for a dipole loop in vacuum. There is no plasma present in this calculation. The fall-off in the EM field far away from the antenna $(r \gg a)$ scales as $1 / r_{0}^{3}$, where $r_{0}$ is the distance from the observer to the center of the wire loop.

The Fourier transform of $\Psi$ is then given by

$$
\hat{\Psi}_{\text {wire }}\left(\rho, k_{\xi}\right)=\int \Psi_{\text {wire }}(\rho, \xi) e^{i k_{\xi} \xi} d \xi
$$




\section{PSI FOR DIPOLE LOOP}
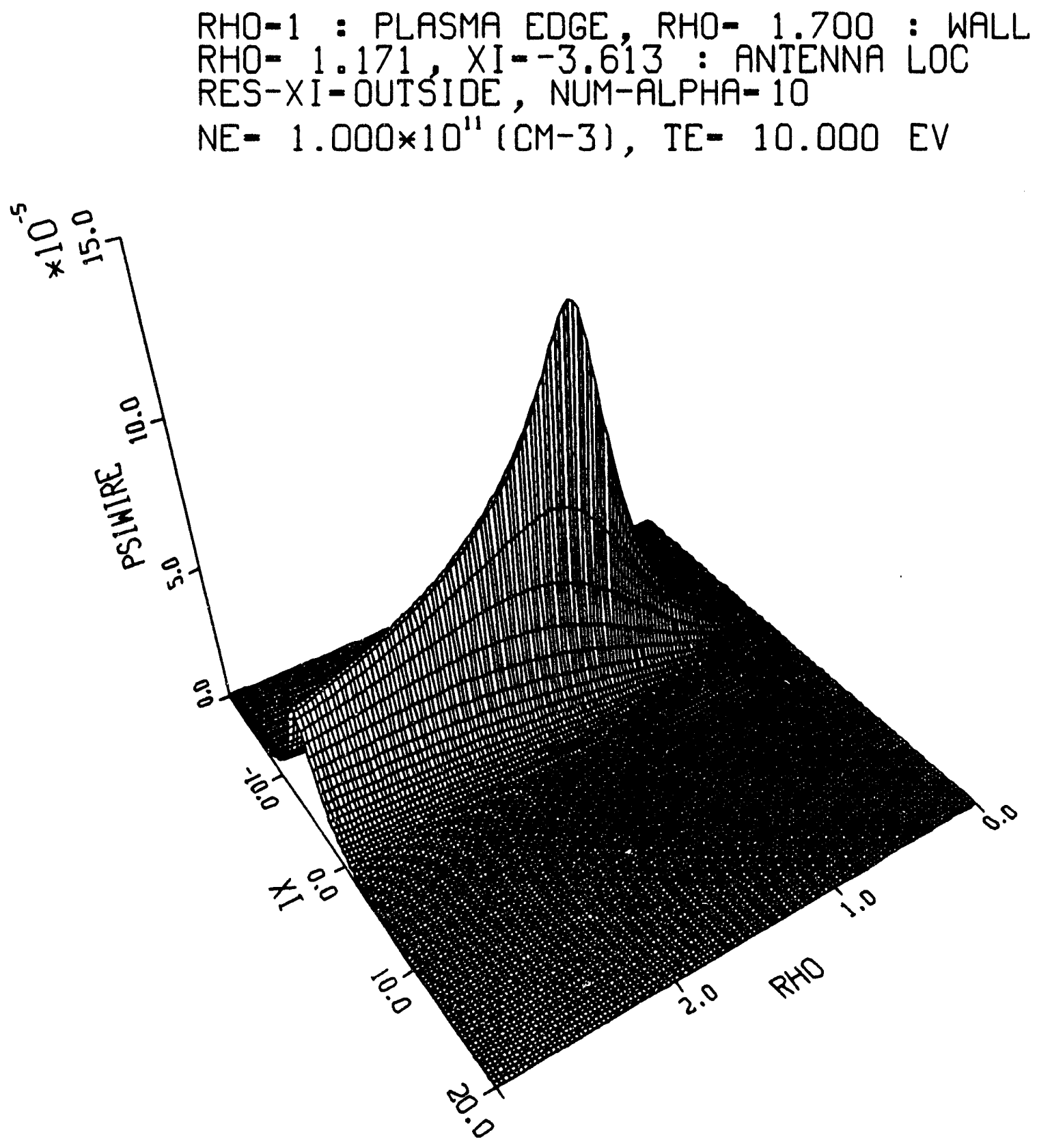

Figure 3-3: $\psi_{\text {wire }}(\rho, \xi)$ in vacuum for the dipole current source described in this chapter and given by Equation 3.17. There is no plasma present in this geometry. 


\subsection{Vacuum Fields}

In this section, the natural mode oscillations of the EM fields in the bounded plasma-vacuum system will be solved. The method of solution is straight forward except that the changing radius of the plasma-vacuum interface and the presence of a conducting wall dictate a coordinate transformation in order to simplify the boundary conditions.

In the vacuum region, assume the following fields exist

$$
\begin{aligned}
& \underline{E}=E_{\theta} \hat{e_{\theta}} \\
& \vec{B}=B_{r} \hat{e_{r}}+B_{z} \hat{e_{z}} \\
& \vec{B}=\nabla \times \vec{A} \\
& \underline{E}=-\frac{\partial \vec{A}}{\partial t} \\
& \vec{A}=A_{\theta} \hat{e_{\theta}}
\end{aligned}
$$

Define

$$
\Psi \equiv r A_{\theta}
$$

where

$$
\Psi=I e^{-i \omega t}\left[\Psi_{\text {wire }}(r, z)+\Psi_{V}\right]
$$

Then

$$
\begin{aligned}
\vec{B} & =\frac{\nabla \Psi \times \hat{e_{\theta}}}{r} \\
E & =-\frac{\partial \vec{A}}{\partial t}=\frac{i \omega \Psi}{r} \hat{e_{\theta}}
\end{aligned}
$$

In vacuum,

$$
\nabla \times \vec{B}=\nabla \times\left(\frac{\nabla \Psi \times \hat{e_{\theta}}}{r}\right)=\mu_{0} I=0
$$

It can be shown that this results in

$$
\hat{\theta}\left(r \frac{\partial}{\partial r} \frac{1}{r} \frac{\partial}{\partial r} \Psi_{V}+\frac{\partial^{2}}{\partial z^{2}} \Psi_{V}\right)=0
$$


where $\Psi_{V}$ is regular everywhere since the source term has ben explicitly separated out.

\subsubsection{Change of Variable}

A change of variable is necessary to simplify the boundary. Because the plasma radius is changing axially by a finite amount, the cylindrical geometry poses difficulties when matching fields across the boundary. Therefore a new set of coordinates is introduced here with the strict purpose of simplifying the boundary conditions.

Let

$$
\rho \equiv \frac{r}{R(z)} \quad ; \quad z^{\prime} \equiv z
$$

where $R(z)$ is the plasma-vacuum boundary. The desired boundary conditions are

1. $\rho=1$ at the plasma-vacuum interface $r=R(z)$

2. $\rho=\frac{b}{R} \equiv \kappa=$ constant at the vacuum chamber wall.

A major assumption is made here that the radius of the conducting wall scales with the radius of the plasma column. This is not an unrealistic assumption since the vacuum chamber necks down in the mirror region of our experimental device as seen in Figure 3-2. This assumption greatly simplifies the location of the boundaries: $\rho=1$ at the plasma-vacuum interface and $\rho=$ $\kappa=$ constant at the vacuum chamber wall.

Carrying out the transformation leads to

$$
\begin{gathered}
\frac{\partial}{\partial z}=\frac{\partial}{\partial z^{\prime}}+\frac{\partial \rho}{\partial z} \frac{\partial}{\partial \rho} \\
\frac{\partial}{\partial r}=\frac{\partial \rho}{\partial r} \frac{\partial}{\partial \rho} \\
r \frac{\partial}{\partial r} \frac{1}{r} \frac{\partial}{\partial r}=\frac{1}{R^{2}(z)} \rho \frac{\partial}{\partial \rho} \frac{1}{\rho} \frac{\partial}{\partial \rho}
\end{gathered}
$$




$$
\begin{gathered}
\frac{\partial^{2}}{\partial z^{2}}=\frac{\partial^{2}}{\partial z^{\prime 2}}+\left(\rho^{\prime}\right)^{2} \frac{\partial^{2}}{\partial \rho^{2}}+\rho^{\prime} \frac{\partial^{2}}{\partial \rho \partial z^{\prime}}+\frac{\partial}{\partial z^{\prime}} \rho^{\prime} \frac{\partial}{\partial \rho} \\
\rho^{\prime} \equiv \frac{\partial \rho}{\partial z}
\end{gathered}
$$

We then have the following differential equation for $\Psi_{V}$

$$
\left\{\frac{\partial^{2}}{\partial z^{\prime 2}}+\left(\rho^{\prime}\right)^{2} \frac{\partial^{2}}{\partial \rho^{2}}+\rho^{\prime} \frac{\partial^{2}}{\partial \rho \partial z^{\prime}}+\frac{\partial}{\partial z^{\prime}} \rho^{\prime} \frac{\partial}{\partial \rho}+\frac{1}{R^{2}(z)} \rho \frac{\partial}{\partial \rho} \frac{1}{\rho} \frac{\partial}{\partial \rho}\right\} \Psi_{V}=0
$$

Now assume that $\rho^{\prime}$ is small compared to the field variation, i.e.

$$
\rho^{\prime} \ll 1
$$

This is a good approximation for the experiment where $\rho^{\prime} \sim a / L \sim 10 \mathrm{~cm} / 400 \mathrm{~cm} \ll$ 1 and is known as the slow-z approximation. In this limit, the differential equation becomes

$$
\frac{\partial^{2}}{\partial z^{2}} \Psi_{V}+\frac{1}{R^{2}(z)} \rho \frac{\partial}{\partial \rho} \frac{1}{\rho} \frac{\partial}{\partial \rho} \Psi_{V}=0
$$

Now make a second substitution of variable (essentially a stretching transformation in $\mathbf{z}$ )

$$
\rho=\rho \quad ; \quad d \xi=\frac{d z^{\prime}}{R\left(z^{\prime}\right)}
$$

This results in

$$
\begin{aligned}
R^{2}(z) \frac{\partial^{2}}{\partial z^{\prime 2}} & =R \frac{\partial}{\partial \xi} \frac{1}{R} \frac{\partial}{\partial \xi} \Psi_{V} \\
& =\frac{\partial^{2} \Psi_{V}}{\partial \xi^{2}}-\frac{\frac{\partial}{\partial \xi} R}{R} \frac{\partial}{\partial \xi} \Psi_{V} \\
& \simeq \frac{\partial^{2} \Psi_{V}}{\partial \xi^{2}}
\end{aligned}
$$

The differential equation is

$$
\rho \frac{\partial}{\partial \rho} \frac{1}{\rho} \frac{\partial}{\partial \rho} \Psi_{V}+\frac{\partial^{2}}{\partial \xi^{2}} \Psi_{V}=O\left(\rho^{\prime}\right) \sim 0
$$

Because the vacuum and plasma fields satisfy different differential equations, it is obvious that the natural $z$-dependence of the plasma and vacuum fields are 
also different. Since the plasma-vacuum interface varies with $z$, the boundary conditions are treated by performing a Fourier transform on both the plasma and vacuum fields. The boundary conditions are then matched in Fourier space and the resulting fields are then inverse Fourier transformed to produce the EM fields in real space. This operation essentially yields field coefficients that are functions of $z$ which are tailored to the boundary condition of the particular plasma-vacuum system.

Take the Fourier Transform

$$
\hat{\Psi}_{V}\left(\rho, k_{\xi}\right)=\int e^{i k_{\xi} \xi} \Psi_{V}(\rho, \xi) d \xi
$$

The differential equation then becomes

$$
\rho \frac{\partial}{\partial \rho} \frac{1}{\rho} \frac{\partial}{\partial \rho} \hat{\Psi}_{V}-k_{\xi}^{2} \hat{\Psi}_{V}=0
$$

Define:

$$
U \equiv \frac{1}{\rho} \frac{\partial}{\partial \rho} \hat{\Psi}_{V}
$$

and taking the derivative of Equation 3.20 with respect to $\rho$ yields

$$
\frac{1}{\rho} \frac{\partial}{\partial \rho} \rho \frac{\partial}{\partial \rho} U-k_{\xi}^{2} U=0
$$

The solution to this is the $I_{0}$ and the $K_{0}$ Bessel Funtions. Solving for $\hat{\Psi}_{V}$ : Equation 3.20 becomes

$$
\begin{gathered}
\rho \frac{\partial}{\partial \rho} U=k_{\xi}^{2} \hat{\Psi}_{V} \\
\hat{\Psi}_{V}=\frac{\rho}{k_{\xi}^{2}} \frac{\partial}{\partial \rho} U \\
=\frac{\rho}{k_{\xi}^{2}} \frac{\partial}{\partial \rho}\left(a_{1} I_{0}\left(k_{\xi} \rho\right)+a_{2} K_{0}\left(k_{\xi} \rho\right)\right) \\
=\frac{\rho k_{\xi}}{k_{\xi}^{2}}\left[a_{1} I_{1}\left(k_{\xi} \rho\right)+a_{2} K_{1}\left(k_{\xi} \rho\right)\right]
\end{gathered}
$$

Therefore

$$
\hat{\Psi}_{v}\left(\rho_{i} k_{\xi}\right)=c_{1}\left(k_{\xi}\right) \rho I_{1}\left(k_{\xi} \rho\right)+c_{2}\left(k_{\xi}\right) \rho K_{1}\left(k_{\xi} \rho\right)
$$


Equation 3.21 will be used in the boundary conditions at $\rho=1$ and $\rho=\kappa$. The two unknowns $c_{1}\left(k_{\xi}\right)$ and $c_{2}\left(k_{\xi}\right)$ in this equation will be completely solved by the boundary conditions to fully determine the vacuum field. 


\subsection{Boundary Condition}

The boundary conditions for this geometry are relatively simple because of the changes of variables that were made. Two boundaries exist in this geometry: at the wall $(\rho=\kappa)$, and at the vacuum-plasma interface $(\rho=1)$. These boundary conditions are discussed in detail in Appendix E.1 and are summarized below

\subsubsection{Boundary I: Vacuum chamber wall $\rho=\kappa$}

At $\rho=\kappa$, the tangential electric field $E_{\tan }$ is zero.

The Fourier-transformed vacuum $\hat{\Psi}_{V}$ is then shown to be

$$
\hat{\Psi}_{V}\left(\rho, k_{\xi}\right)=\frac{c_{1}\left(k_{\xi}\right) \rho}{K_{1}\left(k_{\xi} \kappa\right)} \Delta_{\kappa \rho}-\left(\frac{\rho}{x}\right) \frac{K_{1}\left(k_{\xi} \rho\right)}{K_{1}\left(k_{\xi} \kappa\right)} \hat{\Psi}_{\text {wire }}\left(\kappa, k_{\xi}\right)
$$

The total $\Psi$ is given by $\Psi=\left(\Psi_{v}+\Psi_{\text {wire }}\right)$

$$
\Psi=\left[c_{1}\left(k_{\xi}\right) \frac{\rho \triangle_{\kappa \rho}}{K_{1}\left(k_{\xi} \kappa\right)}-\left(\frac{\rho}{\kappa}\right) \frac{K_{1}\left(k_{\xi} \rho\right)}{K_{1}\left(k_{\xi} \kappa\right)} \hat{\Psi}_{\text {wire }}\left(\kappa, k_{\xi}\right)+\hat{\Psi}_{\text {wire }}\left(\rho, k_{\xi}\right)\right]
$$

where $\Delta_{\kappa \rho}$ is defined as

$$
\begin{aligned}
& \Delta_{\kappa \rho} \equiv K_{1}\left(k_{\xi} \kappa\right) I_{1}\left(k_{\xi} \rho\right)-I_{1}\left(k_{\xi} \kappa\right) K_{1}\left(k_{\xi} \rho\right) \\
& \Delta_{\kappa \rho^{\prime}} \equiv K_{1}\left(k_{\xi} \kappa\right) I_{1}^{\prime}\left(k_{\xi} \rho\right)-I_{1}\left(k_{\xi} \kappa\right) K_{1}^{\prime}\left(k_{\xi} \rho\right)
\end{aligned}
$$

and

$$
I_{1}^{\prime}(z) \equiv \frac{d I_{1}(z)}{d z} \quad \text { and } \quad K_{1}^{\prime}(z) \equiv \frac{d K_{1}(z)}{d z}
$$

\subsubsection{Boundary II: Plasma Vacuum Interface $\rho=1$}

Two conditions exist at the plasma-vacuum interface boundary : $E_{\text {tan }}$ is continuous; $\vec{B}_{\tan }$ is continous.

$\mathbf{E}_{\tan }$ continuous, $\overrightarrow{\mathbf{B}}_{\tan }$ continuous.

Using the continuity of the fields across the plasma-vacuum boundary, the Fourier-transformed plasma $\hat{\phi}_{p}$ is found to be

$$
\hat{\hat{\phi}}_{p}\left(\alpha_{i} k_{\xi}\right)=\frac{1}{\alpha J_{\mathrm{v}}(\alpha)-J_{\mathrm{i}}(\alpha) \frac{\frac{\partial}{\partial_{\beta}} \hat{F}}{\hat{F}}}\left[\frac{\partial}{\partial \rho} \hat{G}-\frac{\frac{\partial}{\partial \rho} \hat{F}}{\hat{F}} \hat{G}\right]_{\hat{\rho}=1}
$$


where $\alpha$ is the perpendicular wave number of the plasma field whose derivation will be detailed in the next section, and

$$
\begin{aligned}
& \hat{F}\left(\rho, k_{\xi}\right) \equiv \frac{\rho \Delta_{\kappa \rho}}{K_{1}\left(k_{\xi} \kappa\right)} \\
& \hat{G}\left(\rho, k_{\xi}\right) \equiv-\left(\frac{\rho}{\kappa}\right) \frac{K_{1}\left(k_{\xi} \rho\right)}{K_{1}\left(k_{\xi} \kappa\right)} \hat{\Psi}_{\text {wire }}\left(\kappa, k_{\xi}\right)+\hat{\Psi}_{\text {wire }}\left(\rho, k_{\xi}\right)
\end{aligned}
$$

The complete solution of the plasma field quantity $\hat{\phi}_{p}$ as a function of the parallel Fourier wave number $k_{\xi}$ and perpendicular wave number $\alpha$ is then known. Once $\hat{\phi}_{p}$ is determined, the self-consistent Maxwell's equations in plasma are then solved to yield a complete set of $\hat{\chi}_{p}$ and $\hat{\phi}_{p}$ and the ICRF EM fields can then be completely determined.

\subsubsection{Curve fitting to $\Psi_{\text {wire }}(\rho=1, \xi)$ and $\partial \Psi_{\text {wire }} / \partial \rho$ for semi-analytical Fourier Transform}

The values of $\Psi_{\text {wire }}(\rho=1, \xi)$ and $\partial \Psi_{\text {wire }} / \partial \rho$ can be found from the previous section. It is possible to simply do a numerical FFT transform of those functions to obtain $\hat{\Psi}\left(\rho=1, k_{\xi}\right)$ and $\partial \hat{\Psi} / \partial \rho$, but the resolution of such a transform can be limiting. Therefore it is desirable to fit a curve to the two functions that can facilitate a simple analytical Fourier transform.

Choose a fitting function $\Psi_{\text {fit }}$ to model the actual $\Psi_{\text {actual }}$ of the wire dipole source as :

$$
\Psi_{f i t}(\xi)=\frac{\Psi_{0}}{\left[1+\left(\frac{\xi-\xi_{w}}{\Delta}\right)^{2}\right]^{5 / 2}}\left[1+c_{1}\left(\xi-\xi_{w}\right)+c_{2}\left(\xi-\xi_{w}\right)^{2}\right]
$$

The following steps can then be taken to minimize the error in this fit and find the resulting $c_{1}, c_{2}, \Delta$ and $\xi_{w}$ as a result of this error minimization.

1. Find $\xi_{w}$ from the maximum point of $\Psi_{\text {actual }}(\rho=1, \xi)$

2. Select $\triangle$ by examining the half-width of $\Psi_{\text {centul }}$ 
3. Define the error $\epsilon$

$$
\epsilon \equiv \int_{-\infty}^{\infty}\left(\Psi_{\text {actual }}(\xi)-\Psi_{f i t}\right)^{2} d \xi
$$

4. Minimize the error $\epsilon$ and determine $c_{1}$ and $c_{2}$ completely

$$
\frac{\partial \epsilon}{\partial c_{1}}=0 \quad \frac{\partial \epsilon}{\partial c_{2}}=0
$$

The details of these derivations are given in Appendix E.2. Using the method outlined above, a sample curve fit to a given dipole current source is computed and presented on Figure 3-4. It is seen that some discrepancies between $\Psi_{f i t}$ and $\Psi_{\text {wire }}$ do exist but in general there is good agreement between the fit and the actual dipole loop generated $\Psi_{\text {wire }}$. 
PS], PS]FII (RHO-1)

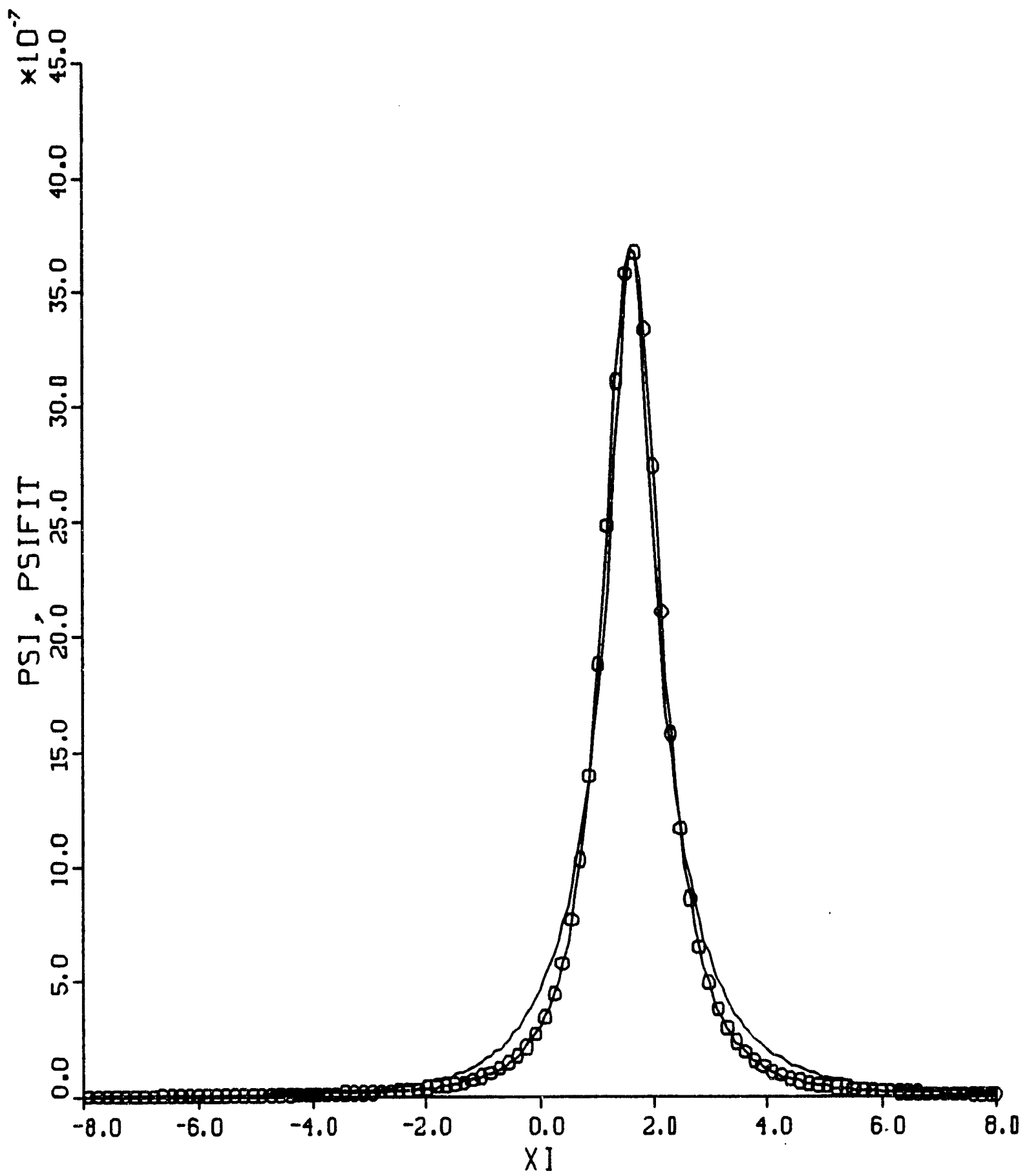

Figure 3-4: $\Psi$ and $\Psi_{\text {fit }}$ for a dipole current source evaluated at different $\xi$ 's along the plasma radius $(\rho=1)$. The solid line is the computed curve for the source term $\Psi_{\text {wire }}$, the line with the open circles is the best-fit curve $\Psi_{\text {fit }}$ obtained using error minimization. 


\section{Fourier Transform of the fitting function $\Psi_{f i t}(\xi)$}

Given that $c_{1}, c_{2}, \Delta$ and $\xi_{w}$ are known, the Fourier transform of $\Psi_{f i t}$ can then be found analytically by

$$
\begin{gathered}
\hat{\Psi}_{f i t}\left(k_{\xi}\right)=\int_{-\infty}^{\infty} \Psi_{f i t}(\xi) e^{i k_{\xi} \xi} d \xi \\
\hat{\Psi}_{f i t}\left(k_{\xi}\right)=\int_{-\infty}^{\infty} \frac{\Psi_{0}}{\left[1+\left(\frac{\xi-\xi_{w}}{\Delta}\right)^{2}\right]^{5 / 2}}\left[1+c_{1}\left(\xi-\xi_{w}\right)+c_{2}\left(\xi-\xi_{w}\right)^{2}\right] e^{i k_{\xi} \xi} d \xi \\
=\Psi_{0} \Delta^{5} e^{i k_{\xi} \xi_{w}} \int_{-\infty}^{\infty} \frac{e^{i k_{\xi}}}{\left[\Delta^{2}+x^{2}\right]^{5 / 2}}\left[1+c_{1} x+c_{2} x^{2}\right] d x
\end{gathered}
$$

where

$$
x \equiv\left(\xi-\xi_{w}\right)
$$

since

$$
\begin{gathered}
e^{i a}=\cos (a)+i \sin (a) \\
\Psi_{f i t}=\Psi_{0} \Delta^{5} e^{i k_{\xi} \xi_{w}} \int_{-\infty}^{\infty} \frac{\cos \left(k_{\xi} x\right)+i \sin \left(k_{\xi} x\right)}{\left[\Delta^{2}+x^{2}\right]^{5 / 2}}\left[1+c_{1} x+c_{2} x^{2}\right] d x
\end{gathered}
$$

Examining the cosine integral first

$$
\begin{aligned}
I_{c o s} & \equiv \int_{-\infty}^{\infty} \frac{\cos \left(k_{\xi} x\right)}{\left[\Delta^{2}+x^{2}\right]^{5 / 2}}\left[1+c_{1} x+c_{2} x^{2}\right] \\
& =\int_{-\infty}^{\infty} \frac{\cos \left(k_{\xi} x\right)}{\left[\Delta^{2}+x^{2}\right]^{5 / 2}}\left[1+c_{2} x^{2}\right] \\
& =2 \int_{0}^{\infty} \frac{\cos \left(k_{\xi} x\right)}{\left[\Delta^{2}+x^{2}\right]^{5 / 2}}\left[1+c_{2} x^{2}\right] d x
\end{aligned}
$$

where the $x$ term has been dropped because its oddness results in its integral being zero.

From Gradshteyn \& Ryzhik [52], it is seen that

$$
\int_{0}^{\infty} \frac{x^{2 m} \cos (a x) d x}{\left(\beta^{2}+x^{2}\right)^{n+\frac{1}{2}}}=\frac{(-1)^{m} \sqrt{\pi}}{2^{n} \beta^{n} \Gamma\left(n+\frac{1}{2}\right)} \cdot \frac{d^{2 m}}{d a^{2 m}}\left\{a^{n} K_{n}(a \beta)\right\}
$$


The $I_{\text {cos }}$ integral can then be shown to be

$$
I_{c o s}=\frac{2 \sqrt{\pi}}{4 \Delta^{2} \Gamma(5 / 2)}\left\{k_{\xi}^{2} K_{2}\left(k_{\xi} \Delta\right)-c_{2} \frac{d^{2}}{d k_{\xi}^{2}}\left[k_{\xi}^{2} K_{2}\left(k_{\xi} \Delta\right)\right]\right\}
$$

The sin component of the integral is given by

$$
\begin{aligned}
I_{\text {sin }} & =\int_{-\infty}^{\infty} \frac{i \sin \left(k_{\xi} x\right)}{\left[\Delta^{2}+x^{2}\right]^{5 / 2}}\left[1+c_{1} x+c_{2} x^{2}\right] d x \\
& =\int_{-\infty}^{\infty} \frac{i \sin \left(k_{\xi} x\right)}{\left[\Delta^{2}+x^{2}\right]^{5 / 2}}\left[c_{1} x\right] d x \\
& =2 \int_{0}^{\infty} \frac{i \sin \left(k_{\xi} x\right)}{\left[\Delta^{2}+x^{2}\right]^{5 / 2}}\left[c_{1} x\right] d x
\end{aligned}
$$

where the $1+c_{2} x^{2}$ term in the integral has been dropped because of its odd property when multiplied by the sine component.

From Gradshteyn,

$$
\int_{0}^{\infty} \frac{x^{2 m+1} \sin (a x) d x}{\left(\beta^{2}+x^{2}\right)^{n+\frac{1}{2}}}=\frac{(-1)^{m+1} \sqrt{\pi}}{2^{n} \beta^{n} \Gamma\left(n+\frac{1}{2}\right)} \cdot \frac{d^{2 m+1}}{d a^{2 m+1}}\left[a^{n} K_{n}(a \beta)\right]
$$

It can therefore be shown that

$$
I_{\text {sin }}=\frac{-2 i c_{1} \sqrt{\pi}}{4 \triangle^{2} \Gamma(5 / 2)} \frac{d}{d k_{\xi}}\left[k_{\xi}^{2} K_{2}\left(k_{\xi} \Delta\right)\right]
$$

The Fourier transform of the fitting function can then be given by

$$
\hat{\Psi}_{f i t}\left(k_{\xi}\right)=\frac{2 \Psi_{0} \Delta^{3}}{3} e^{i k_{\xi} \xi_{w}}\left\{k_{\xi}^{2} K_{2}\left(k_{\xi} \Delta\right)-i c_{1} \frac{d}{d k_{\xi}}\left[k_{\xi}^{2} K_{2}\left(k_{\xi} \Delta\right)\right]-c_{2} \frac{d^{2}}{d k_{\xi}^{2}}\left[k_{\xi}^{2} K_{2}\left(k_{\xi} \Delta\right)\right]\right\}
$$

where the identity $\Gamma(5 / 2)=\frac{3}{4} \Gamma(1 / 2)=\frac{3}{4} \sqrt{\pi}$ was used. Also using the identity (Abramowitz \& Stegun)

$$
e^{\nu \pi i} K_{\nu}^{\prime}(z)=e^{(\nu-1) \pi i} K_{\nu-1}(z)-\frac{\nu}{z} e^{\nu \pi i} K_{\nu}(z)
$$

It is then easily shown that

$$
\frac{d}{d k_{\xi}}\left(k_{\xi}^{2} K_{2}(a)\right)=-k_{\xi} a K_{1}(a)
$$




$$
\frac{d^{2}}{d k_{\xi}^{2}}\left(k_{\xi}^{2} K_{2}(a)\right)=-a K_{1}(a)+a^{2} K_{0}(a)
$$

where here $a$ is defined as

$$
a \equiv k_{\xi} \triangle
$$

So $\hat{\Psi}_{f i t}$ can be seen to be

$$
\hat{\Psi}_{f i t}\left(k_{\xi}\right)=\frac{2 \Psi_{0} \Delta^{3}}{3} e^{i k_{\xi} \xi_{w}}\left\{k_{\xi}^{2} K_{2}(a)+i c_{1} k_{\xi}^{2} \Delta K_{1}(a)+c_{2}\left[\Delta k_{\xi} K_{1}(a)-\left(\Delta k_{\xi}\right)^{2} K_{0}(a)\right]\right\}
$$

From the above equation, both an oscillatory component $e^{i k_{6} \xi_{w}}$ and decaying components $K_{n}\left(k_{\xi} \Delta\right)$ are apparent. The resulting $\hat{\Psi}_{f i t}\left(k_{\xi}\right)$ is computed and given on Figure 3-5. It is seen that the source term decays off from a peak at $k_{\xi}=0$ to almost negligible values beyond $k_{\xi} \simeq 5$. This sets the approximate upper and lower bounds on the range of $k_{\xi}$ which are important to the solution of this problem. 


\section{FFT OF PSI(RHO-1)}

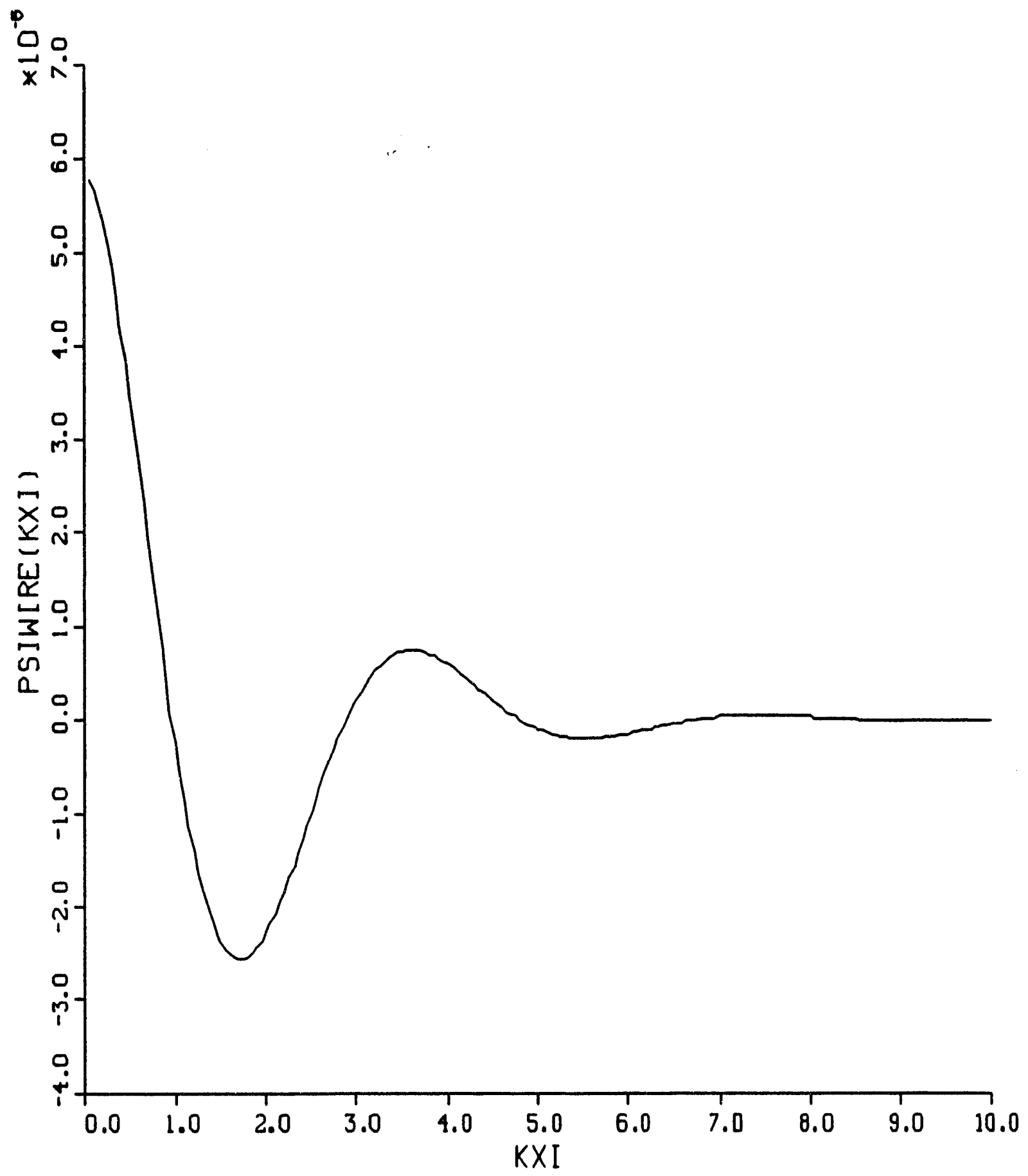

Figure 3-5: Fourier Transform of $\Psi_{\text {fit }}$ for a Dipole Current Source. $\Psi_{\text {fit }}$ is computed using Equation 3.28. Note that $\Psi\left(k_{\xi}\right)$ falls off to negligible levels for $k_{\xi}>5$. This sets the upper bound on the range of interest of $k_{\xi}$. 


\subsection{Plasma Fields}

This section will present and solve the ICRF EM fields in the plasma medium. The same techniques presented in the previous section for coordinate transformation from $(r, z)$ to $(\rho, \xi)$ is used to treat the plasma fields. Special residue calculus techniques are employed to treat the resonance poles that occur as $\omega \rightarrow \omega_{c i}$. The resulting two coupled integral equations are solved simultaneously by linear algebra techniques and a figure of merit is introduced and computed. Using this method, several parametric scans are also performed.

The fundamental equations used are Maxwell's equation:

$$
\begin{gathered}
\nabla \times \underline{E}=-\frac{\partial \vec{B}}{\partial t}=i \omega \vec{B} \\
\nabla \times \vec{B}=\frac{1}{c^{2}} \frac{\partial \underline{E}}{\partial t}+\mu_{0} J=-i \frac{\omega}{c^{2}} \underline{K} \cdot \underline{E}
\end{gathered}
$$

where

$$
\underline{K} \equiv\left|\begin{array}{ccc}
K_{1} & K_{2} & 0 \\
-K_{2} & K_{1} & 0 \\
0 & 0 & K_{3}
\end{array}\right|
$$

Using Assumption $1(m=0)$, it is seen that

$$
\begin{aligned}
\nabla \times \nabla \underline{E} & =-\hat{r} \frac{\partial}{\partial z}\left[\frac{\partial E_{r}}{\partial z}-\frac{\partial E_{z}}{\partial r}\right]+\hat{\theta}\left[\frac{\partial}{\partial z}\left(-\frac{\partial E_{\theta}}{\partial z}\right)-\frac{\partial}{\partial r} \frac{1}{r} \frac{\partial r E_{\theta}}{\partial r}\right]+\hat{z}\left[\frac{1}{r} \frac{\partial}{\partial r} r\left(\frac{\partial E_{r}}{\partial z}-\frac{\partial E_{z}}{\partial r}\right)\right] \\
& =k_{0}^{2}\left[\hat{r}\left(K_{1} E_{r}+K_{2} E_{\theta}\right)+\hat{\theta}\left(-K_{2} E_{r}+K_{1} E_{\theta}\right)+\hat{z} E_{z}\right]
\end{aligned}
$$

Matching the different components yield

$$
\begin{aligned}
-\frac{\partial^{2}}{\partial z^{2}} E_{r}+\frac{\partial}{\partial z} \frac{\partial}{\partial r} E_{z} & =k_{0}^{2}\left(K_{1} E_{r}+K_{2} E_{\theta}\right) \\
-\frac{\partial^{2} E_{\theta}}{\partial z^{2}}-\frac{\partial}{\partial r} \frac{1}{r} \frac{\partial}{\partial r}\left(r E_{\theta}\right) & =k_{0}^{2}\left(-K_{2} E_{r}+K_{1} E_{\theta}\right) \\
\frac{1}{r} \frac{\partial}{\partial r} r \frac{\partial}{\partial z} E_{r}-\frac{1}{r} \frac{\partial}{\partial r} r \frac{\partial}{\partial r} E_{z} & =k_{0}^{2} K_{3} E_{z}
\end{aligned}
$$


Using Assumption $3\left(E_{\mathrm{z}} \ll E_{r, \theta}\right)$, the following is obtained

$$
\begin{aligned}
-\frac{\partial^{2}}{\partial z^{2}} E_{r} & =k_{0}^{2}\left(K_{1} E_{r}+K_{2} E_{\theta}\right) \\
-\frac{\partial^{2} E_{\theta}}{\partial z^{2}}-\frac{\partial}{\partial r} \frac{1}{r} \frac{\partial}{\partial r}\left(r E_{\theta}\right) & =k_{0}^{2}\left(-K_{2} E_{r}+K_{1} E_{\theta}\right)
\end{aligned}
$$

Define:

$$
\begin{aligned}
& E_{\theta} \equiv i \omega \frac{\phi_{p}}{r} \\
& E_{r} \equiv i \omega \frac{\chi_{p}}{r}
\end{aligned}
$$

where $\phi_{p}$ and $\chi_{p}$ are now the depeadent variables. This substitution is made in order to allow matching to the corresponding vacuum solutions across the boundary.

The differential equations then become

$$
\begin{aligned}
-\frac{\partial^{2}}{\partial z^{2}} \chi_{p} & =k_{0}^{2}\left(K_{1} \chi_{p}+K_{2} \phi_{p}\right) \\
-\frac{\partial^{2}}{\partial z^{2}} \phi_{p}-r \frac{\partial}{\partial r} \frac{1}{r} \frac{\partial}{\partial r}\left(\phi_{p}\right) & =k_{0}^{2}\left(-K_{2} \chi_{p}+K_{1} \phi_{p}\right)
\end{aligned}
$$

\subsubsection{Change of Variable}

In order to produce a set of simple boundary conditions for a plasma cylinder of varying radius, a change of variables is required. This change of variables is performed to specifically treat the problem of the changing radius.

Select the following change of variables:

$$
\begin{array}{ccc}
r=R(y) \rho & \Rightarrow & \rho=\frac{r}{R(y)} \\
z=y & \Rightarrow & y=z
\end{array}
$$

where $R(y)$ is the y dependent radius of the plasma boundary. This choice conveniently sets the plasma-vacuum boundary at $\rho=1$ and results in the partial derivatives becoming 


$$
\begin{aligned}
\frac{\partial}{\partial r} & =\frac{\partial}{\partial \rho} \frac{\partial \rho}{\partial r}=\frac{1}{R(y)} \frac{\partial}{\partial \rho} \\
\frac{\partial}{\partial z} & =\frac{\partial \rho}{\partial z} \frac{\partial}{\partial \rho}+\frac{\partial y}{\partial z} \frac{\partial}{\partial y} \\
& =r \frac{\partial}{\partial z} R^{-1}(z) \frac{\partial}{\partial \rho}+\frac{\partial}{\partial y} \\
& =-\frac{r R^{\prime}}{R^{2}} \frac{\partial}{\partial \rho}+\frac{\partial}{\partial y} \\
& =-\frac{\rho}{R} R^{\prime} \frac{\partial}{\partial \rho}+\frac{\partial}{\partial y}
\end{aligned}
$$

By the same token, the second derivatives are seen to be

$$
\begin{gathered}
\frac{\partial^{2}}{\partial r^{2}}=\frac{1}{R^{2}(y)} \frac{\partial^{2}}{\partial \rho^{2}} \\
\frac{\partial^{2}}{\partial z^{2}}=\frac{\partial}{\partial z}\left[-\frac{\rho}{R} R^{\prime} \frac{\partial}{\partial \rho}+\frac{\partial}{\partial y}\right] \\
=\frac{\partial^{2}}{\partial y^{2}}-2 \rho \frac{R^{\prime}(y)}{R(y)} \frac{\partial^{2}}{\partial \rho \partial y}+\left(\frac{\rho R^{\prime}}{R}\right)^{2} \frac{\partial^{2}}{\partial \rho^{2}}+\left(\frac{R^{\prime}}{R}\right)^{2} \rho \frac{\partial}{\partial \rho}
\end{gathered}
$$

Recall that

$$
\frac{\partial}{\partial y} \sim k_{y}=\frac{1}{\lambda_{y}} \quad \text { and } \quad \frac{R^{\prime}}{R} \sim \frac{1}{L_{B}}
$$

\section{Slow-z Approximation}

Because the magnetic field is assumed to be a slowly varying function of $z$, it can be easily shown that the wave number of the ICRF waves in plasma is much larger than the inverse of the field variation scaling length, in other words $\lambda_{y} \ll L_{B}$, where $L_{B}$ is the field variation scaling length. This is because the elements of the plasma dielectric tensor have a much faster axial variation than the field when resonance is approached. Therefore terms with $\frac{\partial^{2}}{\partial \rho \partial y}$ and $\rho \frac{\partial}{\partial \rho}$ can be dropped in the $\partial^{2} / \partial z^{2}$ equation.

$$
\frac{\partial^{2}}{\partial \underline{z}^{2}} \simeq \frac{\partial^{2}}{\partial y^{2}}+\left(\frac{\rho R^{\prime}}{R}\right)^{2} \frac{\partial^{2}}{\partial \rho^{2}}
$$


The basic equations reduce to

$$
\begin{gathered}
\frac{\partial^{2}}{\partial y^{2}} \chi_{p}+k_{0}^{2} K_{1} \chi_{p}+k_{0}^{2} K_{2} \phi_{p}=0 \\
\frac{\partial^{2}}{\partial y^{2}} \phi_{p}+\frac{\rho}{R^{2}} \frac{\partial}{\partial \rho} \frac{1}{\rho} \frac{\partial}{\partial \rho} \phi_{p}+k_{0}^{2} K_{1} \phi_{p}-k_{0}^{2} K_{2} \chi_{p}=0
\end{gathered}
$$

The equations can be further simplified by a stretching transformation on $y$, defined by

$$
d \xi \equiv \frac{d y}{R(y)}
$$

This yields

$$
\begin{aligned}
R^{2}(y) \frac{\partial^{2}}{\partial y^{2}} & =R \frac{\partial}{\partial \xi} \frac{1}{R} \frac{\partial}{\partial \xi} \\
& =\frac{\partial^{2}}{\partial \xi^{2}}-\frac{\frac{\partial}{\partial \xi}}{R} \frac{\partial}{\partial \xi} \\
& \simeq \frac{\partial^{2}}{\partial \xi^{2}}
\end{aligned}
$$

The differential equations then become

$$
\begin{gathered}
\frac{\partial^{2}}{\partial \xi^{2}} \chi_{p}+k_{0}^{2} K_{1 R 2}(\xi) \chi_{p}+k_{0}^{2} K_{2 R 2}(\xi) \phi_{p}=0 \\
\frac{\partial^{2}}{\partial \xi^{2}} \phi_{p}+\rho \frac{\partial}{\partial \rho} \frac{1}{\rho} \frac{\partial}{\partial \rho} \phi_{p}+k_{0}^{2} K_{1 R 2}(\xi) \phi_{p}-k_{0}^{2} K_{2 R 2}(\xi) \chi_{p}=0
\end{gathered}
$$

where

$$
K_{1 R 2}(\xi) \equiv K_{1}(\xi) R^{2}(\xi) \quad ; \quad K_{2 R_{2}}(\xi) \equiv K_{2}(\xi) R^{2}(\xi)
$$

This is the desired from of the differential equations. These equations are now solved by a combination of techniques involving separation of variables and Fourier analysis.

\section{Fourier Analysis}

Define the Fourier transforms $\hat{\phi}_{p}, \hat{\chi}_{p}$, and $\hat{K}_{1,2 R 2}$ as

$$
\hat{\phi}_{p}\left(\rho, k_{\xi}\right) \equiv \int e^{i k \xi} \phi_{p}(\rho, \xi) d \xi
$$




$$
\begin{gathered}
\hat{\chi}_{p}\left(\rho, k_{\xi}\right) \equiv \int e^{i k \xi} \chi_{p}(\rho, \xi) d \xi \\
\hat{K}_{1,2 R 2}\left(k_{\xi}\right) \equiv \int e^{i k \xi} R^{2}(\xi)\left[K_{1}(\xi), K_{2}(\xi)\right] d \xi
\end{gathered}
$$

After performing the Fourier transform, equations 3.34 and 3.35 become

$$
\begin{gathered}
-k_{\xi}^{2} \hat{\hat{\chi}}_{p}\left(\alpha, k_{\xi}\right)+k_{0}^{2}\left(\begin{array}{c}
+\int \hat{K}_{1 R 2}\left(k_{\xi}-k_{\xi}^{\prime}\right) \hat{\hat{\chi}}_{p}\left(\alpha, k_{\xi}^{\prime}\right) d k_{\xi}^{\prime} \\
+\int \hat{K}_{2 R 2}\left(k_{\xi}-k_{\xi}^{\prime}\right) \hat{\hat{\phi}}_{p}\left(\alpha, k_{\xi}^{\prime}\right) d k_{\xi}^{\prime}
\end{array}\right)=0 \\
\left(-k_{\xi}^{2}-\rho \frac{\partial}{\partial \rho} \frac{1}{\rho} \frac{\partial}{\partial \rho} \phi_{p}\right) \hat{\hat{\phi}}_{p}\left(\rho, k_{\xi}\right)+k_{0}^{2}\left(\begin{array}{c}
+\int \hat{K}_{1 R 2}\left(k_{\xi}-k_{\xi}^{\prime}\right) \hat{\hat{\phi}}_{p}\left(\rho, k_{\xi}^{\prime}\right) d k_{\xi}^{\prime} \\
-\int \hat{K}_{2 R 2}\left(k_{\xi}-k_{\xi}^{\prime}\right) \hat{\hat{\chi}}_{p}\left(\rho, k_{\xi}^{\prime}\right) d k_{\xi}^{\prime}
\end{array}\right)=0
\end{gathered}
$$

The method of separation of variables can be employed to solve the above equations The radial dependence of $\hat{\phi}_{p}, \hat{\chi}_{p}$ is found by assuming solutions of the form:

$$
\rho \frac{\partial}{\partial \rho} \frac{1}{\rho} \frac{\partial}{\partial \rho} \hat{\phi}_{p}=-\alpha^{2} \hat{\phi}_{p}
$$

where $\alpha$ is a separation constant.

The radial solution to the above differential equation is $\rho J_{1}(\alpha \rho)$. Thus, we can write

$$
\begin{aligned}
& \hat{\phi}_{p}\left(\rho, k_{\xi}\right) \equiv \hat{\hat{\phi}}_{p}\left(\alpha, k_{\xi}\right) \rho J_{1}(\alpha \rho) \\
& \hat{\chi}_{p}\left(\rho, k_{\xi}\right) \equiv \hat{\hat{\chi}}_{p}\left(\alpha, k_{\xi}\right) \rho J_{1}(\alpha \rho)
\end{aligned}
$$

The differential equations for $\phi_{p}$ and $\chi_{p}$ then can be written as

$$
-k_{\xi}^{2} \hat{\hat{\chi}}_{p}\left(\alpha, k_{\xi}\right)+k_{0}^{2}\left(\begin{array}{c}
+\int \hat{K}_{1 R 2}\left(k_{\xi}-k_{\xi}^{\prime}\right) \hat{\hat{\chi}}_{p}\left(\alpha, k_{\xi}^{\prime}\right) d k_{\xi}^{\prime} \\
+\int \hat{K}_{2 R 2}\left(k_{\xi}-k_{\xi}^{\prime}\right) \hat{\hat{\phi}}_{p}\left(\alpha, k_{\xi}^{\prime}\right) d k_{\xi}^{\prime}
\end{array}\right)=0
$$




$$
\left(-k_{\xi}^{2}-\alpha^{2}\right) \hat{\hat{\phi}}_{p}\left(\alpha, k_{\xi}\right)+k_{0}^{2}\left(\begin{array}{c}
+\int \hat{K}_{1 R 2}\left(k_{\xi}-k_{\xi}^{\prime}\right) \hat{\hat{\phi}}_{p}\left(\alpha, k_{\xi}^{\prime}\right) d k_{\xi}^{\prime} \\
-\int \hat{K}_{2 R 2}\left(k_{\xi}-k_{\xi}^{\prime}\right) \hat{\hat{\chi}}_{p}\left(\alpha, k_{\xi}^{\prime}\right) d k_{\xi}^{\prime}
\end{array}\right)=0
$$

where the $\rho J_{1}(\alpha \rho)$ term is common to all terms and is simply dropped.

Equations 3.36 and 3.37 are a set of coupled integral equations where the integral terms arise because of the z-dependence of the equilibrium. We will see that $\phi_{p}$ is explicitly determined from the boundary conditions. One of the equations is used to eliminate $\chi_{p}$ in terms of $\phi_{p}$, the other equation then gives the "dispersion relation" $\alpha=\alpha\left(k_{\xi}\right)$

\subsubsection{Fourier Transform of $\hat{K}_{1 R 2}$ and $\hat{K}_{2 R 2}$ terms}

The evaluation of the transformed functions of $\hat{K}_{1 R 2}$ and $\hat{K}_{2 R 2}$ is now addressed. In order to allow for a rapidly converging Fourier series, a series of steps are taken as listed below.

\section{A. Subtraction of infinity value}

Recall Equations 3.34 and 3.35 and rewrite $K_{1 R 2}$ and $K_{2 R_{2}}$ as follows

$$
\begin{aligned}
k_{0}^{2} K_{1 R 2} & \equiv k_{0}^{2} K_{1}(\xi) R^{2}(\xi) \\
& =\underbrace{k_{0}^{2} K_{1}(\xi) R^{2}(\xi)-k_{0}^{2} K_{1, \infty} R_{\infty}^{2}}_{I}+k_{0}^{2} K_{1, \infty} R_{\infty}^{2} \\
k_{0}^{2} K_{2 R 2} & \equiv k_{0}^{2} K_{2}(\xi) R^{2}(\xi) \\
& =\underbrace{k_{0}^{2} K_{2}(\xi) R^{2}(\xi)-k_{0}^{2} K_{2, \infty} R_{\infty}^{2}}_{I}+k_{0}^{2} K_{2, \infty} R_{\infty}^{2}
\end{aligned}
$$

The subtraction of the values of $K_{1 R 2}$ and $K_{2 R 2}$ as $\xi \rightarrow \infty$ implies that the term (I) in the functions $K_{1 R 2}, K_{2 R 2}$ converges to zero as $\xi \rightarrow \infty$. This results in a rapid convergence of the integrals containing the (I) terrh. The remaining constant term is integrated analytically. 
Recalling that $K_{1} \equiv S, K_{2} \equiv-i D$ in conventional dispersion terms, and taking the same Fourier transform as defined before, the transformed differential equations 3.34 and 3.35 become

$$
\begin{aligned}
& -k_{\xi}^{2} \hat{\hat{र}}_{p}\left(\alpha, k_{\xi}\right)+k_{0}^{2}\left(\begin{array}{l}
+\int \tilde{S}_{R 2}\left(k_{\xi}-k_{\xi}^{\prime}\right) \hat{\hat{\chi}}_{p}\left(\alpha, k_{\xi}^{\prime}\right) d k_{\xi}^{\prime} \\
-i \int \tilde{D}_{R 2}\left(k_{\xi}-k_{\xi}^{\prime}\right) \hat{\hat{\phi}}_{p}\left(\alpha, k_{\xi}^{\prime}\right) d k_{\xi}^{\prime} \\
+S_{\infty} \hat{\hat{\chi}}_{p}\left(\alpha, k_{\xi}\right) \\
-i D_{\infty} \hat{\hat{\phi}}_{p}\left(\alpha, k_{\xi}\right)
\end{array}\right)=0 \\
& \left(-k_{\xi}^{2}-\alpha^{2}\right) \hat{\hat{\phi}}_{p}\left(\alpha, k_{\xi}\right)+k_{0}^{2}\left(\begin{array}{l}
+\int \tilde{S}_{R 2}\left(k_{\xi}-k_{\xi}^{\prime}\right) \hat{\hat{\phi}}_{p}\left(\alpha, k_{\xi}^{\prime}\right) d k_{\xi}^{\prime} \\
+i \int \tilde{D}_{R 2}\left(k_{\xi}-k_{\xi}^{\prime}\right) \hat{\hat{\chi}}_{p}\left(\alpha, k_{\xi}^{\prime}\right) d k_{\xi}^{\prime} \\
+S_{\infty} \hat{\hat{\phi}}_{p}\left(\alpha, k_{\xi}\right) \\
+i D_{\infty} \hat{\hat{\chi}}_{p}\left(\alpha, k_{\xi}\right)
\end{array}\right)=0
\end{aligned}
$$

where

$$
\begin{gathered}
\tilde{S}_{R 2}\left(k_{\xi}\right) \equiv \int e^{i k_{\xi} \xi}\left[R^{2}(\xi) K_{1}(\xi)-R_{\infty}^{2} K_{1, \infty}\right] d \xi \\
\tilde{D}_{R 2}\left(k_{\xi}\right) \equiv i \int e^{i k_{\xi} \xi}\left[R^{2}(\xi) K_{2}(\xi)-R_{\infty}^{2} K_{2, \infty}\right] d \xi \\
S_{\infty} \equiv R_{\infty}^{2} K_{1 \infty} \\
D_{\infty} \equiv i R_{\infty}^{2} K_{2 \infty}
\end{gathered}
$$

\section{B. Pole Removal}

The second step in obtaining a rapidly converging Fourier Series of $K_{1 R 2}$ and $K_{2 R 2}$ involves the analytic removal of the pole in $\tilde{S}_{R 2}$ and $\tilde{D}_{R 2}$ as $\omega \rightarrow \Omega_{i}$. This results in a smooth function across the singularity which again leads to a faster convergence of the Fourier series. The peaked function which is subtracted is then transformed analytically by Residue Calculus [53].

Recall that in the sharp boundary model, the magnetic flux through a circle of radius $R$ is given by

$$
\Phi=B(z) R^{2}(z)=\text { constant }
$$


Using the conservation of magnetic flux, the cyclotron frequency can be expressed as

$$
\omega_{c i}^{2}=\omega_{0}^{2} \frac{B^{2}}{B_{0}^{2}}=\omega_{c i}^{2} \frac{R_{\alpha}^{4}}{R^{4}}
$$

where $R_{\alpha}$ is the radius at the location of the $\omega=\omega_{c i}$ resonance.

Writing $K_{1 R 2}$ and $K_{2 R 2}$ out fully

$$
\begin{gathered}
K_{1 R 2}=R^{2}(\xi)\left[1-\frac{\omega_{p i}^{2}}{\omega^{2}-\omega_{c i}^{2}+2 i \nu_{i e} \omega}\right] \\
K_{2 R 2}=-i R^{2}(\xi)\left[\frac{\omega_{p i}^{2} \omega_{c i}}{\omega\left(\omega^{2}-\omega_{c i}^{2}+2 i \nu_{i e} \omega\right)}\right]
\end{gathered}
$$

The resonant portion of both $K_{1 R 2}$ and $K_{2 R 2}$ are:

$$
\mathcal{R}=\frac{1}{\omega^{2}-\omega_{c i}^{2}+2 i \nu \omega}
$$

Substituting for $\omega_{c i}^{2}$ yields

$$
\begin{aligned}
\mathcal{R} & =\frac{R^{4}}{\omega^{2} R^{4}-\omega^{2} R_{\alpha}^{4}+2 i \nu \omega R^{4}} \\
& =\frac{R^{4} / \omega^{2}}{R^{4}-R_{\alpha}^{4}+2 i \frac{\nu}{\omega} R^{4}}
\end{aligned}
$$

To proceed further, we specify a function $R_{(\xi)}$ that conforms to the geometry of the system.

Selection of $z(\xi), R(\xi)$

The function $z(\xi)$ must conform to the following conditions

1. As $\xi \rightarrow 0, R(z) \rightarrow R_{0}$

2. As $\xi \rightarrow \infty, R(z) \rightarrow R_{\infty}$

3. At $\xi=\xi_{1}, R_{1}=\frac{1}{2}\left(R_{0}+R_{\infty}\right)$ 
where $R_{0}$ is the radius at $z=0, R_{\infty}$ is the radius at $z \rightarrow \infty$, and $\xi_{1}$ the $\xi$ at which the plasma radius is half way between $R_{0}$ and $R_{\infty}$.

Select the following function to model the experimental situation

$$
R(\xi)=\frac{c_{1}+c_{2} \xi^{2}}{1+c_{3} \xi^{2}}
$$

Using the first two conditions listed above yields

and

$$
R(\xi)=\frac{R_{0}+c_{2} \xi^{2}}{1+c_{3} \xi^{2}}
$$

Rearranging $R(\xi)$ yields

$$
\frac{c_{2}}{c_{3}}=R_{\infty}
$$

$$
R(\xi)=\frac{R_{0}-R_{\infty}}{1+c_{3} \xi^{2}}+R_{\infty}
$$

The third condition given above yields

$$
c_{3}=\frac{1}{\xi_{1}^{2}}
$$

This results in

$$
R(\xi)=R_{\infty}+\frac{R_{0}-R_{\infty}}{1+\frac{\xi^{2}}{\xi_{1}^{2}}}
$$

which is the desired expression for $R(\xi)$. Now consider $z(\xi)$. From the definition of $\xi$, it is seen that

$$
d \xi \equiv \frac{d z}{R}
$$

Then $z$ as a function of $\xi$ can be found by

$$
d z=R(\xi) d \xi
$$

or

$$
z(\xi)=R_{\infty} \xi+\xi_{1}\left(R_{0}-R_{\infty}\right) \tan ^{-1}\left(\frac{\xi}{\xi_{1}}\right)
$$

The computed results of $R(\xi)$ and $z(\xi)$ are plotted on Figure 3-6. Note that $\xi$ is very similar to $z$ except at the origin. 


\section{The Resonance Function}

Now recall that the resonance location is given by $R=R_{\alpha}$. The $\xi$ location of resonance is given by

$$
\begin{gathered}
R=R_{\alpha}=R_{\infty}+\frac{R_{0}-R_{\infty}}{1+\frac{\xi_{a}^{2}}{\xi_{1}^{2}}} \\
\Rightarrow \xi_{\alpha}^{2}=\xi_{1}^{2}\left(\frac{R_{0}-R_{\alpha}}{R_{\alpha}-R_{\infty}}\right)
\end{gathered}
$$

If we recall that $\mathcal{R}$ is given in Equation 3.43 by

$$
\mathcal{R}=\frac{R^{4} / \omega^{2}}{R^{4}-R_{\alpha}^{4}+2 i \frac{\nu}{\omega} R^{4}}
$$

then this can be rewritten as

$$
\mathcal{R}=\frac{c_{0}}{f(\xi)+i \epsilon}
$$

where $c_{0}$ is a constant, and

$$
\begin{aligned}
f(\xi) & \equiv 1-\frac{R_{\alpha}^{4}}{R^{4}(\xi)} \\
\epsilon & \equiv 2 \frac{\nu}{\omega}
\end{aligned}
$$

The constant of proportionality $c_{0}$ will be ignored for now as we examine the $\xi$ dependence of $\mathcal{R}$. Therefore let

$$
\mathcal{R} \equiv \frac{1}{f(\xi)+i \epsilon}
$$

Using a Taylor expansion for $\mathcal{R}$ near resonance

$$
R . \simeq \frac{1}{f_{0}^{\prime \prime}(\xi) \frac{\xi^{2}}{2}+f_{0}^{\prime}(\xi) \hat{\xi}+i \epsilon}
$$

and assuming a subtracting function of the form

$$
\mathcal{R}_{\text {rub }}=\frac{1}{\left(1+\epsilon c_{1}\right) f_{0}^{\prime} \hat{\xi}+i \epsilon\left(1+c_{2} \epsilon\right)}
$$

then

$$
\mathcal{R}-\mathcal{R}_{\text {sub }} \simeq \frac{-\frac{f_{0}^{\prime \prime}}{2}\left(\hat{\xi}^{2}-2 \hat{\xi} \epsilon c_{1} \frac{f_{0}^{\prime}}{f_{0}^{\prime \prime}}-2 i \frac{\epsilon^{2} c_{2}}{f_{0}^{\prime \prime}}\right)}{\left(f_{0}^{\prime}\right)^{2}\left[\hat{\xi}^{2}+\frac{2 i \epsilon}{f_{0}^{\prime}} \hat{\xi}-\frac{\epsilon^{2}}{\left(f_{0}^{\prime}\right)^{2}}\right]}
$$


$R(x i)$ lowiro ve. $x i$

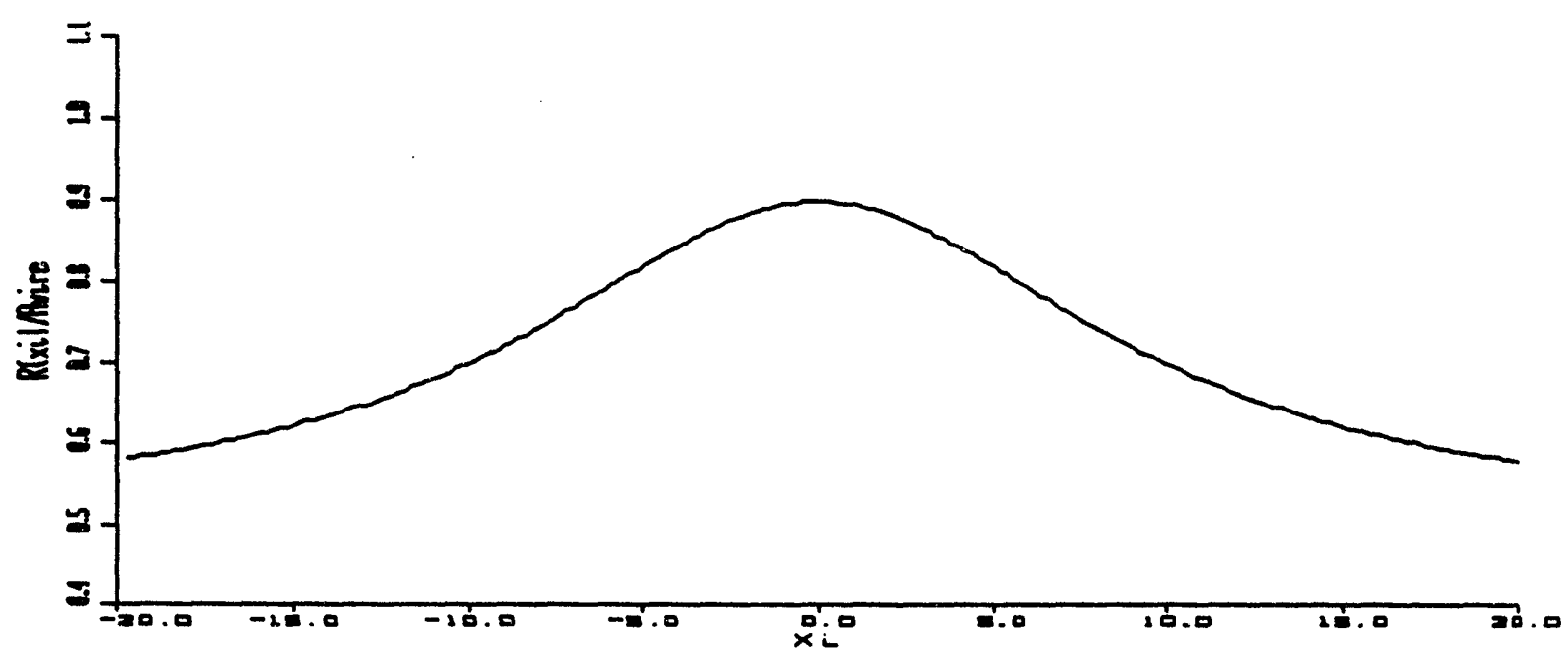

$Z(X I) /$ AWIRE VS. XI

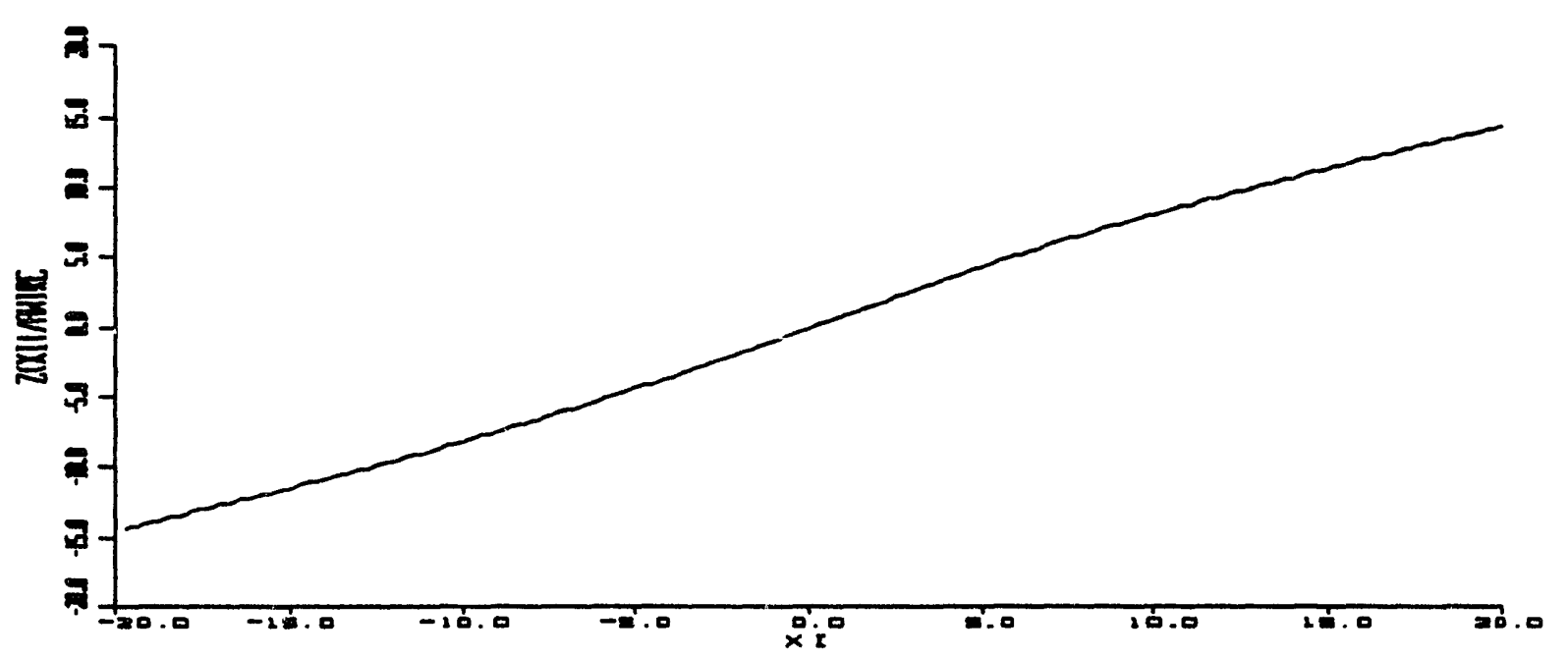

Figure 3-6: $R(\xi)$ and $z(\xi)$ for geometry modeled in analysis as given by Equations 3.44 and 3.45 
where

$$
\begin{aligned}
f_{0}^{\prime} & =\left.\frac{\partial}{\partial \xi}\left(1-\frac{R_{\alpha}^{4}}{R^{4}(\xi)}\right)\right|_{\xi=\xi_{\alpha}} \\
& =\left.\frac{4 R_{\alpha}^{4}}{R^{5}(\xi)} \frac{\partial R}{\partial \xi}\right|_{\xi=\xi_{\alpha}} \\
& =\frac{4}{R_{\alpha}} \frac{\partial}{\partial \xi} R\left(\xi=\xi_{\alpha}\right) \\
f_{0}^{\prime \prime}= & \left.\frac{\partial^{2}}{\partial \xi^{2}}\left(1-\frac{R_{\alpha}^{4}}{R^{4}(\xi)}\right)\right|_{\xi=\xi_{\alpha}} \\
= & \frac{4 R_{\alpha}^{4}}{R^{5}(\xi)}\left[\frac{-5}{R}\left(\frac{\partial R}{\partial \xi}\right)^{2}+\frac{\partial^{2} R}{\partial \xi^{2}}\right]_{\xi=\xi_{\alpha}} \\
= & \frac{-20}{R_{\alpha}^{2}}\left(\frac{\partial R}{\partial \xi}\right)^{2}+\frac{4}{R_{\alpha}} \frac{\partial^{2} R}{\partial \xi^{2}}
\end{aligned}
$$

It is assumed here that $f_{0}^{\prime} \neq 0$, i.e. the resonance does not occur at the exact center of the machine. The case of $f_{0}^{\prime}=0$ is treated shortly.

If we select $c_{1}$ and $c_{2}$ such that the coefficients of $\hat{\xi}$ and $\hat{\xi}^{2}$ are equal in the numerator and denominator,

$$
\begin{aligned}
& c_{1}=\frac{-i f_{0}^{\prime \prime}}{\left(f_{0}^{\prime}\right)^{2}} \\
& c_{2}=\frac{-i f_{0}^{\prime \prime}}{2\left(f_{0}^{\prime}\right)^{2}}
\end{aligned}
$$

It is seen that in the limit of $\hat{\xi} \rightarrow 0$,

$$
\lim _{\hat{\boldsymbol{\xi}} \rightarrow 0}\left[\mathcal{R}-\mathcal{R}_{\text {rub }}\right]=-\frac{f_{0}^{\prime \prime}}{2\left(f_{0}^{\prime}\right)^{2}}
$$

and is finite.

The entire $\mathcal{R}_{\text {oub }}$ must then contain two portions, one taking care of the singularity at $\xi=+\xi_{\alpha}$, the other eliminating the poie at $\xi=-\xi_{\alpha}$, in other words

$$
\mathcal{R}_{\text {sub }}=\frac{1}{\left(1+\epsilon c_{11}\right) \hat{f}_{0}^{\prime} \hat{\xi}+i \epsilon\left(1+c_{12} \epsilon\right)}+\frac{1}{\left(1+\epsilon c_{21}\right) \hat{\hat{f}}_{0}^{\prime} \hat{\hat{\xi}}+i \epsilon\left(1+c_{22} \epsilon\right)}
$$


where

$$
\begin{gathered}
\hat{\xi} \equiv \xi-\xi_{\alpha} \\
\hat{\hat{\xi}} \equiv \xi+\xi_{\alpha} \\
\hat{f}_{0}^{\prime} \equiv f_{0}^{\prime}\left(\xi=+\xi_{\alpha}\right) \\
\hat{\hat{f}}_{0}^{\prime} \equiv f_{0}^{\prime}\left(\xi=-\xi_{\alpha}\right)
\end{gathered}
$$

So the actual subtracted peak function are

$$
\begin{aligned}
S_{\text {sub }}(\xi) & =R_{\alpha}^{2} \frac{-\omega_{p i}^{2}}{\omega^{2}}\left[\frac{1}{\hat{f}_{0}^{\prime} \hat{\xi}+i \epsilon}+\frac{1}{\hat{\hat{f}}_{0}^{\prime} \hat{\hat{\xi}}+i \epsilon}\right] \\
& =\frac{-\omega_{p i}^{2}}{\omega^{2}} R_{\alpha}^{2} \mathcal{R}_{\text {sub }} \\
D_{\text {sub }}(\xi) & =R_{\alpha}^{2} \frac{\omega_{p i}^{2} \omega_{c i}}{\omega^{3}}\left[\frac{1}{\hat{f}_{0}^{\prime} \hat{\xi}+i \epsilon}+\frac{1}{\hat{\hat{f}}_{0}^{\prime} \hat{\hat{\xi}}+i \epsilon}\right] \\
& =\frac{\omega_{\text {pi }}^{2} \omega_{c i}}{\omega^{3}} R_{\alpha}^{2} R_{\text {sub }} \simeq \frac{\omega_{p i}^{2}}{\omega^{2}} R_{\alpha}^{2} R_{\text {sub }}
\end{aligned}
$$

The Fourier transform of $S_{\text {sub }}$ and $D_{\text {sub }}$ are then seen to be

$$
\begin{gathered}
\hat{S}_{\text {sub }}\left(k_{\xi}\right)=\frac{-\omega_{p i}^{2}}{\omega^{2}} R_{\alpha}^{2} \hat{\mathcal{R}}_{\text {sub }}\left(k_{\xi}\right) \\
\hat{D}_{\text {sub }}\left(k_{\xi}\right)=\frac{\omega_{p i}^{2}}{\omega^{2}} R_{\alpha}^{2} \hat{\mathcal{R}}_{\text {sub }}\left(k_{\xi}\right) \\
\hat{\mathcal{R}}_{\text {sub }}\left(k_{\xi}\right)=\int_{-\infty}^{\infty} \frac{e^{i k_{\xi} \xi} d \xi}{\hat{f}_{0}^{\prime}\left(\xi-\xi_{\alpha}\right)+i \epsilon}+\int_{-\infty}^{\infty} \frac{e^{i k_{t} \xi} d \xi}{\hat{f}_{0}^{\prime}\left(\xi+\xi_{\alpha}\right)+i \epsilon} \\
=e^{i k_{\ell} \xi_{\alpha}} \int_{-\infty}^{\infty} \frac{e^{i k_{\xi} x} d x}{\hat{f}_{0}^{\prime} x+i \epsilon}+e^{-i k_{\xi} \xi_{\alpha}} \int_{-\infty}^{\infty} \frac{e^{i k_{\xi} x^{\prime}} d x^{\prime}}{\hat{\hat{f}}_{0}^{\prime} x^{\prime}+i \epsilon}
\end{gathered}
$$

where

$$
x \equiv \xi-\xi_{\alpha} \quad ; \quad x^{\prime} \equiv \xi-\xi_{\alpha}
$$

103 
and

$$
\begin{gathered}
\hat{f}_{0}^{\prime}=\frac{4}{R_{\alpha}} \frac{d R\left(\xi=\xi_{\alpha}\right)}{d \xi}<0 \\
\hat{\hat{f}}_{0}^{\prime}=\frac{4}{R_{\alpha}} \frac{d R\left(\xi=-\xi_{\alpha}\right)}{d \xi}>0
\end{gathered}
$$

Depending on the sign of $k_{\xi}$, the $\xi$-integral will be closed in either the upper half of the complex $\xi$ plane or the lower half.

1. $k_{\xi}>0$ - For $k_{\xi}>0$, the $\xi$-integral would be closed in the upper half plane and includes only the $\xi=\xi_{\alpha}-i \epsilon / \hat{f}_{0}^{\prime}$ root. The resulting $\hat{\mathcal{R}}_{\text {sub }}$ is then

$$
\hat{\mathcal{R}}_{\text {sub }}=\frac{\pi i}{\hat{f}_{0}^{\prime}} e^{i k_{g} \xi_{a}} e^{k_{\varepsilon} e / \hat{f}_{0}^{\prime}}
$$

2. $k_{\xi}<0$ - For $k_{\xi}<0$, the $\xi$-integral is closed in the lower half $\xi$-plane and includes only the $\xi=-\xi_{\alpha}-i \epsilon / \hat{\hat{f}}_{0}^{\prime}$ root. Because the integral path is reversed, the resulting $\hat{\boldsymbol{R}}_{\text {rub }}$ is

$$
\hat{\mathcal{R}}_{\text {sub }}=\frac{-\pi i}{\hat{\hat{f}}_{0}^{\prime}} e^{-i k_{\xi} \xi_{a}} e^{k_{\varepsilon} e / \hat{f}_{0}^{\prime}}
$$

Typical order of magnitude estimates of $\epsilon$ and $\hat{f}_{0}^{\prime}, \hat{\hat{f}}_{0}^{\prime}$ show that

$$
\epsilon \sim 10^{-3} \quad \hat{f}_{0}^{\prime} \sim-0.2 \quad ; \quad \hat{\hat{f}}_{0}^{\prime} \sim+0.2
$$

From the Fourier spectrum of the best-fit curve of the source term $\Psi_{\text {wire }}$ on Figure $3-5$, it is seen that the range of $k_{\xi}$ that is relevant to this problem is approximately $k_{\xi} \leq 5$. In other words

$$
e^{ \pm k_{\mathrm{t}} \varepsilon / \hat{f}_{0}^{\prime}} \simeq 1
$$

Therefore

$$
\begin{aligned}
& k_{\xi}>0 \Rightarrow \quad \hat{\mathcal{R}}_{\text {oub }}\left(k_{\xi}\right) \simeq \frac{\pi i}{\hat{f}_{0}^{\prime}} e^{i k_{\ell} \xi_{\alpha}} \\
& k_{\xi}<0 \Rightarrow \quad \hat{\mathcal{R}}_{\text {oub }}\left(k_{\xi}\right) \simeq \frac{-\pi i}{\hat{\hat{f}}_{0}^{\prime}} e^{-i k_{\xi} \xi_{a}}
\end{aligned}
$$



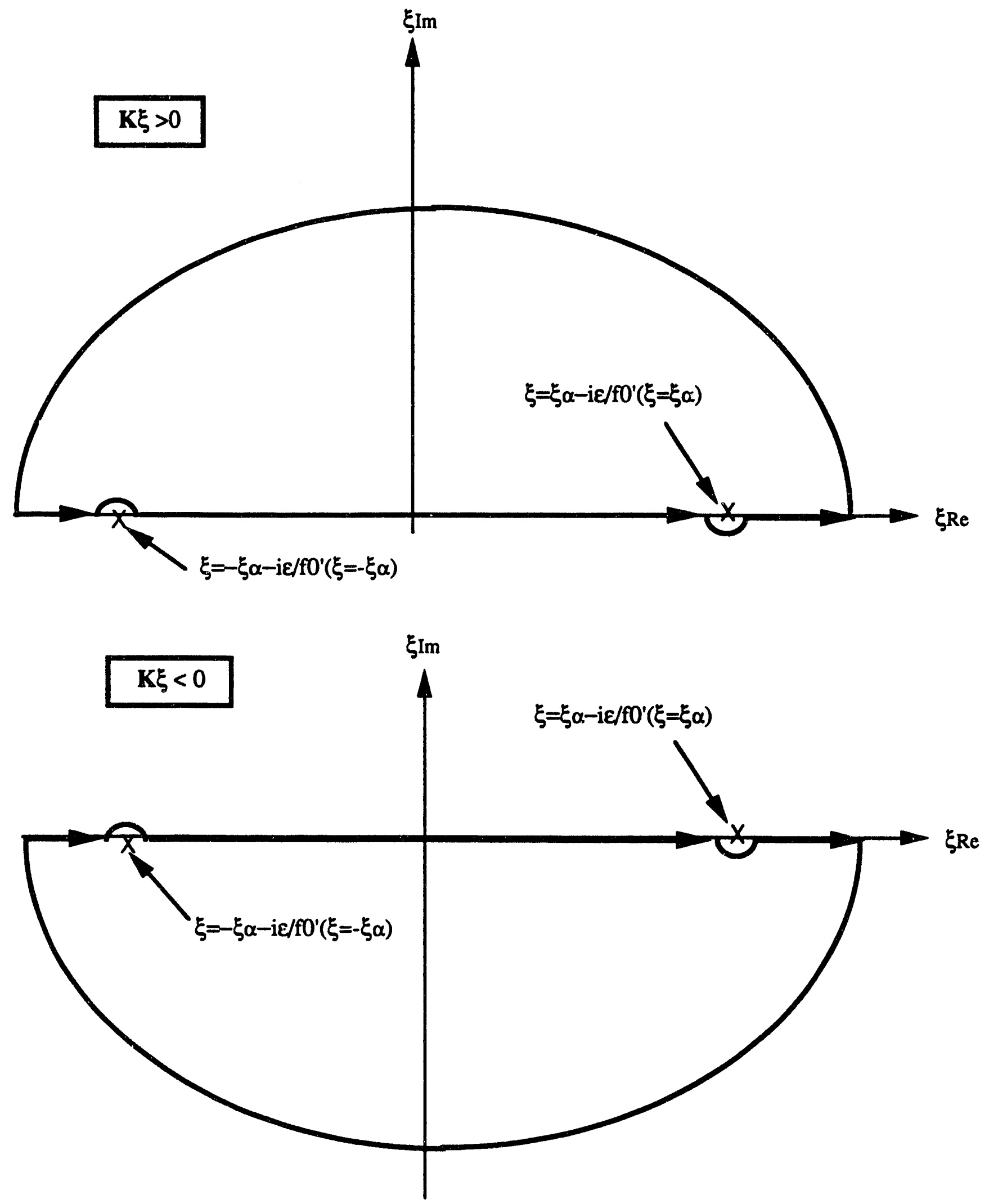

Figure 3-7: Residue integral path used in the extraction of poles of $K_{1 R_{2}}$ and $K_{2 R 2}$. Top case is for $k_{\xi}>0$, Bottom is for $k_{\xi}<0$. Note that different poles are inside the contour path for each case. 
$\mathcal{R}_{\text {oub }}$ for resonance at center of machine

If the resonance should occur at the EXACT center of the machine, this implies the slope of $R$ is zero. The old expansion and $R$ and $\mathcal{R}_{\text {sub }}$ is no longer valid. Therefore a special set of formulation is required to handle this case.

Taking the Taylor series expansion of $\mathcal{R}$ for $f_{0}^{\prime}=0$ and $f_{0}^{\prime \prime \prime}=0$ in a symmetric machine yields

$$
\mathcal{R} \simeq \frac{1}{f_{0}^{\prime \prime} \frac{\xi^{2}}{2}+f_{0}^{I V} \frac{\xi^{4}}{24}+i \epsilon}
$$

Selecting the following subtraction function

$$
\mathcal{R}_{\text {sub }}=\frac{1}{f_{0}^{\prime \prime}\left(1+\epsilon c_{1}\right) \frac{\epsilon^{2}}{2}+i \epsilon\left(1+\epsilon c_{2}\right)}
$$

then leads to

$$
\begin{gathered}
\mathcal{R}-\mathcal{R}_{\text {sub }}=\frac{\left(-\frac{f_{0}^{I V}}{24}\right)\left[\xi^{4}-\frac{12 f_{0}^{\prime \prime}}{f_{0}^{\prime V}} \epsilon c_{1} \xi^{2}-\frac{24 i \epsilon^{2} c_{0}}{f_{0}^{I V}}\right]}{\left(\frac{f_{0}^{\prime \prime}}{2}\right)^{2}\left[\xi^{4}+2 i \epsilon \frac{f_{0}^{\prime \prime} \xi^{2}}{\left(f_{0}^{\prime \prime}\right)^{2}}-4 \frac{\epsilon^{2}}{\left(f_{0}^{\prime \prime}\right)^{2}}\right]} \\
\text { and } \quad\left(\mathcal{R}-\left.\mathcal{R}_{\text {sub }}\right|_{\xi \rightarrow 0}=\frac{-1}{6} \frac{f_{0}^{I V}}{\left(f_{0}^{\prime \prime}\right)^{2}}\right.
\end{gathered}
$$

Equating the coefficients of $\xi$ yields

$$
\begin{aligned}
& c_{1}=\frac{-i f_{0}^{I V}}{6\left(f_{0}^{\prime \prime}\right)^{2}} \\
& c_{2}=\frac{-i f_{0}^{I V}}{6\left(f_{0}^{\prime \prime}\right)^{2}}
\end{aligned}
$$

The transform of this fitting function is then

$$
\begin{aligned}
\hat{\mathcal{R}}_{\text {sub }}\left(k_{\xi}\right) & =\int_{-\infty}^{\infty} \frac{e^{i k_{t} \xi} d \xi}{a_{1} \xi^{2}+b_{1}} \\
& =\frac{1}{a_{1}} \int_{-\infty}^{\infty} \frac{e^{i k_{t} \xi} d \xi}{\xi^{2}+\frac{b_{1}}{a_{1}}} \\
& =\frac{1}{a_{1}} \int_{-\infty}^{\infty} \frac{e^{i k_{t} \xi} d \xi}{\left(\xi-i \frac{b_{1}}{a_{1}}\right)\left(\xi+i \frac{b_{1}}{a_{1}}\right)} \\
& =\frac{1}{2 a_{1} \sqrt{\frac{b_{1}}{a_{1}}}} \int e^{i k_{t} \xi} d \xi\left[\frac{1}{\xi-\sqrt{\frac{b_{1}}{\alpha_{1}}}}-\frac{1}{\xi+\sqrt{\frac{b_{1}}{\alpha_{1}}}}\right]
\end{aligned}
$$


where

$$
\begin{aligned}
& a_{1} \equiv \frac{f_{0}^{\prime \prime}}{2}\left(1+\epsilon c_{1}\right) \\
&= \frac{f_{0}^{\prime \prime}}{2}\left(1-i \epsilon \frac{f_{0}^{I V}}{6\left(f_{0}^{\prime \prime}\right)^{2}}\right)<0 \\
& \alpha_{1} \equiv-a_{1}>0 \\
& b_{1} \equiv i \epsilon\left(1+\epsilon c_{2}\right) \\
&=i \epsilon\left(1-\epsilon \frac{f_{0}^{I V}}{6\left(f_{0}^{\prime \prime}\right)^{2}}\right)
\end{aligned}
$$

The poles and the contour integral paths for $k_{\xi}>0$ and $k_{\xi}<0$ are plotted on Figure 3-8. From the residue contours, it is seen that

$$
\begin{aligned}
& k_{\xi}>0 \Rightarrow \quad \hat{\mathcal{K}}_{\text {oub }}\left(k_{\xi}\right)=\frac{\pi i}{2 a_{1} \sqrt{\frac{b_{1}}{\alpha_{1}}}} e^{i k_{\xi} \sqrt{\frac{b_{1}}{a_{1}}}} \\
& k_{\xi}<0 \Rightarrow \quad \hat{\mathcal{R}}_{\text {rub }}\left(k_{\xi}\right)=\frac{-\pi i}{2 a_{1} \sqrt{\frac{b_{1}}{\alpha_{1}}}} e^{-i k_{\xi} \sqrt{\frac{b_{1}}{a_{1}}}}
\end{aligned}
$$

The negative sign for the $k_{\xi}<0$ case is due to the reversed direction of the integral contour.

In the limit of $\epsilon \rightarrow 0, b_{1} \rightarrow 0$ and it can be shown that

$$
\begin{aligned}
& k_{\xi}>0 \Rightarrow \hat{\mathcal{R}}_{\text {rub }} \simeq \frac{-(1+i) \pi}{2\left(-\epsilon f_{0}^{\prime \prime}\right)^{1 / 2}} \\
& k_{\xi}<0 \Rightarrow \hat{\mathcal{R}}_{\text {rub }} \simeq \frac{+(1+i) \pi}{2\left(-\epsilon f_{0}^{\prime \prime}\right)^{1 / 2}}
\end{aligned}
$$



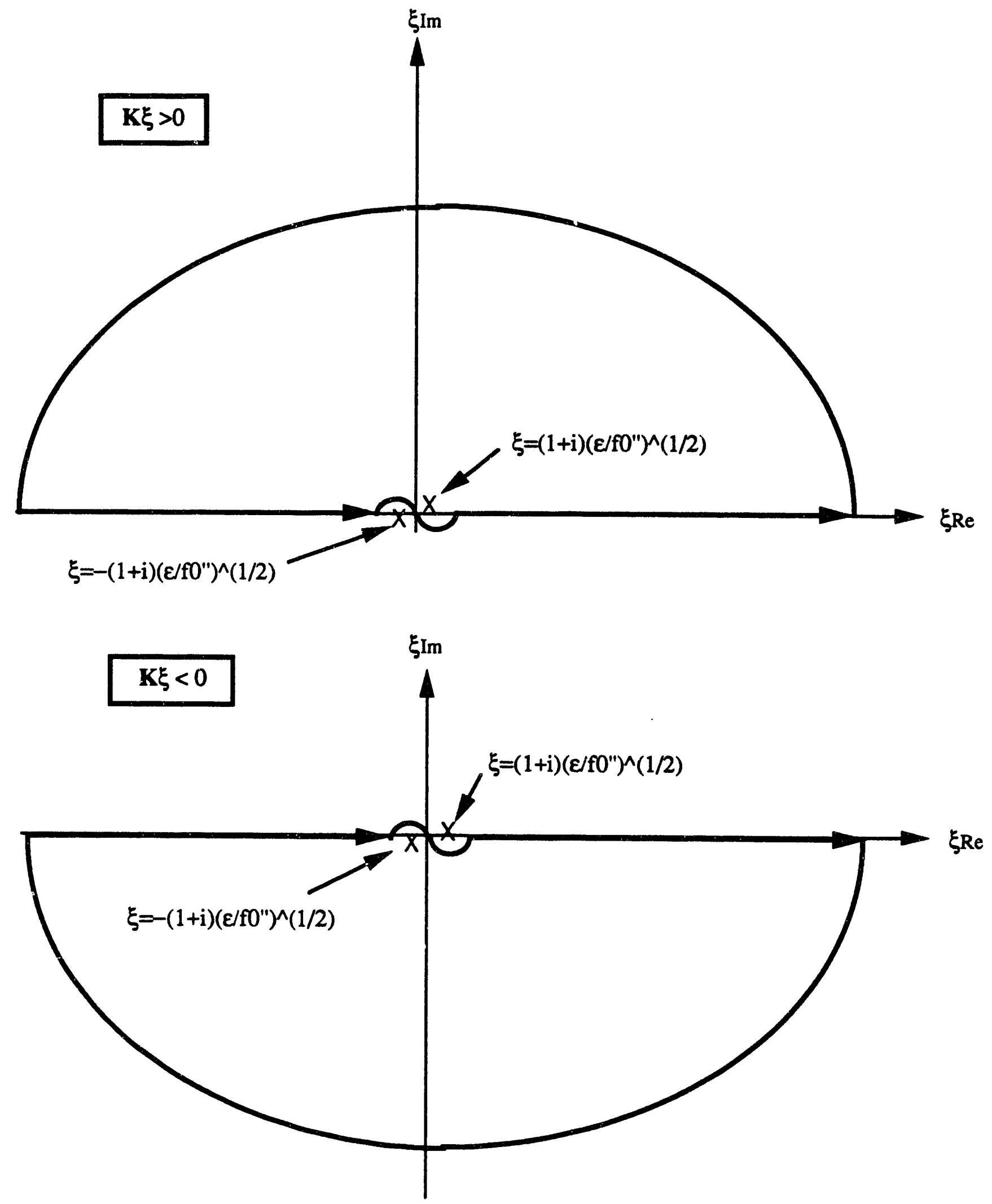

Figure 3-8: Residue integral path used in the extraction of poles of $K_{1 R 2}$ and $K_{2 R 2}$ for the case of resonance at $\xi=0$. Top case is for $k_{\xi}>0$, Bottom is for $k_{\xi}<0$. Note that different poles are inside the contour path for each case. 
Substituting these results into the basic equations yield

$$
\begin{aligned}
& -k_{\xi}^{2} \hat{\hat{\chi}}_{p}\left(\alpha, k_{\xi}\right)+k_{0}^{2}\left(\begin{array}{l}
+\int\left[\tilde{S}_{s m}\left(k_{\xi}-k_{\xi}^{\prime}\right)+\bar{S}_{\text {sub }}\left(k_{\xi}-k_{\xi}^{\prime}\right)\right] \hat{\hat{\chi}}_{p}\left(\alpha, k_{\xi}^{\prime}\right) d k_{\xi}^{\prime} \\
-i \int\left[\tilde{D}_{s m}\left(k_{\xi}-k_{\xi}^{\prime}\right)+\tilde{D}_{s u b}\left(k_{\xi}-k_{\xi}^{\prime}\right)\right] \hat{\hat{\phi}}_{p}\left(\alpha, k_{\xi}^{\prime}\right) d k_{\xi}^{\prime} \\
+S_{\infty} \hat{\hat{\chi}}_{p}\left(\alpha, k_{\xi}\right) \\
-i D_{\infty} \hat{\hat{\phi}}_{p}\left(\alpha, k_{\xi}\right)
\end{array}\right)=0 \\
& \left(-k_{\xi}^{2}-\alpha^{2}\right) \hat{\hat{\phi}}_{p}\left(\alpha, k_{\xi}\right)+k_{0}^{2}\left(\begin{array}{l}
+\int\left[\tilde{S}_{s m}\left(k_{\xi}-k_{\xi}^{\prime}\right)+\tilde{S}_{s u b}\left(k_{\xi}-k_{\xi}^{\prime}\right)\right] \hat{\hat{\phi}}_{p}\left(\alpha, k_{\xi}^{\prime}\right) d k_{\xi}^{\prime} \\
+i \int\left[\tilde{D}_{s m}\left(k_{\xi}-k_{\xi}^{\prime}\right)+\tilde{D}_{s u b}\left(k_{\xi}-k_{\xi}^{\prime}\right)\right] \hat{\hat{x}}_{p}\left(\alpha, k_{\xi}^{\prime}\right) d k_{\xi}^{\prime} \\
+S_{\infty} \hat{\hat{\phi}}_{p}\left(\alpha, k_{\xi}\right) \\
+i D_{\infty} \hat{\hat{\chi}}_{p}\left(\alpha, k_{\xi}\right)
\end{array}\right)=0
\end{aligned}
$$

where

$$
\begin{gathered}
\tilde{S}_{R 2}\left(k_{\xi}-k_{\xi}^{\prime}\right) \equiv\left[\tilde{S}_{o m}\left(k_{\xi}-k_{\xi}^{\prime}\right)+\tilde{S}_{a u b}\left(k_{\xi}-k_{\xi}^{\prime}\right)\right] \\
\tilde{D}_{R 2}\left(k_{\xi}-k_{\xi}^{\prime}\right) \equiv\left[\tilde{D}_{m m}\left(k_{\xi}-k_{\xi}^{\prime}\right)+\tilde{D}_{s u b}\left(k_{\xi}-k_{\xi}^{\prime}\right)\right]
\end{gathered}
$$

$\tilde{S}_{\text {sub }}$ and $\tilde{D}_{\text {sub }}$ are the Fourier transforms of the subtracted terms whose kernals are derived in this section and seen in Equation 3.47. $\bar{S}_{o m}$ and $\tilde{D}_{o m}$ are the smooth functions that result after subtracting out the $\tilde{S}_{\text {sub }}, \tilde{D}_{\text {sub }}$ terms from $\tilde{S}_{R 2}$ and $\tilde{D}_{R 2}$. 


\subsubsection{Solution of Coupled Integral Equations}

By the procedure outlined in the previous section, it is possible to find the Fourier transforms $\bar{S}_{R 2}$ and $\tilde{D}_{R 2}$. These would then, in principle, allow for the solution of the coupled integral equations given by Equations 3.40 and 3.41. The unknowns are $\alpha$ and $\hat{\hat{\chi}}_{p}$. Note that $\hat{\hat{\phi}}_{p}$ is found from the boundary condition and is given by Equation 3.24.

\section{Method of Solution}

The two equations involved are

$$
\begin{aligned}
-k_{\xi}^{2} \hat{\hat{\chi}}_{p}\left(\alpha, k_{\xi}\right)+k_{0}^{2}\left(\begin{array}{l}
+ \\
-i \int\left[\tilde{S}_{s m}\left(k_{\xi}-k_{\xi}^{\prime}\right)+\tilde{S}_{s u b}\left(k_{\xi}-k_{\xi}^{\prime}\right)\right] \hat{\hat{\chi}}_{p}\left(\alpha, k_{\xi}^{\prime}\right) d k_{\xi}^{\prime} \\
+S_{\infty} \hat{\hat{\chi}}_{p}\left(\alpha, k_{\xi}\right) \\
-i D_{\infty} \hat{\hat{\phi}}_{p}\left(\alpha, k_{\xi}\right)
\end{array}\right)=0 \\
\left(-k_{\xi}^{2}-\alpha^{2}\right) \hat{\hat{\phi}}_{p}\left(\alpha, k_{\xi}\right)+k_{0}^{2}\left(\begin{array}{l}
\hat{\phi}_{p}\left(\alpha, k_{\xi}^{\prime}\right) d k_{\xi}^{\prime} \\
+i \int\left[\tilde{D}_{s m}\left(k_{\xi}-k_{\xi}^{\prime}\right)+\tilde{D}_{s u b}\left(k_{\xi}-k_{\xi}^{\prime}\right)\right] \hat{\hat{\chi}}_{p}\left(\alpha, k_{\xi}^{\prime}\right) d k_{\xi}^{\prime} \\
+S_{\infty} \hat{\hat{\phi}}_{p}\left(\alpha, k_{\xi}\right) \\
+i D_{\infty} \hat{\hat{\chi}}_{p}\left(\alpha, k_{\xi}\right)
\end{array}\right)=0
\end{aligned}
$$

We solve the two coupled integral equations by a simple finite differencing procedure

Let

$$
\begin{gathered}
k_{j} \equiv k_{\xi} \quad ; \quad k_{l} \equiv k_{\xi}^{\prime} \\
\chi_{j l} \equiv \chi_{p}\left(\alpha\left(k_{j}\right), k_{l}\right) \\
\phi_{j l} \equiv \phi_{p}\left(\alpha\left(k_{j}\right), k_{l}\right) \\
S_{j-l} \equiv\left[\tilde{S}_{\text {sm }}\left(k_{j}-k_{l}\right)+\tilde{S}_{\text {oub }}\left(k_{j}-k_{l}\right)\right] \\
D_{j-l} \equiv\left[\tilde{D}_{\text {om }}\left(k_{j}-k_{l}\right)+\tilde{D}_{\text {oub }}\left(k_{j}-k_{l}\right)\right]
\end{gathered}
$$

The equations above then become 


$$
\begin{gathered}
-k_{j}^{2} \chi_{j j}+k_{0}^{2}\left[\sum_{l} S_{j-l} \chi_{j l} \Delta k_{l}-i \sum_{l} D_{j-l} \phi_{j l} \Delta k_{l}\right]+k_{0}^{2}\left(S_{\infty} \chi_{j j}-i D_{\infty} \phi_{j j}\right)=0 \\
-\left(k_{j}^{2}+\alpha_{j}^{2}\right) \phi_{j j}+k_{0}^{2}\left[\sum_{l} S_{j-l} \phi_{j l} \Delta k_{l}+i \sum_{l} D_{j-l} \chi_{j l} \Delta k_{l}\right]+k_{0}^{2}\left(S_{\infty} \phi_{j j}+i D_{\infty} \chi_{j j}\right)=0
\end{gathered}
$$

The steps to be followed to solve these two coupled difference equations are as follows:

1. Pick an array of $k_{1}, k_{2} \ldots k_{n}$

2. Focus on a particular longitudinal wave number $k_{j}$ and corresponding perpendicular wavenumber $\alpha_{j}$ which is as yet unknown. An initial guess for $\alpha_{j}$ is determined by using Equations 3.34 and 3.35 and letting $\frac{\theta^{2}}{\partial \xi^{2}}=-k_{\xi}^{2}$. The resulting equations can then be simultaneously be solved to yield $\alpha_{j}$ in terms of $k_{j}$ and is seen to be

$$
\alpha_{j}^{2}=-k_{j}^{2}+k_{0}^{2} S_{\infty}+\frac{k_{0}^{4} D_{\infty}^{2}}{k_{0}^{2} S_{\infty}-k_{j}^{2}}
$$

3. Solve for $\phi_{j l}$ array using Equation 3.24

4. Substitute $\alpha_{j}, k_{j}$ 's, and $\phi_{j l}$ array into Equation 3.48 to obtain the matrix equation for $\chi_{j l}$

$$
\underline{\underline{M}} \cdot \chi \underline{\underline{b} a}
$$

5. Calculate $\chi_{j l}$. The procedure selected for solving the system of simultaneous linear equations is the LU-decomposition method as outlined by Press et al. in Numerical Recipes [54].

The matrix representation of the problem is 


$$
\underline{\underline{A}} \cdot \vec{x}=\vec{b}
$$

If $\underline{\underline{A}}$ is represented by $\underline{\underline{A}}=\underline{\underline{L}} \cdot \underline{\underline{U}}$, then

$$
\underline{\underline{A}} \cdot \vec{x}=(\underline{\underline{L}} \cdot \underline{\underline{U}}) \cdot \vec{x}=\underline{\underline{L}} \cdot(\underline{\underline{U}} \cdot \vec{x}) \equiv \underline{\underline{L}} \cdot \vec{y}=b
$$

Vector $\vec{y}$ can first be solved from

$$
\underline{\underline{L}} \cdot \vec{y}=\vec{b}
$$

and then the vector $\vec{x}$ can be solved from

$$
\underline{\underline{U}} \cdot \vec{x}=\vec{y}
$$

The advantage of this method is that $\underline{\underline{L}}$ is a matrix that has nonzero elements on the diagonal and below, while $\underline{\underline{U}}$ is a matrix that has nonzero elements on the diagonal and above. This makes the forward and backward substitution required very simple to implement.

6. Substitute $\chi_{j l}$ into Equation 3.49 to iterate for new $\alpha_{j}$. If we rewrite Equation 3.49 as

$$
-\left(k_{j}^{2}+\alpha_{j}^{2}\right) \phi_{j j}+k_{0}^{2} C_{2}+k_{0}^{2}\left(S_{\infty} \phi_{j j}+i D_{\infty} \chi_{j j}\right)=0
$$

then the following equation is obtained for $\alpha_{j}$ :

$$
\alpha_{j}^{2}=-k_{j}^{2}+\frac{k_{0}^{2} C_{2}}{\phi_{j j}}+\frac{k_{0}^{2}\left(S_{\infty} \phi_{j j}+i D_{\infty} \chi_{j j}\right)}{\phi_{j j}}
$$

7. Reiterate to step 3 until convergence is obtained.

8. Pick the next longitudinal wave number $k_{j+1}$ and continue starting with Step 2 
9. Regenerate ICRF EM fields by inverse Fourier transform. After solving for the $\alpha_{j}$ that corresponds to each $k_{\xi j}$, the resulting arrays of $\phi$ and $\chi$ can then be obtained. The plasma electric fields can then be found by

$$
\begin{aligned}
& \hat{E}_{\theta}\left(\rho, k_{\xi}\right)=\hat{E}_{\theta}\left(\alpha, k_{\xi}\right) \rho J_{1}(\alpha \rho) \equiv i \omega \frac{\hat{\hat{\phi}}_{p}\left(\alpha, k_{\xi}\right)}{r} \rho J_{1}(\alpha \rho) \\
& \hat{E}_{r}\left(\rho, k_{\xi}\right)=\hat{E}_{r}\left(\alpha, k_{\xi}\right) \rho J_{1}(\alpha \rho) \equiv i \omega \frac{\hat{\hat{\chi}}_{p}\left(\alpha, k_{\xi}\right)}{r} \rho J_{1}(\alpha \rho)
\end{aligned}
$$

The ICRF EM fields as a function of $(\rho, \xi)$ is found by inverse Fouriertransforming to yield

$$
\begin{aligned}
& E_{\theta}(\rho, \xi)=\frac{1}{2 \pi} \int \hat{E}\left(\rho, k_{\xi}\right) e^{-i k_{\xi} \xi} d k_{\xi} \\
& =\frac{i \omega}{2 \pi \rho R(\xi)} \int_{-\infty}^{\infty} \hat{\hat{\phi}}_{p}\left(\alpha, k_{\xi}\right) \rho J_{1}(\alpha \rho) e^{-i k_{\xi} \xi} d k_{\xi} \\
& =\frac{i \omega}{2 \pi R(\xi)} \int_{-\infty}^{\infty} \hat{\hat{\phi}}_{p}\left(\alpha, k_{\xi}\right) J_{1}(\alpha \rho) e^{-i k_{\xi} \xi} d k_{\xi} \\
& E_{r}(\rho, \xi)=\frac{1}{2 \pi} \int_{-\infty}^{\infty} \hat{E}_{r}\left(\rho, k_{\xi}\right) e^{-i k_{\xi} \xi} d k_{\xi} \\
& =\frac{i \omega}{2 \pi \rho R(\xi)} \int_{-\infty}^{\infty} \hat{\hat{\chi}}_{p}\left(\alpha, k_{\xi}\right) \rho J_{1}(\alpha \rho) e^{-i k_{\xi} \xi} d k_{\xi} \\
& =\frac{i \omega}{2 \pi R(\xi)} \int_{-\infty}^{\infty} \hat{\hat{\chi}}_{p}\left(\alpha, k_{\xi}\right) J_{1}(\alpha \rho) e^{-i k_{\xi} \xi} d k_{\xi} \\
& \begin{aligned}
i \omega B_{z}(\rho, \xi) & =\nabla \times \underline{E} \\
i \omega B_{z}(\rho, \xi) & =\frac{1}{r} \frac{\partial r E_{\theta}}{\partial r}-\underbrace{\frac{1}{r} \frac{\partial E_{r}}{\partial \theta}}_{=0}=\frac{i \omega}{r} \frac{\partial \phi_{p}}{\partial r} \\
& =\frac{i \omega}{\rho R(\xi) R(\xi)} \frac{\partial \phi_{p}(\rho, \xi)}{\partial \rho} \\
B_{z}(\rho, \xi) & =\frac{1}{\rho R^{2}(\xi)} \frac{\partial}{\partial \rho}\left[\hat{\hat{\phi}}_{p}(\alpha, \xi) \rho J_{1}(\alpha \rho)\right]
\end{aligned}
\end{aligned}
$$




$$
\begin{aligned}
& =\frac{1}{2 \pi} \int_{-\infty}^{\infty} e^{-i k_{\xi} \xi} d k_{\xi} \frac{\hat{\hat{\phi}}_{p}\left(\alpha, k_{\xi}\right)}{R^{2}(\xi)}\left[\alpha J_{1}^{\prime}(\alpha \rho)+\frac{J_{1}(\alpha \rho)}{\rho}\right] \\
& =\frac{1}{2 \pi R^{2}(\xi)} \int_{-\infty}^{\infty} e^{-i k_{\xi} \xi} \hat{\hat{\phi}}_{p}\left(\alpha, k_{\xi}\right) \alpha J_{0}(\alpha \rho) d k_{\xi}
\end{aligned}
$$

In evaluating these integral, it is implicitly assumed that $\alpha=\alpha\left(k_{\xi}\right)$ has been determined as described above. 


\subsubsection{Alternative Method of Solution of $\alpha$}

Equation 3.48 can be expressed as a matrix equation

$$
\underline{A}_{\chi} \cdot \chi=\underline{\underline{A}}_{\phi} \cdot \phi
$$

Then $\chi$ can be expressed as

$$
\chi=\left(\underline{\underline{A}}^{-1} \cdot \underline{\underline{A}}_{\phi}\right) \cdot \underline{\phi}
$$

The second coupled matrix equation (Equation 3.49) can then be written as

$$
\begin{aligned}
\left(k_{0}^{2} S_{\infty}-k_{j}^{2}-\alpha_{j}^{2}\right) \phi_{j}+k_{0}^{2} \Delta k_{l} \vec{S}_{j m l} \cdot \underline{\phi} & +i k_{0}^{2} D_{\infty}\left(\hat{e}_{j} \cdot \underline{\underline{A}}_{x}^{-1} \cdot \underline{\underline{A}}_{\phi}\right) \cdot \underline{\phi} \\
& +i k_{0}^{2} \Delta k_{l} \vec{D}_{j m l} \cdot\left(\underline{\underline{A}}_{x}^{-1} \cdot \underline{\underline{A}}_{\phi}\right) \cdot \phi=0
\end{aligned}
$$

where

$$
\begin{gathered}
\underline{A}_{x}(i, j)=\left(-k_{j}^{2}+k_{0}^{2} S_{\infty}\right) \delta(i-j)+k_{0}^{2} S_{i-j} \Delta k_{l} \\
\underline{\underline{A}}_{\phi}(i, j)=i k_{0}^{2} D_{\infty} \delta(i-j)+i k_{0}^{2} D_{i-j} \Delta k_{l}
\end{gathered}
$$

It can be seen that both $\underline{A}_{\alpha}$ and $\underline{A}_{\phi}$ are independent of $\alpha$. Therefore they only have to be calculated once when searching for the optimum $\alpha$. Therefore an iteration for $\alpha$ using the second coupled matrix equation given above is the only step necessary for solving the perpendicular mode $\alpha$ that corresponds to each $k_{\xi}$.

The rational for using an alternative method of solution of the coupled equations is to speed up the calculations. Instead of performing two sets of matrix operations, this alternative method performs one set of matrix inversion at the onset of calculations, and subsequently requires only one set of matrix operation to solve the $\alpha_{j}, \chi_{j}$, and $\phi_{j}$. Implementation of this scheme on the CRAY resulted in a 10 fold increase in speed. 


\subsubsection{Figure of Merit Calculation}

The figure of merit to be used in the cylindrical geometry case is one that is commonly used in literature $[55,56,57,58]$ to determine optimal coupling, namely the antenna impedence. It can be easily shown $[59,44]$ that the absorbed power is calculated by

$$
P=\frac{1}{2} \operatorname{Re} \int E \cdot I^{*} d V
$$

The volume integral in cylindrical geometry can be expressed in terms of the $(\rho, \xi)$ coordinate as follows:

$$
d V=r d r d z d \theta=\underbrace{\rho R(\xi)}_{r}\left|\begin{array}{ll}
\frac{\partial_{r}}{\partial \xi} & \frac{\partial_{r}}{\partial \rho} \\
\frac{\partial_{z}}{\partial \xi} & \frac{\partial_{z}}{\partial \rho}
\end{array}\right| d \rho d \xi d \theta
$$

where

$$
d z \equiv R(\xi) d \xi \quad ; \quad r \equiv \rho R(\xi)
$$

After some algebra, it is seen that

$$
d V=\rho R^{3}(\xi) d \rho d \xi d \theta
$$

Thus the power absorption integral becomes

$$
\begin{aligned}
P & =\frac{1}{2} \operatorname{Re} \int \underline{E} \cdot \underline{I}^{*} d V \\
& =\operatorname{Re} \int \frac{-i \pi \omega}{\mu_{0} c^{2}} E(\rho, \xi) \cdot\left(\underline{K^{*}}(\xi)-\underline{I}\right) \cdot E^{*}(\rho, \xi) \rho R^{3}(\xi) d \rho d \xi
\end{aligned}
$$

Expressing $E$ and $L$ in terms of their Fourier coefficient:

$P=\operatorname{Re} \iiint \int \frac{-i \pi \omega}{\mu_{0} c^{2}}\left\{\begin{array}{l}\rho R^{3}(\xi)\left[E\left(\alpha, k_{\xi}\right) e^{-i k_{\xi} \xi} \rho J_{1}(\alpha \rho)\right] \cdot\left[\underline{K}^{*}(\xi)-\underline{I}\right] \cdot \\ {\left[\underline{E}^{*}\left(\alpha^{\prime}, k_{\xi}^{\prime}\right) e^{+i k_{\xi}^{\prime} \xi} \rho J_{1}\left(\alpha^{\prime} \rho\right)\right]}\end{array}\right\} d k_{\xi} d k_{\xi}^{\prime} d \rho d \xi$

Expanding the dielectric tensor yields

$$
\begin{aligned}
P= & \operatorname{Re} \int_{\rho=0}^{1} \frac{-i \pi \omega}{\mu_{0} c^{2}} \rho^{3} J_{1}(\alpha \rho) J_{1}\left(\alpha^{\prime} \rho\right) d \rho \int_{-\infty}^{\infty} d k_{\xi} \int_{-\infty}^{\infty} d k_{\xi}^{\prime} \int_{-\infty}^{\infty} R^{3}(\xi) e^{-i \xi\left(k_{\xi}-k_{\xi}^{\prime}\right)} d \xi \\
& \left\{\begin{array}{l}
E_{r}\left(\alpha, k_{\xi}\right)\left[(S(\xi)-1) E_{r}\left(\alpha^{\prime}, k_{\xi}^{\prime}\right)-i D(\xi) E_{\theta}\left(\alpha^{\prime}, k_{\xi}^{\prime}\right)\right]^{*}+ \\
E_{\theta}\left(\alpha, k_{\xi}\right)\left[i D(\xi) E_{r}\left(\alpha^{\prime}, k_{\xi}^{\prime}\right)+(S(\xi)-1) E_{\theta}\left(\alpha^{\prime}, k_{\xi}^{\prime}\right)\right]^{*}+ \\
E_{z}\left(\alpha, k_{\xi}\right)\left[(P(\xi)-1) E_{z}\left(\alpha^{\prime}, k_{\xi}^{\prime}\right)\right]^{*}
\end{array}\right.
\end{aligned}
$$


Again the last term involving $E_{z}$ is dropped because $E_{z}$ is much smaller than $E_{r}, E_{\theta}$.

Recall that

$$
\begin{aligned}
& E_{r}(\rho, \xi)=\frac{i \omega}{\rho R(\xi)} \chi_{p}(\rho, \xi) \\
& E_{\theta}(\rho, \xi)=\frac{i \omega}{\rho R(\xi)} \phi_{p}(\rho, \xi)
\end{aligned}
$$

The absorbed power can then be expressed in terms of $\chi$ and $\phi$

$$
\begin{aligned}
P= & \frac{\pi k_{0}^{2} \omega}{\mu_{0}} \operatorname{Re} \int_{\rho=0}^{1}-i \rho J_{1}(\alpha \rho) J_{1}\left(\alpha^{\prime} \rho\right) d \rho \int_{-\infty}^{\infty} d k_{\xi} \int_{-\infty}^{\infty} d k_{\xi}^{\prime} \int_{-\infty}^{\infty} R(\xi) e^{-i \xi\left(k_{\xi}-k_{\xi}^{\prime}\right)} d \xi \\
& \left\{\begin{array}{l}
\chi_{p}\left(\alpha, k_{\xi}\right)\left[(S(\xi)-1) \chi_{p}\left(\alpha^{\prime}, k_{\xi}^{\prime}\right)-i D(\xi) \phi_{p}\left(\alpha^{\prime}, k_{\xi}^{\prime}\right)\right]^{*}+ \\
\phi_{p}\left(\alpha, k_{\xi}\right)\left[i D(\xi) \chi_{p}\left(\alpha^{\prime}, k_{\xi}^{\prime}\right)+(S(\xi)-1) \phi_{p}\left(\alpha^{\prime}, k_{\xi}^{\prime}\right)\right]^{*}
\end{array}\right\}
\end{aligned}
$$

Two types of $\xi$ integrals have to be performed:

$$
\begin{gathered}
I_{\bullet}(a) \equiv \int_{-\infty}^{\infty} e^{i a \xi} R(\xi)\left[S^{*}(\xi)-1\right] \\
I_{d}(a) \equiv \int_{-\infty}^{\infty} e^{i a \xi} R(\xi) D^{*}(\xi)
\end{gathered}
$$

where $a$ in this case is simply $k_{\xi}^{\prime}-k_{\xi}$.

This transform is similar to what was calculated previously with the Fourier transform of $R^{2}(\xi) S(\xi)$ and $R^{2}(\xi) D(\xi)$. The same procedure for obtaining the Fourier transform of $R^{2} D, S$ as outlined in the previous section is employed.

Define the following transformed functions

$$
\begin{gathered}
\hat{S_{R 1}(a)} \equiv \int_{\nu_{-\infty}}^{\infty} R(\xi)\left[S^{*}(\xi)-1\right] e^{i a \xi} d \xi \\
\hat{D_{R 1}}(a) \equiv \int_{-\infty}^{\infty} R(\xi) D^{*}(\xi) e^{i a \xi} d \xi \\
K_{R \infty S_{\infty}} \equiv R(\infty)\left[S^{*}(\infty)-1\right]
\end{gathered}
$$




$$
K_{R \infty D_{\infty}} \equiv R(\infty) D^{*}(\infty)
$$

and using the well-known identity

$$
\int_{-\infty}^{\infty} e^{i a x} d x=\delta(a)
$$

The equation for the absorbed power then becomes

$$
\begin{aligned}
P= & \frac{\pi k_{0}^{2} \omega}{\mu_{0}} \operatorname{Re} \int_{0}^{1}-i \rho J_{1}(\alpha \rho) J_{1}\left(\alpha^{\prime} \rho\right) d \rho \int_{-\infty}^{\infty} d k_{\xi} \int_{-\infty}^{\infty} d k_{\xi}^{\prime} \\
& \left\{\begin{array}{l}
{\left[\hat{S}_{R 1}\left(k_{\xi}^{\prime}-k_{\xi}\right)+K_{R \infty S_{\infty}} \delta\left(k_{\xi}^{\prime}-k_{\xi}\right)\right]\left[\chi_{p}\left(\alpha, k_{\xi}\right) \chi_{p}^{*}\left(\alpha^{\prime}, k_{\xi}^{\prime}\right)+\phi_{p}\left(\alpha, k_{\xi}\right) \phi_{p}^{*}\left(\alpha^{\prime}, k_{\xi}^{\prime}\right)\right]+} \\
{\left[i \hat{D}_{R 1}\left(k_{\xi}^{\prime}-k_{\xi}\right)+i K_{R \infty D \infty} \delta\left(k_{\xi}^{\prime}-k_{\xi}\right)\right]\left[\chi_{p}\left(\alpha, k_{\xi}\right) \phi_{p}^{*}\left(\alpha^{\prime}, k_{\xi}^{\prime}\right)-\phi_{p}\left(\alpha, k_{\xi}\right) \chi_{p}^{*}\left(\alpha^{\prime}, k_{\xi}^{\prime}\right)\right]}
\end{array}\right\}
\end{aligned}
$$

The above equation can be separated into two components: $P \equiv P_{1}+P_{2}$

$$
\begin{aligned}
P_{1}= & \frac{\pi k_{0}^{2} \omega}{\mu_{0 \prime}} \operatorname{Im} \int_{0}^{1} \rho J_{1}^{2}(\alpha \rho) d \rho \int_{-\infty}^{\infty} d k_{\xi} \\
& \left\{\begin{array}{l}
K_{R \infty S_{\infty}}\left[\left|\chi_{p}\left(\alpha, k_{\xi}\right)\right|^{2}+\left|\phi_{p}\left(\alpha, k_{\xi}\right)\right|^{2}\right] \\
+i K_{R \infty D \infty}\left[\chi_{p}\left(\alpha, k_{\xi}\right) \phi^{*}\left(\alpha, k_{\xi}\right)-\phi_{p}\left(\alpha, k_{\xi}\right) \chi^{*}\left(\alpha, k_{\xi}\right)\right]
\end{array}\right\} \\
P_{2}= & \frac{\pi k_{0}^{2} \omega}{\mu_{0}} \operatorname{Im} \int_{0}^{1} \rho J_{1}(\alpha \rho) J_{1}\left(\alpha^{\prime} \rho\right) d \rho \int_{-\infty}^{\infty} d k_{\xi} \int_{-\infty}^{\infty} d k_{\xi}^{\prime} \cdot \\
& \left\{\begin{array}{l}
\hat{S}_{R 1}\left(k_{\xi}-k_{\xi}^{\prime}\right)\left[\chi_{p}\left(\alpha, k_{\xi}\right) \chi_{p}^{*}\left(\alpha^{\prime}, k_{\xi}^{\prime}\right)+\phi_{p}\left(\alpha, k_{\xi}\right) \phi_{p}^{*}\left(\alpha^{\prime}, k_{\xi}^{\prime}\right)\right] \\
+i \hat{D}_{R 1}\left(k_{\xi}-k_{\xi}^{\prime}\right)\left[\chi_{p}\left(\alpha, k_{\xi}\right) \phi_{p}^{*}\left(\alpha^{\prime}, k_{\xi}^{\prime}\right)-\phi_{p}\left(\alpha, k_{\xi}\right) \chi_{p}^{*}\left(\alpha^{\prime}, k_{\xi}^{\prime}\right)\right]
\end{array}\right\}
\end{aligned}
$$

It can be shown that

$$
\begin{aligned}
\int \rho J_{1}^{2}(\alpha \rho) d \rho & =\frac{1}{\alpha^{2}} \int(\alpha \rho) J_{1}^{2}(\alpha \rho) d(\alpha \rho) \\
& =\frac{1}{\alpha^{2}} \int z J_{1}^{2}(z) d z \\
& =\frac{1}{\alpha^{2}}\left\{\frac{z^{2}}{2}\left(J_{0}^{2}(z)+J_{1}^{2}(z)\right)-z J_{0}(z) J_{1}(Z)\right\}
\end{aligned}
$$

Therefore 


$$
\int_{0}^{1} \rho J_{1}^{2}(\alpha \rho) d \rho=\frac{1}{\alpha^{2}}\left\{\frac{\alpha^{2}}{2}\left[J_{0}^{2}(\alpha)+J_{1}^{2}(\alpha)\right]-\alpha J_{0}(\alpha) J_{1}(\alpha)\right\}
$$

$P_{1}$ is seen to be

$$
\begin{aligned}
P_{1}= & \frac{\pi k_{0}^{2} \omega}{\mu_{0}} \operatorname{Im} \int_{-\infty}^{\infty} d k_{\xi}\left[\frac{1}{2}\left[J_{0}^{2}(\alpha)+J_{1}^{2}(\alpha)\right]-\frac{1}{\alpha} J_{0}(\alpha) J_{1}(\alpha)\right] \\
& \left\{\begin{array}{l}
K_{R \infty S \infty}\left[\left|\chi_{p}\left(\alpha, k_{\xi}\right)\right|^{2}+\left|\phi_{p}\left(\alpha, k_{\xi}\right)\right|^{2}\right] \\
+i K_{R \infty D \infty}\left[\chi_{p}\left(\alpha, k_{\xi}\right) \phi^{*}\left(\alpha, k_{\xi}\right)-\phi_{p}\left(\alpha, k_{\xi}\right) \chi^{*}\left(\alpha, k_{\xi}\right)\right]
\end{array}\right\}
\end{aligned}
$$

From Gradshteyn \& Ryzhik [52], it is seen that

$$
\int x Z_{p}(a x) B_{p}(\beta x) d x=\frac{\beta x Z_{p}(a x) B_{p-1}(\beta x)-a x Z_{p-1}(a x) B_{p}(\beta x)}{\alpha^{2}-\beta^{2}}
$$

vhere $Z_{p}(x)$ and $B_{p}(x)$ are arbitrary Bessel functions.

Therefore the $\alpha$ integral in part $P_{2}$ is seen to be

$$
\begin{aligned}
\int_{0}^{1} \rho J_{1}(\alpha \rho) J_{1}\left(\alpha^{\prime} \rho\right) d \rho & =\left.\frac{\alpha^{\prime} \rho J_{1}(\alpha \rho) J_{0}\left(\alpha^{\prime} \rho\right)-\alpha \rho J_{0}(\alpha \rho) J_{1}\left(\alpha^{\prime} \rho\right)}{\alpha^{2}-\alpha^{\prime 2}}\right|_{\rho=0} ^{1} \\
& =\frac{\rho}{\alpha^{2}-\alpha^{22}}\left[\alpha^{\prime} J_{1}(\alpha \rho) J_{0}\left(\alpha^{\prime} \rho\right)-\alpha J_{0}(\alpha \rho) J_{1}\left(\alpha^{\prime} \rho\right)\right]_{\rho=0}^{1} \\
& =\frac{1}{\alpha^{2}-\alpha^{22}}\left[\alpha^{\prime} J_{1 \alpha^{2}} J_{0 \alpha^{\prime}}-\alpha J_{0 \alpha} J_{1 \alpha^{\prime}}\right]
\end{aligned}
$$

The second component of the power interal $P_{2}$ is seen to be

$$
\begin{aligned}
P_{2}= & \frac{\pi k_{0}^{2} \omega}{\mu_{0}} \operatorname{Im} \int_{-\infty}^{\infty} d k_{\xi} \int_{-\infty}^{\infty} d k_{\xi}^{\prime}\left(\frac{1}{\alpha^{2}-\alpha^{\prime 2}}\right)\left[\alpha^{\prime} J_{1 \alpha} J_{0 \alpha^{\prime}}-\alpha J_{0 \alpha} J_{1 \alpha^{\prime}}\right] \\
& \left\{\begin{array}{l}
\hat{S}_{R 1}\left(k_{\xi}-k_{\xi}^{\prime}\right)\left[\chi_{p}\left(\alpha, k_{\xi}\right) \chi_{p}^{*}\left(\alpha^{\prime}, k_{\xi}^{\prime}\right)+\phi_{p}\left(\alpha, k_{\xi}\right) \phi_{p}^{*}\left(\alpha^{\prime}, k_{\xi}^{\prime}\right)\right] \\
+i \hat{D}_{R 1}\left(k_{\xi}-k_{\xi}^{\prime}\right)\left[\chi_{p}\left(\alpha, k_{\xi}\right) \phi_{p}^{*}\left(\alpha^{\prime}, k_{\xi}^{\prime}\right)-\phi_{p}\left(\alpha, k_{\xi}\right) \chi_{p}^{*}\left(\alpha^{\prime}, k_{\xi}^{\prime}\right)\right]
\end{array}\right\}
\end{aligned}
$$

This section performed the power integral in Fourier space. While it might appear that performing the volume integral in real space $(\rho, \xi)$ is equally valid and much simpler, it is not done here due to the presence of the $\omega=\omega_{c i}$ resonance in the geometry. Some elements of the dielectric tensor behave asymptotically as 
resonance is approached in $\xi$ and render the volume integral in $(\rho, \xi)$ space highly unstable. It is for this reason that the power integration here is performed in Fourier space.

After solving for the absorbed power $P$, the plasma impedence could be calculated by

$$
R_{p l}=\frac{2 P}{I^{2}}
$$

where $I$ is the current of the dipole source loop. It is this quantity $R_{p l}$ that will be examined as the figure of merit in the analysis and comparisons performed in the next few sections. 


\subsection{Code Benchmarking and Comparison to Lit- erature}

A code CYLWAVE implementing the theoretical methodology outlined in this chapter has been written as part of this thesis. The calculations performed by the CYLWAVE code are benchmarked to ensure $t$ uracy of the results. Both benchmarking and comparison with literature were performed and are listed below.

1. Benchmark: Straight Cylinder Case. The fields for a straight cylinder are formulated, computed and cornpared to the CYLWAVE code results. The straight cylinder fields are relatively easy to solve becaues the integral equations become algebraic. This yields a precise quantitative benchmark against which CYLWAVE results can be compared.

2. Comparison to literature. Because the analysis undertaken in this chapter is unique in that it handles both the field inhomogeneity and the cylindricul geometry, it is difficult to benchmark against the existing literature quantitatively. However, qualitative order-cf-magnitude comparisons with other well known theoretical and analytical studies are made.

\subsubsection{Benchmark: Straight Cylinder Case}

The fields in the case of the straight cylinder are relatively simple so formulate because the convolution integral become delta functions when $K_{1,2}$ and $R$ are no longer functions of $\xi$. In other words, the two coupled integral equations 3.36 and 3.37 become simple algebraic ones:

$$
\begin{gathered}
-k_{\xi}^{2} \hat{\hat{\chi}}_{p}\left(k_{\xi}\right)+k_{0}^{2} K_{1} R_{0}^{2} \hat{\hat{\chi}}_{p}\left(k_{\xi}\right)+k_{0}^{2} K_{2} R_{0}^{2} \hat{\hat{\phi}}_{p}\left(k_{\xi}\right)=0 \\
\left(-k_{\xi}-\alpha^{2}\right) \hat{\hat{\phi}}_{p}\left(k_{\xi}\right)+k_{0}^{2} K_{1} R_{0}^{2} \hat{\hat{\phi}}_{p}\left(k_{\xi}\right)-k_{0}^{2} K_{2} R_{0}^{2} \hat{\hat{\chi}}_{p}\left(k_{\xi}\right)=0
\end{gathered}
$$


In 0 der for both equations to hold, the determinent must then be zero, yielding a dispersion relation for $\alpha\left(k_{\xi}\right)$ :

$$
\begin{gathered}
\left(k_{\xi}^{2}+\alpha^{2}-k_{0}^{2} R_{0}^{2} K_{1}\right)\left(k_{\xi}^{2}-k_{0}^{2} R_{0}^{2} K_{1}\right)+k_{0}^{4} R_{0}^{4} K_{2}^{2}=0 \\
\Rightarrow \alpha^{2}=\frac{\mathcal{L}^{2}+k_{0}^{4} R_{0}^{4} K_{2}^{2}}{-\mathcal{L}}
\end{gathered}
$$

where

$$
\mathcal{L} \equiv k_{\xi}^{2}-k_{0}^{2} R_{0}^{2} K_{1}
$$

$\hat{\hat{\chi}}_{p}$ can then be determined in terms of $\hat{\hat{\phi}}_{p}$

$$
\hat{\hat{\chi}}_{p}\left(k_{\xi}\right)=\frac{k_{0}^{2} R_{0}^{2} K_{2}}{k_{\xi}^{2}-k_{0}^{2} R_{0}^{2} K_{1}} \dot{\hat{\phi}}_{p}\left(k_{\xi}\right)
$$

Recall that the $\phi$ could be found in terms of the source term $\Psi$ from Equation 3.24

$$
\hat{\hat{\phi}}_{p}\left(\alpha, k_{\xi}\right)=\frac{1}{\alpha J_{0}(\alpha)-J_{1}(\alpha) \frac{\frac{\partial}{\rho} \hat{F}}{\hat{F}}}\left[\frac{\partial}{\partial \rho} \hat{G}-\frac{\frac{\partial}{\partial \rho} \hat{F}}{\hat{F}} \hat{G}\right]_{\rho=1}
$$

The ICRF $B_{z}$ fields for an almost-straight case $\left(R_{0}=0.9 a, R_{\infty}=0.89 a\right)$ and the analytical limit of the straight cylinder case were computed on the surface of the plasma cylinder are are plotted on Figure 3-9. It is seen that the agreement between the CYLWAVE case and the analytic limit of the straight cylinder is very good. 


\section{BZ AND BZFLAT FOR BENCHMAR}

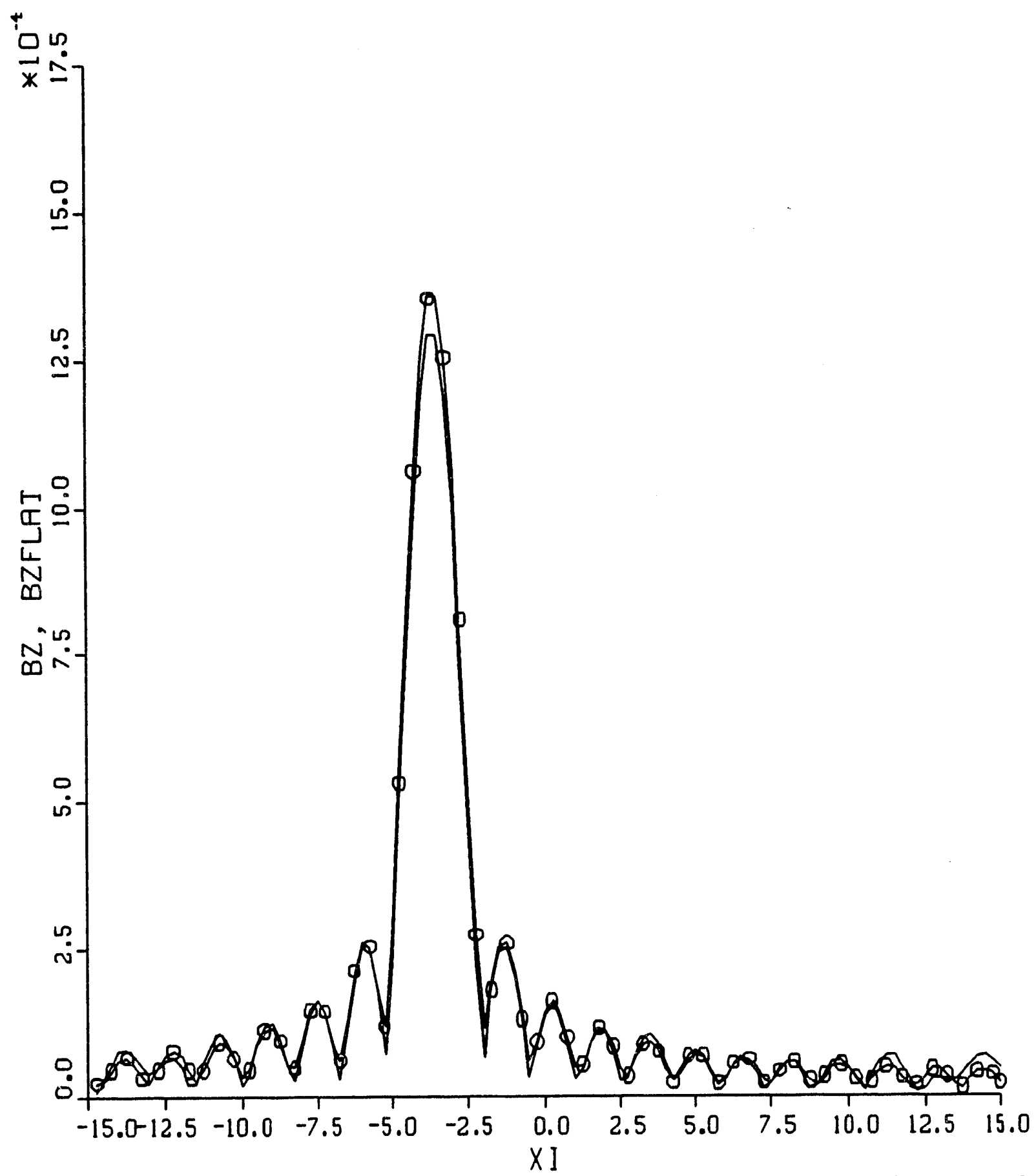

Figure 3-9: ICRF $B_{z}$ plots along the surface of the plasma cylinder for a straight cylinder case. Both $B_{z}$ as computed by CYLWAVE (Solid line), and the $B_{z}$ computed from the analytical limits of a straight cylinder (open circle line) are plotted versus the axial coordinate $\xi$. Waves are launched from antenna located at $\rho=1.171, \xi=-3.613$. For this case, ICRF resonance location is located OUTSIDE the chamber. 


\subsubsection{Comparison of CYLWAVE results with literature}

In the early 60 's, Hooke et al. [60] performed experiments on the B-66 machine using a pickup loop to pick up the cross-sectional area integrated ICRF $\int B_{z} d A$. The antenna used in the experiment is approximately a multiple turn loop. The results of the $\int B_{z} d A$ obtained from the experiment is shown on the top half of Figure 3-10. For the geometry shown, the antenna is located at approximately $z=+20 \mathrm{~cm}$. It is seen that the $\int B_{z} d A$ decays as the resonance is approached.

The $\int B_{z} d A$ is also computed using the CYLWAVE program. It is seen that the results obtained from CYLWAVE agree in general with the results of the B-66 experiment. The differences in the launching antenna and geometry (the conducting wall does NOT scale as the plasma cylinder) probably account for much of the difference in the results. 


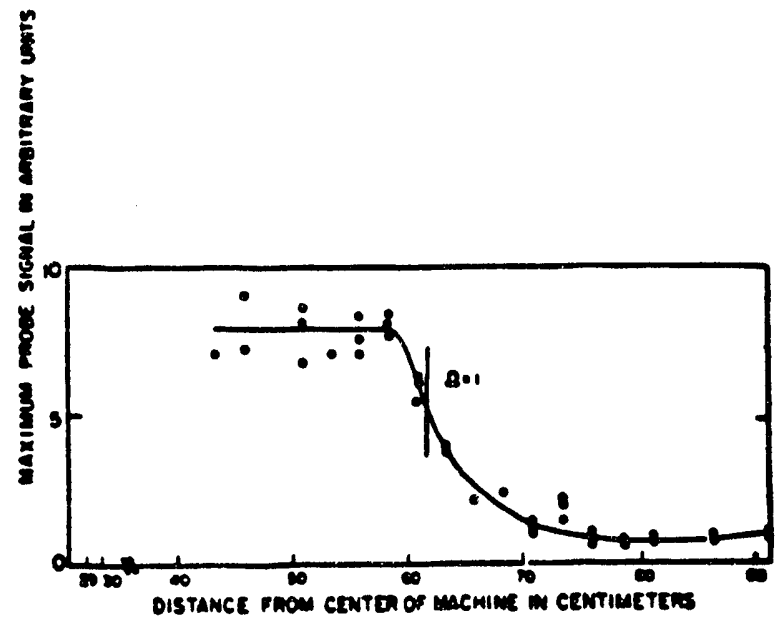

INT BZ DA VS DIST FROM ANTENNA

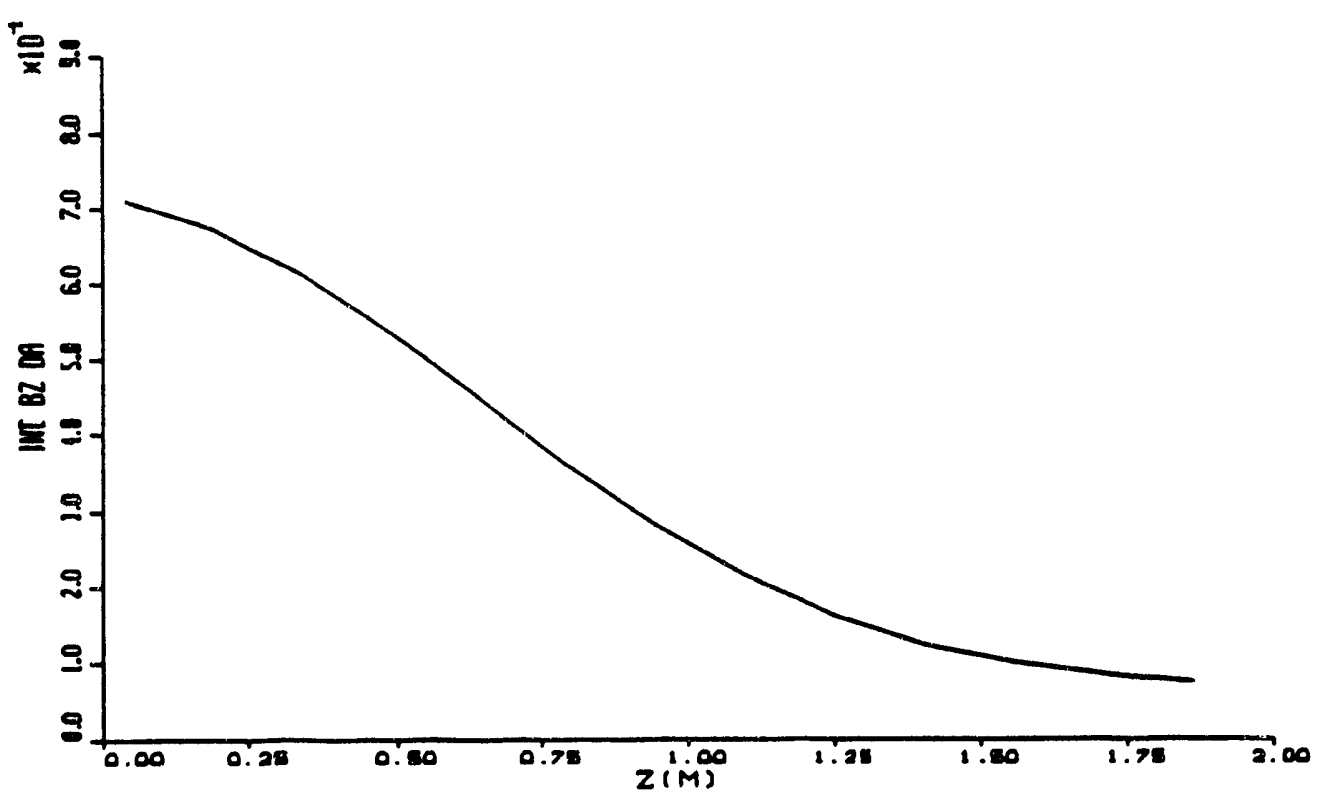

Figure 3-10: Comparison of $\int B_{z} d A$ versus distance from the centerline for the B66 experiment[60] (Top) and versus the distance from the antenna for the CYLWAVE code (Bottom). The location of the antenna in the B-66 experiment is approximately at $z=20 \mathrm{~cm}$. 


\subsection{Discussion of Results}

Several cases of interest were examined for relevant physics. The cases are

\subsubsection{Resonance in chamber vs. Resonance out of cham- ber}

Both the Resonance-cut-of-chamber (ROC) case and the Resonance-in-chamber (RIC) case are computed and shown in Figures 3-13 and 3-16. Several qualitative differences are visible:

1. The structure of the perpendicular mode numbers $\alpha$ are quite difference between the two cases. The $\alpha$ 's for the RIC case as seen in Figure 3-14 exhibit a behavior which is very similar to the infinite plasma dispersion relation. The $\alpha$ 's for the ROC case as seen in Figure 3-11, on the other hand, exhibit a markedly different behavior from the infinite plasma dispersion relation.

2. The small amplitude oscillations that are prevalent in the ICRF $B_{z}$ fields of the ROC case as given in Figures 3-12 and 3-13 are practically absent in the RIC case shown on Figures 3-15 and 3-16. This agrees well with the assumption that a resonance located in the chamber would damp out most of the field oscillations as the waves propagate towards resonance. 


\section{ALPHARE, ALPHAIM VS KXI}

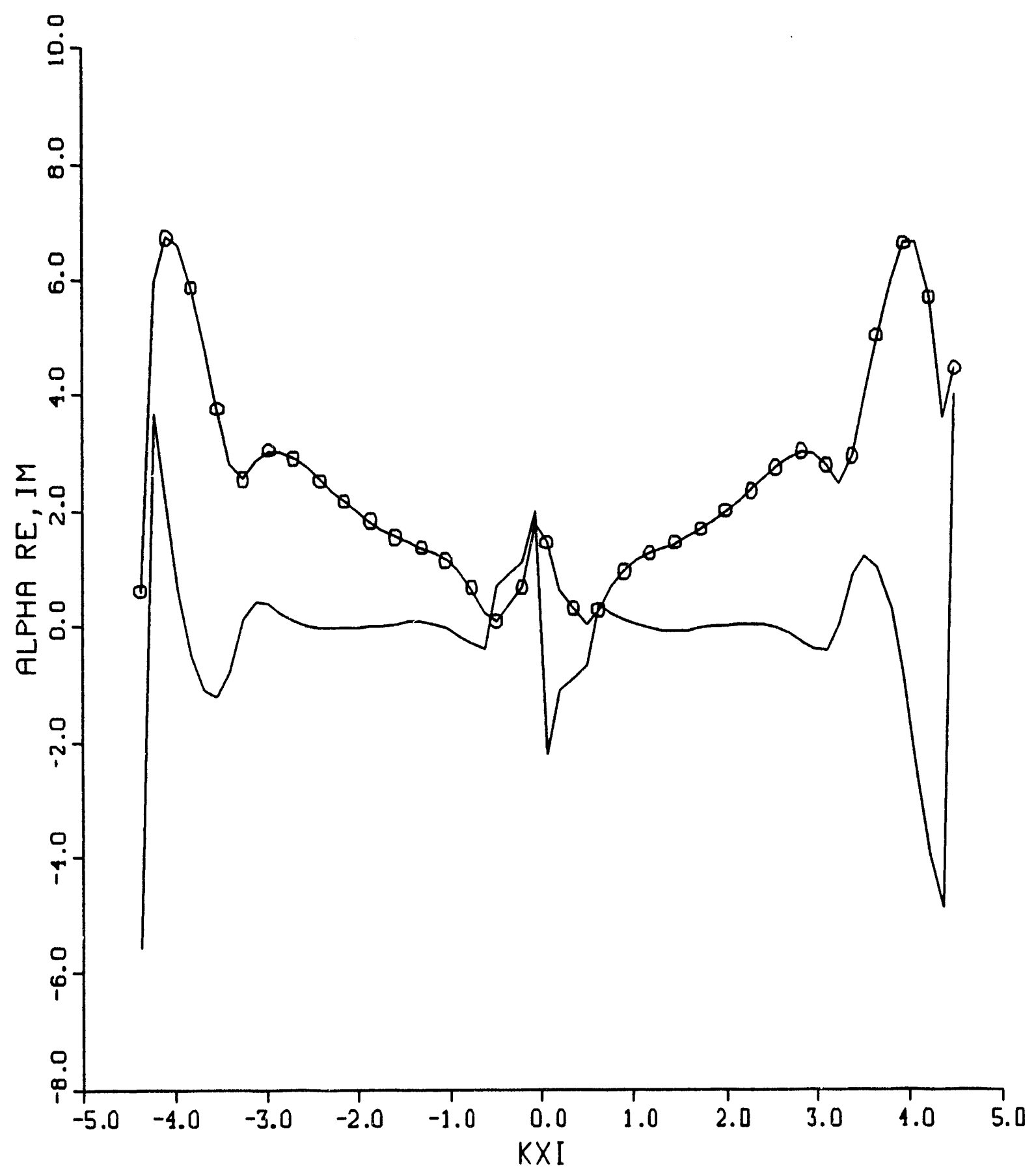

Figure 3-11: Real and Imaginary components of the perpendicular mode number $\alpha$ versus $k_{\xi}$ for case of plasma density $n_{e}=1 \times 10^{11} \mathrm{~cm}^{-3}: \alpha_{R e}$ (Line plot) $\alpha_{I m}$ (Open circles). Note that the behavior of $\alpha$ for the ROC case is quite different from that of the infinite plasma dispersion relation. 
$B Z(R H O=1)$ VS. XI

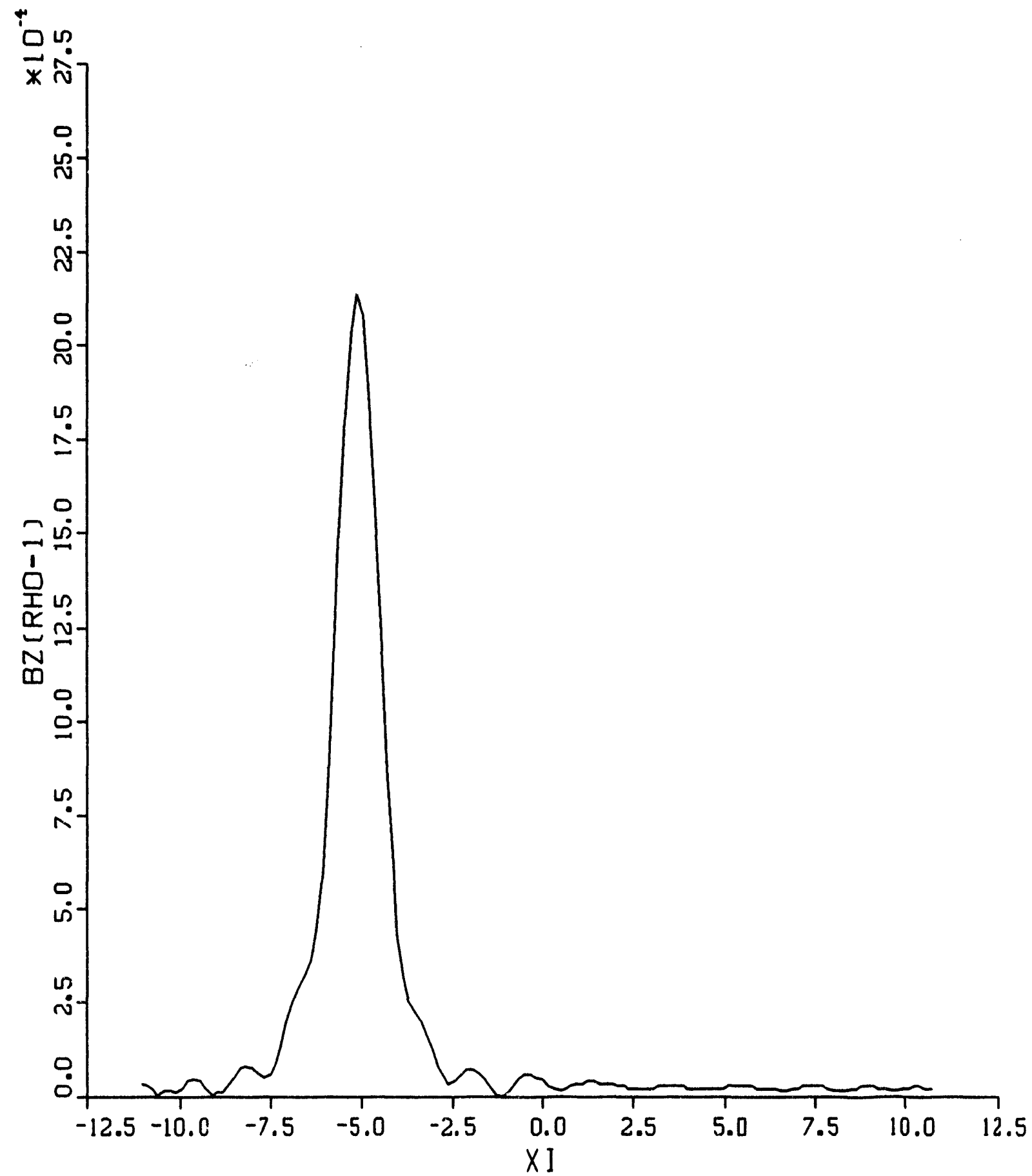

Figure 3-12: ICRF $B_{z}$ at $\rho=1$ versus $k_{\xi}$ for case of plasma density $n_{e}=1 \times$ $10^{11} \mathrm{~cm}^{-3}$. ICRF resonance location is OUTSIDE the chamber. This is the same case as Figure 3-11. The peak corresponds to the axial location of the antenna at $\xi=-5.00$. 


\section{BZ}

RHO-1 : PLASMA EDGE, RHO- 2.500 : WALL RHO- $1.397, X I--5.000$ : ANTENNA LOC RES-X]-OUTSIIDE, NUM-ALPHA-65 $\mathrm{NE}=1.000 * 10^{11}(\mathrm{CM}-3), \mathrm{TE}=10.000 \mathrm{EV}$

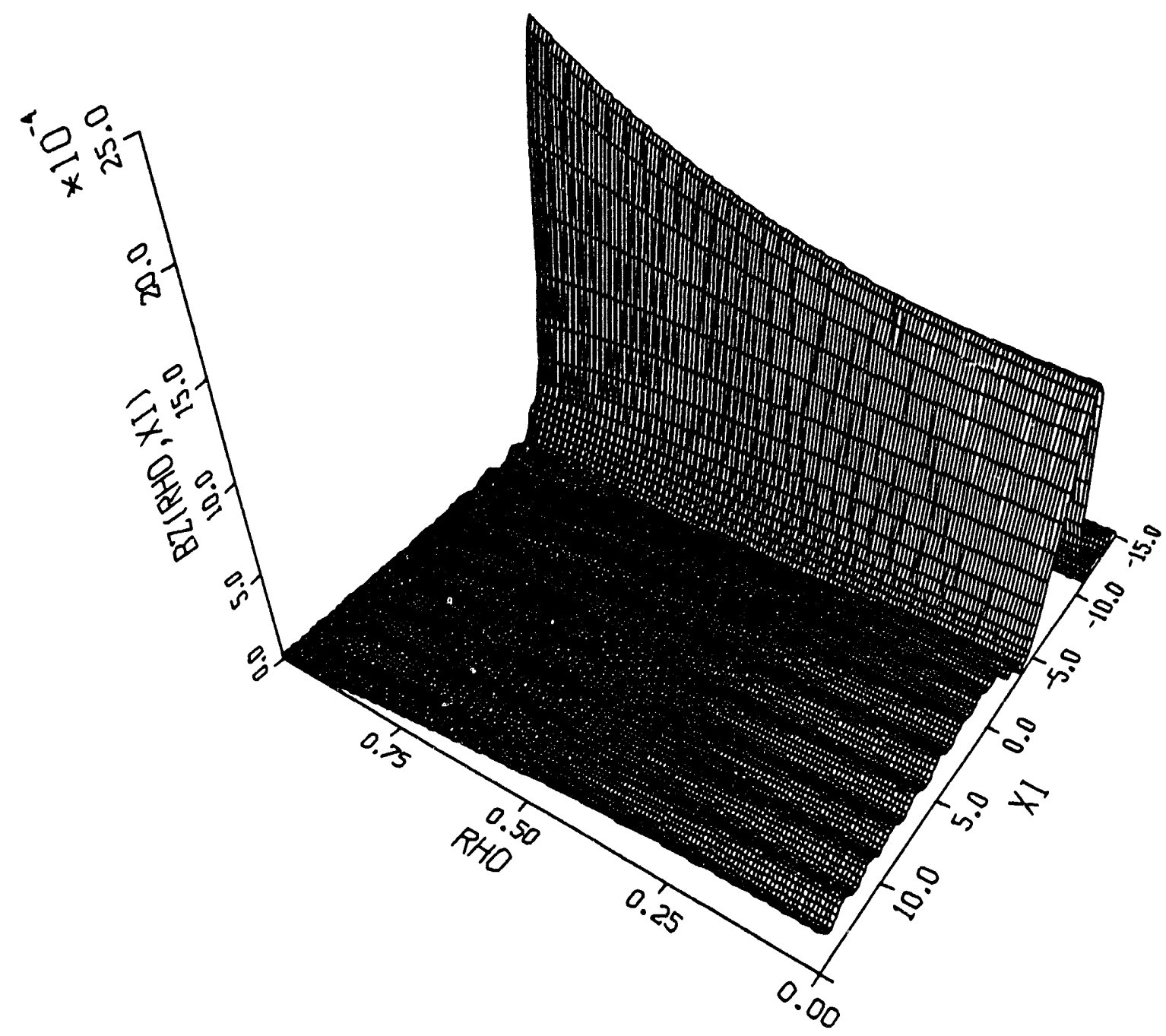

Figure 3-13: ICRF $B_{z}$ plot as function of $(\rho, \xi)$. Waves are launched from antenna located at $\rho=1.397, \xi=-5.000$. For this case, ICRF resonance location is OUTSIDE the machine. $65 k_{\xi}$ spectral points were taken to generate this profile. The geometry is $R_{0}=0.77 a, R_{\infty}=0.50 a$. Note the presence of small-amplitude oscillations away from the antenna. 
ALPHARE, ALPHAIM FOR INF DISP

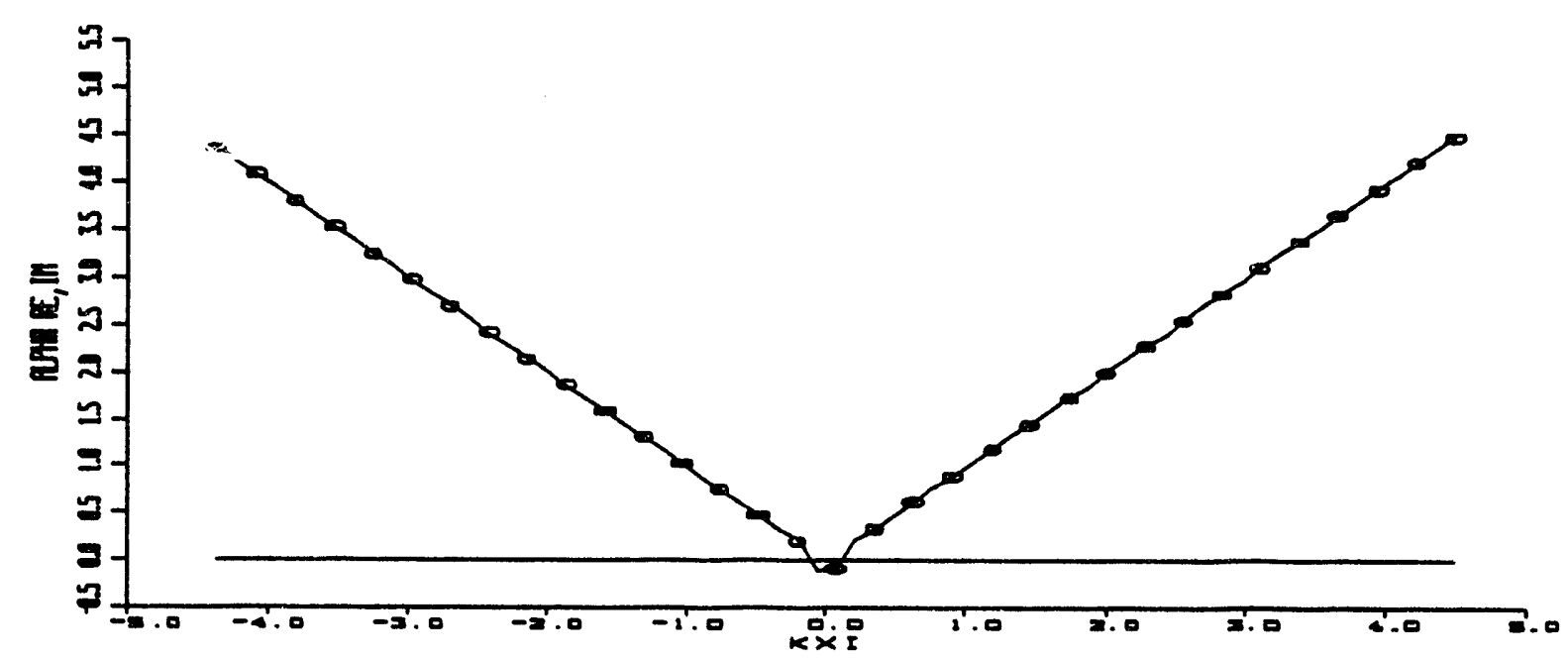

pLPHARE, ALPHAIM VS KXI

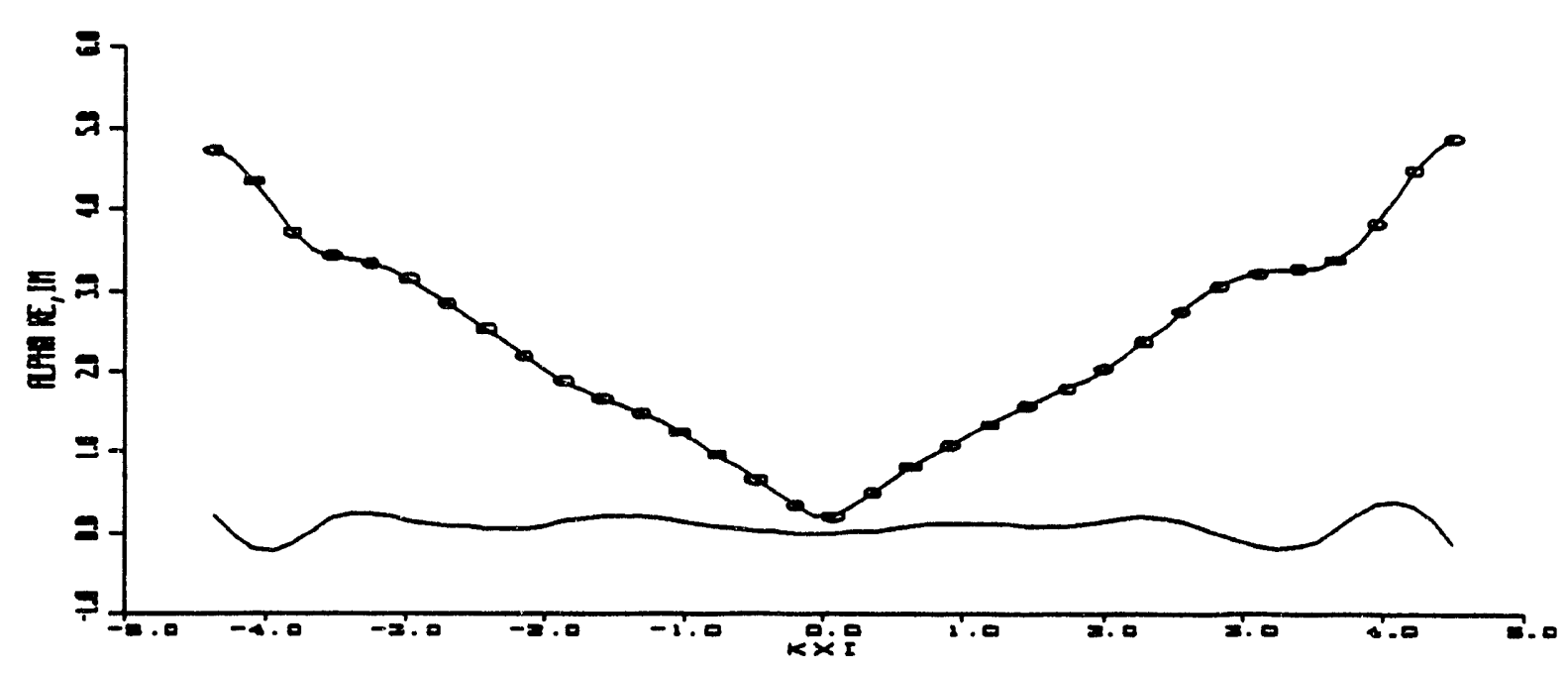

Figure 3-14: Real and Imaginary components of the perpendicular mode number $\alpha$ versus $k_{\xi}$ for case of plasma density $n_{e}=1 \times 10^{11} \mathrm{~cm}^{-3}: \alpha_{\text {Re }}$ (Line plot) $\alpha_{I m}$ (Open circles). Top plot is the case of the infinite plasma dispersion relation. Bottom plot shows the $\alpha$ eigenmodes calculated for a Resonance-In-Chamber (RIC) case. Note that the behavior of $\alpha$ for the RIC case is similar to the $\alpha$ behavior of the infinite plasma dispersion relation. 


\section{$B Z(R H O=1) \quad V S . X I$}

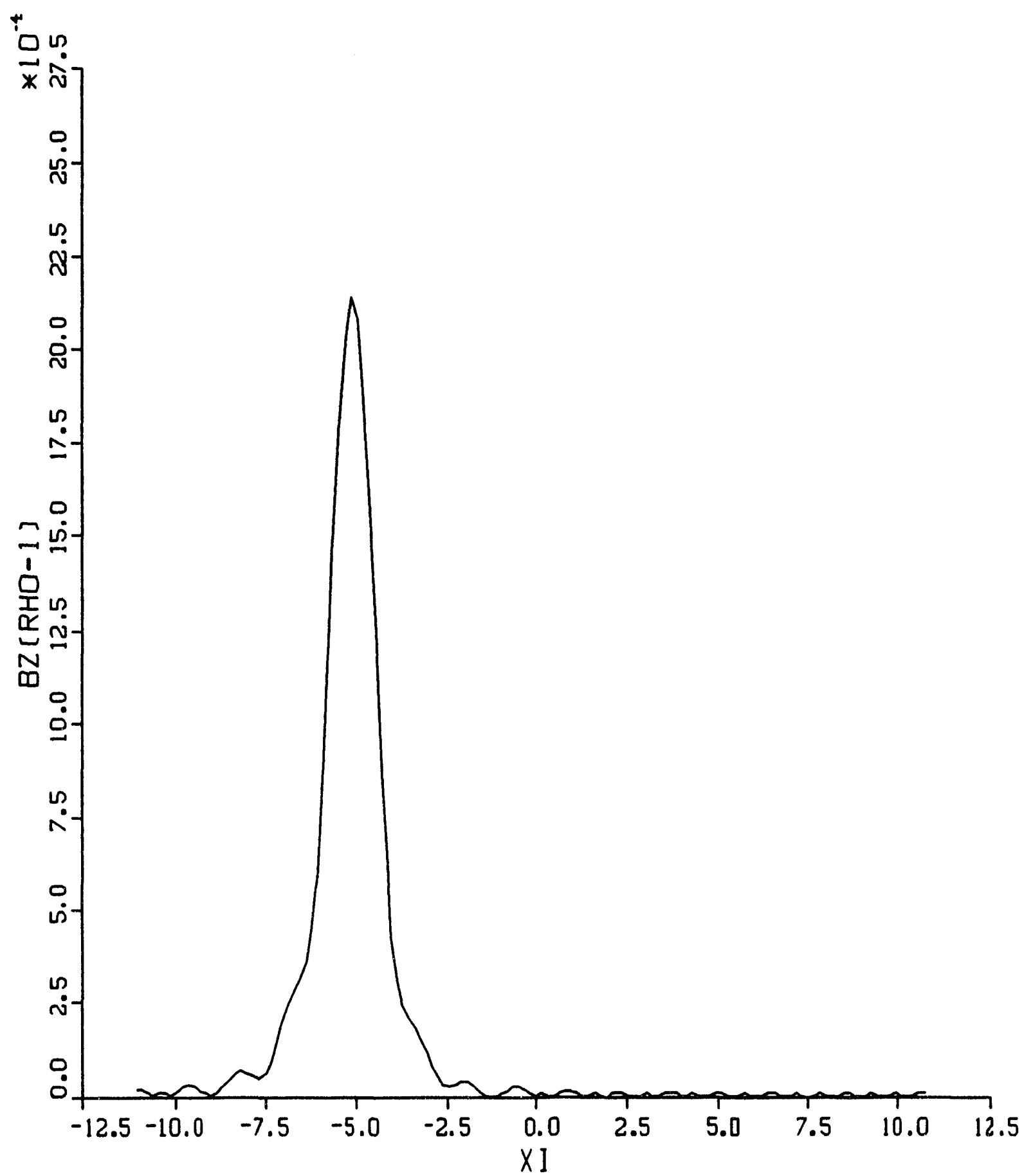

Figure 3-15: ICRF $B_{z}$ at $\rho=1$ versus $k_{\xi}$ for case of plasma density $n_{a}=1 \times$ $10^{11} \mathrm{~cm}^{-3}$. ICRF resonance location is located IN the chamber at $\xi= \pm 0.977$. This is the same case as Figure 3-14. The peak corresponds to the axial location of the antenna at $\xi=-5.00$. 


\section{BZ}
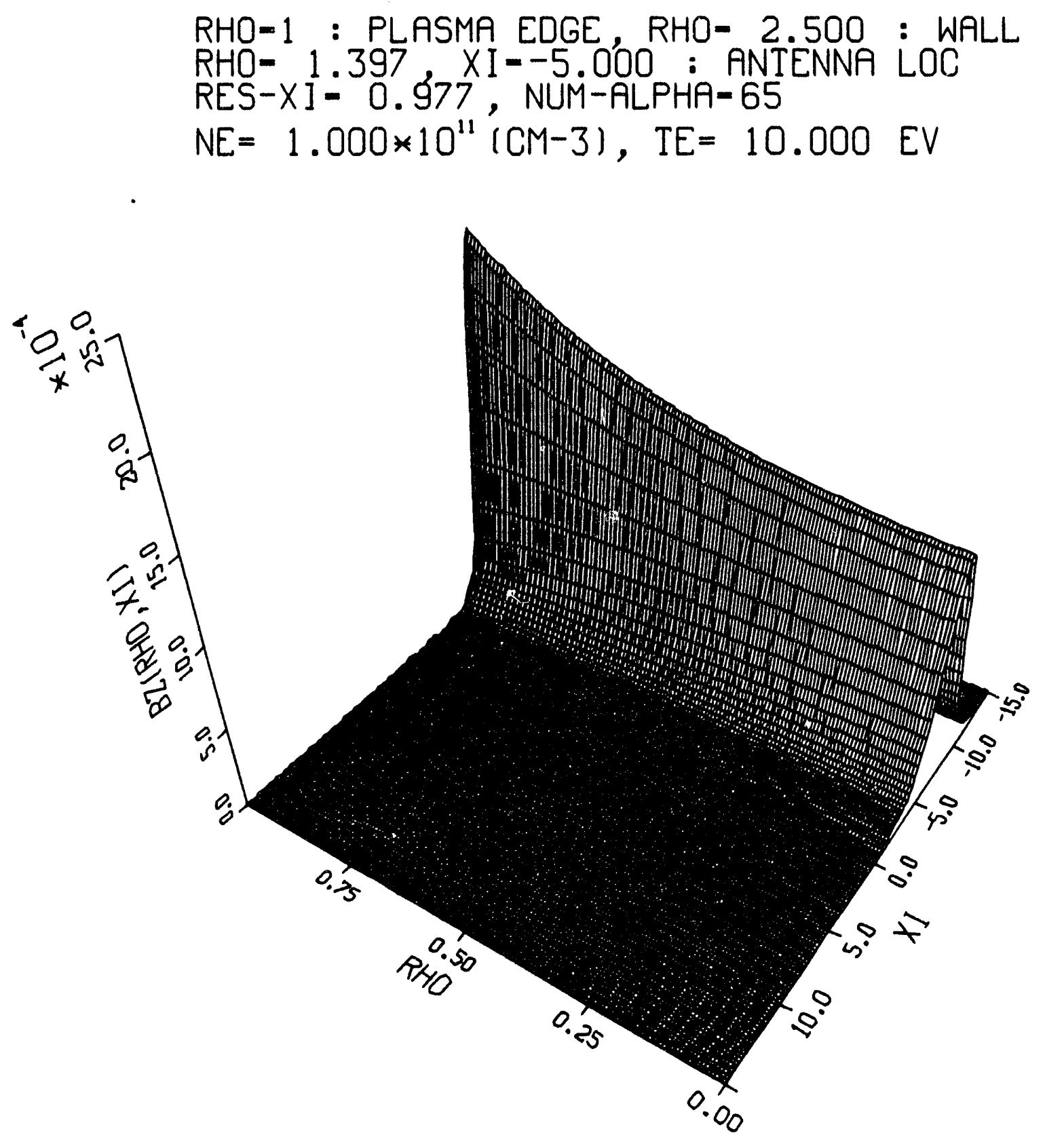

Figure 3-16: ICRF $B_{z}$ plot as function of $(\rho, \xi)$. Waves are launched from antenna located at $\rho=1.397, \xi=-5.00$. For this case, ICRF resonance location is located IN the chamber at $\xi= \pm 0.977$. The geometry is $R_{0}=0.77 a, R_{\infty}=0.5 a$. Note the absence of small-scale oscillations away from the antenna relative to Figure 3-12. 


\subsubsection{Mirror Ratio Scan}

A scan of the mirror ratio and the resulting $R_{p l}$ allows the determination of the optimum mirror ratio to maximize RF heating. However, it must be kept in mind that in a magnetic mirror the confinement degrades with lower mirror ratio because of the loss cone.

A scan is performed using different values of mirror ratio $M$ where

$$
M \equiv \frac{B_{\max }}{B_{\min }}=\frac{R_{0}^{2}}{R_{\infty}^{2}}
$$

The $R_{0}$ is kept constant and only the $R_{\infty}$ is varied in the calculation of the mirror ratio. $R_{\infty}$ is varied from $0.2 a$ to $0.76 a$ while $R_{0}$ is kept at $0.77 a$. The resulting mirror ratio then varies from 1.054 to about 14.83 . The normalized impedence results from the scan are plotted on Figure 3-17.It is seen that as the mirror ratio decreases, the plasma loading impedence $R_{p l}$ increases.

A resonance volume theory [61] has been proposed which simply states that the coupling resistance is proportional to the amount of plasma volume close to resonance. When viewed in this context, the result of the analytical analysis conform to predictions because as the mirror ratio approaches 1, larger portions of the plasma become closer to resonance, resulting in more damping. By comparing a normalized "resonance volume" to the actual plasma impedence, one can verify this. The volume close to resonance can be approximated by

$$
\Delta V=2 \pi R \Delta L=2 \pi R \frac{\Delta B}{\left(\frac{d B}{d \xi}\right)}
$$

where $\Delta B$ is the determination of how close to resonance the volume should be, and $d B / d \xi$ is the slope of the macroscopic axial magnetic field at resonance. Therefore it is seen that this resonance-volume is inversely proportional to $d B / d \xi$.

The normalized resonance volume has been computed and plotted (open circle) on Figure 3-17. Comparing the CYLWAVE results (solid line) to the resonance volume, it is apparent that the general behavior of the plasma loading impedence at different mirror ratios agrees with that of the resonance-volume. The difference 
between the two curves is probably attributable to the difference in incident field intensity that is sampled by the resonance volume since the resonance layer moves when $R_{\infty}$ changes.

The results of the mirror scan show that the maximization of the plasma loading impedence is accomplished by lowering the slope at the resonance point, in other words lower the mirror ratio. However, the trade off is that in a mirror geometry, lower mirror ratios result in worse confinement. Therefore a careful examination of the confinement-heating dilemma should be made (but is beyond the scope of this thesis) in order to produce good heating yet not contribute to excessive loss of confinement. 
RPL, NORM (1/DRDXI) VS. MR

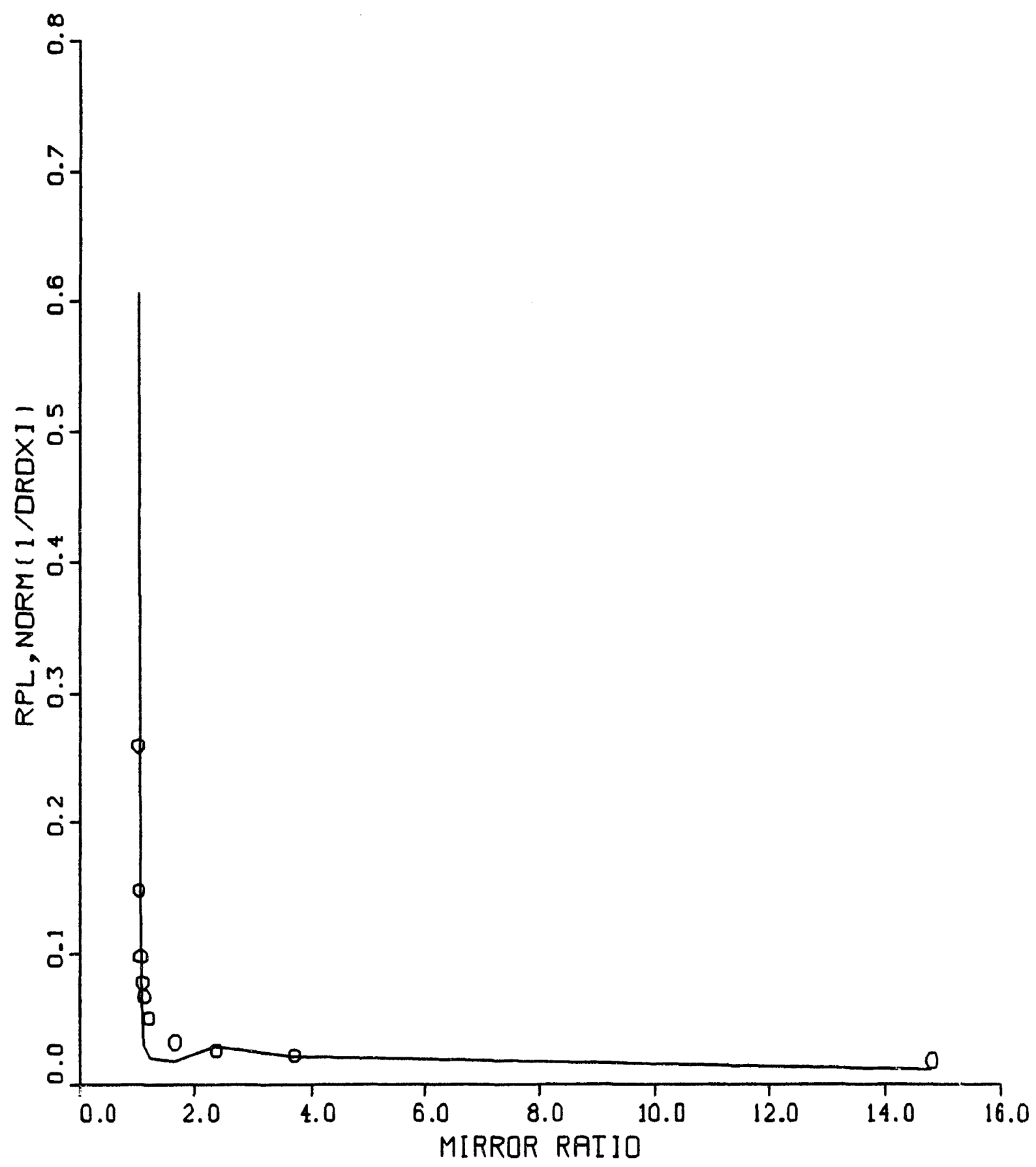

Figure 3-17: Plasma Impedence $R_{p l}$ (Solid line) and Normalized Resonancevolume (Open Circles) calculations performed for different mirror ratios. ICRF antenna is located at $\xi=5.0$. Plasma parameters are: $n_{e}=1.0 \times 10^{11} \mathrm{~cm}^{-3}$, $T_{e}=10 \mathrm{eV}$. 


\subsubsection{Resonance Location Scan}

The behavior of the antenna-plasma coupling impedence as a function of resonance location is also performed.

For plasma paramaterse $n_{e}=1 \times 10^{11} \mathrm{~cm}^{-3}$ and $T_{e}=10 \mathrm{eV}$, the computed plasma coupling impedence $R_{p l}$ at different resonance locations $\xi_{\text {res }}$ are plotted on Figure 3-18. The coupling impedence is seen to peak at two locations - resonance at plasma center, and resonance directly underneath the antenna. In fact, the resonance-under-antenna case shows just as large a peak in plasma loading impedence as the resonance-at-center case. This indicates that, depending on the mirror ratio, locating the resonance close to the antenna may be just as important for coupling as locating the resonance at the plasma center. Thus an optimum coupling condition, from a wave-propagation point of view, would be to have the resonance located either directly underneath the antenna or at the center of the machine. 


\section{RPL VS. RESONANCE LOCATION}

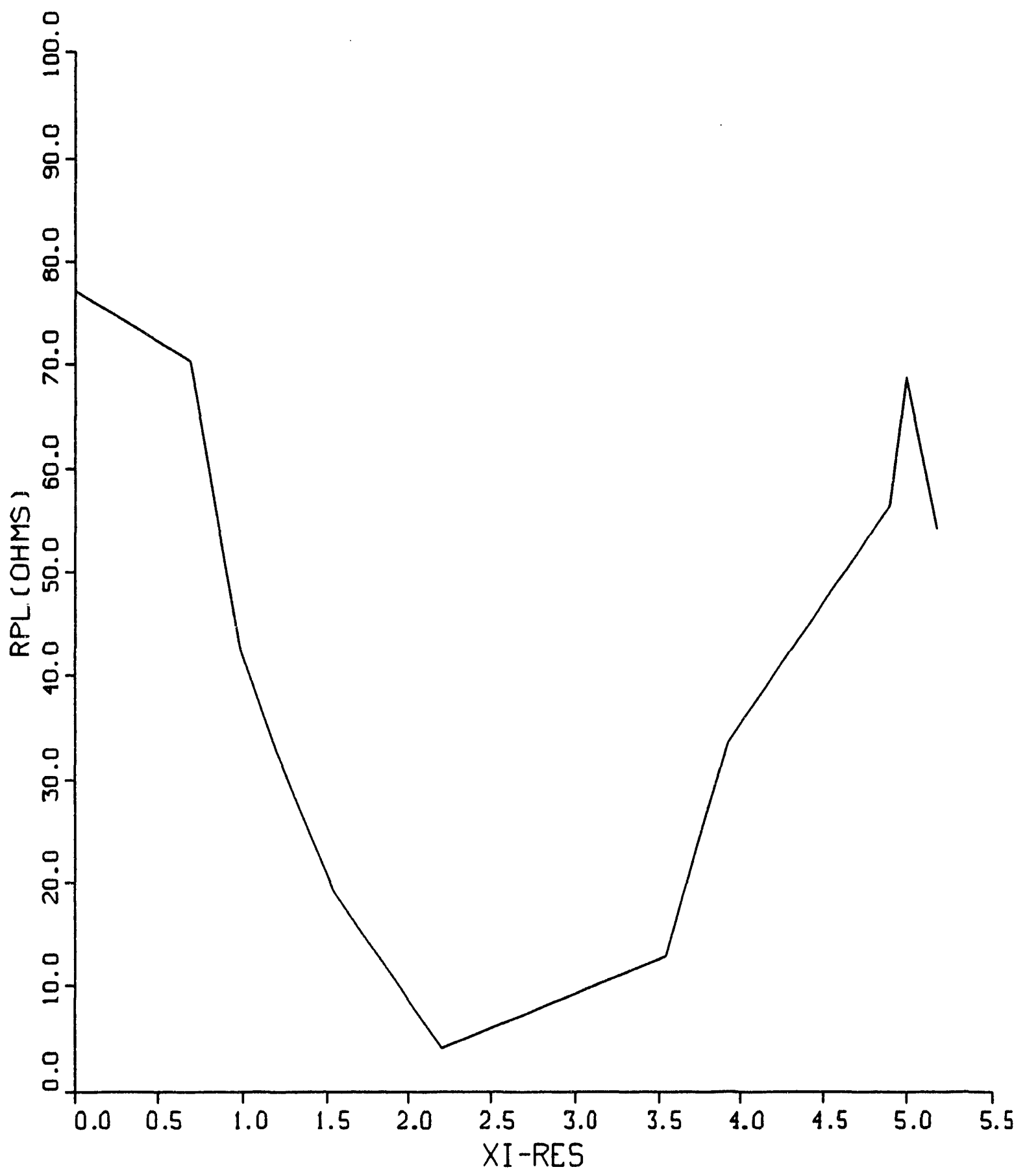

Figure 3-18: Plasma Impedence $R_{p l}$ versus resonance location $\xi_{\text {res. }}$ Plasma parameters are: $n_{e}=1.0 \times 10^{11} \mathrm{~cm}^{-3}, T_{e}=10 \mathrm{eV}$. Note that the peaks in $R_{p l}$ are located at $\xi=0$ field minimim, and $\xi=5.000$ antenna location. 


\subsubsection{Density Scan}

A density scan is performed in which different plasma densities are used to calculate the resulting plasma impedence. The case chosen is for a case of $R_{0, p l}=0.77 a$, $R_{\infty, p l}=0.50 a$, and $r_{\kappa} \equiv R_{w a l l} / R_{p l}=1.75$. The temperature is held constant at $10 \mathrm{eV}$ and the density is scanned from $10^{11} \mathrm{~cm}^{-3}$ to $3 \times 10^{12} \mathrm{~cm}^{-3}$. The resulting plasma impedence is plotted (open circle) versus density on Figure 3-19. The computed $R_{p l}$ is compared to a $1 / \sqrt{n_{e}}$ dependence (solid line). The reason that $R_{p l}$ is compared to a $1 / \sqrt{n_{e}}$ behavior is that a simple examination of the $P_{\text {dise }}$ shows that $P_{\text {dise }}$ scales as $1 / \alpha$. Since the perpendicular wave number $\alpha$ behaves like that of the infinite plasma dispersion which behaves as

$$
\alpha^{2} \propto(S, D) \propto \omega_{p i}^{2} \propto n_{e}
$$

it is seen that a $1 / \sqrt{n_{e}}$ behavior is the expected scaling of the plasma impedence. This is a very simple analogy, but it is seen to be valid when the comparison is made. 
NORM (1/NE**0.5), RPL VS. NE

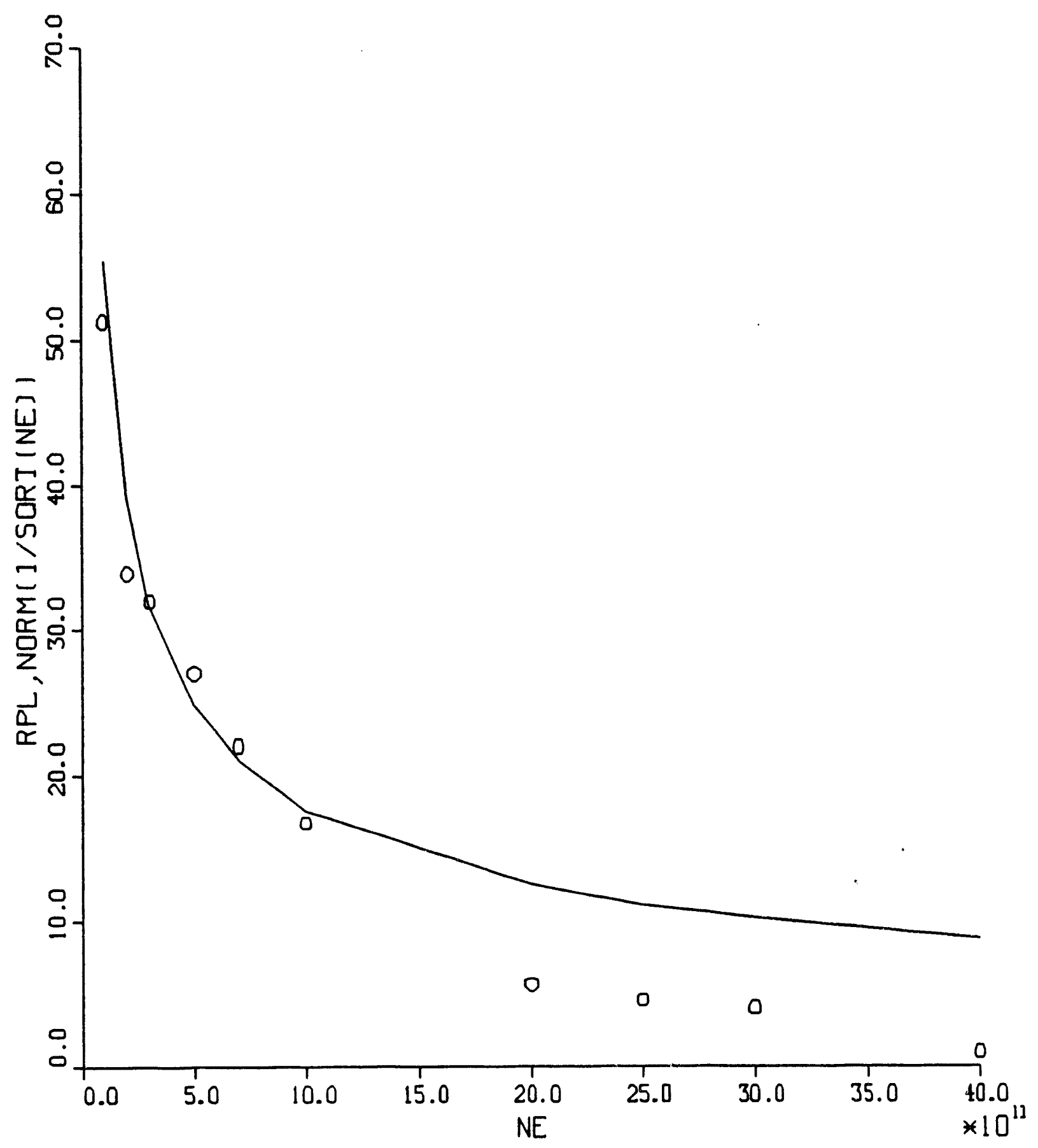

Figure 3-19: CYLWAVE computed $R_{p l}$ (Open circle) and a normalized $1 / \sqrt{n_{e}}$ (Solid line) are plotted versus the plasma density $n_{e}$. It is seen that the plasma impedence $R_{p l}$ agrees well with the $1 / \sqrt{n_{e}}$ scaling. 


\subsection{Conclusion}

From the developement of an ICRF theory and the results obtained from this theory, several conclusions can be drawn:

1. A new model of the ICRF-plasma wave coupling problem in cylindrical geometry with inhomogeneous $B_{z}$ is examined. The ICRF $B_{z}$ fields computed from the analysis are consistent with benchmark.

2. Although differences in geometry, physics, and antenna geometry make quantitative comparisons impossible, it is nevertheless seen that the value of the plasma impedence $R_{p l}$ computed by this approach is of comparable order to both literature and experimental values.

3. The resonance-in-chamber (RIC) case contains much less small-scale oscillations than the resonance-out-of-chamber (ROC) case. This is due to the presence of the resonance which damps out the small-scale oscillations as the waves propagate outward from the antenna in the $+\hat{\xi}$ direction towards resonance.

4. Impedence calculations at different mirror ratios reveal that the plasma loading impedence $R_{p l}$ generally agrees with the "resonance-volume" model and is roughly proportional to $1 /(d B / d \xi)$. The ramification is that a trade-off between mirror confinement and heating is necessary to obtain good heating without sacrificing too much confinement loss due to particle diffusion into the loss cone.

5. Results from the plasma impedence calculations at different resonance locations show that, depending upon the mirror ratio, locating the resonance directly beneath the antenna could be just as important to maximizing $R_{p l}$ as locating the resonance at the field minimum in the center of the plasma. 
The theoretical work discussed in this chapter is by no means an end, but rather a simplified illustration of a powerful new technique of addressing the ICRF wave-plasma coupling problem. Much work still remains to be done to refine this technique to be applicable to existing antenna geometries and to include the other mode (TM). These issues and more are addressed in Chapter 6.

The analytical study described in this chapter has two significant advantages over other studies. First, the axial inhomogeneity and cylindrical geometry are both treated in a theoretically rigorous and consistent manner, and secondly, the subtraction and proper treatment of the resonance pole allow the ICRF waves to propagate up to and through the resonance. These advantages demonstrate the power of this new method of analysis. 


\section{Chapter 4}

\section{Computational ICRF Analysis}

The work performed in this chapter is a simulation of ICRF wave behavior as it propagates towards a magnetic beach resonance. The concept of a magnetic beach upon which to damp electromagnetic waves is not a new idea. First proposed by Furth in 1959 [62] and continuing in the 60's with the B-66 machine and through the C-Stellerator $[3,63,64]$, the magnetic beach concept has been the subject of many theoretical and experimental studies. The thrust of this chapter will to build upon these pioneering work and later work by McVey [65] and Myer[66], culminating in the development of a new methodology to treat the problem of the axial inhomogeneity.

A multimodal analysis is undertaken in this Chapter, resulting in the development of the BEACH computational code. The chapter is organized as follows: Section 4.1 develops the basic theory underlying the $\mathrm{BEACH}$ code development. Section 4.2 offers a brief description of the BEACH code and some background on the theory and algorithm of the BEACH code. Some physics issues that are of particular importance and relevance to the BEACH code are discussed in Section 4.3. Section 4.4 then provides the results obtained from the BEACH computational analysis for various combinations of plasma properties. Next, the experimental results of the legendary B-66 machine are compared to the computational simulation performed by the BEACH code. Finally, the key differences between the 
BEACH and the CYLWAVE analyses are discussed in Section 4.5. Note that comparison to the experimental results obtained from the PPEX machine is provided in Chapter 5, the experimental chapter.

This chapter presents a different approach to simulating the propagation of ICRF waves towards a magnetic beach. The approach taken is different from the previous chapter in the way that the field inhomogeneity is treated. The work performed breaks the inhomogeneous axial magnetic field into a set of discrete homogeneous axial slices, and within each slice the uniform field is responsible for generating a set of discrete field eigenmodes. It is a more localized approach than the previous chapter. However, this chapter allows the treatment of both modes ("almost TE" and "almost TM") of the dispersion as well as a simulation of more realistic ICRF antennas. It is yet another step in the evolution of a complete solution of the cylindrical ICRF wave-plasma coupling problem. 


\subsection{BEACH Code Theory}

\subsubsection{Formulation of Field Solution}

As mentioned before, previous work on ICRF often ignore either the cylindrical geometry or the axial field inhomogeneity. The BEACH code represents the firstof-its-kind analysis that includes both the axially inhomogeneous magnetic field and the cylindrical geometry in its treatment of ICRF propagation. BEACH code models an axially varying cylindrical geometry. The advantage of this geometry is that it accurately simulates the geometry of the experiments of interest. The basic geometry used in the cylindrical geometry analysis is seen in Fig. 4-1

The analysis of the ICRF-plasma coupling problem is presented in the context of the procedure outlined in Section 3.2.2. The development of the theory is similar to the approach taken by McVey [4]. First, the functional dependences of the vacuum and plasma fields are solved, then the boundary conditions are used to couple together the field solutions in different regions. Finally, plasma power absorption will be examined.

\section{VACUUM FIELD SOLUTION}

Starting from Maxwell's equation, it can be readily shown [4] that the vacuum field solutions in a straight cylindrical geometry reduce to the following differential equation:

$$
\begin{aligned}
& \frac{d^{2}}{d r^{2}} E_{z}+\frac{1}{r} \frac{d}{d r} E_{z}-\left(\nu^{2}+\frac{n^{2}}{r^{2}}\right) E_{z}=0 \\
& \frac{d^{2}}{d r^{2}} H_{z}+\frac{1}{r} \frac{d}{d r} H_{z}-\left(\nu^{2}+\frac{n^{2}}{r^{2}}\right) H_{z}=0
\end{aligned}
$$

where $n$ is the azimuthal mode number and appears in the fields in the functional form $e^{\text {in } \theta}$. The solutions to the above differential equations are the Bessel functions $I_{n}(\nu r)$ and $K_{n}(\nu r)$.

PLASMA FIELDS 


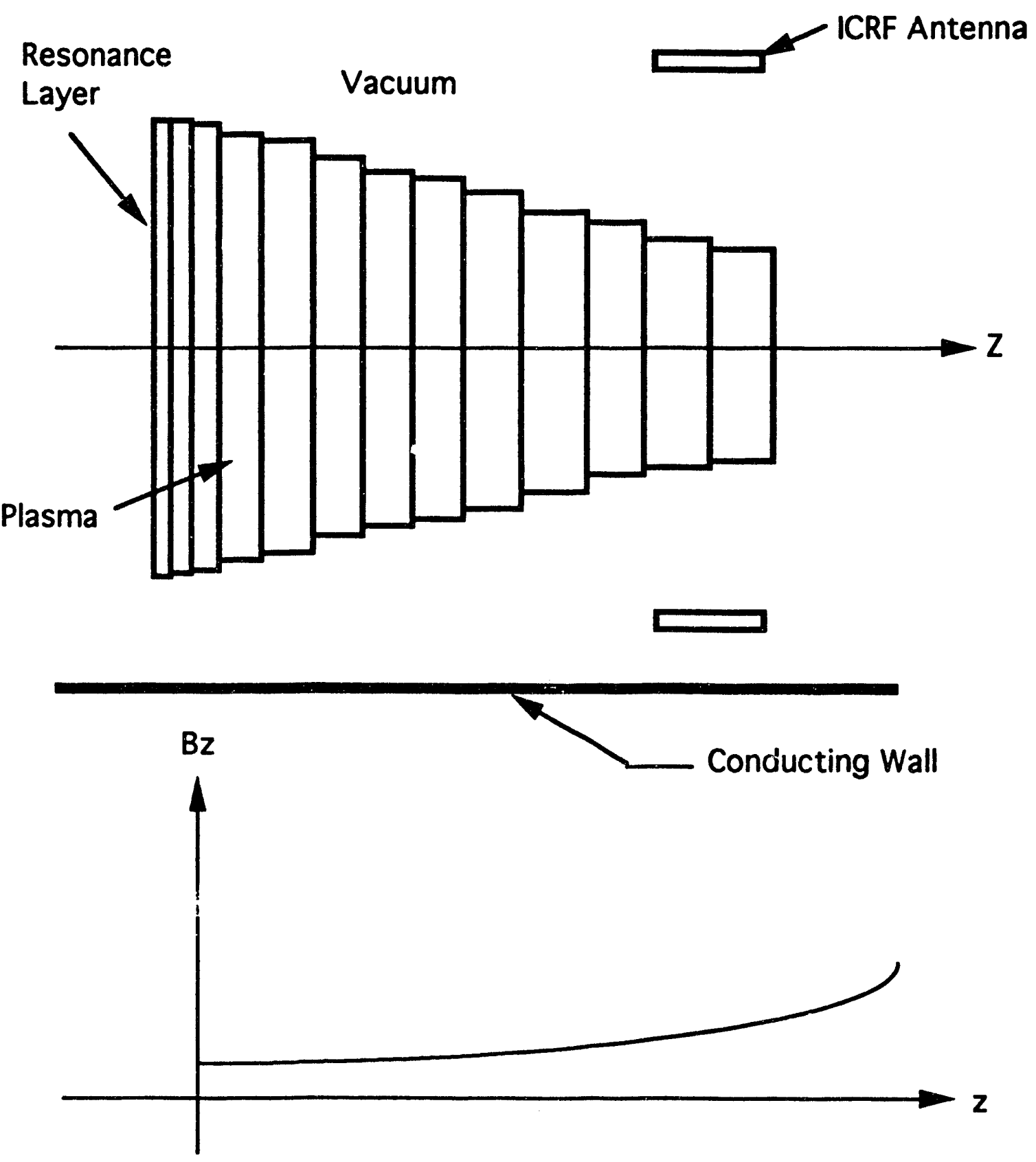

Figure 4-1: Cylindrical ICRF geometry model used in the BEACH code. The axially varying magnetic field plotted at the bottom is simulated by slicing up the cylinder into adjacent axial slices each of which assumes a constant $B_{\mathbf{z}}$. 
It has been shown that Maxwell's equations can be decoupled into two component solutions in a vacuum: TE and TM. While the presence of a plasma prevents a complete separation into the two modes, Maxwell's equations in plasma are shown by McVey [4] and Myer [67] to be simplifiable into two similar modes: the "nearly" TE mode, and the "nearly" TM mode:

From Maxwell's equations, it can be shown that

$$
\nabla \times(\nabla \times \vec{E})=k_{0}^{2} \underline{\underline{\epsilon}}(z) \cdot \vec{E}
$$

where $\underline{\epsilon}$ is the dielectric tensor.

If axial gradients of plasma properties are allowed, the following equations for $E_{z}$ and $H_{z}$ are obtained:

$$
\begin{gathered}
\nabla_{\perp}^{2} E_{z}+k_{0}^{2} P E_{z}+\frac{\partial}{\partial z}\left(\frac{1}{S} \frac{\partial\left(P E_{z}\right)}{\partial z}\right)=-\mu_{0} \omega \frac{\partial}{\partial z}\left(\frac{D H_{z}}{S}\right) \\
\nabla_{\perp}^{2} H_{z}+k_{0}^{2} \frac{R L}{S} H_{z}+\frac{\partial^{2}}{\partial z^{2}} H_{z}=\frac{\epsilon_{0} \omega D}{S} \frac{\partial\left(P E_{z}\right)}{\partial z}
\end{gathered}
$$

where $S, D, P, R, L$ are elements of the dielectric tensor.

Assuming

$$
\nabla_{\perp}^{2} E_{z}, H_{z}=-k_{\perp}^{2} E_{z}, h_{z}
$$

leads to

$$
\begin{aligned}
& P E_{z}=\sum_{n} e^{i n \phi}\left(J_{n}\left(k_{1} r\right) \mathcal{E}_{n 1}+J_{n}\left(k_{2} r\right) \mathcal{E}_{n 2}\right) \\
& H_{z}=\sum_{n} e^{i n \phi}\left(J_{n}\left(k_{1} r\right) \mathcal{H}_{n 1}+J_{n}\left(k_{2} r\right) \mathcal{H}_{n 2}\right)
\end{aligned}
$$

Where $k_{j}, \varepsilon_{n j}$, and $\mathcal{H}_{n j}$ are slowly varying functions of $\mathbf{z}$ compared to the parallel wave number, in other words

$$
\left|\frac{d}{d z}\left(k_{j}, \varepsilon_{n j}, \mathcal{H}_{n j}\right)\right| \ll\left|k_{j}\left(k_{j}, \mathcal{E}_{n j}, \mathcal{H}_{n j}\right)\right|
$$

This permits simplification of the differential equations when ordering 


\section{WKB Solution}

If the $k_{\perp}$ varies slowly as a function of $z$, the following is obtained

$$
\begin{gathered}
\left(\frac{\varepsilon_{n j}^{\prime}}{S}\right)^{\prime}+\left(k_{0}^{2}-k_{j}^{2} / P\right) \varepsilon_{n j}=-\mu_{0} \omega\left(\frac{D \mathcal{H}_{n j}}{S}\right)^{\prime} \\
\mathcal{H}_{n j}^{\prime \prime}-\left(k_{j}^{2}-k_{0}^{2} \frac{R L}{S}\right) \mathcal{H}_{n j}=\frac{\epsilon_{0} \omega D}{S} \varepsilon_{n j}^{\prime}
\end{gathered}
$$

If the plasma properties are not functions of of $z$, the differential equations can be shown to result in two sets of identical 4th order differential equation:

$$
\begin{gathered}
\mathcal{H}_{n j}^{I V}+\mathcal{H}_{n j}^{\prime \prime}\left(S \mathcal{L}_{\mathrm{p}}-\mathcal{L}_{R L S}+\frac{k_{0}^{2} D^{2}}{S}\right)-\mathcal{L}_{p} \mathcal{L}_{R L S} S \mathcal{H}_{n j}=0 \\
\varepsilon_{n j}^{I V}+\mathcal{E}_{n j}^{\prime \prime}\left(S \mathcal{L}_{p}-\mathcal{L}_{R L S}+\frac{k_{0}^{2} D^{2}}{S}\right)-\mathcal{L}_{p} \mathcal{L}_{R L S} S \varepsilon_{n j}=0
\end{gathered}
$$

where $\mathcal{L}_{P}$ and $\mathcal{L}_{R L S}$ are defined as

$$
\begin{gathered}
\mathcal{L}_{P} \equiv\left(k_{0}^{2}-k_{j}^{2} / P\right) \\
\mathcal{L}_{R L S} \equiv\left(k_{j}^{2}-k_{0}^{2} \frac{R L}{S}\right)
\end{gathered}
$$

Since the two equations are identical, and the boundary conditions at the plasmavacuum interface are the same, the functional dependences of $\mathcal{H}_{n j}(z)$ and $\varepsilon_{n j}(z)$ are identical. Thus $\mathcal{H}_{n j}$ and $\varepsilon_{n j}$ differ by only a constant of proportionality. This constant of proportionality is found by substituting $\mathcal{H}_{n j}=h_{1} \varepsilon_{n j}$ into Equations

\section{1 and 4.2 .}

Assuming WKB solutions [68] of the form

$$
\varepsilon_{n j}=f \psi, \mathcal{H}_{n j}=g \psi
$$

where

$$
\psi \equiv e^{i \int^{z} k_{x}\left(z^{\prime}\right) d z^{\prime}}
$$


This WKB approximation is valid for small field gradients

$$
\frac{d l n B_{0}}{d z} \ll k_{z}
$$

It must be noted that the WKB approximation fails near resonance and turning points because the above small field gradient requirement is violated. This means that the $\mathrm{BEACH}$ code is not valid for propagation very close to resonance.

\section{Ordering}

To Lowest Order in $f$ and $g$ : assume no functional dependence of $f$ and $g$, Equations 4.2 and 4.1 simply yield the dispersion relation:

$$
S k_{\perp}^{4}+\left[k_{z}^{2}(S+P)-k_{0}^{2}(S P+R L)\right] k_{\perp}^{2}+P\left(k_{z}^{2}-k_{0}^{2} R\right)\left(k_{z}^{2}-k_{0}^{2} L\right)=0
$$

To First Order $O\left(f^{\prime}, g^{\prime}\right)$ : It can be shown $[67,4]$ that

$$
\begin{aligned}
& H_{z}=H_{p} J_{n}\left(k_{r 1} r\right)+y_{2} E_{p} J_{n}\left(k_{r 2} r\right) \\
& E_{z}=z_{1} H_{p} J_{n}\left(k_{r 1} r\right)+E_{\mathrm{r}} J_{n}\left(k_{r 2} r\right)
\end{aligned}
$$

where

$$
\begin{aligned}
& z_{1}=\frac{i \omega \mu_{0} k_{z} D}{P\left(k_{z}^{2}-k_{0}^{2} S\right)+k_{r 1}^{2} S} \\
& y_{2}=\frac{-i \omega \epsilon_{0} k_{z} P D}{S\left(k_{r 2}^{2}+k_{z}^{2}\right)-R L k_{0}^{2}}
\end{aligned}
$$

Th $H_{p}$ terms of both equations correspond to the Magnetic Modes, or the Nearly TE Mode, while the $E_{p}$ terms correspond to the Electric Modes, or the Nearly TM Mode.

\subsubsection{Boundary Conditions}

As mentioned before, the axial inhomogeneity is treated by separating the plasmavacuum cylinder into discrete axial slices. In each axial slice, all plasma properties and axial magnetic field are constant. For the geometry of Fig 4-1, boundary conditions are imposed for each individual axial slice. 
1. At $\mathbf{r}=\mathbf{a}$ (plasma-vacuum interface)

$$
\begin{aligned}
& E_{\phi}^{1}(a)=E_{\phi}^{2}(a) \\
& E_{z}^{1}(a)=E_{z}^{2}(a) \\
& H_{\phi}^{1}(a)=H_{\phi}^{2}(a) \\
& H_{z}^{1}(a)=H_{z}^{2}(a)
\end{aligned}
$$

2. At $r=b$ (Antenna location)

$$
\begin{gathered}
E_{\phi}^{2}(b)=E_{\phi}^{3}(b) \\
E_{z}^{2}(b)=E_{z}^{3}(b) \\
H_{\phi}^{3}(b)-H_{\phi}^{2}(b)=J_{z}(b) \\
H_{z}^{2}(b)-H_{z}^{3}(b)=J_{\phi}(b)
\end{gathered}
$$

3. At $r=c$ (Conducting Wall)

$$
\begin{aligned}
& E_{\phi}^{3}(c)=0 \\
& E_{z}^{3}(c)=0
\end{aligned}
$$

Using the above boundary conditions, the field coefficients of the three regions can be fully determined and are given in Appendix C.

\subsubsection{Absorbed Power Calculation}

Having solved for the field coefficients, an estimation of the power absorbed by the plasma can be made. The power $\mathcal{P}$ absorbed by the plasma is given by:

$$
\begin{gathered}
\mathcal{P}\left(w / m^{2}\right)=\frac{1}{2} \operatorname{Re}\left(\vec{E} \cdot \vec{J}^{*}\right) \\
\mathcal{P}=\frac{1}{2} \operatorname{Re}\left(E_{r} J_{+}^{*}+E_{\theta} \cdot J_{\theta}^{*}+E_{z} J_{z}^{*}\right)
\end{gathered}
$$


The plasma current is found by the product of the conductivity tensor and the electric field. The conductivity tensor $\underline{\underline{\sigma}}$ can be expressed in terms of the dielectric tensor $\underline{\underline{K}}$.

$$
\begin{aligned}
\nabla \times \vec{B} & =\frac{1}{c^{2}} \frac{\partial \vec{E}}{\partial t}+\mu_{0} \vec{J} \\
& =\frac{-i \omega}{c^{2}} \underline{K} \cdot \vec{E}
\end{aligned}
$$

Since $\vec{J}=\underline{\underline{g}} \cdot \vec{E}$,

$$
\begin{gathered}
\frac{-i \omega}{c^{2}} \underline{\underline{K}} \cdot \vec{E}=\frac{-i \omega}{c^{2}} \underline{\underline{I}} \cdot \vec{E}+\mu_{0} \underline{\underline{g}} \cdot \vec{E} \\
\underline{\underline{\sigma}}=\frac{-i \omega}{c^{2}} \frac{(\underline{\underline{\underline{K}}-\underline{\underline{I}})}}{\mu_{0}}
\end{gathered}
$$

According to Myer [69], the $E_{z} J_{z}^{*}$ contribution to the dissipated power is mostly absorbed by the electrons. This is due to the fact that electrons will act to flow along the field lines and zero out any $E_{\|}$. In fact, current drives in tokamaks take advantage of this fact by launching favorably shaped $E_{\mathbf{z}}$ spectra to couple to and drive fast electrons. However, in the context of this thesis, this energy absorbed by the electrons will not be examined. 


\subsection{BEACH Code}

The goal of the computational analysis is to provide an alternative method of modeling the wave-plasma coupling problem than the analytical approach taken in the previous chapter. While the analytical approach examined a continuous spectrum of axial wavenumbers $k_{\xi}$ and their corresponding perperndicular wavenumbers $\alpha$, the BEACH code solves for the discrete eigenmodes that exist in each individual axial slice. In other words there no longer exists a continuum of $k_{z}$ in each slice, but rather an infinite number of DISCRETE modes. However, this computational analysis is not simply a purely numerical simulation. Much analytical work is done to reduce the fields to functional form, i.e. $I_{n}\left(k_{r} r\right), K_{n}\left(k_{r} r\right), J_{n}\left(k_{\perp 1} r\right)$ before the numerical "number-crunching" is handed over to the codes.

BEACH is a code that was developed as a major part of the thesis. It has, the major feature of being able to take into consideration the axial magnetic field inhomogeneity. Thus, the thesis places great emphasis on the BEACH code as well as the CYLWAVE code because of their ability to simulate propagation in an inhomogeneous axial magnetic field, a topic of great importance to ICRF heating.

The unique feature of the BEACH and CYLWAVE analysis undertaken as part of this thesis lies in the generality of their approachs. Other studies [33, 34, 32] have been performed which either look at the $k_{z}(z)$ variation as it impacts upon amplitude transport in a WKB [68] sense:

$$
|\mathcal{P}+\mathcal{T}|_{0}=|\mathcal{P}+\mathcal{T}|_{1} e^{-i \int k_{z}(z) d z}
$$

or have relied upon a single mode solution of the boundary conditions coupled with the above WKB analysis. However, no attempts have been made to examine both the variation of the field amplitudes as well as the wave number $k_{z}(z)$. The BEACH and CYLWAVE codes take into consideration all these factors and are thus more complete treatments than other approaches before them. Together $\mathrm{BEACH}$ and CYLWAVE demonstrate two different approaches to simulating the same problem. 


\subsubsection{Background}

BEACH is a code that was initially begun by Myer at MIT to examine the ICRF wave propagation problem taking into consideration the axial magnetic field profile. A major portion of the work accomplished in this thesis was to complete the BEACH code and apply it to model the magnetic geometry of PPEX and of other machines to examine ICRF wave propagation and plasma heating.

The BEACH code assumes a radially uniform density profile. How would that affect the results of the computation? It must be noted here that one of the significant results that emerged from the theoretical and experimental work on wave coupling by Sigmann and Reinmann [70] and Hosea and Sinclair [33] was that coupling to homogeneous plasma very nearly equals that of an inhomogeneous plasma if one uses the volume average density of the inhomogeneous plasma for the homogeneous plasma case. This result suggests that the use of a radially constant profile is a plausible starting point for the development of new techniques.

\subsubsection{Theory \& Algorithm}

The BEACH code extends the analytical approach of cylindrical geometry solution seen in Section 4.1

The BEACH code solves for the ICRF wave propagation in an inhomogeneous axial magnetic field. The inhomogeneous axial magnetic field $B_{x 0}(z)$ is handled by separating the axial length into discrete axial "slices", each of which contains a homogeneous axial magnetic field. The eigenmodes in each slice can then be calculated as if the field in that slice were constant.

The code first finds the eigenmodes that exist in each axial slice by satisfying both the infinite plasma dispersion relation and the boundary condition. The boundary condition of the plasma-vacuum system is obtained from the boundary conditions as seen in Equations 4.4 through 4.13 and is shown by McVey [65] to be given by 


$$
\begin{aligned}
& c_{1} H_{p}+c_{2} E_{p}=e_{1} J_{\phi} \\
& c_{3} H_{p}+c_{4} E_{p}=e_{2} J_{\phi}
\end{aligned}
$$

The solution to the above are:

$$
\begin{gathered}
H_{p}=\frac{\left(e_{1} c_{4}-e_{2} c_{2}\right)}{D} J_{\phi} \\
E_{p}=\frac{\left(e_{2} c_{1}-e_{1} c_{3}\right)}{D} J_{\phi} \\
D=c_{1} c_{4}-c_{2} c_{3}
\end{gathered}
$$

where the elements $c_{1}, \ldots, c_{4}$ and $e_{1}, e_{2}$ are defined in Appendix C. The term $D$ in Equation 4.18 represents the boundary condition of the bounded plasma-vacuum system. Solving for the zero of this boundary condition coupled with the infinite plasma dispersion relation (Equation 4.3 ) yields the eigenmodes of the cylindrical system.

After solving for the eigenmodes of the system, the code then computes the excitation coefficients of the initial axial slice as driven by the source term (Antenna). The excitation coefficient of the next axial slice can then be determined by imposing two conditions of continuity on the boundary between the two axial slices: conservation of the integrated axially propagating Poynting Flux $\int \vec{S}_{\mathbf{z}} r d r$, and the continuity of wave phase. These two conditions uniquely determine the excitation coefficients of the different eigenmodes in the next axial slice. This analysis is then performed for all axial slices.

As this chapter shows, it is evident that in order to adequately reproduce the ICRF field profiles the solution of about 30-50 eigenmodes is required. These eigenmodes, upon summation, form the resulting field solutions. The reason that so many modes are required is that there is no one dominant mode that is excited 
to an amplitude that is orders of magnitude higher than others. Instead, what is seen is that a multitude of modes is excited, each of whose excitation coefficient is about the same order as the others. Thus, for completeness, it is found that the summation of at least $\mathbf{3 0}$ modes is required for the inverse Fourier transform in order for the solutions to converge.

The program algorithm is shown on Fig. 4-2.

A sample page of output from the BEACH code illustrates the functionality of the code. Part A (Upper left figure) of Figure 4-3 presents the eigenmodes that are found to exist in this system, part $B$ (Upper right figure) shows the integrated axially propagating Poynting flux for each mode. Parts C \& D (lower two plots) give the excitation coefficients $E_{p}$ and $H_{p}$ which are the field coefficients given in Equations 4.16 and 4.17 .

\subsubsection{BEACH Subcodes}

The basic algorithm of the BEACH code is seen on Figure 4-2. BEACH consists of three subcodes - $x$ disp, $x$ field, and xplot. The xdisp code solves for the finite discreet eigenmodes for the bounded plasma-vacuum system, xfield solves for the excitation amplitudes in different axial slices, and xplot plots out the results.

\subsection{Physics Issues}

The formulation of the BEACH code took into consideration several important physical phenomena. Some of these are discussed below.

\section{POWER CONSERVATION}

As mentioned before, BEACH was seen not to conserve energy in earlier versions. Changes were then undertaken as part of this thesis to correct the code so that
energy is conserved. 


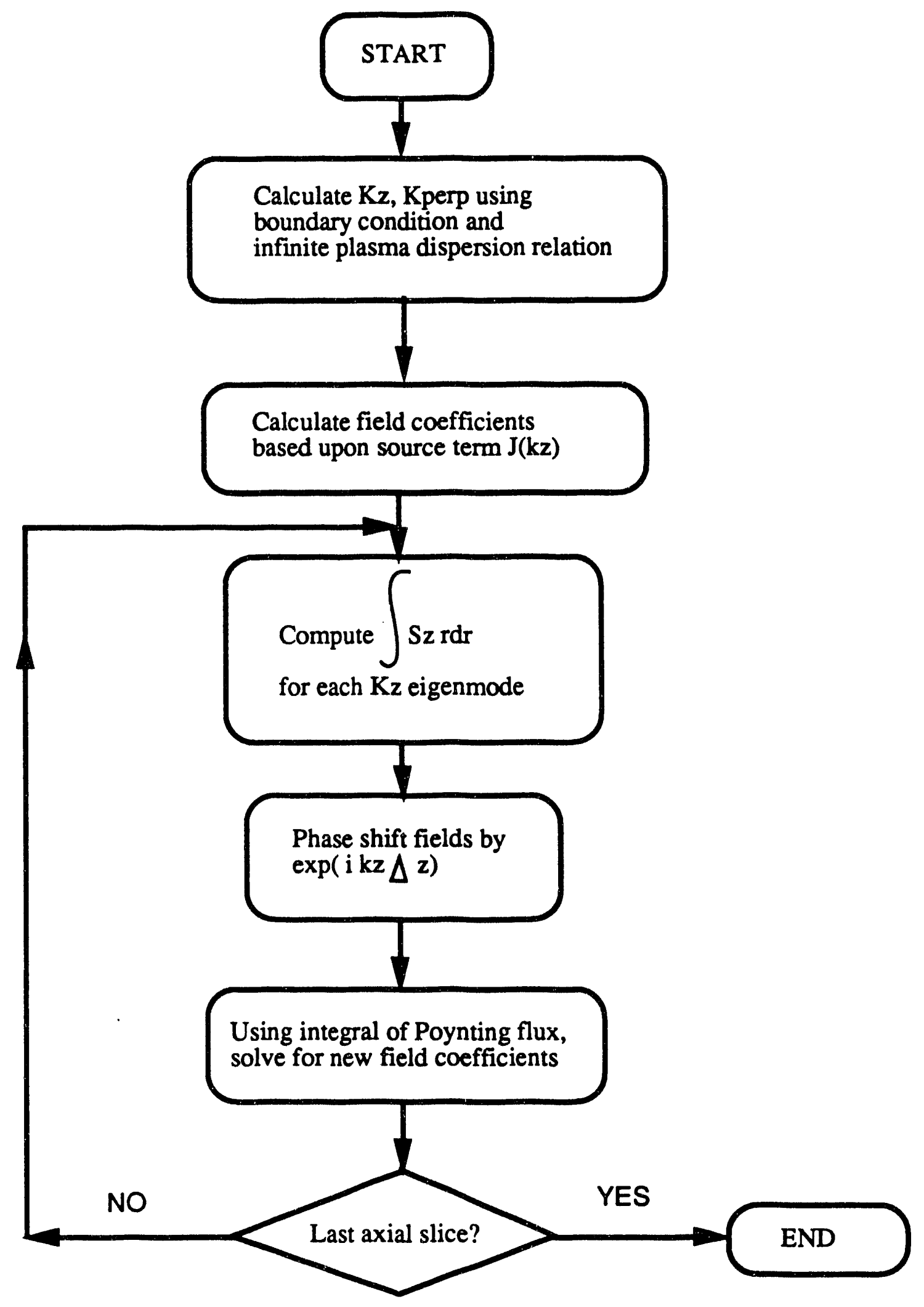

Figure 4-2: BEACH Code Algorithm 

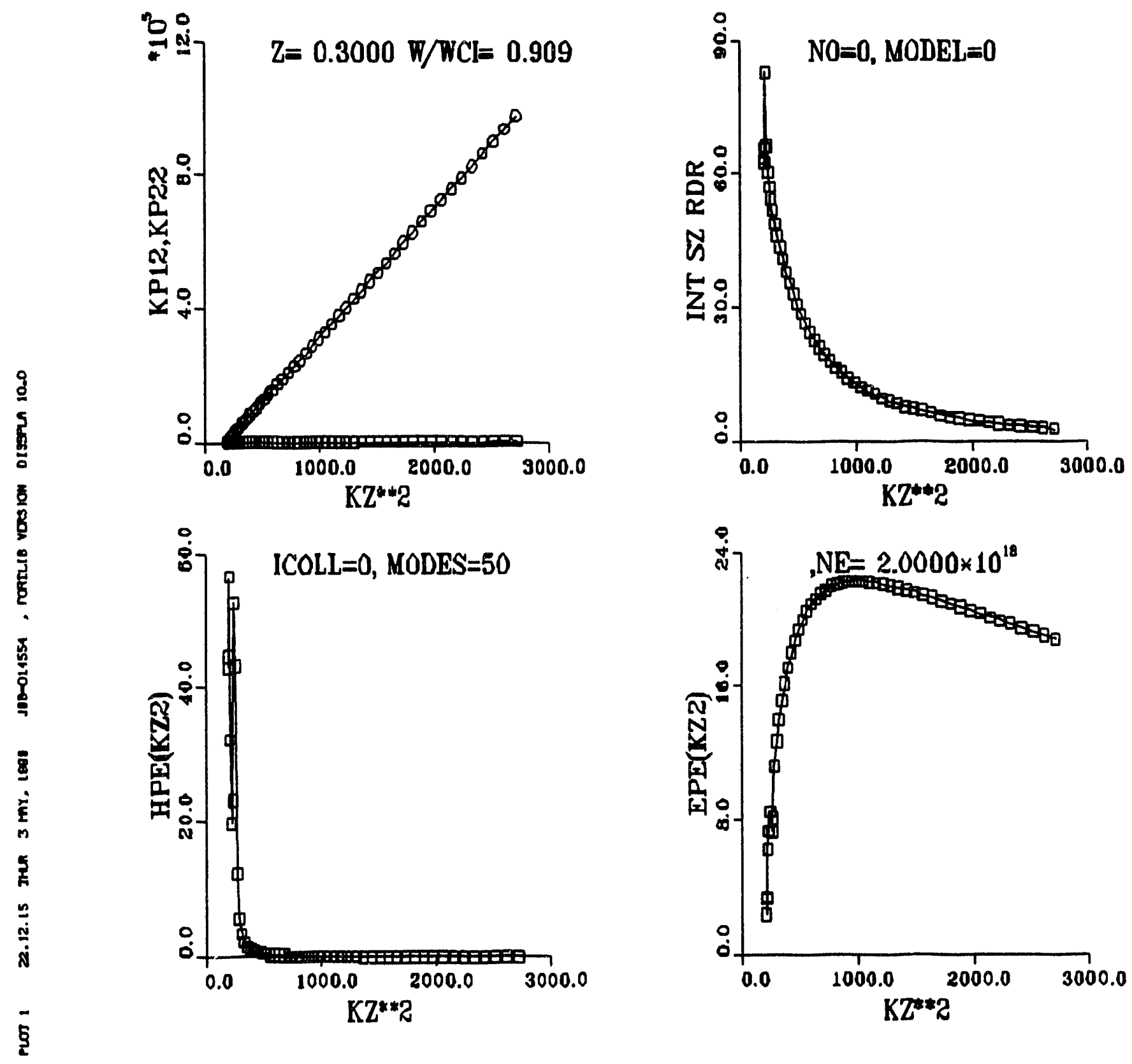

Figure 4-3: Sample output from BEACH Code 
In order to conserve energy, the parallel propagating Poynting Flux for each individual eigenmode is first calculated. From the fields calculated by the geometries, the Poynting Flux power flow per cross-sectional area can be calculated by $[44,47]$ :

$$
\vec{S}\left(w / m^{2}\right)=\frac{1}{2} \operatorname{Re}(\vec{E}(\vec{r}) \times \vec{H}(\vec{r}))
$$

The integrated axial power flow is then found by

$$
P_{z}\left(w / m^{2}\right)=\int \vec{S} \cdot d \vec{A}=\int \frac{1}{2} \operatorname{Re}\left\{\vec{E}(\vec{r}) \times \vec{H}^{*}(\vec{r})\right\} \cdot d \vec{A}
$$

This $P_{z}$ is then held to be a conserved quantity across the interface between the different axial "slices" of the geometry. This ensures the conservation of flowing energy across axial boundaries.

\section{Power Conservation Across Adjacent Axial Slices}

The power conservation across adjacent axial slices is formulated here.

From the expression for the vacuum and plasma fields found in Appendix B, the field coefficients $E_{1} \ldots E_{4}, H_{1} \ldots H_{4}$ can be determined in terms of the plasma field coefficients $H_{p}$ and $E_{p}$. From Equation 4.16 and 4.17 , it is seen that for a sourceless slice, $E_{p}=-\frac{c_{1}}{c_{2}} H_{p}$. Thus, the transverse fields in each region can all be expressed in terms of $H_{p}$ and are given below

Vacuum Regions I $(b<r<c)$

$$
\begin{aligned}
& E_{r}(r)=H_{p} C_{E r 1}(r) \\
& E_{\phi}(r)=H_{p} C_{E \phi 1}(r) \\
& H_{r}(r)=H_{p} C_{H r 1}(r) \\
& H_{\phi}(r)=H_{p} C_{H \phi 1}(r)
\end{aligned}
$$

where

$$
C_{E r 1}(r) \equiv i \frac{k_{z} \Delta_{c r^{\prime}}}{\nu \Delta_{a c}}\left(J_{n 1 a} z_{1}-J_{n 2 a} \frac{C_{1}}{C_{2}}\right)+\frac{\omega \mu_{0}}{\nu^{2}} \frac{n}{r} \frac{\Delta_{c^{\prime} r}}{\Delta_{a c^{\prime}}}\left(J_{n 1 a}-y_{2} J_{n 2 a} \frac{C_{1}}{C_{2}}\right)
$$




$$
\begin{aligned}
C_{E \phi 1}(r) & \equiv \frac{-k_{z} n}{\nu^{2} r} \frac{\Delta_{c r}}{\Delta_{a c}}\left(J_{n 1 a} z_{1}-J_{n 2 a} \frac{C_{1}}{C_{2}}\right)-\frac{i \omega \mu_{0}}{\nu} \frac{\Delta_{c^{\prime} r^{\prime}}}{\Delta_{a c^{\prime}}}\left(J_{n 1 a}-y_{2} J_{n 2 a} \frac{C_{1}}{C_{2}}\right) \\
C_{H r 1}(r) & \equiv \frac{\omega \epsilon_{0} n}{\nu^{2} r} \frac{\Delta_{c r}}{\Delta_{a c}}\left(J_{n 1 a} z_{1}-J_{n 2 a} \frac{C_{1}}{C_{2}}\right)+\frac{i k_{z}}{\nu} \frac{\Delta_{c^{\prime} r^{\prime}}}{\Delta_{a c^{\prime}}}\left(J_{n 1 a}-y_{2} J_{n 2 a} \frac{C_{1}}{C_{2}}\right) \\
C_{H \phi 1}(r) & \equiv \frac{i \omega \epsilon_{0}}{\nu} \frac{\Delta_{c r^{\prime}}}{\Delta_{a c}}\left(J_{n 1 a} z_{1}-J_{n 2 a} \frac{C_{1}}{C_{2}}\right)-\frac{k_{z} n}{\nu^{2} r} \frac{\Delta_{c^{\prime} r}}{\Delta_{a c^{\prime}}}\left(J_{n 1 a}-y_{2} J_{n 2 a} \frac{C_{1}}{C_{2}}\right)
\end{aligned}
$$

Where $\Delta_{a b^{\prime}}$ is defined in Apppendix C, Equation C.20.

Vacuum Region II $(b<\mathbf{r}<\mathbf{a})$

Using a similar approach

$$
\begin{aligned}
& E_{r}(r)=H_{p} C_{E r 2}(r) \\
& E_{\phi}(r)=H_{p} C_{E \phi 2}(r) \\
& H_{r}(r)=H_{p} C_{H r 2}(r) \\
& H_{\phi}(r)=H_{p} C_{H \phi 2}(r)
\end{aligned}
$$

Where $\mathcal{C}(r)$ 's are likewise defined.

Plasma Region, $(\mathbf{r}<\mathbf{a})$

Substituting for $E_{p}$ in Equations B.15 - B.18 in Appendix B, the transverse plasma fields are found to be

$$
\begin{aligned}
\hat{E}_{r}=H_{p}\left[e_{r 1}(r)-\frac{C_{1}}{C_{2}} e_{r 2}(r)\right] & \equiv H_{p} \mathcal{D}_{E r}(r) \\
\hat{E}_{\phi}=H_{p}\left[e_{\phi 1}(r)-\frac{C_{1}}{C_{2}} e_{\phi 2}(r)\right] & \equiv H_{p} \mathcal{D}_{E \phi}(r) \\
\hat{H}_{r}=H_{p}\left[h_{r 1}(r)-\frac{C_{1}}{C_{2}} h_{r 2}(r)\right] & \equiv H_{p} \mathcal{D}_{H r}(r) \\
\hat{H}_{\phi}=H_{p}\left[h_{\phi 1}(r)-\frac{C_{1}}{C_{2}} h_{\phi 2}(r)\right] & \equiv H_{p} \mathcal{D}_{H \phi}(r)
\end{aligned}
$$

where the variables $e_{r 1}(r)$ through $h_{r 2}(r)$ are defined in Appendix C.

Using these fields then, the axial propagating Poynting Flux $S_{z}$ can be found as

$$
S_{z}=\frac{1}{2} \operatorname{Re}\left(E_{r} H_{\phi}^{*}-E_{\phi} H_{r}^{*}\right)
$$


Since $(A B)^{*} \equiv A^{*} B^{*}$, it is seen that

$$
\begin{aligned}
S_{z, \text { vac }, 1} & =H_{p} H_{p}^{*}\left[C_{E r 1} C_{H \phi 1}^{*}-C_{E \phi 1} C_{H r 1}^{*}\right] \\
S_{z, \text { vac }, 2} & =H_{p} H_{p}^{*}\left[C_{E r 2} C_{H \phi 2}^{*}-C_{E \phi 2} C_{H r 2}^{*}\right] \\
S_{z, \text { pla }} & =H_{p} H_{p}^{*}\left[D_{E r} D_{H \phi}^{*}-\mathcal{D}_{E \phi} D_{H r}^{*}\right]
\end{aligned}
$$

where

$$
\begin{gathered}
H_{p}=H_{p, R e}+i H_{p, i m} \\
H_{p} H_{P}^{*}=\left(H_{p, R e}^{2}+H_{p, i m}^{2}\right)
\end{gathered}
$$

Thus the continuity of power flow across the boundary between axial slices yields a continuity equation in terms of the real and imaginary components of the plasma magnetic field coefficient $H_{p}$. A second boundary condition is required to fully solve for both the real and imaginary component of $H_{p}$.

\section{Conservation of Wave Phase}

This second boundary condition at the interface between axial slices is the conservation of wave phase, which states that the wave phase across the boundary is continuous. This means that even though $H_{p}$ can change, the ratio

$$
\frac{H_{p, i m}}{H_{p, \text { Re }}}=\tan \left(\theta_{\text {wave }}\right)
$$

cannot change across the boundary. This condition is the standard electromagnetic continuity condition across the boundary between two different dielectric media detailed by Kong [59].

The conservation of the area-integrated Poynting Flux $\int \vec{S}_{z} \cdot d \vec{A}$ along with the conservation of the phase angle of the wave, $\theta=\tan ^{-1}\left(H_{p, \text { im }} / H_{p, R_{e}}\right)$ yield a set of solutions of the excitation coefficients $H_{p}$ and $E_{p}$ that uniquely satisfy the boundary condition and the dispersion relation. 


\section{DISSIPATION vs. NO DISSIPATION}

In solving for the eigenmodes of the system, different methods of solution are used for the dissipation and dissipationless models. Solving for a dissipationless case requires only solving for real $k_{z}$ roots, while solving a dissipation case requires finding imaginary $k_{z}$ roots. A straightline linear interpolation scheme is used to find the $k_{z}$ roots in the dissipationless case, while Müller's Method [54] is used (via the IMSL subroutine ZANLY) in finding the complex roots for the dissipational case. Comparison of execution times shows that a sample dissipationless case of 50 roots takes about 5 minutes of CRAY (XMP) cpu time, while the dissipation case takes about 100 minutes.

\section{BOUNDARY CONDITION ISSUES}

The discretizational modeling of the inhomogeneous axial magnetic field $B_{z 0}$ raises a major question - that of the non-divergence of $\vec{B}$. Namely, is $\nabla \cdot \vec{B}=0$ ?

The approach taken in the BEACH code is to conserve the total magnetic flux in the system. Thus, when the magnetic field in region 1 is GREATER than $B_{\mathbf{z}}$ in region 2, the cross sectional area of the plasma is SHRUNK so as to conserve the flux contained in the plasma tube. The magnetic flux in the vacuum region is not conserved. This non-conservation of the magnetic flux is then a source of error in the code. In order to examine this error, the BEACH code maintains an option to allow the physical geometry, i.e. vacuum chamber wall, to expand and contract with the magnetic field $B_{z 0}(z)$ in order to conserve the magnetic flux. After comparing the results from the two cases (one with fixed wall geometry, other with variable wall geometry), it was concluded that the error involved in keeping the geometry stationary had negligible effect on the propagation modes. 


\subsection{Computational Results}

The aim of the computational analysis is to make comparisons between different cases of differing plasma properties and to make recommendations on how to maximize the antenna-wave-plasma coupling.

Several different studies are performed in this computational analysis to examine the effects of various plasma characteristics on ICRF wave propagation. These are listed below:

1. Collisionless vs. collisional plasma

2. Full Turn Loop vs. Dual Half Turn Loop Antennas

3. Electron density $-n_{e}$. (Collisional only)

4. Temperature dependence. (Collisional only)

5. $\omega / \omega c i$ at launch point (Collisional only)

Comparisons to experimental data are also made. Special attention is given to two experiments: the B-66 experiment as reported by Uman \& Hooke [3], and the PPEX experiment that was constructed as the experimental portion of this thesis. Comparison with the PPEX experimental results will be provided in the next Chapter on Experimental Results.

Because the analysis undertaken here is a cold plasma treatment, the validity of temperature dependence must be delimited. The type of plasma for which the current treatment is valid is a cold collisional plasma. Since the PPEX machine operates at relatively low temperatures $(<1 \mathrm{KeV})$, the cold collisional plasma approximation is valid. However, it must be recognized that the cold collisional approximation may not be valid for the higher temperature regimes of the B-66 machine.

The Computational Results section is organized as follows: Section 4.4.1 will describe the baseline model and magnetic geometry used in the analysis. Section 
4.4.2 then gives the comparison of results obtained for a collisional vs. a collisionless plasma model. Section 4.4.3 presents the comparison between the fields generated by a Full Turn Loop vs. a Dual Half Turn Loop antenna. An analysis of the effect of density on propagation is then examined in Section 4.4.4, followed by a temperature analysis in Section 4.4.5. An analysis of the launch frequency is also examined in 4.4.6, and finally comparison to experimental results from the B-66 machine is made in Section 4.4.7.

\subsubsection{Baseline Model}

The computational simulations for all subsections (except the B-66 section) were performed using the magnetic field geometry of the PPEX machine, as shown on Figure 4-4.

The baseline model is driven by a Full Turn Loop (FTL) antenna. Because it is symmetric in $\theta$, the full turn loop antenna excites only the $n=0$ mode. ' $i$ 'he $J_{\theta}\left(k_{z}\right)$ spectrum is calculated for the FTL system and plotted on Figure 4-5.

The BEACH code is used to compute the propagational profiles of the $B_{2}$ and $E_{z}$ ICRF fields and the results are seen on Figure 4-6 $\left(B_{z}\right)$ and 4-7 $\left(E_{z}\right)$. 


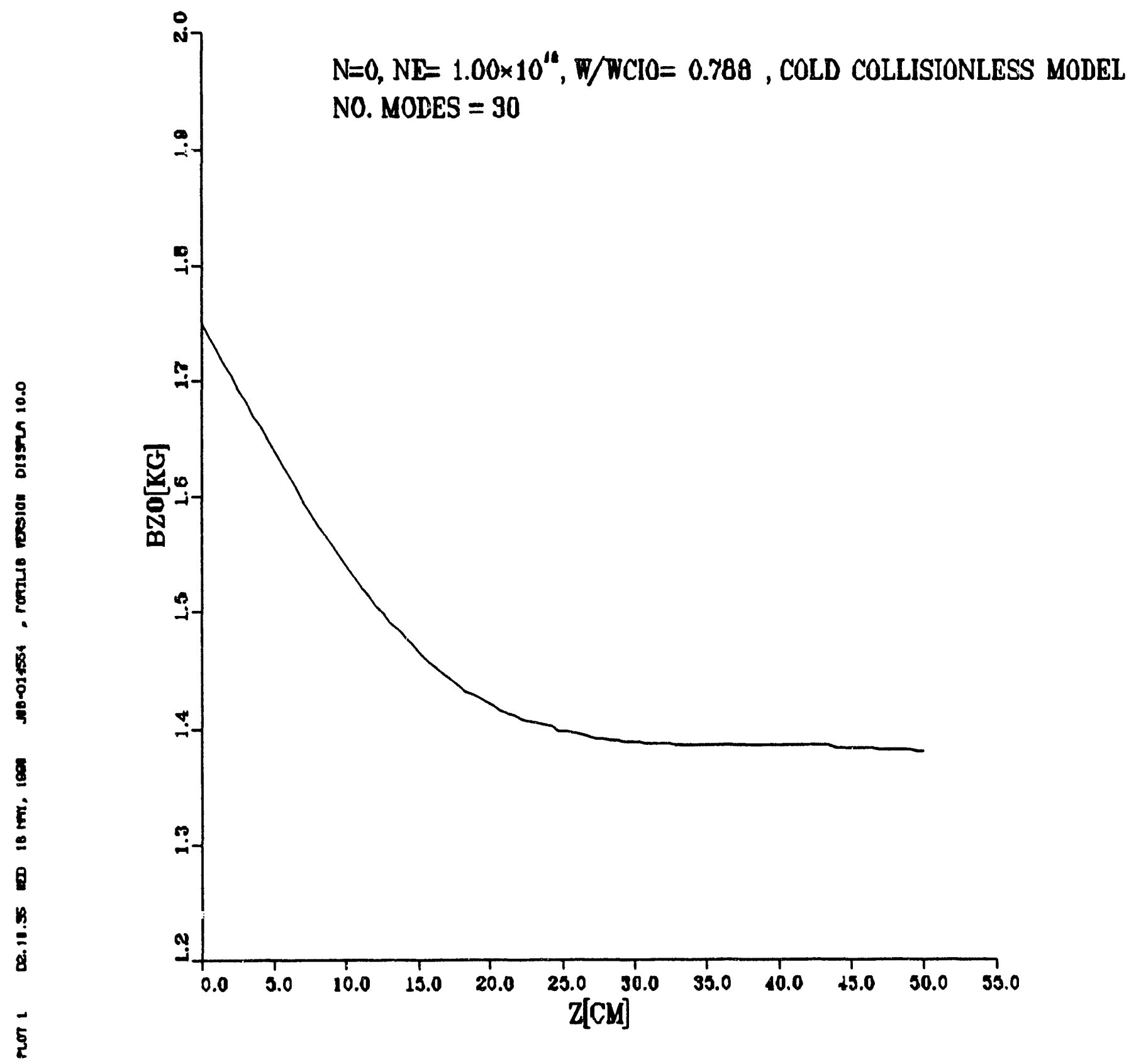

Figure 4-4: PPEX axial magnetic field $B_{z 0}(z)$ profile as modelled by BEACH code 


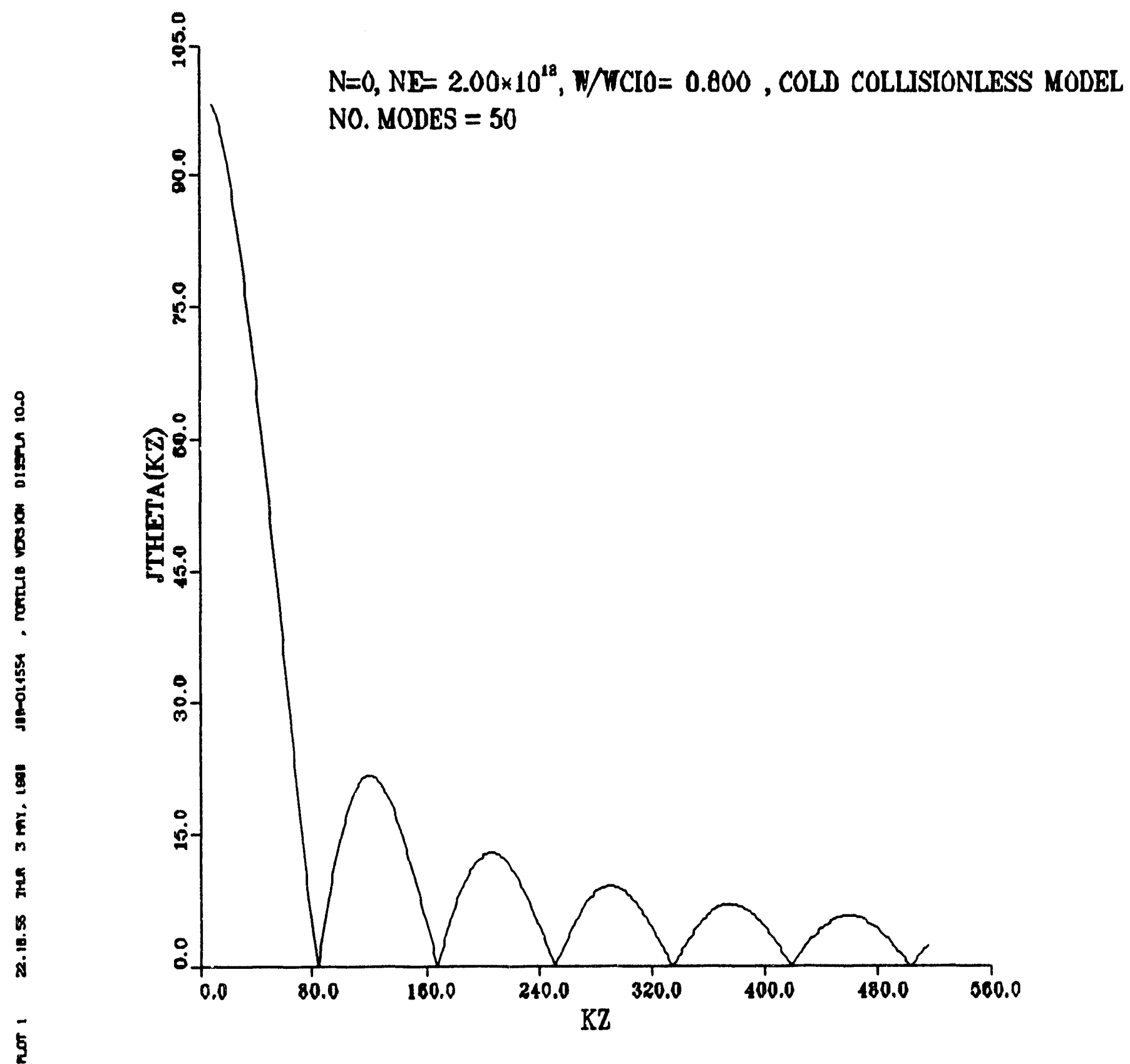

Figure 4-5: $J_{\theta}\left(k_{z}\right)$ Spectrum for the Baseline Full Turn Loop Antenna 
$\mathrm{N}=0, \mathrm{NE}=1.0 \mathrm{C} \times 10^{18}, \mathrm{~W} / \mathrm{WCI} 0=0.788$, COLD COLLISIONLESS MODEL NO. MODES $=30$

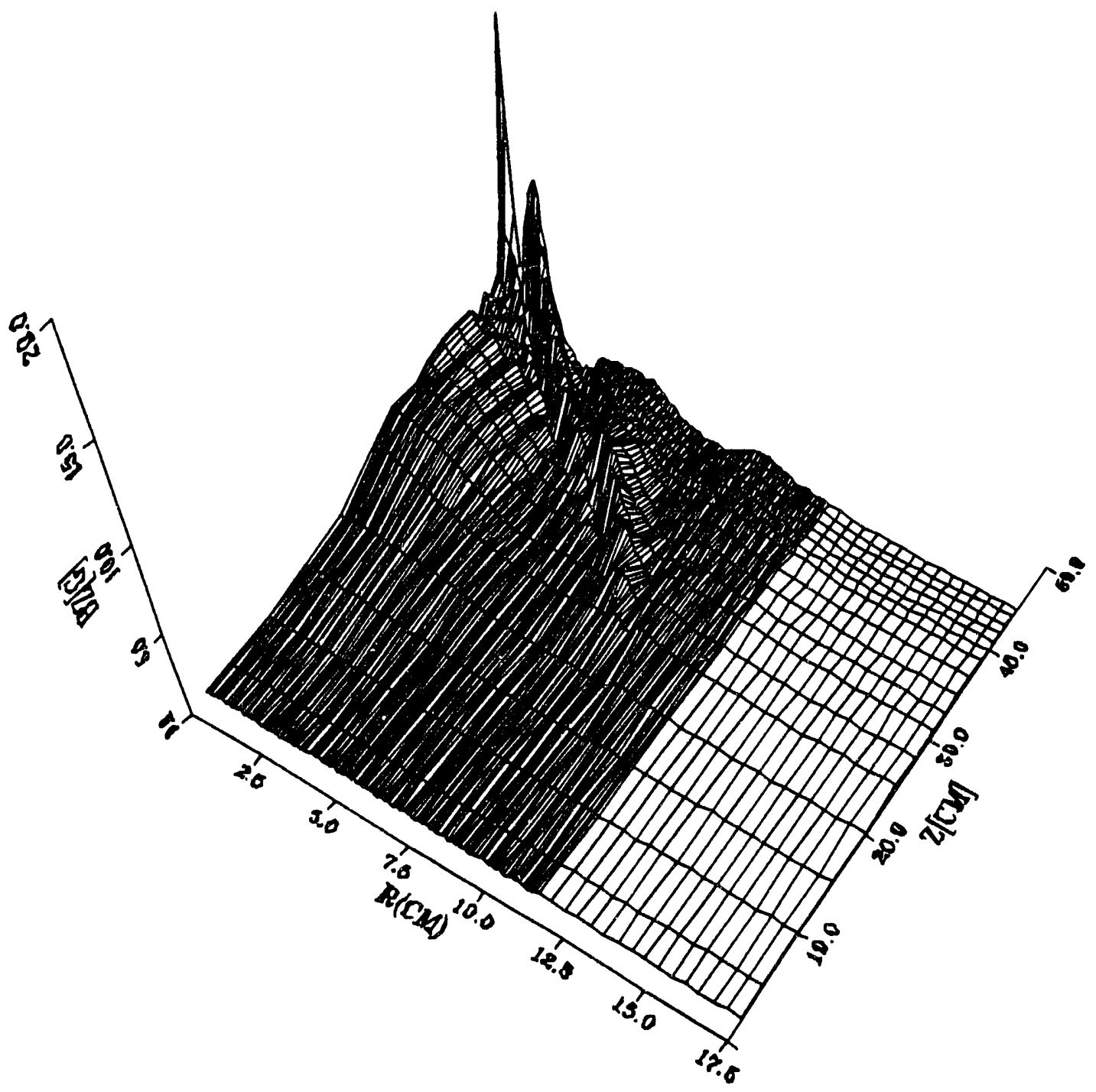

Figure 4-6: ICRF $\mathbf{B}_{\mathbf{z}}$ Propagation in a Cold Collisionless Plasma, Full Turn Loop Antenna, $\omega / \omega c i 0=0.788$. Waves are launched by the ICRF antenna at $z=0 \mathrm{~cm}$ and propagate towards resonat 65 at $z=50 \mathrm{~cm}$. 


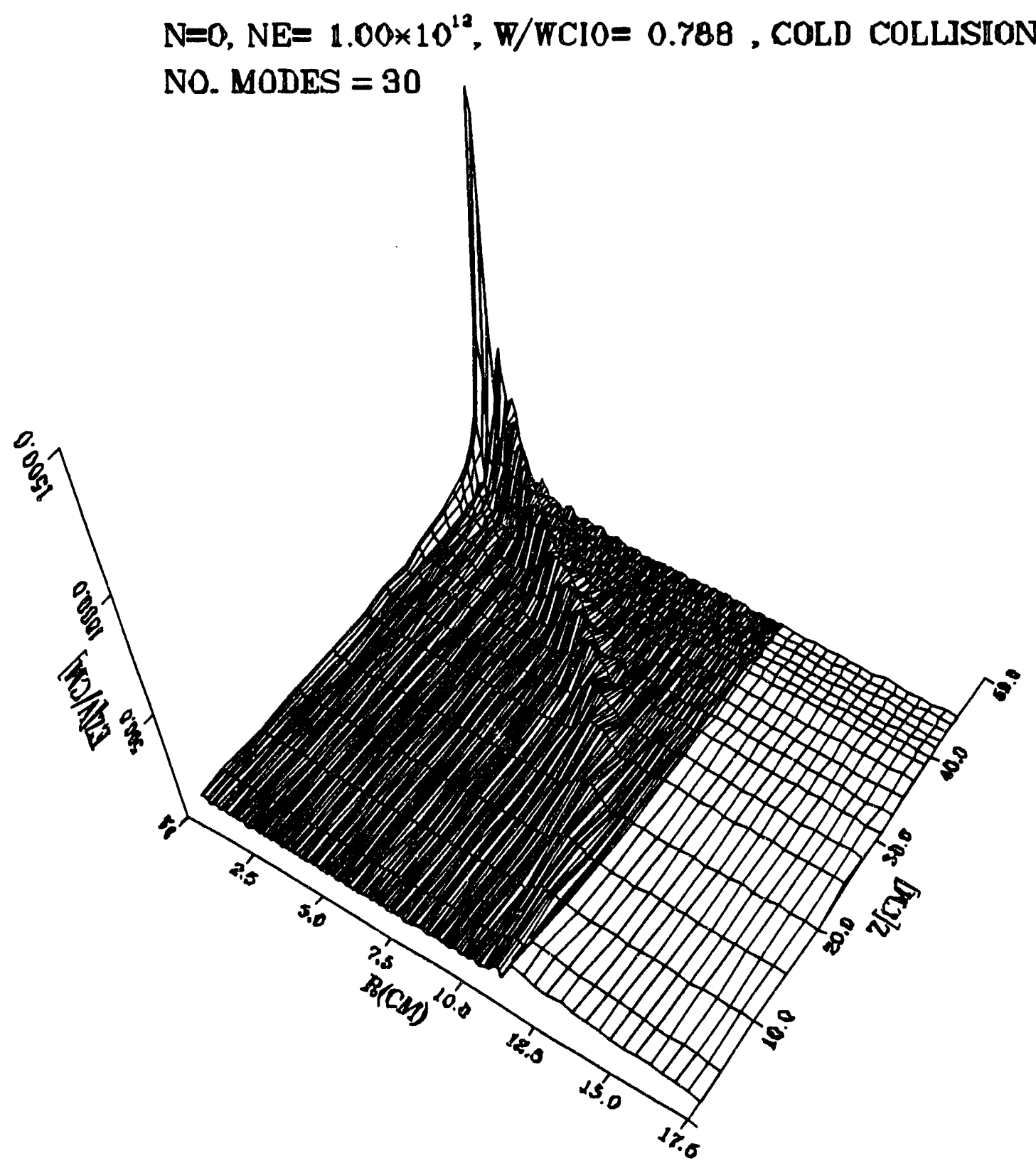

Figure 4-7: ICRF $\mathbf{E}_{\mathbf{z}}$ Propagation in a Cold Collisionless Plasma, Full Turn Loop Antenna, $\omega / \omega c i 0=0.788$ 


\subsubsection{Collisionless vs. Collisional}

The collisionless vs. collisional analysis performed here uses the baseline plasma properties as given in Section 4.4.1. The launching antenna used in the collisionless vs. collisional analysis is the Full Turn Loop antenna. Thus, the collisionless results are already given in Figures 4-6 and 4-7.

\section{COLLISIONLESS}

The calculation of the $k_{z}$ eigenmodes of the system allows a selection of either a collisional or a collisionless model. Selecting a collisionless model allows the code to make the following assumptions:

- $S, P$, and D (Elements of the dielectric tensor $\underline{\underline{\epsilon}}$ ) are real.

$$
\begin{gathered}
S=1-\sum_{\alpha} \frac{\omega_{p i}^{2}}{\omega^{2}-\omega_{c i}^{2}} \\
D=\sum_{\alpha} \frac{\Omega_{c \alpha} \omega_{p \alpha}^{2}}{\omega\left(\omega^{2}-\Omega_{c \alpha}\right)} \\
P=1-\sum_{\alpha} \frac{\omega_{p i}^{2}}{\omega^{2}}
\end{gathered}
$$

- Only real $k_{z}$ eigenmodes exist, i.e. no damping modes can exist

The collisionless model is provided here as a baseline against which the damping results of the collisional model could be compared. A cold plasma model is also useful as a check to see if the code results conforms to basic physical insight. Because there is no dissipation mechanism (Cold plasma, no collisions), some effects are expected physically from this simple propagation.

Upon examination of the propagation results as given in Figure 4-6 and 47 , three important results are evident as the waves propagate towards resonance 
- Decrease in axial and radial wavelengths, buildup of $\left|B_{z}\right|$ as resonance is approached, and the inward propagation of the large edge $E_{z}$ field. These results are discussed below.

1. $\left|B_{z}\right|$ Increases - This is an expected result. Stix showed [2] that for a homogeneous lossless medium, the group velocity is

$$
v_{0}=\frac{\mathcal{P}+\boldsymbol{T}}{W_{0}}
$$

where $\mathcal{P}$ is the Poynting Flux, $\mathcal{T}$ the nonelectromagnetic energy flux due to the coherent motion of the charge carriers $\left(\mathcal{T}=0\right.$ for cold plasma), and $W_{0}$ the wave energy density.

When resonance is approached, the group velocity slows down, resulting in an increased energy density $W_{0}$. This increased energy density is apparent in the increase in $\left|B_{z}\right|$, since the energy density varies as

$$
W_{0}=\mu_{0} \frac{|B|^{2}}{2}+\epsilon_{0} \frac{|E|^{2}}{2}
$$

2. Increase in axial and radial $k_{z}$ - As resonance $\left(\omega=\omega_{c i}\right)$ is approached, the left hand cut-off $\left(k_{z}^{2}=k_{0}^{2} L\right)$ increases, causing an increase in the resulting $k_{z}$ eigenmodes. This is consistent with the slowing down of the group and phase velocities as resonance is approached.

3. High edge electric fields $E_{+}, E_{-}$and $E_{z}$ that propagate inward - This is a result that could be consistent with experimental observation. Machuzak [71] indicated that although a high edge electric field is predicted by the ANTENA code [72], experimentally no edge ICRF electric field downstream from the antenna is detectable. The $\mathrm{BEACH}$ simulation shows that the reason that no edge electric field is detectable is that the edge spike moves radially inward into the plasma as resonance is approached. 


\section{COLLISIONAL}

Although a cold plasma is the medium in which the theory is performed here, collisions may be added to the analysis to allow wave-plasma energy trasnfer and to better simulate a realistic medium.

The model used here takes the expansion of the plasma Z-function by excluding finite temperature effects and including terms up to order $O\left(\nu_{i} / \omega\right)$. The code results for the case of ICRF propagation in a cold collisional plasma is seen below in Figure 4-8. What the result shows is that in the presence of collisions, the wave amplitude initially rises as before, but that the wave amplitude also decays as resonance is approached. The decay in field amplitude is due to the damping of the waves from collisions. This decay differs markedly from the collisionless results which shows a continuos growth of the wave amplitude as resonance is approached. Likewise the results for the propagation of the ICRF electric field in the presence of collisions shown on Figure 4-9 also shows a decay as the wave propagates towards resonance.

The presence of collisions manifests itself in a complex dielectric tensor. Recall that the dielectric tensor is given by Equation 3.3

$$
\underline{\epsilon} \equiv\left[\begin{array}{ccc}
S & -i D & 0 \\
i D & S & 0 \\
0 & 0 & P
\end{array}\right]
$$

Where

$$
\begin{aligned}
& S=\frac{1}{2}(R+L) \\
& D=\frac{1}{2}(R-L)
\end{aligned}
$$

As seen in Appendix A, the presence of collisions in a cold plasma produces the following expressions for $S, D$, and $P$

$$
S \simeq 1-\sum_{\alpha} \frac{\omega_{p \alpha}^{2}}{\omega D_{D}}\left\{\begin{array}{c}
\omega\left(\omega^{2}-\omega_{c i}^{2}+\nu_{i}^{2}\right) \\
-i \nu_{i}\left(\omega^{2}+\omega_{c i}^{2}+\nu_{i}^{2}\right)
\end{array}\right\}
$$


$\mathrm{N}=0, \mathrm{NE}=1.00 \times 10^{18}, \mathrm{~W} / \mathrm{WCIO}=0.788$, COLD COLLISIONAL MODEL $\mathrm{TI}-\mathrm{PERP}=10.00, \mathrm{TE}-\mathrm{PERP}=10.00, \mathrm{TI}-\mathrm{Z}=10.00, \mathrm{TE}-\mathrm{Z}=10.00$ NO. MODES $=\mathbf{3 0}$

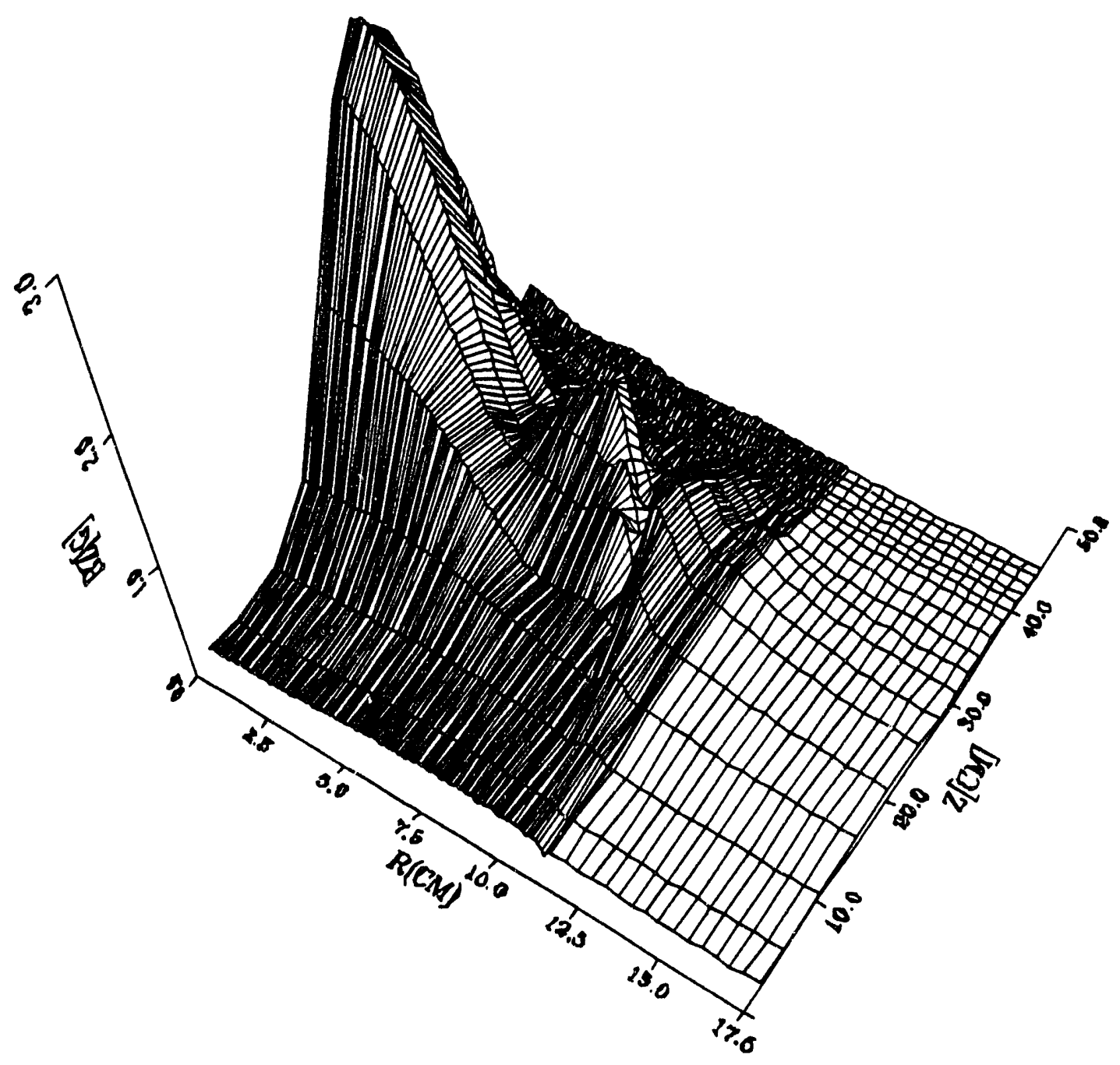

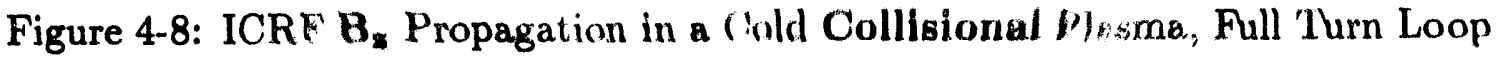
Antenna, $\omega / \omega_{i}=0.788$ 
$\mathrm{N}=0, \mathrm{NE}=1.00 \times 10^{16}, \mathrm{~W} / \mathrm{WCIO}=0.780$, COLD COLLISIONAL MODEL $\mathrm{Tl}$-PERP $=10.00, \mathrm{TE}-\mathrm{PERP}=10.00, \mathrm{TI}-\mathrm{Z}=10.00, \mathrm{TE}-\mathrm{Z}=10.00$ NO. MODES $=\mathbf{3 0}$

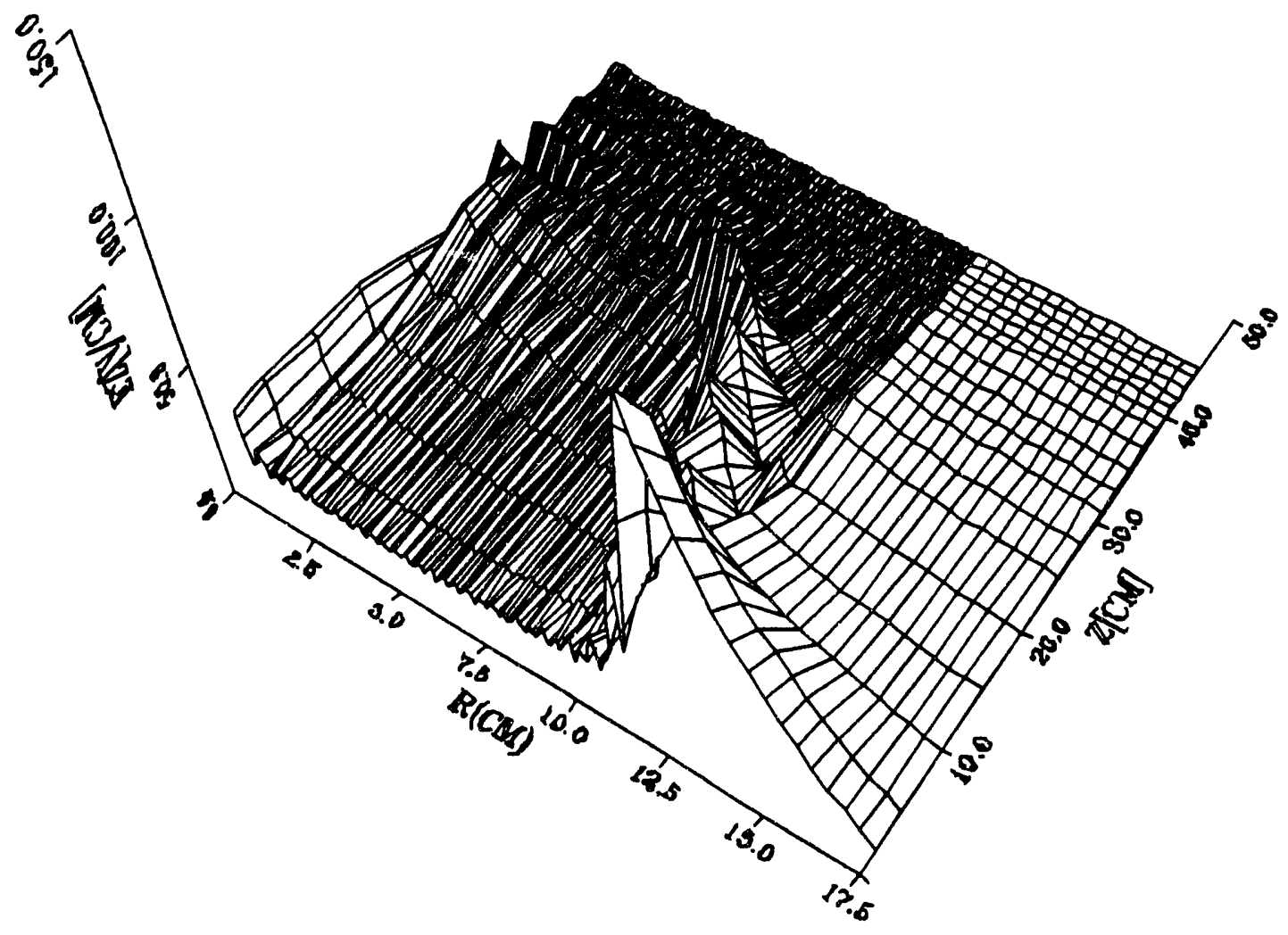

Figure 4-9: ICRF $\mathrm{E}_{\mathbf{z}}$ Propagation in a Cold Collisional Plasma, Full Turn Loop Antenna, $\omega / \omega_{\text {cio }}=0.788$ 


$$
\begin{gathered}
D \simeq \frac{\omega_{p i}^{2} \omega_{c i}}{\omega} \frac{\left(\omega^{2}-\omega_{c i}^{2}-\nu_{i}^{2}-2 i \omega \nu_{i}\right)}{\left(\omega^{2}-\omega_{c i}^{2}-\nu_{i}^{2}\right)^{2}+4 \omega^{2} \nu_{i}^{2}} \\
P \simeq 1-\sum_{\alpha} \frac{\omega_{p \alpha}^{2}}{\omega\left(\omega+i \nu_{\alpha}\right)}
\end{gathered}
$$

Using these complex elements of the dielectric tensor, the dispersion relation can be solved with the geometric boundary condition to yield a set of eigenmode solutions for this plasma-vacuum geometry. The difference between these solutions and those of the cold collisionless plasma is that the $k_{z}$ eigenmodes are now complex - these waves are now damped as they propagate towards the cyclotron resonance. This damping transfers energy from the waves to the plasma, causing plasma heating.

\section{COLLISIONLESS vs. COLLISIONAL}

The propagation characteristics of the collisional case resemble, for the most part, that of the cold collisionless model. However, some key differences are evident. These differences are given in Table 4.1 and are discussed below.

The differences in propagation characteristics between the collisional and collisionless case is evident upon comparing the propagating ICRF fields.

The collisional vs. collisionless results for the ICRF $\int \vec{B}_{z} \cdot d \vec{A}$ of a full turn antenna are compared in Figure 4-10. The factor $\int \overrightarrow{B_{z}} \cdot d \vec{A}$ is used here as an indication of the amount of damping that an ICRF wave encounters. An examination of Figure 4-10 shows that the damping mechanism introduced by the collisional process is able to remove the energy buildup as resonance is approached. Thus, for the collisional case, although there is initial growth in $\int \vec{B}_{z} \cdot d \vec{A}$, the damping that sets in as resonance is approached is able to greatly reduce the propagating field.

The ideal nondamping case shows a conservation of the axially propagating Poynting flux as well. This is to be expected since there is no damping mechanism 
to decrease this flowing power. However, the collisional case shows that this integrated Poynting flux does decrease over the length of an axial slice. (Recall that the integrated Poynting flux is conserved across the boundary between two adjacent slices) This again is due to the fact that collisions introduce an axial damping mechanism which removes energy from the propagating Poynting flux.

The results obtained agree with intuition. Collisionless propagation does not decrease the ICRF $B_{x}$ because there is no mechanism for dissipational damping. Collisional propagation, on the other hand, contains dissipation mechanisms to damp the energy buildup of the waves as they propagate towards resonance, hence the decrease in $\tilde{B}_{z}$. 

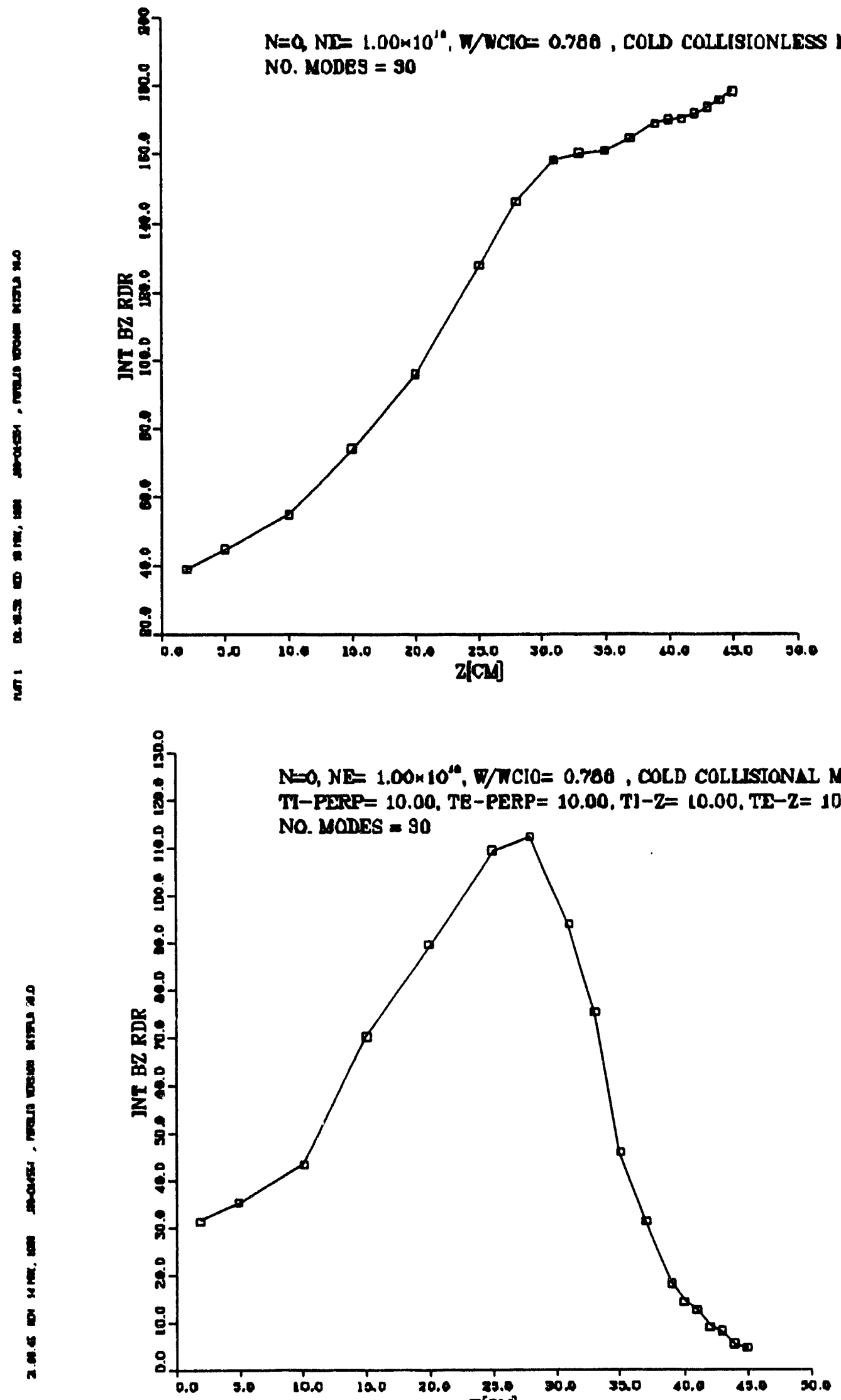

Figure vs. Collisional Plasma (Bottom) for a Full Turn Loop Antenna, $\omega / \omega_{\text {cio }}=0.788$ 


\subsubsection{Full Turn Loop vs. Dual Half Turn Loop}

The Full Turn Loop(FTL) and the Dual Half Turn Loop(DHTL) are the two antenna examined in this thesis whose geometries are shown in Figure 4-11.

The FTL is symmetric about $\theta$ and thus it does not have any azimuthal dependence $e^{i n \theta}$. Therefore, the FTL geometry is sometimes also referred to as the $n=0$ geometry, since the only mode that the FTL antenna excites is the $n=0$ mode.

The DHTL is asymmetric in $\theta$ and thus it has a finite azimuthal dependence $e^{i n \theta}$. The largest azimuthal mode that the DHTL excites is the $n=1$ mode, thus the DHTL geometry is also referred to as the $n=1$ geometry in this thesis.

The plasma characteristics used for the comparison of the Full Turn Loop and the Dual Half Turn Loop are identical : $n_{e}=2 \times 10^{12} \mathrm{~cm}^{-3}, 30$ modes, and an initial launch frequency of $\omega / \omega_{\text {cio }}=0.788$.

\section{FULL TURN LOOP, $n=0$ Mode}

Figures 4-12 and 4-13 show the propagation characteristic of the ICRF $B_{z}$ and $E_{z}$ fields as the waves propagate from the launch point $(z=0)$ towards the resonance point downstream. The main features of this propagation are that the waves again peak as resonance is approached.

\section{DUAL HALF TURN LOOP, $n=1$ Mode}

The solution of the dual half turn loop (DHTL) geometry poses a more difficult problem than the full turn loop (FTL) solution. The main difference between the DHTL and the FTL lies in the $\vec{J}\left(k_{z}\right)$ driving term, specifically the radial feeders. This is different from the Full Turn Loop (FTL) geometry in which the contribution from the radial feeders cancel out (Since one leg is entering and the other is exiting, net $J_{r}$ is zero). The modelling of the driving term $\vec{J}\left(k_{z}\right)$ for the DHTL is detailed in Appendix B. 

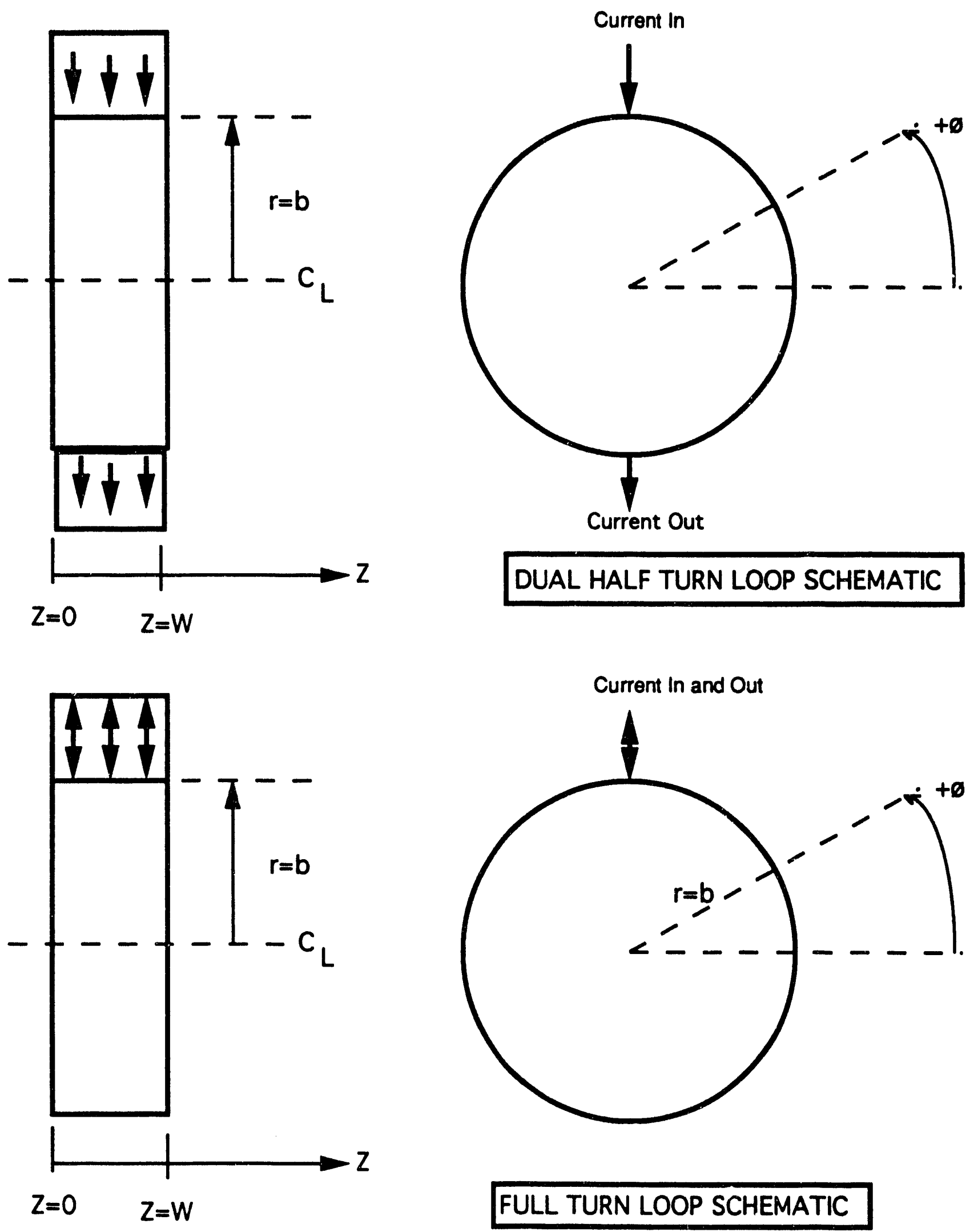

Figure 4-11: Full Turn Loop and Dual Half Turn Loop Schematic 
$\mathrm{N}=0, \mathrm{NE}=1.50 \times 10^{12}, \mathrm{~W} / \mathrm{WCLO}=0.788$, COLD COLLISIONLESS MODEL NO. MODES $=\mathbf{3 0}$

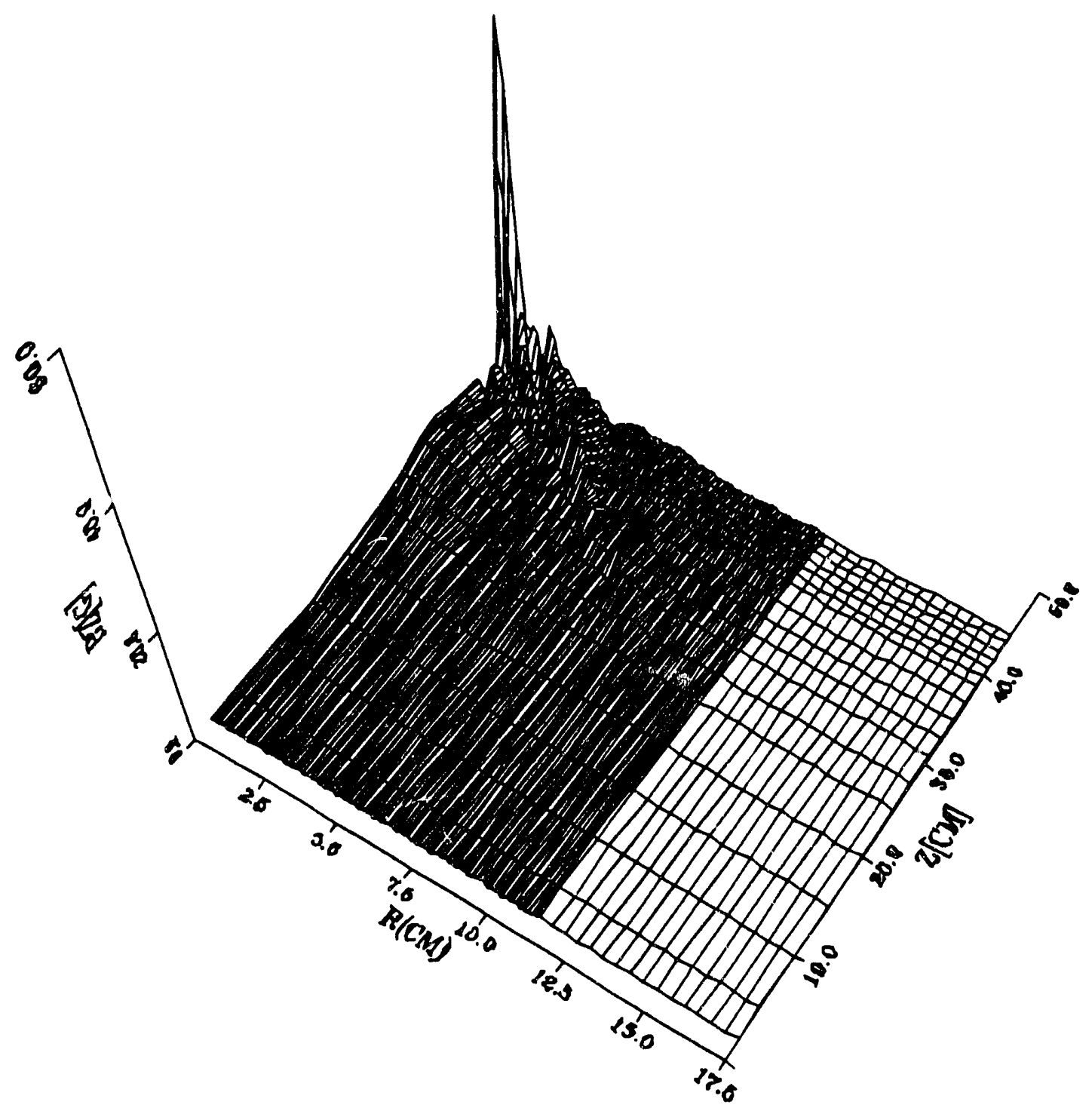

Figure 4-12: ICRF $\mathbf{B}_{\mathbf{z}}$ Propagation in a Cold Collisionless Plasma, Full Turn Loop Antenna, $\omega / \omega c i 0=0.788$. Waves are launched by the ICRF antenna at $z=0 \mathrm{~cm}$ and propagate towards resonaht? at $z=50 \mathrm{~cm}$. 
$\mathrm{N}=0, \mathrm{NE}=1.50 * 10^{12}, \mathrm{~W} / \mathrm{WCIO}=0.788, \mathrm{COLD}$ COLLISIONILSS MODEL NO. MODES $=\mathbf{3 0}$

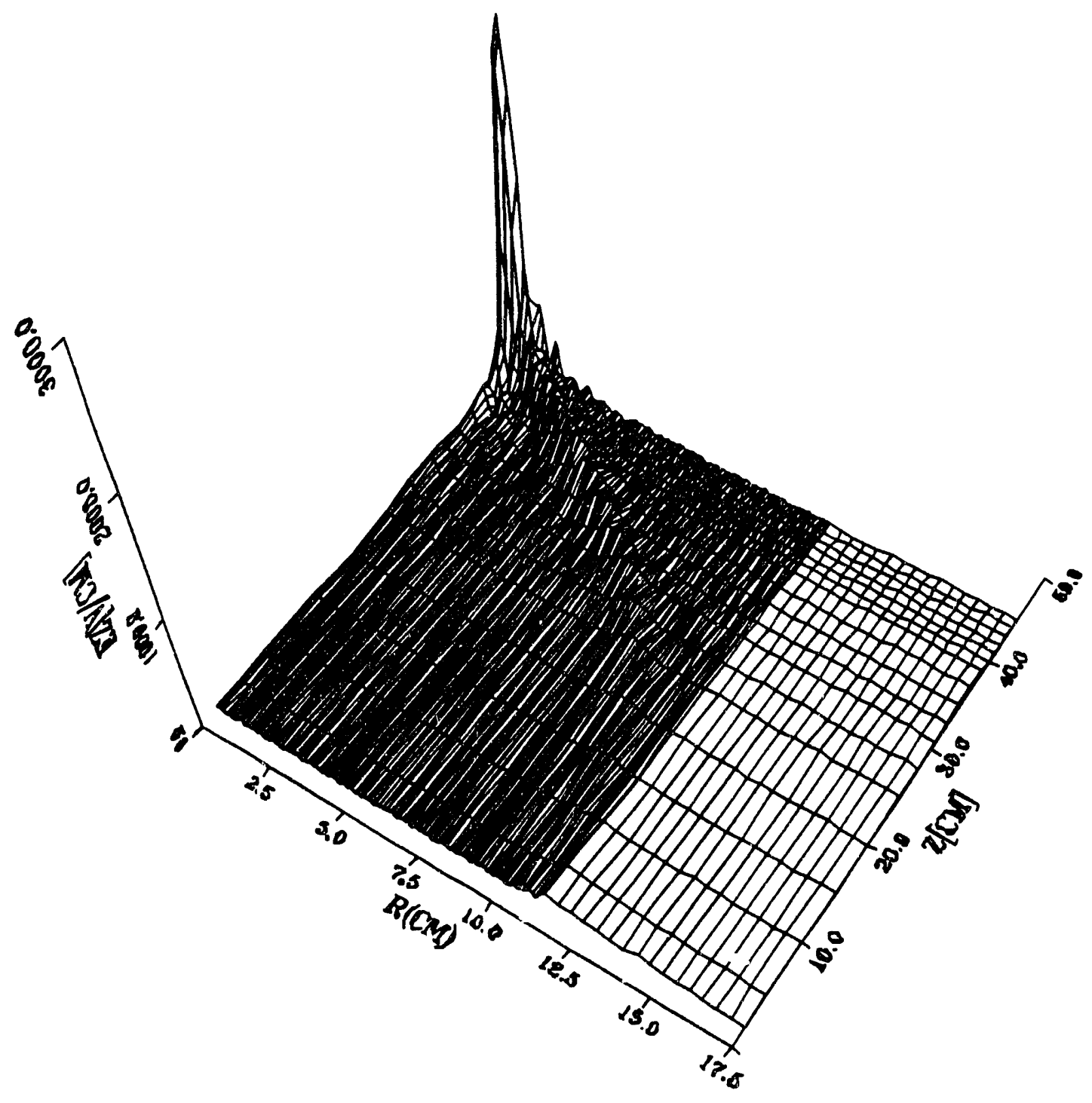

Figure 4-13: ICRF $\mathbf{E}_{\mathbf{z}}$ Propagation in a Cold Collisionless Plasma, Full Turn Loop Antenna, $\omega / \omega c i 0=0.788$ 
The computational simulation of the $n=1$ mode ICRF wave propagation is shown on Figure 4-14. From Figure 4-14, the basic characteristics of ICRF propagation towards resonance are similar to the FTL $(\mathrm{n}=0)$ case, i.e. increasing $k_{z}$ and $k_{\perp}$, and increasing $B_{z}$ amplitude. The main difference is the larger $k_{\perp}$ and $k_{\mathrm{z}}$ for the DHTL case, which is to be expected since the asymmetric (about $\mathrm{r}=0$ ) DHTL excitation necessitates radial eigenmodes that have smaller perpendicular wavelengths (larger $k_{\perp}$ ) and hence larger $k_{z}$.

The peaking of the edge electric fields can be seen on Figure 4-15. The $E_{z}$ field appears to be similar in structure to the FTL case, with an initial edge peaked $E_{z}$ that propagates towards the center of the plasma as resonance is approached. As mentioned before, the CYLWAVE analysis performed in the previous chapter differs from BEACH in that CYLWAVE waves do not change $k_{\xi}$ and therefore do not change their phase velocity. This constant phase velocity means that there is no buildup of wave energy due to the slowing down of phase velocity. Hence the key difference between the BEACH and the CYLWAVE results is that BEACH shows initial peaking and subsequent decay of the ICRF wave amplitude, while CYLWAVE results contain only the decaying portion. 


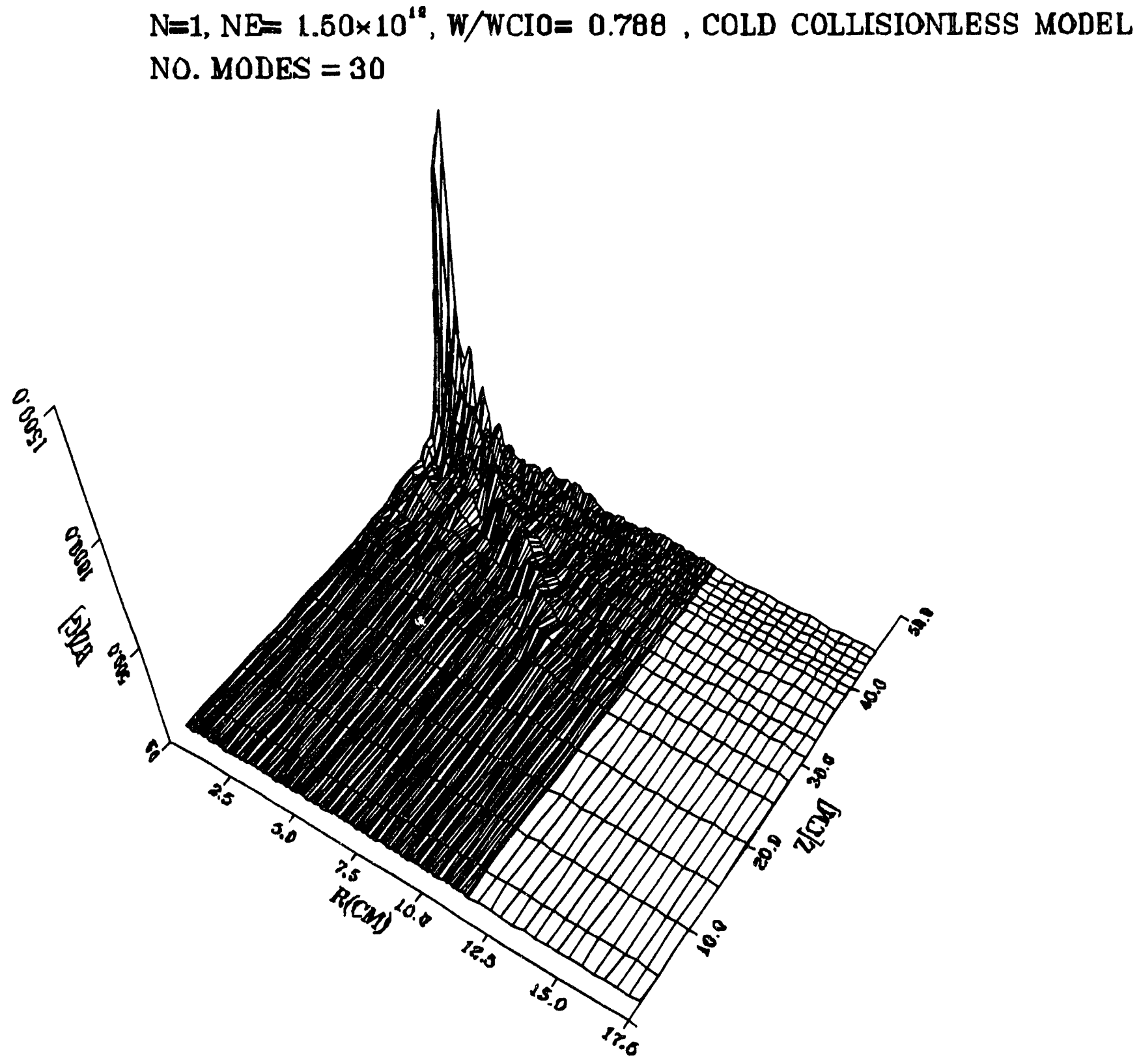

Figure 4-14: ICRF $\mathbf{B}_{\mathbf{z}}$ Propagation in a Cold Collisionless Plasma, Dual Half Turn Loop Antenna, $\omega / \omega c i 0=0.788$ 
$\mathrm{N}=1, \mathrm{NE}=1.50 * 10^{12}, \mathrm{~W} / \mathrm{WCIO}=0.788$, COLD COLLISIONLESS MODEL NO. MODES $=30$

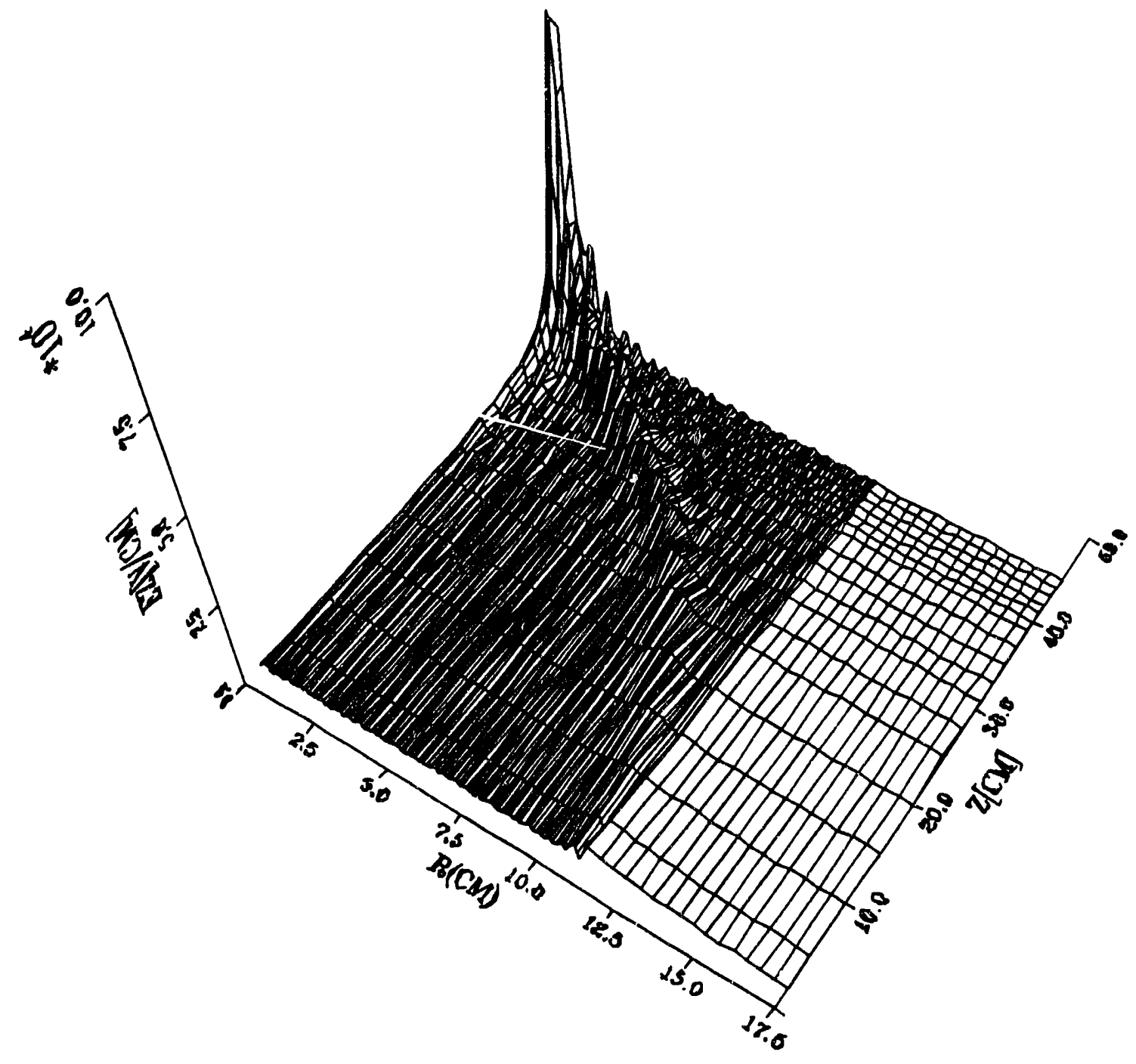

Figure 4-15: ICRF Ez Propagation in a Cold Collisionless Plasma,Dual Half Turn Loop Antenna, $\omega / \omega c i 0=0.788$ 


\section{SIMILARITIES \& DIFFERENCES}

As mentioned before, there are some basic characteristics to ICRF propagation towards resonance that show up in both the $n=0$ and $n=1$ cases. The characteristics that are inherent in both the $n=0$ and $n=1$ cases are basically those of the collisionless model listed in the Section 4.4.2- $\left|B_{z}\right|$ increase towards resonance, and $\left|E_{z}\right|$ peak moves inward. The key difference between the $\mathrm{n}=1$ and $\mathrm{n}=0$ modes is, as mentioned before, the higher $k_{z}$ 's and $k_{\perp}$ 's that are generated by the $n=1$ mode antenna. This finding again agrees with physical insight which dictates that the asymmetric boundary conditions imposed by the $n=1$ antenna would result in higher mode numbers and thus a larger number of field oscillations in the plasma. An examination of the radial field profiles readily shows more radial oscillations for the $\mathrm{n}=1$ mode. The radial field profile for the $n=0$ mode is computed and shown on Figure 4-16. Contrasted with the $n=1$ mode on Figure 4-17, the increased oscillations for the $n=1$ mode is clearly evident. 

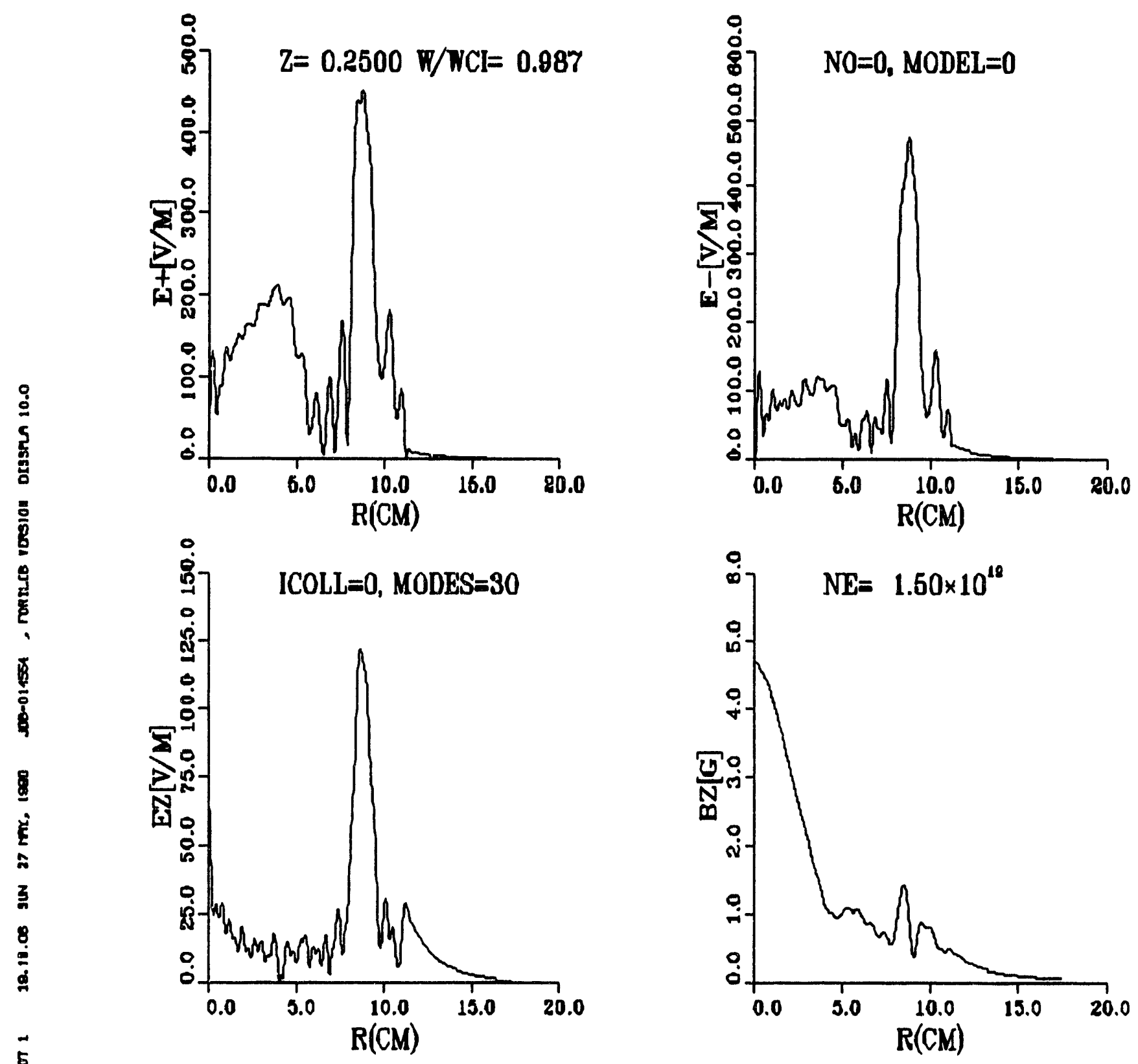

Figure 4-16: Radial Field profiles for $E_{+}, E_{-}, E_{z}, B_{z}$, Cold Collisionless Plasma, Full Turn Loop Antenna, $\omega / \omega c i 0=0.788$ 

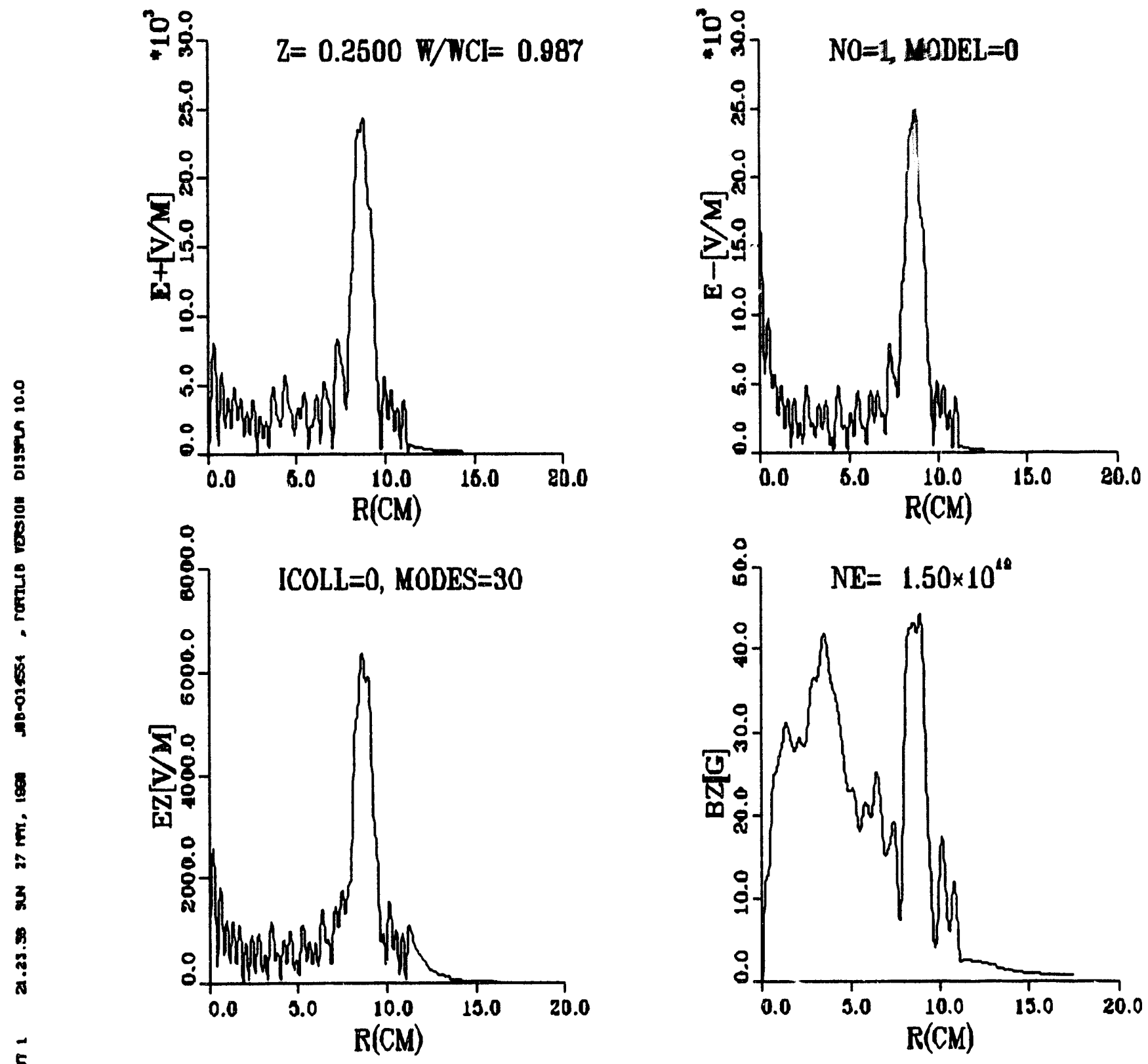

Figure 4-17: Radial Field profiles for $E_{+}, E_{-}, E_{z}, B_{z}$, Cold Collisionless Plasma, Dual Half Turn Loop Antenna, $\omega /$ wci0 $=0.788$ 
Table 4.1: Cold Collisionless vs. Cold Collisional Plasma as $\omega \rightarrow \omega_{c i}$

\begin{tabular}{||l|l|l||}
\hline COLLISIONLESS & COLLISIONAL & COMMENTS \\
\hline $\int S_{\mathbf{z}} r d r$ remains constant & $\int S_{\mathbf{z}} r d r$ decreases & $\begin{array}{l}\text { Damping due to collisions } \\
\text { decreases the propagating } \\
\text { Poynting Flux }\end{array}$ \\
\hline $\begin{array}{l}B_{\mathbf{z}} \text { increases } \\
\text { towards resunance }\end{array}$ & $\begin{array}{l}B_{\mathbf{z}} \text { increases initially } \\
\text { but then decreases } \\
\text { towards resonance }\end{array}$ & $\begin{array}{l}\text { Collisional Damping more } \\
\text { than compensates for } \\
\text { increasing stored field } \\
\text { energy }\end{array}$ \\
\hline $\begin{array}{l}\text { Edge peaking of } \\
E_{z}, E_{+}, E_{-} . \\
\text {moves inward towards } \\
\text { center of plasma }\end{array}$ & $\begin{array}{l}\text { Initial edge peaking of } \\
E_{z}, E_{+}, E_{-} \text {near launch } \\
\text { point moves radially inward } \\
\text { as } \omega \rightarrow \omega_{c i} \text { much faster } \\
\text { than collisionless case }\end{array}$ & $\begin{array}{l}\text { Agrees with experiments } \\
\text { which show no large } \\
\text { ICRF E-field at edge } \\
\text { downstream from antenna }\end{array}$ \\
\hline
\end{tabular}




\subsubsection{Effects of Density on Propagation}

Changes in plasma density affects the propagation characteristics both by changing the boundary conditions as well as altering the eigenmodes that exist in the plasma-vacuum system. Different densities were used in different runs to examine the effect of density on propagation.

One way to examine the effect of density on propagation is to look at the element $S$ of the dielectric tensor $\underline{\underline{\epsilon}}$. From Appendix A, it was shown that

$$
S=1+\sum_{\alpha} \frac{\omega_{p \alpha}^{2}}{\omega D_{D}}\left\{\omega\left(\omega^{2}-\omega_{c i}^{2}+\nu_{i}^{2}\right)-i \nu_{i}\left(\omega^{2}+\omega_{c i}^{2}+\nu_{i}^{2}\right)\right\}
$$

where

$$
D_{D} \equiv\left(\omega^{2}-\omega_{c i}^{2}-\nu_{i}^{2}\right)^{2}+4 \omega^{2} \nu_{i}^{2}
$$

Since $S \propto \omega_{p \alpha}^{2}, S$ is proportional to the plasma density $n_{e}$. (assuming $n_{i}=n_{c}$ ) The dispersion relation shown on Figure 3-1, $k_{\perp}\left(k_{z}\right)$, shows the point $k_{z}^{2}=k_{0}^{2} S$. A preliminary eigenmode $k_{z}$ scan showed that no waves exist at $k_{z}^{2}<k_{0}^{2} S$ for the geometries examined (PPEX, B-66). Thus, within the context of the geometries examined, the point $k_{0}^{2} S$ represents the minimal point below which the waves cannot propagate. As the density $n_{e}$ increased, the location of the point $k_{0}^{2} S$ begins to move up on the $k_{z}^{2}$ axis, pushing up the lowest possible $k_{z}$ eigenmode. Thus, an increase in density would precipitate higher $k_{z}$ eigenmodes and thus more radial and axial oscillations in the field amplitude.

Two different density simulations are presented on Figure 4-18. The upper graph shows the propagation of the integrated $B_{z}$ for a plasma of density $1 \times$ $10^{12} \mathrm{~cm}^{-3}$ while the lower graph shows propagation at $5 \times 10^{12} \mathrm{~cm}^{-3}$. The main difference to note is the difference in the damping of the wave amplitude. The $5 \times 10^{12} \mathrm{~cm}^{-3}$ plasma peaks earlier than the $1 \times 10^{12} \mathrm{~cm}^{-3}$ plasma, but then damps out much faster as well.

The damping aspect of higher density can be observed by examining the $k_{z}$ roots for the first and last axial slices. Figure 4-19 shows a comparison of the 
$n_{e}=1 \times 10^{12}$ density case versus the $n_{e}=5 \times 10^{12}$ case. The upper graphs show the complex $k_{z}$ roots for the $n_{e}=10^{12}$ density case. The upper left graph is the complex $k_{z}$ roots found for the first axial slice, $\omega / \omega_{c i}=0.788$. The upper right graph is the complex $k_{z}$ roots found for the last axial slice, $\omega / \omega_{c i}=0.997$. As can be observed, the imaginary part of $k_{z}$ becomes much larger as resonance is approached. The lower two graphs again show the complex $k_{z}$ roots for the $n_{e}=5 \times 10^{12}$ case. Comparing the results for the first and last slices with that of the $n_{e}=10^{12}$ case shows that the imaginary $k_{z}$ is much larger for the higher density case, thus resulting in more damping of the fields and greater energy transfer to the plasma.

The issue of higher density raises an interesting question. Since higher density results in larger $k_{0}^{2} S$ and therefore larger $k_{z}$ roots, the modal excitation coefficients should, in principle, be excited to a lower level because the $k_{z}$ spectrum of the launching aitenna ( $\mathrm{n}=0$, Full Turn Loop) favors lower $k_{z}$. So shouldn't a higher density result in less efficient coupling to the plasma instead of better coupling as claimed before? The answer is no. The $n=0$ antenna spectrum on Figure 4-5 shows the first minimum in $J_{\theta}\left(k_{z}\right)$ at approximately $k_{z}=80 \mathrm{~m}^{-1}$. As seen on Figure 4-19, the initial $k_{z, R e}$ roots for the $n_{e}=10^{12} \mathrm{~cm}^{-3}$ (Upper Left) and $n_{e}=5 \times 10^{12} \mathrm{~cm}^{-3}$ (Lower Left) plasma cases show that even though the density increased fivefold, the minimum $k_{z}$ root only increased by a factor of about 2 , which is to be expected since $k_{z}=k_{0} \sqrt{S} \propto \sqrt{n_{e}}$. However, although the minimum $k_{z}$ root has increased by a factor of 2 , the remaining roots do not grow likewise. So what is seen is that the entire $k_{z}$ eigenmode spectrum for the $n_{e}=5 \times 10^{12} \mathrm{~cm}^{-3}$ case is upshifted in $k_{z}$ by $k_{z, \min }$ (where $k_{z, \min }$ is the $n_{e}=10^{12} \mathrm{~cm}^{-3}$ case's minimum $k_{z}$ root) over the $n_{e}=10^{12} \mathrm{~cm}^{-3}$ eigenmode spectrum. Figure 4-19 shows that although the $n_{c}=5 \times 10^{12} \mathrm{~cm}^{-3}$ eigenmode spectrum is upshifted by this amount, the 30 th $k_{z}$ mode found is approximately $35 \mathrm{~m}^{-1}$, which is still well less than the first minimum of the $J_{\theta}\left(k_{z}\right)$ spectrum located at $k_{z}=80 m^{-1}$. Thus, the upshift in the $k_{z}$ spectrum due to higher density is seen to not have much of an effect on 
the modal excitation coefficient.

Another interesting effect that is observable is the peaking location of the ICRF $B_{x}$ field. Figure 4-18 shows that the peaking location for the $n_{e}=5 \times 10^{12} \mathrm{~cm}^{-3}$ plasma is located axially earlier than that of the $n_{e}=10^{12} \mathrm{~cm}^{-9}$ plasma. This difference could be explained in terms of the difference in the collision frequency due to density. The higher density case would have a higher collision frequency since $\nu \propto n_{e}$. Because the collision term often appears as part of the factor $\left(\omega-\omega_{c i}+i \nu_{i}\right)$, it is seen that the damping effect of collisions is not really felt until $\left|\omega-\omega_{c i}\right| \sim \nu_{i}$. Since a higher density plasma. dictates a higher collision frequency, this means that $\omega_{c i}(z)$ can be farther away from $\omega$ before collisional effects are felt. Thus, it is seen that a HIGHER density plasma results in collisional damping effects that begin to set in EARLIER on in the propagation than a lower density plasma, hence an earlier peaking of the ICRF $B_{z}$ field for higher density is expected. 

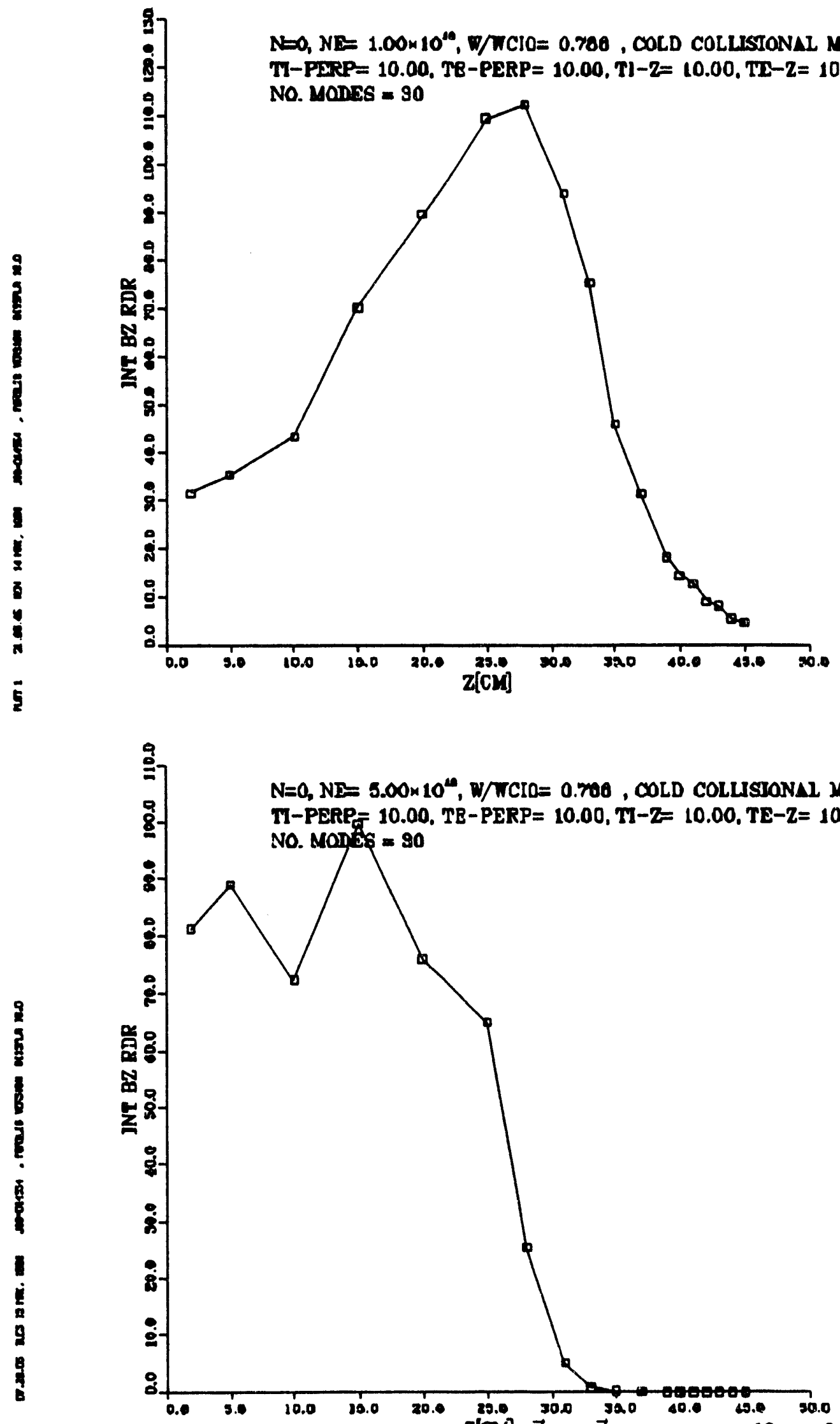

Figise 4-18: BEACH code computed ICrith $\overrightarrow{B_{z}} \cdot d \vec{A}$ for a $1 \times 10^{12} \mathrm{~cm}^{-3}$ plasma (Top) and a $5 \times 10^{12} \mathrm{~cm}^{-3}$ plasma (Bottom). Full Turn Loop Antenna, $\omega / \omega_{\text {cio }}=$ 0.788 

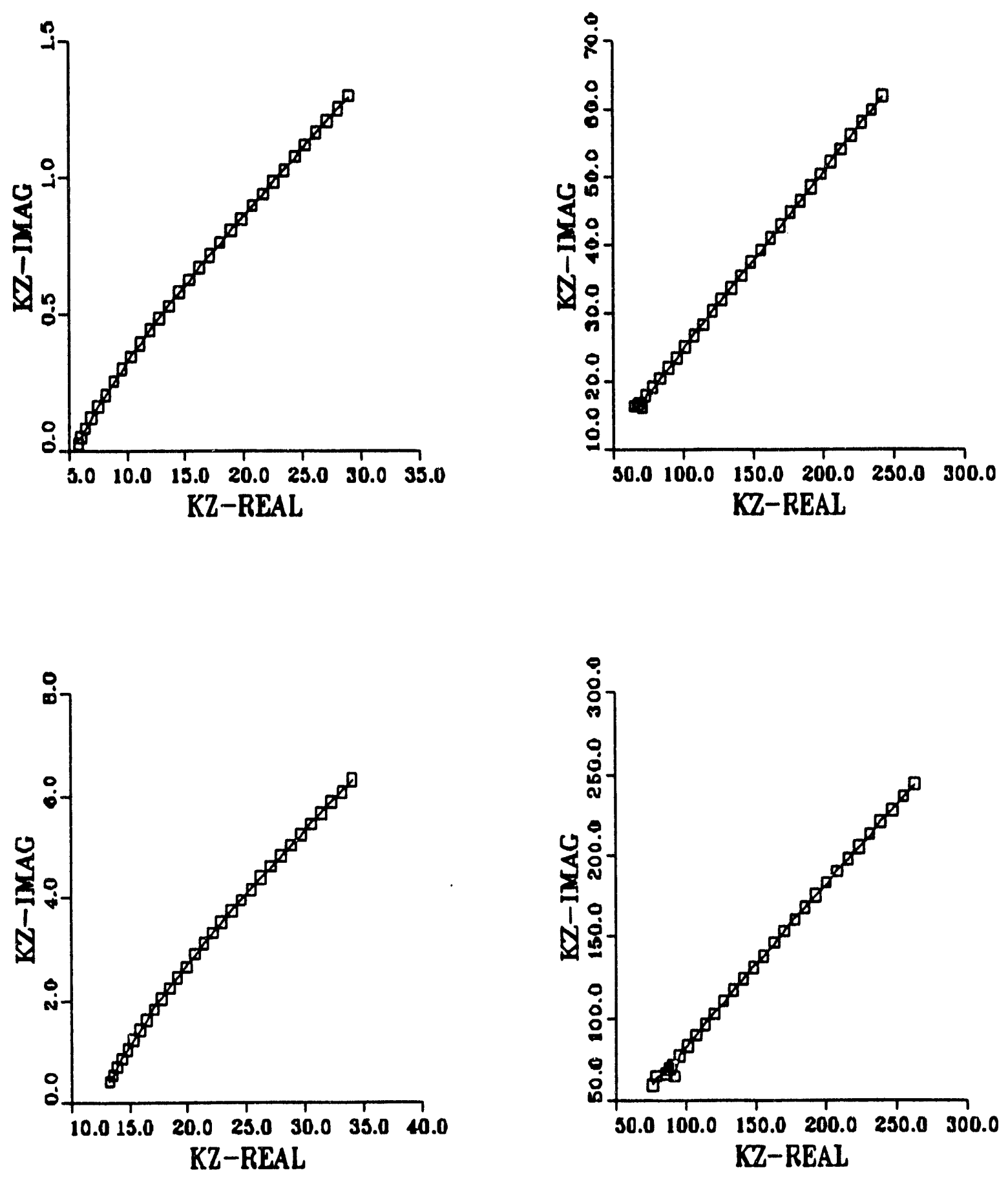

Figure 4-19: BEACH code computed $k_{z}$ roots for a $1 \times 10^{12} \mathrm{~cm}^{-3}$ plasma $-\omega / \omega_{c i}=$ 0.788 (Upper Left), $\omega / \omega_{c i}=0.997$ (Upper Right) and a $5 \times 10^{12} \mathrm{~cm}^{-3}$ plasma $-\omega / \omega_{c i}=0.788$ (Lower Left), $\omega / \omega_{c i}=0.997$ (Lower Right). Full Turn Loop Antenna 


\subsubsection{Effects of Temperature on Propagation}

The examination of the effect of different plasma temperatures on propagation is again limited to a low temperature treatment, i.e. $\omega>k_{x} v_{a}$, where $v_{a}$ is given by [3]

$$
v_{a}=\left(\frac{k T_{\|}}{m_{i}}\right)^{1 / 2}
$$

This means that the propagation analysis is valid for low temperatures $(<$ $100 \mathrm{eV}$ ), but will not be valid at higher temperatures (1keV and up).

Again, intuition would indicate that since the collisional frequency $\nu \propto T^{-3 / 2}$, the dissipation due to collisions would DECREASE as temperature is increased. Therefore, less damping of the wave fields would be expected as the temperature is raised. A comparison of results generated by BEACH for two different temperature plasmas appears to agree with the above analysis. Figure 4-20 shows that the lower temperature (10ev) plasma appears to be more effective at damping the wave amplitude, while the higher temperature $(50 \mathrm{eV})$ plasma appears to be less effective at removing the buildup of energy as resonance is approached.

The damping aspect of different temperature plasmas can be observed, as for the densities, by examining the $k_{z}$ roots for the first and last axial slices. Figure 4-21 shows a comparison of che $10 \mathrm{eV}$ case versus a $50 \mathrm{eV}$ case. The top two graphs show the complex $k_{z}$ roots for the $10 \mathrm{eV}$ case. The upper left graph is the complex $k_{z}$ roots found for the first axial slice, $\omega / \omega_{c i}=0.788$. The upper right graph is the complex $k_{z}$ roots found for the last axial slice, $\omega / \omega_{c i}=0.997$. Again, the imaginary part of $k_{z}$ becomes much larger as resonance is approached. The lower two graphs show the complex $k_{z}$ roots for first and last slices of the $50 \mathrm{eV}$ plasma case. Comparing the results for the first and last slices with that of the $10 \mathrm{eV}$ case shows that the imaginary $k_{z}$ is much larger for the lower temperature case, thus resulting in more damping of the fields and greater energy transfer to the plasma.

There is one major difference between varying density and varying temperature - changing the temperature of the plasma does not affect the REAL $k_{z}$ solution. 
This is obvious because temperature doesn't affect the minimum limit $k_{z}=k_{0} \sqrt{S}$ since $S$ is not a function of temperature. However, as seen on Figure 4-21, varying temperature does affect the imaginary $k_{z}$, which is again expected since collisional dissipation $\nu_{i}$ is dependent upon temperature.

The peaking location of the ICRF $\int B_{z} r d r$ for different temperature plasmas behaves as expected. Figure 4-20 shows that the peaking location for the $10 \mathrm{eV}$ plasma is located axially earlier than that of the 50ev plasma. Again, this difference could be explained in terms of the difference in the collision frequency due to temperature. The higher temperature case would have a lower collision frequency since $\nu \propto T^{-3 / 2}$. Because the collision term often appears as part of the factor $\left(\omega-\omega_{c i}+i \nu_{i}\right)$, the damping effect of collisions is not really felt until $\left|\omega-\omega_{c i}\right| \sim \nu_{i}$. Since a higher temperature plasma dictates a lower collision frequency, the $\omega_{c i}(z)$ must be closer to $\omega$ before collisional effects are felt. Thus, it is seen that a higher temperature plasma results in collisional damping effects that begin to set in LATER on in the propagation than a lower temperature plasma, hence a later peaking of the ICRF $B_{z}$ field for higher temperature is expected. 

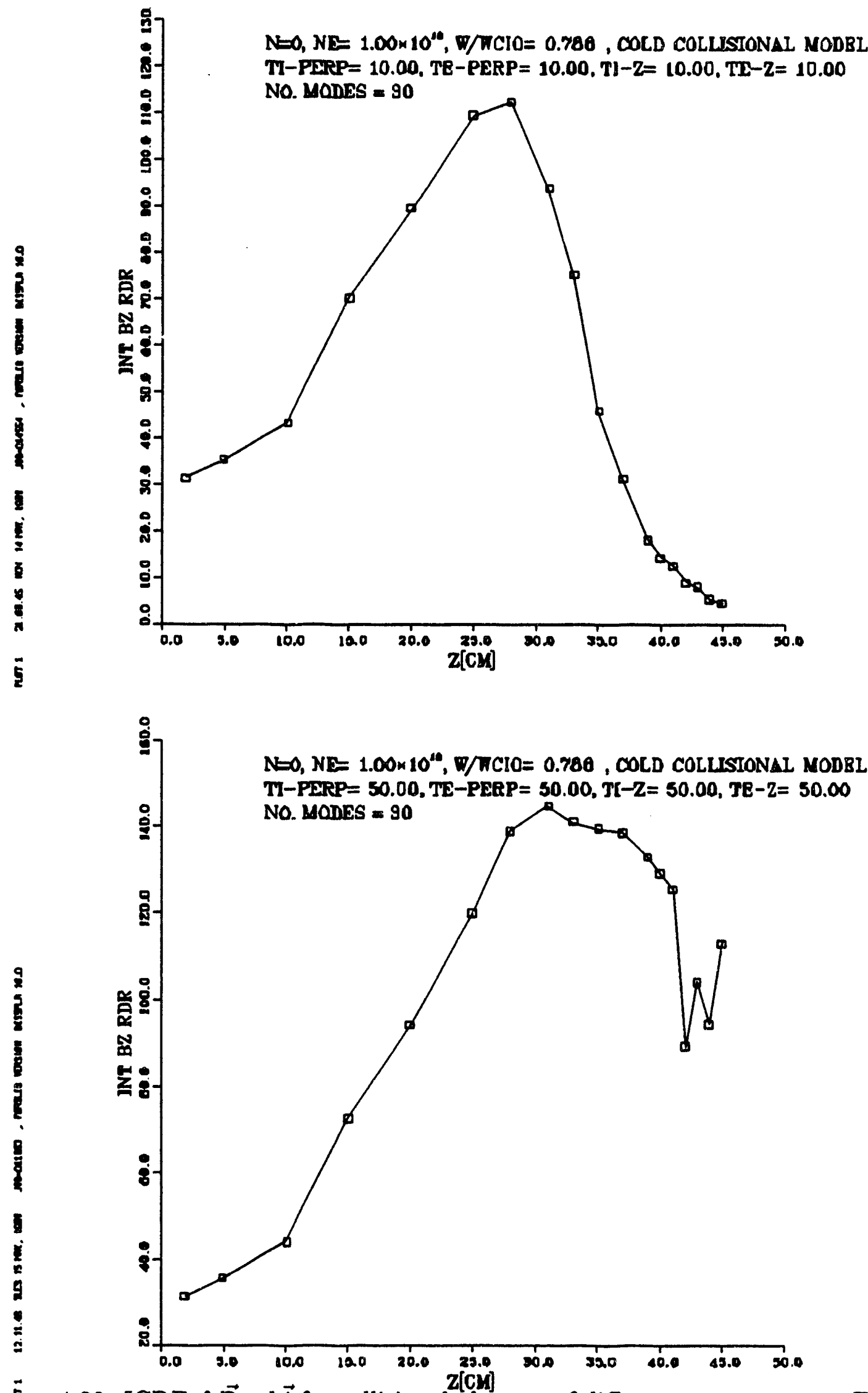

Fighre 4-20: ICRF $\int \overrightarrow{B_{z}} \cdot d \vec{A}$ for collisional plasmas of different temperatures: Top view $-10 \mathrm{eV}$ plasma, Bottom view $-50 \mathrm{eV}$ plasma. $\omega / \omega_{\text {cio }}=0.788$ 

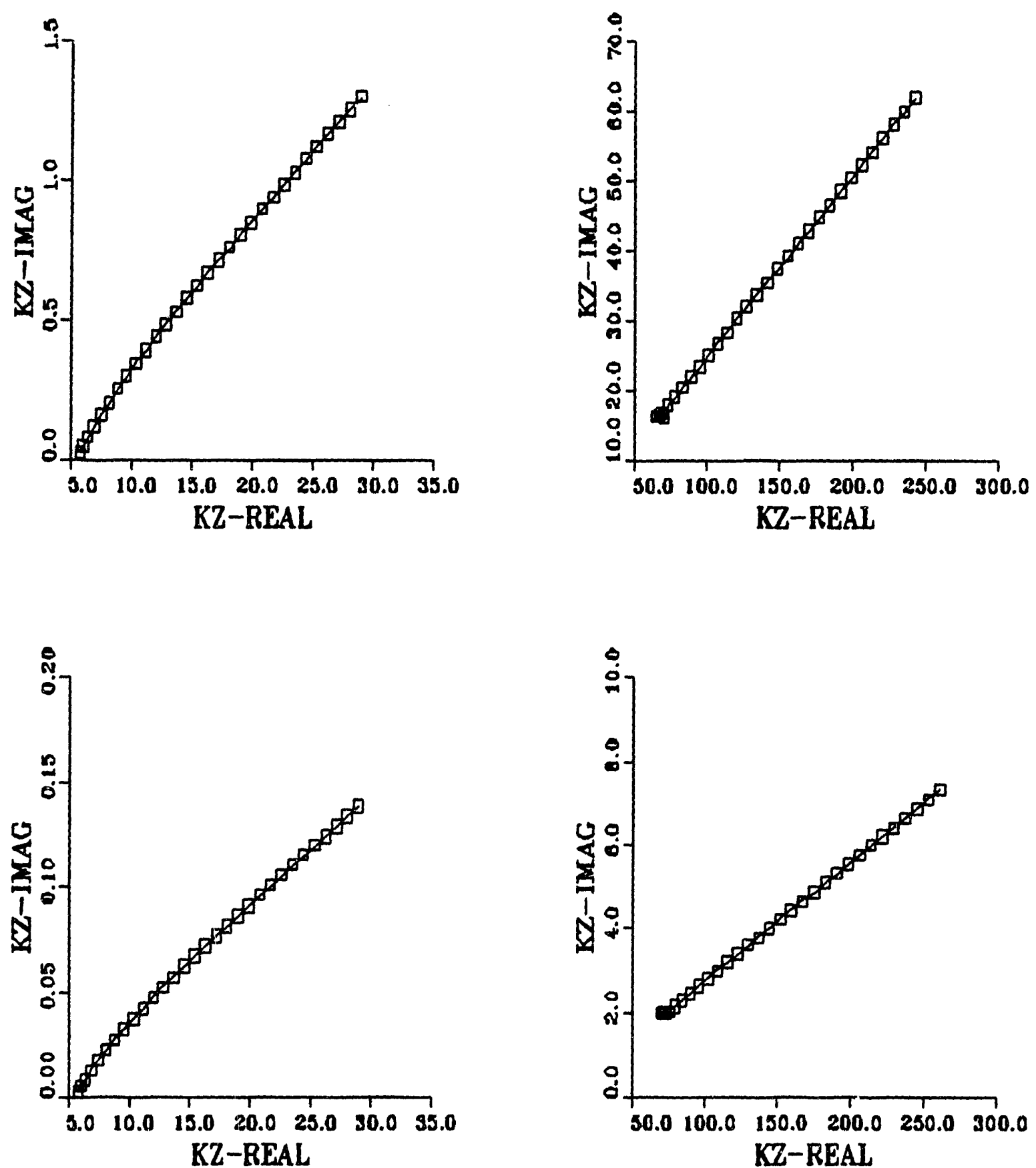

Figure 4-21: BEACH code computed $k_{z}$ roots for a $10 \mathrm{eV}$ plasma $-\omega / \omega_{c i}=0.788$ (Upper Left), $\omega / \omega_{c i}=0.997$ (Upper Right) and a $50 \mathrm{eV}$ plasma $-\omega / \omega_{c i}=0.788$ (Lower Left), $\omega / \omega_{c i}=0.997$ (Lower Right). Full Turn Loop Antenna, $n_{e}=$ $1 \times 10^{12} \mathrm{~cm}^{-3}$ 


\subsubsection{Effects of $\omega / \omega_{c i}$ on Propagation}

The difference in $\omega / \omega_{\mathcal{c}}$ at the antenna location affects the resulting propagation and heating characteristics of the ICRF wave. Simulations for different $\omega / \omega_{\text {cio }}$ are shown on Figure 4-22.

There appears to be an optimal $\omega / \omega_{c i}$ for launching ICRF waves. BEACH simulation has been run for one magnetic geometry but three different launch locations (and therefore different $\omega / \omega_{c i}$ ). Note that because the magnetic geometry of the three cases are identical, the imaginary $k_{z}$ damping mode numbers are also identical. Thus, a $k_{z}$ comparison is inappropriate for determining the optimal $\omega / \omega_{c i}$ for launching ICRF waves. Two other characteristics are therefore examined $-\int B_{z} r d r$ of the ICRF $B_{z}$ field, and the modal excitation coefficient of each of the eigenmodes. By comparing the $\int B_{z} r d r$, one can see which of the $\omega / \omega_{c i}$ cases generates the largest energy buildup and decay. The modal excitation coefficient directly shows how each mode is excited by the $J_{\theta}\left(k_{z}\right)$ driving term.

The $\int B_{z} r d r$ results are shown in Figure 4-22. As can be seen, it appears that $\int B_{z} r d r$ is largest for the $\omega / \omega_{c i}=0.788$ case and steadily decreases for large $\omega / \omega_{c i}$

The reason that lower launching values of $\omega / \omega_{c i}$ fare better than larger $\omega / \omega_{c i}$ is not difficult to understand. Examination of the driving source term $J_{\theta}\left(k_{z}\right)$ (Figure 4-5) clearly shows that the Full Turn Loop antenna favors lower $k_{z}$ modes. For the specific antenna geometry in question, if $k_{z}>80 m^{-1}$, it is seen that less drive is available to the eigenmodes. Since lower initial $\omega / \omega_{c i}$ results in lower initial $k_{z}$ (Since $k_{0}^{2} S$ is less, the lowest $k_{z}$ roots will be less), these lower $k_{z}$ 's couple better to the FTL antenna spectrum and therefore result in larger modal excitation coefficients. On the other hand, the larger initial $\omega / \omega_{c i}$ results in larger initial $k_{z}$ 's which don't couple as well to the antenna spectrum. Therefore, it is seen that lower $\omega / \omega_{c i}$ allows better coupling to the power spectrum of the $n=0$ Full Turn Loop antenna. 

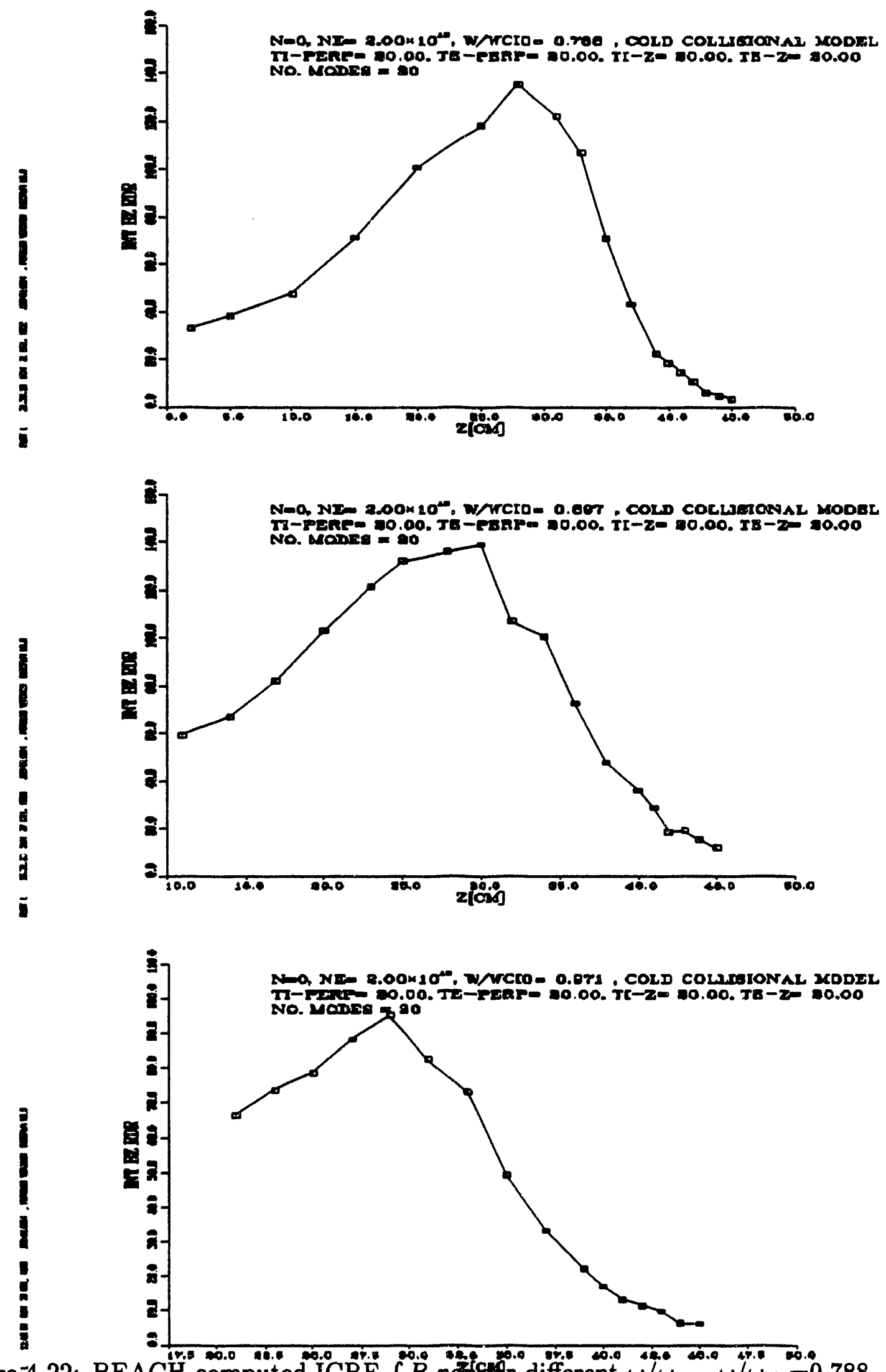

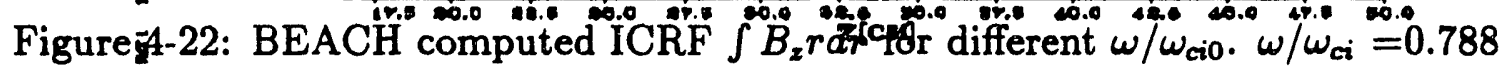
(Top), $\omega / \omega_{c i}=0.897$ (Middle), and $\omega / \omega_{c i}=0.971$ (Bottom). Full Turn Loop Antenna 


\subsubsection{Comparison of BEACH Results to Other Experi- mental Results - B-66}

The BEACH code results qualitatively reproduce many of the experimental results obtained on other machines. Two machines were modelled - the B-66 machine and the results obtained on it by Uman[3], and the MIT-PPEX machine as described in Chapter 2.

The two experiments were selected for the main purpose of verifying the BEACH code. The B-66 machine provides good AXIAL measurements of the ICRF $B_{2}$ field, while the PPEX experiment provided RADIAL measurements of the ICRF $B_{z}$. Thus these two experiments combined give a good set of empirical data against which BEACH could be benchmarked. The comparison of BEACH to the experimental results of the PPEX machine are presented in the radial $B_{z}$ results section in Chapter 5. This section will concentrate on the B-66 machine.

\section{B-66}

The B-66 machine [3] was a linear magnetic mirror device that was one of the first to examine the magnetic beach heating phenomenon. The plasma is generated inside a glass vacuum tube by microwave breakdown. The surrounding magnetic field coils generate a maximum ficld of 2.6 Tesla with a minimum center cell field of about 1 Tesla. ICRF power (16-MHz, 1-MW) is fed to the plasma via a multiturn induction coil. ICRF $B_{z}$ is detected using a wave probe that consists of "one turn of solid-jacketed, $50-\Omega$ coaxial cable with a symmetrically placed $\frac{1}{4}$-in. break in the jacket"[3] which is embedded in ceramic. The loop thus completely surrounds the plasma and is capable of picking up only $n=0$ ICRF oscillations.

The B-66 device was selected as a benchmark for BEACH both for its relatively simple geornetry as well as for its easily modelled diagnostic data, $\int B_{z} r d r$. Since there is currently no axial measurement of the ICRF $b_{z}$ on the PPEX experiment, the B-66 is selected to provide experimental axial field profiles for comparison to 
BEACH results.

The basic schematic of the B-66 machine is shown in Figure 4-23. ICRF waves are launched by an antenna located at $z=0 \mathrm{~cm}$ and propagate towards $\omega=\omega_{c i}$ at $z=61.2 \mathrm{~cm}$.

As mentioned before, the B-66 experimental B-dot probe data was obtained using a loop that completely surrounded the plasma. Thus the signals detected were the $\mathrm{n}=0$ mode and were also integrated over the entire cross-sectional area of the plasma, $\int \overrightarrow{B_{z}} \cdot d \vec{A}$.

The $B_{z 0}(z)$ magnetic field geometry of the B-66 machine that is modelled by BEACH is plotted on Figure 4-24. 


\section{BEACH vs. B-66}

Using the magnetic geometry of the B-66 machine, the ICRF $\int \overrightarrow{B_{z}} \cdot d \vec{A}$ is computed by BEACH for a collisionless case and plotted on Figure 4-25. Again, as in the cases seen in Section 4.4.2, the field amplitude grows larger as resonance is approached. This can be compared to the collisional case shown on Figure 4-28, in whicn collisional damping is able to reduce much of the energy buildup that is characteristic of undampened propagation towards resonance. Nevertheless, the wave amplitude is still able to begin to increase as $\omega$ gets closer to $\omega_{c i}$, although at a much lower rate than the collisionless case.

Note that even with the inclusion of finite temperature effects (Single mode calculation), the simulations conducted by Uman \& Hooke fared no better than the cold plasma approach undertaken in this thesis in attempting to fit to the experimental data. The results of Uman \& Hooke's analysis are shown on Figure 4-27. For a plasma with a measured temperature of $100 \mathrm{eV}$, Uman had to use a simulation temperature of $T_{\|}=20 \mathrm{eV}$ to adequately match to the data, while a $55 \mathrm{eV}$ plasma required a simulation temperature of $T_{\|}=10 \mathrm{eV}$.

The experimental B-66 data are plotted against the results obtained from the BEACH simulation. Figure 4-28 shows the results for a $T_{\|}=5 \mathrm{eV}, n_{e}=1.75 \times 10^{13}$ simulation. Figures 4-26 and 4-29 the results of $7 \mathrm{eV}$ and $10 \mathrm{eV}$ plasma simulations for $n_{e}=2 \times 10^{13}$. There is a qualitative fit for most of the cases but divergence between B-66 results and BEACH simulation occurs for the higher temperature simulations $(10 \mathrm{eV})$ as resonance is approached. This is again intuitive because higher temperature results in lower collisional damping which in turn results in less removal of the field energy that is building up. 


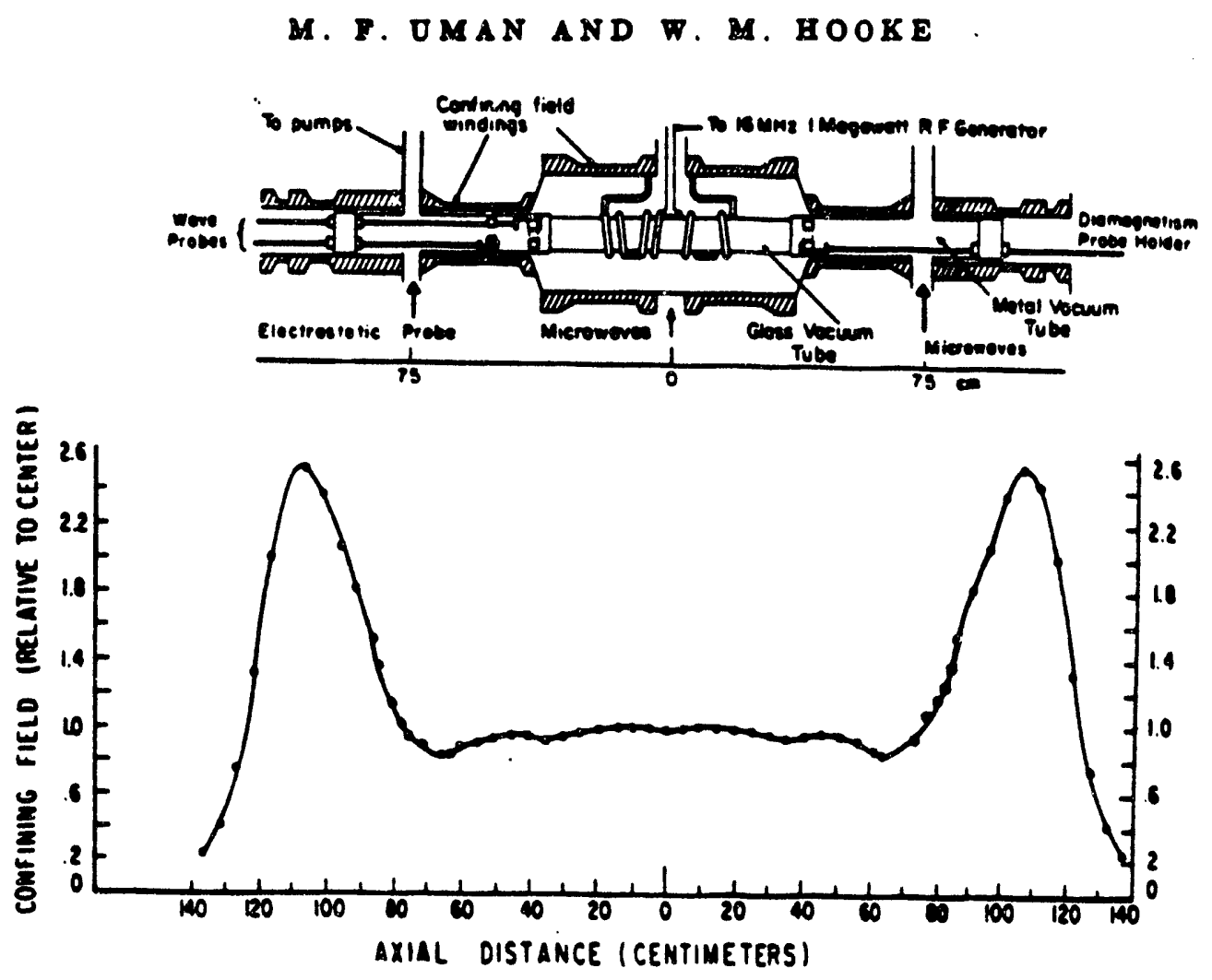

Ho. 3. Schematic diacram of the B-66 device aboring the axid mequatic feld profla.

Figure 4-23: B-66 Experimental Schematic and Magnetic Geometry [3] 


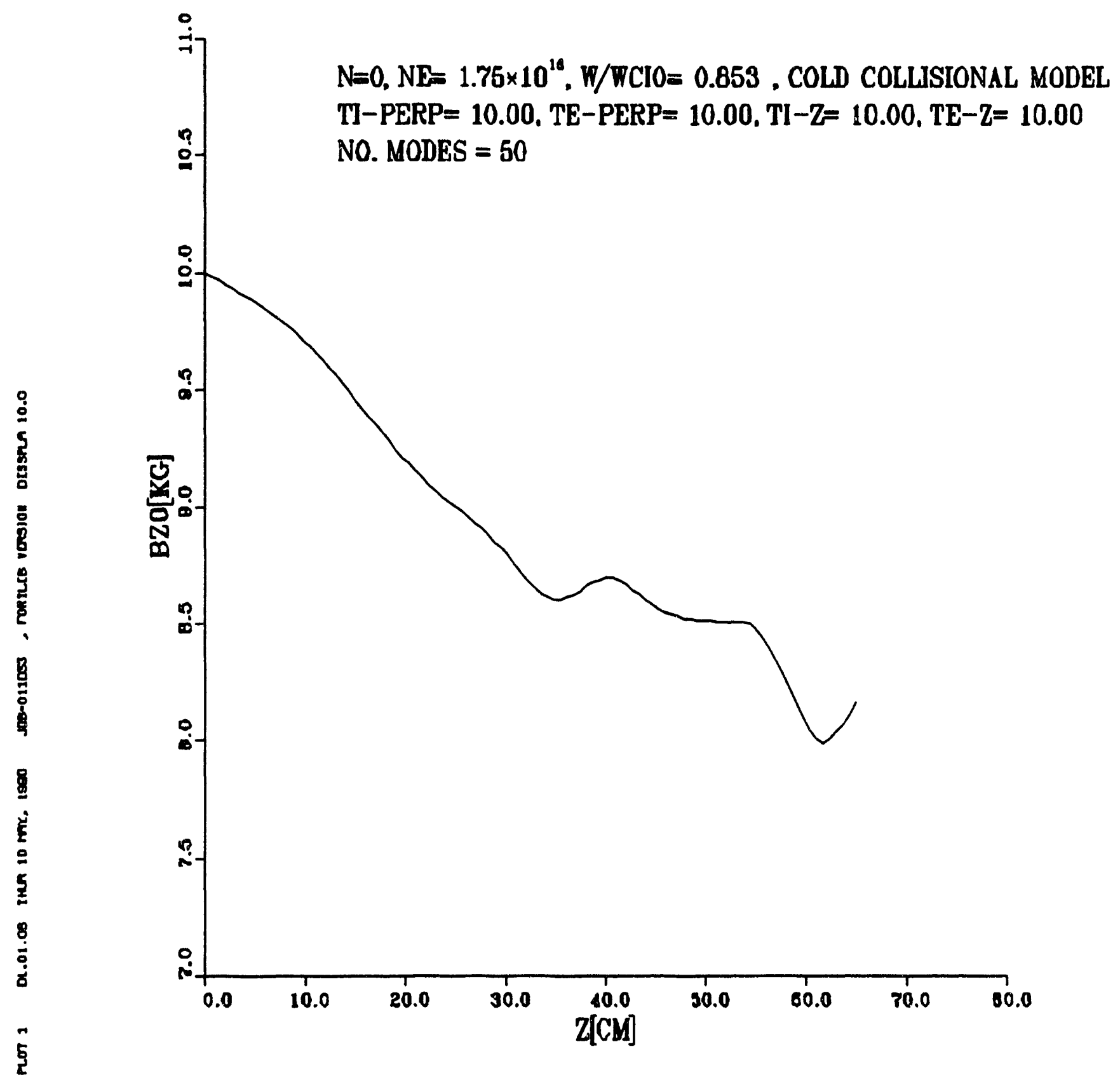

Figure 4-24: B-66 $B_{z 0}(z)$ Field Profile 


\section{Possible Sources of Error in B-66 Simulation}

Althogh much effort was spent on trying to simulate the geometry of the B-66 device as closely as possible, some differences between the BEACH simulation of the geometry and the real B-66 geometry remain.

The first and perhaps major difference is the antenna. The simulation of the B-66 uses a simple Full Turn Loop antenna to generate the $n=0$ mode ICRF waves. The real B-66 antenna is a multiturn antenna which spans some $30 \mathrm{~cm}$. This difference in the geometry of the wave launcher could account for some of the differences between the BEACH simulation results and the B-66 experimental data.

The second difference is the location of the conducting wall. The B-66 machine used a glass vacuum tube as its vacuum chamber, while the real metal container is located at a larger radial location. The BEACH simulation uses this larger radial location as its conducting wall. However the real B-66 has a break in this conducting wall for the ICRF leads. This could also introduce a difference in the field propagation characteristic. 


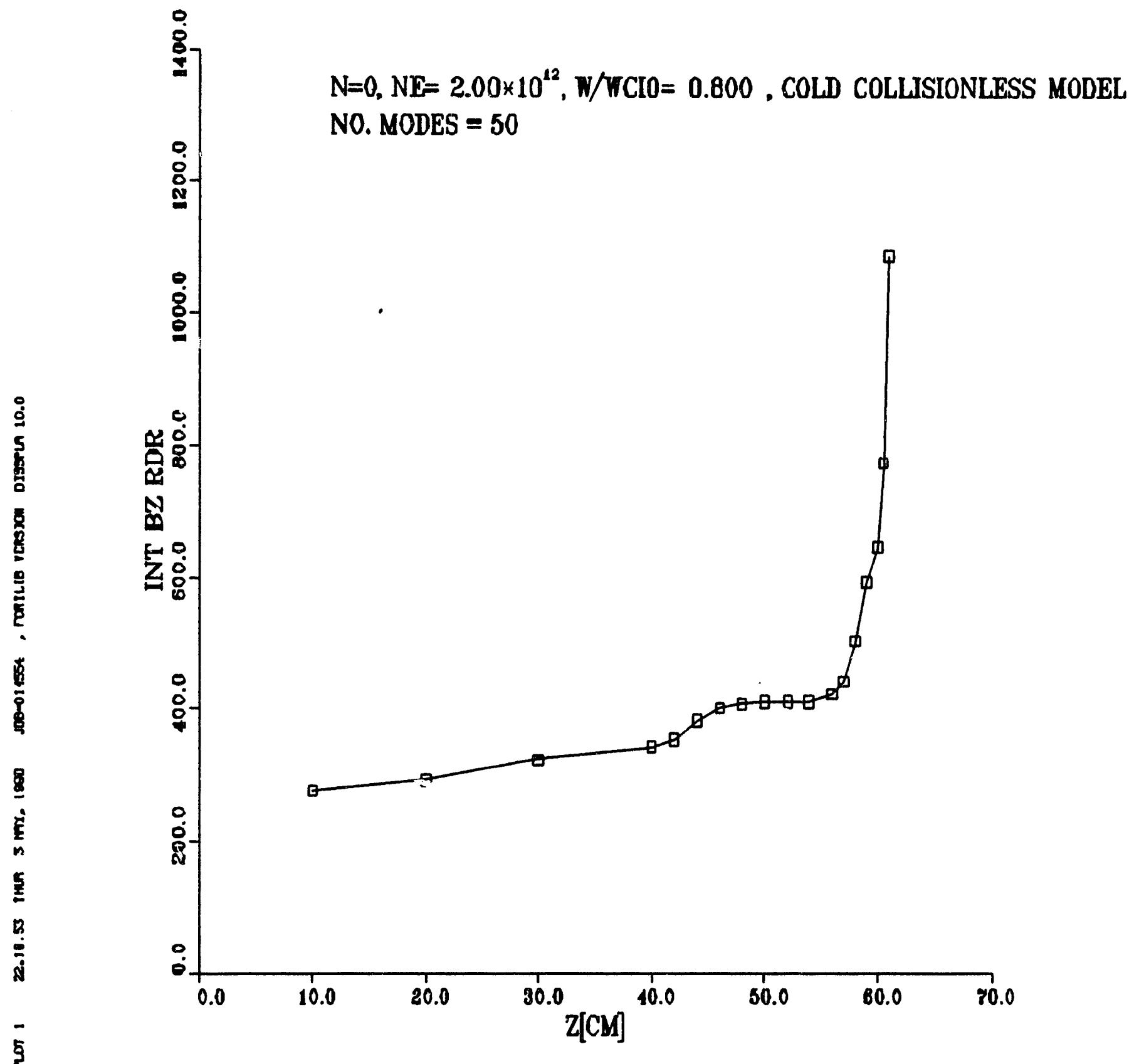

Figure 4-25: ICRF Wave $\int \vec{B}_{z} \cdot d \vec{A}$ for Magnetic Field Gecmetry of the B-66 Machine, Collisionless 


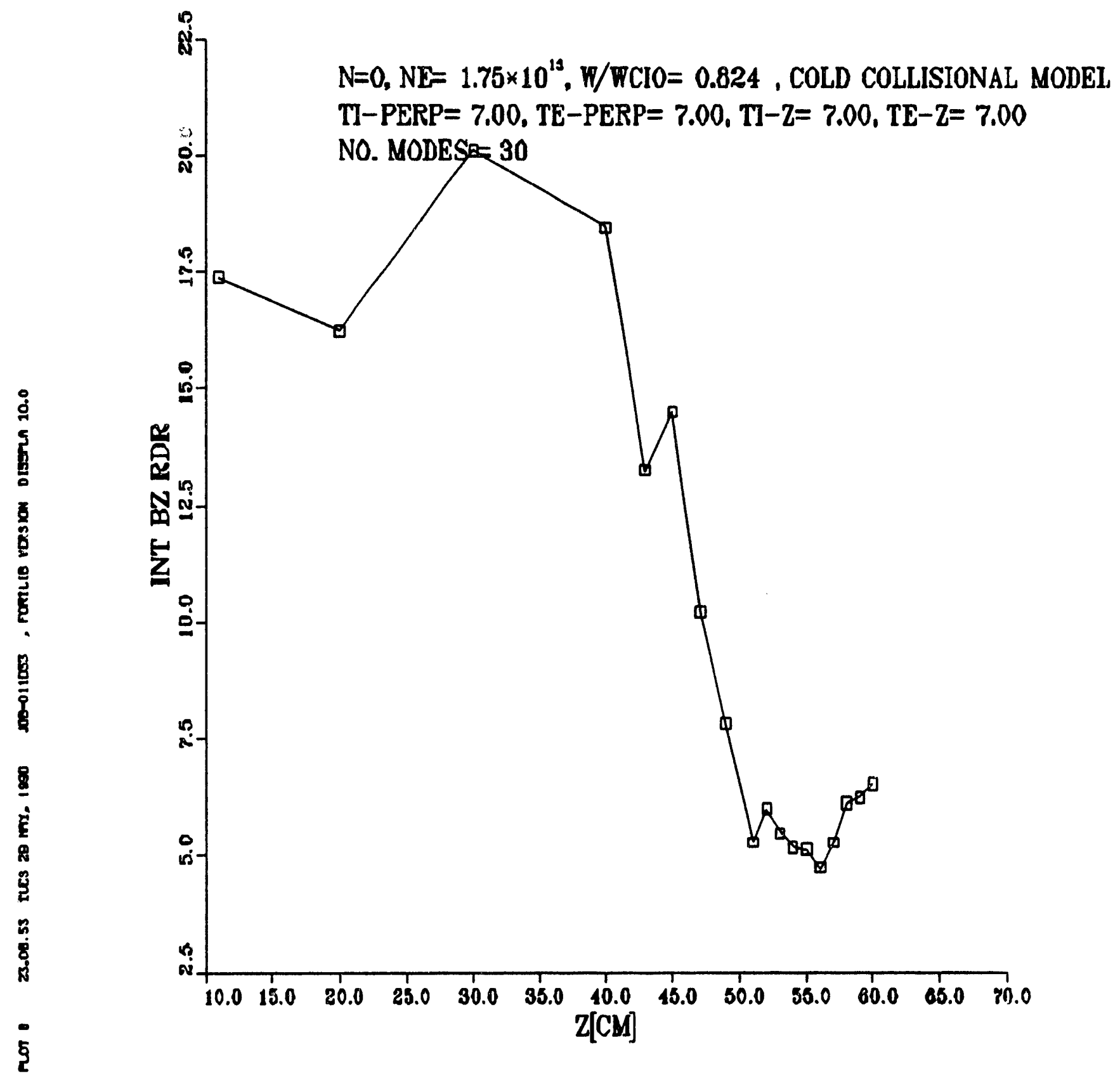

Figure 4-26: $\mathrm{BEACH}$ code $\int B_{z} r d r$ simulation for the B-66 geometry. Plasma properties are: $n_{\mathrm{e}}=1.75 \times 10^{13} \mathrm{~cm}^{-3} 3, T_{i, e}=7 \mathrm{eV}$. Full Turn Loop Antenna. 


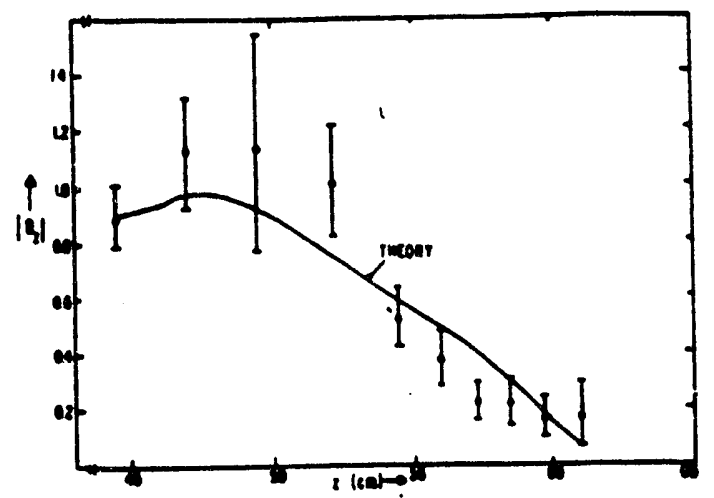

Fra. 7. Relative wave amplitude vernu arial pooition for dinmenetic temperature of $110 \circ \mathrm{V}$. The colid line i the theoretical prediction for ${ } T_{1}-20 \circ V$ and $n,-1.75 \times$ $10 \mathrm{~cm}-1$. The obeerved value of $2 \pi T_{1}=110 \cdot V$.

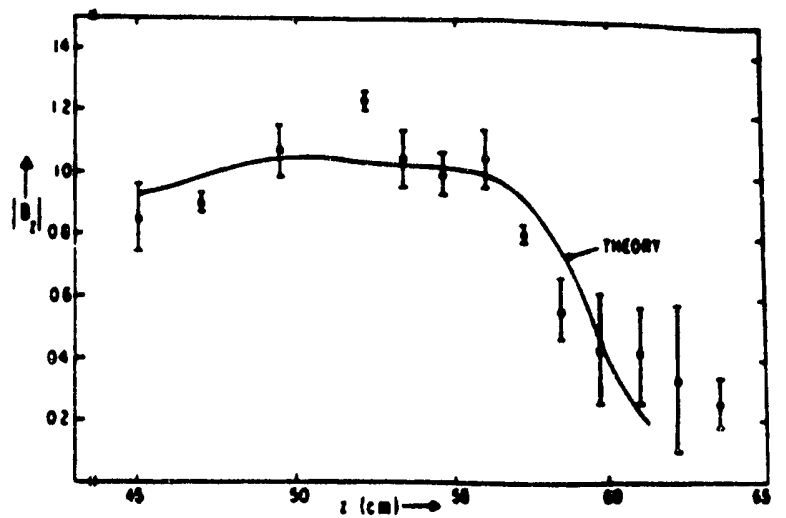

F10. 8. Relative wave smplitude veru arial position for diamarnetic temperature of $35 \mathrm{eV}$. The colid line is tho theoretical prediction for $\varepsilon T_{1}=10 \bullet \mathrm{V}$ and $n_{0}-1.73 \times$ $10^{21} \mathrm{~cm}^{-1}$. The obeerved value of $2 \times T_{1}=55 \mathrm{ov}$.

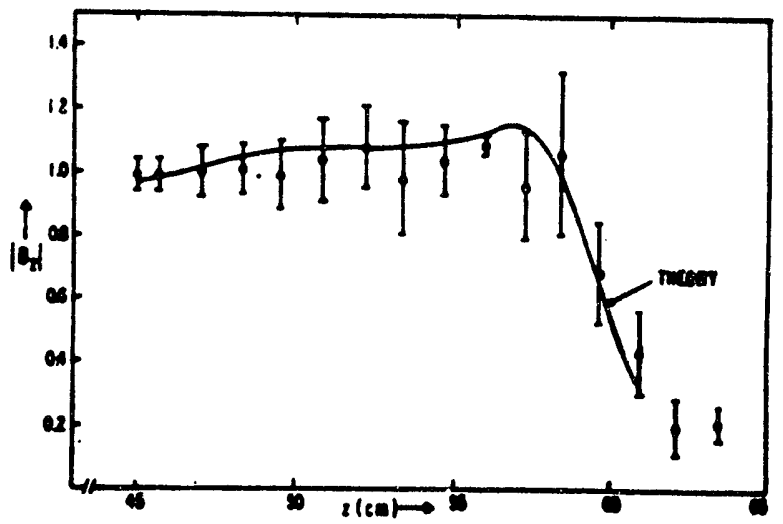

Fio. 9. Relative wave umplitude verou axid parition for dinmagnetic temperature of 10 ov. The colid tine is the theoretical prediction for $T_{1}=5 \cdot V$ and $-1.75 \times$ $10 \mathrm{~cm}^{-1}$. The obeerved valuo of $2 \mathrm{~s} T_{1}=10 \mathrm{eV}$.

Figure 4-27: B-66 Data and Finite Temperature Modeling [3]. Upper: $T_{\|}=$ $110 \mathrm{eV}, n_{\mathrm{e}}=1.6 \times 10^{12} \mathrm{~cm}^{-3}$; Middle: $T_{\|}=55 \mathrm{eV}, n_{\mathrm{e}}=1.25 \times 10^{12} \mathrm{~cm}^{-3}$; Lower: $T_{\|}=10 \mathrm{el}^{\prime}, n_{e}=0.9 \times 10^{12} \mathrm{~cm}^{-3}$ 


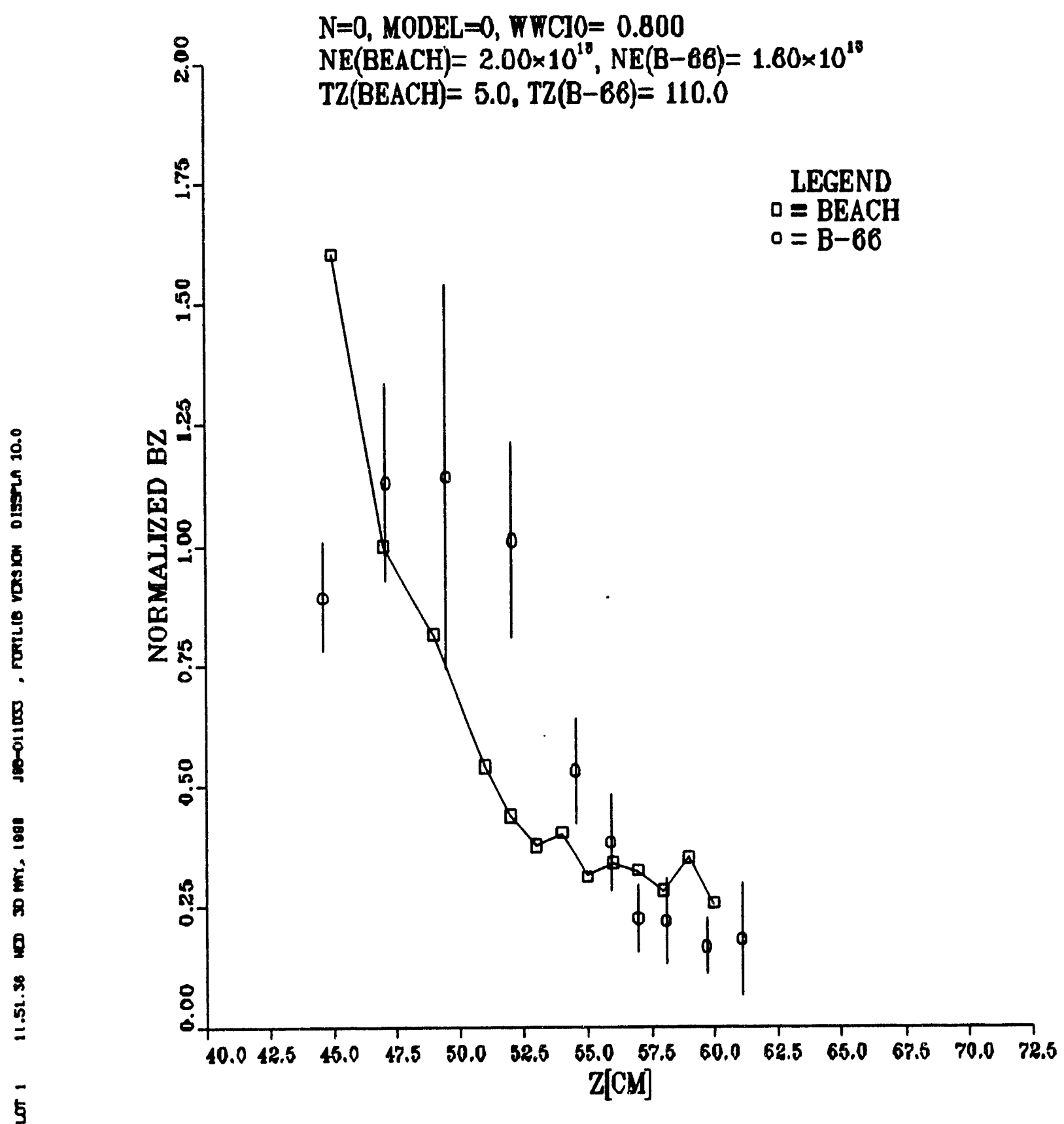

Figure 4-28: BEACH code $\int B_{z} r d r$ vs. Experimental B-66 Data. BEACH plasma properties are: $n_{e}=2 \times 10^{13} \mathrm{~cm}^{-3}, T_{i, e}=5 \mathrm{eV}$. B-66 plasma properties are: $n_{e}=1.6 \times 10^{13} \mathrm{~cm}^{-} 3, T_{i, e}=110 \mathrm{eV}$. Full Turn Loop Antenna. 


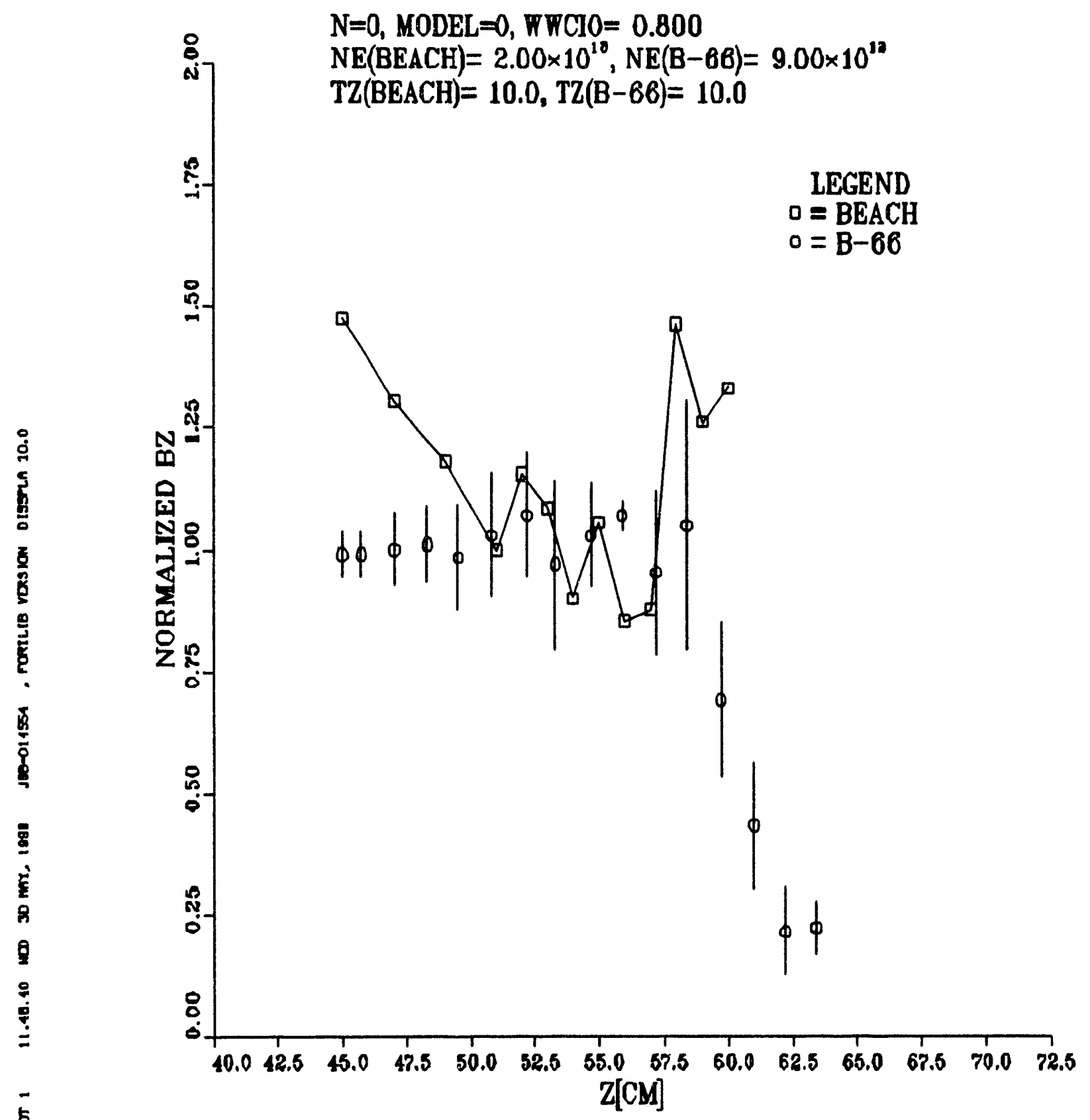

Figure 4-29: BEACH code $\int B_{z} r d r$ vs. Experimental B-66 Data. BEACH plasma properties are: $n_{e}=2 \times 10^{13} \mathrm{~cm}^{-3}, T_{i, e}=10 \mathrm{eV}$. B-66 plasma properties are: $n_{e}=0.9 \times 10^{13} \mathrm{~cm}^{-3}, T_{i, e}=10 \mathrm{eV}$. Full Turn Loop Antenna. 


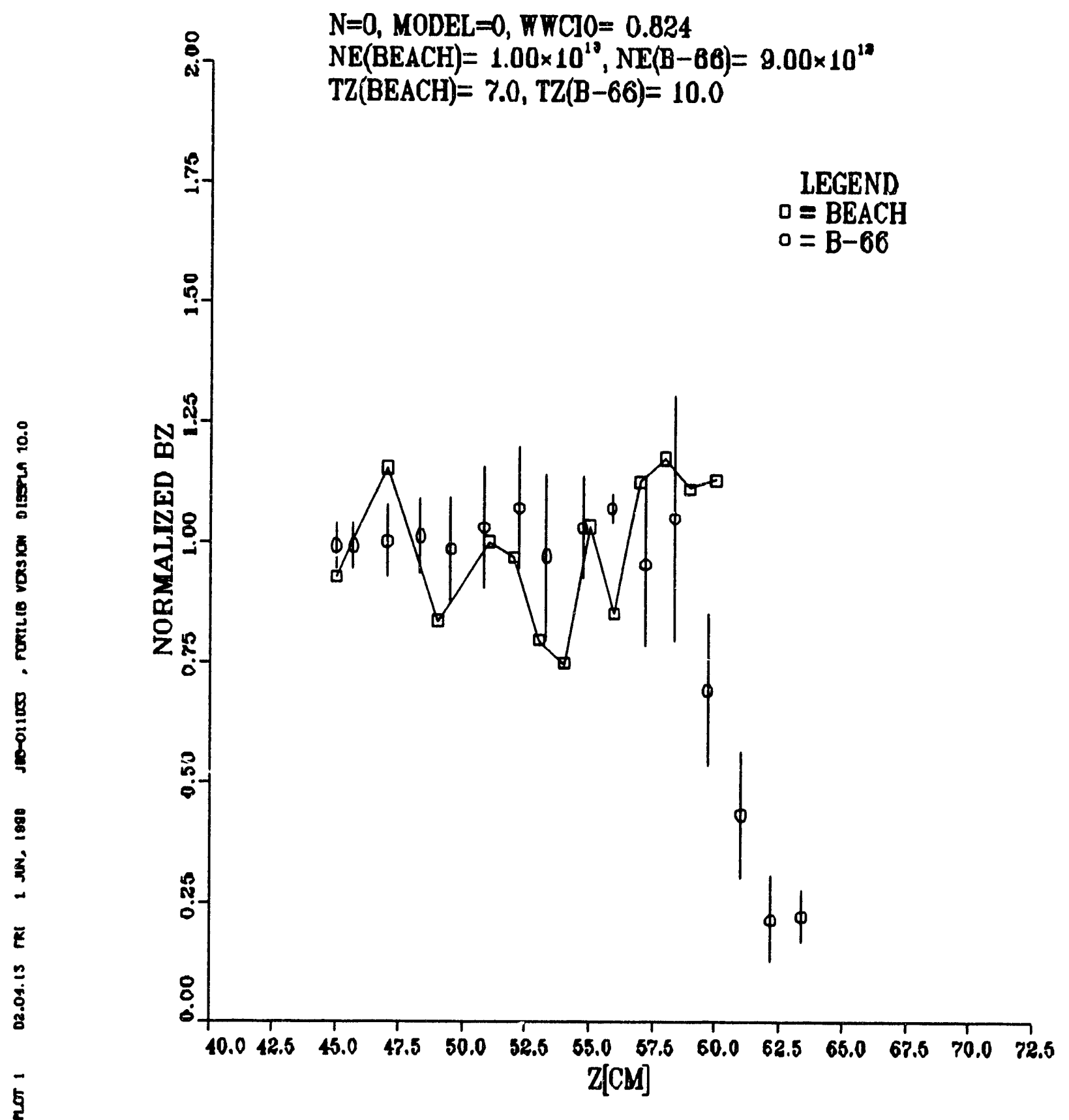

Figure 4-30: BEACH code $\int B_{z} r d r$ vs. Experimental B-66 Data. BEACH plasma properties are: $n_{e}=1.00 \times 10^{13} \mathrm{~cm}^{-} 3, T_{i, e}=7 \mathrm{eV}$. B-66 plasma properties are: $n_{e}=0.9 \times 10^{13} \mathrm{~cm}^{-3}, T_{i, e}=10 \mathrm{eV}$. Full Turn Loop Antenna. 


\subsection{Differences between BEACH and CYLWAVE}

There are some key differences between the CYLWAVE analytical theory of the previous chapter and the BEACH computations presented in this chapter. These are

1. BEACH is a local propagation analysis, while CYLWAVE is a global analysis. This means that the ICRF waves in the BEACH analysis "sees" only the $k_{z}$ of the slice they currently are propagating in, and the physics changes according to the "local" conditions. CYLWAVE, on the other hand, treats the geometry in a global manner. The parallel wave number $k_{\xi}$ and the perpendicular wave number $\alpha$ refiect the OVERALL geometry of the system and do not change with the wave's phase front.

2. The BEACH code is an axial propagation code, while the CYLWAVE is a standing wave code. The BEACH code tracks the propagation of the waves towards resonance, altering wave $k_{z}$ to match the changing plasma conditions that are encountered. The CYLWAVE code analyzes the overall effect of the spatially dependent dielectric tensor and computes the ICRF fields in the presence of this dielectric tensor. CYLWAVE does not track wave fronts or change $k_{z}$. This difference is evident in Figure 4-31 which shows the BEACH computed $\int B_{z} d A$ peak up due to the increased wave energy resulting from a slowing down of the parallel phase velocity. CYLWAVE, on the other hand, exhibits no such buildup because of its constant $k_{\xi}$.

3. The two approaches differ on their treatment of the axial inhomogeneity in $B_{z}$ and the changing plasma radius. $\mathrm{BEACH}$ splits the cylinder of changing radius into many constant radii slices, while the CYLWAVE code performs a coordinate transform and a stretching transform in order to simplify the boundary condition. 
4. BEACH is invalid close to resonance and cutoff, while CYLWAVE can handle resonances and cutoffs. The WKB approximation used in the BEACH code becomes invalid when approaching resonance. CYLWAVE handles the resonance by performing a Fourier transform and analytically integrating the poles.

5. BEACH contains both the almost-TE and the almost-TM modes, while CYLWAVE contains only the almost-TE mode. BEACH is able to decouple the almost-TE mode and the almost-TM mode because of the constant plasma parameters it assumes for each axial slice. CYLWAVE, on the other hand, contains elements of the dielectric tensor that are strong functions of $\xi$. This prevents a simple decoupling of the differential equations that couple the TE and TM modes together. 


\section{BEACH/CYLWAVE INT BZ}

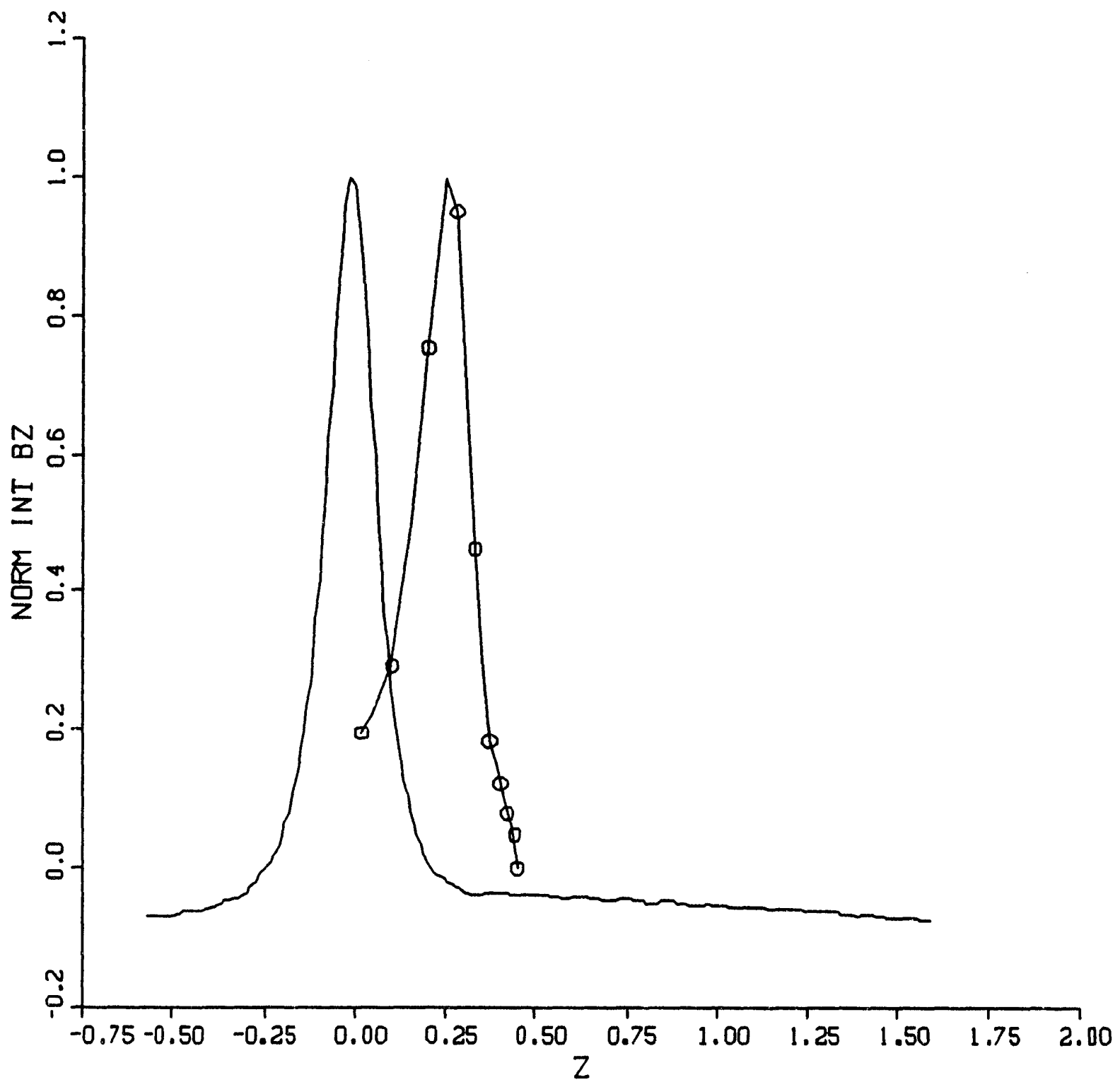

Figure 4-31: ICRF $\int B_{z} r d r$ Propagation in a Cold Collisional Plasma. CYLWAVE results (Solid line) and BEACH results (Open circle line) are plotted versus axial distance from antenna. BEACH assumes a full turn loop antenna, while CYLWAVE uses a dipole loop antenna located at $z=0$. 


\subsection{Summary of Computational Study}

In order to complement the analytical model, a computational multimodal eigenvalue simulation of ICRF propagation and heating is undertaken in this chapter. The simulation uses a computer code, $\mathrm{BEACH}$, that was developed as part of this thesis. BEACH takes into account the axially inhomogeneous $B_{z 0}$ by dividing up the axial length into different slices., each of which has a constant axial magnetic field. This allowed the simplification of the EM plasma fields into a fourth order equation which could then be separated into two coexisting modes - the "almost TE" and the "almost TM" modes. BEACH first solves for a finite number of $K_{z}$ eigenmodes for each slice (usually 30-50 modes are taken), and then solves for the excitation coefficient of each of these modes as driven by the antenna driving

source $\vec{J}\left(r, n, k_{z}\right)$. Two conditions - Poynting power conservation and wave phase conservation are used to match fields across the boundary between two adjacent axial slices. This solves for the set of unique excitation coefficients for the next (non-driven) axial slice. After all the fields are solved for each slice, the 30-50 modes are then inverse-Fourier transformed to obtain the full EM fields.

\section{RESULTS}

The results of the BEACH computer simulation indicates that

1. Higher density results in higher eigenmodes. Although these modes have only slightly higher REAL parts, the imaginary $k_{z}$ of these modes are significantly increased as a result of higher density. Thus, BEACH code found that higher density is better for ICRF heating.

2. Lower Temperatures generates larger $k_{z, i m}$, causing the ICRF waves to damp out more and coupling more energy to the plasma. Thus, LOW TEMPERATURE is better for ICRF heating. This finding, however, is only valid in the context of a cold plasma approximation $\left(T_{\|} \lesssim 100 \mathrm{eV}\right)$. 
3. There is an optimal $\omega / \omega_{c i}$ for launching ICRF waves. This optimal $\omega / \omega_{c i}$ depends mainly upon the $k_{z}$ spectrum of the launching mechanism, i.e. antenna. For the Full Turn Loop antenna, it was found that lower launch $\omega / \omega_{c i}$ is more suitable for coupling to the ICRF waves.

4. Comparison of the BEACH results with the B-66 experimental results show a qualitative fit, however the two results begin to diverge as $\omega / \omega_{c i} \rightarrow 1$. It is seen that the BEACH results often did not contain enough dissipation mechanisms (BEACH contains only collisional dissipation) to completely damp out the rapidly growing field energy.

5. There are many differences between the BEACH analysis and the CYLWAVE analysis. BEACH uses a "local" propagation approach, while CYLWAVE uses a "global" approach. BEACH separates the geometry into discrete axial slices of constant plasma properties, while CYLWAVE employs coordinate transformations and Fourier transform to handle the axial inhomogeneity. BEACH contains both the almost-TE and almost-TM modes, while the CYLWAVE contains only the almost-TE mode. Each approach has its strength and weakness. Future improvements of either analysis must incorporate features of the other in order to best simulate a realistic physical geometry.

In conclusion, the study of ICRF wave propagation undertaken in this chapter is able to generate intuitive results for ICRF wave propagation and damping, as well as produce results that qualitatively agree with the experimental data obtained from the B-66 experiment. BEACH and CYLWAVE are seen to differ on several fundamental issues. The best features of both should be incorporated into the next generation analysis. 


\section{Chapter 5}

\section{Experimental Results}

As indicated in Chapter 2, the experiments were conducted on the MIT-PPEX mirror machine that was constructed as part of this thesis. It is the goal of the experimental work to try to verify some of the predictions of the simulation results. Thus, the main focus of this chapter is on the experimental results obtained from the PPEX machine, in particular the B-dot probe's pickup of the ICRF $B_{z}$. The results from radial scan of the ICRF $B_{z}$ will be compared to predictions of the BEACH code.

PPEX offers many "controls" to the experimentalist in order to tailor a plasma discharge of the desired plasma parameters. These controls are given in Table 5.1.

Two sets of ICRF antenna are used in the heating of the PPEX machine: one in the anchor at $7.5 \mathrm{MHz}$, the other in the center cell at $3 \mathrm{MHz}$. Measurements of plasma properties commence before the firing of the ICRF shot and continue well beyond the end of the ICH pulse. The ICRF $B_{z}$ measurements by the B-dot probe usually takes place during the ICRF shot because of the short scan period dictated by the limited memory of the digitization module (512K of RAM corresponds to $\sim 0.5 \mathrm{~ms}$ at the $100 \mathrm{MHz}$ digitization rate).

Over 2400 shots were taken from the inception of computer assisted data acquisition to the present time. Of these 2400 shots, over 1000 shots were with ICRF. 
Two types of ICRF shots are identified in this thesis - Type I and Type II. Type I shots are those for which the ICRF frequency of the antenna is greater than the cyclotron frequency at the antenna location, i.e. $\omega>\omega_{c i, a n t}$. Type II shots are the opposite, i.e. $\omega<\omega_{\text {ci,ant. }}$ Type II results are the results that will be simulated by the BEACH code, thus emphasis will be placed on the B-dot probe's ICRF $B_{z}$ measurements from the TYPE II shots.

This chapter is organized into several sections. Section 5.1 will first present the ECRH plasma shots. The next section will provide the rationale for the separation of the ICRF shots into two types: $\omega>\omega_{c i}$ (TYPE I) and $\omega<\omega_{c i}$ (TYPE II). Section 5.3 will present the results obtained for a TYPE I ICRF discharge, followed by Section 5.4 which presents the results for the TYPE II ICRF discharge, including the radial $B_{z}$ scan that will be matched against the theoretical predictions of the eigenmode code $\mathrm{BEACH}$ as described in the previous chapter. Finally, Section 5.5 will summarize the experimental results obtained.

\subsection{Baseline ECRH Shots}

As mentioned in Chapter 2, the PPEX machine has three heating systems : one Electron Cyclotron Resonance Heating (ECRH) system at $2.1 \mathrm{GHz}$, and two Ion Cyclotron Range of Frequency (ICRF) systems at 3 and $7.5 \mathrm{MHz}$. The results presented in this section are obtained from plasma shots heated by solely the ECRH system. There are two reason that these results are presented. First, to provide a set of baseline plasma characteristics that can be obtained without use of ICRF power, and secondly, to provide sensitivity calibration of the interferometer and Langmuir probe diagnostics

The ECRH discharge is created by running the center cell and anchor coils at lower currents $\left(I_{c c}=200 \mathrm{~A}, I_{\text {mirr }}=400 \mathrm{~A}\right.$ versus $I_{c c} \simeq 600 \mathrm{~A}, I_{\text {mirr }} \simeq 1600 \mathrm{~A}$ for typical ICRF discharges). This is done to produce the $750 \mathrm{G}$ field required in order to locate the first harmonic ECH resonance close to the ECRH wave 
launcher. However, lower current and thus lower axial field $B_{\mathbf{z} 0}$ results in much larger Larmor radii for both the electrons and ions, hence a lower density.

The diagnostic results obtained from an ECH plasma shot is shown on Figure 5-1. The top graph shows the Langmuir Probe's response. The middle shows the microwave interferometer's phase shift response, and the lower graph is the $H_{\alpha}$ light emission from the plasma discharge.

The Langmuir piobe allows a good estimate of the edge plasma density and electror temperature. From the linear region of the I vs. V plot of the Langmuir probe response curve, the average electron temperature for an ECH discharge is estimated to be $18 \pm 3 \mathrm{eV}$. The density can be found using the following equation:

$$
n_{e}=\frac{I_{s i} e^{\frac{l}{2}}}{A, q} \sqrt{\frac{m_{i}}{T_{e}}}
$$

From the ion saturation current measurement of $I_{s i} \sim 1 \mathrm{~mA}$ and an electron temperature of $18 \mathrm{eV}$, a plasma density of $7.9(+1.3,-0.6) \times 10^{10} \mathrm{~cm}^{-3}$ is found.

The $H_{\alpha}$ light emission shows the heating of the plasma from the ECRH source. The H- $\alpha$ emission data is used as one further check to verify the operations of the other two diagnostics.

The microwave's phase difference digitization is performed with the Jorway J1808 phase digitizer. For a mean plasma radius of $10 \mathrm{~cm}$, this level of interferometer response corresponds to a maximum chord-averaged ECH discharge density of $8.1 \pm 2.0 \times 10^{10} \mathrm{~cm}^{-3}$.

From the data acquired from the Langmuir Probe and Interferometer diagnostics, it is seen that the Langmuir Probe's density of $7.9 \times 10^{10} \mathrm{~cm}^{-3}$ agrees quite well with the interferometer's maximum density of $8.1 \times 10^{10}$. Both diagnostics used in combination thus could provide relatively good and mutually verifiable measurements of the chord-average electron density. 

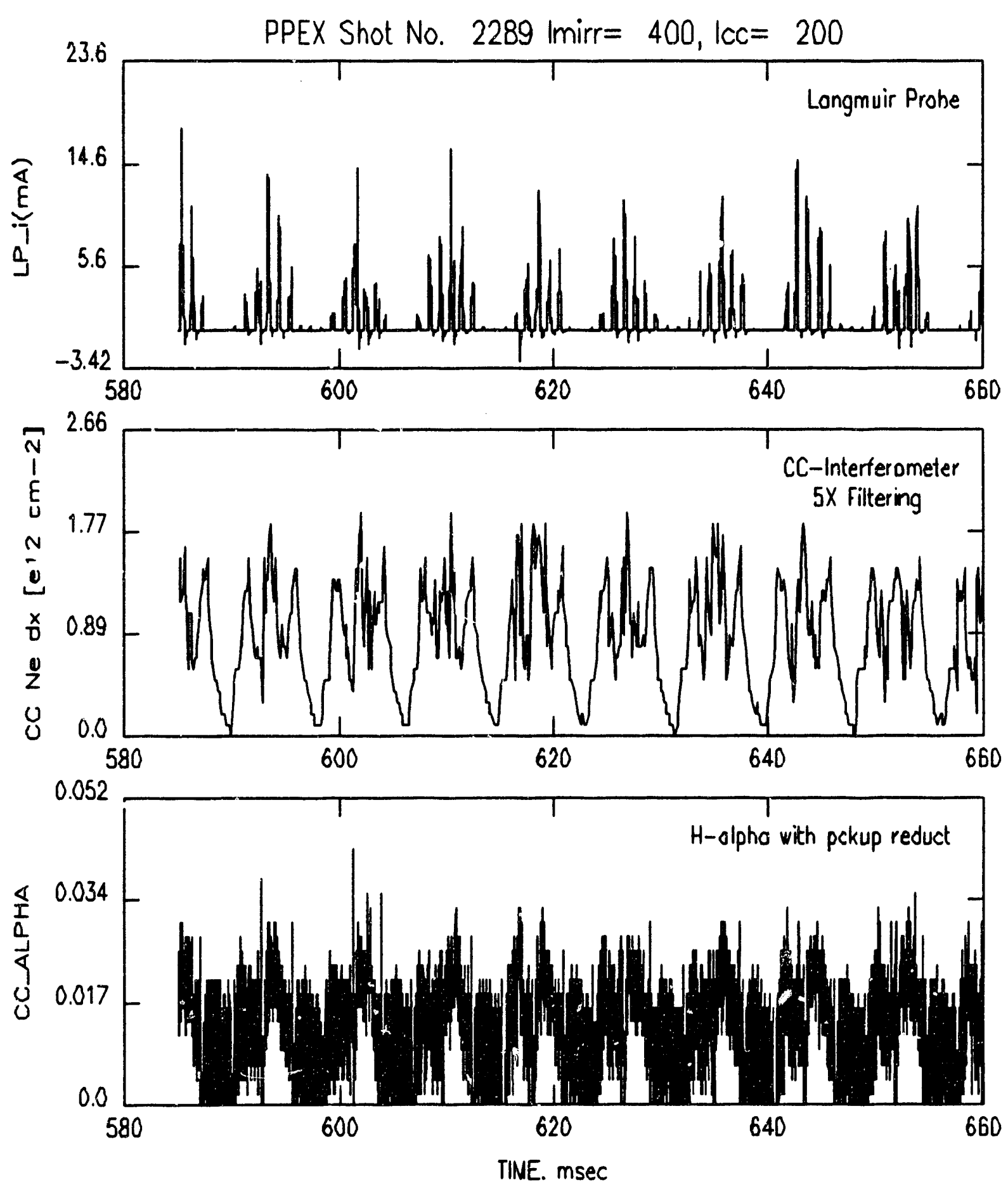

Figure 5-1: Processed PPEX ECH Shot Data. From Top to bottom : Langmuir Probe Current, Interferometer Line Density, and H- $\alpha$ Light Emissions. The Langmuir Probe current is obtained by sawtooth scanning the LP Voltage at $\pm 300 \mathrm{~V}$, $1 \mathrm{kHz}$. 


\subsection{ICRF Shots}

Although the PPEX machine has ICRF antennas located in both the center cell and the mirror cell, only the CC ICRF is used in the experiments described in this chapter. This is because the mirror ICRF antenna launches waves that are damped in the mirror cells, therefore the B-dot pickup probe in the center of the machine has difficulty picking up adequate signals. The mirror ICRF antenna is unable to launch waves at lower frequencies because its matchbox was designed for operation at frequencies of resonance in the mirror cell $(\sim 7 \mathrm{MHz})$ and not for center cell resonance ( $\sim 3 \mathrm{MHz})$.

CC ICRF shots are used to extract information about ICRF wave damping and wave energy absorption by the plasma. Special emphasis is placed on the set of experimental results obtained from the B-dot probe, the Langmuir probe, and the interferometer to obtain a set of plasma properties $n_{e}, T_{i}$, as well as ICRH heating and propagation characteristics.

A representative CC-ICRH discharge has the time sequencing and setting shown in Table 5.2 .

\subsubsection{Classification of ICRF Discharge}

The ICRF discharges to be examined in this thesis are basically grouped into two types - TYPE I is the class of discharges with $\omega>\omega_{c i}$, and TYPE II is the class of discharges with $\omega<\omega_{c i}$. The magnetic geometry of the two systems are seen in Figure 5-2. There are two reasons why the discharges are thus classified - the difference in level of plasma oscillation, and the different physics inherent in the two types.

The difference in the oscillation level between the two discharges can be seen on Figure 5-3, which shows the H- $\alpha$ acquired during TYPE I and II discharges. From the figure, it is obvious that TYPE I discharge is much noisier. This noisiness makes it difficult to reproduce ICRF shots with similar plasma characteristic and 

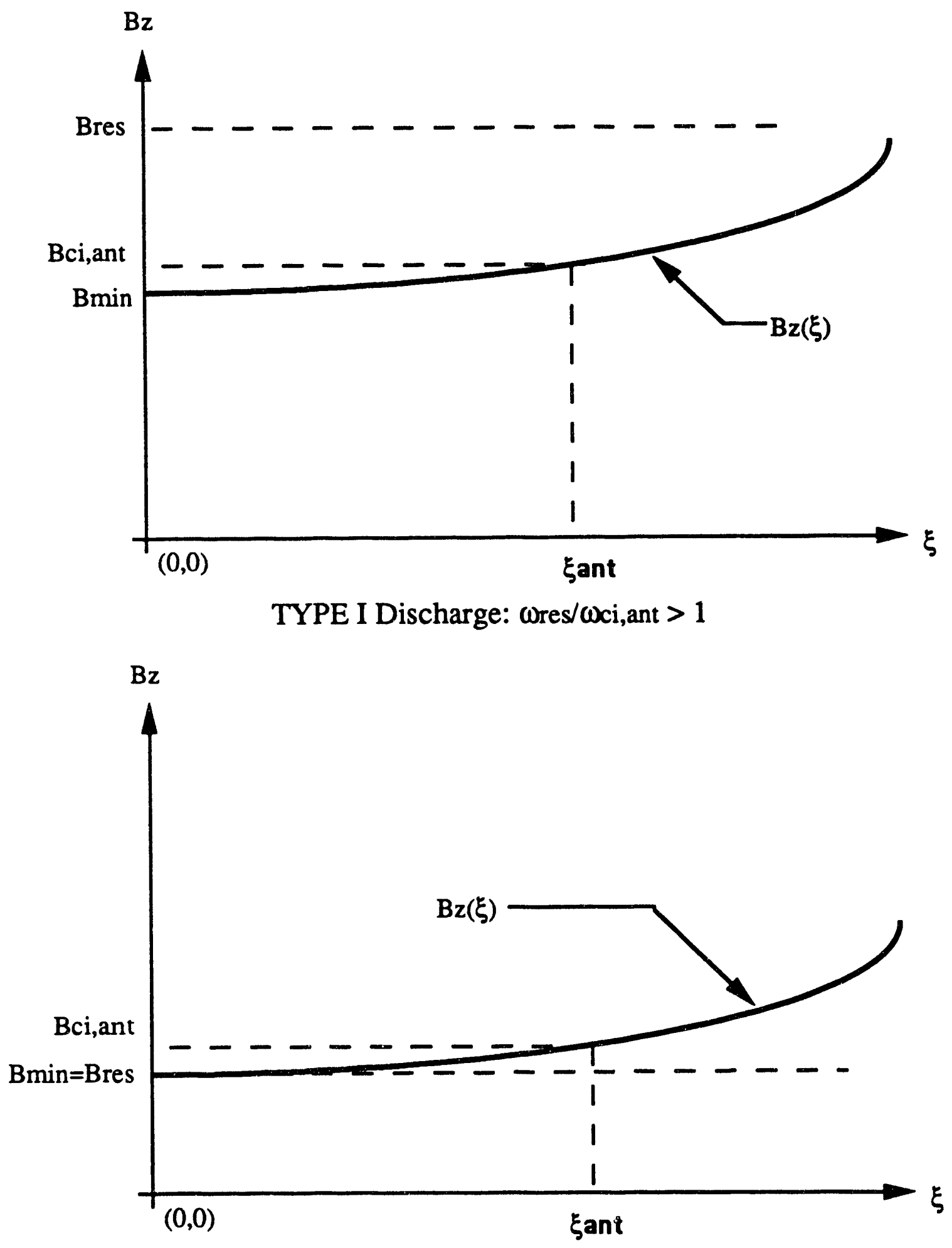

TYPE II Discharge: $\omega$ res $/ \omega c i$, ant $<1$

Figure 5-2: Difference in geometry between TYPE I and TYPE II discharges. Geometry of TYPE I (Top) shows that the launching frequency is HIGHER than the cyclotron frequency at the antenna. This results in resonance that is not located between the antenna and the field minimum. TYPE II (Bottom) shows that the launching frequency $\omega_{\text {res }}$ is LOWER than the cyclotron frequency at the antenna location $\omega_{\text {ci,ant }}$. This allows for an IC.RF resonance layer that could be located either between the antenna analgine center of the machine on out of the machine. 
is one of the two reasons that the radial $B_{z}$ scan is performed for the TYPE II plasma.

The second reason that TYPE II is chosen to perform the radial scan is that for $\omega>\omega_{c i}$, the ion cyclotron resonance at $\omega=\omega_{c i}$ is not crossed. Crossing the $\omega=\omega_{c i}$ resonance layer alters the physics of the propagation because the $L$ in the dielectric tensor becomes negative, and thus there is no longer the $k_{z}^{2}=k_{0}^{2} L$ cutoff on Figure 3-1. This results in the elimination of the TE mode and leaves just one $k_{\perp}$ for every $k_{\|}$. Hence, the propagational physics is different if the $\omega=\omega_{c i}$ resonance is cross. The TYPE I discharge has only one $k_{\perp}$ associated with each $k_{z}$ eigenmode, while the TYPE II discharge has two $k_{\perp}$ 's associated with each $k_{z}$. The BEACH code simulations is performed assuming both $k_{\perp}$ modes exist, which is similar to the TYPE II discharge. Thus, in order to maintain similar modal characteristic with the $\mathrm{BEACH}$ code, the radial $B_{z}$ scan is only performed for a TYPE II discharge.

\subsubsection{Verification of the BEACH code}

To compare the results of the PPEX experiment to the $\mathrm{BEACH}$ code, the plasma density must be known. The plasma density is required for $\mathrm{BEACH}$ to produce the radial profile that can be compared with the PPEX results. Accurate knowledge of the ion and electron temperatures is not required in producing the radial profile because their contribution to the wave propagation comes in through the collision term, which affects the axial profile (through the $e^{-k_{z, i m} z}$ term) much more than the radial profile.

Given that the main plasma property required for cornparison with the BEACH code is the plasma density, the great majority of the diagnostic effort is devoted towards obtaining a set of independent measurements of plasma density. Three diagnostics are used to provide density data: Interferometer, $\mathrm{H}-\alpha$ detector, and Langmuir Probe. The interferometer is used to provide the line average density of the plasma. The H- $\alpha$ detector is not absolutely calibrated, due to a lack of an 

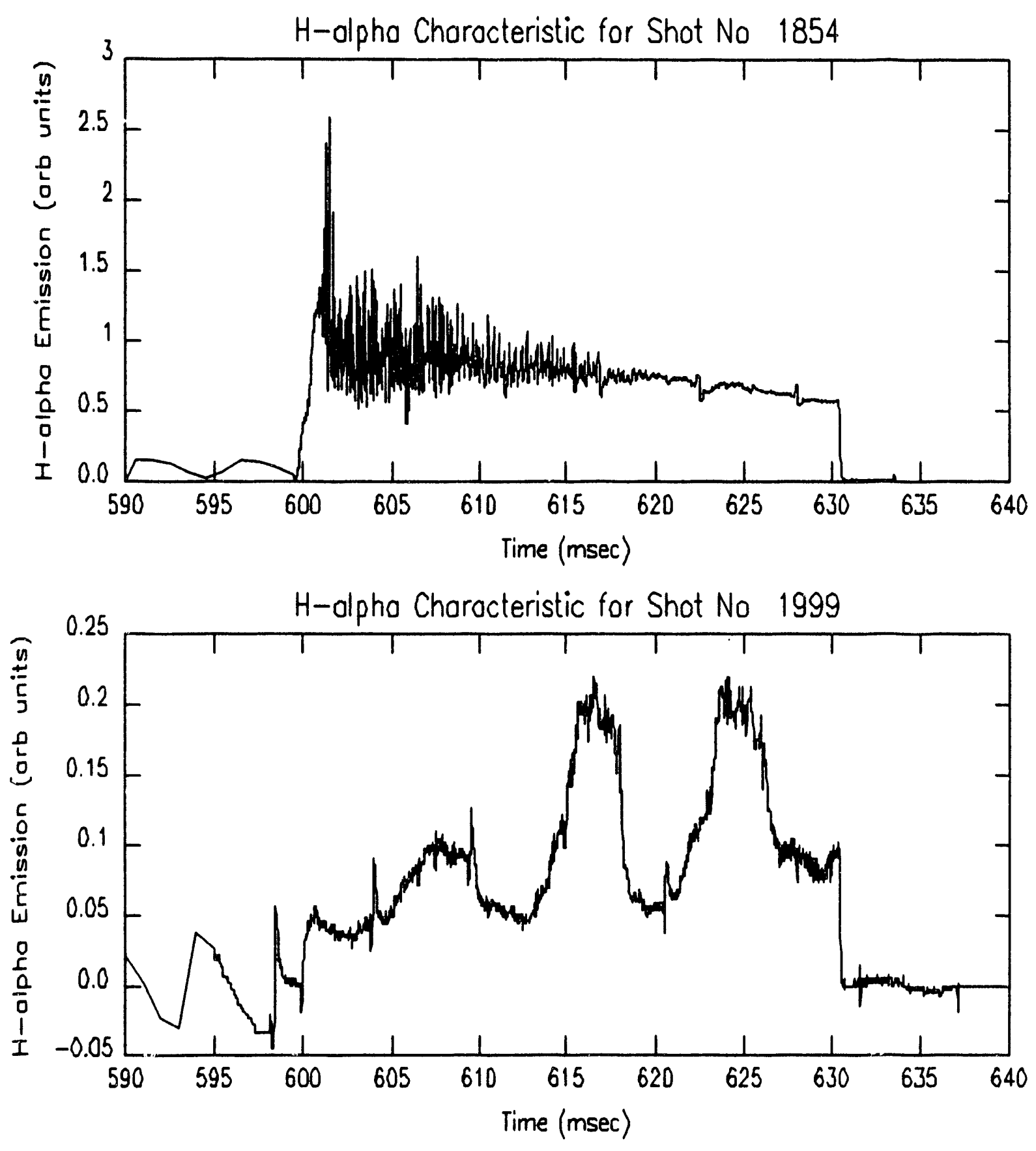

Figure 5-3: Comparison between the H- $\alpha$ output of TYPE I and TYPE II discharges. TYPE I (Top) shows a much higher light emission level as well as the three different regions identified as Ramp-up, Oscillatory, and Quiescence. TYPE II (Bottom) shows little light emission and is relatively quiescent 
absolute light source. Therefore, its purpose is to verify the temporal behavior of the interferometer and Langmuir probe diagnostics. Because the time resolution of the interferometer system used ( $8 \mathrm{kHz}$ digitization rate) is sometimes unable to catch the fast timescale changes in density, resulting in missing one or more $2 \pi$ radian phase shifts, the $\mathrm{H}-\alpha$ emission is monitored to determine if the interferometer is still phase-locked on the microwave signal. The H- $\alpha$ light output should have the same temporal characteristic as the interferometer density measurement. Finally, the Langmuir probe is used to provide edge density measurements that could be compare to the interferometer. If both the Langmuir probe and interferometer yield similar results for density, then the measurements can be seen to be self consistent.

However, it must be pointed out that the radial dependence of the ICRF $B_{z}$ field is NOT a strong function of density. BEACH code simulations show that density variation by a factor of two does not affect the characteristic radial profile very much. Thus, the accuracy of the density diagnostics is not extremely crucial to provide adequate modeling of the ICRF $B_{z}(r)$ radial field profile.

A radial scan is performed to compare the PPEX experimental results against the predictions of the $\mathrm{BEACH}$ code. As mentioned before, this scan is only done for TYPE II discharges. The B-dot probe [25] is inserted into the plasma and the ICRF $B_{z}$ fields are measured during an ICRF pulse. At least five shots are taken at each radial location before the probe is moved to another radial location. After a complete scan is performed, the results are then compared against the BEACH predictions. The B-dot probe's pickup loops have a diameter of $\frac{1}{4}$. This imposes a limit on the resolution of the $B_{z}(r)$ measurements because according to Hutchinson [73], any field structure whose scale length is less than size of the pickup loop (Diam $=\frac{1}{4}$ ") will be distorted by the presence of the probe. Thus, the fine structure of the $B_{z}(r)$ field will not be accurately represented. However, field structures with sizes greater than $\frac{1}{4}$ " can be resolved. 


\subsection{TYPE I Discharge $-\omega>\omega_{\mathrm{ci}}$}

As mentioned before, BEACH code results are comparable in magnetic geometry to the TYPE II discharges. Therefore TYPE I discharges were not considered in the theory portion of this thesis. However, several interesting characteristics of TYPE I discharges were found during the course of experiments and are presented in this section both for completeness and also as a lead-in to possible future work.

The data acquired during a sample TYPE I ICRH shot is shown in Figure 5-4. The three diagnostics whose data are given in Figure 5-4 are (Top to bottom): Langmuir Probe Current Interferometer, and H- $\alpha$ light emission.

\subsubsection{H- $\alpha$ and Interferometry Results}

Light emission from the $\mathrm{H}-\alpha$ is a good indicator of the ionization process. The interferometer data yields an indication of the chord-averaged electron density, which is essential in the context of this thesis to examine ICRF propagation. Taken together, the $\mathrm{H}-\alpha$ and the interferometer data yield a correlatable and coherent measurement of plasma density.

Analysis of the $\mathrm{H}-\alpha$ output shows that there is significant plasma ionization during the ICRF shot, but that both the $\mathrm{H}-\alpha$ and the microwave interferometer output drop rapidly to zero after the ICRF is turned off. This indicates a low confinement time which is estimated from the density decay time at $\tau_{\text {particle }}<$ $0.5 \mathrm{~ms}$ 。

The H- $\alpha$ and interferometry results obtained from TYPE I discharges lead the PPEX team to identify tinree distinctly different regimes in an ICRF discharge - Ramp-up, Oscillation, and Quiescent. As seen in Figure 5-4, the H- $\alpha$ output is seen to initially rise slowly, with very little noise. This is identified as the Ramp-up phase. After a plateau is reached, the $\mathrm{H}-\alpha$ then goes through a period of intensifying oscillations (Oscillation), culminating in a final Quiescent period of almost no noise before ICRF power is cut off. 

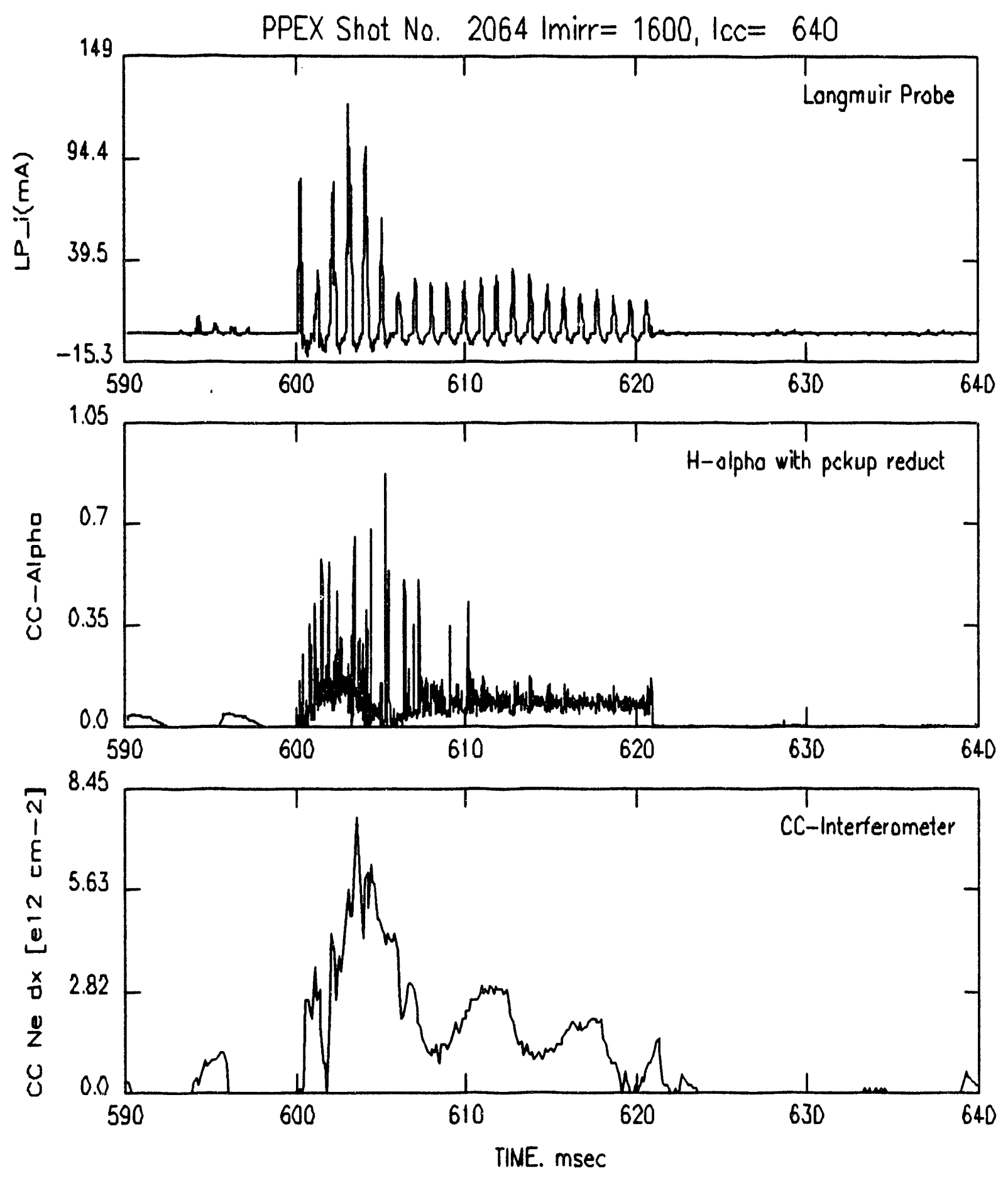

Figure 5-4: Diagnostic data obtained during a TYPE I ICRF shot. Diagnostics are (Top to bottom): Langmuir probe current, Interferometer, and H- $\alpha$ Light Emission 
Interferometer data shows peak line densities of approximately $5 \pm 0.85 \times$ $10^{12} \mathrm{~cm}^{-2}$, or densities of $2.5 \pm 0.42 \times 10^{11} \mathrm{~cm}^{-3}$. Average line density is approximately $2 \pm 0.85 \times 10^{12} \mathrm{~cm}^{-2}$ or density of approximately $1 \pm 0.42 \times 10^{11} \mathrm{~cm}^{-3}$.

$\mathrm{H}-\alpha$ Light emissions are at a level that is generally much higher than for ECH emissions. For the Quiescent phase, light emissions are typically about 2.8 times larger than the ECH emissions.

\subsubsection{Langmuir Probe Results}

The Langmuir Probe data is shown in Figure 5-5.

The Langmuir probe results clearly show the chaotic nature of the TYPE I discharge. Two distinctly different sets of data are evident - the coherent set of data points labeled as Set B and the rest of the points, labeled as Set A. Set A is the Langmuir probe response during the Ramp-up and Oscillatory phases, while Set B consists of data obtained during the Quiescent phase of ICRF discharge. The Langmuir probe tip has a diameter of $1.78 \mathrm{~mm}$. Based upon the Langmuir Probe data, the electron temperature at $6.67 \mathrm{~cm}$ is $T_{e}=45 \pm 10 \mathrm{eV}$, while the density is estimated at $n_{e}=0.86(+0.11,-0.076) \times 10^{11} \mathrm{~cm}^{-3}$.

Note that the Langmuir probe density results are in fairly good agreement with the interferometry result $-n_{e}(L P)=0.86(+0.11,-0.076) \times 10^{11} \mathrm{~cm}^{-3}$ versus $n_{e}(I F)=1 \pm 0.42 \times 10^{11} \mathrm{~cm}^{-3}$.

\subsubsection{B-Dot Probe Results for $\omega>\omega_{c i}$}

The ICRF $B_{z}$ data obtained are analyzed by Fast Fourier Transforming the signal into its frequency components. This allows an examination of the level of excitation of the base $3 \mathrm{MHz}$ frequency as well as of any other harmonics.

The $B_{z}$ data are presented as follows. Section 5.3.3 introduces and examines the spectral broadening phenomenon that is predicted and observed. Subsection 5.3.4 discusses the results obtained in each of the three regions of the ICRF dis- 


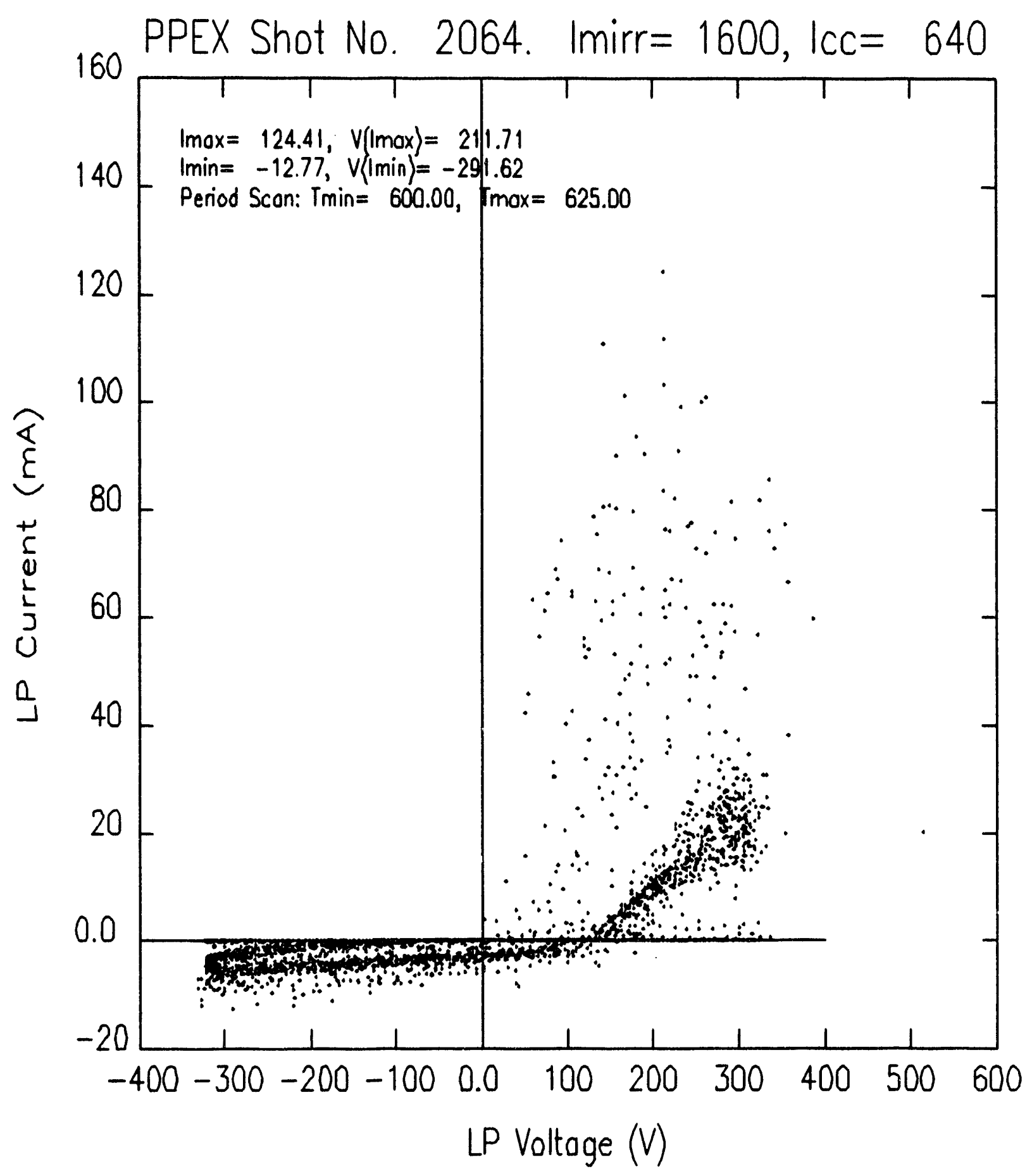

Figure 5-5: Diagnostic data obtained from the CC-Langmuir Probe during a TYPE I ICRF shot. Note the two distinctly different types of data labelled A and $\mathrm{B} . \mathrm{A}$ is the Langmuir probe response during the Ramp-up and Oscillatory periods. B (dense cluster of data points) is the LP response during the Quiescent period. For set $\mathrm{B}, T_{e} \simeq 45 \pm 10 \mathrm{eV}, n_{e}=0.86(+0.11,-0.076) \times 10^{11} \mathrm{~cm}^{-3}$ 
charge as discussed previously in the $\mathrm{H}-\alpha$ Section.

\section{Frequency Spectral Broadening}

One of the predictable effects of plasma loading is the detuning of the resonant tuning circuit by the addition of plasma impedence and resistance. Recall that the $Q$-factor of a tuned circuit is given by

$$
Q=\frac{\omega L}{R}
$$

An additional resistance due to plasma loading will significantly lower the Q-factor and broaden the frequency spectral profile of the ICRF output. Thus, one would expect significant broadening of the ICRF spectra during maximal antenna-plasma coupling.

To observe the spectral broadening phenomenon, a comparison of a vacuum shot to a plasma shot is necessary. The Fourier spectrum of the blank shot is seen in the top half of Figure 5-6. The $3 \mathrm{MHz}$ base ICRF frequency is clearly evident at about 1000 times the amplitude of the largest of the other detectable frequencies. Other non-3MHz-multiple frequencies are clearly also excited in the vacuum, most likely generated by the imperfection of the ICRF amplifier (The Continental Electric Co's Transmitters). Several noteworthy features of the vacuum $B_{z}$ spectrum and are listed below

1. The $3 \mathrm{MHz}$ base ICRF frequency (Also referred to as the primary mode) is prominently excited at a normalized magnitude of 1 .

2. Multiples of the primary $3 \mathrm{MHz}$ mode $(6,9 \mathrm{MHz}$, etc) are also excited, although their amplitudes are much lower than the primary mode. Typically these amplitudes are about $0.1 \%$ of the primary mode amplitude.

3. Non-harmonic modes are generated (See next subsection). 
The Fourier $B_{z}$ spectrum of a plasma shot is seen in the lower half of Figure 5-6. The relative magnitude of the $3 \mathrm{MHz}$ primary mode remains unchanged from the vacuum shot. However, several significant differences can be observed.

1. For the plasma shot, the background level of the Fourier spectrum has increased a factor of 10 over the blank shot.

2. In the presence of a plasma, significant broadening of the vacuum ICRF spectrum was observed. This is hereby referred to as the Log-Q Effect since the broadening is readily apparent not so much on the linear scale as on the logarithmic scale.

\section{NON-HARMONIC MODES}

As observable in the vacuum shot of Figure 5-6, non-harmonic frequencies (nonmultiples of the base $3 \mathrm{MHz}$ frequency) are generated and picked up by the B-dot probe. This is an indication of the imperfection of the ICRF transmitters. An examination of the signal produced from two $-70 \mathrm{~dB}$ couplers on a dummy load shows that this is indeed an artifact that is generated by the ICRF transmitters used and NOT produced by the geometry of the system. The cause of this artifact is the fact that a high power RF amplifier tube operates in Class $\mathrm{C}$ mode with a conduction angle of less than 180 degrees. Thus the amplifier system relies upon an ideal output tuning system to regenerate a perfect sinusoid and tune out other harmonics. However, the tuning system of the transmitters are difficult to tune and are hardly ideal. Thus, some non-harmonic frequencies are also produced, e.g. the mode at $\sim 4 \mathrm{MHz}$ in Figure 5-6. Note that these modes are excited at much lower amplitude than the base frequency, typically at $10^{-4}$ times the $3 \mathrm{MHz}$ amplitude. 

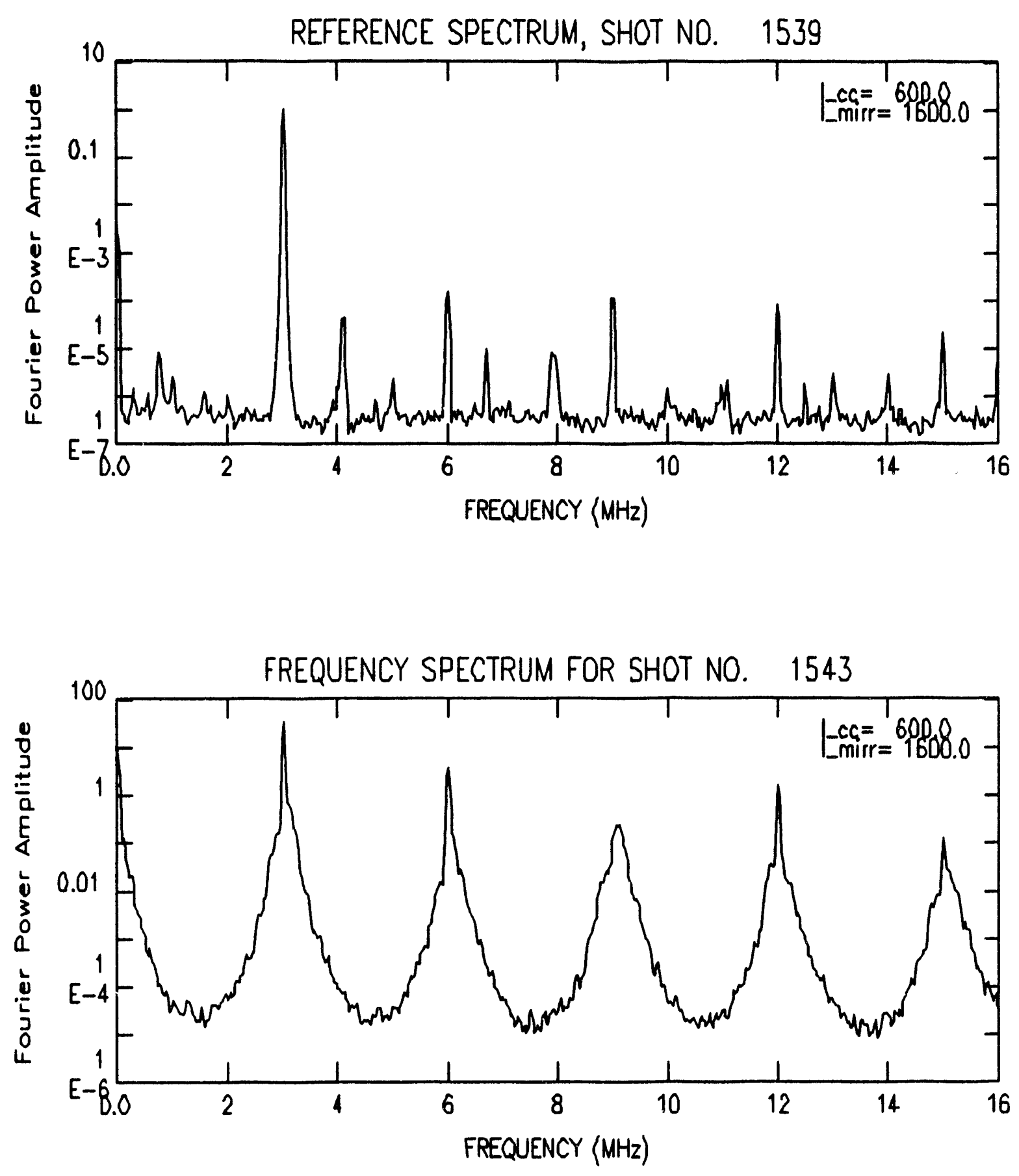

Figure 5-6: Fourier Spectral Broadening of a Plasma 3MHz CC-ICRF Shot. Both the Reference vacuum shot (Top) and the Plasma shot (Bottom) are shown 


\subsection{4 $B_{z}$ Measurements in Three Regions of ICRF dis- charge}

As identified in Section 5.3.1, the ICRF discharge can be temporally separated into three regions: Ramp-up, Oscillation, and Quiescence. In order to obtain self-consistent results, B-dot probe measurements are taken in each of the three different temporal regions. As seen in Figure 5-7, there is significant qualitative difference between the three regions.

The first region is identified as the Ramp-up region. This is the region in which the H- $\alpha$ emission increases linearly with little oscillation. The Fast Fourier Transform (FFT) $[74,75]$ of the ICRF $B_{z}$ as seen in Figure 5-7 shows significant spectral broadening during this phase of the discharge. As explained in Section 5.3.3, this indicates that the wave-plasma coupling is maximum in the Ramp-up region.

The second Oscillatory region is one in which the oscillations in both the light emission and the interferometer measurements are seen to oscillate, initially with very large amplitude and frequency, but with gradually lower amplitude and frequency. The FFT of the ICRF $B_{z}$ obtained for this regime (See Center Figure in Figure 5-7) shows a spectrum with very little broadening lower amplitude than the Ramp-up region.

Finally, the Quiescent region is also seen on Figure 5-7. Again, as for the Oscillatory region, there is minimal spectral broadening. The $3 \mathrm{MHz}$ signal amplitude is seen to be considerably less than that of the Oscillatory region.

Because the broadening is only visible on a logarithmic basis, the $Q$-value of the spectra could not be resolved very accurately due to the limited resolution of the FFT Fourier spectrum. However, it is estimated that $Q>40$ for all the cases and regions examined. This means that given the frequency of the ICRF system $(3 \mathrm{MHz})$ and the inductance of the antenna $(\sim 1 \mu \mathrm{H})$, the plasma resistance is less than $1 \Omega$ for all cases examined. This value of plasma resistance agrees 
well with other studies and experiments $[11,12,4]$. 

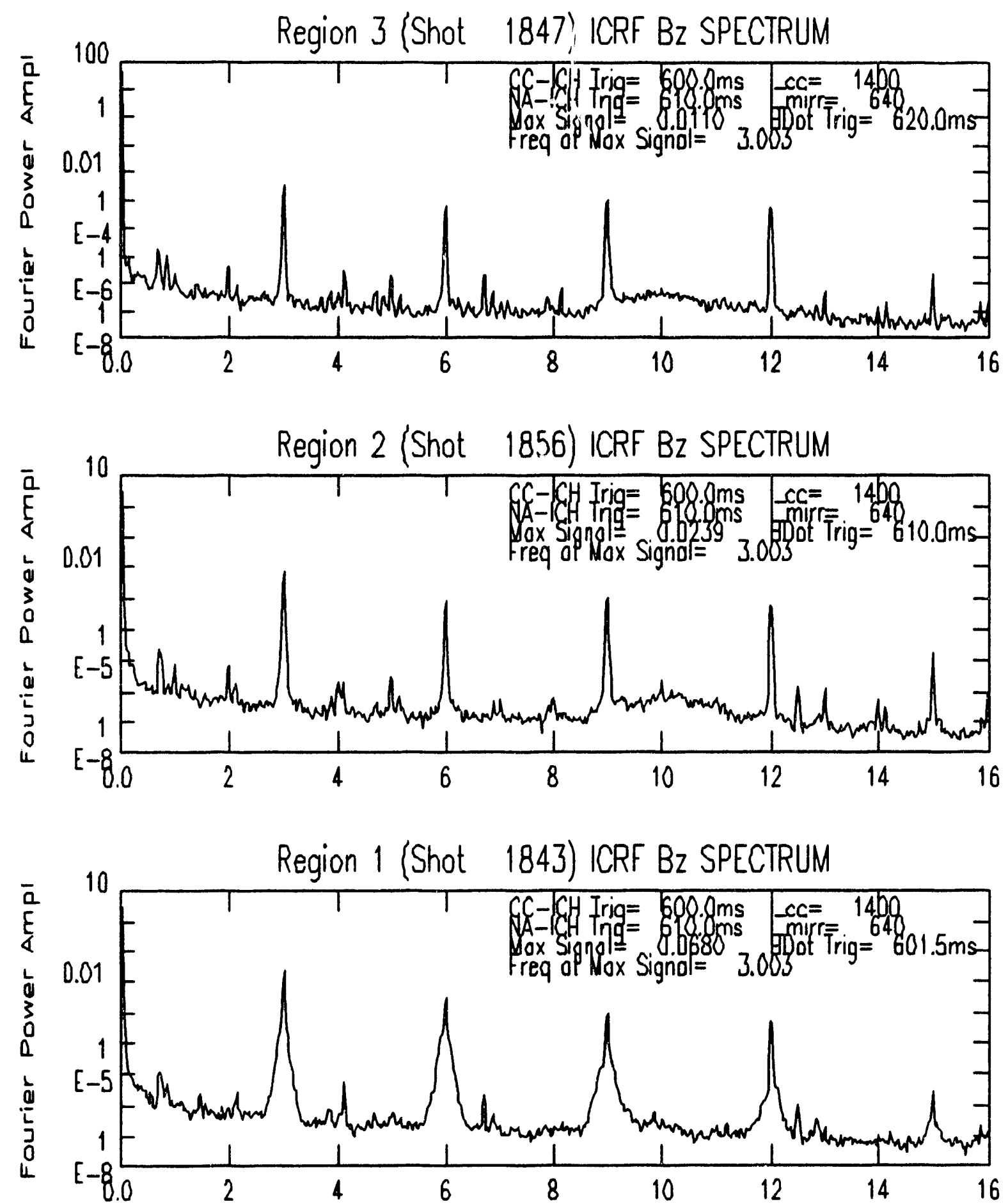

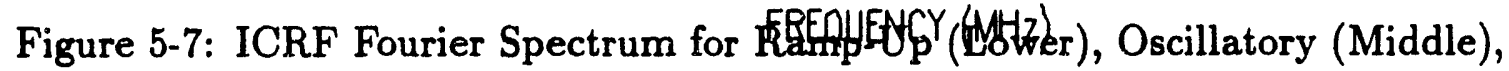
and Quiescence (Upper) Regions for a TYPE I $\left(\omega<\omega_{c i}\right)$ Discharge 
Table 5.1: PPEX Control Parameters

\begin{tabular}{|l|l|l|}
\hline Control & Typical Range & Plasma Properties Affected \\
\hline Neutral Gas Pressure $P_{0}$ & $10^{-5} \rightarrow 10^{-4}$ Torr & $n_{e}, \tau_{C X}$ \\
ECH Switch-On Time & $0-200 \mathrm{~ms}$ & $n_{e}$ \\
ECH Duration & $0-700 \mathrm{~ms}$ & $n_{e}$ \\
ICRF Switch On Time & $300-500 \mathrm{~ms}$ & $\omega_{c i}$ \\
ICRF Duration & $2-100 \mathrm{~ms}$ & $n_{e}, n_{i}, T_{i}, T_{e}$ \\
ICRF Cathode High Voltage & $1-10 \mathrm{kV}$ & $n_{e}, n_{i}, T_{i}, T_{e}$ \\
ICRF Frequency & $3-7 \mathrm{MHz}$ & ICRF Resonance Location \\
CC Coil Current & $120-800 \mathrm{~A}$ & $\omega=\omega_{c i}$ location, $n_{e}$ \\
Mirror Coil Current & $200-2000 \mathrm{~A}$ & $\omega=\omega_{c i}$ location, $n_{e}$ \\
\hline
\end{tabular}


Table 5.2: CC-ICRH Sequence of Events

\begin{tabular}{||l|l|l|l||}
\hline $\begin{array}{l}\text { TIME } \\
(\mathrm{ms})\end{array}$ & EVENT & SETTING & $\begin{array}{l}\text { DURATION } \\
(\mathrm{ms})\end{array}$ \\
\hline 0 & $I_{C C}, I_{M I R R}$ ON & $I_{C C}=680 \mathrm{~A}, I_{M I R R}=1600 \mathrm{~A}$ & 1000 \\
0 & Diamagnetic Loop ON & DAQ at 1-100KHz & 2000 \\
100 & ECH ON & Freq=2.1GHz, Power $=2 \mathrm{KW}$ & 700 \\
580 & Interferometer ON & $\begin{array}{l}\text { Freq=35GHz base, } \\
\Delta f=80 \mathrm{KHz} \text { scanning freq }\end{array}$ & 500 \\
580 & Langmuir Probe DAQ ON & V=士200V & 70 \\
600 & CC-ICH ON & HV=8kV & $10-30$ \\
615 & B-Dot Probe DAQ ON & DAQ at $100 \mathrm{MHz}$ & 0.6 \\
630 & CC-ICH OFF & & \\
\hline
\end{tabular}




\subsection{TYPE II Discharge $-\omega<\omega_{c i}$}

The TYPE II discharge $\left(\omega<\omega_{c i}\right)$ is of greater relevance to the simulations performed in this thesis. Because the waves do not cross the $\omega=\omega_{c i}$ resonance, there will always exist two roots (TE and TM) for each eigenmode. Since the BEACH code expects that both roots to exist, the TYPE II discharge is therefore more similar than TYPE I to the theoretical analysis performed in the previous chapter.

The experimental results obtained for TYPE II discharges will be presented as follows. Section 5.4.1 will present the density results as obtained through the $\mathrm{H}-\alpha$ and interferometry measurements. Section 5.4.2 gives the Langmuir probe results both before and during an ICH discharge to provide edge density and electron temperature measurements. Finally, the B-dot probe measurements will be discussed in the context of the radial scan.

\subsubsection{H- $\alpha$ and Interferometry Results}

Data collected during a typical TYPE II discharge is seen on Figure 5-8 As evidenced by the H- $\alpha$ and interferometry data on Figure 5-8, the TYPE II discharge is much more quiescent than the TYPE I discharge. However, it must be noted that both discharges have a much lower frequency oscillation (period of about $10 \mathrm{~ms}$ ) that is much more pronounced in TYPE II discharge. This oscillation is seen to be caused by the periodic nature of the ECRH heating pulse. Recall that the ECRH heating pulse is a half-rectified sine oscillation with a mean period of about $10 \mathrm{~ms}$. This oscillating source of heating corresponds well to the oscillation in both the density and H- $\alpha$ output. It is thus seen that although the ECRH by itself could not generate large plasma densities, ECRH coupled with ICRF produced a much larger plasma density than could ECRH or ICRF alone.

From the interferometer measurements, the $\omega>\omega_{c i}$ discharges generate densities that are lower than the $\omega<\omega_{c i}$ discharges. Peak line densities obtained from 
the interferometer is approximately $3.2 \pm 0.85 \times 10^{12} \mathrm{~cm}^{-2}$, which corresponds to a plasma density of approximately $1.6 \pm 0.42 \times 10^{11} \mathrm{~cm}^{-3}$. Average line density is lower at approximately $2.6 \pm 0.85 \times 10^{12} \mathrm{~cm}^{-2}$ or about $1.3 \pm 0.42 \times 10^{11} \mathrm{~cm}^{-3}$. The uncertainty is due to both pickup as well as the J1808 phase digitizer's resolution limit of $1 / 16$ fringe.

\subsubsection{Langmuir Probe Results for TYPE II Discharge}

The Langmuir probe measurement can be seen as part of the shot data on Figure 5-8. The Langmuir probe results are seen to be consistent with the $H-\alpha$ and interferometer output in their temporal behavior, i.e. all three diagnostics show peaking when the ECH heating source is on. As seen in Figure 5-8, the abrupt increase in Langmuir probe current occurred with the commencement of the ICRF pulse at $600 \mathrm{~ms}$ and continues until the end of the ICRF pulse at $630 \mathrm{~ms}$. Again, as with the $\mathrm{H}-\alpha$ and interferometry data, the Langmuir probe showed the ECRH modulation of $10 \mathrm{~ms}$.

The Langmuir probe response during ICRF is examined in Figure 5-9. From the Langmuir probe data, the average electron temperature is calculated to be approximately $30 \pm 5 \mathrm{eV}$ The average ICH ion saturation current is seen in Figure 5-9 to be approximately $6 \mathrm{~mA}$. Given this level of $I_{s i}$ and the electron temperature of about $30 \pm 5 \mathrm{eV}$, a rough estimate of the maximum edge density during ICRF discharge can be obtained via

$$
n_{e}=\frac{I_{s i} e^{\frac{1}{2}}}{A, q} \sqrt{\frac{m_{i}}{T_{e}}}
$$

For the probe tip diameter of $1.78 \mathrm{~mm}$, this results in a density of about $n_{e} \simeq$ $1.06(+0.10,-0.08) \times 10^{11} \mathrm{~cm}^{-3}$ at the probe location $\left(r_{\text {probe }}=6.7 \mathrm{~cm}\right)$. This density result is in fairly good agreement with the interferometer's average density (from the previous section) of $1.3 \pm 0.42 \times 10^{11} \mathrm{~cm}^{-3}$. 

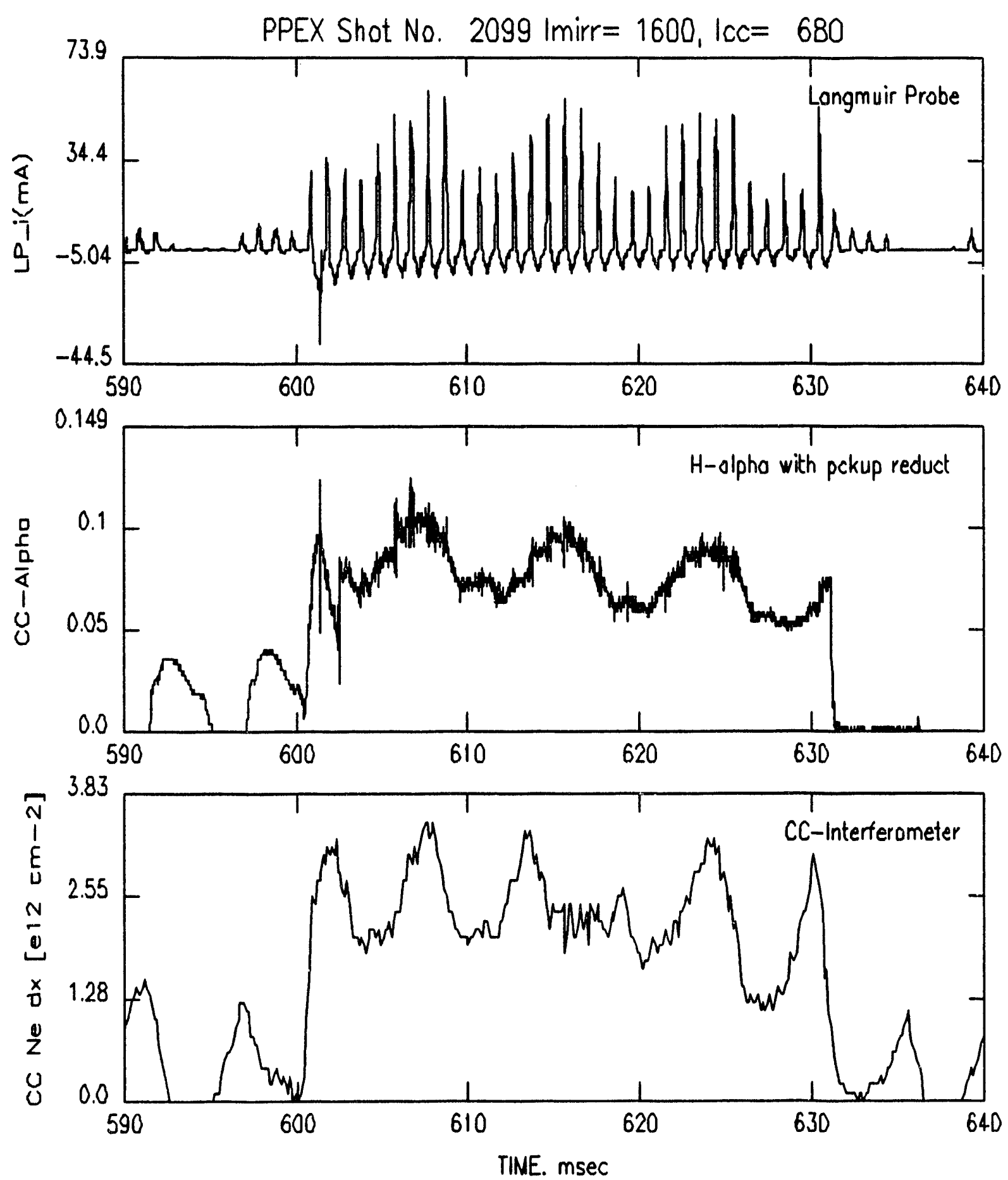

Figure 5-8: Processed Diagnostic results from a TYPE II $\left(\omega>\omega_{c i}\right)$ CC-ICRF shot. Diagnostics are (Top to bottom): Diamagnetic loop voltage, Langmuir probe current(mA), Interferometer line density, and $\mathrm{H}-\alpha$ Detector. Note that for this particular shot, the ICRF power starts at $600 \mathrm{~ms}$ and ends at $630 \mathrm{~ms}$ 


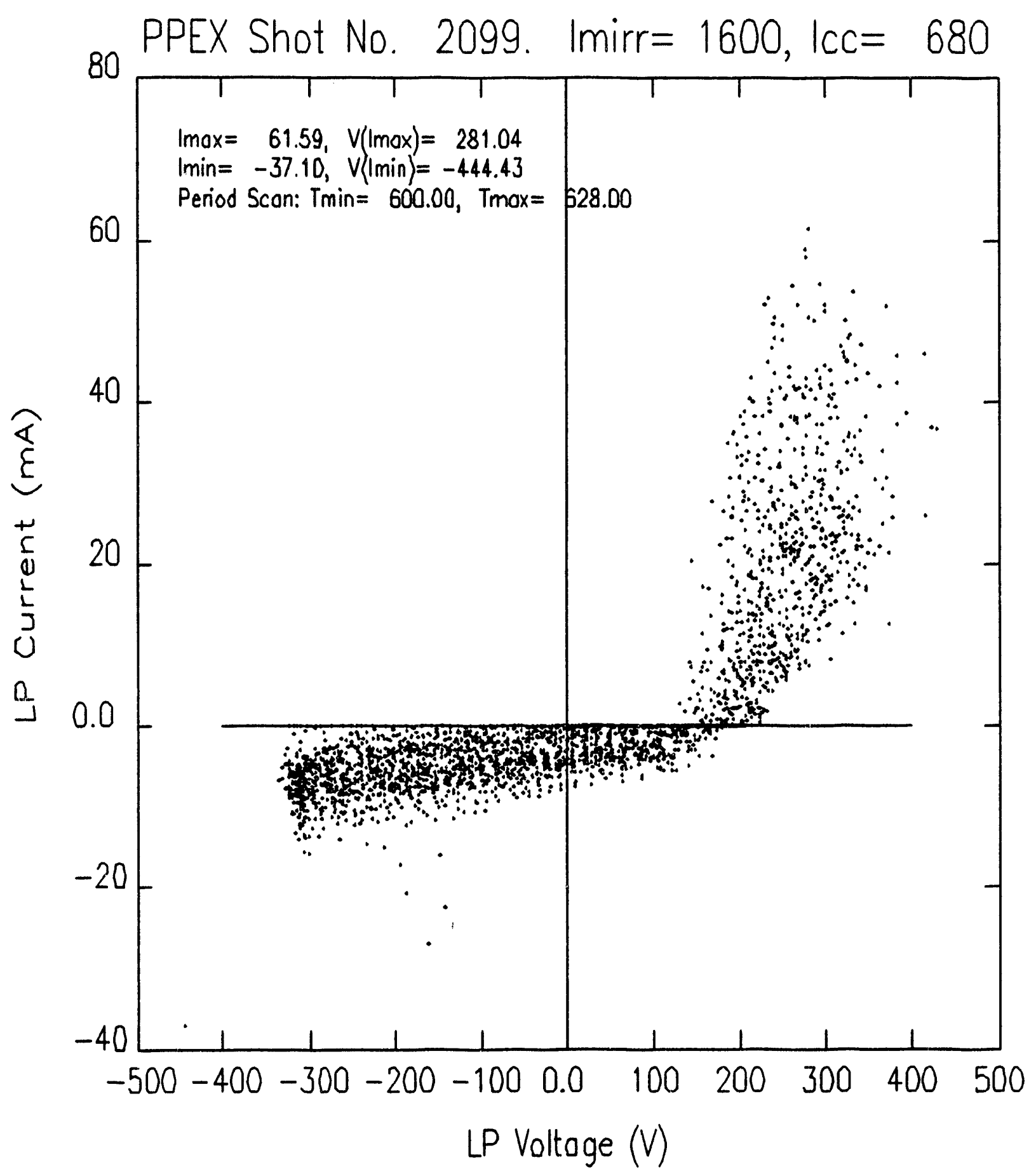

Figure 5-9: Langmuir Probe Data obtained for a TYPE II $\left(\omega>\omega_{c i}\right)$ CC-ICRF shot. The points shown are experimental data obtained during an ICRF shot. Coil Currents are: $I_{\mathrm{cc}}=680 \mathrm{~A}, I_{\text {mirr }}=1600 \mathrm{~A}$. Average $T_{\mathrm{e}} \simeq 30 \pm 5 \mathrm{eV}$, Average $n_{e} \simeq 1.06(+0.10,-0.08) \times 10^{11} \mathrm{~cm}^{-3}$ 


\subsubsection{B-Dot Probe Results - The Radial Scan}

The B-Dot Probe data obtained from a typical CC-ICRH shot is obtained by inserting a B-Dot probe (The details of this particular B-dot probe is given by Chen [25]) into the plasma at a radius of between 0 to $12 \mathrm{~cm}$. The adjustability of the radial location provides flexibility in obtaining radial profiles of the ICH $B_{z}$ field. Because the pickup coils of the B-dot probe used in the PPEX experiment were located in the tip of the probe, multiple shots had to be taken to obtain a radial profile of the ICRF magnetic fields. The radial scan is performed for the TYPE II plasma $\left(\omega<\omega_{c i}\right)$ and not for the TYPE I $\left(\omega>\omega_{c i}\right)$ plasma mainly because the BEACH code simulation assumes that $\omega<\omega_{c i}$. This is done so as to ensure that there are two roots to the solution of the infinite plasma dispersion relation. If $\omega>\omega_{c i}$ in any region of the plasma, the TE wave would be cut off, leaving only the TM wave. Since the BEACH code requires that both TE and TM branches exist, a radial scan is performed in the $\omega<\omega_{c i}$ case for comparison to the BEACH simulation.

Given the rationale for performing the radial field scan during TYPE II discharge, the scan is then performed for two different central cell currents. This is to examine the effect of proximity to resonance on the radial field profile. Because BEACH predicts that the ICRF $B_{z}$ contains a large edge peak that propagates inward as resonance is approached, raising the central cell current effectively moves the resonance further away from the probe located at the $\mathrm{z}=0$ field minimum of the machine. In other words, INCREASING $I_{c c}$ effectively DECREASES $\omega / \omega_{c i}$ at the B-dot probe location $(z=0)$. Thus, what is predicted by BEACH is that increasing $I_{c c}$ causes the edge peak to be located closer to the edge of the plasma.

The result of the ICRF $B_{z}$ radial scan is now presented. Figure 5-10 shows the B-dot probe's pickup of the ICRF $B_{z}$ 's $3 \mathrm{MHz}$ signal at various radial locations. The magnetic coil current settings are $I_{c c}=680 \mathrm{~A}$ and $I_{\text {mirr }}=1600 \mathrm{~A}$, The neutral pressure is maintained at between 5 to $9 \times 10^{-5}$ Torr. The ICRF cathode high voltage was set at approximately $5 \mathrm{kV}$. Length of the ICRF pulses was $30 \mathrm{msec}$. 
Each radial point represents at least 5 shots. The vertical error bars represent the statistical $\sigma$ spread in the experimental results. The horizontal error bar represents both the size of the coil $\left(\operatorname{Diam}=\frac{1}{4}{ }^{\prime \prime}\right)$ and the error in measuring the location of B-dot probe relative to plasma centerline. Each data point is obtained from the processed $3 \mathrm{MHz}$ signal level of the Fourier spectrum of the B-dot probe signal.

The ICRF $B_{z}$ measurements taken by the B-Dot probe for the $I_{c c}=680 \mathrm{~A}$ case are shown on Figure 5-10 plotted against the BEACH code results. The value of $\omega / \omega_{c i}=0.94$ was selected because of the center cell current $I_{c c}$ setting. Since resonance of $\omega / \omega_{c i}=1$ at $3 \mathrm{MHz}$ corresponds to $I_{c c}=640 \mathrm{~A}$, an $I_{c c}$ current of $680 \mathrm{~A}$ corresponds to $\omega / \omega_{c i}$ of 0.94 . The main feature to note on both the simulation results and the experimental results is the presence of a peak near the edge of the plasma. The amplitude of this peak is seen experimentally to be about 3 to 4 times larger than the $B_{z}$ data for the other radial locations. The BEACH code is seen to predict the presence of this peak, although the amplitude of the simulation differs somewhat from that of the experiment. The $B_{z}$ measurements at smaller radii appear to differ from the prediction as well. This difference could be attributed to the larger disturbance to the plasma due to the larger physical presence of the B-Dot probe in the plasma when taking small radii measurements of $B_{\boldsymbol{z}}$. 


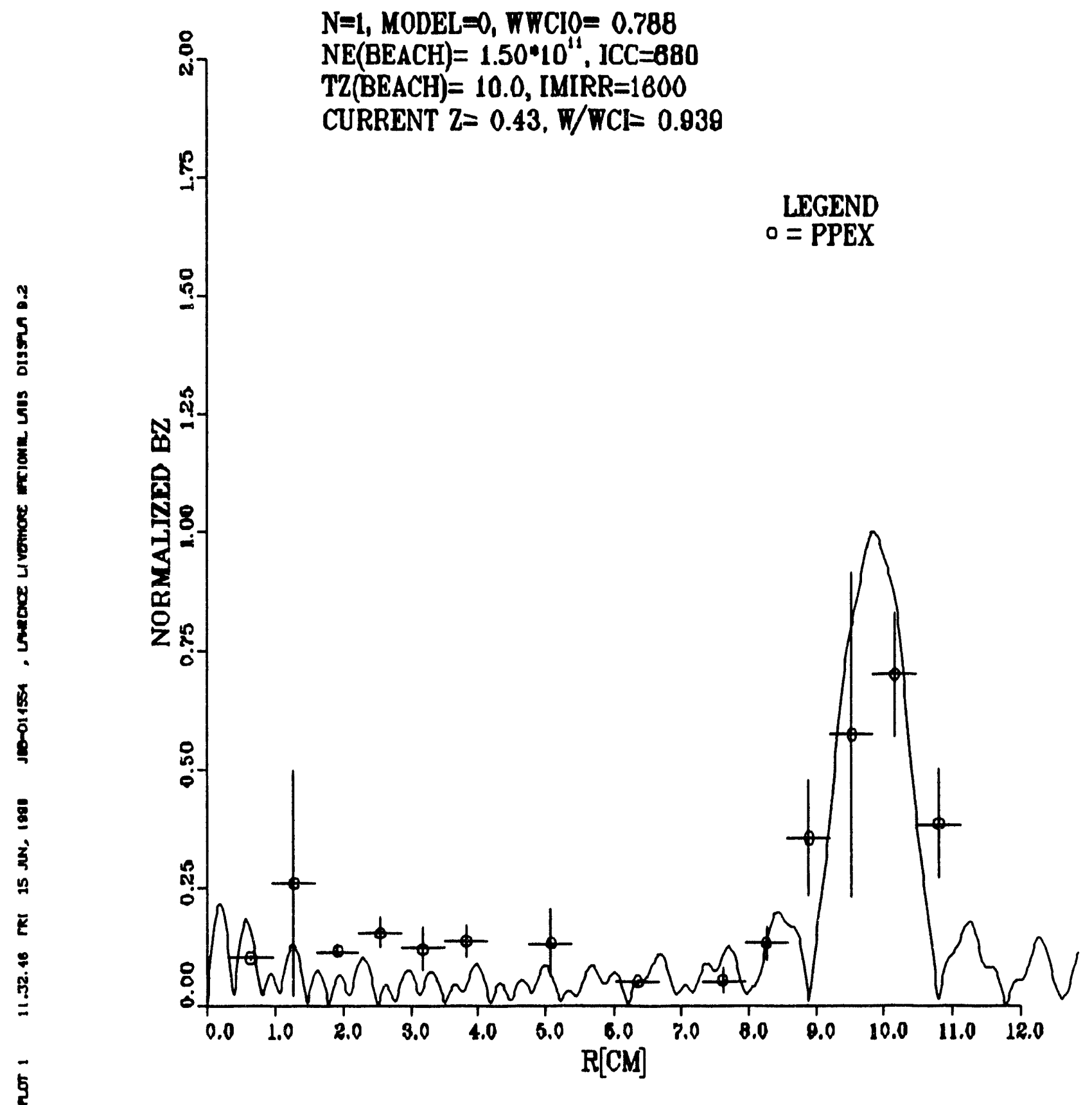

Figure 5-10: PPEX Experimental Results vs. BEACH Predictions. $I_{c c}=680 \mathrm{~A}$, $I_{\text {mirr }}=1600 \mathrm{~A}$. Horizontal error bar includes allowance for finite size $\left(1 / 4^{\prime \prime} \mathrm{Diam}\right)$ of the B-Dot Probe pickup loops as well as for measurement errors. 


\subsection{Summary of Experimental Results}

A magnetic mirror machine, PPEX, was designed, constructed and made operational as part of this thesis. The machine has 3 heating systems - one microwave electron cyclotron heating $(\mathrm{ECH})$ unit $(2.1 \mathrm{GHz})$ in the Center Cell, one ion cyclotron heating unit $(7.5 \mathrm{MHz})$ in the North Anchor, and one ICH unit (3MHz) in the Center Cell. Up to $200 \mathrm{~kW}$ of power is available to each ICH units, while the $\mathrm{ECH}$ unit is capable of putting out $2 \mathrm{~kW}$. Diagnostics currently in place include a Langmuir Probe, a $35 \mathrm{GHz}$ microwave interferometer, an $\mathrm{H}$-alpha detector, a diamagnetic loop, and a B-dot probe. Measurements of the ICRF $B_{2}$ field during a plasma discharge are taken using the B-dot probe to obtain a radial profile of the $B_{z}$ field. The baseline ECH results obtained from $I_{c c}=200 \mathrm{~A}, I_{\text {mirr }}=400 \mathrm{~A}$ produced an electron temperature of $T_{e} \simeq 18 \mathrm{eV}, n_{e}<8 \times 10^{10} \mathrm{~cm}^{-3}$.

For the ICRF discharge, two types of discharges are identified : TYPE I $\left(\omega>\omega_{c i}\right.$ somewhere in the plasma) and TYPE II $\left(\omega<\omega_{c i}\right.$ everywhere in the plasma).

Typical TYPE I discharges are created by setting $I_{c c}=640 \mathrm{~A}, I_{\text {mirr }}=1600 \mathrm{~A}$. Experimental Langmuir probe results obtained during TYPE I discharge indicate that $T_{e} \simeq 45 \pm 10 \mathrm{eV}$ and density of $n_{e} \simeq 0.86(+0.11,-0.076) \times 10^{11} \mathrm{~cm}^{-3}$, while interferometry yields a chord-averaged density of $n_{e}=1 \pm 0.42 \times 10^{11} \mathrm{~cm}^{-3}$. The TYPE I discharge is characterized by an initially large amount of oscillations in the plasma density. The total ICH heating period can be classified into three temporal regions or phases: Ramp-up, Oscillatory, and Quiescence. These 3 regimes are selected because of their characteristic $\mathrm{H}$-alpha and density output. During the Ramp-up phase, the $\mathrm{H}$-alpha output grows linearly without much noise. After reaching a plateau, the Oscillation regime is entered, characterized initially by large amplitude (up to $20 \%$ of the plateau level) and high frequency ( 10K oscillations which gradually decrease in both amplitude and frequency. After a certain point, the oscillations cease abruptly, followed by a Quiescent phase of 
quiescent plateau, ending with the shut-off of the ICH heating power.

ICRF $B_{z}$ obtained via the B-dot probe is taken in each of the three different phases identified above. Significant differences in their spectral characteristics are noticeable. In region 1 (Ramp-up), the $B_{z}$ signal appears to be growing and the fundamental $3 \mathrm{MHz}$ frequency is very much broadened. The data obtained during the Oscillatory phase indicate that the $B_{z}$ signals have plateaued and show signs of losing the spectral broadening observed during the initiation phase. Finally, phase 3 (Quiescence) $B_{z}$ show that the amplitude has indeed plateaued and may even start decreasing. However, it has not been established whether this decrease is due to the $e^{-t / \tau}$ decay of the RF amplifier's high voltage capacitors, or due to wave-plasma coupling. The spectral broadening observed in phase 1 is almost nonexistent in phase 3. Based upon these results, it is believed that maximum ICRF wave-plasma coupling occurred in the Ramp-Up region.

For the TYPE II discharge, the electron temperature obtained from Langmuir probe measurements is $T_{e} \simeq 30 \pm 5 \mathrm{eV}$, and the density was found to be approximately $n_{e} \simeq 1.06(+0.10,-0.08) \times 10^{11} \mathrm{~cm}^{-3}$. Again, the Langmuir probe density results are in good agreement with the interferometer result which yields a chord-averaged density of $1.3 \pm 0.42 \times 10^{11} \mathrm{~cm}^{-3}$.

A radial scan of $B_{z}$ is performed on the PPEX machine to examined the $B_{z}(r)$ radial profile. The scan was performed for the standard magnet coil current setting of $I_{c c}=680 \mathrm{~A}$ and $I_{\text {mirr }}=1600 \mathrm{~A}$. The major characteristic observed in the radial scan was an edge peak, which was predicted by the BEACH code. The BEACH code results produced a very good fit to the experimental data. 


\section{Chapter 6}

\section{Conclusions and}

\section{Recommendations}

\subsection{Conclusions}

This thesis examines the characteristics of the ICRF propagating wave in a magnetic mirror geometry. The su dy consists of three parts - an analytical study, a multi-eigenmode expansion computational study, and an experimental study. Some conclusions can be drawn from the studies performed in this thesis.

\subsubsection{Analytical Study}

A theoretical analysis was undertaken in Chapter 3. The unique and new contribution of this analysis is in its inclusion of both the nonuniform axial magnetic field and a cylindrical geometry in its solution of the wave-plasma coupling problem. The analysis also treated the resonance poles in a coherent manner that permitted the examination of the entire plasma-vacuum system in a global manner that included the resonance poles. The analysis assumes a dipole loop as the ICRF antenna and includes the "almost TE mode" as the field considered (the "almost TM" mode is NOT considered). The results of this study presented in 
Chapter 3 show that the ICRF waves are indeed damped as they leave the antenna and propagate towards the resonant beach. This damping is more pronounced if the resonance is located in the nuachine. Examination of two cases - one with the ICRF resonance inside the machine, one outside - shows that there is significant damping associated with the presence of a resonance inside the machine. The case with resonance in the machine has very little oscillations in the ICRF $B_{z}$

field as the wave propagates away from the antenna, while the case with resonance OUTSIDE the machine shows considerable axial oscillations away from the antenna.

Plasma coupling impedence $R_{p l}$ was observed to rise as the mirror ratio is decreased. It is postulated that this is due to the increased volume of plasma close to resonance as the slope of the field is lowered. A scan of a normalized resonance-volume versus the mirror ratio was performed and compared to a scan of the plasma coupling impedence $R_{p l}$ versus the mirror ratio. The result appear to confirm this assumption. The two curves show surprisingly good although not total agreement. The difference could be attributed to many factors such as the changing source fields experienced by the resonant volume as the mirror ratio is lowered.

The behavior of plasma coupling impedence versus the resonance location $\xi_{\text {res }}$ was examined. It was seen that the plasma coupling impedence peaked in two locations: when the resonance is directly under the antenna, and when the resonance is in the center of the machine. Thus an optimum heating condition, from a wave-propagation point of view, would be to have the antenna located directly above the resonance.

\subsubsection{Computational ICRF Study}

The multi-eigenmodal analysis uses a different approach from the analytical study of the previous chapter in its modeling of the axial inhomogeneity. The approach simulated the inlomogeneous axial magnetic field by separating the cylindrical 
plasma-vacuum systems into axial slices and assuming a homogeneous field in each slice. The analysis includes collisions as the damping source. The multimodal analysis found

1. Higher density results in larger $k_{z}$ eigenmodes with slightly higher REAL parts $k_{z, R e}$ and much larger imaginary parts $k_{z, i m}$. This larger $k_{z, i m}$ results in significantly increased damping for higher density. Thus, it is recommended that higher density operation will enhance power transfer from the waves to the plasma.

2. Lower Temperatures do not change the real $k_{z}$ wave number much (5\% at most), but generates much larger imaginary $k_{z, i m}$, causing the ICRF waves to damp out more and coupling more energy to the plasma. Thus, lower temperature is seen to allow more ICRF power coupling to the plasma. This finding, however, is only valid in the context of a cold plasma approximation $\left(T_{\|} \leqslant 100 \mathrm{eV}\right)$.

3. There is an optimal $\omega / \omega_{c i}$ for launching ICRF waves. This optimal $\omega / \omega_{c i}$ depends mainly upon the $k_{z}$ spectrum of the launching mechanism, i.e. antenna. For the Full and Dual Half Turn Loop antenna, it was found that lower launch $\omega / \omega_{c i}$ is more suitable for coupling to the ICRF waves in that it allowed the eigenmodes to be excited at higher levels by the antenna power spectrum $\vec{J}\left(r, n, k_{z}\right)$.

The results of the $\mathrm{BEACH}$ code are also benchmarked against the experimental results obtained on the B-66 machine by Uman [3]. It is seen that a qualitative fit to the experimental data could be obtained, although the BEACH code uses lower temperatures than the B-66 experimental results in order to obtain adequate profile agreement.

The results of the BEACH code were compared to that of the CYLWAVE for a full-turn loop. Although some differences were seen, the major agreement is 
that both codes predict a decay as the waves propagate towards resonance. It is difficult to compare the results of both codes because of the different antenna geometries (full-turn loop for BEACH, dipole loop for CYLWAVE), and different physics (both TE and TM modes for BEACH, only TE mode for CYLWAVE) that separate these two codes.

\subsubsection{Experimental Results}

The experimental phase includes the construction of a linear magnetic mirror. The PPEX machine was brought from the design stage to the current data acquisition stage. One ECRH and two ICRF systems are employed for plasma heating. Two types of discharges are identified. TYPE I $\left(\omega>\omega_{c i}\right)$ and TYPE II $\left(\omega<\omega_{c i}\right)$. The TYPE I discharges is seen to be separable into three distinct temporal regions during ICRF discharge - Ramp-up, Oscillatory, and Quiescent. The ICRF spectrum obtained during the Ramp-up showed significantly more spectral broadening than in either the Oscillatory and the Quiescent regions. Because spectral broadening is also an indication of the degree of detuning of the frequency resonant system, the large spectral broadening indicates introduction of maximal plasma resistance. This plasma resistance allows coupling of energy from the antenna to the plasma. Hence, the largest wave-plasma power coupling occurs during the Ramp-up regime.

The experimental work conducted for TYPE II discharges involves obtaining a set of radial ICRF $B_{z}$ measurements near resonance. A B-Dot probe is used to scan multiple radial locations. The results from the probe show a large peak in the $B_{z}$ at the edge of the plasma which is seen to agree well with the BEACH predicted profile. BEACH simulations show that the ICRF edge field peaks were more prominent for lower density plasmas than higher density plasmas. This could be explained by examining the role of density in shorting out the parallel electric field. The higher the density, the easier it is to shield out the parallel electric field, hence the smaller the $E_{z}$. Because the $E_{z}$ fields constitute the TM modes, or the 
edge modes as described in Chapter 3, the lower density plasmas would have a larger fraction of the fields being made up of these TM Edge modes. Thus, lower density plasmas are seen to have a larger edge component of the ICRF fields than higher density plasmas.

\subsubsection{General Conclusion}

In conclusion, this thesis attempts to model ICRF propagation and to verify the modeling experimentally. A new analytical method of ICRF analysis is proposed and developed as part of this thesis which includes both the inhomogeneous axial magnetic field as well as the cylindrical geometry. A multimodal analysis is also undertaken which shows that many modes are required to adequately simulate the propagating $B_{z}$ field profiles. Both theoretical analyses show that achieving opirimal coupling requires the optimization of density, temperature, mirror ratio, and resonance and antenna locations. The two theoretical analyses examined in Chapters 3 and 4 each has its own advantages and disadvantages. The ultimate goal is to reconcile both approaches to include the advantages of both.

The experimental results obtained from the PPEX machine that was constructed as part of this thesis appear to qualitatively agree with the BEACH analysis predictions. The PPEX radial $B_{z}$ results agree very well with the BEACH code, while the experimental axial $B_{z}$ profiles from the B-66 machine agree qualitatively well with $\mathrm{BEACH}$ predictions. 


\subsection{Recommendations for Further Studies}

\subsubsection{Theoretical - Analytical (CYLWAVE)}

The theoretical approach put forth in this thesis in Chapter 3 is an elegant new method of including both an inhomogeneous axial magnetic field and a cylindrical geometry in the solution of ICRF propagation and heating problems. Several changes and modifications could be made to improve the sophistication of this approach. These include

1. Inclusion of finite temperature effects. The dielectric tensor would be modified for inclusion of finite temperature effects. This would permit a more realistic simulation of finite temperature plasmas.

2. Inclusion of almost-TM mode $\left(E_{z} \neq 0\right)$. This would result in a set of FOUR coupled integral-differential equations instead of TWO. However, the general approach would remain unaltered. This would permit simulation of both the almost-TE and almost-TM modes.

3. Simulation of more realistic antenna geometries. This would allow the code to be able to simulate actual experiments. Antennas such as the full turn loop, dual half-turn loop, and other more exotic ones could be simulated.

\subsubsection{Theoretical - Computational (BEACH)}

The BEACH code could be improved by the addition of

1. Finite temperature effects. Again, the elements of the dielectric tensor would have to be modified.

2. Examination of resonance. Further examination of the behavior of waves up to and through resonance should be examined. This would require an extensive reformulation of the WKB formalism used. 


\subsubsection{Experimental}

The PPEX is an extremely versatile machine that is ideally suited for studying atomic and plasma physics phenomena. It is ideal for the experimentalist because it is small enough to be run by only two people, yet powerful enough to do interesting physics studies. The experiments conducted in this thesis have only scratched the surface of the capabilities of this machine. From the experimental results obtained during the ICRF runs, several interesting phenomena have been identified will be briefly discussed below as possible projects for future studies.

\section{Plasma Broadening of ICRF Spectra}

One of the results obtained during this thesis is the broadening (by the plasma) of the ICRF frequency spectra during a shot. Although the PPEX team has identified this as indicative of maximal ICRF coupling to the plasma, the cause of this phenomenon is still unknown. Much work could be done to uncover the exact mechanism of this ICRF spectra broadening.

\section{Oscillations During TYPE I ICH Discharge}

The H-alpha detector produced some interesting data during some $\mathrm{ICH}$ discharges. On some shots, the $\mathrm{H}$-alpha light grew up to a plateau, then oscillations in the light level began to grow until about $20 \%$ of the peak value, then at some point abruptly ends, resulting in a quiescent plateau level of H-alpha light output which dies off upon the shut-off of the ICH heating power. Many postulates have been made by the PPEX team as to the exact nature of this oscillation, but more studies and diagnostics would have to be in place to determine the exact mechanism and cause of this oscillation. 
Blowout of the plasma at high RF cathode voltage

When the plate voltage of the ICRF amplifier was set at above $6 \mathrm{kV}$, it was seen that the discharge would initiate, but after one milisecond would extinguish. This effect is similar to the "candle effect" that was observed by Goodman [76] on Constance. It would be useful to find out the exact mechanism of this blowout. 


\section{Appendix A}

\section{Useful Plasma Definitions}

\section{A.1 Plasma Dispersion Function $Z(\xi)$}

The plasma dispersion function $Z(\xi)$ was first tabulated by Fried and Conte[46] and has been extended by Landau and others $[77,78]$ and is defined by

$$
Z(\xi)=\pi^{-\frac{1}{2}} \int_{-\infty}^{+\infty} d t \frac{e^{-t^{2}}}{t-\xi}=2 i \exp \left(-\xi^{2}\right) \int_{-\infty}^{i \xi} d t \exp \left(-t^{2}\right)
$$

where $\xi \equiv \frac{v_{p h}}{v_{t h}}=\frac{\omega}{k v_{t h}}$

The derivative of $Z(\xi)$ can be shown to be

$$
\frac{d Z}{d \xi}=-2(1+\xi Z)
$$

The plasma dispersion function also has the following properties

$$
\begin{gathered}
Z(0)=i \pi^{\frac{1}{2}} \\
Z(x)=\exp \left(-x^{2}\right)\left(i \pi^{\frac{1}{2}}-2 \int_{0}^{x} d t \exp \left(t^{2}\right)\right) \\
Z(i y)=i \pi^{\frac{1}{2}} \exp \left(y^{2}\right)[1-\operatorname{erf}(y)]
\end{gathered}
$$

The series expansion of $Z(\xi)$ are : 
1. Power Series (Small Argument $\xi$ )

$$
Z(\xi)=i \pi^{\frac{1}{2}} \exp \left(-\xi^{2}\right)-2 \xi\left(1-\frac{2 \xi^{2}}{3}+\frac{4 \xi^{4}}{15}-\frac{8 \xi^{8}}{105}+\ldots\right)
$$

2. Asymptotic Series $(\xi \gg 1)$

$$
Z(\xi)=i \pi^{\frac{1}{2}} \sigma \exp \left(-\xi^{2}\right)-\xi^{-1}\left(1+\frac{1}{2 \xi^{2}}+\frac{3}{4 \xi^{4}}+\frac{15}{8 \xi^{8}}+\ldots\right)
$$

where

$$
\sigma= \begin{cases}0 & y \\ 1 & |y|<|x|^{-1} \\ 2 & y<|x|^{-1} \\ & <-|x|^{-1}\end{cases}
$$

\section{A.2 The Plasma Dielectric Tensor}

The elements of the plasma dielectric tensor given here are derived in the kinetic limit as shown by Ichimaru [79]. The plasma dielectric tensor $\underline{\underline{\epsilon}}$ is given by

$$
\underline{\underline{\epsilon}} \equiv\left[\begin{array}{ccc}
S & -i D & 0 \\
i D & S & 0 \\
0 & 0 & P
\end{array}\right]
$$

whose elements are defined as:

$$
\begin{gathered}
S=\frac{1}{2}(R+L) \\
D=\frac{1}{2}(R-L) \\
R=1+\sum_{\alpha} \frac{\omega_{p \alpha}^{2}}{\omega k_{z} v_{\alpha}} Z\left(\xi_{1}\right) \\
L=1+\sum_{\alpha} \frac{\omega_{p \alpha}^{2}}{\omega k_{z} v_{\alpha}} Z\left(\xi_{-1}\right)
\end{gathered}
$$




$$
\begin{gathered}
P=1-\sum_{\alpha} \frac{\omega_{p \alpha}^{2}}{\left(k_{z} v_{\alpha}\right)^{2}} \frac{Z^{\prime}\left(\xi_{0}\right)}{1+\frac{i{ }_{0}}{k_{z} v_{\alpha}} Z\left(\xi_{0}\right)} \\
\xi_{n}=\frac{\omega+i \nu_{\alpha}+n \omega_{c \alpha}}{k_{z} v_{\alpha}}
\end{gathered}
$$

Where $\nu_{\alpha}$ is the collision frequency using the Krook collisional factor. $Z\left(\xi_{n}\right)$ is given in the previous section.

\section{A.2.1 Expansion of the Plasma Dielectric Tensor for a cold collisional plasma}

For a cold collisional plasma, the elements of the dielectric tensor can be expanded by taking expansions of the plasma dispersion function. In the cold plasma limit, $k_{\|} v_{t i} \ll \omega$, therefore, $\xi=$ Large. So taking the expansion of $Z(\xi)$ for large $\xi$,

$$
Z(\xi)=i \sqrt{\pi} \epsilon e^{-\xi^{2}}-\xi^{-1}\left(1+\frac{1}{2 \xi}+\frac{3}{4 \xi^{4}}+\cdots\right)
$$

Recall that $D$ and $S$ are defined as

$$
\begin{aligned}
& D \equiv \frac{1}{2}(R-L) \\
& S \equiv \frac{1}{2}(R+L)
\end{aligned}
$$

Solving for D:

$$
D=\frac{1}{2}\left[\sum_{\alpha} \frac{\omega_{p \alpha}^{2}}{\omega k_{z} v_{\alpha}}\left(Z\left(\xi_{1}\right)-Z\left(\xi_{-1}\right)\right)\right]
$$

Expanding $Z\left(\xi_{1}\right)-Z\left(\xi_{-1}\right)$

$$
Z\left(\xi_{1}\right)-Z\left(\xi_{-1}\right) \simeq-\frac{1}{\xi_{1}}+\frac{1}{\xi_{-1}}-\frac{1}{2 \xi_{1}^{3}}+\frac{1}{2 \xi_{-1}^{3}}+\cdots
$$

It can be shown that

$$
D \simeq \frac{\omega_{p i}^{2} \omega_{c i}}{\omega} \frac{\omega^{2}-\omega_{c i}^{2}-\nu_{i}^{2}-2 i \omega \nu_{i}}{\left(\omega^{2}-\omega_{c i}^{2}-\nu_{i}^{2}\right)^{2}+4 \omega^{2} \nu_{i}^{2}}
$$

Two limits can then be constructed: $\omega \neq \omega_{c i}$, and $\omega \rightarrow \omega_{c i}$. 
$\underline{\omega} \neq \omega_{c i}$

For this case, since $\omega \gg \nu_{i}$, drop all terms of order $O\left(\nu_{i}^{2} / \omega^{2}\right)$ :

$$
D \simeq \frac{\omega_{p i}^{2} \omega_{c i}}{\omega}\left(\frac{\omega^{2}-\omega_{c i}^{2}-2 i \omega \nu_{i}}{\left(\omega^{2}-\omega_{c i}^{2}\right)^{2}}\right)
$$

Separating into Real and Imaginary components, where

$$
\begin{gathered}
D \equiv D_{R e}+i D_{i m} \\
D_{R e}=\frac{\omega_{p i}^{2} \omega_{c i}}{\omega\left(\omega^{2}-\omega_{c i}^{2}\right)} \\
D_{i m}=\frac{-2 \nu_{i} \omega_{p i}^{2} \omega_{c i}}{\omega\left(\omega^{2}-\omega_{c i}^{2}\right)^{2}}
\end{gathered}
$$

$\underline{\omega} \rightarrow \omega_{c i}$

In this limit, it is seen that $\left(\omega^{2}-\omega_{c i}^{2}\right) \rightarrow 0$, and thus

$$
D=\frac{\omega_{p i}^{2} \omega_{c i}}{\omega} \frac{-n u_{i}^{2}-2 i \omega \nu_{i}}{\nu_{i}^{4}+4 \omega^{2} \nu_{i}^{2}}
$$

Dropping terms to $\mathrm{O}\left(\nu_{i}^{2} / \omega^{2}\right)$

$$
D \simeq \frac{\omega_{p i}^{2} \omega_{c i}}{\omega} \frac{\left(1+\frac{2 i \omega}{\nu_{i}}\right)}{4 \omega^{2}}
$$

Separating into components $D \equiv D_{R e}+i D_{i m}$

$$
\begin{aligned}
& D_{R e}=\frac{-\omega_{p i}^{2} \omega_{c i}}{4 \omega^{3}} \\
& D_{i m}=\frac{-2 \omega_{p i}^{2} \omega_{c i}}{4 \nu_{i} \omega^{2}}
\end{aligned}
$$

Next, solve for $\mathbf{S}$ in these limits

$$
S=1+\frac{1}{2}\left[\sum_{\alpha} \frac{\omega_{p \alpha}^{2}}{\omega k_{z} v_{\alpha}}\left(Z\left(\xi_{1}\right)+Z\left(\xi_{-1}\right)\right)\right]
$$


Expanding $Z\left(\xi_{1}\right)+Z\left(\xi_{-1}\right)$

$$
Z\left(\xi_{1}\right)-Z\left(\xi_{-1}\right) \simeq-\frac{1}{\xi_{1}}-\frac{1}{\xi_{-1}}-\frac{1}{2 \xi_{1}^{3}}-\frac{1}{2 \xi_{-1}^{3}}+\cdots
$$

Keeping terms to $O(1 / \xi)$

$$
Z\left(\xi_{1}\right)+Z\left(\xi_{-1}\right) \simeq \frac{-2 k_{\|} v_{t}}{D_{D}}\left\{\omega\left(\omega^{2}-\omega_{c i}^{2}+\nu_{i}^{2}\right)-i \nu_{i}\left(\omega^{2}-\omega_{c i}^{2}+\nu_{i}^{2}\right)\right\}
$$

where $D_{D}$ is defined as

$$
D_{D} \equiv\left(\omega^{2}-\omega_{c i}^{2}-\nu_{i}^{2}\right)^{2}+4 \omega^{2} \nu_{i}^{2}
$$

Therefore $\mathrm{S}$ is

$$
S=1+\sum_{\alpha} \frac{\omega_{p \alpha}}{\omega D_{D}}\left\{\omega\left(\omega^{2}-\omega_{c i}^{2}+\nu_{i}^{2}\right)-i \nu_{i}\left(\omega^{2}+\omega_{c i}^{2}+\nu_{i}^{2}\right)\right\}
$$

Now taking limits

$\underline{\omega \neq \omega_{c i}}$

In this limit, we can drop $O\left(\nu_{i}^{2} / \omega^{2}\right)$ and obtain

$$
S=1-\sum_{\alpha} \omega_{p \alpha} \frac{\left[\omega\left(\omega^{2}-\omega_{c i}^{2}\right)-i \nu_{i}\left(\omega^{2}+\omega_{c i}^{2}\right)\right]}{\omega\left(\omega^{2}-\omega_{c i}^{2}\right)^{2}}
$$

Separating into real and imaginary components : $S=S_{R e}+i S_{i m}$

$$
\begin{gathered}
S_{R e}=1-\sum_{\alpha} \frac{\omega_{p \alpha}^{2}}{\left(\omega^{2}-\omega_{c i}^{2}\right)} \\
S_{i m}=\sum_{\alpha} \frac{\omega_{p \alpha}^{2}}{\left(\omega^{2}-\omega_{c i}^{2}\right)} \frac{\nu_{i}}{\omega} \frac{\omega^{2}+\omega_{c i}^{2}}{\omega^{2}-\omega_{c i}^{2}}
\end{gathered}
$$

$\underline{\omega \rightarrow \omega_{c i}}$

In this limit, we cannot drop $O\left(\nu_{i}^{2} / \omega^{2}\right)$ until we have eliminated the $\omega^{2}-\omega_{c i}^{2}$ terms. It can be shown that

$$
S=1-\sum_{\alpha} \frac{\omega_{p \alpha}}{\omega^{2}}\left\{\frac{1}{4}-i \frac{\nu_{i}\left(\omega^{2}+\omega_{c i}^{2}\right)}{4 \omega \nu_{i}^{2}}\right\}
$$


Separating into components $S=S_{R e}+i S_{i m}$

$$
\begin{gathered}
S_{R e}=1-\sum_{\alpha} \frac{\omega_{p \alpha}^{2}}{4 \omega^{2}} \\
S_{i m}=\sum_{\alpha} \frac{\omega_{p \alpha}^{2}\left(\omega^{2}+\omega_{c i}^{2}\right)}{4 \omega^{3} \nu_{i}}
\end{gathered}
$$


The derivation of elements of the plasma dielectric tensor in the cold plasma collisional limit is presented here. The results derived show that including the collisional frequency results in the coupling of the ion and electron terms which invalidates the simple substitution of $m \rightarrow m(1+i \nu / \omega)$. Note that the collisional frequencies used are given by the NRL Plasma Formulary [80] as

$$
\begin{gathered}
\nu_{e}=2.91 \times 10^{-6} n_{e} \ln \Lambda T_{a}^{-3 / 2} \sec ^{-1} \\
\nu_{i}=4.80 \times 10^{-8} Z^{4} \mu^{-1 / 2} n_{i} \ln \Lambda T_{i}^{-3 / 2} \sec ^{-1}
\end{gathered}
$$

where $n_{e}, n_{i}$ are given in $\mathrm{cm}^{-3}, T_{i}, e$ are given in $e V$, and $\mu \equiv m_{i} / m_{p}$.

The continuity equation for species $j$ is given by

$$
m_{j} n_{j} \frac{\partial \overrightarrow{v_{j}}}{\partial t}=n_{i} q_{j}\left(\vec{E}+\overrightarrow{v_{j}} \times \overrightarrow{B_{0}}\right)-\nabla P_{j}-m_{j} n_{j} \nu_{j h}\left[\overrightarrow{v_{j}}-\overrightarrow{v_{h}}\right]
$$

In the cold plasma limit $\left(\nabla P_{j} \simeq 0\right)$

Ion

$$
m_{i} n_{i} \frac{\partial \vec{v}_{i}}{\partial t}=n_{i} q_{i}\left(\vec{E}+\overrightarrow{v_{i}} \times \overrightarrow{B_{0}}\right)-m_{i} n_{i} \nu_{i e}\left[\overrightarrow{v_{i}}-\overrightarrow{v_{e}}\right]
$$

Electron

$$
m_{e} n_{e} \frac{\partial \vec{v}_{e}}{\partial t}=n_{e} q_{e}\left(\vec{E}+\vec{v}_{e} \times \vec{B}_{0}\right)-m_{e} n_{e} \nu_{e i}\left[\vec{v}_{e}-\vec{v}_{i}\right]
$$

Substituting in for the plasma current $\vec{J}$, where

$$
\vec{J} \equiv e n\left(\overrightarrow{v_{i}}-\overrightarrow{v_{e}}\right)
$$

Separating into components

Ions

$\hat{\mathbf{x}} \quad-i \omega v_{i x}=\frac{q_{i}}{m_{i}}\left(E_{1 x}+v_{i y} B_{0}\right)-\nu_{i e} J_{x} / e n$

$\hat{\mathbf{y}} \quad-i \omega v_{i y}=\frac{g_{i}}{m_{i}}\left(E_{1 y}-v_{i x} B_{0}\right)-\nu_{i e} J_{y} / e n$

$\hat{\mathbf{z}} \quad-i \omega v_{i z}=\frac{g_{i}}{m_{i}} E_{1 z}-\nu_{i e} J_{z} / e n$ 


\section{Electrons}

$\hat{\mathbf{x}} \quad-i \omega v_{e x}=\frac{q_{e}}{m_{e}}\left(E_{1 x}+v_{e y} B_{0}\right)+\nu_{e i} J_{x} / e n$

$\hat{\mathbf{y}} \quad-i \omega v_{e y}=\frac{q_{e}}{m_{e}}\left(E_{1 y}-v_{e x} B_{0}\right)+\nu_{e i} J_{y} / e n$

$\hat{\mathbf{z}} \quad-i \omega v_{e z}=\frac{g_{e}}{m_{e}} E_{1 z}+\nu_{e i} J_{z} / e n$

Combining the ion $\hat{x}$ and $\hat{y}$ equations yield

$$
V_{i x}\left(-\omega^{2}+\Omega_{i}^{2}\right)=-\frac{i \omega q_{i}}{m_{i}} E_{1 x}+\frac{q_{i}}{m_{i}} \Omega_{i} E_{1 y}-\frac{\Omega_{i} \nu_{i e}}{e n} J_{y}+\frac{i \omega \nu_{i e}}{e n} J_{x}
$$

The electron terms yield

$$
V_{e x}\left(-\omega^{2}+\Omega_{e}^{2}\right)=-\frac{i \omega q_{e}}{m_{e}} E_{1 x}+\frac{q_{e}}{m_{e}} \Omega_{e} E_{1 y}+\frac{\Omega_{e} \nu_{e i}}{e n} J_{\nu}-\frac{i \omega \nu_{e i}}{e n} J_{x}
$$

DEFINE: $U_{j} \equiv \Omega_{j}^{2}-\omega^{2}$

Combining to form $J_{x}=e n\left(v_{i x}-v_{e x}\right)$

$$
\begin{aligned}
J_{x}\left(1-i \omega\left[\frac{\nu_{i e}}{U_{i}}+\frac{\nu_{e i}}{U_{e}}\right]\right) & =E_{1 x}(i \omega e n)\left[\frac{-q_{i}}{U_{i} m_{i}}+\frac{-q_{e}}{U_{e} m_{e}}\right] \\
& +E_{1 y}(e n)\left[\frac{-q_{i} \Omega_{i}}{U_{i} m_{i}}+\frac{-q_{e} \Omega_{e}}{U_{e} m_{e}}\right] \\
& +J_{y}\left(\frac{-\Omega_{i} \nu_{i e}}{U_{i}}-\frac{\Omega_{e} \nu_{e i}}{U_{e}}\right)
\end{aligned}
$$

Likewise, the ion $\hat{x}$ and $\hat{y}$ equations can be combined to find $V_{i y}, V_{e y}$ :

$$
V_{i y}\left(-\omega^{2}+\Omega_{i}^{2}\right)=-\frac{i \omega q_{i}}{m_{i}} E_{1 y}-\frac{q_{i}}{m_{i}} \Omega_{i} E_{1 x}-\frac{\Omega_{i} \nu_{i e}}{e n} J_{x}+\frac{i \omega \nu_{i e}}{e n} J_{y}
$$

The electron terms yield

$$
V_{e y}\left(-\omega^{2}+\Omega_{e}^{2}\right)=-\frac{i \omega q_{e}}{m_{e}} E_{1 y}-\frac{q_{e}}{m_{e}} \Omega_{e} E_{1 x}-\frac{\Omega_{e} \nu_{e i}}{e n} J_{x}-\frac{i \omega \nu_{e i}}{e n} J_{y}
$$

Again, using the identity $J_{y} \equiv e n\left(V_{i y}-V_{e y}\right)$ yields 


$$
\begin{aligned}
J_{y}\left(1-i \omega\left[\frac{\nu_{i e}}{U_{i}}+\frac{\nu_{e i}}{U_{e}}\right]\right) & =E_{1 y}(-i \omega e n)\left[\frac{-q_{i}}{U_{i} m_{i}}-\frac{-q_{e}}{U_{e} m_{e}}\right] \\
& +E_{1 x}(e n)\left[\frac{-q_{i} \Omega_{i}}{U_{i} m_{i}}+\frac{-q_{e} \Omega_{e}}{U_{e} m_{e}}\right] \\
& +J_{x}\left(\frac{\Omega_{i} \nu_{i e}}{U_{i}}+\frac{\Omega_{e} \nu_{e i}}{U_{e}}\right)
\end{aligned}
$$

Defining

$$
\begin{aligned}
& a_{1} \equiv \frac{\nu_{i e} \Omega_{i}}{U_{i}}+\frac{\nu_{e i} \Omega_{e}}{U_{e}} \\
& a_{2} \equiv-1+i \omega\left(\frac{\nu_{i e}}{U_{i}}+\frac{\nu_{e i}}{U_{e}}\right) \\
& a_{3} \equiv i \omega e n\left[-\frac{q_{i}}{m_{i} U_{i}}+\frac{q_{e}}{m_{e} U_{e}}\right] \\
& a_{4} \equiv \text { en }\left[-\frac{q_{i} \Omega_{i}}{m_{i} U_{i}}+\frac{q_{e} \Omega_{e}}{m_{e} U_{e}}\right]
\end{aligned}
$$

The currrents $J_{x}$ and $J_{y}$ can then be expressed in terms of $a_{1} \ldots a_{4}$

$$
\begin{gathered}
J_{x}=\frac{1}{a_{1}^{2}+a_{2}^{2}}\left[-E_{1 x}\left(a_{1} a_{4}+a_{2} a_{3}\right)+E_{1 y}\left(-a_{1} a_{3}+a_{2} a_{4}\right)\right] \\
J_{y}=\frac{1}{a_{1}^{2}+a_{2}^{2}}\left[E_{1 x}\left(a_{1} a_{3}-a_{2} a_{4}\right)+E_{1 y}\left(-a_{1} a_{4}+a_{2} a_{3}\right)\right]
\end{gathered}
$$

Now since

$$
\begin{aligned}
\nabla \times \vec{B} & =\frac{1}{c^{2}} \frac{\partial \vec{E}}{\partial t}+\mu_{0} \vec{J} \\
& =-\frac{i \omega}{c^{2}} \underline{K} \cdot \vec{E}
\end{aligned}
$$

Therefore

$$
\underline{\underline{K}} \cdot \vec{E}=\underline{\underline{I}} \cdot \vec{E}+i \frac{\vec{J}}{\epsilon_{0} \omega}
$$

Recall that $\underline{K}$ is defined as 


$$
\underline{\underline{K}} \equiv\left[\begin{array}{ccc}
S & -i D & 0 \\
i . D & S & 0 \\
0 & 0 & F
\end{array}\right]
$$

Therefore, S, D and P are found to be

$$
S=1+\frac{A_{T}}{A_{B}}
$$

Where

$$
\begin{aligned}
& A_{T} \equiv \omega_{p i}^{2}+\omega_{p e}^{2} \frac{U_{i}}{U_{e}}+\frac{i}{\omega}\left[\omega_{p i}^{2} \nu_{i e}+\omega_{p e}^{2} \nu_{e i} \frac{U_{i}}{U_{i}}+\frac{\left(\Omega_{i} \Omega_{e}-\omega^{2}\right)}{U_{e}}\right] \\
&= \omega_{p i}^{2}\left(1+i \frac{\nu_{i e}}{\omega}\right)+\omega_{p e}^{2} \frac{U_{i}}{U_{e}}\left(1+i \frac{\nu_{e i}}{\omega}\right) \\
& \quad+\frac{i}{\omega U_{e}}\left[\left(\Omega_{i} \Omega_{e}-\omega^{2}\right)\left(\omega_{p i}^{2} \nu_{e i}+\omega_{p e}^{2} \nu_{i e}\right)\right] \\
& A_{B} \equiv \Omega_{i}^{2}-\omega^{2}-2 i \omega\left(\nu_{i e}+\nu_{e i} \frac{U_{i}}{U_{e}}\right)+\nu_{i e}^{2}+\nu_{e i}^{2} \frac{U_{i}}{U_{e}}+2 \frac{\nu_{i e} \nu_{e i}}{U_{e}}\left[\Omega_{i} \Omega_{e}-\omega^{2}\right]
\end{aligned}
$$

And $D$ is found to be

$$
D=-\frac{D_{T}}{A_{B}}
$$

Where

$$
D_{T} \equiv \frac{\omega_{p i}^{2} \Omega_{i}}{\omega}+\frac{\omega_{p e}^{2} \Omega_{e}}{\omega} \frac{U_{i}}{U_{e}}
$$

Finally, $\mathrm{P}$ is found to be

$$
P=1-\frac{\omega_{p i}^{2}+\omega_{p e}^{2}}{\omega^{2}\left[1+i \frac{\nu_{i e}}{\omega}+i \frac{\nu_{\alpha i}}{\omega}\right]}
$$

Dropping terms to $O\left(m_{e} / m_{i}\right)$ and simplifying

$$
S \simeq 1+\frac{\omega_{p i}^{2}\left[1-i \frac{\nu_{i j} \omega}{\omega_{i i}^{2}}\right]}{\Omega_{i}^{2}-\omega^{2}-2 i \omega \nu_{i e}-\nu_{i e}^{2}\left(\omega^{2} / \omega_{c i}^{2}\right)}
$$


Likewise for D

$$
D \simeq \frac{\omega_{p i}^{2} \omega}{\omega_{c i}\left[\omega^{2}-\Omega_{i}^{2}+2 i \omega \nu_{i e}+\nu_{i e}^{2}\left(\omega^{2} / \omega_{c i}^{2}\right)\right]}
$$

Compare this to a simple substitution in for $\nu$

$$
\begin{gathered}
S=1-\sum_{j} \frac{\omega_{p j}^{2}}{\omega} \frac{\left(\omega+i \nu_{j}\right)}{\omega^{2}-\omega_{c j}^{2}-\nu_{j}^{2}+2 i \nu_{j} \omega} \\
D=\sum_{j} \frac{\omega_{p j}^{2} \omega_{c j}}{\omega\left[\omega^{2}-\omega_{c j}^{2}-\nu_{j}^{2}+2 i \nu_{j} \omega\right]}
\end{gathered}
$$

There are some qualitative differences, although in the limit of $\omega \rightarrow \omega_{c i}$, the differences approach zero.

\section{A.2.2 Real and Imaginary parts}

The real and imaginary parts of $S$ and $D$ are hereby examined.

From the previous derivation, it was seen that

$$
\begin{gathered}
S \simeq 1-\frac{\omega_{p i}^{2}\left[1-i \frac{\nu_{i e} \omega}{\Omega_{i}^{2}}\right]}{\omega^{2}-\Omega_{i}^{2}+2 i \omega \nu_{i e}+\nu_{i e}^{2}\left(\omega^{2} / \Omega_{i}^{2}\right)} \\
S=1-\frac{\omega_{p i}^{2}\left[1-i \frac{\nu_{i e} \omega}{\Omega_{i}^{2}}\right]\left[\omega^{2}-\Omega_{i}^{2}+\nu_{i e}^{2}\left(\omega^{2} / \Omega_{i}^{2}\right)-2 i \omega \nu_{i e}\right]}{\left[\omega^{2}-\Omega_{i}^{2}-\nu_{i e}^{2}\left(2-\omega^{2} / \Omega_{i}^{2}\right)\right]^{2}+4 \omega^{2} \nu_{i e}^{2}} \\
=1-\frac{\omega_{p i}^{2}}{A_{C}}\left\{\omega^{2}-\Omega_{i}^{2}-\nu_{i e}^{2}\left(\omega^{2} / \Omega_{i}^{2}\right)-i \nu_{i e} \omega\left[1+\frac{\omega^{2}}{\Omega_{i}^{2}}+\nu_{i e}^{2} \frac{\omega^{2}}{\Omega_{i}^{4}}\right]\right\}
\end{gathered}
$$

where

$$
A_{C} \equiv\left[\omega^{2}-\Omega_{i}^{2}\right]^{2}+2 \omega^{2} \nu_{i e}^{2}\left(1+\frac{\omega^{2}}{\Omega_{i}^{2}}\right)
$$

Likewise, $\mathrm{D}$ was found to be

$$
D \simeq \frac{\omega_{p i}^{2} \omega}{\omega_{c i}\left[\omega^{2}-\Omega_{i}^{2}+2 i \omega \nu_{i e}+\nu_{i e}^{2} \frac{\omega^{2}}{\Pi_{i}^{2}}\right]}
$$


Expanding,

$$
\begin{aligned}
D & =\frac{\omega_{p i}^{2} \omega}{\omega_{c i}\left[\omega^{2}-\Omega_{i}^{2}+2 i \omega \nu_{i e}+\nu_{i e}^{2}\left(\frac{\omega^{2}}{n_{i}^{2}}\right)\right]} \\
& =\frac{1}{A_{C}} \frac{\omega_{p i}^{2} \omega}{\Omega_{i}}\left[\omega^{2}-\Omega_{i}^{2}+\nu_{i e}^{2}\left(\omega^{2} / \Omega_{i}^{2}\right)-2 i \omega \nu_{i e}\right]
\end{aligned}
$$

Separating into real and imaginary components

$$
\begin{gathered}
S \equiv S_{R e}+i S_{I m} \\
D \equiv D_{R e}+i D_{I m} \\
S_{R e}=1-\frac{\omega_{p i}^{2}}{A_{C}}\left[\omega^{2}-\Omega_{i}^{2}-\nu_{i e}^{2}\left(\omega^{2} / \Omega_{i}^{2}\right)\right] \\
S_{I m}=\frac{\omega_{p i}^{2} \nu_{i e} \omega}{\Omega_{i}^{2} A_{C}}\left(1+\omega^{2} / \Omega_{i}^{2}+\nu_{i e}^{2} \frac{\omega^{2}}{\Omega_{i}^{2}}\right) \\
D_{R e}=\frac{\omega_{p i}^{2} \omega}{A_{C} \Omega_{i}}\left[\omega^{2}-\Omega_{i}^{2}+\nu_{i e}^{2}\left(\omega^{2} / \Omega_{i}^{2}\right)\right] \\
D_{I m}=-2 \frac{\omega_{p i}^{2} \omega^{2} \nu_{i e}}{A_{C} \Omega_{i}}
\end{gathered}
$$

For $\omega<\Omega_{i}$, it is seen that

$$
\begin{aligned}
& S_{R e}>0 \\
& S_{I m}>0 \\
& D_{R e}<0 \\
& D_{I m}<0
\end{aligned}
$$

\section{A.2.3 Perpendicular wave number $K_{x}$ for TE Mode}

Recall that the perpendicular wave number $k_{x}$ was found from Maxwell's equation to be 


$$
k_{x}^{2}=k_{0}^{2} \frac{\left(S^{2}-D^{2}\right)}{S}-k_{y}^{2}
$$

Expanding the term $\frac{S^{2}-D^{2}}{S}$

$$
\begin{gathered}
\left.\frac{\left(S^{2}-D^{2}\right)}{S}=\frac{1}{S_{R e}+i S_{I m}}\left(S_{R e}+i S_{I m}\right)^{2}-\left(D_{R e}+i D_{I m}\right)^{2}\right) \\
\frac{\left(S^{2}-D^{2}\right)}{S}=\frac{1}{S_{R e}^{2}+S_{I m}^{2}}\left(S_{R e}-i S_{I m}\right)\left\{\begin{array}{c}
S_{R e}^{2}+2 i S_{r e} S_{I m}-S_{I m}^{2} \\
-\left[D_{R e}^{2}+2 i D_{R e} D_{I m}-D_{I m}^{2}\right]
\end{array}\right\} \\
=\frac{1}{S_{R e}^{2}+S_{I m}^{2}}\left\{\begin{array}{c}
S_{R e}\left(S_{R e}^{2}-D_{R e}^{2}\right)+S_{R e} S_{I m}^{2} \\
+D_{I m}\left(S_{R e} D_{I m}-2 D_{R e} S_{I m}\right) \\
+i\left[S_{I m}\left(S_{R e}^{2}+D_{R e}^{2}\right)-2 S_{R e} D_{R e} D_{I m}\right. \\
\left.+S_{I m}\left(S_{I m}^{2}-D_{I m}^{2}\right)\right]
\end{array}\right\}
\end{gathered}
$$

It is seen that the real part of this is positive since $\left|S_{R e}\right|>\left|D_{R e}\right|$ and $S_{R e}, D_{R e} \gg$ $S_{I m}, D_{I m}$. The imaginary part of the above is now examined. Recall also that $\left|S_{I m}\right|<\left|D_{I m}\right|, D_{R e}, D_{I m}<0$ and $S_{R e}, S_{I m}>0$.

$$
\begin{aligned}
\operatorname{Im}\left(\frac{S^{2}-D^{2}}{S}\right) & =S_{I m}\left(S_{R e}^{2}+D_{R e}^{2}\right)+S_{I m}\left(S_{I m}^{2}-D_{I m}^{2}\right)-2 S_{R e} D_{R e} D_{I m} \\
& =S_{I m}\left(S_{R e}+D_{R e}\right)^{2}-2 S_{R e} D_{R e}\left(S_{I m}+D_{I m}\right) \\
& =\oplus+\ominus \\
& =? ?
\end{aligned}
$$

From this, a criterion for $\operatorname{Im}\left(k_{x}^{2}\right)>0$ can be obtained. It is important to have $\operatorname{Im}\left(k_{x}^{2}\right)>0$ in order for the EM waves to damp and transfer their energy to the plasma. If $\operatorname{Im}\left(k_{x}^{2}\right)<0$, the sign of $\operatorname{Re}\left(k_{x}\right)$ would be different from the sign of $\operatorname{Im}\left(k_{x}\right)$, and the wave would no longer be damped.

$$
\operatorname{Im}\left(k_{x}^{2}\right) \propto \operatorname{Im}\left(\frac{S^{2}-D^{2}}{S}\right)>0
$$




$$
\begin{aligned}
\operatorname{Im}\left(\frac{S^{2}-D^{2}}{S}\right) & =S_{I m}\left(S_{R e}^{2}+D_{R e}^{2}\right)-2 S_{R e} D_{R e} D_{I m}+S_{I m}\left(S_{I m}^{2}-D_{I m}^{2}\right)>0 \\
S_{I m}\left(S_{R e}^{2}+D_{R e}^{2}\right) & >2 S_{R e} D_{R e} D_{I m}
\end{aligned}
$$

\section{Limit: $\omega \rightarrow \omega_{\mathrm{cl}}$}

In this limit, we assume that $\omega$ is so close to $\omega_{c i}$ that $\omega^{2}-\omega_{c i}^{2}<\nu_{i e}^{2}$.

$$
\begin{gathered}
S_{R e} \simeq 1+\frac{\omega_{p i}^{2}}{4 \omega^{2}} \\
S_{I m} \simeq \frac{\omega_{p i}^{2}}{2 \omega \nu_{i e}} \\
D_{R e} \simeq \frac{\omega_{p i}^{2} \omega}{4 \Omega_{i}^{2}} \\
D_{I m} \simeq-\frac{\omega_{p i}^{2}}{2 \nu_{i e} \omega_{c i}}
\end{gathered}
$$

So the $\operatorname{Im}\left(k_{x}\right)>0$ criterion becomes

$$
\begin{aligned}
\operatorname{Im}\left(\frac{S^{2}-D^{2}}{S}\right) & =S_{I m}\left(S_{R e}^{2}+D_{R e}^{2}\right)-2 S_{R e} D_{R e} D_{I m} \\
& \simeq S_{I m}\left(S_{R e}^{2}+D_{R e}^{2}\right)+2 S_{R e} D_{R e} S_{I m} \\
& =S_{I m}\left(S_{R e}+D_{R e}\right)^{2}
\end{aligned}
$$

Since $S_{I m}>0$, it is seen that in the limit of $\omega \rightarrow \omega_{c i}, \operatorname{Im}\left(\frac{S^{2}-D^{2}}{S}\right)>0$, therefore both $\operatorname{Re}\left(k_{x}\right)$ and $\operatorname{Im}\left(k_{x}\right)$ are positive.

2. Limit: $\left|\omega^{2}-\Omega_{i}^{2}\right| \gg \nu_{i e}^{2}$

In this limit, the frequency is sufficiently far away from resonance such that terms of order $O\left(\nu_{i e}^{2} / \omega^{2}\right)$ can be ignored 


$$
\begin{gathered}
S_{R e} \simeq 1-\frac{\omega_{p i}^{2}}{\left[\omega^{2}-\Omega_{i}^{2}\right]} \\
S_{I m} \simeq \frac{\omega_{p i}^{2} \omega \nu_{i e}\left[\Omega_{i}^{2}+\omega^{2}\right]}{\Omega_{i}^{2}\left[\omega^{2}-\Omega_{i}^{2}\right]^{2}} \\
D_{R e} \simeq \frac{\omega_{p i}^{2} \omega}{\Omega_{i}\left(\omega^{2}-\Omega_{i}^{2}\right)} \\
D_{I m} \simeq-2 \frac{\omega_{p i}^{2} \omega^{2} \nu_{i e}}{\Omega_{i}\left[\omega^{2}-\Omega_{i}^{2}\right]^{2}}
\end{gathered}
$$

Therefore it is seen that

$$
\frac{D_{I m}}{S_{I m}}=-2 \frac{\omega \Omega_{i}}{\left(\Omega_{i}^{2}+\omega^{2}\right)}
$$

Sowthe imaginary part of $k_{x}$ is proportional to

$$
\begin{aligned}
\operatorname{Im}\left(\frac{S^{2}-D^{2}}{S}\right) & =S_{I m}\left[S_{R e}^{2}+D_{R e}^{2}-2 S_{R e} D_{R e}\left(\frac{-2 \omega \Omega_{i}}{\Omega_{i}^{2}+\omega^{2}}\right)\right] \\
& =S_{i m}\left[\left(S_{R e}+D_{R e}\right)^{2}-2 S_{R e} D_{R e} \frac{\left(\Omega_{i}-\omega\right)^{2}}{\Omega_{i}^{2}+\omega^{2}}\right]
\end{aligned}
$$

Since $S_{R e}$ and $D_{R e}$ have opposite signs, it is seen that $k_{\mathrm{x}}>0$ for practically all cases. 


\section{Appendix B}

\section{Current Driven Boundary}

\section{Conditions}

The presence of a current source (antenna) at $r=b$ is what drives the fluctuating EM fields. The formulation of the source terms is hereby examined. Section B.1 shows the formulation of the Full Turn Loop source, section B.2 shows the development of the Dual Half-Turn Loop Source term, while Section B.3 examines the problem of the radial feeder in detail.

\section{B.1 Source Term, Full Turn Loop}

The geometry under consideration is shown in Figure B-1. The derivation of the source term of the full turn loop antenna is derived by Myer [personal communications] and presented here for completeness.

The full turn loop antenna is essentially a source of $J_{\theta}$ current given by

$$
J_{\theta}(r, \phi, z)=\frac{I}{W} \delta_{r}(b) U_{z}( \pm W / 2)
$$

where $\mathrm{W}$ is the width of the loop, $\delta_{r}(b) \equiv \delta(r-b), U_{z}( \pm W / 2)$ is the step function $U(z+W / 2, z-W / 2)$.

The Fourier transform of the above source is then performed by 


$$
J_{\phi}\left(r, n, k_{z}\right)=\frac{1}{2 \pi} \int_{0}^{2 \pi} d \phi \int_{-\infty}^{\infty} d z e^{-i n \phi} e^{-i k_{z}} \frac{I}{W} \delta_{r}(b) U_{z}( \pm W / 2)
$$

After some algebra, it is seen that the Fourier-transformed antenna source term for a full turn loop antenna is given by:

$$
J_{\phi}\left(r, n=0, k_{z}\right)=\frac{I}{\frac{k_{z} W}{2}} \delta_{r}(b) \sin \left(\frac{k_{z} W}{2}\right)
$$

\section{B.2 Source Term, Half Turn Loop}

The problem of the dual half turn loop can be considered as a summation of two half-turn loops. Examining the right half turn loop shows that the driving source term is given by

$$
\begin{gathered}
J_{r}=\frac{I}{W} \frac{U(r-b)}{r}[\delta(\theta=-\pi / 2)-\delta(\theta=\pi / 2)] U_{z}( \pm W / 2) \\
J_{\theta}=-\frac{I}{W} \delta(r=b) U_{\theta}(-\pi / 2, \pi / 2) U_{z}( \pm W / 2)
\end{gathered}
$$

Since

$$
\nabla \cdot \vec{J}=-\frac{d \rho}{d t}
$$

the formulation must be such that $\nabla \cdot \vec{J}=0$, otherwise there would be free charge buildup in the system.

The left half loop can be likewise formulated. The $J_{r}$ remains the same, however, the $J_{\theta}$ term is changed to

$$
J_{\theta}=\frac{I}{W} \delta(r=b) U_{\theta}(\pi / 2,3 \pi / 2) U_{z}( \pm W / 2)
$$

So the dual half turn loop driving term is simply the summation of these two halves 


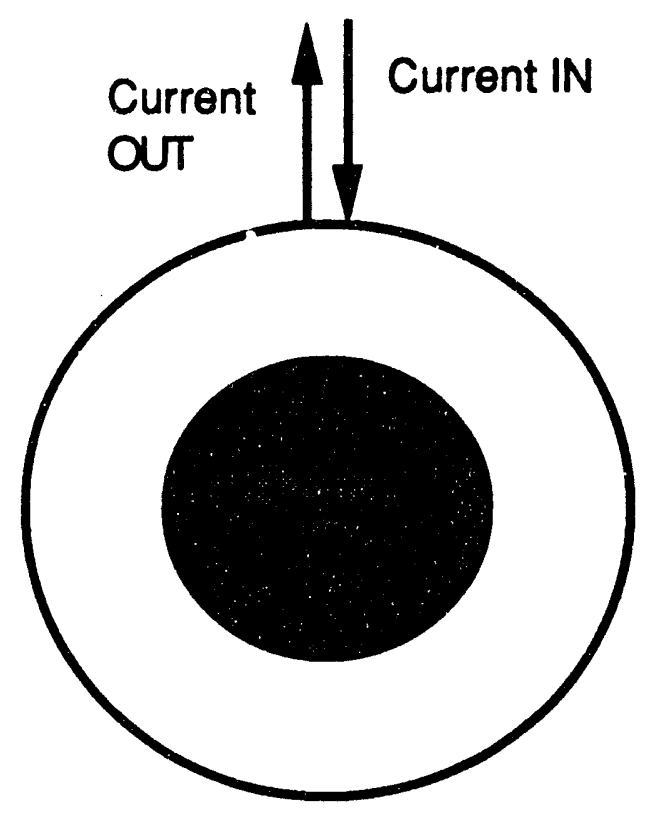

Full Turn Loop (FTL) Schematic

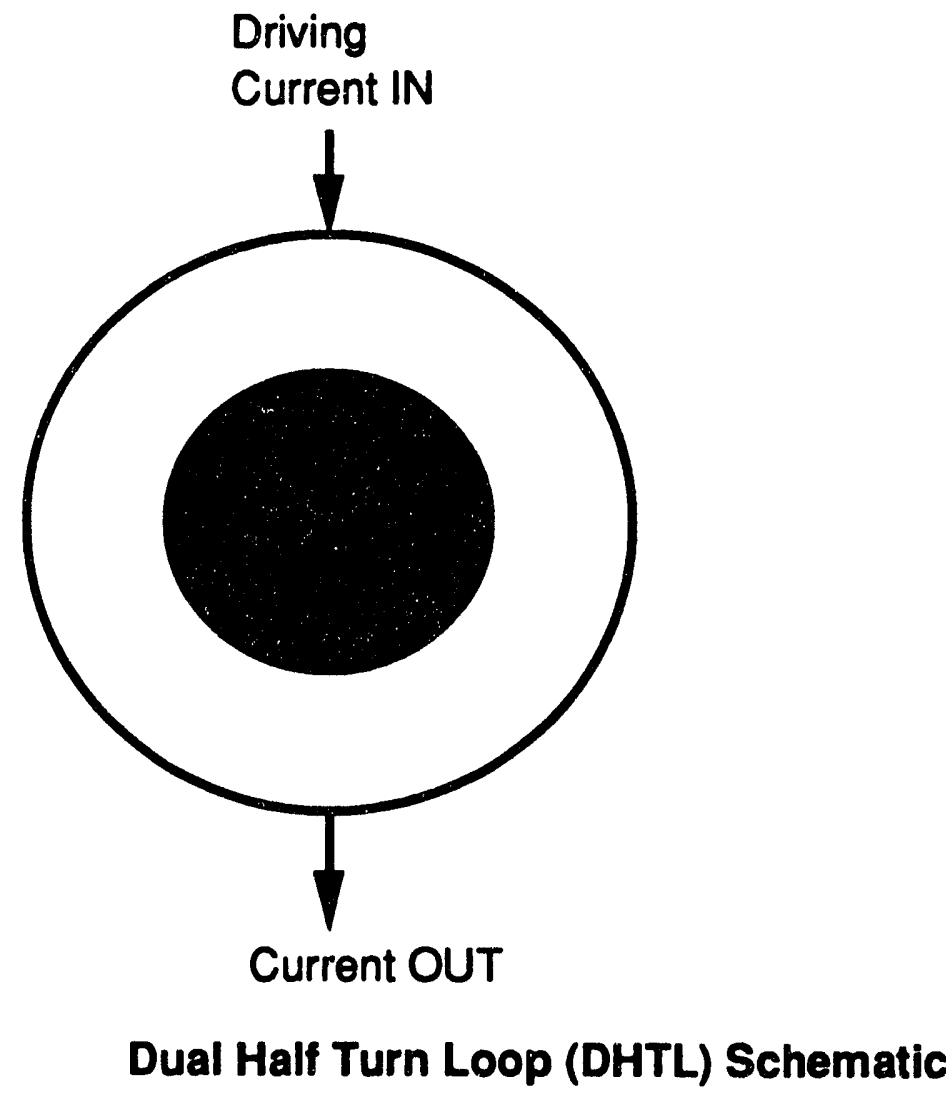

Figure B-1: Geometry of Current Source 


$$
\begin{gathered}
J_{r}=\frac{2 I}{W} \frac{U(r-b)}{r}\left[\delta_{\theta}(-\pi / 2)-\delta_{\theta}(\pi / 2) U_{z}( \pm W / 2)\right] \\
J_{\theta}=\frac{I}{W} \delta_{r}(b) U_{z}( \pm W / 2)\left[-U_{\theta}(-\pi / 2, \pi / 2)+U_{\theta}(\pi / 2,3 \pi / 2)\right]
\end{gathered}
$$

The Laplace transform is then found by

$$
E\left(r, n, K_{\|}\right) \equiv \frac{1}{2 \pi} \int_{0}^{2 \pi} e^{-i n \theta} \int_{-\infty}^{\infty} e^{i k_{\|} z} E(r, \theta, z)
$$

It can be shown that this generates the Laplace transformed driving terms

$$
\begin{gathered}
J_{r}\left(r, n, k_{\|}\right)=\frac{I}{\pi} \frac{U(r-b)}{r}(i)^{n} \frac{\sin \left(\frac{k_{\|} W}{2}\right)}{\left(\frac{k_{\|} W}{2}\right)}\left[1+(-1)^{n+1}\right] \\
J_{\theta}=\frac{I}{2 \pi} \frac{\delta_{r}(b)}{n}(i)^{n+1} \frac{\sin \left(\frac{k_{\|} W}{2}\right)}{\left(\frac{k_{\|} W}{2}\right)}\left[1+(-1)^{n+1}\right]
\end{gathered}
$$

\section{B.3 The Radial Current Feeder Problem}

The radial current feed problem arises in the physical modeling of an ICRF system. An antenna always has an input and an output feeder that has an r-component $\left(\overrightarrow{J_{f d}}=J_{0}(r) \hat{r}\right)$. This extra radial driving term significantly alters the nature of the problem and is often ignored in theoretical ICRF analyses. Work has been done by both McVey $[4,72]$ and Myer [personal communication] to include the radial feeders as part of the EM fields' driving term to better simulate reality. The steps taken in this analysis are shown below.

\section{B.3.1 Modified Solution of the Vacuum-Plasma Eigen- modes}

Using the basic Plasma-Vacuum Geometry as seen in Figure 4-1, Maxwell's equations can be solved in regions I, II, and III using boundary conditions across $\mathrm{r}=\mathrm{a}, \mathrm{r}=\mathrm{b}, \mathrm{r}=\mathrm{c}$. 
Taking into consideration the radial feeders requires altering the solutions of the vacuum field in region I $(a<r<b)$. Maxwell's equations are

$$
\begin{gathered}
\nabla \times \vec{E}=-\frac{d}{d t} \vec{B} \\
\nabla \times \vec{B}=\mu_{0}\left(\vec{J}+\epsilon_{0} \vec{E}\right)
\end{gathered}
$$

After some simple manipulation

$$
\begin{gathered}
\nabla^{2} \vec{E}-\frac{1}{c^{2}} \frac{\partial^{2} \vec{E}}{\partial t^{2}}=\mu_{0} \frac{\partial}{\partial t} \vec{J} \\
\nabla^{2} \vec{B}-\frac{1}{c^{2}} \frac{\partial^{2} \vec{B}}{\partial t^{2}}=-\mu_{0} \nabla \times \vec{J}
\end{gathered}
$$

Taking the $\hat{z}$ components of the above two equations yields

$$
\begin{gathered}
\nabla^{2} E_{z}+k_{0}^{2} E_{z}=0 \\
\nabla^{2} B_{z}+k_{0}^{2} B_{z}=\frac{\mu_{0}}{r} \frac{\partial J_{r}}{\partial \phi}
\end{gathered}
$$

Taking the Fourier-transform

$$
\begin{gathered}
\hat{E}_{z}\left(r, n, k_{\|}\right)=\frac{1}{2 \pi} \int_{0}^{2 \pi} d \phi \int_{-\infty}^{\infty} d z E_{z}(r, \phi, z) e^{-i n \phi} e^{-i k_{\| z}} \\
\frac{d^{2}}{d r^{2}} \hat{E}_{z}+\frac{1}{r} \frac{d \hat{E}_{z}}{d r}-\left(\nu^{2}+\frac{n^{2}}{r^{2}}\right) \hat{E}_{z}=0 \\
\frac{d^{2}}{d r^{2}} \hat{B}_{z}+\frac{1}{r} \frac{d \hat{B}_{z}}{d r}-\left(\nu^{2}+\frac{n^{2}}{r^{2}}\right) \hat{B}_{z}=\frac{\mu_{0}}{r} i n \hat{J}_{r}
\end{gathered}
$$

The above equation can be solved using the method of variation of parameters.

$$
y(r)=y_{H}(r)+y_{2}(r) \int^{r} \frac{f(t) y_{1}(t)}{W(y 1, y 2)} d t-y_{1}(r) \int^{r} \frac{f(t) y_{2}(t)}{W(y 1, \dot{y} 2)} d t
$$




$$
\begin{gathered}
y_{H}(r)=H_{3} K_{n}(\nu r)+H_{4} I_{n}(\nu r) \\
y_{1}(r)=K_{n}(\nu r) \quad y_{2}(r)=I_{n}(\nu r) \quad W(y 1, y 2)=\frac{1}{\nu r} \\
f(t)=\mu_{0} \frac{i n}{r} \hat{J}_{r}\left(t, n, k_{\|}\right)
\end{gathered}
$$

Using the method of variation of parameters as outlined above, the solutions to Equations B.3 and B.4 are found to be

$$
\begin{gathered}
\hat{E}_{z}=E_{3} K_{n}(\nu r)+E_{4} I_{n}(\nu r) \\
\hat{H}_{2}=H_{3} K_{n}(\nu r)+H_{4} I_{n}(\nu r)+g(r)
\end{gathered}
$$

where $\mathcal{G}(r)$ and its derivative $\mathcal{G}^{\prime}(r)$ are defined as

$$
\begin{gathered}
\mathcal{G}(r) \equiv i n \nu\left[I_{n}(\nu r) \int_{b}^{r} K_{n}(\nu t) \hat{J}_{r}\left(t, n, k_{\|}\right) d t-K_{n}(\nu r) \int_{b}^{r} I_{n}(\nu t) \hat{J}_{r}\left(t, n, k_{\|}\right) d t\right] \\
\mathcal{G}^{\prime}(r) \equiv \frac{1}{\nu} \frac{d \mathcal{G}}{d r}=i n \nu\left[I_{n}^{\prime}(\nu r) \int_{b}^{r} K_{n}(\nu t) \hat{J}_{r}\left(t, n, k_{\|}\right) d t-K_{n}^{\prime}(\nu r) \int_{b}^{r} I_{n}(\nu t) \hat{J}_{r}\left(t, n, k_{\|}\right) d t\right]
\end{gathered}
$$

The solutions to Equations B.3 and B.4 are

$$
\begin{gathered}
\hat{E}_{z}=E_{3} K_{n}(\nu r)+E_{4} I_{n}(\nu r) \\
\hat{H}_{z}=H_{3} K_{n}(\nu r)+H_{4} I_{n}(\nu r)+g(r)
\end{gathered}
$$

The inclusion of the term $\mathcal{G}(r)$ significantly alters the boundary value problem of the system. While the boundary conditions per se remain unchanged, the field coefficients of the initial driven eigenmode solutions will all contain this $\mathcal{G}(\boldsymbol{r})$ driving term.

The fields in the other regions remain unchanged. 
Region 2, b $<\mathbf{r}<\mathbf{a}$

$$
\begin{aligned}
& {\hat{E_{z}}}^{2}=E_{1} K_{n}(\nu r)+E_{2} I_{n}(\nu r) \\
& \hat{H}_{z}{ }^{2}=H_{1} K_{n}(\nu r)+H_{2} I_{n}(\nu r)
\end{aligned}
$$

Plasma Region, $r<$ a

$$
\begin{aligned}
& \hat{E}_{z}^{p}=z_{1} H_{p} J_{n}\left(k_{\perp 1} r\right)+E_{p} J_{n}\left(k_{\perp 2} r\right) \\
& \hat{H}_{z}{ }^{\varnothing}=H_{p} J_{n}\left(k_{\perp 1} r\right)+y_{2} E_{p} J_{n}\left(k_{\perp 2} r\right)
\end{aligned}
$$

The transverse vacuum fields are then

$$
\begin{gathered}
\hat{E}_{r}=\frac{-i k_{z}}{\nu^{2}} \frac{d \hat{E}_{z}}{d r}+\frac{\omega \mu_{0}}{\nu^{2}} \frac{n}{r} \hat{H}_{z} \\
\hat{E}_{\phi}=\frac{k_{z}}{\nu^{2}} \frac{n}{r} \hat{E}_{z}+\frac{i \omega \mu_{0}}{\nu^{2}} \frac{n}{r} \frac{d \hat{H}_{z}}{d r} \\
\hat{H}_{r}=\frac{\omega \epsilon_{0}}{\nu^{2}} \frac{n}{r} \hat{E}_{z}-\frac{i k_{z}}{\nu^{2}} \frac{d \hat{H}_{z}}{d r} \\
\hat{H}_{\phi}=\frac{-i \omega \epsilon_{0}}{\nu^{2}} \frac{d \hat{E}_{z}}{d r}+\frac{k_{z}}{\nu^{2}} \frac{n}{r} \hat{H}_{z}
\end{gathered}
$$

From Maxwells equations, it can be shown that the transverse plasma fields are

$$
\begin{aligned}
& \hat{E}_{r}=e_{r 1}\left(r, H_{p}+e_{r 2}(r) E_{p}\right. \\
& \hat{E}_{\phi}=e_{\phi 1}(r) H_{p}+e_{\phi 2}(r) E_{p} \\
& \hat{H}_{r}=h_{r 1}(r) H_{p}+h_{r 2}(r) E_{p} \\
& \hat{H}_{\phi}=h_{\phi 1}(r) H_{p}+h_{\phi 2}(r) E_{p}
\end{aligned}
$$




\section{Appendix C}

\section{Transverse Plasma Fields and \\ Excitation Coefficient Definitions}

(Source: McVey [65])

Using Maxwell's equations and the bounded plasma boundary condition, the transverse plassia fields can be expressed in terms of the coefficients of the $E_{x}$ (TM) and the $H_{z}$ (TE) fields.

$$
\begin{aligned}
& E_{r}=e_{r 1} H_{p}+e_{r 2} E_{p} \\
& E_{\theta}=e_{\phi 1} H_{p}+e_{\phi 2} E_{p} \\
& H_{r}=h_{r 1} H_{p}+h_{r 2} E_{p} \\
& H_{\phi}=3_{\phi 1}^{3} H_{p}+h_{\phi 2} E_{p}
\end{aligned}
$$

where the coefficients are defined

$$
\begin{gathered}
e_{r 1}=\omega \mu_{0} k_{r 1} \Lambda^{-1}\left\{\begin{array}{c}
{\left[k_{0}^{2} D+\epsilon_{1}\left(k_{z}^{2}-k_{0}^{2} S\right)\right] J_{n}^{\prime}\left(k_{r 1} r\right)} \\
+\left[\left(k_{z}^{2}-k_{0}^{2} S\right)+\epsilon_{1} k_{0}^{2} D\right] \frac{n J_{n}\left(k_{r 1} r\right)}{k_{r+1} r}
\end{array}\right\} \\
e_{r 2}=-i k_{z} k_{r 2} \Lambda^{-1}\left\{\begin{array}{c}
{\left[\left(k_{z}^{2}-k_{0}^{2} S\right)+\epsilon_{2} k_{0}^{2} D\right] J_{n}^{\prime}\left(k_{r 2} r\right)} \\
+\left[k_{0}^{2} D+\epsilon_{2}\left(k_{z}^{2}-k_{0}^{2} S\right)\right] \frac{n_{r_{u}}\left(k_{r 2} r\right)}{k_{r 2} r}
\end{array}\right\}
\end{gathered}
$$




$$
\begin{aligned}
& e_{\phi 1}=i \omega \mu_{0} k_{r 1} \Lambda^{-1}\left\{\begin{array}{c}
{\left[\left(k_{z}^{2}-k_{0}^{2} S\right)+\epsilon_{1} k_{0}^{2} D\right] J_{n}^{\prime}\left(k_{r 1} r\right)} \\
+\left[k_{0}^{2} D+\epsilon_{1}\left(k_{z}^{2}-k_{0}^{2} S\right)\right] \frac{n J_{n}\left(k_{r 1} r\right)}{k_{r 1} r}
\end{array}\right\} \\
& e_{\phi 2}=k_{z} k_{r 2} \Lambda^{-1}\left\{\begin{array}{c}
{\left[k_{0}^{2} D+\epsilon_{2}\left(k_{z}^{2}-k_{0}^{2} S\right)\right] J_{n}^{\prime}\left(k_{r 2} r\right)} \\
+\left[\left(k_{z}^{2}-k_{0}^{2} S\right)+\epsilon_{2} k_{0}^{2} D\right] \frac{n_{n}\left(k_{r 2} r\right)}{k_{r 2} r}
\end{array}\right\} \\
& h_{r 1}=-i k_{z} k_{r 1} \Lambda^{-1}\left\{\begin{array}{c}
{\left[\left(k_{z}^{2}-k_{0}^{2} S\right)+\delta_{1} k_{0}^{2} D\right] J_{n}^{\prime}\left(k_{r 1} r\right)} \\
+\left[k_{0}^{2} D+\delta_{1}\left(k_{z}^{2}-k_{0}^{2} S\right)\right] \frac{n J_{n}\left(k_{r 2} r\right)}{k_{r 1} r}
\end{array}\right\} \\
& h_{r 2}=-\omega \epsilon_{0} k_{r 2} \Lambda^{-1}\left\{\begin{array}{c}
{\left[k_{z}^{2} D+\delta_{2}\left(k_{z}^{2}-k_{0}^{2} S\right)\right] J_{n}^{\prime}\left(k_{r 2} r\right)} \\
+\left[\left(k_{z}^{2} S-k_{0}^{2} R L\right)+\delta_{2} k_{0}^{2} D\right] \frac{n J_{n}\left(k_{r 2 r} r\right)}{k_{r 2} r}
\end{array}\right\} \\
& h_{\phi 1}=k_{z} k_{r 1} \Lambda^{-1}\left\{\begin{array}{c}
{\left[k_{0}^{2} D+\delta_{1}\left(k_{z}^{2} S-k_{0}^{2} R L\right)\right] J_{n}^{\prime}\left(k_{r 1} r\right)} \\
+\left[\left(k_{z}^{2}-k_{0}^{2} S\right)+\delta_{1} k_{0}^{2} D\right] \frac{n J_{n}\left(k_{r 1} r\right)}{k_{r 1} r}
\end{array}\right\} \\
& h_{\phi 2}=-i \omega \epsilon_{0} k_{r 2} \Lambda^{-1}\left\{\begin{array}{c}
{\left[\left(k_{z}^{2} S-k_{0}^{2} R L\right)+\delta_{2} k_{0}^{2} D\right] J_{n}^{\prime}\left(k_{r 2} r\right)} \\
+\left[k_{z}^{2} D+\delta_{2}\left(k_{z}^{2}-k_{0}^{2} S\right)\right] \frac{n J_{n}\left(k_{r 2} r\right)}{k_{r 2} r}
\end{array}\right\}
\end{aligned}
$$

Where

$$
\begin{gathered}
\epsilon_{1}=\frac{-i k_{z}}{\omega \mu_{0}} z_{1} \\
\epsilon_{2}=\frac{i \omega \mu_{0}}{k_{z}} y_{2} \\
\delta_{1}=\frac{-i \omega \epsilon_{0}}{k_{z}} z_{1} \\
\delta_{2}=\frac{i k_{z}}{\omega \mu_{0}} y_{2}
\end{gathered}
$$

The excitation coefficients are seen as

$$
\begin{aligned}
& c_{1}=i e_{\phi 1}(r=a)+\frac{\omega \mu_{0}}{\nu}\left(\frac{\Delta_{a^{\prime} c^{\prime}}}{\Delta_{a c^{\prime}}}+\frac{\epsilon_{1} n}{\nu a}\right) J_{n}\left(k_{r 1} a\right) \\
& c_{2}=i e_{\phi 2}(r=a)-i \frac{k_{z}}{\nu}\left(\frac{n}{\nu a}+\epsilon_{2} \frac{\Delta_{a^{\prime} c^{\prime}}}{\Delta_{a c^{\prime}}}\right) J_{n}\left(k_{r 2} a\right) \\
& c_{3}=h_{\phi 1}(r=a)-\frac{k_{z}}{\nu}\left(\frac{n}{\nu a}+\delta_{1} \frac{\Delta_{a^{\prime} c}}{\Delta_{a c}}\right) J_{n}\left(k_{r 1} a\right) \\
& c_{4}=h_{\phi 2}(r=a)+\frac{\omega \epsilon_{0}}{\nu}\left(\frac{\Delta_{a^{\prime} c}}{\dot{\Delta}_{a c}}+\delta_{2} \frac{n}{\nu a}\right) J_{n}\left(k_{r 2} a\right)
\end{aligned}
$$




$$
\begin{gathered}
e_{1}=\frac{\omega \mu_{0} b}{\nu a} \frac{\Delta_{b}^{\prime} c}{\Delta_{a c^{\prime}}} \\
e_{2}=\frac{-k_{0}^{2}}{k_{z} \nu^{2}} \frac{n}{a} \frac{\Delta_{b c}}{\Delta a c} \\
\Lambda=\left(k_{0}^{2} R-k_{z}^{2}\right)\left(k_{0}^{2} L-k_{z}^{2}\right) \\
\Delta_{a b^{\prime}} \equiv K_{n a} I_{n b}^{\prime}-K_{n b}^{\prime} I_{n a}
\end{gathered}
$$




\section{Appendix D}

\section{ICRF Wave Propagation in Slab}

\section{Geometry}

The propagation of ICRF wave in slab geometry has been studied in great detail r-er the course of the past 30 years $[59,45,81]$. This Appendix will demonstrate the steps required in the solution of such a problem. Two cases are examined the $k_{y}=0$ case and the $k_{y} \neq 0$ case. It will be seen that different physics are inherent in each case. For the $k_{y}=0$ case, it is seen that the TE and TM modes are decoupled in the plasma, allowing a relatively straightforward solution of the fields. The $k_{y} \neq 0$ case couples the TE and TM modes in the plasma, thus making the boundary conditions more difficult to handle. 


\section{D.1 Full Slab Model: $k_{z}=0$}

The first geometry to be analyzed is the slab geometry with $k_{z}=0$. The approach taken in this analysis is the standard solution of electromagnetic fields as given in Kong [59] in which the waves are separated into two independent components, TE and TM. It will be shown that in the limit of $k_{z}=0$, these two modes are effectively decoupled from each other and form two independent solutions to the problem.

The geometry under consideration is a semiinfinite slab in which EM waves are incident from source at $x=-\infty$ upon the vacuum-plasma interface at $x=0$. The plasma is infinite in the $y$ and $z$ extent.

\section{D.1.1 Field Formulation}

Recall Maxwell's equations:

Vacuum

$$
\begin{aligned}
& \nabla \times \vec{E}=-\frac{\partial \vec{B}}{\partial t} \\
& \nabla \times \vec{B}=\frac{1}{c^{2}} \frac{\partial \vec{E}}{\partial t}
\end{aligned}
$$

Plasma

$$
\begin{gathered}
\nabla \times \vec{E}=-\frac{\partial \vec{B}}{\partial t} \\
\nabla \times \vec{B}=\frac{1}{c^{2}} \frac{\partial \vec{E}}{\partial t}+\mu_{0} \vec{J}=-\frac{i \omega}{c^{2}} \underline{K} \cdot \vec{E}
\end{gathered}
$$

Assuming no propagation in $\hat{z}: k_{z}=0$

$$
\nabla^{2} \rightarrow \frac{\partial^{2}}{\partial x^{2}}+\frac{\partial^{2}}{\partial y^{2}}
$$

Vacuum: 
$\hat{\mathbf{x}} \quad B_{x}=\frac{k_{x}}{\omega} E_{z}$

$\hat{\mathbf{y}} \quad B_{y}=-\frac{k_{x}}{\omega} E_{z}$

$\hat{\mathbf{z}} \quad \omega B_{z}=k_{x} E_{y}-k_{y} E_{x}$

Ampere's Law:

$\hat{\mathbf{x}} \quad E_{x}=-\frac{k_{x} c^{2}}{\omega} B_{z}$

$\hat{\mathbf{y}} \quad E_{y}=\frac{k_{n} c^{2}}{\omega} B_{z}$

$\hat{\mathbf{z}} \quad \omega E_{z}=-c^{2}\left[k_{x} B_{y}-k_{y} B_{x}\right]$

Combining

$$
\frac{\partial^{2}}{\partial x^{2}} B_{z}, E_{z}=\left(k_{y}^{2}-k_{0}^{2}\right) B_{z}, E_{z}
$$

The solution to the above is

$$
B_{z}, E_{z}=B_{z 0}, E_{z 0} e^{i k_{z} x+i k_{y} y-i \omega t}
$$

Where

$$
k_{x}^{2} \equiv k_{0}^{2}-k_{y}^{2}
$$

The vacuum solutions for Incident Wave is then given by

$$
\begin{aligned}
& E_{x}=-\frac{k_{y} c^{2}}{\omega} B_{z 0 I} e^{i k_{x I} x+i k_{y} y} \\
& E_{y}=\frac{k_{x} c^{2}}{\omega} B_{x 0 I} e^{i k_{x I} x+i k_{y} y} \\
& E_{x}=E_{z 0 I} e^{i k_{y I} x+i k_{y} y} \\
& B_{x}=\frac{k_{y}}{\omega} E_{z 0 I} e^{i k_{x I} x+i k_{y} y} \\
& B_{y}=-\frac{k_{x}}{\omega} E_{x 0 I} e^{i k_{x I} x+i k_{y} y} \\
& B_{z}=B_{z 0 I} e^{i k_{x I} x+i k_{y} y}
\end{aligned}
$$




\section{Reflected Wave}

The reflected wave is directed in the $-\hat{x}$ direction, so substitution of $k_{x} \rightarrow-k_{x}$ yields the following set of Reflected Wave fields:

$$
\begin{aligned}
& E_{x}=-\frac{k_{y} c^{2}}{\omega} B_{z 0 R} e^{-i k_{x} x+i k_{y} y} \\
& E_{y}=-\frac{k_{x} c^{2}}{\omega} B_{z 0 R} e^{-i k_{z} y+i k_{y} y} \\
& E_{z}=E_{z 0 R} e^{-i k_{y} x+i k_{y} y} \\
& B_{x}=\frac{k_{y}}{\omega} E_{z 0 R} e^{-i k_{y} x+i k_{y} y} \\
& B_{y}=\frac{k_{x}}{\omega} E_{z 0 R} e^{-i k_{y} x+i k_{y} y} \\
& B_{z}=B_{z 0 R} e^{-i k_{x} x+i k_{y} y}
\end{aligned}
$$

Note: $k_{x I}=k_{x R}$ because the medium is the same (vacuum).

Defining a reflection coefficient $R$ such that

$$
\begin{aligned}
& B_{z 0 R}=R_{T E} B_{z 0 I} \\
& E_{z 0 R}=R_{T M} E_{z 0 I}
\end{aligned}
$$

the entire vacuum field can be seen to be

$$
\begin{aligned}
& \text { TE Mode }\left\{\begin{array}{l}
E_{x}=-\frac{k_{y} c^{2}}{\omega} B_{z o I}\left[e^{i k_{x} x}+R_{T E} e^{-i k_{x} x}\right] \\
E_{y}=-\frac{k_{x} c^{2}}{\omega} B_{z o I}\left[e^{i k_{x} x}-R_{T E} e^{-i k_{x} x}\right] \\
B_{z}=B_{z o I}\left[e^{i k_{x} x}+R_{T E} e^{-i k_{x} x}\right]
\end{array}\right\} \\
& \text { TM Mode }\left\{\begin{array}{l}
B_{x}=\frac{k_{y}}{\omega} E_{z o I}\left[e^{i k_{x} x}+R_{T M} e^{-i k_{x} x}\right] \\
B_{y}=-\frac{k_{x}}{\omega} E_{z o I}\left[e^{i k_{x} x}-R_{T M} e^{-i k_{x} x}\right] \\
E_{z}=E_{z o I}\left[e^{i k_{y} x}+R_{T M} e^{-i k_{x} x}\right]
\end{array}\right\}
\end{aligned}
$$

\section{Plasma Fields}

Gauss's Law still remain the same:

$\hat{\varkappa} \quad B_{x}=\frac{k_{x}}{\omega} E_{x}$ 
$\hat{\mathbf{y}} \quad B_{y}=-\frac{k_{n}}{\omega} E_{z}$

$\hat{\mathbf{z}} \quad \omega B_{z}=k_{x} E_{y}-k_{y} E_{x}$

Ampere's Law yields:

$\hat{\mathbf{x}} \quad B_{z}=-\frac{\omega}{c^{2} k_{y}}\left[S E_{x}-i D E_{y}\right]$

$\hat{\mathbf{y}} \quad B_{z}=\frac{\omega}{c^{2} k_{\mathrm{z}}}\left[i D E_{x}+S E_{y}\right]$

$\hat{\mathbf{z}} \quad-\frac{\omega P}{c^{2}} E_{z}=\left[k_{x} B_{y}-k_{y} B_{x}\right]$

It is seen that the solutions can be decoupled into two sets of independent solutions, the TE and TM modes

TE Mode

$$
\begin{aligned}
& E_{y}=\frac{c^{2}}{\omega} \frac{S k_{x B}+i D k_{y}}{S^{2}-D^{2}} B_{z} \\
& E_{x}=-\frac{c^{2}}{\omega} \frac{S k_{\nu}-i D k_{x B}}{S^{2}-D^{2}} B_{z}
\end{aligned}
$$

TM Mode

$$
\begin{gathered}
B_{y}=-\frac{k_{x E}}{\omega} E_{z} \\
B_{x}=\frac{k_{y}}{\omega} E_{z}
\end{gathered}
$$

TOTAL PLASMA FIELD

So the plasma fields are :

$$
\begin{aligned}
& \text { TE Mode }\left\{\begin{array}{ll}
E_{x}=-\frac{c^{2}}{\omega} \frac{\left(S k_{y}-i D k_{x B}\right)}{S^{2}-D^{2}} & B_{z 0 P} e^{i k_{x B} x+i k_{y} y} \\
E_{y}=\frac{c^{2}}{\omega} \frac{\left(S k_{y B}+i D k_{y}\right)}{S^{2}-D^{2}} & B_{z 0 P e^{i k_{x} x+i k_{y} y}} \\
B_{z}= & B_{z 0 P} e^{i k_{x B} x+i k_{y} y}
\end{array}\right\} \\
& \text { TM Mode }\left\{\begin{array}{ll}
B_{x}=\frac{k_{y}}{\omega} & E_{z O P} e^{i k_{x} B x+i k_{y} y} \\
B_{y}=\frac{k_{x}}{\omega} & E_{z O P} e^{i k_{x B x+i k_{y} y}} \\
E_{z}= & E_{z O P} e^{i k_{x} \Delta x+i k_{y} y}
\end{array}\right\}
\end{aligned}
$$




\section{D.1.2 Plasma Dispersion Relation for TE and TM Modes}

\section{TE Mode}

Recall that the TE mode fields are given in terms of $B_{z}$ as

$$
\begin{aligned}
& E_{y}=\frac{c^{2}}{\omega} \frac{S k_{x B}+i D k_{y}}{S^{2}-D^{2}} B_{z} \\
& E_{x}=-\frac{c^{2}}{\omega} \frac{S k_{y}-i D k_{x B}}{S^{2}-D^{2}} B_{z}
\end{aligned}
$$

Substituting the above equations, into the Gauss's Law yields

$$
\begin{gathered}
\frac{\omega^{2}}{c^{2}} B_{z}=\frac{k_{x B}\left(S k_{x B}+i D k_{y}\right)}{S^{2}-D^{2}} B_{z}+k_{y} \frac{\left(S k_{y}-i D k_{x B}\right)}{S^{2}-D^{2}} B_{z} \\
\Rightarrow \quad k_{0}^{2}\left(S^{2}-D^{2}\right)=S\left(k_{x B}^{2}+k_{y}^{2}\right)
\end{gathered}
$$

Finally, the dispersion relation is found to be

$$
k_{x B}^{2}=k_{0}^{2} \frac{\left(S^{2}-D^{2}\right)}{S}-k_{y}^{2}
$$

Compared to $k_{x}^{2}=k_{0}^{2}-k_{y}^{2}$ for the vacuum solution.

\section{TM Mode}

Recall that the TM mode fields are given in terms of $E_{z}$ as

$$
\begin{gathered}
B_{y}=-\frac{k_{x E}}{\omega} E_{z} \\
B_{x}=\frac{k_{y}}{\omega} E_{z}
\end{gathered}
$$

Substituting into the third Ampere's law yields the TM Plasma dispersion relation of

$$
k_{x E}^{2}=k_{0}^{2} P-k_{y}^{2}
$$

\section{D.1.3 Boundary Conditions}

The boundary conditions are as follows: 
1. $E_{\|}$Continuous

2. $D_{\perp}$ Continuous. This arises from the zero surface charge condition.

3. $B_{\|}$Continous. This condition valid only if no surface current is present.

4. $B_{\perp}$ condinous. Valid since no magnetic monopoles.

\section{TE Mode}

Assuming

$$
\begin{aligned}
& B_{z 0 R} \equiv R B_{z 0 I} \\
& B_{z 0 P} \equiv T B_{z 0 I}
\end{aligned}
$$

1. $E_{\|}$Continuous:

$$
1-R=\frac{1}{k_{x}} \frac{\left(S k_{x B}+i D k_{y}\right)}{S^{2}-D^{2}} T
$$

2. $H_{\|}$Continous:

$$
1+R=T
$$

Cornbining:

$$
\begin{gathered}
T_{T E}=\frac{2 k_{x}\left(S^{2}-D^{2}\right)}{\left(S^{2}-D^{2}\right) k_{x}+S k_{x B}+i D k_{y}} \\
R_{T E}=T_{T E}-1=\frac{k_{x}\left(S^{2}-D^{2}\right)-S k_{x B}-i D k_{y}}{\left(S^{2}-D^{2}\right) k_{x}+S k_{x B}+i D k_{y}}
\end{gathered}
$$

TM Mode

1. $E_{\|}$Continous

$$
1+R=T
$$


2. $H_{\|}$Continuous

$$
1-R=\frac{k_{x E}}{k_{x}} T
$$

Combining

$$
\begin{gathered}
T_{T M}=\frac{2 k_{x}}{k_{x}+k_{\triangleright E}} \\
R_{T M}=T_{T M}-1=\frac{k_{x}-k_{x E}}{k_{x}+k_{x E}}
\end{gathered}
$$

Rewriting both

$$
\begin{aligned}
R_{T E} & =\frac{1-\psi_{T E}}{1+\psi_{T E}} \\
T_{T E} & =\frac{2}{1+\psi_{T E}} \\
R_{T M} & =\frac{1-\psi_{T M}}{1+\psi_{T M}} \\
T_{T M} & =\frac{2}{1+\psi_{T M}}
\end{aligned}
$$

Where

$$
\begin{aligned}
\psi_{T E} & \equiv \frac{S k_{x B}+i D k_{y}}{k_{x}\left(S^{2}-D^{2}\right)} \\
\psi_{T M} & \equiv \frac{k_{x E}}{k_{x}}
\end{aligned}
$$

These results are identical in form to the results obtained on p.113 of Kong $[59]$. 


\section{D.1.4 Dissipation and Figure of Merit for TE Mode}

Although conventional literature uses the plasma impedence $R_{\text {plas }}$ as the standard measurement of heating efficiency $[55,56,57]$, it is not used as the figure of merit in this analysis because the launching structure is not considered as part of the problem. In this section a different figure of merit is developed and computed for various plasma properties.

The time averaged Poynting vector is shown [59] to be given by

$$
<\vec{S}>=\frac{1}{2} \operatorname{Re}\left\{\vec{E} \times \vec{H}^{*}\right\}
$$

The incident, reflected and transmitted Poynting Flux are thus given by

$$
\begin{gathered}
<\vec{S}>_{i}=\frac{\left|B_{x 0}\right|^{2} c^{2}}{2 \mu_{0} \omega}\left(k_{x 0} \hat{x}+k_{y} \hat{y}\right) \\
<\vec{S}>_{R}=\frac{|R|^{2}\left|B_{z 0}\right|^{2} c^{2}}{2 \mu_{0} \omega}\left(-k_{x 0} \hat{x}+k_{y} \hat{y}\right) \\
<\vec{S}>_{T}=\frac{|T|^{2}\left|B_{z 0}\right|^{2} c^{2}}{2 \mu_{0} \omega}\left(\operatorname{Re}\left(C_{y}\right) \hat{x}+\operatorname{Re}\left(C_{x}\right) \hat{y}\right)
\end{gathered}
$$

where

$$
\begin{gathered}
C_{y} \equiv \Psi_{T E} k_{x 0} \\
C_{x} \equiv \frac{\left(S k_{y}-i D k_{x B}\right)}{\left(S^{2}-D^{2}\right)}
\end{gathered}
$$

Looking at the geometry of the system in Figure D-1 shows that the energy that is actually transmitted through the interface is the perpendicular Poynting flux.

Then a reflectivity $r$ can be defined [59] as the ratio of the $-\hat{x}$ directed reflected Poynting flux to the $+\hat{x}$ directed incident Poynting flux. Likewise a transmitivity $\boldsymbol{t}$ can be defined as the ratio of the $+\hat{x}$ directed incident Poynting flux to the $+\hat{x}$ directed incident Poyntinğ fiux. 


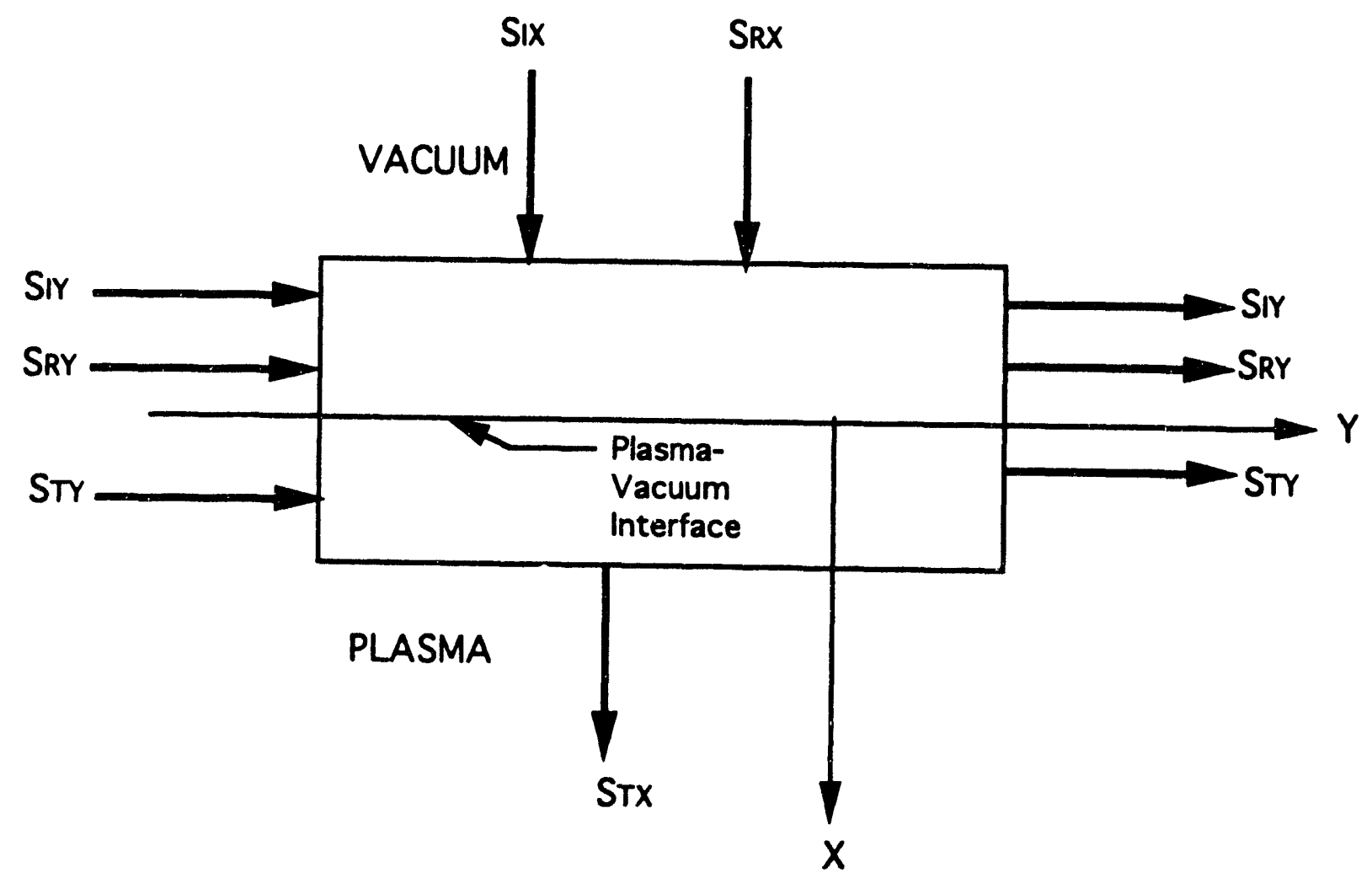

Figure D-1: Geometry of TE and TM wave incident on vacuum-plasma interface for $k_{z}=0$ case. 


$$
\begin{gathered}
r \equiv \frac{\hat{x} \cdot\left\langle\vec{S}>_{R}\right.}{-\hat{x} \cdot\left\langle\vec{S}>_{I}\right.}=|R|^{2} \\
t \equiv \frac{-\hat{x} \cdot\left\langle\vec{S}>_{T}\right.}{-\hat{x} \cdot\left\langle\vec{S}>_{I}\right.}=\operatorname{Re}\left(\psi_{T E}\right)|T|^{2}
\end{gathered}
$$

Since it has been shown that

$$
\begin{aligned}
& R_{T E}=\frac{1-\psi_{T E}}{1+\psi_{T E}} \\
& T_{T E}=\frac{2}{1+\psi_{T E}}
\end{aligned}
$$

It is easily seen that

$$
r+t=1
$$

and this is indicative of the conservation of the normal power flow across a boundary.

Therefore a figure of merit $\mathcal{F}$ can be defined as

$$
\mathcal{F} \equiv t \equiv \frac{-\hat{x} \cdot\left\langle\vec{S}>_{T}\right.}{-\hat{x} \cdot\left\langle\vec{S}>_{I}\right.}=\operatorname{Re}\left(\psi_{T E}\right)|T|^{2}
$$


RE (PSITE)

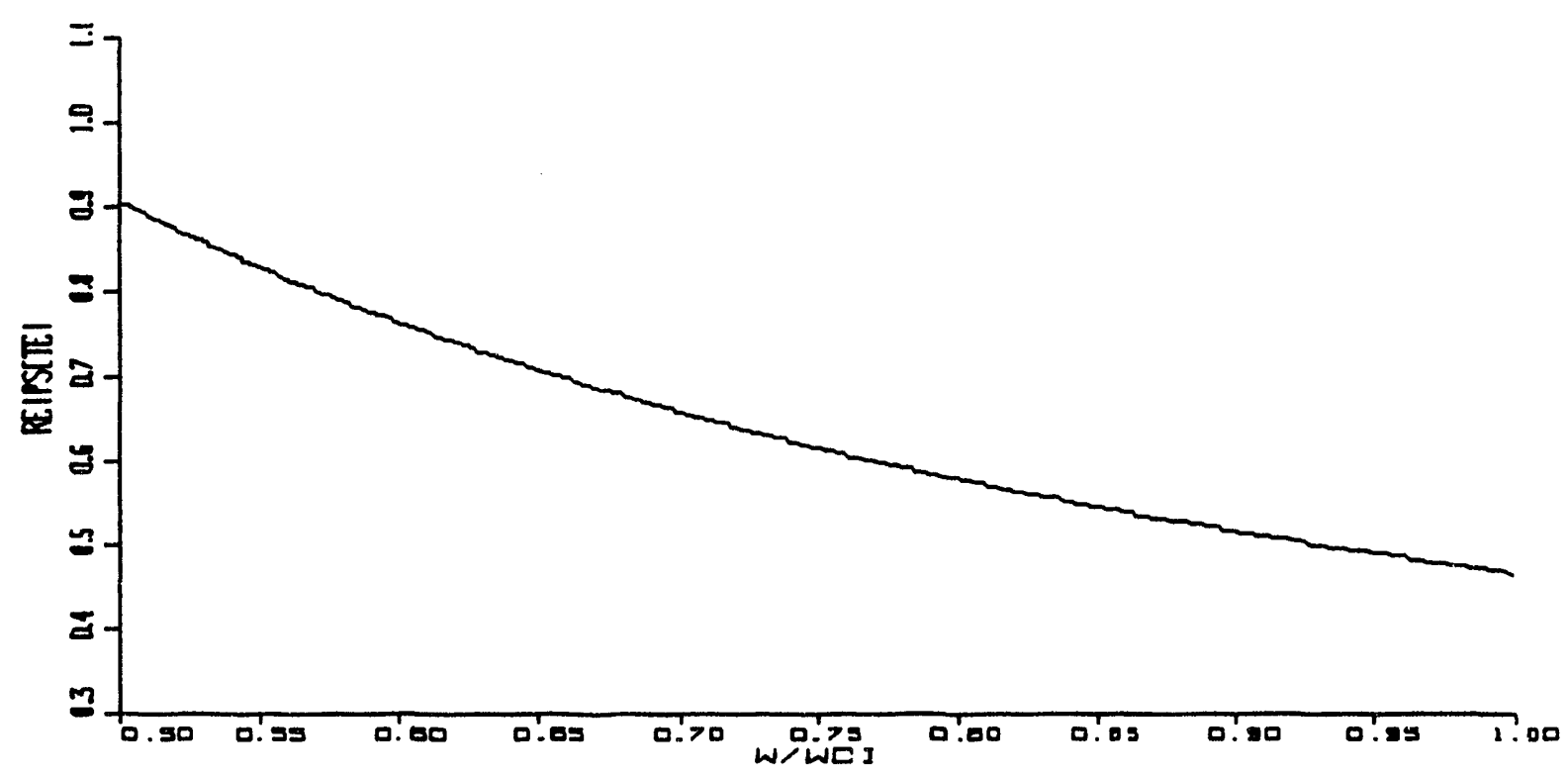

(I) $\times 2$ VS W/WCI

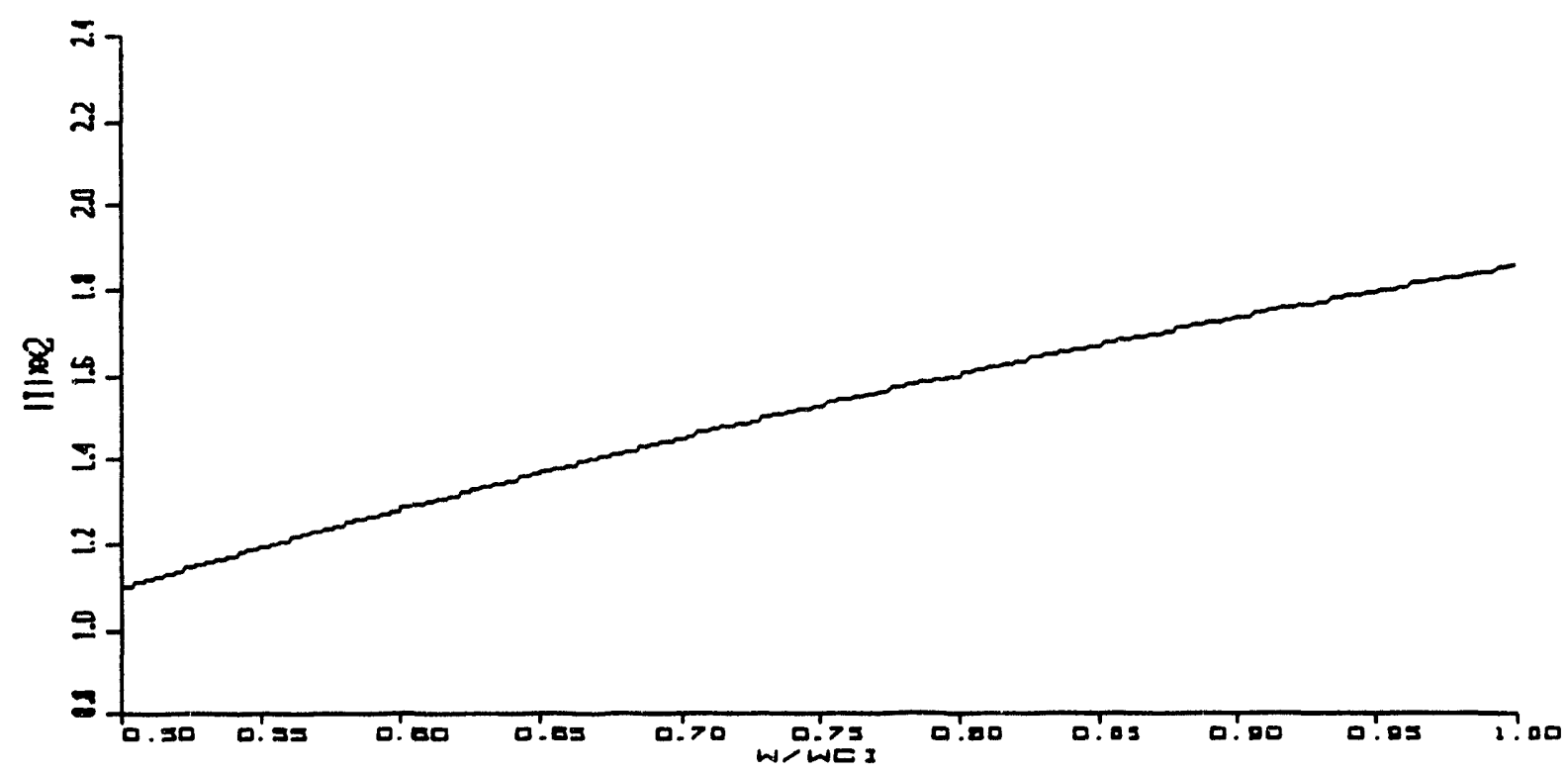

Figure D-2: $\operatorname{Re}\left(\psi_{T E}\right)$ (Top) and $|T|^{2}$ (Bottom) versus $\omega_{c i}$ for $k_{\xi}=0$ case. Plasma density of this scan was fixed at $n_{e}=1.0 \times 10^{10} \mathrm{~cm}^{-3}$. 


\section{FIGURE OF MERII CALCULATION}

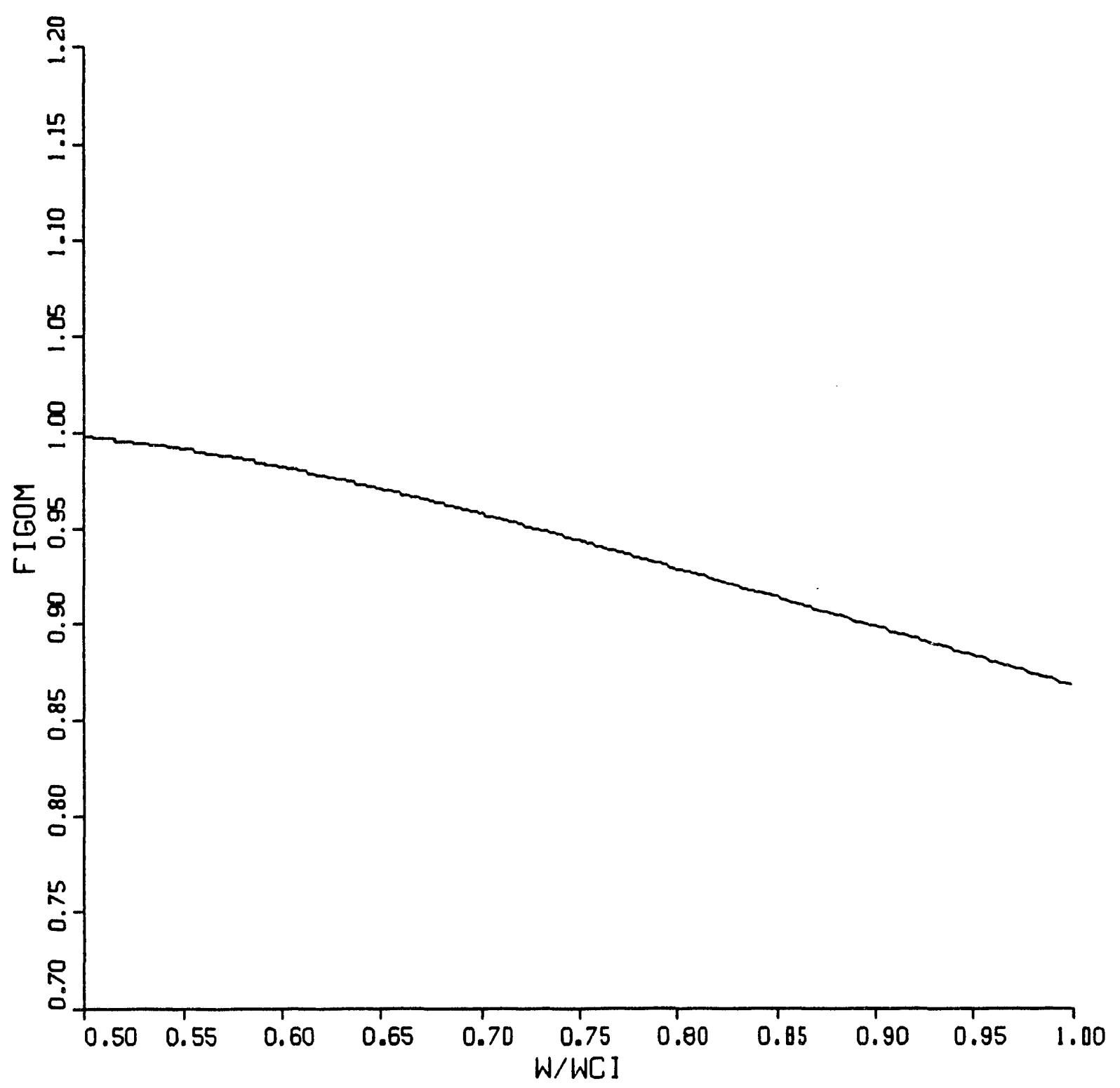

Figure D-3: Figure of Merit calculation for $k_{z}=0$ case at different ion cyclotron frequencies $\omega_{c i}$. Plasma density of this scan was fixed at $n_{e}=1.0 \times 10^{10} \mathrm{~cm}^{-3}$. 
RE (PSIIE)

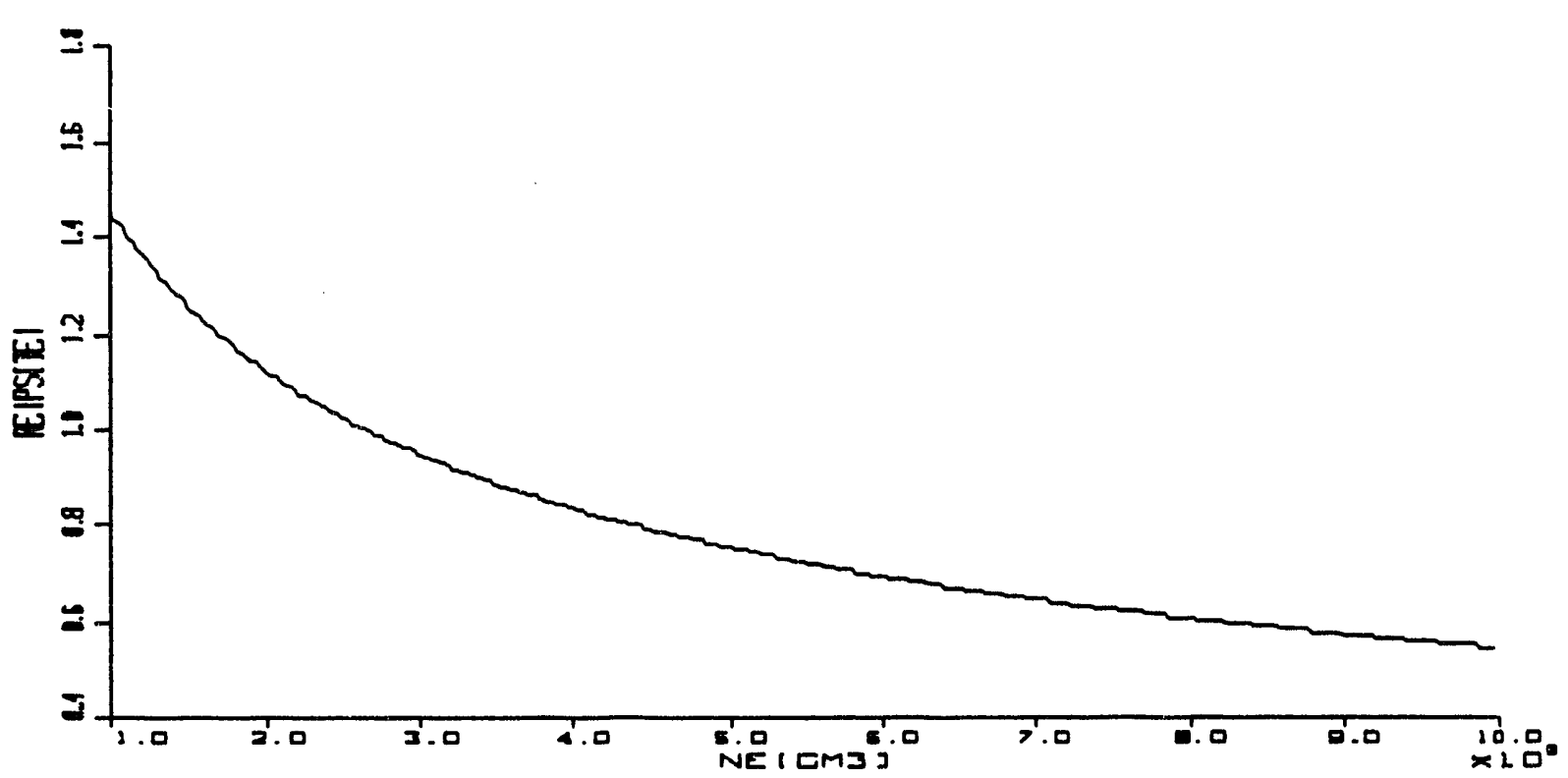

(I) 2 VS NE

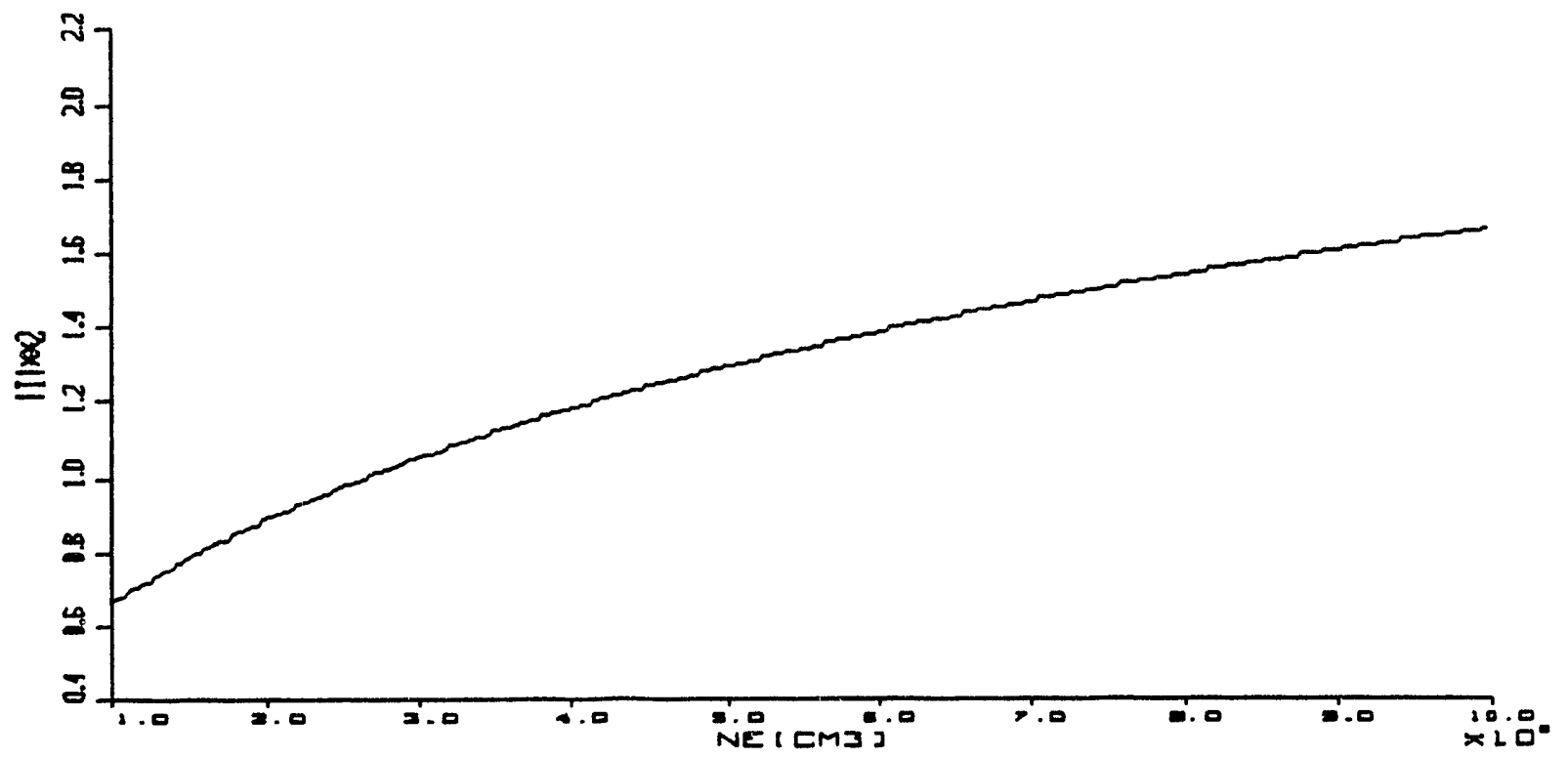

Figure D-4: $\operatorname{Re}\left(\psi_{T E}\right)$ (Top) and the transmission coefficient $|T|^{2}$ (Bottom) calculation for $k_{z}=0$ case at different densities. Frequency ratio of this scan was fixed at $\omega / \omega_{c i}=0.85$. 


\section{FIGURE OF MERIT CALCULATION}

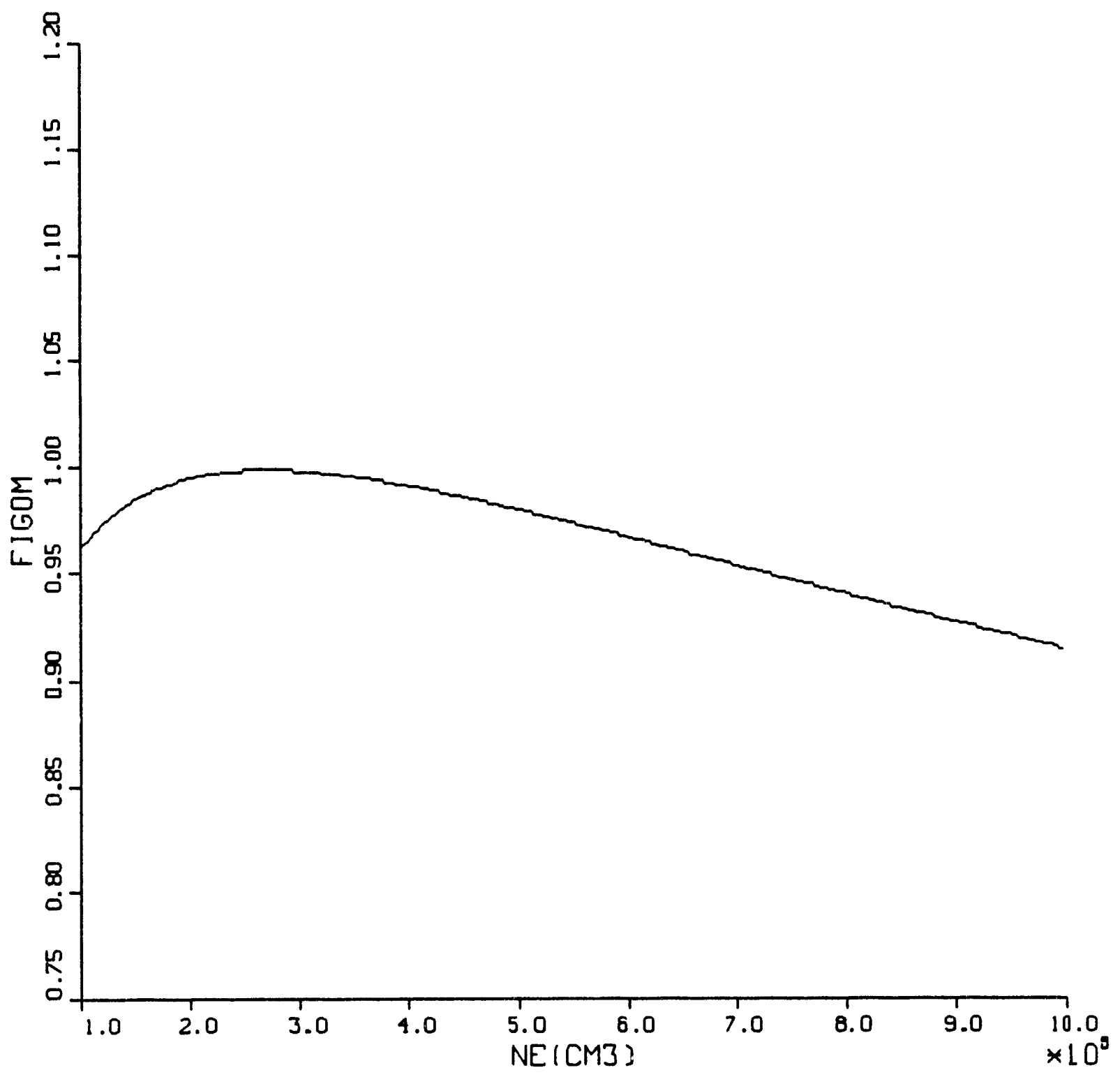

Figure D-5: Figure of Merit calculation for $k_{z}=0$ case at different densities. Frequency ratio of this scan was fixed at $\omega / \omega_{c i}=0.85$. 


\section{D.1.5 Discussion of Results}

The results of efficiency calculation performed in this section show that

1. The lower the $\omega / \omega_{c i}$ the higher the figure of merit, and

2. there exists an optimum density that produces an optimum figure of merit.

These results are consistent with expectations. Because of the infinite slab geometry, all the Poynting Flux that is transmitted across the boundary is eventually absorbed by the small damping introduced by collisions. Thus the only issue is essentially the transmission that occurs across the boundary. 


\section{D.2 Full Slab Theory of $k_{z} \neq 0$ TE/TM Waves}

The previous section dealt with the case of $k_{z}=0$. The next logical extension of the problem is for a wave that is allowed to propage in the $\mathrm{z}$ direction.

As will be seen, there will be two modes that are found to exist in this system, the TE and the TM mode. However, these two modes will be shown to be no longer independent of each other. The TE and the TM modes are still separate and independent waves in the vacuum region, but they now coupled together via the plasma.

The basic formulation is arrived at in much the same manner as the previous section. However, linear algebra methods will be used to provide better insight into the solution of the problem.

\section{D.2.1 Formulation}

Maxwell's equation is used to express the transverse fields in terms of the parallel fields $B_{z}$ and $E_{z}$. It can be shown that:

\section{Incident Vacuum Field}

$$
\begin{aligned}
& E_{x}=\frac{e^{i k_{\mathrm{z}} x}}{k_{0}^{2}-k_{z}^{2}}\left[-\omega k_{y} B_{z 0}-k_{x} k_{z} E_{z 0}\right] \\
& E_{y}=\frac{e^{i k_{x} x}}{k_{0}^{2}-k_{z}^{2}}\left[\omega k_{x} B_{z 0}-k_{y} k_{z} E_{z 0}\right] \\
& E_{z}=E_{z 0} e^{i k_{x} x} \\
& B_{x}=\frac{k_{0}^{2} e^{i k_{x} x}}{k_{0}^{2}-k_{z}^{2}}\left[-\frac{k_{x} k_{z}}{k_{0}^{2}} B_{x 0}+\frac{k_{y}}{\omega} E_{z 0}\right] \\
& B_{y}=\frac{k_{0}^{2} e^{i k_{x} x}}{k_{0}^{2}-k_{z}^{2}}\left[-\frac{k_{y} k_{z}}{k_{0}^{2}} B_{z 0}-\frac{k_{x}}{\omega} E_{z 0}\right] \\
& B_{z}=B_{z 0} e^{i k_{x} x}
\end{aligned}
$$

Likewise, the reflected fields are expressable in terms of the incident fields as well: 


\section{Reflected Vacuum Field}

$$
\begin{aligned}
& E_{x}=\frac{e^{-i k_{x} x}}{k_{0}^{2}-k_{z}^{2}}\left[-\omega k_{y} R_{B} B_{z 0}+k_{x} k_{z} R_{E} E_{z 0}\right] \\
& E_{y}=\frac{e^{-i k_{x} x}}{k_{0}^{2}-k_{z}^{2}}\left[-\omega k_{x} R_{B} B_{z 0}-k_{y} k_{z} R_{E} E_{x 0}\right] \\
& E_{z}=R_{E} E_{z 0} e^{i-k_{x} x} \\
& B_{x}=\frac{k_{0}^{2} e^{-i k_{x} x}}{k_{0}^{2}-k_{z}^{2}}\left[+\frac{k_{x} k_{z}}{k_{0}^{2}} R_{B} B_{z 0}+\frac{k_{y}}{\omega} R_{E} E_{z 0}\right] \\
& B_{y}=\frac{k_{0}^{2} e^{-i k_{x} x}}{k_{0}^{2}-k_{z}^{2}}\left[-\frac{k_{y} k_{z}}{k_{0}^{2}} R_{B} B_{z 0}+\frac{k_{x}}{\omega} R_{E} E_{x 0}\right] \\
& B_{z}=R_{B} B_{z 0} e^{-i k_{x} x}
\end{aligned}
$$

The plasma fields are more difficult to obtain but the methodology is the same as for the vacuum fields. It has been shown $[82,4]$ that the fields are given by

\section{Plasma Fields}

$$
\begin{aligned}
& B_{z}=B_{z 1} e^{i k_{x 1} x}+y_{2} \mu_{0} E_{z 2} e^{i k_{z 2} x} \\
& E_{z}=\frac{z_{1}}{\mu_{0}} B_{z 1} e^{i k_{z 1} x}+E_{z 2} e^{i k_{z 2} x} \\
& E_{x}=\omega\left(\delta_{0} B_{z}^{\prime}+\delta_{1} k_{y} B_{z}\right)-i k_{z}\left(\delta_{1} E_{z}^{\prime}+\delta_{0} k_{y} E_{z}\right) \\
& \equiv e_{x b 1} B_{x 1} e^{i k_{x 1} x}+e_{x e 2} E_{z 2} e^{i k_{x 2 x}} \\
& E_{y}=i \omega\left(\delta_{1} B_{z}^{\prime}+\delta_{0} k_{y} B_{z}\right)+k_{z}\left(\delta_{0} E_{z}^{\prime}+k_{y} \delta_{1} E_{z}\right) \\
& \equiv e_{y b 1} B_{z 1} e^{i k_{z 3} x}+e_{y e 2} E_{z 2} e^{i k_{z 2} x} \\
& B_{\mathrm{a}}=-i k_{z}\left(\delta_{1} B_{z}^{\prime}+\delta_{0} k_{y} B_{z}\right)-\frac{\omega}{c^{2}}\left(\delta_{z} E_{z}^{\prime}+\delta_{2} k_{y} E_{z}\right) \\
& \equiv b_{x i 1} B_{z 1} e^{i k_{x 1} \infty}+b_{x c 2} E_{x 2} e^{i k_{x 2 \infty}} \\
& B_{y}=k_{z}\left(\delta_{0} B_{z}^{\prime}+k_{y} \delta_{1} B_{z}\right)-i \frac{\omega}{c^{2}}\left(\delta_{2} E_{z}^{\prime}+\delta_{z} k_{y} E_{z}\right) \\
& \equiv b_{y b 1} B_{z 1} e^{i k_{z 1} x}+b_{y e 2} E_{z 2} e^{i k_{z 2} x}
\end{aligned}
$$

Where

$$
B_{z}^{\prime}=i k_{x 1} B_{z 1} e^{i k_{x 1} x}+i k_{x 2} y_{2} \mu_{0} E_{z 2} e^{i k_{x 2} x}
$$




$$
\begin{gathered}
E_{z}^{\prime}=\frac{i k_{x 1} z_{1}}{\mu_{0}} B_{z 1} e^{i k_{x 1} x}+i k_{x 2} E_{z 2} e^{i k_{x 2} x} \\
\delta_{3} \equiv\left(k_{0}^{2} R-k_{z}^{2}\right)\left(k_{0}^{2} L-k_{z}^{2}\right) \\
\delta_{0} \equiv \frac{k_{0}^{2} D}{\delta_{3}} \\
\delta_{z} \equiv \frac{k_{z}^{2} D}{\delta_{3}} \\
\delta_{1} \equiv \frac{k_{z}^{2}-k_{0}^{2} S}{\delta_{3}} \\
\delta_{2} \equiv \frac{k_{z}^{2} S-k_{0}^{2} R L}{\delta_{3}} \\
z_{1} \equiv \frac{i \omega \mu_{0} k_{z} D}{P\left(k_{z}^{2}-k_{0}^{2} S\right)+k_{x 1}^{2} S} \\
e_{x b 1}=i k_{x 1} \omega \delta_{0}+\omega \delta_{1} k_{y}+\frac{-i \omega \epsilon_{0} k_{z} P D}{S\left(k_{x 2}^{2}+k_{z}^{2}\right)-R L k_{0}^{2}} \\
e_{x e 2}=i \omega \delta_{0} y_{2} \mu_{0} k_{x 2}+\omega \delta_{1} k_{y} y_{2} \mu_{0}+k_{z} \delta_{1} k_{x 2}-i k_{y} k_{z} \delta_{0} \\
e_{y b 1}=-
\end{gathered}
$$

\section{D.2.2 Plasma Dispersion for $k_{z} \neq 0$}

Recall Maxwell's equation

$$
\nabla \times \underline{E}=-\frac{\partial \underline{B}}{\partial t}
$$




$$
\begin{gathered}
\nabla \times \underline{B}=-\frac{1}{c^{2}} \frac{\partial \underline{B}}{\partial t}+\mu_{0} \underline{I} \\
\nabla \times \nabla \times \underline{E}=\nabla(\nabla \cdot \underline{E})-\nabla^{2} \underline{E}=i \omega\left(\frac{-i \omega}{c^{2}} E+\mu_{0} \mathcal{J}\right) \\
\nabla(\nabla \cdot \underline{E})-\nabla^{2} \underline{E}=\frac{\omega^{2}}{c^{2}} \underline{\epsilon} \cdot \underline{E}
\end{gathered}
$$

Grouping into components

$$
\begin{gathered}
E_{x}\left(k_{y}^{2}+k_{z}^{2}-k_{0}^{2} S\right)+E_{y}\left(i D k_{0}^{2}-k_{x} k_{y}\right)-k_{x} k_{z} E_{x}=0 \\
E_{x}\left(-k_{x} k_{y}-i D k_{0}^{2}\right)+E_{y}\left(k_{x}^{2}+k_{z}^{2}-k_{0}^{2} S\right)-k_{y} k_{z} E_{x}=0 \\
-k_{x} k_{x} E_{x}-k_{y} k_{x} E_{y}+\left(k_{x}^{2}+k_{y}^{2}-k_{0}^{2} P\right) E_{z}=0
\end{gathered}
$$

The determinant of this $3 \times 3$ system is then zero

$$
\left|\begin{array}{ccc}
k_{y}^{2}+k_{z}^{2}-k_{0}^{2} S & -k_{x} k_{y}+i k_{0}^{2} D & -k_{x} k_{z} \\
-k_{x} k_{y}-i k_{0}^{2} D & k_{x}^{2}+k_{z}^{2}-k_{0}^{2} S & -k_{y} k_{z} \\
-k_{x} k_{z} & -k_{y} k_{z} & k_{x}^{2}+k_{y}^{2}-k_{0}^{2} P
\end{array}\right|=0
$$

Grouping in terms of $k_{x}^{2}$ yields

$$
k_{x}^{4}\left(-k_{0}^{2} S\right)+k_{x}^{2}\left\{\begin{array}{c}
-a_{22} k_{z}^{2}+2 a_{23} k_{y} k_{z}-a_{33} k_{y}^{2} \\
+a_{11} a_{33}+a_{11} a_{22}+a_{13}^{2}
\end{array}\right\}+\left(a_{11} a_{22} a_{33}+a_{13}^{2} a_{33}-a_{11} a_{23}^{2}\right)=0
$$

where

$$
\begin{gathered}
a_{11} \equiv k_{y}^{2}+k_{z}^{2}-k_{0}^{2} S \\
a_{22} \equiv k_{z}^{2}-k_{0}^{2} S \\
a_{23} \equiv-k_{y} k_{z} \\
a_{33} \equiv k_{y}^{2}-k_{0}^{2} P \\
a_{13}=-i D k_{0}^{2}
\end{gathered}
$$

Solving the above quadratic for $k_{x}^{2}$ yields two roots, $k_{x 1}^{2}$ and $k_{x 2}^{2}$. 


\section{D.2.3 Vectorization}

The transverse fields $E_{x}, E_{y}, B_{x}, B_{y}$ can be formulated in terms of the longitudinal fields $B_{z}$ and $E_{z}$.

$$
\underline{U}=\underline{A} \cdot \hat{V}
$$

where

$$
\begin{gathered}
\underline{U} \equiv\left[\begin{array}{l}
E_{x} \\
E_{y} \\
B_{x} \\
B_{y}
\end{array}\right] \\
\underline{\underline{A}} \equiv\left[\begin{array}{ll}
a_{11} & a_{12} \\
a_{21} & a_{22} \\
a_{31} & a_{32} \\
a_{41} & a_{42}
\end{array}\right] \\
\hat{V}=\left[\begin{array}{l}
E_{x 0} \\
B_{z 0}
\end{array}\right]
\end{gathered}
$$

Written in this form, the incident and reflected wave's transverse field components can be written as

$$
\begin{gathered}
\underline{U}_{i}=\underline{\underline{A}}_{+}\left(\omega, k_{x}, k_{y}, k_{z}\right) \cdot \hat{\underline{V}} e^{i k_{z} k} \\
\underline{U}_{R}=\underline{\underline{A}}\left(\omega,-k_{x}, k_{y}, k_{z}\right) \cdot \underline{\hat{W} e^{-i k_{z} k}} \\
\underline{\hat{W}} \equiv\left[\begin{array}{c}
R_{E} E_{z 0} \\
R_{B} B_{z 0}
\end{array}\right]
\end{gathered}
$$

The total vacuum wave is then the sum of both these components

$$
\underline{U}_{V}=\underline{U}_{i}+\underline{U}_{R}=\underline{\underline{A}}_{+} \cdot \hat{\underline{V}}+\underline{\underline{A}}-\underline{\hat{W}}
$$


Now the plasma transverse field coefficients can also be expressed as

$$
\underline{U}_{P}=\underline{\underline{A}}_{P} \cdot \underline{V}_{\mathrm{p}}
$$

where

$$
V_{p} \equiv\left[\begin{array}{c}
E_{z 2} \\
B_{z 1}
\end{array}\right]
$$

\section{Elements of the $A$ matrix}

From Section D.2.1, the elements of the $A$ matrices for the vacuum and the plasma regions can be found.

Recall that the incident transverse matrix consists of

$$
\underline{U}_{i}=\underline{\underline{A}}_{+} \cdot \hat{\underline{V}}
$$

It can be seen then that $\underline{\underline{A}}_{+}$is given by

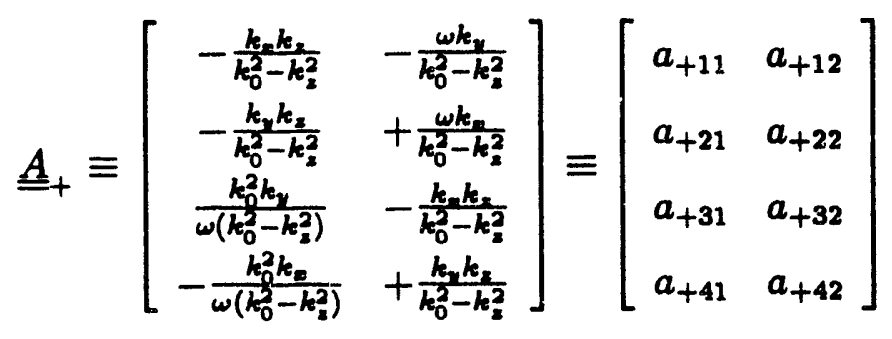

Likewise the reflected transverse matrix is defined by

$$
\underline{U}_{R}=\underline{A}-\underline{\hat{W}}
$$

It can be seen then that $\underline{\underline{A}}_{-}$is given by

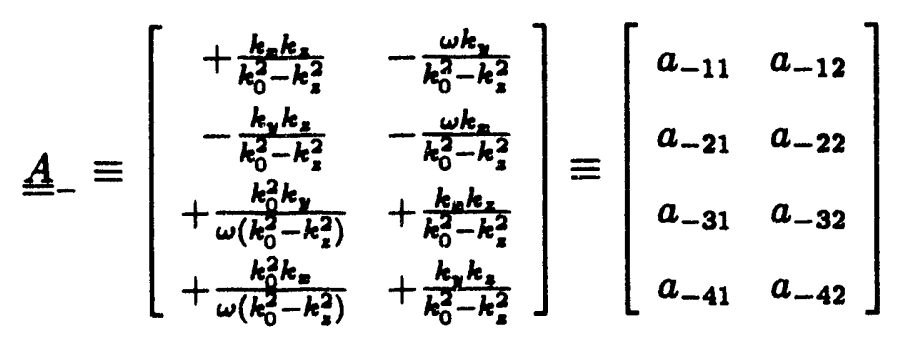

Using the definitions given in Section D.2.1, the vector $\underline{\underline{A}}_{p}$ can be constructed as well 


$$
\stackrel{A}{\Rightarrow} \equiv\left[\begin{array}{ll}
e_{x e 2} & e_{x b 1} \\
e_{y e 2} & e_{y b 1} \\
b_{x e 2} & b_{x b 1} \\
b_{y e 2} & b_{y b 1}
\end{array}\right] \equiv\left[\begin{array}{ll}
a_{p 11} & a_{p 12} \\
a_{p 21} & a_{y 22} \\
a_{p 31} & a_{p 32} \\
a_{p 41} & a_{p 42}
\end{array}\right]
$$

where $e_{x b 1} \ldots b_{y \in 2}$ are defined previously.

\section{D.2.4 Boundary Condition}

Next, the boundary conditions at the vacuum-plasma interface is utilized:

1. $E_{z}, B_{z}$ continuous

2. $E_{y}, B_{y}$ continuous

$\mathrm{BC}$ (1) $\mathrm{E}_{\mathrm{z}}, \mathrm{B}_{\mathrm{z}}$ continuous

$$
\begin{array}{ll}
E_{z} \rightarrow & E_{z 0}+R_{E} E_{z 0}=E_{z p}=E_{z 2}+\frac{z_{1}}{\mu_{0}} B_{z 1} \\
B_{z} \rightarrow \quad & B_{z}+R_{B} B_{z 0}=B_{z p}=B_{z 1}+\mu_{0} y_{2} E_{z 2}
\end{array}
$$

The result of this is

$$
\underline{V}_{p}=\underline{\underline{a}} \cdot \underline{\hat{V}}
$$

where

$$
\underline{\underline{a}} \equiv\left(\frac{1}{1-y_{2} z_{1}}\right)\left[\begin{array}{cc}
\left(1+R_{E}\right) & -\frac{z_{1}}{\mu_{0}}\left(1+R_{B}\right) \\
-\mu_{0} y_{2}\left(1+R_{E}\right) & \left(1+R_{B}\right)
\end{array}\right] \equiv\left[\begin{array}{ll}
a_{11} & a_{12} \\
a_{21} & a_{22}
\end{array}\right]
$$

This results in

$$
\begin{gathered}
\underline{U}_{p}=\underline{A}_{p} \cdot \underline{V}_{p}=\underline{A}_{p} \cdot(\underline{q} \cdot \hat{V}) \\
\underline{U}_{V}=\underline{\underline{A}}_{+} \cdot \hat{V}+\underline{\underline{A}} \underline{\underline{W}}
\end{gathered}
$$

BC (2) $E_{y}, B_{y}$ continuous 
Define

$$
\begin{aligned}
& P_{1} \equiv\left[\begin{array}{llll}
0 & 1 & 0 & 0
\end{array}\right] \\
& \underline{P}_{2} \equiv\left[\begin{array}{llll}
0 & 0 & 0 & 1
\end{array}\right]
\end{aligned}
$$

$E_{y}$ continuity condition becomes:

$$
\underline{P}_{1} \cdot\left[\underline{A}_{p} \cdot(\underline{\underline{a}} \cdot \hat{V})\right]=\underline{P}_{1} \cdot\left[\underline{\underline{A}}_{+} \cdot \hat{\underline{V}}+\underline{\underline{A}}_{-} \cdot \hat{\hat{W}}\right]
$$

$B_{y}$ continuity condition becomes:

$$
\underline{P}_{2} \cdot\left[\underline{A}_{p} \cdot(\underline{\underline{a}} \cdot \hat{V})\right]=\underline{P}_{2} \cdot\left[\underline{A}_{+} \cdot \hat{V}+\underline{\underline{A}}_{-} \cdot \hat{W}\right]
$$

Since $\hat{\underline{W}}=\underline{R} \cdot \hat{\underline{V}}$, where

$$
\underline{\underline{R}}=\left[\begin{array}{cc}
R_{E} & 0 \\
0 & R_{B}
\end{array}\right]
$$

Therefore

$$
\left\{\underline{P}_{1} \cdot\left[\underline{A}_{p} \cdot \underline{\underline{a}}\right]-\underline{P}_{1} \cdot\left[\underline{\underline{A}}_{+}+\underline{\underline{A}}_{-} \cdot \underline{\underline{R}}\right]\right\} \cdot \hat{\hat{V}}=0
$$

Likewise

$$
\left\{\underline{P}_{2} \cdot\left[\underline{A}_{p} \cdot \underline{\underline{a}}\right]-\underline{P}_{2} \cdot\left[\underline{\underline{A}}_{+}+\underline{\underline{A}} \cdot \underline{\underline{R}}\right]\right\} \cdot \hat{\underline{V}}=0
$$

Recall that $\underline{A}_{\mathrm{p}}$ is defined as

$$
\stackrel{A_{p}}{=}\left[\begin{array}{cc}
a_{p 11} & a_{p 12} \\
a_{p 21} & a_{p 22} \\
a_{p 31} & a_{p 32} \\
a_{p 41} & a_{p 42}
\end{array}\right]
$$

Therefore

$$
\underline{A}_{p} \cdot \underline{\underline{a}}=\left[\begin{array}{cc}
a_{p 11} a_{11}+a_{p 12} a_{21} & a_{p 11} a_{12}+a_{p 12} a_{22} \\
a_{p 21} a_{11}+a_{p 22} a_{21} & a_{p 21} a_{12}+a_{p 22} a_{22} \\
a_{p 31} a_{11}+a_{p 32} a_{21} & a_{p 31} a_{12}+a_{p 32} a_{22} \\
a_{p 41} a_{11}+a_{p 42} a_{21} & a_{p 41} a_{12}+a_{p 42} a_{22}
\end{array}\right] \equiv \underline{A}_{r p s}
$$


Also

$$
\begin{aligned}
& \underline{\underline{A}}_{+}+\underline{\underline{A}} \cdot \underline{\underline{R}}=\left[\begin{array}{ll}
a_{+11}+R_{E} a_{-11} & a_{+12}+R_{B} a_{-12} \\
a_{+21}+R_{E} a_{-21} & a_{+22}+R_{B} a_{-22} \\
a_{+31}+R_{E} a_{-31} & a_{+32}+R_{B} a_{-32} \\
a_{+41}+R_{E} a_{-41} & a_{+32}+R_{B} a_{-42}
\end{array}\right] \\
& \underline{P}_{1} \cdot\left(\underline{\underline{A}}_{+}+\underline{\underline{A}} \cdot \underline{\underline{R}}\right)=\left[\begin{array}{ll}
a_{+21}+R_{E} a_{-21} & a_{+22}+R_{B} a_{-22}
\end{array}\right] \\
& \underline{P}_{2} \cdot\left(\underline{A}_{+}+\underline{\underline{A}} \cdot \underline{\underline{R}}\right)=\left[\begin{array}{ll}
a_{+41}+R_{E} a_{-41} & a_{+42}+R_{B} a_{-42}
\end{array}\right] \\
& \underline{P}_{1} \cdot(\underline{\underline{A}} \cdot \underline{\underline{a}})=\left[\left(\frac{1+R_{E}}{1-y_{2} z_{1}}\right)\left[a_{\mathrm{p} 21}-\mu_{0} y_{2} a_{\mathrm{p} 22}\right] \quad\left(\frac{1+R_{B}}{1-y_{2} z_{1}}\right)\left[-\frac{z_{1}}{\mu_{0}} a_{p 21}+a_{p 22}\right]\right] \\
& \underline{P}_{2} \cdot\left(\underline{A}_{p} \cdot \underline{\underline{a}}\right)=\left[\left(\frac{1+R_{E}}{1-y_{2} z_{1}}\right)\left[a_{p 41}-\mu_{0} y_{2} a_{p 42}\right] \quad\left(\frac{1+R_{B}}{1-y_{2} z_{1}}\right)\left[-\frac{z_{1}}{\mu_{0}} a_{p 41}+a_{p 42}\right]\right]
\end{aligned}
$$

So then Equations D.2 and D.3 become

$$
\begin{aligned}
& \hat{E}_{z 0}\left\{\begin{array}{l}
\left(\frac{1+R_{E}}{1-y_{22}}\right)\left[a_{p 21}-\mu_{0} y_{2} a_{p 22}\right] \\
-\left(a_{+21}+R_{E} a_{-21}\right)
\end{array}\right\}=-\hat{B}_{z 0}\left\{\begin{array}{l}
\left(\frac{1+R_{B}}{1-y_{22}}\right)\left(a_{p 22}-\frac{z_{1}}{\mu_{0}} a_{p 21}\right) \\
-\left(a_{+22}+R_{B} a_{-22}\right)
\end{array}\right\} \\
& \hat{E}_{z 0}\left\{\begin{array}{l}
\left(\frac{1+R_{R}}{1-y_{2} z_{1}}\right)\left[a_{p 41}-\mu_{0} y_{2} a_{p 42}\right] \\
-\left(a_{+41}+R_{E} a_{-41}\right)
\end{array}\right\}=-\hat{B}_{z 0}\left\{\begin{array}{l}
\left(\frac{1+R_{B}}{1-y_{2} z_{1}}\right)\left(a_{p 42}-\frac{z_{1}}{\mu_{0}} a_{p 41}\right) \\
-\left(a_{+42}+R_{B} a_{-42}\right)
\end{array}\right\}
\end{aligned}
$$

This can be written as

$$
\left(\begin{array}{ll}
l_{11} & l_{12} \frac{B_{x 0}}{E_{x 0}} \\
l_{21} & l_{22} \frac{B_{x 0}}{E_{x 0}}
\end{array}\right)\left(\begin{array}{l}
R_{E} \\
R_{B}
\end{array}\right)=\left(\begin{array}{l}
r_{1}+r_{1 B E} \frac{B_{x 0}}{E_{x 0}} \\
r_{2}+r_{2 B E} \frac{B_{x 0}}{E_{x 0}}
\end{array}\right)
$$

where

$$
l_{11}=\frac{a_{p 21}-\mu_{0} y_{2} a_{p 22}}{1-y_{2} z_{1}}-a_{-21}
$$




$$
\begin{aligned}
l_{12} & =\left[\frac{a_{p 22}-\frac{z_{1}}{\mu_{0}} a_{p 21}}{1-y_{2} z_{1}}-a_{-22}\right] \\
l_{21} & =\frac{a_{p 41}-\mu_{0} y_{2} a_{p 42}}{1-y_{2} z_{1}}-a_{-41} \\
l_{22} & =\left[\frac{a_{p 42}-\frac{z_{1}}{\mu_{0}} a_{p 41}}{1-y_{2} z_{1}}-a_{-42}\right] \\
r_{1} & =\frac{\mu_{0} y_{2} a_{p 22}-a_{p 21}}{1-y_{2} z_{1}}+a_{+21} \\
r_{1 B E} & =\left[\frac{-a_{p 22}+\frac{z_{1}}{\mu_{0}} a_{p 21}}{1-y_{2} z_{1}}+a_{+22}\right] \\
r_{2} & =\frac{\mu_{0} y_{2} a_{p 42}-a_{p 41}}{1-y_{2} z_{1}}+a_{+41} \\
r_{2 B E} & =\left[\frac{-a_{p 42}+\frac{z_{1}}{\mu_{0}} a_{p 41}}{1-y_{2} z_{1}}+a_{+42}\right]
\end{aligned}
$$

The reflection coefficients can then be calculated to be

$$
\begin{gathered}
R_{E}=\frac{\left(l_{22} r_{1}-l_{12} r_{2}\right)+\frac{B_{00}}{E_{00}}\left(l_{22} r_{1 B E}-l_{12} r_{2 B E}\right)}{|D|} \\
R_{B}=\frac{\left(l_{11} r_{2 B E}-l_{21} r_{1 B E}\right)+\frac{E_{20}}{B_{, 0}}\left(l_{11} r_{2}-l_{21} r_{1}\right)}{|D|} \\
|D| \equiv l_{11} l_{22}-l_{12} l_{21}
\end{gathered}
$$

Defining $C_{R E 1}, C_{R E 2}$ and $C_{R B 1}, C_{R B 2}$ as

$$
\begin{aligned}
& R_{E}=C_{R E 1}+C_{R E 2} \frac{B_{z 0}}{E_{z 0}} \\
& R_{B}=C_{R B 1}+C_{R B 2} \frac{E_{z 0}}{B_{z 0}}
\end{aligned}
$$

where

$$
\begin{aligned}
C_{R E 1} & \equiv \frac{l_{22} r_{1}-l_{12} r_{2}}{|D|} \\
C_{R E 2} & \equiv \frac{l_{22} r_{1 B E}-l_{12} r_{2 B E}}{|D|} \\
C_{R B 1} & \equiv \frac{l_{11} r_{2 B E}-l_{21} r_{1 B E}}{|D|} \\
C_{R B 2} & \equiv \frac{l_{11} r_{2}-l_{21} r_{1}}{|D|}
\end{aligned}
$$




\section{D.2.5 Figure of Merit Calculation}

The figure of merit in the $k_{z} \neq 0$ case is similar to that $k_{z}=0$ case. The ratio of the transmitted $x$-directed Poynting flux to the incident $x$-directed Poynting flux is used as a figure of merit, i.e.

$$
\mathcal{F} \equiv \frac{\hat{x} \cdot\langle\vec{S}\rangle_{T}}{\hat{x} \cdot\left\langle\vec{S}>_{I}\right.}
$$

where we recall that the time-averaged Poynting flux is given by

$$
<\vec{S}>=\frac{1}{2} \operatorname{Re}\left(\vec{E} \times \vec{H}^{*}\right)
$$

\section{Incident Poynting Flux}

The $\mathrm{x}$-directed incident Poynting flux is seen to be

$$
<\vec{S}>_{I x}=\frac{1}{2 \mu_{0}} \operatorname{Re}\left[E_{y} B_{z}^{*}-E_{z} B_{y}^{*}\right]
$$

Using the vacuum fields from Section D.2.1

$$
\begin{aligned}
<\vec{S}>_{I x} & =\frac{1}{2 \mu_{0}\left(k_{0}^{2}-k_{Z}^{2}\right)} \operatorname{Re}\left[B_{z 0}^{*}\left(\omega k_{x} B_{z 0}-k_{y} k_{z} E_{z 0}\right)+E_{z 0}\left(k_{y} k_{z} B_{z 0}^{*}+\frac{k_{0}^{2} k_{x}}{\omega} E_{z 0}^{*}\right)\right] \\
& =\frac{\omega k_{x}}{2 \mu_{0}\left(k_{0}^{2}-k_{z}^{2}\right)} \operatorname{Re}\left\{\left|B_{z 0}\right|^{2}+\frac{\left|E_{z 0}\right|^{2}}{c^{2}}\right\}
\end{aligned}
$$

\section{Reflected Poynting Flux}

Similarly the $\mathrm{x}$-directed Reflected Poynting flux can be shown to be given by

$$
<\vec{S}>_{R X}=\frac{-\omega k_{z}}{2 \mu_{0}\left(k_{0}^{2}-k_{z}^{2}\right)}\left[\left|R_{B}\right|^{2}\left|B_{z 0}\right|^{2}+\frac{\left|E_{z 0}\right|^{2}\left|R_{E}\right|^{2}}{c^{2}}\right]
$$

\section{Transmitted Poynting Flux}

Likewise the $x$-directed Transmitted Poynting flux can be shown to be given by

$$
<\vec{S}>_{T_{x}}=\frac{1}{2 \mu_{0}} \operatorname{Re}\left\{e_{y b 1}\left|T_{B}\right|^{2}\left|B_{x 0}\right|^{2}-b_{y e z}^{*}\left|T_{E}\right|^{2}\left|E_{z 0}\right|^{2}\right\}
$$


The unknowns are now $T_{E}$ and $T_{B}$. (Recall that $R_{E}$ and $R_{B}$ are solved previously.

An examination of the boundary conditions at the plasma-vacuum interface shows that $T_{E}$ and $T_{B}$ can be expresed in terms of $R_{E}$ and $R_{B}$.

$$
T_{E}=\frac{1}{1-y_{2} z_{1}}\left[\left(1+R_{E}\right)-\frac{z_{1}}{\mu_{0}}\left(1+R_{B}\right) \frac{B_{z 0}}{E_{x 0}}\right]
$$

expanding $R_{E}$ and $R_{B}$ :

$$
T_{E}=\frac{1}{1-y_{2} z_{1}}\left[\left(1+C_{R E 1}-\frac{z_{1}}{\mu_{0}} C_{R B 2}\right)+\frac{B_{x 0}}{E_{x 0}}\left(C_{R E 2}-\frac{z_{1}}{\mu_{0}}-\frac{z_{1}}{\mu_{0}} C_{R B 1}\right)\right]
$$

or

$$
T_{E}=C_{T E}+C_{T E R} \frac{B_{z 0}}{E_{z 0}}
$$

where

$$
\begin{aligned}
C_{T E} & \equiv \frac{1}{1-y_{2} z_{1}}\left(1+C_{R E 1}-\frac{z_{1}}{\mu_{0}} C_{R B 2}\right) \\
C_{T E R} & \equiv \frac{1}{1-y_{2} z_{1}}\left(C_{R E 2}-\frac{z_{1}}{\mu_{0}}-\frac{z_{1}}{\mu_{0}} C_{R B 1}\right)
\end{aligned}
$$

Likewise it can be shown that

$$
T_{B}=\frac{1}{1-y_{2} z_{1}}\left[\left(1+C_{R B 1}-\mu_{0} y_{2} C_{R E 2}\right)+\frac{E_{z 0}}{B_{z 0}}\left(C_{R B 2}-\mu_{0} y_{2}-\mu_{0} y_{2} C_{R E 1}\right)\right]
$$

or

$$
T_{B}=C_{T B}+C_{T B} \frac{E_{z 0}}{B_{z 0}}
$$

where

$$
\begin{aligned}
C_{T B} & \equiv \frac{1}{1-y_{2} z_{1}}\left(1+C_{R B 1}-\mu_{0} y_{2} C_{R E 2}\right) \\
C_{T B R} & \equiv \frac{1}{1-y_{2} z_{1}}\left(C_{R B 2}-\mu_{0} y_{2}-\mu_{0} y_{2} C_{R E_{1}}\right)
\end{aligned}
$$

Next, a reflectivity $r$ and a transmisivity $t$ can be calculated (Note that our figure of merit is the transmitivity $t$ ).

$$
r \equiv \frac{-\hat{x} \cdot<\vec{S}>_{R}}{\hat{x} \cdot\left\langle\vec{S}>_{I}\right.} \quad ; \quad t \equiv \frac{\hat{x} \cdot<\vec{S}>_{T}}{\hat{x} \cdot\left\langle\vec{S}>_{I}\right.}
$$




$$
\begin{aligned}
& r=\frac{\left|R_{B}\right|^{2}+\left|R_{E}\right|^{2} \frac{\left|E_{0,0}\right|^{2}}{\left.\left.c^{2}||\right|_{x 0}\right|^{2}}}{1+\frac{1}{c^{2}} \frac{\left|E_{80}\right|^{2}}{\left|B_{x 0}\right|^{2}}} \\
& t=\left(\frac{k_{0}^{2}-k_{z}^{2}}{\omega k_{x}}\right)\left[\frac{e_{y b 1 R e}\left|T_{B}\right|^{2}-b_{y e 2 R_{e}}\left|T_{E}\right|^{2}\left|\frac{E_{s e}}{B_{x 0}}\right|^{2}}{\left(1+\frac{1}{c^{2}}\left|\frac{E_{x 0}}{B_{x 0}}\right|^{2}\right)}\right]
\end{aligned}
$$




\section{D.2.6 Variational Solution of $\eta$}

In order to find the optimal $\eta$, a variational technique is employed here to find the optimum $\frac{E_{\mathrm{e}}}{B_{\mathrm{z}}}$ and then $\eta_{\text {opt }}$.

Recall from previous section that the figure of merit $\mathcal{F}$ was found to be

$$
\mathcal{F}=t=\frac{c_{1}}{1+|\mathcal{X}|^{2}}\left[e_{y b 1 R e}\left|C_{T B}+C_{T B R} c \mathcal{X}\right|^{2}-b_{y e 2 R e}\left|\left(C_{T E}+\frac{C_{T E R}}{c \mathcal{X}}\right)(c \mathcal{X})\right|^{2}\right]
$$

where

$$
c_{1} \equiv \frac{k_{0}^{2}-k_{Z}^{2}}{\omega k_{x}} \quad ; \quad \mathcal{X} \equiv \frac{E_{z 0}}{c B_{z 0}}
$$

This can be rewritten as

$\mathcal{F}=\frac{c_{1}}{1+|\mathcal{X}|^{2}}\left\{\begin{array}{c}e_{y 1 b e}\left(\left|C_{T B}\right|^{2}+c C_{T B}^{*} C_{T B R} \mathcal{X}+c C_{B T} C_{T B R}^{*} \mathcal{X}^{*}+c^{2}\left|C_{T B R}\right|^{2}|\mathcal{X}|^{2}\right) \\ -b_{\text {ye2Re }}\left(\left|C_{T E R}\right|^{2}+c C_{T E} C_{T E R}^{*} \mathcal{X}+c C_{T E}^{*} C_{T E R} \mathcal{X}^{*}+c^{2}\left|C_{T E}\right|^{2}|\mathcal{X}|^{2}\right)\end{array}\right\}$

Separating $\mathcal{F}$ into a numerator and a denominator $\left(\mathcal{F} \equiv \frac{N}{D}\right)$ yields

$$
N-D \mathcal{F}=0
$$

and applying variational technique

$$
\mathcal{X} \rightarrow \mathcal{X}_{0}+\delta \mathcal{X}
$$

and keeping terms of $\mathrm{O}(\delta \mathcal{X})$, the following is obtained

$$
\left(\begin{array}{c}
e_{y b 1 R e}\left(c C_{T B}^{*} C_{T B R} \delta \mathcal{X}+c C_{T B} C_{T B R}^{*} \delta \mathcal{X}^{*}+c^{2}\left|C_{T B R}\right|^{2}\left[\mathcal{X} \delta \mathcal{X}^{*}+\mathcal{X}^{*} \delta \mathcal{X}\right]\right) \\
-b_{y e 2 R e}\left(c C_{T E} C_{T E R}^{*} \delta \mathcal{X}+c C_{T E}^{*} C_{T E R} \delta \mathcal{X}^{*}+c^{2}\left|C_{T E}\right|^{2}\left[\mathcal{X} \delta \mathcal{X}^{*}+\mathcal{X}^{*} \delta \mathcal{X}\right]\right) \\
-\frac{F}{c_{1}}\left[\mathcal{X} \delta \mathcal{X}^{*}+\mathcal{X}^{*} \delta \mathcal{X}\right]
\end{array}\right)=0
$$

This can be written as

$\delta . \mathcal{X}\left\{\begin{array}{c}e_{y b 1 R e}\left(c C_{T B}^{*} C_{T B R}+c^{2}\left|C_{T B R}\right|^{2} \mathcal{X}_{0}^{*}\right) \\ -b_{y e 2 R e}\left(c C_{T E}^{*} C_{T E R}+c^{2}\left|C_{T E}\right|^{2} \mathcal{X}_{0}^{*}\right) \\ -\frac{t}{c_{1}} X_{0}^{*}\end{array}\right\}+\delta \mathcal{X}^{*}\left\{\begin{array}{c}e_{y b 1 R e}\left(c C_{T B} C_{T B R}^{*}+c^{2}\left|C_{T B R}\right|^{2} \mathcal{X}_{0}\right) \\ -b_{y e 2 R e}\left(c C_{T E} C_{T E R}^{*}+c^{2}\left|C_{T E}\right|^{2} \mathcal{X}_{0}\right) \\ -\frac{t}{c_{1}} \mathcal{X}_{0}\end{array}\right\}=0$ 
Since the $\delta \mathcal{X}$ term is simply the complex conjugate of the $\delta \mathcal{X}^{*}$ term, it is seen that the optimal $\mathcal{F}$ is found to be $\mathcal{F}_{\text {opt }}=\frac{c_{1}}{\mathcal{X}_{0}^{*}}\left[c\left(e_{y b 1 R e} C_{T B}^{*} C_{T B R}-b_{y e 2 R e} C_{T E} C_{T E R}^{*}\right)+c^{2} \mathcal{X}_{0}^{*}\left(e_{y b 1 R e}\left|C_{T B R}\right|^{2}-b_{y e 2 R e}\left|C_{T E}\right|^{2}\right)\right]$

The optimal $\mathcal{X}$ can be found by substituting in for $\mathcal{F}$ from Equation D.9 $\frac{c C_{1}}{\mathcal{X}_{0}^{*}}\left(\begin{array}{c}e_{y b 1 R e} C_{T B}^{*} C_{T B R}-b_{y e 2 R e} C_{T E} C_{T E R}^{*} \\ +c \mathcal{X}_{0}^{*}\left(e_{y b 1 R e}\left|C_{T B R}\right|^{2}-b_{y e 2 R e}\left|C_{T E}\right|^{2}\right)\end{array}\right)=\frac{c_{1}}{1+|\mathcal{X}|^{2}}\left[\begin{array}{c}e_{y 1 b e}\left(\left|C_{T B}\right|^{2}+c C_{T B}^{*} C_{T B R} \mathcal{X}+c C_{B T^{\prime}}\right. \\ -b_{y e 2 R e}\left(\left|C_{T E R}\right|^{2}+c C_{T E} C_{T E R}^{*} \mathcal{X}+c C\right.\end{array}\right.$ The result of this is an equation for the optimal value of $\mathcal{X}^{*}$ :

$$
N_{1}\left(\mathcal{X}_{\text {opt }}^{*}\right)^{2}+N_{2} \mathcal{X}_{\text {opt }}^{*}+N_{3}=0
$$

where

$$
\begin{aligned}
& N_{1} \equiv c\left(e_{y b 1 R_{e}} C_{T B} C_{T B R}^{*}-b_{y e 2 R e} C_{T E}^{*} C_{T E R}\right) \\
& N_{2} \equiv E_{y 1 b R e}\left|C_{T B}\right|^{2}-b_{y e 2 R_{e}}\left|C_{T E R}\right|^{2}-c^{2}\left(e_{y b 1 R e}\left|C_{T B R}\right|^{2}-b_{y e 2 R e}\left|C_{T E}\right|^{2}\right) \\
& N_{3} \equiv c\left(b_{y e 2 R e} C_{T E} C_{T E R}^{*}-e_{y b 1 R e} C_{T B}^{*} C_{T B R}\right)
\end{aligned}
$$

This can then be solved quadratically to yield the optimum value of $\mathcal{X}^{*}$. 


\section{W Scan}

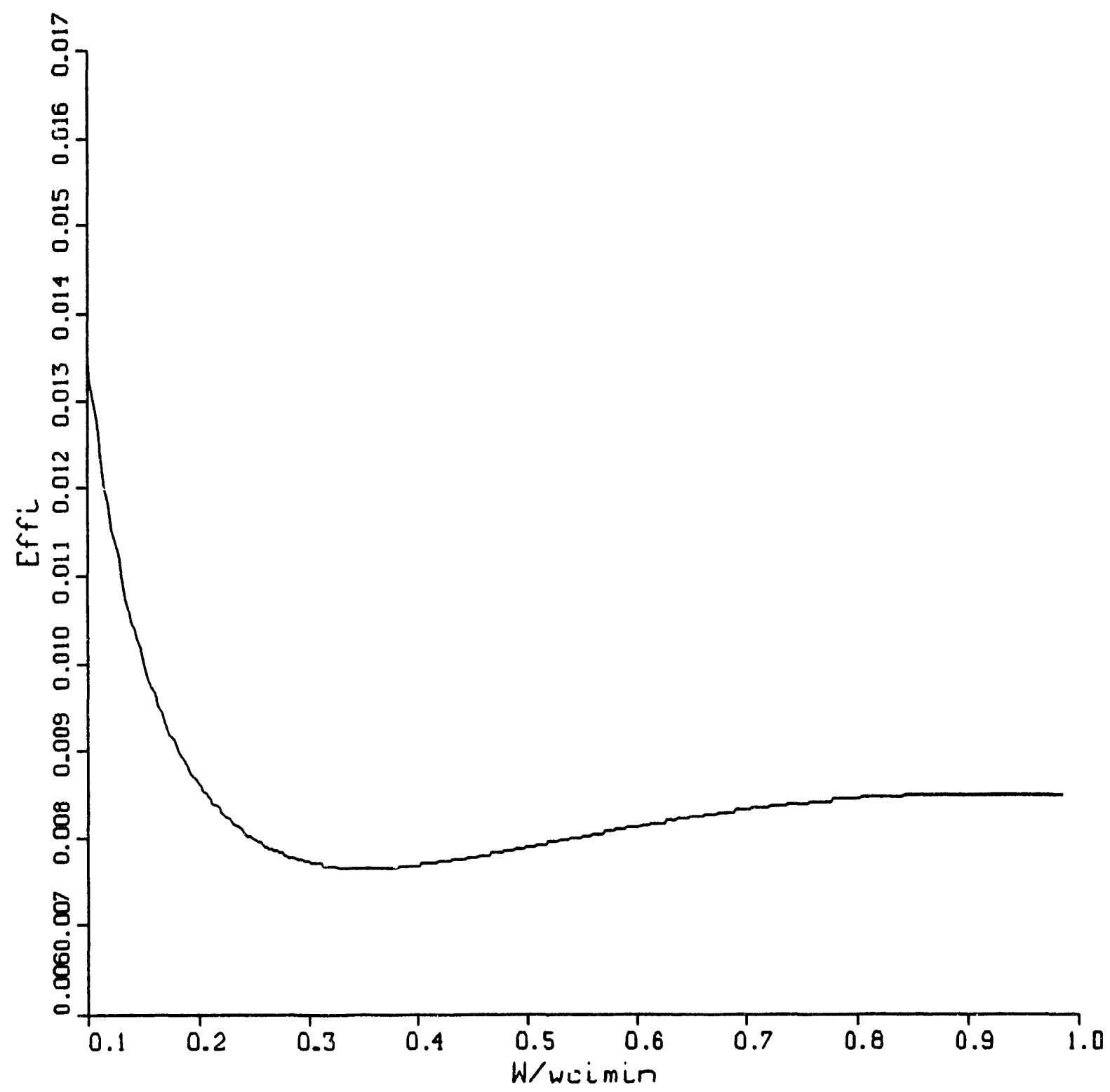

Figure D-6: Figure of Merit calculation for $k_{z} \neq 0$ case at different ion cyclotron frequency ratios $\omega / \omega_{c i}$. Plasma density of this scan was fixed at $n_{e}=1.0 \times$ $10^{10} \mathrm{~cm}^{-3}$. Note that for the $\omega$ scan the optimum $\mathcal{X}$ is recalculated at each value of $\omega / \omega_{c i}$. 


\section{DENSITY SCAN}

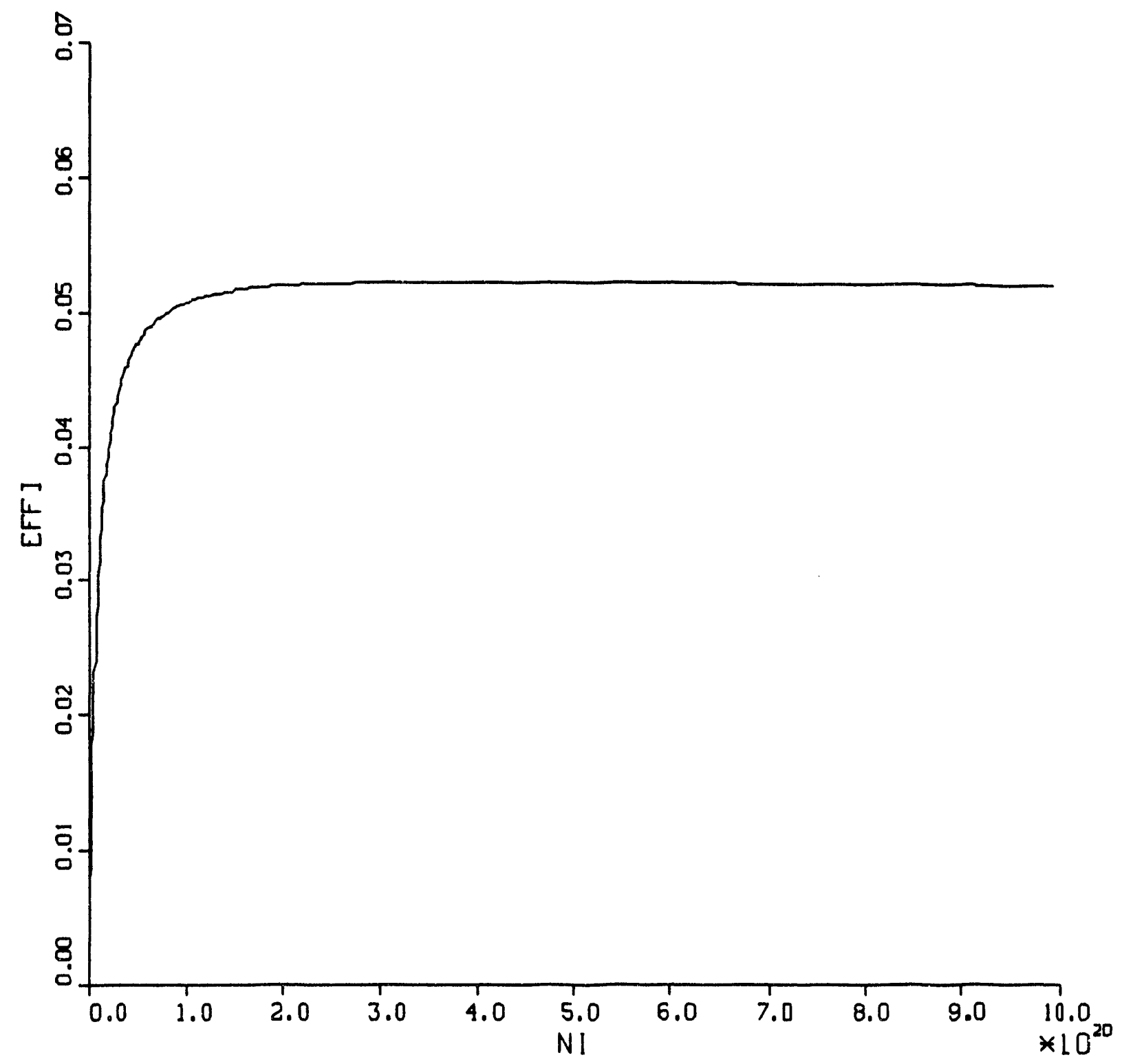

Figure D-7: Figure of Merit calculation for $k_{z} \neq 0$ case at different plasma densities $n_{e}$. The value of $\omega / \omega_{c i}$ of this scan was fixed at $\omega / \omega_{c i}=0.1$. Note that for this density scan the optimum $\mathcal{X}$ is recalculated at each value of $\omega / \omega_{c i}$. Unit of density is $\left[m^{-3}\right]$. 


\section{Appendix E}

\section{Derivations used in the}

\section{Analytical ICRF Analysis}

This appendix includes the detailed derivations whose results are shown in Chapter 3 .

\section{E.1 Boundary Condition for the Analytical Model}

Two boundaries exist in the analytical model described in Chapter 3. The first boundary is the plasma-vacuum interface at $\rho=1$. The second boundary is the conducting wall located at $\rho=\kappa$. This appendix will describe these boundary conditions in detail.

\section{E.1.1 Boundary I: Vacuum chamber wall $\rho=\kappa$}

At $\rho=\kappa$, the tangential electric field $\mathbf{E}_{\tan }$ is zero.

Recall from Equation 3.15

$$
E=\frac{i \omega \psi}{r} \hat{e_{\theta}}
$$

At the vacuum chamber wall

$$
\left.\Psi\right|_{\text {wall }}=\left[\Psi_{V}+\Psi_{\text {wire }}\right]_{\text {wall }}=0
$$


Taking the Fourier transform of yields

$$
\hat{\Psi}_{V}\left(\kappa, k_{\xi}\right)+\hat{\Psi}_{\text {wire }}\left(\kappa, k_{\xi}\right)=0
$$

where the Fourier Transform of $\Psi_{\text {wire }}(\rho, \xi)$ is defined as:

$$
\hat{\Psi}_{\text {wire }}\left(\rho=\kappa, k_{\xi}\right) \equiv \int e^{i k_{\xi} \xi} \Psi_{\text {wire }}(\rho=\kappa, \xi) d \xi
$$

Since the source term is fully known, $\hat{\Psi}_{\text {wire }}$ is also known. This allows the expression of one of the two constants in terms of the other.

$$
c_{2}\left(k_{\xi}\right)=\frac{1}{K_{1}\left(k_{\xi} \kappa\right)}\left[-\frac{1}{\kappa} \hat{\Psi}_{w i r e}\left(\kappa, k_{\xi}\right)-c_{1}\left(k_{\xi}\right) I_{1}\left(k_{\xi} \kappa\right)\right]
$$

Thus

$\hat{\Psi}_{V}\left(\rho, k_{\xi}\right)=\frac{c_{1} \rho}{K_{1}\left(k_{\xi} \kappa\right)}\left[K_{1}\left(k_{\xi} \kappa\right) I_{1}\left(k_{\xi} \rho\right)-I_{1}\left(k_{\xi} \kappa\right) K_{1}\left(k_{\xi} \rho\right)\right]-\left(\frac{\rho}{\kappa}\right) \frac{K_{1}\left(k_{\xi} \rho\right)}{K_{1}\left(k_{\xi} \kappa\right)} \hat{\Psi}_{\text {wire }}\left(\kappa, k_{\xi}\right)$

Define

$$
\begin{aligned}
& \Delta_{\kappa \rho} \equiv K_{1}\left(k_{\xi} \kappa\right) I_{1}\left(k_{\xi} \rho\right)-I_{1}\left(k_{\xi} \kappa\right) K_{1}\left(k_{\xi} \rho\right) \\
& \triangle_{\kappa \rho^{\prime}} \equiv K_{1}\left(k_{\xi} \kappa\right) I_{1}^{\prime}\left(k_{\xi} \rho\right)-I_{1}\left(k_{\xi} \kappa\right) K_{1}^{\prime}\left(k_{\xi} \rho\right)
\end{aligned}
$$

where

$$
I_{1}^{\prime}(z) \equiv \frac{d I_{1}(z)}{d z} \quad \text { and } \quad K_{1}^{\prime}(z) \equiv \frac{d K_{1}(z)}{d z}
$$

Then

$$
\hat{\Psi}_{V}\left(\rho, k_{\xi}\right)=\frac{c_{1}\left(k_{\xi}\right) \rho}{K_{1}\left(k_{\xi} \kappa\right)} \Delta_{\kappa \rho}-\left(\frac{\rho}{\kappa}\right) \frac{K_{1}\left(k_{\xi} \rho\right)}{K_{1}\left(k_{\xi} \kappa\right)} \hat{\Psi}_{\text {wire }}\left(\kappa, k_{\xi}\right)
$$

So the total $\Psi$ is given by $\Psi=\left(\Psi_{v}+\Psi_{\text {wire }}\right)$

$$
\Psi=\left[c_{1}\left(k_{\xi}\right) \frac{\rho \triangle_{\kappa \rho}}{K_{1}\left(k_{\xi} \kappa\right)}-\left(\frac{\rho}{\kappa}\right) \frac{K_{1}\left(k_{\xi} \rho\right)}{K_{1}\left(k_{\xi} \kappa\right)} \hat{\Psi}_{\text {wire }}\left(\kappa, k_{\xi}\right)+\hat{\Psi}_{\text {wire }}\left(\rho, k_{\xi}\right)\right]
$$

\section{E.1.2 Boundary II: Plasma Vacuum Interface $\rho=1$}

Two conditions exist at the plasma-vacuum interface boundary : $E_{\tan }$ is continuous; $\vec{B}_{\tan }$ is continous.

$\mathrm{E}_{\text {tan }}$ continuous 
Assuming that the corresponding plasma $\Psi$ is known, and defined by $\Psi_{\text {plas }} \equiv$ $\phi_{p}\left(\rho, k_{\xi}\right)$, the boundary condition is expressed as

$$
\begin{gathered}
\hat{\phi}_{p}\left(\rho=1, k_{\xi}\right)=\hat{\Psi}_{V}\left(\rho=1, k_{\xi}\right)+\hat{\Psi}_{\text {wire }}\left(\rho=1, k_{\xi}\right) \\
\Rightarrow \quad \hat{\phi}_{p}\left(1, k_{\xi}\right)=c_{1}\left(k_{\xi}\right) \hat{F}\left(1, k_{\xi}\right)+\hat{G}\left(1, k_{\xi}\right)
\end{gathered}
$$

where

$$
\begin{aligned}
\hat{F}\left(\rho, k_{\xi}\right) & \equiv \frac{\rho \Delta_{\kappa \rho}}{K_{1}\left(k_{\xi} \kappa\right)} \\
\hat{G}\left(\rho, k_{\xi}\right) & \equiv-\left(\frac{\rho}{\kappa}\right) \frac{K_{1}\left(k_{\xi} \rho\right)}{K_{1}\left(k_{\xi} \kappa\right)} \hat{\Psi}_{\text {wire }}\left(\kappa, k_{\xi}\right)+\hat{\Psi}_{\text {wire }}\left(\rho, k_{\xi}\right)
\end{aligned}
$$

sh def

$\vec{B}_{\text {tan }}$ continuous

Since the B-field is given by Equation 3.14

$$
\vec{B}=\frac{\nabla \Psi \times \hat{e_{\theta}}}{r}
$$

By the slow-z approximation

$$
B_{\text {tan }} \simeq B_{z}=\frac{1}{r} \frac{\partial}{\partial r} \Psi
$$

Thus we have

$$
\begin{gathered}
\frac{\partial}{\partial \rho} \hat{\phi}_{p}\left(1, k_{\xi}\right)=\frac{\partial}{\partial \rho} \hat{\Psi}_{V}\left(\rho=1, k_{\xi}\right)+\frac{\partial}{\partial \rho} \hat{\Psi}_{\text {wire }}\left(\rho=1, k_{\xi}\right) \\
\frac{\partial}{\partial \rho} \hat{\phi}_{p}\left(1, k_{\xi}\right)=c_{1} \frac{\partial}{\partial \rho} \hat{F}\left(1, k_{\xi}\right)+\frac{\partial}{\partial \rho} \hat{G}\left(1, k_{\xi}\right)
\end{gathered}
$$

Using Equations ?? and ??

$$
\begin{aligned}
\hat{\phi}_{p}\left(1, k_{\xi}\right)-\hat{G}\left(1, k_{\xi}\right) & =c_{1} \hat{F}\left(1, k_{\xi}\right) \\
\frac{\partial}{\partial \rho}\left(\hat{\phi}_{p}\left(1, k_{\xi}\right)-\hat{G}\left(1, k_{\xi}\right)\right) & =c_{1} \frac{\partial}{\partial \rho} \hat{F}\left(1, k_{\xi}\right)
\end{aligned}
$$

Dividing one by the other yields

$$
\left.\frac{\frac{\partial}{\partial \rho}\left(\hat{\phi}_{p}-\hat{G}\right)}{\hat{\phi}_{p}-\hat{G}}\right|_{\rho=1}=\left.\frac{\frac{\partial}{\partial \rho_{p}} \hat{F}}{\hat{F}}\right|_{\rho=1}
$$


This results in

$$
\left.\frac{\partial}{\partial \rho} \hat{\phi}_{\mathrm{p}}\right|_{1}-\left.\frac{\frac{\partial}{\partial \rho} \hat{F}}{\hat{F}} \hat{\phi}_{p}\right|_{1}=\left.\frac{\partial}{\partial \rho} \hat{G}\right|_{1}-\left.\frac{\frac{\partial}{\partial \rho} \hat{F}}{\hat{F}} \hat{G}\right|_{1}
$$

Since $\hat{F}$ and $\hat{G}$ are known, Equation ?? can be explicitly solved for $\hat{\phi}_{p}\left(1, k_{\xi}\right)$.

Assume $\hat{\phi}_{p}\left(\rho, k_{\xi}\right)$ is given by

$$
\hat{\phi}_{p}\left(\rho, k_{\xi}\right)=\hat{\hat{\phi}}_{p}\left(\alpha, k_{\xi}\right) \rho J_{1}(\alpha \rho)
$$

(This assumption will be shown to be valid in Section 3.7)

Then, using the identity

$$
\frac{\partial}{\partial \rho}\left(\rho J_{1}(\alpha \rho)\right)=\alpha \rho J_{0}(\alpha \rho)
$$

$\hat{\hat{\phi}}_{p}$ is found to be

$$
\hat{\hat{\phi}}_{p}\left(\alpha, k_{\xi}\right)=\frac{1}{\alpha J_{0}(\alpha)-J_{1}(\alpha) \frac{\frac{\theta}{\partial \rho} \hat{F}}{\hat{F}}}\left[\frac{\partial}{\partial \rho} \hat{G}-\frac{\frac{\partial}{\partial \rho} \hat{F}}{\hat{F}} \hat{G}\right]_{\rho=1}
$$

where $\hat{F}$ and $\hat{G}$ are given in Equations 3.21 and 3.22. Their derivatives are seen to be

$$
\begin{gathered}
\frac{\partial}{\partial \rho} F\left(\rho, k_{\xi}\right)=\frac{\rho k_{\xi} \Delta_{\kappa \rho^{\prime}}}{K_{1}\left(k_{\xi} \kappa\right)}+\frac{\Delta_{\kappa \rho}}{K_{1}\left(k_{\xi} \kappa\right)} \\
\left.\Rightarrow \frac{\partial F / \partial \rho}{F^{\prime}}\right|_{\rho=1}=1+\left.k_{\xi} \frac{\Delta_{\kappa \rho^{\prime}}}{\Delta_{\kappa \rho}}\right|_{\rho=1} \\
\frac{\partial}{\partial \rho} G\left(\rho, k_{\xi}\right)=\hat{\Psi}_{\text {wire }}\left(\kappa, k_{\xi}\right)\left[-\frac{1}{\kappa} \frac{K_{1}\left(k_{\xi} \rho\right)}{K_{1}\left(k_{\xi} i \kappa\right)}-\frac{k_{\xi} \rho}{\kappa} \frac{K_{1}^{\prime}\left(k_{\xi} \rho\right)}{K_{1}\left(k_{\xi} \kappa\right)}\right]+\frac{\partial}{\partial \rho} \hat{\Psi}_{\text {wire }}\left(\rho, k_{\xi}\right)
\end{gathered}
$$

Since

$$
\alpha J_{0}(\alpha)-J_{1}(\alpha)=\alpha J_{1}^{\prime}(\alpha)
$$

$\hat{\hat{\Phi}}_{p}$ can then be expressed as

$$
\hat{\hat{\Phi}}_{p}\left(\alpha, k_{\xi}\right)=\frac{1}{\alpha J_{1}^{\prime}(\alpha)-J_{1}(\alpha) k_{\xi} \frac{\Delta_{\alpha \rho^{\prime}}}{\Delta_{r \rho}}}\left[\frac{\partial}{\partial \rho} \hat{G}-\frac{\frac{\partial}{\partial \rho} \hat{F}}{\hat{F}} \hat{G}\right]_{\rho=1}
$$


Examining the last term

$$
\begin{aligned}
\frac{\partial}{\partial \rho} \hat{\Psi}_{\text {wire }}\left(\rho, k_{\xi}\right) & =\frac{\partial}{\partial \rho} \int \Psi_{\text {wire }}(\rho, \xi) e^{i k_{\ell} \xi} d \xi \\
& =\int \frac{\partial}{\partial \rho} \Psi_{\text {wire }}(\rho, \xi) e^{i k_{\ell} \xi} d \xi
\end{aligned}
$$

Recall that $\Psi(\rho, \xi)$ is given by Equation 3.13 as

$$
\Psi_{\text {wire }}(\rho, \xi)=\underbrace{\frac{4 \mu_{0} I a \rho R(\xi)}{\sqrt{a^{2}+\rho^{2} R^{2}(\xi)+z^{2}(\xi)+2 a \rho R(\xi)}}}_{\Psi_{w 1}} \underbrace{\left[\frac{\left(2-k_{A}^{2}\right) K\left(k_{A}\right)-2 E\left(k_{A}\right)}{k_{A}^{2}}\right]}_{\Psi_{w 2}}
$$

Therefore

$$
\begin{aligned}
\frac{\partial}{\partial \rho} \Psi_{w i r e}(\rho, \xi) & =\Psi_{w 2} \frac{\partial}{\partial \rho} \Psi_{w 1}+\Psi_{w 1} \frac{\partial}{\partial \rho} \Psi_{w 2} \\
& =\Psi_{w 2} \frac{\partial}{\partial \rho} \Psi_{w 1}+\Psi_{w 1}\left(\frac{\partial k_{A}}{\partial \rho}\right) \frac{\partial \Psi_{w 2}}{\partial k_{A}}
\end{aligned}
$$

where it can be shown that

$$
\frac{\partial \Psi_{w 1}}{\partial \rho}=\frac{4 \mu_{0} I a R(\xi)\left[a^{2}+z^{2}+a \rho R(\xi)\right]}{\left(a^{2}+\rho^{2} R^{2}(\xi)+z^{2}(\xi)+2 a \rho R(\xi)\right)^{3 / 2}}
$$

Jackson's notation [44] defined $k_{A}$ as

$$
\begin{aligned}
k_{A}^{2} & \equiv \frac{4 a \rho R(\xi)}{a^{2}+\rho^{2} R^{2}(\xi)+z(\xi)^{2}+2 a \rho R(\xi)} \\
\Rightarrow \quad k_{A} & =\sqrt{\frac{4 a \rho R(\xi)}{a^{2}+\rho^{2} R^{2}(\xi)+z(\xi)^{2}+2 a \rho R(\xi)}}
\end{aligned}
$$

It can be shown then that

$$
\frac{\partial k_{A}}{\partial \rho}=\frac{a R(\xi)}{\rho} \frac{a^{2}-\rho^{2} R^{2}(\xi)+z^{2}(\xi)}{\left[a^{2}+\rho^{2} R^{2}(\xi)+z(\xi)^{2}+2 a \rho R(\xi)\right]^{3} / 2}
$$

Next

$$
\begin{aligned}
\frac{\partial \Psi_{w 2}}{\partial k_{A}}= & \frac{\partial}{\partial k_{A}}\left[\frac{\left(2-k_{A}^{2}\right) K\left(k_{A}\right)-2 E\left(k_{A}\right)}{k_{A}^{2}}\right] \\
= & \frac{1}{k_{A}^{4}}\left\{k_{A}^{2}\left(-2 k_{A} K\left(k_{A}\right)+\left(2-k_{A}^{2}\right) K^{\prime}\left(k_{A}\right)\right)-2 k_{A}^{2} E^{\prime}\left(k_{A}\right)\right. \\
& \left.-2 k_{A}\left[\left(2-k_{A}^{2}\right) K\left(k_{A}\right)-2 E\left(k_{A}\right)\right]\right\} \\
= & \frac{1}{k_{A}^{3}}\left[-4 K\left(k_{A}\right)+k_{A}\left(2-k_{A}^{2}\right) K^{\prime}\left(k_{A}\right)+4 E\left(k_{A}\right)-2 k_{A} E^{\prime}\left(k_{A}\right)\right]
\end{aligned}
$$


From Gradshteyn and Ryzhik [52], it is seen that

$$
\begin{gathered}
\frac{d K\left(k_{A}\right)}{d k_{A}}=\frac{E\left(k_{A}\right)}{k_{A}\left(1-k_{A}^{2}\right)}-\frac{K\left(k_{A}\right)}{k_{A}} \\
\frac{d E\left(k_{A}\right)}{d k_{A}}=\frac{E\left(k_{A}\right)-K\left(k_{A}\right)}{k_{A}}
\end{gathered}
$$

It can then be easily shown that

$$
\frac{\partial \Psi_{w 2}}{\partial k_{A}}=\frac{1}{k_{A}^{3}}\left\{\left(\frac{4-3 k_{A}^{2}}{1-k_{A}^{2}}\right) E\left(k_{A}\right)+K\left(k_{A}\right)\left(k_{A}^{2}-4\right)\right\}
$$

So then we can plug back in and solve for $\partial \Psi_{\text {wire }} / \partial \rho$ by

$$
\frac{\partial \Psi_{\text {wire }}}{\partial \rho}=\frac{\partial \Psi_{\text {wire }}}{\partial k_{A}} \frac{\partial k_{A}}{\partial \rho}
$$

\section{E.2 Curve Fitting of Vacuum Field Source Terms}

A fitting function $\Psi_{f i t}$ is chosen to model the actual $\Psi$ of the wire dipole antenna.

An error $\epsilon$ can be defined as

$$
\begin{aligned}
& \epsilon \equiv \int_{-\infty}^{\infty}\left(\Psi_{\text {actual }}(\xi)-\Psi_{f i t}\right)^{2} d \xi \\
& =\int_{-\infty}^{\infty}\left[\Psi_{\text {actual }}^{2}(\xi)-2 \Psi_{\text {actual }}(\xi) \Psi_{f i t}+\Psi_{f i t}^{2}\right] d \xi \\
& =\int_{-\infty}^{\infty}\left\{\Psi_{\text {actual }}^{2}(\xi)-\frac{2 \Psi_{\text {actual }}(\xi) \Psi_{0}}{\left[1+\left(\frac{\xi-\xi_{w}}{\Delta}\right)^{2}\right]^{5 / 2}}\left[1+c_{1}\left(\xi-\xi_{w}\right)+c_{2}\left(\xi-\xi_{w}\right)^{2}\right]\right. \\
& \left.+\frac{\Psi_{0}^{2}}{\left[1+\left(\frac{\xi-\xi_{w}}{\Delta}\right)^{2}\right]^{5}}\left[\begin{array}{l}
1+2 c_{1}\left(\xi-\xi_{w}\right)+2 c_{2}\left(\xi-\xi_{w}\right)^{2}+c_{1}^{2}\left(\xi-\xi_{w}\right)^{2} \\
+c_{2}^{2}\left(\xi-\xi_{w}\right)^{4}+2 c_{1} c_{2}\left(\xi-\xi_{w}\right)^{3}
\end{array}\right]\right\} d \xi \\
& =a_{1}+a_{2} c_{1}+a_{3} c_{2}+a_{4} c_{1}^{2}+a_{5} c_{2}^{2}+a_{6} c_{1} c_{2}
\end{aligned}
$$

where the coefficients $a_{1} \ldots a_{6}$ are defined as

$$
a_{1} \equiv \int_{-\infty}^{\infty}\left[\Psi_{\text {actual }}^{2}(\xi)-\frac{2 \Psi_{\text {actual }}(\xi) \Psi_{0}}{\left[1+\left(\frac{\xi-\xi_{w}}{\Delta}\right)^{2}\right]^{5 / 2}}+\frac{\Psi_{0}^{2}}{\left[1+\left(\frac{\xi-\xi_{w}}{\Delta}\right)^{2}\right]^{5}}\right] d \xi
$$




$$
\begin{gathered}
a_{2} \equiv \int_{-\infty}^{\infty}\left[\frac{-2\left(\xi-\xi_{w}\right) \Psi_{\text {actual }}(\xi) \Psi_{0}}{\left[1+\left(\frac{\xi-\xi_{w}}{\Delta}\right)^{2}\right]^{5 / 2}}+\frac{2\left(\xi-\xi_{w}\right) \Psi_{0}^{2}}{\left[1+\left(\frac{\xi-\xi_{w}}{\Delta}\right)^{2}\right]^{5}}\right] d \xi \\
a_{3} \equiv \int_{-\infty}^{\infty}\left[\frac{-2\left(\xi-\xi_{w}\right)^{2} \Psi_{\text {actual }}(\xi) \Psi_{0}}{\left[1+\left(\frac{\xi-\xi_{w}}{\Delta}\right)^{2}\right]^{5 / 2}}+\frac{2\left(\xi-\xi_{w}\right)^{2} \Psi_{0}^{2}}{\left[1+\left(\frac{\xi-\xi_{w}}{\Delta}\right)^{2}\right]^{5}}\right] d \xi \\
a_{4} \equiv \int_{-\infty}^{\infty} \frac{\left(\xi-\xi_{w}\right)^{2} \Psi_{0}^{2}}{\left[1+\left(\frac{\xi-\xi_{w}}{\Delta}\right)^{2}\right]^{5}} d \xi \\
a_{5} \equiv \int_{-\infty}^{\infty} \frac{\left(\xi-\xi_{w}\right)^{4} \Psi_{0}^{2}}{\left[1+\left(\frac{\xi-\xi_{w}}{\Delta}\right)^{2}\right]^{5}} d \xi \\
a_{6} \equiv \int_{-\infty}^{\infty} \frac{2\left(\xi-\xi_{w}\right)^{3} \Psi_{0}^{2}}{\left[1+\left(\frac{\xi-\xi_{w}}{\Delta}\right)^{2}\right]^{5}} d \xi
\end{gathered}
$$

The error $\epsilon$ is minimized in order to obtain the $c_{1}$ and $c_{2}$ that best fits the actual $\Psi_{\text {wire }}$.

$$
\frac{\partial \epsilon}{\partial c_{1}}=0 \quad \frac{\partial \epsilon}{\partial c_{2}}=0
$$

This results in $c_{1}$ and $c_{2}$ being

$$
\begin{aligned}
& c_{1}=\frac{a_{3} a_{6}-2 a_{2} a_{5}}{4 a_{4} a_{5}-a_{6}^{2}} \\
& c_{2}=\frac{2 a_{4} a_{3}-a_{2} a_{6}}{a_{3}^{2}-4 a_{4} a_{5}}
\end{aligned}
$$

From Gradshteyn \& Ryzhik [52], it is seen that

$$
\int_{-\infty}^{\infty} \frac{d x}{\left(1+x^{2}\right)^{5}}=\frac{35 \pi}{128}
$$




$$
\begin{gathered}
\int_{-\infty}^{\infty} \frac{x}{\left(1+x^{2}\right)^{5}} d x=0 \\
\int_{-\infty}^{\infty} \frac{x^{2}}{\left(1+x^{2}\right)^{5}} d x=\frac{5 \pi}{128} \\
\int_{-\infty}^{\infty} \frac{x^{3}}{\left(1+x^{2}\right)^{5}} d x=0 \\
\int_{-\infty}^{\infty} \frac{x^{4}}{\left(1+x^{2}\right)^{5}} d x=\frac{3 \pi}{128}
\end{gathered}
$$

Therefore we get

$$
\begin{gathered}
a_{1} \equiv \int_{-\infty}^{\infty}\left[\Psi_{\text {actual }}^{2}(\xi)-\frac{2 \Psi_{\text {actual }}(\xi) \Psi_{0}}{\left[1+\left(\frac{\xi-\xi_{w}}{\Delta}\right)^{2}\right]^{5 / 2}}\right] d \xi+\frac{35 \pi \Psi_{0}^{2} \Delta}{128} \\
a_{2} \equiv \int_{-\infty}^{\infty} \frac{-2\left(\xi-\xi_{w}\right) \Psi_{\text {actual }}(\xi) \Psi_{0}}{\left[1+\left(\frac{\xi-\xi_{w}}{\Delta}\right)^{2}\right]^{5 / 2}} d \xi \\
a_{3} \equiv \int_{-\infty}^{\infty} \frac{-2\left(\xi-\xi_{w}\right)^{2} \Psi_{\text {actual }}(\xi) \Psi_{0}}{\left[1+\left(\frac{\xi-\xi_{w}}{\Delta}\right)^{2}\right]^{5 / 2}} d \xi+2 a_{4} \\
a_{4}=\frac{5 \pi}{128} \Delta^{3} \Psi_{0}^{2} \\
a_{5}=\frac{3 \pi}{128} \Delta^{5} \Psi_{0}^{2} \\
a_{6}=0
\end{gathered}
$$


and

$$
\begin{gathered}
c_{1}=\frac{-a_{2}}{2 a_{4}} \\
c_{2}=\frac{-a_{3}}{2 a_{5}} \\
\epsilon=a_{1}-\frac{a_{2}^{2}}{2 a_{4}}-\frac{a_{3}^{2}}{2 a_{5}}+\frac{a_{4} a_{2}^{2}}{4 a_{4}^{2}}+\frac{a_{5} a_{3}^{2}}{4 a_{5}^{2}}+\frac{a_{6} a_{2} a_{3}}{4 a_{4} a_{5}} \\
=a_{1}-\frac{a_{2}^{2}}{4 a_{4}}-\frac{a_{3}^{2}}{4 a_{5}}
\end{gathered}
$$




\section{Appendix F}

\section{Diagnostic Theory \& Calibration}

Since the basic theory of plasma diagnostics have been quite thoroughly researched over the past 30 years, a brief summary of diagnostic theory will be given in this appendix. More thorough treatment could be found in works by Huddleston\& Leonard [83], Hutchinson [73], Meuth [84], and Auciello [85].

Calibration of a diagnostic is essential for ascertaining the accuracy of the data that it acquires. Much effort was expended in the calibration of the diagnostics that were used on the PPEX machine. Somc diagnostics could be calibrated out of the machine, while others had to be calibrated in-situ. The calibration methods that were used are detailed below.

\section{F.1 Diamagnetic Loop}

\section{F.1.1 Basic Theory}

\section{BASIC PRINCIPLES}

The diamagnetic loop is a magnetic field probe that is positioned around the plasma. The loop detects the magnetic flux that it surrounds and the voltage induced on the loop is subsequently measured and processed to yield the plasma beta. 
Using Maxwell's equations and the MHD Equilibrium condition, it can be shown that the magnetic field inside a plasma of finite $\beta$ is given by

$$
B_{i}=\sqrt{1-\beta} B_{0}
$$

where $\beta$ is defined as the ratio of the plasma pressure to the magnetic field pressure, $\beta=P /\left(B^{2} / 2 \mu_{0}\right)$. The flux that passes through the diamagnetic loop is then found to be

$$
\phi_{z, \text { Total }}=N\left(\int_{0}^{r_{p}} B_{i} 2 \pi r d r+\int_{r_{p}}^{r_{L}} B_{0} 2 \pi r d r\right)
$$

The plasma contribution is then seen to be

$$
\phi_{z, P}=\frac{N \beta B_{0}^{2} \pi r_{P}^{2}}{2}
$$

The output voltage that is induced in the loop is RC integrated to yield the magnetic flux and is seen to be

$$
V_{0}=\frac{\phi_{z, P}}{R C}
$$

Thus the plasma $\beta$ can be found directly from this output voltage and is seen to be

$$
\beta=\frac{2 R C}{N B_{0}^{2} \pi r_{p}^{2}} \gamma V_{0}
$$

where the factor $\gamma$ takes into account the frequency response of the diamagnetic loop [86].

\section{F.1.2 Calibration}

The diamagnetic loop is deployed in the center cell of the PPEX machine. Its output is processed through an integration circuit before being digitized by an Aurora A-12 unit.

The in-situ calibration is performed by a "blank discharge". A blank discharge is a plasmaless shot that is produced by simply discharging a known current 
through the central cell and mirror coils WITHOUT any auxilliary heating power. The known magnetic field that is generated by this discharge (Calculated by the magnetic field code EFFI) is correlated to the resulting integrated diamagnetic loop signal. The magnitude of the integrated diamagnetic signal is then calibrated to the known magnetic field.

\section{F.2 Spectrometer}

\section{F.2.1 Basic Theory}

The Doppler shift due to a source moving parallel (with velocity $v_{\bullet}$ ) to the direction of observation is

$$
\Delta \lambda= \pm \frac{v_{\bullet}}{c} \lambda
$$

It can be shown (H\&L, p.268) that for sources of Maxwellian velocity distribution,

$$
I(\Delta \lambda)=\frac{I_{t}}{\pi^{\frac{1}{2}} \Delta \lambda_{D}} \exp \left[-\left(\frac{\Delta \lambda}{\Delta \lambda_{D}}\right)\right]
$$

where $I_{t}$ is the total line intensity. The intensity is half its maximum value when the exponential reaches $\frac{1}{2}$. The full width half $\max$ (FWHM) (H\& L. p.269) line width is found to be

$$
\Delta \lambda_{\frac{1}{2}, D}=7.16 \times 10^{-7} \lambda(T / M)^{\frac{1}{2}}
$$

( $T_{i}$ is in degrees Kelvin, $\mathrm{M}$ is the molecular wt of the ion) Therefore the ion temperature is found to be

$$
T_{i}=\frac{\Delta \lambda_{\frac{1}{2} D}^{2}}{\left(7.16 \times 10^{-7} \lambda\right)^{2}}
$$

The rotating mirror assembly is constructed to convert the J-A Monochrometer Spectrometer into a scanning spectrometer. The reason for using a rotating mirror 
assembly is to translate the incoming beam by a glass slide, hence shifting the wavelength entering the photomultiplier slit.

Using Snell's Law, the amount of beam translation is found to be

$$
\delta x=t \sin \theta_{i}\left(1-\frac{\cos \theta_{i}}{\sqrt{n^{2}-\left(\sin \theta_{i}\right)^{2}}}\right)
$$

By translating the beam with a rotating glass slide, the wavelength that is being examined by the photomultiplier assembly is thus caused to vary.

\section{F.2.2 Calibration}

The spectrometer that was used on the PPEX machine was a Jarrel-Ashe 82000 Spectrometer $(0.5 \mathrm{~m}$ focal length). The spectrometer was acquired from a source that did not maintain it very well, and as a result, much effort was expended in obtaining proper mirror adjustment and focus.

The calibration light source used were a hydrogen lamp and a mercury lamp. Both the mercury blue line $(4358.3 \AA)$ and the $H_{\beta}$ were used to calibrate the mirror focus. A He-Ne laser was used to calibrate the vertical positioning of the slit and diffraction grating.

\section{F.3 Interferometer}

\section{F.3.1 Basic Theory}

The basic principle of plasma interferometry utilizes the phase shifting of the plasma $\mathrm{O}$-mode wave whose dispersion relation is

$$
\omega^{2}=\omega_{p e}^{2}+c^{2} k^{2}
$$

where

$$
\omega_{p e}^{2}=\frac{e^{2} n_{0}}{\epsilon_{0} m_{e}}
$$


is the electron plasma frequency. The index of refraction can then be defined as :

$$
N \equiv \frac{c k}{\omega}=\left[1-\frac{\omega_{p}^{2}}{\omega}\right]^{\frac{1}{2}}
$$

The phase change due to the wave propagating in the plasma medium of length $l$ (in addition to its normal propagation in vacuum) is then

$$
\Delta \phi=k l(N-1)
$$

From the measured value of the $\Delta \phi$, the line averaged electron density can be found by:

$$
n_{e}=\frac{\omega^{2} \epsilon_{0} m_{e}}{e^{2}}\left(1-\left[1-\frac{\Delta \phi}{k l}\right]^{2}\right)
$$

\section{FREQUENCY SWEPT OPERATION}

The above is for a homodyne interferometer. The interferometer used in the PPEX experiment is a frequency-swept interferometer. The basic principle of the phase shift is the same as that of the homodyne interferometer, the difference being that the frequency of the interferometer is swept over a fixed range. Sweeping frequencies from $100 \mathrm{KHz}$ to $1 \mathrm{MHz}$ have been used. The interferometer in the PPEX experiment utilizes a $80 \mathrm{KHz}$ ramp generator to produce the sweeping frequency.

For such a frequency swept interferometer, Meuth [84] shows that the phase shift in radians is given by

$$
\Delta \phi=2 \pi \times 1.34 \times 10^{-16} \frac{1}{f_{0}(\mathrm{GHz})} \int n_{e}(x, t) d x\left(m^{-2}\right)
$$

For the $35 \mathrm{GHz}$ interferometer used on the PPEX, this works out to be

$$
\Delta \phi=2.405 \times 10^{-17} \overline{n_{e}} \Delta x
$$

or

$$
\overline{n_{e}} \Delta x=\Delta \phi \times\left(4.158 \times 10^{16} m^{-2}\right)
$$


where $\Delta \phi$ is the phase shift in radians. If a plasma radius of $10 \mathrm{~cm}$ is assumed, this becomes

$$
\overline{n_{e}}=\Delta \phi \times\left(2.08 \times 10^{11} \mathrm{~cm}^{-3}\right)
$$

\section{F.3.2 Calibration}

The interferometer used on the PPEX machine was previously deployed on the TARA tandem mirror machine. TARA deployed two types of interferometers : $35 \mathrm{GHz}$ and $60 \mathrm{GHz}$. The $35 \mathrm{GHz}$ version was not used much on TARA because of the amount of refraction that the dense TARA plasma $\left(>4 \times 10^{12} \mathrm{~cm}^{-3}\right)$ generated. The refraction limited the upper density limit of operations for the $35 \mathrm{GHZ}$ units to approximately $2 \times 10^{12} \mathrm{~cm}^{-3}[84]$.

\section{F.3.3 Problems}

Problems were encountered in attempting to calibrate the interferometer, specifically, in calibrating the IF output of the mixer. The IF output is supposed to mix a referene leg with the plasma leg. This output is then supposed to be tuned to resemble a sinusoidal waveform. However, the ramp generator that was to be adjusted for this purpose often drifted during the course of minutes, forcing a recalibration that was often imprecise (due to the errors in judging the likeness of the IF output to a sinusoid). This created significant problems in providing a consistent set of electron density data.

\section{F.4 Langmuir Probe}

\section{F.4.1 Basic Theory}

Basic Langmuir probe theory states that at negative voltages, the probe should attract only ions at an ion saturation flux given by Huddleston \& Leonard [83] to be 


$$
I_{i, \text { sat }}=\frac{1}{2} n_{0} A\left(\frac{k T_{e}}{M}\right)^{\frac{1}{2}}
$$

where $\mathrm{A}$ is the probe area, $n_{0}$ the plasma density, and $\mathrm{M}$ the ion mass.

As the probe voltage is increased, more and more electrons can penetrate the negative potential until a floating potential $V_{f}$ is eventually reached. The probe being at the floating potential means that the net inward electron flux equals the net inward ion flux. As the voltage is continuously raised, the probe now attains a linear regime in which the electron current behaves in an exponential manner, assuming a Maxwellian electron distribution [83]. This is known as the transition region and has the characteristic:

$$
I=A_{p} n_{0}\left(\frac{k T}{2 \pi m}\right)^{\frac{1}{2}} e^{\frac{k V}{k T}}
$$

From experimental observations of the current-voltage characteric, the electron temperature $T_{e}$ and density $n_{e}$ can be found. To calculate the electron temperature, the slope of the transition region is used. Taking the region of constant slope in this region, Hutchinson shows [73] that the electron temperature can be found from

$$
T_{e}=\frac{e\left(I-I_{s i}\right)}{d I / d V}
$$

where $I$ is the Langmuir probe current in the middle of the linear region, $\frac{d I}{d V}$ is the slope of the Langmuir Probe I-V characteristic of the linear region, and $I_{s i}$ the ion saturation current.

From thic value of the electron temperature, the edge plasma density can be found from the ion saturation current $I_{s i}$ (Hutchinson, p.60):

$$
I_{s i}=n_{\infty} A, C, q e^{-\frac{1}{2}}
$$

where $A_{\mathrm{a}}$ is the area of the Langmuir Probe tip, $C_{0}$ the ion sound speed $\left(C_{0}=\right.$ $\left.\left(T_{e} / m_{i}\right)^{\frac{1}{2}}\right)$. The density is then found to be

$$
n_{\infty}=\frac{I_{a i} e^{\frac{1}{2}}}{A, C, q}
$$




\section{F.4.2 Calibration}

\section{F.5 B-dot Probe}

\section{F.5.1 Basic Theory}

The B-Dot probe picks up the ICRF fluctuating magnetic fields that are either propagating in the plasma or evanescent but still detectable. The B-Dot Probe works with a similar principle to the Diamagnetic Loop, namely that the timevarying $R F$ field is picked up and read as an induced loop voltage $V$, where

$$
V=\frac{d}{d t} \int \vec{B} \cdot d \vec{A}
$$

This induced loop voltage can then be detected and digitized.

\section{F.5.2 Calibration}

The B-dot probe was calibrated using a Helmholtz coil calibration apparatus that is similar to the one described by Philips [87]. A Helmholtz coil is wound around a G-10 insulation spool in which three orthogonal holes have been drilled. The B-Dot probe has 3 pickup loops arranged orthogonally at the tip. By inserting the probe in each of the three orthogonal holes and measuring the voltage from the pickup loops, the B-Dot probe can be calibrated.

The maximum signal that each probe picks up during calibration is recorded and compared to the other probes that are used as well as compared to the known magnetic field that the Helmholtz setup should produce. By doing this, one can obtain both the absolute as well as the relative calibrations. 


\section{Appendix G}

\section{Problems Encountered during PPEX Experiment}

As with any cxperiment, the PPEX team encountered difficulties with several aspects of the experiment. Several of the more noteworthy difficulties are listed below, along with the actions that were taken to resolve or minimize these problems.

\section{G.1 Heating Systems}

\section{G.1.1 Incorrect ECRH Microwave frequency}

The field necessary for Ion Cyclotron Resonance at the center cell field minimum is about $2 \mathrm{kG}$, which is much higher than the resonant field of the ECH heating unit (750G). This nonresonant heating results in an inability to produce plasma breakdowns. This lack of preionization then creates a significant problem during ICH discharges. If there is an insufficient amount of plasma present, the ICH will not couple any power into the plasma and thus will not heat the plasma.

The easiest solution to this problem is to obtain an ECH unit that does resonate at the field of the mirror central cell. However, no suitable unit was located 
within the constiaints of the experimental budget. The remedy taken in the experiment was to leave the ionization gauge turned on during the shot. This generated a source of "seed" electrons that could be picked vip and amplified by the ECH unit. Although this improvisation resulted in shcrt ion gauge filament lifetimes, it proved to be an effective method of generating a source of preionized plasma.

\section{G.1.2 Power output of the ECH Unit}

The second problem is also related to the ECH system. The system is a Raytheon microwave unit outputting $2 \mathrm{~kW}$ of power at a frequency of $2.1 \mathrm{GHz}$. The output of the unit was factory designed to be a half-rectified waveform. This half-rectified power envelope restricts the length of time a preionized source of electrons is available to the ICRF waves. This proved to be a problem at lower power levels $(<30 \mathrm{~kW})$ because the ICRF was seen to have difficulty sustaining a discharge. Higher levels of ICRF power appeared to be able to sustain the discharge and thus minimized the problem. However, this half-rectified waveform did affect the ICRF wave-plasma coupling because at the time of its initial turn-on, the ICRF may not see much plasma and thus will not begin to couple to the plasma until well into ics pulse when the ECH power begins to ramp on.

\section{G.1.3 ICR.F System}

The Continental ICRF Amplifiers used in the PPEX experiment are vintage 1967 US Navy communications equipment. As such, the electronics and plumbing are fragile, old, and decaying. Although attempts were made to service the systems, many problems cropped up which interrupted experimentation. Some of these problems were: recirculation water line leakage, burnout of a high voltage charging supply due to a failed capacitor, breaker failure, timer failure, and many stuck switches. As such, it is not recommended that the further operations of the PPEX machine be contingent upon having an operational Continental amplifier. 
It is recommended that a new ICRF source be either built or acquired with as much solid state equipment as possible to minimize component failure.

\section{G.2 Diagnostics}

Most of the diagnostics used on the PPEX were obtained from the now defunct TARA tandem mirror experiment at MIT. Although much effort was made to recalibrate and fix the instruments to obtain the best results, many problems were encountered in getting accurate data out of some of the diagnostics. Of these diagnostics, the interferometer was the most troublesome. Initially, the interferometer yielded phase data which were undecipherable. After much troubleshooting, the main problem was found to be with the gunn oscillator and mixer units. After the gunn oscillator and mixer units were replaced, the results appeared to be consistent with the other diagnostic results. However, vibration of the chamber due to the magnets discharging cause the horns to move, thereby creating a source of error and noise that could not be eliminated.

\section{G.3 Ground Loop}

Many of the diagnostics suffered from ground loop because of insufficient isolation. Although care was taken to isolate the physical parts of the diagnostic from the machine (where possible), the power supply ground was not isolated from the machine, thereby creating a large source of ground loop. The solution was to physically isolate as much of the diagnostics from the machine, isolate the power supply ground, and isolate the Andrews cable coax from the instrumentation ground. After doing this, most of the ground loop pickup was eliminated. 


\section{Bibliography}

[1] G.R. Smith and M.J. Gerver. Propagation of waves generated in tandem mirror end cells. Technical Report UCRL-87034, Lawrence Livermore National Laboratory, 1981.

[2] T.H. Stix. The Theory of Plasma Waves. McGraw-Hill, 1962.

[3] M.F. Uman and W.M. Hooke. Observation of ion cyclotron waves in a hot plasma. Phys Fluids, 12(5):1072-1080, 1969.

[4] B.D. McVey. Icrf antenna coupling theory for a cylindrically stratified plasma. Technical Report PFC/RR-84-12, MIT Plasma Fusion Center, 1984.

[5] J. Kesner, B.D. McVey, R.S. Post, and D.K. Smith. A tandem mirror with axisymmetric central cell ion confinement. Technical Report PFC/JA-81-11, MIT Plasma Fusion Center, 1981.

[6] J. Kesner, M.J. Gerver, B.G. Lane, B.D. McVey, P.J. Catto, D.A. D'Ippolito, and J.R. Myra. Introduction to tandem mirror physics. Technical Report PFC/RR-83-35, MIT Plasma Fusion Center, 1983.

[7] J.N. Strohmayer. Preliminary design of a tandem mirror reactor. Master's thesis, MIT, 1984.

[8] R.S.Post et al. Recent results from the tara tandem mirror and constance-b mirror. In Plasma Physics and Controlled Thermonuclear Research Conference Proceedings, volume 2, pages 251-262, Kyoto, Japan, 1986. 
[9] S.N.Golovato et al. In Seventh Topical Conference on the Application of Radio-Frequency Power to Plasmas, page 254, Kissimmee, Florida, 1987. American Institute of Physics.

[10] B. Lane. Results from the tara tandem mirror experiment. In International School of Plasma Physics, volume 1, Varenna, Italy, 1987.

[11] G. Dimonte et al. Ion cyclotron heating in tmx-u. Technical Report UCRL94788, Lawrence Livermore National Laboratory, 1986.

[12] A.W. Molvik et al. Initial tmx central cell icrh experiments. Technical Report UCID-18866, Lawrence Livermore National Laboratory, 1980.

[13] M. Inutake et al. Phys. Rev. Lett., 55:939, 1985.

[14] M.Ichimura et al. In Fourth Kiev International Conference on Plasma Theory and Fourth International Congress on Waves and Instabilities in Plasmas, volume 1, page 350, Nagoya, Japan, 1980.

[15] N.Hershkowitz, R. Majeski, and J. Ferron. Experimental results from phaedrus-b. In E.Sindoni S.Ortolani, editor, Physics of Mirros, Reverse Field Pinches and Compact Tori, pages 855-871, Varenna, Italy, 1987.

[16] S.J. Sackett. Effi: A code for calculating the electromagnetic field force and inductance in coil systems of arbitrary geometry. Technical Report UCRL52402, Lawrence Livermore National Laboratory, 1978.

[17] N. Hershkowitz, R. Majeski, and J. Ferron. Ponderomotive stability of mhd modes in mirrors. In E.Sindoni S.Ortolani, editor, Physics of Mirrors, Reverse Field Pinches, and Compact Tori, pages 751-769, Varenna, 1987.

[18] J.J.Browning, N.Hershkowitz, T.Intrator, R.Majeski, and S.Meassick. Radiofrequency interchange stability experiments below the ion cyclotron frequency. Phys. Fluids B, 1(8):1692, 1989. 
[19] J. R. Ferron, N. Hershkowitz, R. A. Breun, S. N. Golovato, and R. Goulding. Rf stabilization of an axisymmetric tandem mirror. Phys. Rev. Lett., 51(21):1955, 1983.

[20] S.N.Golovato et al. Stability of plasmas sustained by icrf in the central cell of the tara tandem mirror. Technical Report PFC/JA-88-20, MIT Plasma Fusion Center, 1988.

[21] K. Brau, R. S. Post, and E. Sevillano. Gas injection system in the tara center cell. Technical Report PFC/CP-86-2, MIT Plasma Fusion Center, 1986.

[22] The American Radio Relay League. The ARRL Handbook for the Radio Amateur. The American Radio Relay League, 1988.

[23] T.W. Fredian and J.A. Stillerman. Mds. Rev. Sci. Instrum., 57:1907, 1986.

[24] B.Labombard. Personal communications, December 1990.

[25] X.Chen. Magnetic probes for small signal detection in a large background field. Technical Report PFC/JA-87-27, MIT Plasma Fusion Center, 1987.

[26] Research Systems, Inc. Introduction to IDL, 1987 edition, 1987.

[27] T.H.Stix. Absorption of plasma waves. Phys. Fluids, 3(1):19, 1960.

[28] T.H.Stix. Generation and thermalization of plasma waves. Phys. Fluids, 1(4):308, 1958.

[29] T.H.Stix. Oscillations of a cylindrical plasma. Phys. Rev., 106(6):1146, 1955.

[30] D.G. Swanson. Hot plasma in a cylindrical waveguide. Phys Fluids, 10(2):428, 1967.

[31] I.B. Bernstein and S.K. Trehan. Plasma oscillations. Nuclear Fusion, 1(1):3, 1960. 
[32] M.A. Rothman, R.M. Sinclair, and I.G. Sinclair. Ion cyclotron heating in the model c stellerator. Phys Fluids, 12(10):2211-2224, 1969.

[33] J.C.Hosea and R.M.Sinclair. Ion cyclotron wave generation in the model c stellerator. Phys Fluids, 3(13):709-711, 1970.

[34] A. Iiyoshi, H. Yamato, and S. Yoshikawa. Limitations on ion cyclotron heating in the local mirrors of the $c$ stellerator. Phys Fluids, 4(10):749-755, 1967.

[35] A.M. Messiaen, P.E. Vandenplas, R.R. Weynants, and R.Koch. Boundedplasma theory of radio-frequency heating of toroidal machines. Nuclear Fusion, 15:75, 1975.

[36] A. Bers, L.P. Harten, and A. Ram. Antenna-plasma coupling theory for icrf heating of large tokamaks. Technical Report PFC/RR-81-7, MIT, 1981.

[37] T.H.Stix. Phys. Rev. Lett., 15:737, 1955.

[38] D.L.Grekov et al. Mode conversion and wave damping in the low frequency range. In Second Joint Grenoble-Varenna International Symposium on Heating in Toroidal Plasmas, page 519, Villa Olmo, Como, Italy, 1981. International School of Plasma Physics.

[39] P.L.Colestock and R.J.Kashuba. The theory of mode conversion and wave damping near the ion cyclotron frequency. Technical Report PPPL-1930, Princeton Plasma Physics Laboratory, 1982.

[40] D.G.Swanson. Radio frequency heating in the ion cyclotron range of frequencies. Phys. Fluids, 28(9):2645, 1985.

[41] B.D.McVey. A ray-tracing analysis of fast-wave heating of tokamaks. Nuclear Fusion, 19(4):461, 1979.

[42] A.Bers, J.Jacquinot, and G.Lister. Field and ray analysis of antenna excitations in icrf heating of large tokamaks. In Second Joint Grenoble-Varenna 
International Symposium on Heating in Toroidal Plasmas, page 569, Villa Olmo, Como, Italy, 1981. international School of Plasma Physics.

[43] N.T.Lam, J.L.Lee, J.Scharer, and R.J.Vernon. Analysis of dielectric-filled waveguide coupling to plasmas in the icrf. IEEE Transactions on Plasma Sciences, PS-14(3):271, 1986.

[44] J.D.Jackson. Classical Electrodynamics. John Wiley and Sons, 2 edition, 1975.

[45] D. R. Nicholson. Introduction to Plasma Theory. John Wiley and Sons, 1983.

[46] B. D. Fried and S. D. Conte. The Plasma Dispersion Function. Academic Press, 1961.

[47] Paul Lorrain and Dale Corson. Electromagnetic Fields and Waves. W.H. Freeman and Company, 2 edition, 1970.

[48] J. F. Freidberg. Ideal magnetohydrodynamic theory of magnetic fusion systems. Review of Modern Physics, 54(3):801-902, 1982.

[49] J. F. Freidberg. Ideal Magnetohydrodynamics. Plenum Press, 1987.

[50] J.D.Kraus. Antennas. McGraw-Hill, 1988.

[51] M. Abramowitz and I. Stegun. Handbook of Mathematical Functions. Dover Publications, Inc., 1972.

[52] I. S. Gradshteyn and I. M. Ryzhik. Table of Integrals, Series and Products. Academic Press, 1983.

[53] Francis Hildebrand. Advanced Calculus for Applications. Prentice-Hall, 1976.

[54] Press, Flannery, Teukolsky, and Vetterling. Numerical Recipes, The Art of Scientific Computing. Cambridge University Press, 1989. 
[55] H.H.Kuehl. Resistance of a short antenna in a warm plasma. Radio Science, $1(8): 971,1966$.

[56] S.Puri. Surface impedance for coupling at the ion cyclotron range of frequencies. In Third Joint Grenoble-Varenna International Symposium on Heating in Toroidal Plasmas, page 391, Centre D'Etudes Nucleaires de Grenoble, France, 1982. International School of Plasma Physics.

[57] K.Balmain. Impedance of a short dipole in a compressible plasma. Radio Sci. H. Res., NBS 69D(4):559, 1965.

[58] S.C. Chiu, C.P. Moeller, V.S. Chan, and F.P. Blau. Study of the slow-wave structure as an icrf launcher. Nuclear Fusion, 24:717, 1984.

[59] J.A. Kong. Electromagnetic Wave Theory. Wiley Interscience, 1985.

[60] W.M.Hooke, F.H.Tenney, M.H.Brennan, H.M. Hill, and T.H.Stix. Experiments on ion cyclotron waves. Physics of Fluids, 4(9):1131, 1961.

[61] O. Eldridge. Electron cyclotron heating as resonant diffusion. Phys. Fluids, 15(4):676, 1972.

[62] H.P. Furth. Ion cyclotron waves in mirror geometry. Technical Report UCRL5423-T, LLNL, 1959.

[63] W.M.Hooke, P. Avivi, M. Brennan, M. A. Rothman, and T. H. Stix. Nuclear Fusion, 3:1083, 1962.

[64] S. Yoshikawa, M.A. Rothman, and R.M. Sinclair. Absorption of ion cyclotron waves by one component of a two-ion plasma. Phys. Rev. Letters, 14(7):214, 1965.

[65] B.D. McVey. Excitation theory of the inductive drive. Technical Report TASK II-2740, TRW, 1984. 
[66] R.C. Myer and S.N. Golovato. Modeling of icrh experiments in the tara tandem mirror. Technical Report PFC/CP-87-6, MIT Plasma Fusion Center, 1987.

[67] R. Myer. Unpublished internal report, 1987.

[68] Carl M. Bender and Steven A. Orszag. Advanced Mathematical Methods for Scientists and Engineers. McGraw-Hill, 1978.

[69] R.C.Myer. Personal communications, February 1990.

[70] D. R. Sigman and J. J. Reinmann. Technical Report D-4058, NASA, 1967.

[71] J. Machuzak. Personal communications, 1989.

[72] B.D. McVey. Antena user guide. Technical Report PFC/RR-84-13, MIT Plasma Fusion Center, 1984.

[73] I.H. Hutchinson. Principles of Plasma Diagnostics. Cambridge University Press, 1987.

[74] E.O. Brigham. The Fast Fourier Transform. Prentice Hall, 1 edition, 1974.

[75] D.E. Smith, E.J. Powers, and G.S. Caldwell. Fast-fourier transform spectralanalysis techniques as a plasma fluctuation diagnostic tool. IEEE Transactions on Plasma Science, PS-2:261, 1974.

[76] D. Goodman. A model for potential modification and loss of equilibrium during ion cyclotron heating in constance.(memorandum to the constance group,unpublished), September 1988.

[77] B. D. Fried, C.L. Hedrick, and J. McCune. Two pole approximation for the plasma dispersion function. Phys Fluids, 11:249, 1968.

[78] R.W. Landau and S. Cuperman. Stability of anisotropic plasmas to almostperpendicular magnetosonic waves. J. Plasma Phys., 6:495, 1971. 
[79] S.Ichimaru. Basic Principles of Plasma Physics. Benjamin/Cummings Publishing Co, 1973.

[80] D.L. Book. Nrl plasma formulary. Technical Report NRL 177-4405, Naval Research Laboratory, 1990.

[81] T.H. Stix and D.G. Swanson. Propagation and mode conversion for waves in nonuniform plasmas. Technical Report PPPL-1903, Princeton Plasma Physics Laboratory, June 1982.

[82] R. Myer, S. Golovato, A. Ram, D. Smith, and A. Bers. Theoretical modeling of central cell icrf on tara, (unpublished), February 1983.

[83] R.H. Huddleston and S.L. Leonard. Plasma Diagnostic Techniques. Academic Press, 1965.

[84] H. Meuth and E. Sevillano. Microwave plasma diagnostics. Technical Report PFC/JA-87-42, MIT Plasma Fusion Center, 1987.

[85] O. Auciello and D.L. (Ed) Flamm. Plasma Diagnostics, volume 1. Academic Press, 1988.

[86] J.Machuzak. Phd thesis. Technical report, MIT Plasma Fusion Center, 1990.

[87] R. Philips and T. Intrator. Methods for construction and calibration of a b-dot probe for use in phaedrus $b$ tandem mirror. Technical Report PTMR88-2, Department of Nuclear Engineering, University of Wisconsin, 1988. 

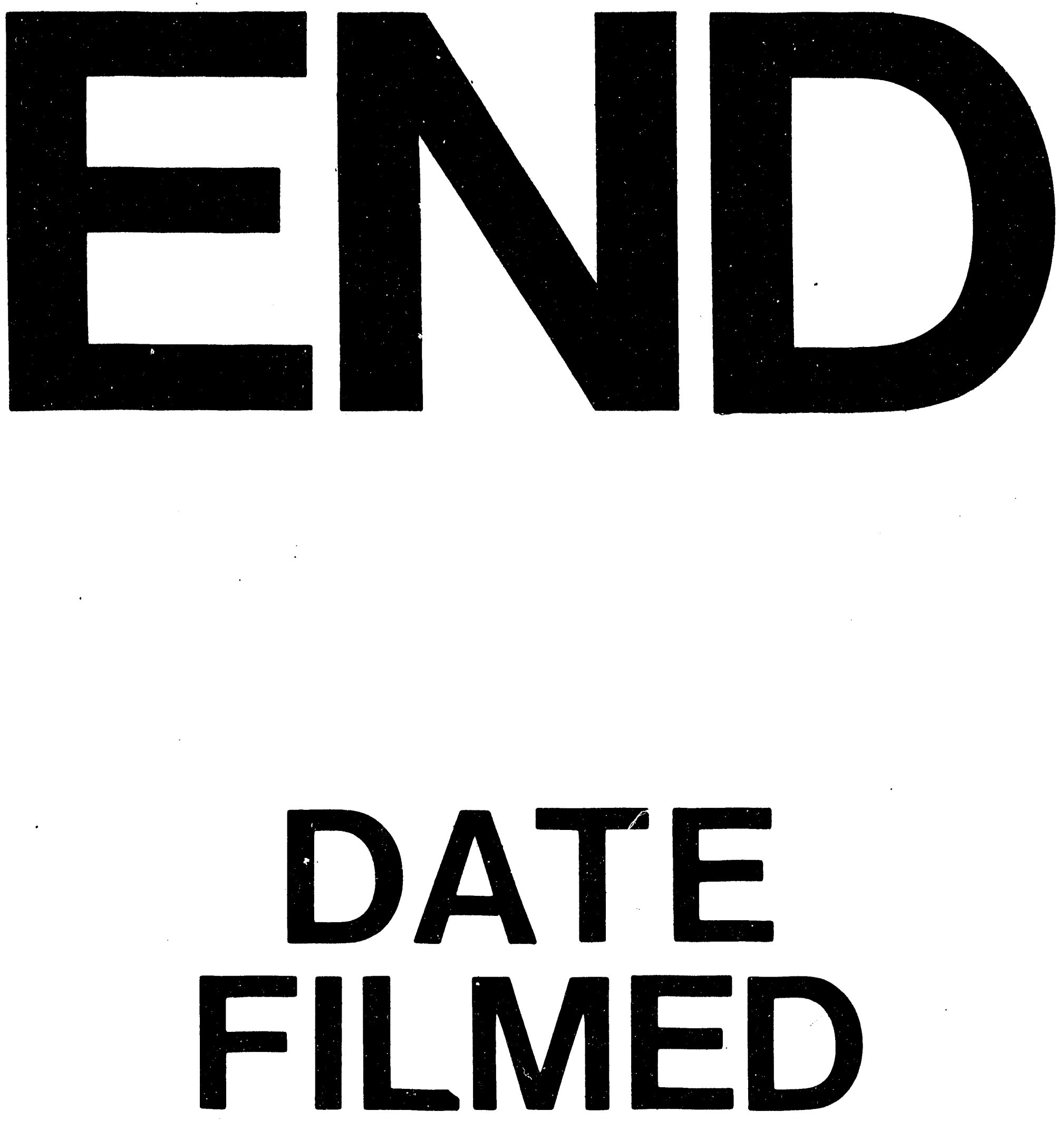

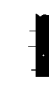

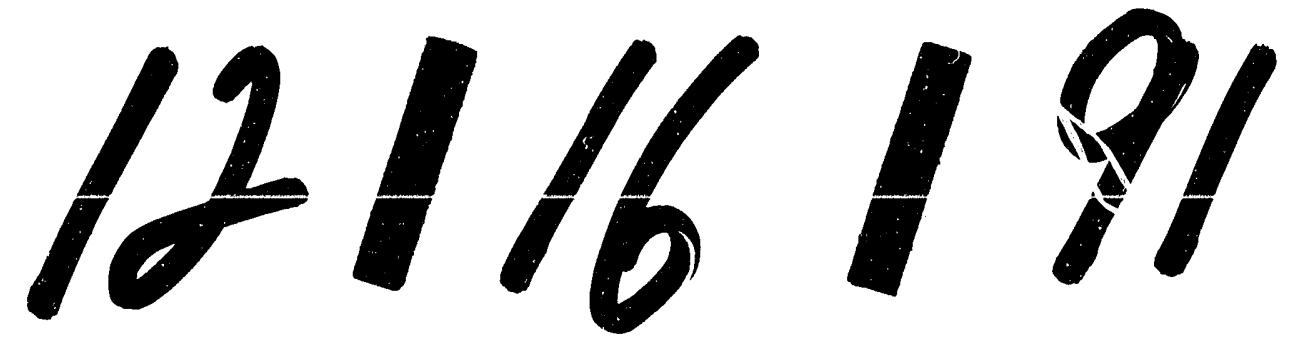

TI 
